\title{
Report on Carcinogens
}

\section{MONOGRAPH ON TRICHLOROETHYLENE}

ROC MONOGRAPH 05

JANUARY 2015 


\title{
Report on Carcinogens Monograph on Trichloroethylene
}

\author{
RoC Monograph 05
}

January 2015

\author{
National Toxicology Program
}

Public Health Service

U.S. Department of Health and Human Services ISSN: 2331-267X

Research Triangle Park, North Carolina, USA 


\section{Foreword}

The National Toxicology Program (NTP), established in 1978, is an interagency program within the Public Health Service of the U.S. Department of Health and Human Services. Its activities are executed through a partnership of the National Institute for Occupational Safety and Health (part of the Centers for Disease Control and Prevention), the Food and Drug Administration (primarily at the National Center for Toxicological Research), and the National Institute of Environmental Health Sciences (part of the National Institutes of Health), where the program is administratively located. NTP offers a unique venue for the testing, research, and analysis of agents of concern to identify toxic and biological effects, provide information that strengthens the science base, and inform decisions by health regulatory and research agencies to safeguard public health. NTP also works to develop and apply new and improved methods and approaches that advance toxicology and better assess health effects from environmental exposures.

The Report on Carcinogens Monograph series began in 2012. Report on Carcinogens Monographs present the cancer hazard evaluations of environmental agents, substances, mixtures, or exposure circumstances (collectively referred to as "substances") under review for the Report on Carcinogens. The Report on Carcinogens is a congressionally mandated, sciencebased, public health document that provides a cumulative list of substances that pose a cancer hazard for people in the United States. Substances are reviewed for the Report on Carcinogens to (1) be a new listing, (2) reclassify the current listing status, or (3) be removed.

NTP evaluates cancer hazards by following a multistep process and using established criteria to review and integrate the scientific evidence from published human, experimental animal, and mechanistic studies. General instructions for the systematic review and evidence integration methods used in these evaluations are provided in the Handbook for the Preparation of Report on Carcinogens Monographs. The handbook's instructions are applied to a specific evaluation via a written protocol. The evaluation's approach as outlined in the protocol is guided by the nature, extent, and complexity of the published scientific information and tailored to address the key scientific issues and questions for determining whether the substance is a potential cancer hazard and should be listed in the Report on Carcinogens. Draft monographs undergo external peer review before they are finalized and published.

The Report on Carcinogens Monographs are available free of charge on the NTP website and cataloged in PubMed, a free resource developed and maintained by the National Library of Medicine (part of the National Institutes of Health). Data for these evaluations are included in the Health Assessment and Workspace Collaborative. Information about the Report on Carcinogens is also available on the NTP website.

For questions about the monographs, please email NTP or call 984-287-3211. 


\section{Table of Contents}

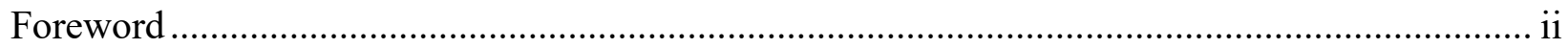

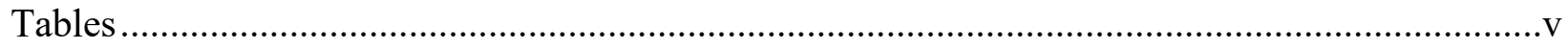

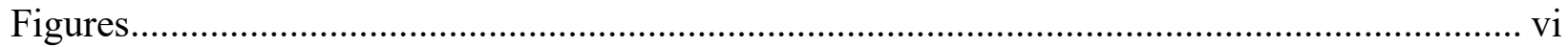

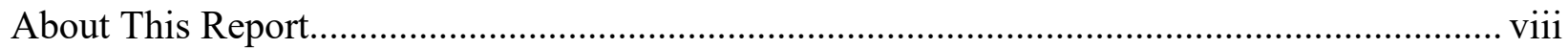

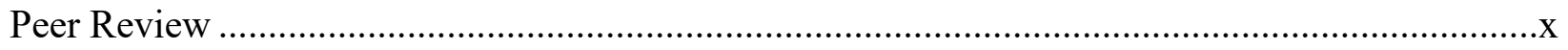

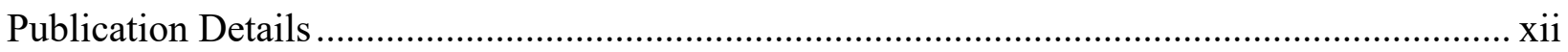

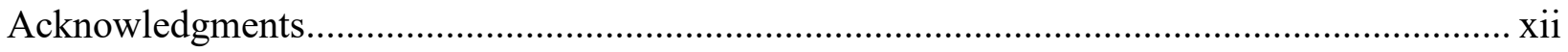

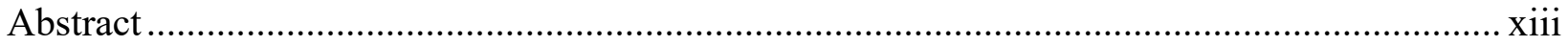

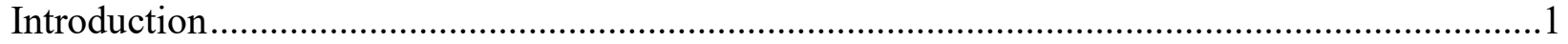

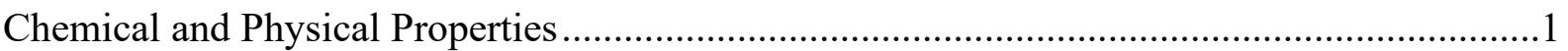

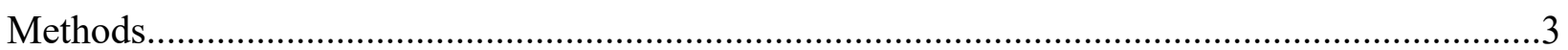

Monograph Contents and Development ……………….................................................

Key Scientific Questions and Issues Relevant for the Cancer Evaluation............................

Methods for Preparing the Monograph...............................................................................5

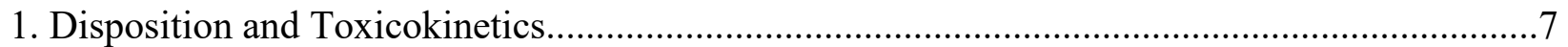

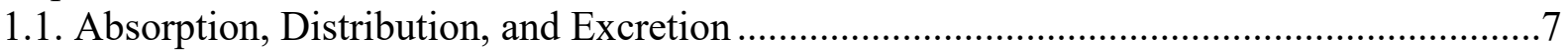

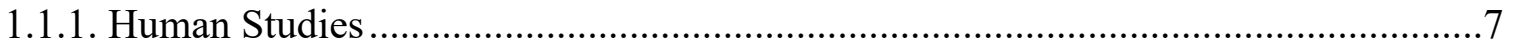

1.1.2. Laboratory Animal Studies ..........................................................................

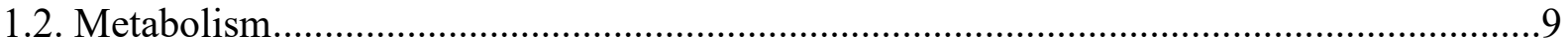

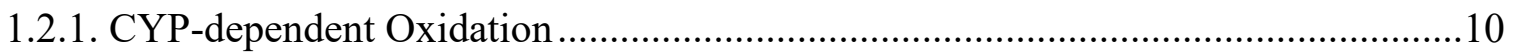

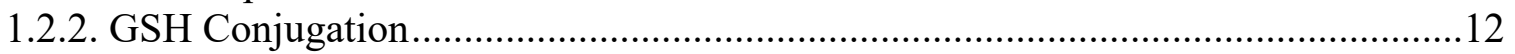

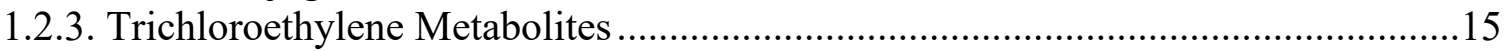

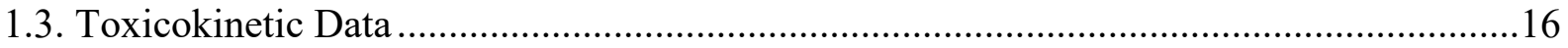

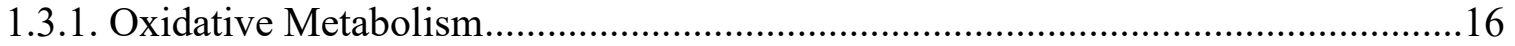

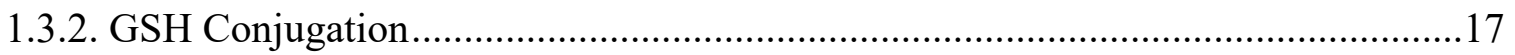

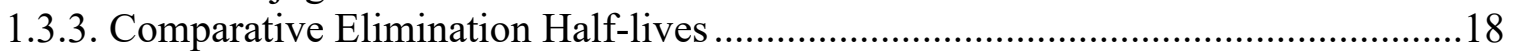

1.3.4. Relative Roles of the CYP and GSH Pathways ...................................................18

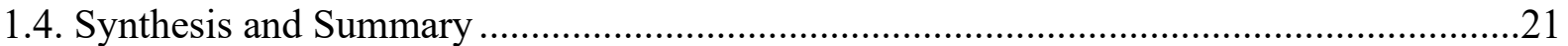

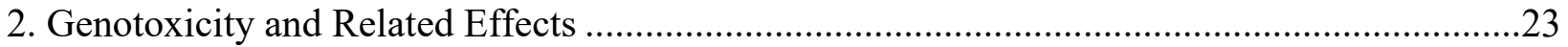

2.1. In Vitro Mutagenicity Studies of Trichloroethylene in Bacteria .......................................23

2.2. In Vitro Genotoxicity Studies of Trichloroethylene in Non-mammalian

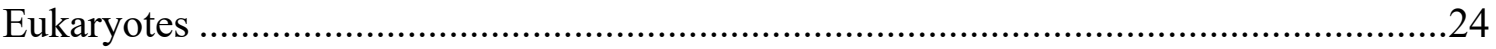

2.3. In Vitro Studies of Genotoxicity and Related Effects of Trichloroethylene in

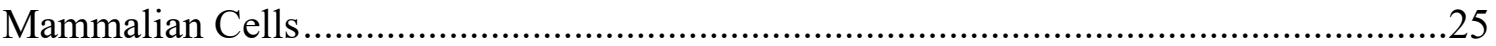

2.4. Studies of Nucleic Acid and Protein Binding of Trichloroethylene ..................................26

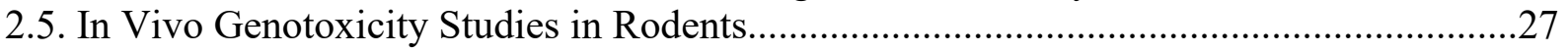

2.6. Studies of Genotoxicity in Humans Exposed to Trichloroethylene ...................................28

2.7. Genotoxic and Related Effects of the Metabolites of Trichloroethylene...........................28

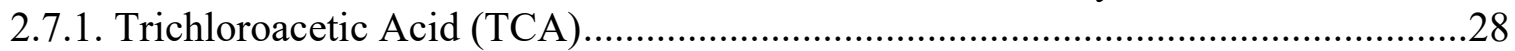




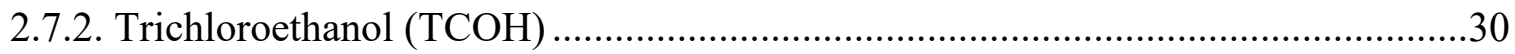

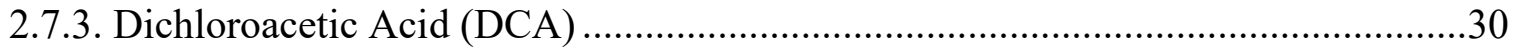

2.7.4. Chloral Hydrate $(\mathrm{CH})$.................................................................................

2.7.5. $S$-(1,2-dichlorovinyl)-L-cysteine (DCVC), $S$-(1,2-dichlorovinyl)glutathione

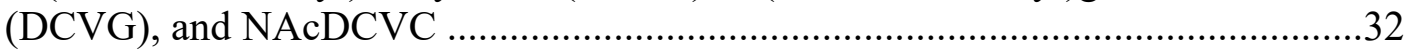

2.8. Summary of Genotoxicity and Related Effects of Trichloroethylene and Its

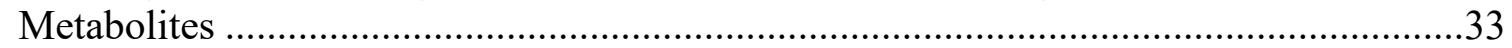

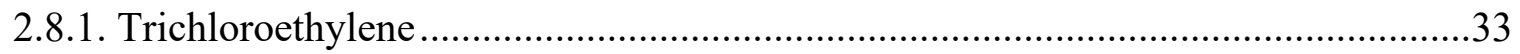

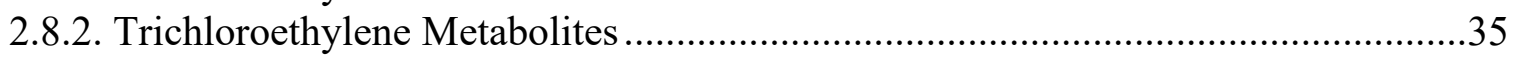

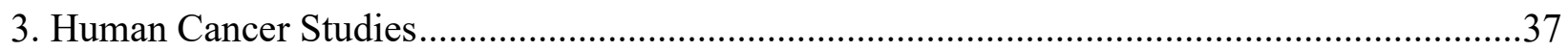

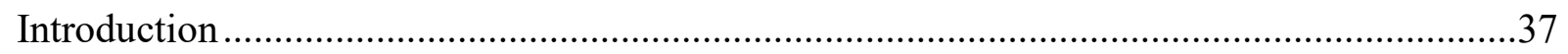

3.1. Literature Search Strategy and Selection of the Relevant Literature ...................................37

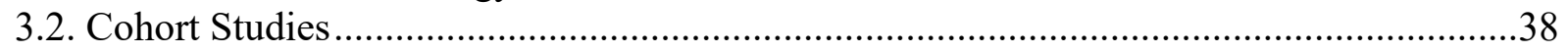

3.2.1. Overview of the Methodologies and Study Characteristics.......................................38

3.2.2. Evaluation of Study Quality and Other Elements Related to the Utility of the Studies to Inform the Cancer Hazard Evaluation................................................42

3.3. Kidney or Liver Cancer Case-control Studies ....................................................................51

3.3.1. Overview of the Methodologies and Study Characteristics.....................................51

3.3.2. Evaluation of Study Quality and Other Elements Related to the Utility of the Studies to Inform the Cancer Hazard Evaluation...............................................53

3.4. Case-control Studies of NHL and Related Subtypes ........................................................58

3.4.1. Overview of the Methodologies and Study Characteristics.....................................58

3.4.2. Evaluation of Study Quality and Other Elements Related to the Utility of the Studies to Inform the Cancer Hazard Evaluation..................................................59

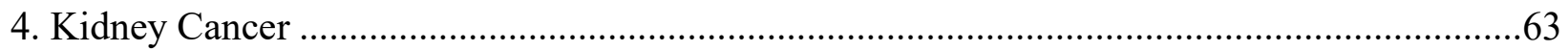

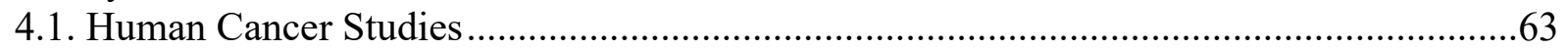

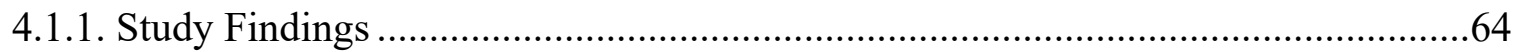

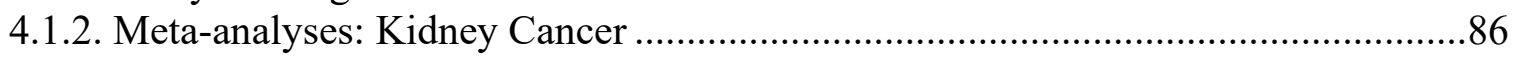

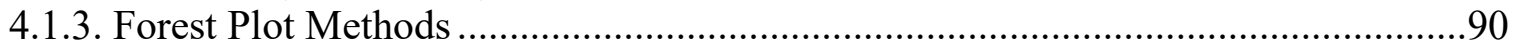

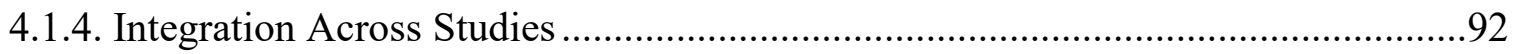

4.2. Mechanistic Data for Kidney Carcinogenicity ..................................................................95

4.2.1. Hypothesized Modes of Action ............................................................................95

4.2.2. The Proposed Role of GSH-conjugation-derived Metabolites in Kidney

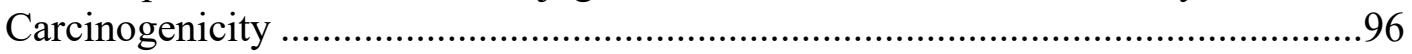

4.2.3. Proposed Modes of Action Associated with Oxidative Metabolites ......................103

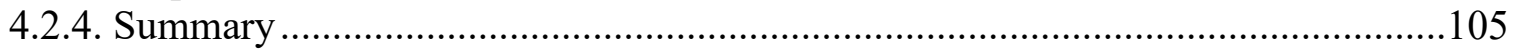

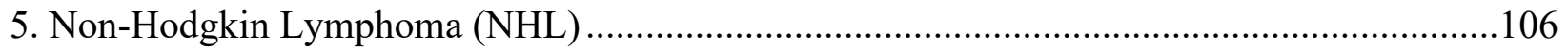

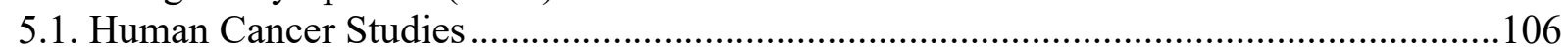

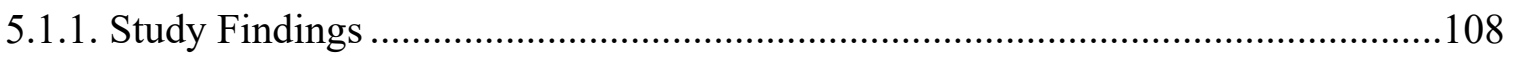

5.1.2. Cohort and Nested Case-control Studies ..............................................................109

5.1.3. Population-based Case-control Studies...................................................................110

5.1.4. Evaluation of Potential Confounding by Occupational Co-exposures or

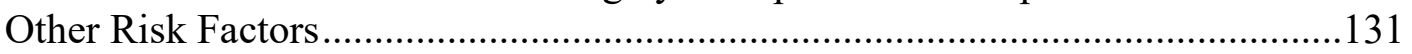

5.1.5. Integration across Studies …………………………………………………....132

5.2. Mechanistic Data for NHL and Related Neoplasms .......................................................135 
5.2.1. Risk Factors for NHL...................................................................................135

5.2.2. Immune Effects of Trichloroethylene ....................................................................136

5.2.3. Possible Modes of Action for Trichloroethylene-induced Immune

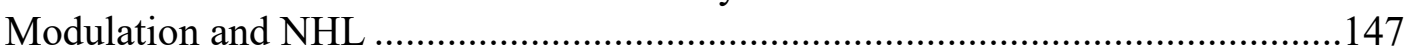

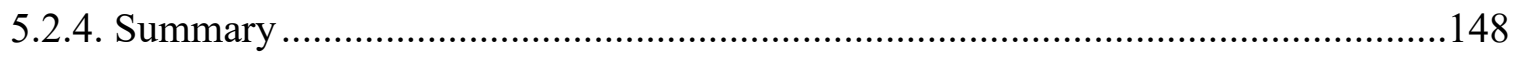

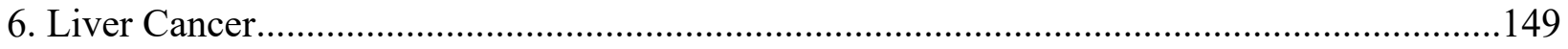

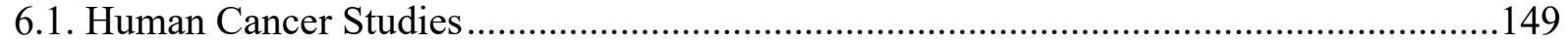

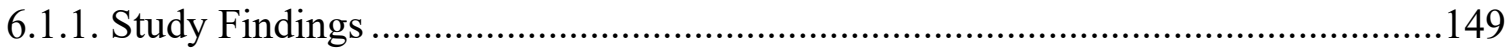

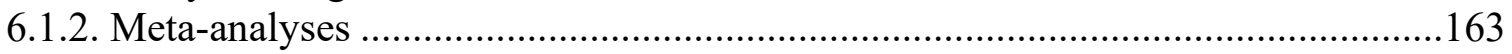

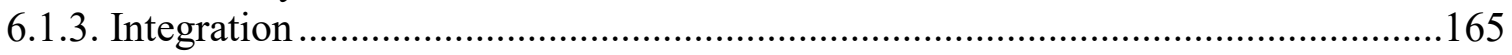

6.2. Mechanistic Data for Liver Carcinogenicity .................................................................166

6.2.1. Hypothesized Modes of Action with Limited Experimental Support ....................167

6.2.2. Hypothesized Modes of Action with Inadequate Support ........................................174

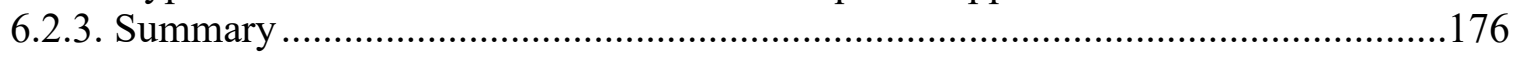

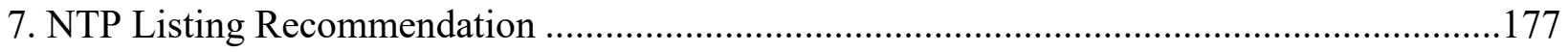

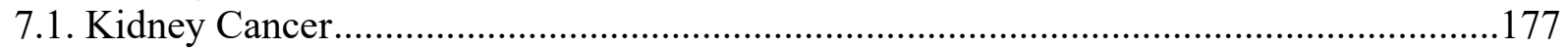

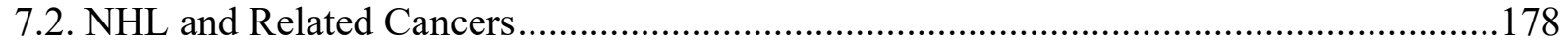

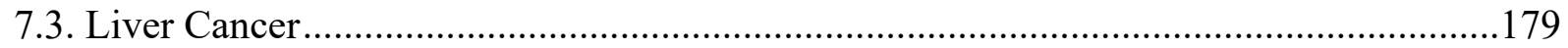

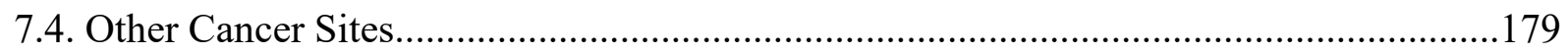

7.5. Toxicological Considerations across End Points ……………………..........................180

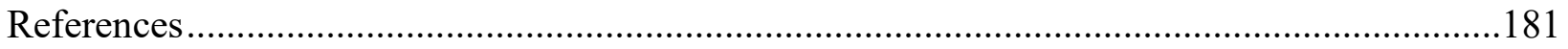

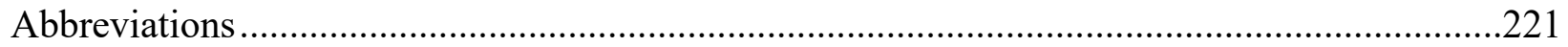

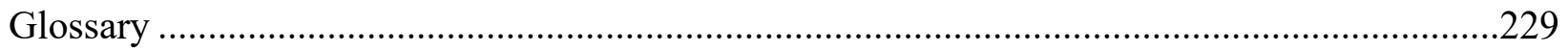

Appendix A. Literature Search Strategy ………………….................................................. A

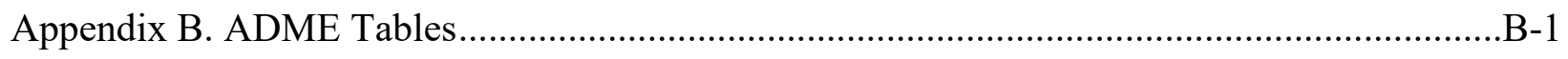

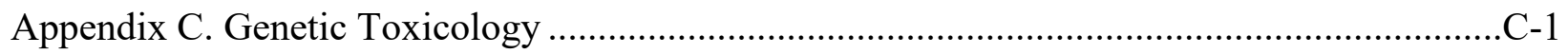

Appendix D. Human Cancer Study Tables ....................................................................... D-1

Appendix E. Immune Effects (Animals) ........................................................................

Appendix F. Mechanisms of Action Tables ……………....................................................

\section{Tables}

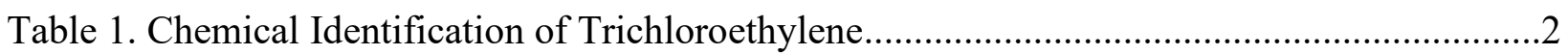

Table 2. Physical and Chemical Properties of Trichloroethylene.....................................................

Table 1-1. Tissue:Blood Partition Coefficients of Trichloroethylene in Rats and Mice ................. 9

Table 1-2. Trichloroethylene Metabolite Formation and Systemic Availability .............................15

Table 2-1. Summary Assessment of Genotoxicity and Related Effects for

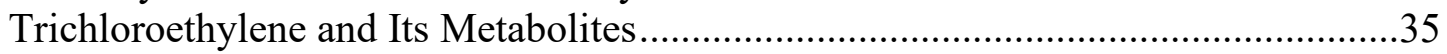

Table 3-1. Cohort and Nested Case-control Studies of Trichloroethylene Exposure ......................40

Table 3-2. Case-control Studies of Trichloroethylene Exposure: Kidney or Liver Cancer............52 
Table 3-3. Case-control Studies of Trichloroethylene Exposure and NHL and Its Subtypes

Table 4-1. Trichloroethylene Cohort and Nested Case-control Studies: Findings for

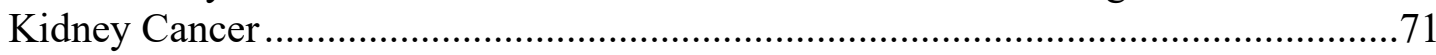

Table 4-2. Case-control Studies of Trichloroethylene Exposure: Findings for Kidney Cancer 79

Table 4-3. Meta-analyses of Kidney Cancer and Trichloroethylene Exposure .87

Table 4-4. Hypothesized Modes of Action and Key Events for Kidney Tumors .96

Table 4-5. Studies of VHL Mutation in Trichloroethylene-exposed Human Subjects with Renal-cell Carcinoma. 100

Table 5-1. Cohort and Nested Case-control Studies and Trichloroethylene Exposure:

Findings for NHL

Table 5-2. Case-control Studies of Trichloroethylene Exposure: Findings for NHL

Table 5-3. Cohort, Nested Case-control, and Population-based Case-control Studies of Trichloroethylene Exposure and NHL Subtypes

Table 5-4. Meta-analyses of Trichloroethylene Exposure and NHL and Related Subtypes .......130

Table 5-5. Case-control Studies of Trichloroethylene Exposure and Autoimmune Diseases in Humans.

Table 5-6. Studies of Trichloroethylene Exposure and Lymphocytes, and Immune Markers in Humans

Table 6-1. Findings for Trichloroethylene and Cancers of the Liver, Biliary Tract, or Gallbladder

Table 6-2. Meta-analyses of Liver Cancer (Including Gall Bladder and Biliary Passages) and Trichloroethylene Exposure

Table 6-3. Possible Modes of Action and Key Events for Trichlorethylene-induced Liver Cancer.

\section{Figures}

Figure 1. Chemical Structure of Trichloroethylene 1

Figure 1-1. Oxidative Metabolism of Trichloroethylene (TCE) ...............................................11

Figure 1-2. Glutathione-dependent Metabolic Pathways of Trichloroethylene...........................13

Figure 1-3. PBPK Model Predictions for the Fraction of Trichloroethylene Intake That Is Metabolized under Continuous Inhalation Exposure in Humans .............................20

Figure 1-4. PBPK Model Predictions for the Fraction of Trichloroethylene Intake That Is Metabolized under Continuous Oral Exposure in Humans......................................20

Figure 3-1. Study Utility Ranking: Cohort Studies ..............................................................50

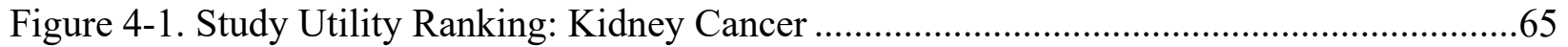

Figure 4-2. Forest Plot-1: Kidney Cancer and Ever Exposure to Trichloroethylene....................91

Figure 4-3. Forest Plot-2: Kidney Cancer and High Exposure to Trichloroethylene ....................92

Figure 4-4. Forest Plot-3: Kidney Cancer and Estimated Exposure Level for Trichloroethylene

Figure 5-1. Study Utility Ranking: NHL 108

Figure 5-2. Forest Plot: NHL and Ever Exposure to Trichloroethylene..................................134

Figure 6-1. Study Utility Ranking: Liver Cancer 151

Figure 6-2. Forest Plot: Liver Cancer and Ever Exposure to Trichloroethylene..... 166 
This report has been reformatted to meet new NTP publishing requirements; its content has not changed. The proposed substance profile is no longer part of the document because it is published in the 14th Report on Carcinogens. 


\section{About This Report}

National Toxicology Program ${ }^{1}$

${ }^{1}$ Division of the National Toxicology Program, National Institute of Environmental Health

Sciences, Research Triangle Park, North Carolina, USA

\section{Collaborators}

Ruth Lunn, Sanford Garner, Stanley Atwood, Andrew Ewens, Neela Guha, Gloria D. Jahnke, Alton Peters, Jennifer Ratcliffe, Diane L. Spencer

Division of the National Toxicology Program, National Institute of Environmental Health Sciences, Research Triangle Park, North Carolina, USA

Led the preparation of the cancer hazard evaluation, including the design, conduct, assessment of studies, technical review, and proposed cancer hazard conclusions

Ruth Lunn, Dr.P.H. (Project Co-lead)

Contributed to the conduct, technical review, assessment of studies and proposed cancer hazard conclusions

Gloria D. Jahnke, D.V.M., DABT

Diane L. Spencer, M.S.

Integrated Laboratory Systems, Inc., Research Triangle Park, North Carolina, USA

Contributed to the conduct and technical review of the cancer hazard evaluation

Sanford Garner, Ph.D. (Project Co-lead)

Stanley Atwood, M.S., DABT

Andrew Ewens, Ph.D., DABT

Alton Peters, M.S.

Jennifer Ratcliffe, Ph.D.

External Advisor

Contributed to the design, conduct, and provided expert input on report preparation and review

Neela Guha, Ph.D., International Agency for Research on Cancer (IARC), Lyon, France

\section{Contributors}

\section{External Advisors}

Provided expert input on report preparation and review

Cheryl Scott, M.S.P.H., U.S. Environmental Protection Agency (U.S. EPA), Washington,

District of Columbia, USA

Patricia Stewart, Ph.D., Stewart Exposure Assessments, LLC, Arlington, Virginia, USA

TCE Webinar "Human Cancer Studies on Exposure to Trichloroethylene: Methods Used to Assess Exposure and Cancer Outcome" (March 17, 2014)

Served as moderators/discussion leaders

Neela Guha, Ph.D., IARC, Lyon, France

Cheryl Scott, M.S.P.H., U.S. EPA, Washington, District of Columbia, USA 
Presented on scientific issues related to evaluating human cancer studies Bernard D. Goldstein, M.D., University of Pittsburgh, Pittsburgh, Pennsylvania, USA Mark Purdue, Ph.D., National Cancer Institute (NCI), Rockville, Maryland, USA Patricia Stewart, Ph.D., Stewart Exposure Assessments, LLC, Arlington, Virginia, USA

\section{TCE Information Group Meeting (April 3, 2014)}

Discussed immune studies on TCE

Dori Germolec, Ph.D. (Moderator), Division of the National Toxicology Program (DNTP), National Institute of Environmental Health Sciences (NIEHS)

Anneclaire De Roos, Ph.D., Drexel University, Philadelphia, Pennsylvania, USA

Patricia Ganey, Ph.D., Michigan State University, East Lansing, Michigan, USA

Kathleen M. Gilbert, Ph.D., University of Arkansas, Little Rock, Arkansas, USA

Robert Luebke, Ph.D., U.S. EPA, Research Triangle Park, North Carolina, USA

Christine Parks, Ph.D., Kelly Government Services, Research Triangle Park, North Carolina, USA

Rafael Ponce, Ph.D., Amgen, Seattle, Washington, USA

Mark Purdue, Ph.D., NCI, Rockville, Maryland, USA

\section{RoC Internal Review Group}

Provided critical scientific review of the draft monograph prior to external peer review and public release

Abee Boyles, Ph.D., Division of Extramural Research and Training (DERT), NIEHS

Caroline Dilworth, Ph.D., DERT, NIEHS

Steve Ferguson, Ph.D., DNTP, NIEHS

Dori Germolec, Ph.D., DNTP, NIEHS

Scott Masten, Ph.D., DNTP, NIEHS

Alex Merrick, Ph.D., DNTP, NIEHS

Arun Pandiri, Ph.D., DNTP, NIEHS

Christine Parks, Ph.D., Kelly Government Services, Research Triangle Park, North Carolina, USA

Cynthia Rider, Ph.D., DNTP, NIEHS

Avima Ruder, Ph.D., National Institute for Occupational Safety and Health, Centers for Disease

Control and Prevention, Cincinnati, Ohio, USA

Suramya Waidyanatha, Ph.D., DNTP, NIEHS

Nigel Walker, Ph.D., DNTP, NIEHS

Lori White, Ph.D., DNTP, NIEHS

Mary Wolfe, Ph.D., DNTP, NIEHS

Integrated Laboratory Systems, Inc., Research Triangle Park, North Carolina, USA

Provided administrative support

Ella Darden, B.S.

Tracy Saunders, B.S. 


\title{
Peer Review
}

Peer review of the Draft RoC Monograph on Trichloroethylene was conducted by an ad hoc expert panel at a public meeting held August 12, 2014, in the Rodbell Auditorium at the National Institute of Environmental Health Sciences, David P. Rall Building, Research Triangle Park, NC (see http://ntp.niehs.nih.gov/go/38854 for materials, minutes, and panel recommendations from the peer-review meeting). The selection of panel members and conduct of the peer review were performed in accordance with the Federal Advisory Committee Act and Federal policies and regulations. The panel members served as independent scientists, not as representatives of any institution, company, or governmental agency.

The charge to the Peer-Review Panel was as follows:

(1) Comment on the draft cancer evaluation c for trichloroethylene, specifically, whether it was technically correct and clearly stated, whether NTP has objectively presented and assessed the scientific evidence, and whether the scientific evidence is adequate for applying the RoC listing criteria,

(2) Comment on the draft substance profile for trichloroethylene, specifically, whether the scientific justification presented in the substance profile supports NTP's preliminary policy decision on the RoC listing status of the substance (available in the 14th edition of the Report on Carcinogens).

The Panel was asked to vote on the following questions:

(1) Whether the scientific evidence supports NTP's preliminary conclusion on the level of evidence for carcinogenicity from human cancer studies for each of the three cancer sites: kidney cancer, non-Hodgkin lymphoma (NHL), and liver cancer.

(2) Whether the scientific evidence supports NTP's preliminary listing decision for trichloroethylene in the RoC.

This RoC monograph on trichloroethylene has been revised based on NTP's review of the Panel's peer-review comments. The Peer-Review Panel Report, which captures the Panel recommendations for listing status of trichloroethylene in the $\mathrm{RoC}$ and their scientific comments, and the NTP Response to the Peer-Review Report are available on the Peer-Review Meeting webpage for trichloroethylene (http://ntp.niehs.nih.gov/go/38854).

\section{Peer Reviewers}

\author{
David A. Eastmond, Ph.D. (Chair) \\ Professor and Department Chair \\ Environmental Toxicology Graduate Program and Department of Cell Biology \& Neuroscience \\ University of California \\ Riverside, California, USA
}

Sarah J. Blossom, Ph.D.

Assistant Professor of Pediatrics and Microbiology and Immunology

UAMS College of Medicine

Arkansas Children's Hospital Research Institute

Little Rock, Arkansas, USA 
Kenneth P. Cantor, Ph.D.

Private Consultant

KP Cantor Environmental, LLC

Silver Spring, Maryland, USA

John M. Cullen, V.M.D., Ph.D., DACVP, FIATP

Alumni Distinguished Undergraduate Professor

Department of Population Health and Pathobiology

Research Building

College of Veterinary Medicine

North Carolina State University

Raleigh, North Carolina, USA

George R. Douglas, Ph.D.

George R. Douglas Consulting

Kanata, Ontario, Canada

S. Katharine Hammond, Ph.D.

Professor of Environmental Health Sciences

School of Public Health

University of California, Berkeley

Berkeley, California, USA

Lawrence H. Lash, Ph.D.

Professor and Associate Chair

Department of Pharmacology

Wayne State University School of Medicine

Detroit, Michigan, USA

Marie-Elise Parent, Ph.D.

Epidemiology and Biostatistics Unit

INRS-Institut Armand-Frappier

Université du Québec

Laval, Québec, Canada

David B. Richardson, Ph.D.

Associate Professor

Department of Epidemiology

School of Public Health

University of North Carolina

Chapel Hill, North Carolina, USA

Paolo Vineis, M.D., M.P.H.

Chair in Environmental Epidemiology

MRC/PHE Centre for Environment and Health

School of Public Health

Imperial College London

London, England, United Kingdom 


\section{Publication Details}

Publisher: National Toxicology Program

Publishing Location: Research Triangle Park, NC

ISSN: 2331-267X

DOI: https://doi.org/10.22427/ROC-MGRAPH-5

Report Series: RoC Monograph Series

Report Series Number: 05

Official citation: National Toxicology Program (NTP). 2015. Report on Carcinogens monograph on trichloroethylene. Research Triangle Park, NC: National Toxicology Program. RoC Monograph 05.

\section{Acknowledgments}

This work was supported by the Intramural Research Program (ES103316 and ES103317) at the National Institute of Environmental Health Sciences, National Institutes of Health and performed for the National Toxicology Program, Public Health Service, U.S. Department of Health and Human Services under contract HHSN273201100004C. 


\section{Abstract}

Introduction: Trichloroethylene (TCE, CASRN 79-01-6) is a volatile, chlorinated alkene used mainly as an intermediate for hydrofluorocarbon production and as a degreaser for metal parts, although its use as a degreaser has decreased in the United States since the 1970s. People are exposed in workplaces that produce or use TCE as a degreaser or solvent, and from drinking contaminated water and breathing contaminated air.

Methods: The National Toxicology Program (NTP) conducted a cancer hazard assessment of TCE, focusing on three types of cancer kidney cancer, non-Hodgkin lymphoma (NHL), and liver cancer. NTP used systematic review methods to identify studies, evaluate study quality, integrate evidence across studies, and integrate evidence across data streams (human, animal, and mechanistic data). Using established criteria, NTP reached conclusions regarding the strength of the evidence for each of the three cancer types and on the recommended listing status of trichloroethylene in the Report on Carcinogens.

\section{Results and Discussion:}

Kidney cancer: Epidemiological studies provide evidence of a causal relationship between exposure to TCE and kidney cancer based on consistent evidence of increased risk of kidney cancer across studies with different study designs, geographical areas, and occupational settings. Kidney cancer risk increased with increasing level or duration of TCE exposure in some individual studies and across studies. Exposure to TCE by inhalation or gavage caused kidney tumors in rats. TCE most likely causes kidney cancer by its metabolism in the kidney to genotoxic and cytotoxic GSH-conjugation-derived metabolites (or their systemic delivery to the kidney).

Non-Hodgkin Lymphoma (NHL): Epidemiological studies provide limited evidence that TCE exposure causes NHL, based on positive associations in several studies and evidence for increased risk of NHL across studies combined in two meta-analyses. However, alternative explanations for the associations, such as chance or confounding, cannot reasonably be ruled out. Exposure to TCE by inhalation or gavage caused lymphoma in mice. TCE causes immunomodulation in both people and animals, and immunomodulation is linked to NHL; however, the potential association between trichloroethylene-induced immune effects and lymphoma has not been tested directly in either humans or animals.

Liver cancer: Epidemiological studies of TCE in humans were inconsistent and the overall data were considered inadequate. Exposure to TCE by inhalation or gavage caused lymphoma in mice.

NTP Cancer Hazard Conclusion. The NTP cancer hazard evaluation was the basis for changing the listing status of TCE from reasonably anticipated to known to be a human carcinogen in the 14th edition of the Report on Carcinogens based on sufficient evidence of carcinogenicity from studies in humans. This conclusion is based on epidemiological studies showing that TCE causes kidney cancer in humans, together with supporting evidence from toxicological, toxicokinetic, and mechanistic studies demonstrating the biological plausibility of its carcinogenicity in humans. 


\section{Introduction}

Trichloroethylene has been listed in the Report on Carcinogens (RoC) as reasonably anticipated to be a human carcinogen since 2000 based on limited evidence of carcinogenicity from studies in humans and sufficient evidence of carcinogenicity from studies in experimental animals. Since that time, several cancer studies in humans have been published in the peer-reviewed literature, and the International Agency for Research on Cancer (2014) has concluded that trichloroethylene is carcinogenic to humans (Group 1). Trichloroethylene has been selected as a candidate substance for review for possible change in listing status in the RoC based on evidence of exposure to a significant number of persons residing in the United States and an adequate database of cancer studies.

Trichloroethylene (TCE, CASRN 79-01-6) is a volatile, chlorinated alkene used mainly as an intermediate for hydrofluorocarbon production and as a degreaser for metal parts (USEPA 2014), although its use as a degreaser has decreased in the United States since the 1970s (Bakke et al. 2007). It also is used as a modifier for polyvinyl chloride polymerization and in clear protective spray coatings for use by arts and craft hobbyists. Past uses of trichloroethylene include use as a solvent in the rubber industry, adhesive formulations, dyeing and finishing operations, printing inks, paints, lacquers, varnishes, adhesives, and paint strippers; in the production of agricultural chemicals such as fungicides and insecticides; as an extraction solvent to remove natural fats and oils from plant materials, to manufacture flavoring extracts from spices and hops, and to decaffeinate coffee; and as an anesthetic and analgesic in obstetrics and for minor surgical procedures.

A significant number of people living in the United States are or have been exposed to trichloroethylene because of its widespread presence in the environment from past and present use, particularly in some drinking-water supplies, and in the workplace. Due to its volatility, the principal route of exposure is through inhalation although absorption from dermal and oral exposure also occurs. Exposure has been documented by direct measurements of trichloroethylene in ambient air in the general environment and in workplaces where it is used. The presence of trichloroethylene in groundwater and drinking-water supplies near sites of past use of trichloroethylene has also been confirmed. Additional information on occupational and environmental exposure to trichloroethylene is described in $\mathrm{RoC}$ substance profile (https://ntp.niehs.nih.gov/ntp/roc/content/profiles/trichloroethylene.pdf).

\section{Chemical and Physical Properties}

Trichloroethylene (Figure 1) is a chlorinated alkene. Table 1 contains some chemical identification information for trichloroethylene.

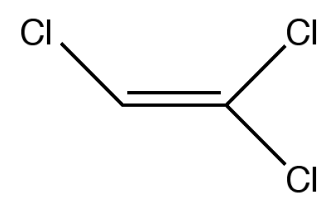

Figure 1. Chemical Structure of Trichloroethylene 
Table 1. Chemical Identification of Trichloroethylene

\begin{tabular}{ll}
\multicolumn{1}{c}{ Characteristic } & \multicolumn{1}{c}{ Information } \\
\hline Chemical Abstracts index name & $1,1,2-$ Trichloroethene \\
CAS Registry number & $79-01-6$ \\
Molecular formula & $\mathrm{C}_{2} \mathrm{HCl}_{3}$ \\
Synonyms & TCE; TRI; 1, 1,2-trichloroethylene; trichloroethene; ethylene \\
& trichloride; acetylene trichloride \\
\hline
\end{tabular}

Source: HSDB (2012), IARC (2014).

Trichloroethylene exists at room temperature as a clear, colorless, nonflammable liquid with an ethereal odor. It is slightly soluble in water, soluble in ethanol, acetone, diethyl ether, and chloroform, and miscible in oil. Trichloroethylene evaporates easily (Dow 2008). It is relatively stable, but oxidizes slowly when exposed to sunlight in air (IARC 1976). Physical and chemical properties of trichloroethylene are listed in Table 2.

Table 2. Physical and Chemical Properties of Trichloroethylene

\begin{tabular}{lc}
\hline \multicolumn{1}{c}{ Property } & Information \\
\hline Molecular weight & 131.4 \\
Specific gravity & 1.4642 at $20^{\circ} \mathrm{C} / 4^{\circ} \mathrm{C}$ \\
Melting point & $-84.7^{\circ} \mathrm{C}$ \\
Boiling point & $87.2^{\circ} \mathrm{C}$ \\
Log $K_{\text {ow }}$ & 2.61 \\
Water solubility & $1.28 \mathrm{~g} / \mathrm{L}$ at $25^{\circ} \mathrm{C}$ \\
Vapor pressure & $69 \mathrm{~mm} \mathrm{Hg}$ at $25^{\circ} \mathrm{C}$ \\
Vapor density relative to air (air $=1)$ & 4.53 \\
\hline Source: HSDB (2012).
\end{tabular}




\section{Methods}

This Report on Carcinogens (RoC) monograph on trichloroethylene reviews the relevant scientific information and assesses its quality, applies the RoC listing criteria to the scientific information, and recommends an RoC listing status for trichloroethylene. Exposure information from the substance profile in the 12th RoC was updated in the substance profile in the 14th RoC (https://ntp.niehs.nih.gov/ntp/roc/content/profiles/trichloroethylene.pdf). The exposure information in the 12 th $\mathrm{RoC}$ meets the criteria for exposure to a significant number of persons residing in the United States in the RoC.

\section{Monograph Contents and Development}

The methods for preparing the draft RoC monograph on trichloroethylene are described in the "Trichloroethylene Protocol" (http://ntp.niehs.nih.gov/ntp/roc/thirteenth/protocols/tce protocol12-31-13 508.pdf). As discussed in the protocol, RoC monograph focuses on the relationship between exposure to trichloroethylene and non-Hodgkin lymphoma (NHL) and its histological subtypes and related cancers, and cancers of the kidney and liver. It provides information on the following topics that are relevant to understanding the relationship between exposure to trichloroethylene and the cancers listed above: chemical and physical properties (Introduction), disposition and toxicokinetics (Section 1), genotoxicity and related effects (Section 2), quality assessment of cancer studies in humans (Section 3), kidney cancer (Section 4), NHL (and related cancers) (Section 5), and liver cancer (Section 6). The information in Section 7 is a synthesis of Sections 1 through 6.

The information reviewed in Sections 1 through 7 (except for information on exposure and properties) must come from publicly available, peer-reviewed sources.

The process for preparing the monograph included approaches for obtaining public and scientific input and using systematic methods (e.g., standardized methods for identifying the literature [see Appendix A], inclusion/exclusion criteria, extraction of data and evaluation of study quality using specific guidelines, and assessment of the level of evidence for carcinogenicity using established criteria).

The Office of the Report on Carcinogens (ORoC) followed the approaches outlined in the concept document, which discusses the scientific issues and questions relevant to the evaluation of trichloroethylene carcinogenicity, the scope and focus of the monograph, and the approaches to obtain scientific and public input to address the key scientific questions and issues for preparing the cancer evaluation component of the draft monograph. The ORoC presented the draft concept document for trichloroethylene to the NTP Board of Scientific Counselors (BSC) at the June 21-22, 2012 meeting that provided opportunity for written and oral public comments and is available on the RoC website (https://ntp.niehs.nih.gov/go/711176), after which the concept was finalized and trichloroethylene was approved by the NTP Director as a candidate substance for review.

Additional scientific input was obtained for exposure, human cancer studies, and immune effects of trichloroethylene. External advisors are identified in the "About This Report" section. 
Key issues identified in the concept document include (1) the need for expert input on the quality of the methods used in the epidemiological studies to assess exposure to trichloroethylene and cancer outcome, and information on trichloroethylene exposure in the studies and (2) the potential role of immune effects of trichloroethylene in human cancer. In order to receive public and scientific input on the epidemiological studies and exposure to trichloroethylene, the ORoC held a webinar titled, "Human Cancer Studies on Exposure to Trichloroethylene (TCE): Methods Used to Assess Exposure and Cancer Outcomes," on March 17, 2014. The ORoC also convened an information group of scientists, with expertise in immunology, cancer, epidemiology, or toxicology, who were asked to provide comments on the body of studies of trichloroethylene exposure and immune effects, and whether these studies are informative for evaluating potential mechanisms for trichloroethylene-related cancers in experimental animals and humans.

Public comments on scientific issues were requested at several times prior to the development of the draft RoC monograph, including the request for information on the nomination, and the request for comment on the draft concept document, which outlined the rationale and approach for conducting the scientific review. In addition, NTP posted its protocol for reviewing the human cancer studies and studies in experimental animals for public input on the ORoC webpage for trichloroethylene (available at

(http://ntp.niehs.nih.gov/ntp/roc/thirteenth/protocols/tce protocol12-31-13 508.pdf) prior to the release of the draft monograph. Two written public comments on trichloroethylene were received from the public as of the date on this document (http://ntp.niehs.nih.gov/go/38854).

\section{Key Scientific Questions and Issues Relevant for the Cancer Evaluation}

The key scientific issues concern the evaluation of cancer studies in humans and experimental animals, and mechanistic data. They are as follows:

\section{Questions Related to the Evaluation of Human Cancer Studies}

- What is the level of evidence (sufficient, limited) for the carcinogenicity of trichloroethylene from studies in humans?

- What are the major strengths and limitations in the individual studies and how do they affect the findings?

- Are the associations between exposure to trichloroethylene and NHL and cancers of the kidney and liver observed in some studies, and in the meta-analyses, credible? Can bias, chance, or confounding be ruled out with reasonable confidence?

\section{Questions Related to the Evaluation of Mechanistic Data}

- What are the potential mechanisms by which trichloroethylene may cause NHL and cancers of the kidney and liver?

- Is there evidence that the mechanisms by which trichloroethylene causes cancer in experimental animals may not occur in humans? If so, what is the level of evidence?

- Is there mechanistic evidence in humans that would support the associations observed in some human cancer studies? If so, what is the level of evidence? Of special interest is the level of evidence for mutagenic and cytogenetic modes of action for kidney cancer. 
- Is there any evidence that trichloroethylene-induced immunologic effects are related to cancer (such as lymphoma or liver cancer) development?

\section{Methods for Preparing the Monograph}

The procedures by which relevant literature was identified, data were systematically extracted and summarized, and the draft monograph was written, together with the processes for scientific review, quality assurance, and assessment and synthesis of data, are described below.

The preparation of the RoC monograph for trichloroethylene began with development of a literature search strategy to obtain information relevant to the topics listed above for Sections 1 through 6 using search terms developed in collaboration with a reference librarian (see Protocol). The citations $(\mathrm{N}=3,543)$ identified from these searches were uploaded to a web-based systematic review software for evaluation by two separate reviewers using inclusion/exclusion criteria, and 473 references were selected for final inclusion in the draft monograph using these criteria. Studies identified from the literature searches but excluded from the review include publications on chemicals other than trichloroethylene (or relevant structurally related compounds such as trichloroethylene metabolites and analogues or byproducts of production of trichloroethylene), and studies involving exposure to trichloroethylene that reported results for topics not covered in this monograph (see Section Monograph Contents).

Information for the relevant cancer and mechanistic sections was systematically extracted in tabular format and/or summarized in the text, following specific procedures developed by ORoC, from studies selected for inclusion in the monograph. All sections of the monograph underwent scientific review and quality assurance (QA) (i.e., assuring that all the relevant data and factual information extracted from the publications have been reported accurately) by a separate reviewer. Any discrepancies between the writer and the reviewer were resolved by mutual discussion in reference to the original data source.

Strengths, weaknesses, and study quality of the cancer studies for trichloroethylene in humans (see Appendix D) were assessed based on a series of a priori considerations (questions and guidelines for answering the questions), which are available in the protocol (available at http://ntp.niehs.nih.gov/ntp/roc/thirteenth/protocols/tce protocol12-31-13 508.pdf). Two reviewers evaluated the quality of each study. Any discrepancies between the two reviewers were resolved by mutual discussion in reference to the original data source. Relevant genotoxicity and mechanistic studies were also assessed for their strengths and weaknesses.

RoC listing criteria (see text box) were applied to the available database of carcinogenicity data to assess the level of evidence (sufficient, limited, or inadequate) for the carcinogenicity of trichloroethylene from studies in humans and the level of evidence (sufficient, not sufficient) from studies in experimental animals. The approach for synthesizing the evidence across studies and reaching a level of evidence conclusion was outlined in the protocol. The evaluation of the mechanistic data included a complete discussion and assessment of the strength of evidence for potential modes of action for trichloroethylene-induced neoplasia, including metabolic activation, cytotoxicity, genetic-related effects, and epigenetic effects. The RoC listing criteria were then applied to the body of knowledge (cancer studies in humans and experimental animals and mechanistic data) for trichloroethylene to reach a listing recommendation. 


\section{RoC Listing Criteria}

\section{Known to Be Human Carcinogen:}

There is sufficient evidence of carcinogenicity from studies in humans*, which indicates a causal relationship between exposure to the agent, substance, or mixture, and human cancer.

\section{Reasonably Anticipated to Be Human Carcinogen:}

There is limited evidence of carcinogenicity from studies in humans*, which indicates that causal interpretation is credible, but that alternative explanations, such as chance, bias, or confounding factors, could not adequately be excluded, OR

there is sufficient evidence of carcinogenicity from studies in experimental animals, which indicates there is an increased incidence of malignant and/or a combination of malignant and benign tumors (1) in multiple species or at multiple tissue sites, or (2) by multiple routes of exposure, or (3) to an unusual degree with regard to incidence, site, or type of tumor, or age at onset, OR

there is less than sufficient evidence of carcinogenicity in humans or laboratory animals; however, the agent, substance, or mixture belongs to a well-defined, structurally related class of substances whose members are listed in a previous Report on Carcinogens as either known to be a human carcinogen or reasonably anticipated to be a human carcinogen, or there is convincing relevant information that the agent acts through mechanisms indicating it would likely cause cancer in humans.

Conclusions regarding carcinogenicity in humans or experimental animals are based on scientific judgment, with consideration given to all relevant information. Relevant information includes, but is not limited to, dose response, route of exposure, chemical structure, metabolism, pharmacokinetics, sensitive sub-populations, genetic effects, or other data relating to mechanism of action or factors that may be unique to a given substance. For example, there may be substances for which there is evidence of carcinogenicity in laboratory animals, but there are compelling data indicating that the agent acts through mechanisms which do not operate in humans and would therefore not reasonably be anticipated to cause cancer in humans.

*This evidence can include traditional cancer epidemiology studies, data from clinical studies, and/or data derived from the study of tissues or cells from humans exposed to the substance in question that can be useful for evaluating whether a relevant cancer mechanism is operating in people. 


\section{Disposition and Toxicokinetics}

Disposition and toxicokinetics refer to how a chemical can enter and leave the body, what happens to it once it is in the body, and the rates of these processes. Disposition includes absorption, distribution, metabolism, and excretion while toxicokinetics refers to the mathematical description of the time course of disposition of a chemical in the body. Section 1.1 discusses the absorption, distribution, and excretion of trichloroethylene; metabolism is discussed in Section 1.2; and toxicokinetic data derived primarily from in vitro studies are presented in Section 1.3. These data show that there are qualitative similarities between rodents and humans. Disposition and toxicokinetic data are important because they describe various factors that affect the toxicity of a chemical. These factors include routes and rates of absorption, tissue concentrations and their temporal changes, reactive metabolites, intoxication and detoxication reactions, routes of elimination, and gender and/or species differences in these factors. The mechanistic implications of these data are discussed in subsequent sections.

\subsection{Absorption, Distribution, and Excretion}

Trichloroethylene is a small, volatile, lipophilic compound that readily crosses cell membranes. The absorption, distribution, and excretion of trichloroethylene in humans and experimental animals has been extensively investigated and reported in several recent high quality reviews published by EPA (2011a), ATSDR (1997; 2013), and IARC (2014). Therefore, this section focuses on the principal findings from those reviews. Overall, the data indicate that trichloroethylene is well absorbed by all routes of exposure, widely distributed, and excreted either unchanged in expired air or as metabolites in the urine.

\subsubsection{Human Studies}

Humans are exposed to trichloroethylene from a variety of sources and by different routes (ATSDR 1997; USEPA 2011a). Occupational exposure occurs primarily by inhalation of vapors and dermal contact with vapors or liquid. Trichloroethylene is a common environmental contaminant; thus, the general population may be exposed from contact with contaminated air, food, and water. Oral absorption in humans is rapid and extensive based on clinical symptoms and measurements of trichloroethylene and its metabolites in urine and blood following accidental or intentional ingestion. However, quantitative estimates of absorption were not possible because the ingested amounts were unknown. Several controlled inhalation and dermal exposure studies have been conducted in humans. Uptake from the lungs is rapid and the absorbed dose is proportional to exposure concentration, duration, and pulmonary ventilation rate. Absorption from the lungs in subjects exposed to trichloroethylene concentrations of 9 to 200 ppm for 30 minutes to 5 hours ranged from about $40 \%$ to $70 \%$ at rest and $25 \%$ to $46 \%$ during exercise. Steady-state concentrations in blood were reached within a few hours after the start of exposure. The resulting concentration in the blood after establishment of equilibrium with alveolar air is determined by the blood-to-air partition coefficient. Measured blood-to-air partition coefficients for trichloroethylene in humans ranged from 8.1 to 11.7. Dermal absorption of trichloroethylene vapors or liquid is rapid (within minutes of application) with peak concentrations in exhaled breath occurring within 15 to 30 minutes. However, a dermal flux rate of $430 \pm 295 \mathrm{nmol} / \mathrm{cm}^{2} /$ minute measured in a study of volunteers exposed to neat liquid for 3 minutes indicated high interindividual variability. Poet et al. (2000) conducted skin absorption 
studies of trichloroethylene in water and soil using human volunteers $(\mathrm{N}=3)$ exposed by hand immersion or forearm patch tests. Mean permeability constants were $0.015 \mathrm{~cm} /$ hour (hand immersion) and $0.019 \mathrm{~cm} /$ hour (patch) in water, and $0.0074 \mathrm{~cm} /$ hour (hand immersion) and $0.0043 \mathrm{~cm} /$ hour (patch) in soil. For the patch tests, $4 \%$ and $0.6 \%$ of the applied trichloroethylene dose was absorbed through the skin from water and soil, respectively. An in vitro study using surgically removed skin samples exposed to trichloroethylene in aqueous solution reported a permeability constant of $0.12 \mathrm{~cm} /$ hour (USEPA $2011 \mathrm{a}$ ).

Once absorbed, trichloroethylene is rapidly distributed throughout the body (USEPA 2011a). Tissue samples collected at autopsy following accidental poisonings or from surgical patients exposed environmentally show that trichloroethylene is distributed to all tested tissues including the brain, muscle, heart, kidney, lung, and liver. Trichloroethylene also crosses the human placenta with ratios of concentrations in fetal:maternal blood ranging from approximately 0.5 to 2. Body distribution is largely determined by solubility in each organ and can be measured by tissue:blood partition coefficient. Partition coefficients reported for human tissues are as follows: fat (63.8 to 70.2 ), liver (3.6 to 5.9), brain (2.6), muscle (1.7 to 2.4), kidney (1.3 to 1.8), and lung ( 0.5 to 1.7$)$. Thus, post-exposure distribution of trichloroethylene is affected by the relative amount of fat tissue in the body, and accumulation of trichloroethylene in fat may prolong internal exposure.

Trichloroethylene is primarily excreted as urinary metabolites (see Section 1.1) or in expired air as the unchanged compound or carbon dioxide (USEPA 2011a). Controlled inhalation studies in humans indicated that $10 \%$ to $20 \%$ is exhaled unchanged while urinary metabolites accounted for about $50 \%$ to $75 \%$ of the retained dose (Bartoníček 1962; IARC 2014; Souček and Vlachová 1960; USEPA 2011a). No quantitative estimates of $\mathrm{CO}_{2}$ elimination in humans were identified. One study reported that $8.4 \%$ of the two primary metabolites (trichloroethanol and trichloroacetic acid) were eliminated in the feces (Bartoníček 1962). Elimination of unchanged trichloroethylene in the urine is minimal. Small amounts of metabolites may be excreted in sweat, milk, and saliva.

\subsubsection{Laboratory Animal Studies}

Trichloroethylene is well absorbed in laboratory animals by all exposure routes (ATSDR 1997; USEPA 2011a). Studies in mice and rats show that absorption of orally administered trichloroethylene may approach 100\%; however, other factors such as stomach contents, vehicle, and dose may affect the degree of absorption. Bioavailability from the gastrointestinal tract is higher in fasted animals, and uptake is faster and more extensive when administered in an aqueous vehicle compared with an oil vehicle. Peak blood levels occurred within minutes of dosing, indicating rapid absorption. Both closed-chamber gas uptake studies and blood concentration measurements following open-chamber experiments demonstrated rapid absorption of trichloroethylene from the respiratory tract of rodents. One study reported that the fractional absorption of trichloroethylene vapors was $>90 \%$ during the initial 5 minutes in rats exposed to 50 or $500 \mathrm{ppm}$ but declined to about $70 \%$ during the second hour of exposure. Studies with guinea pigs and rats indicate that trichloroethylene readily penetrates the skin. Estimated permeability constants in hairless guinea pigs were 0.16 to $0.47 \mathrm{~mL} / \mathrm{cm}^{2} /$ hour (Bogen et al. 1992). (The authors noted that this unit is equivalent to the more commonly used unit of $\mathrm{cm} /$ hour, but they considered it more meaningful for the permeability constant in this context.) Rat skin was shown to be significantly more permeable to trichloroethylene in water or soil than 
human skin with permeability coefficients of $0.31 \mathrm{~cm} /$ hour in water and about $0.09 \mathrm{~cm} /$ hour in soil (Poet et al. 2000).

Detailed tissue distribution studies have been conducted in rodents using different routes of administration (USEPA 2011a). These studies show that trichloroethylene is rapidly distributed throughout the body following inhalation or oral exposure. Tissue:blood partition coefficient values in rats and mice are shown in Table 1-1. The highest tissue concentrations were measured in fat; however, the fat:blood partition coefficients in rats and mice were lower than those reported for humans (63.8 to 70.2, see Section 1.1.1).

Table 1-1. Tissue:Blood Partition Coefficients of Trichloroethylene in Rats and Mice

\begin{tabular}{lccccccc}
\hline \multicolumn{1}{c}{ Species } & Fat & Brain & Liver & Kidney & Lung & Heart & Muscle \\
\hline Rat & $22.7-36.1$ & $0.71-1.29$ & $1.03-2.43$ & $1.0-1.55$ & 1.03 & 1.1 & $0.46-0.84$ \\
Mouse & 36.4 & - & 1.62 & 2.1 & 2.6 & - & 2.36 \\
\hline
\end{tabular}

Source: Adapted from EPA (2011a).

As in humans, laboratory animals primarily excrete trichloroethylene metabolites in the urine (USEPA 2011a). Unchanged trichloroethylene and $\mathrm{CO}_{2}$ are exhaled, and moderate amounts of metabolites are excreted in the feces. The amount of unchanged trichloroethylene exhaled increases with dose in mice and rats, which suggests saturation of metabolic pathways at high doses. In mice, $1 \%$ to $6 \%$ is exhaled unchanged at low doses but increases to $10 \%$ to $18 \%$ at high doses. Rats excrete about $1 \%$ to $3 \%$ unchanged at low doses but show a much higher increase at high doses (43\% to 78\%). At exposures below metabolic saturation, most of the administered trichloroethylene is eliminated as urinary metabolites.

\subsection{Metabolism}

Trichloroethylene metabolism is extensive and complex and most of the toxic effects of this compound have been linked to its metabolites (ATSDR 1997; IARC 2014; USEPA 2011 a). Controlled acute and subacute inhalation studies in humans at trichloroethylene concentrations up to 320 ppm show that $81 \%$ to $92 \%$ of the retained dose is metabolized (Bogen 1988). Saturation of trichloroethylene metabolism occurs at lower doses in rats than in mice, and mathematical simulation models have predicted metabolic saturation in humans at high exposure concentrations (ATSDR 1997). Although there are sex, species, and interindividual differences in metabolism, humans and laboratory animals have in common two distinct pathways: cytochrome P450-dependent oxidation (CYP) and glutathione (GSH) conjugation (USEPA 2011a). Quantitatively, the oxidative pathway predominates in all species studied. Oxidative metabolites have been linked to liver toxicity, while reactive metabolites generated by the GSH pathway have been linked to kidney toxicity. Hepatic first-pass oxidative metabolism is important. In addition to the liver, other important sites of metabolism include the kidney, lung, blood, and male reproductive system (Chiu et al. 2006; Cummings et al. 2001; Lash et al. 2014; Lipscomb et al. 1996). The following sections describe the primary metabolic pathways and metabolites. 


\subsubsection{CYP-dependent Oxidation}

CYP-dependent oxidation occurs in humans and rodents and is illustrated in Figure 1-1. The primary urinary metabolites detected in humans and rodents include trichloroethanol, trichloroethanol-glucuronide, and trichloroacetic acid (Lash et al. 2014; USEPA 2011a). Chloral also is a major oxidative metabolite but has low systemic levels due to rapid transformation to other metabolites (USEPA 2011a). Bradford et al. (2011) reported more than a fourfold difference in peak serum concentrations of trichloroacetic acid in male mice from 15 different strains administered a single oral dose of trichloroethylene. Serum concentrations of dichloroacetic acid varied more than 100-fold between strains but were about 1,000 times lower than trichloroacetic acid concentrations. In vitro data indicate that rodents have a higher capacity to metabolize trichloroethylene than humans, but this has not been verified in vivo (USEPA 2011a). Knadle et al. (1990) reported that rat hepatocytes produced 5 to 20 times more oxidative metabolites of trichloroethylene than human hepatocytes under the same experimental conditions.

Briefly, oxidation in the liver (primarily via CYP2E1) yields a chemically unstable oxygenated trichloroethylene-P450 intermediate that rapidly forms chloral, trichloroethylene oxide, and $N$ (hydroxyacetyl)-aminoethanol. The majority of the flux is towards chloral via chlorine migration (Lash et al. 2014). In body water, chloral is in equilibrium with chloral hydrate. Chloral/chloral hydrate is rapidly reduced by alcohol dehydrogenase or P450 to form trichloroethanol or oxidized by aldehyde dehydrogenase to form trichloroacetic acid. Trichloroethanol production was favored in humans and experimental animals following oral chloral exposure (USEPA 2011a). Trichloroethanol may be oxidized to trichloroacetic acid or form a glucuronide conjugate. Glucuronide conjugates excreted in the bile may be hydrolyzed back to trichloroethanol in the intestine and reabsorbed. In vivo studies in rats showed that enterohepatic circulation of trichloroethanol and subsequent oxidation was responsible for $76 \%$ of the trichloroethanol measured in blood. Although trichloroacetic acid is poorly metabolized it may undergo dechlorination to form dichloroacetic acid. Dichloroacetic acid also may form from trichloroethylene oxide, a short-lived intermediate metabolite. A few in vivo studies in mice have reported that dichloroacetic acid was produced to a very limited extent compared with trichloroacetic acid (Bradford et al. 2011; Kim et al. 2009a; Kim et al. 2009b). Trichloroethylene-oxide was the most likely source (Kim et al. 2009b). However, there is some uncertainty about the sources and amounts of dichloroacetic acid production in vivo, and direct evidence for its formation from trichloroethylene exposure remains equivocal, especially in humans (Lash et al. 2000a; USEPA 2011a). Dichloroacetic acid is difficult to detect in blood because it is rapidly metabolized to monochloroacetic acid by dechlorination or to glyoxylic acid by GST-zeta in hepatic cytosol (Lash et al. 2014; USEPA 2011a). Glyoxylic acid is subsequently converted to oxalic acid, glycine, and carbon dioxide. 


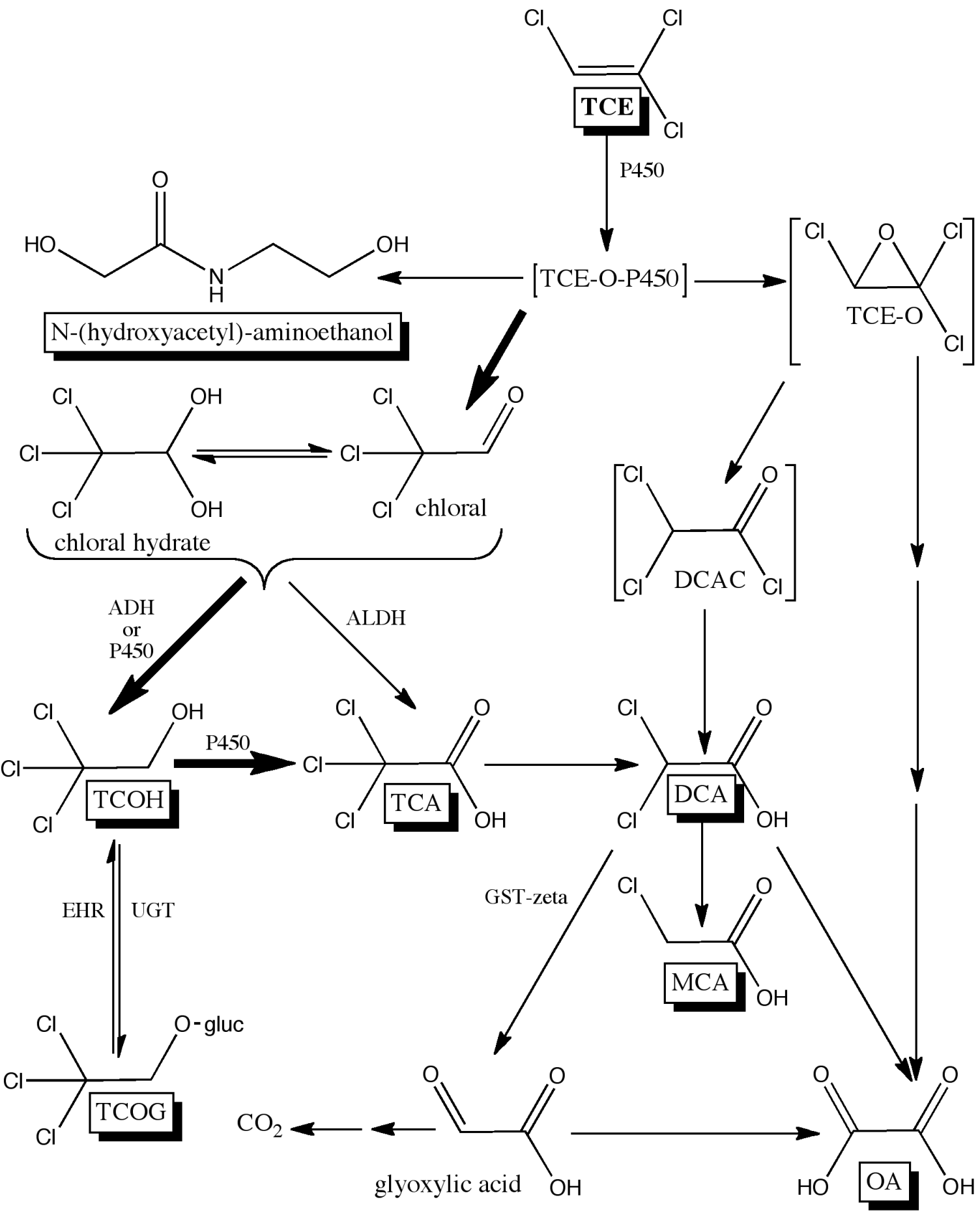

Figure 1-1. Oxidative Metabolism of Trichloroethylene (TCE)

Compounds that are recovered in urine are shown in boxes while chemically unstable or reactive compounds are enclosed in brackets. Heavy arrows indicate primary pathways. $\mathrm{ADH}=$ alcohol dehydrogenase; ALDH = aldehyde dehydrogenase; $\mathrm{DCA}=$ dichloroacetic acid; DCAC = dichloroacetylchloride; $\mathrm{EHR}=$ enterohepatic recirculation; $\mathrm{GST}=$ glutathione-Stransferase; $\mathrm{MCA}=$ monochloroacetic acid; $\mathrm{OA}=$ oxalic acid; $\mathrm{TCA}=$ trichloroacetic acid; $\mathrm{TCE}-\mathrm{O}=$ trichloroethylene oxide; $\mathrm{TCOG}=$ trichloroethanol-glucuronide conjugate; $\mathrm{TCOH}=$ trichloroethanol; UGT = UDP-glucuronosyltransferase. Adapted from: Chiu et al. (2013), Cummings et al. (2001), EPA (2011a), Kim et al. (2009b). 
Lipscomb et al. (1997) determined that CYP2E1 was responsible for more than $60 \%$ of oxidative trichloroethylene metabolism in microsomes from human lymphoblastoid cell lines selectively expressing CYP1A1, CYP1A2, CYP2E1, and CYP3A4. CYP2E1 is highly expressed in human liver and testes but is expressed at very low levels in human kidney (Lash et al. 2014). However, CYP2E1 expression is relatively high in rat kidney (Cummings et al. 2001). Although CYP2E1 is the predominant high-affinity isoform for trichloroethylene oxidation in humans and experimental animals, studies with CYP2E1 knockout mice show that considerable trichloroethylene oxidation occurs in its absence (Ghanayem and Hoffler 2007). Other P450 isozymes involved in the oxidative metabolism of trichloroethylene include CYP1A1/2, CYP2B1/2, and CYP2C11/6 in rat liver and/or kidney and CYP2F4 and CYP2F2 in rat and mouse lung, respectively (Cummings et al. 2001; Nakahama et al. 2001; Tabrez and Ahmad 2013; USEPA 2011a). Other human CYP enzymes that have some activity with trichloroethylene include CYP1A1/1A2, CYP2A6, and CYP3A4 (Lash et al. 2000a; Lash et al. 2014). CYP2E1 activity towards trichloroethylene is approximately 2-fold and 200-fold higher than that of CYP1A2 and CYP3A4, respectively (Lash et al. 2000a). Although liver P450 content is similar across species, mice and rats have higher levels of CYP2E1 than humans (USEPA 2011a). The maximal rate of CYP-dependent oxidative trichloroethylene metabolism is 2- to 4-fold higher in mice than in rats while the maximal rate in humans is 5- to more than 10fold slower than in rats (Lash et al. 2014). Differences in content or expression of the various P450 isoforms could contribute to interspecies differences in susceptibility.

\subsubsection{GSH Conjugation}

Trichloroethylene flux through the GSH conjugation pathway (Figure 1-2) is much less than through the oxidative pathway in humans and experimental animals; however, factors that affect the oxidative pathway indirectly affect the GSH pathway (USEPA 2011a). In vitro studies show that inhibition of P450-mediated oxidation increases GSH conjugation. Reactive metabolites produced several steps downstream from the initial conjugation are thought to cause cytotoxicity and carcinogenicity, particularly in the kidney. Glutathione S-transferase (GST) activity is highest in the liver but appreciable activity also occurs in other tissues including the kidneys (primarily the proximal tubules) (Lash et al. 2014). There is some uncertainty regarding the specific GST isoforms that mediate trichloroethylene conjugation; however, Lash et al. (1999b) reported evidence of high- and low-activity populations among male and female volunteers exposed to trichloroethylene vapors for 4 hours. These data suggest that polymorphisms affect GSH conjugation of trichloroethylene in humans. Several studies have reported that GST polymorphisms modify the risk of renal-cell carcinoma and that specific chemical exposures (including trichloroethylene) can affect the risk (Brüning et al. 1997a; Buzio et al. 2003; Cheng et al. 2012; Moore et al. 2010; Sweeney et al. 2000); however, two recent studies reported no association (Liu et al. 2012; Yang et al. 2013). 


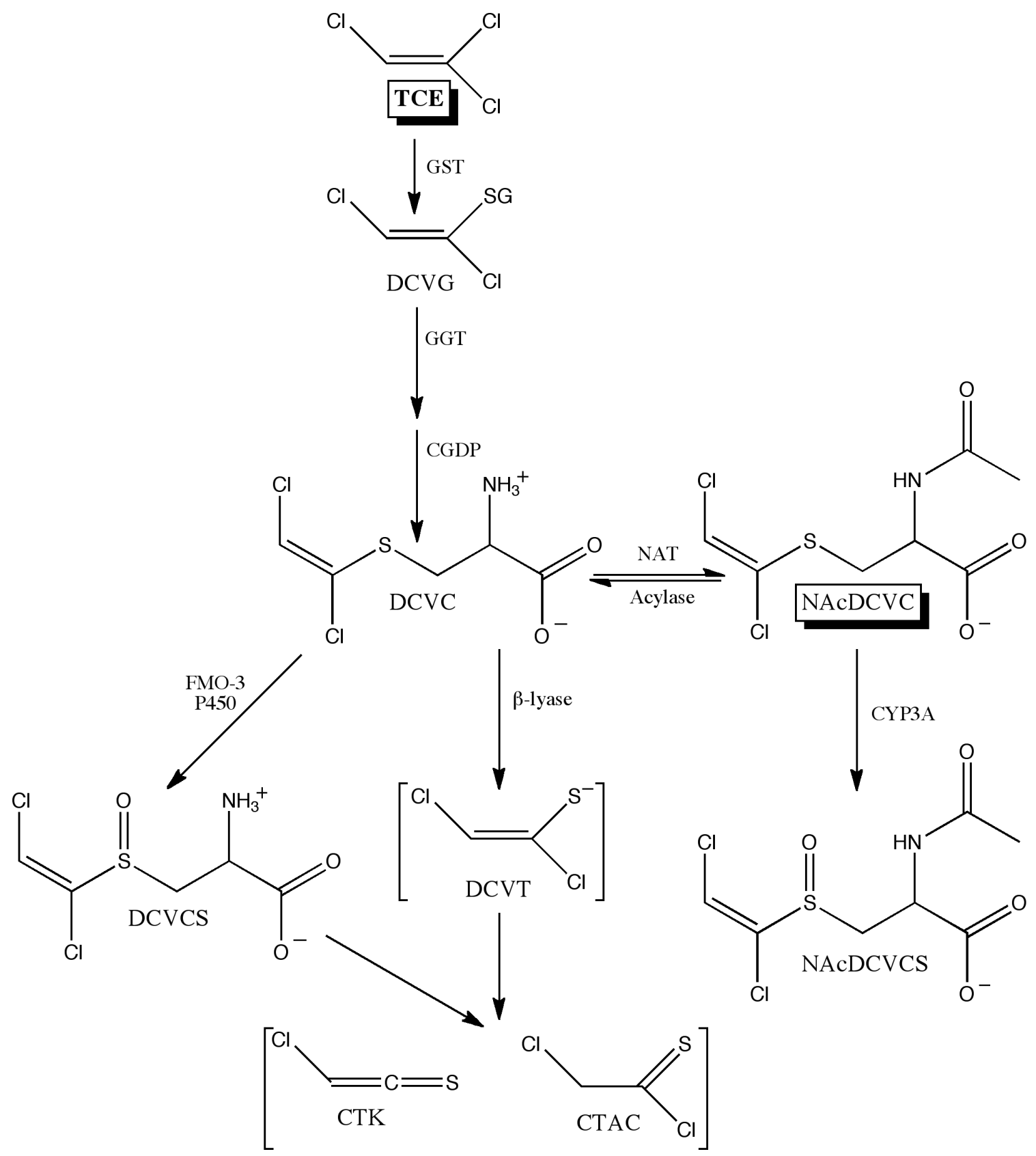

Figure 1-2. Glutathione-dependent Metabolic Pathways of Trichloroethylene

Only 1,2-dichlorovinyl isomers shown but 2,2-dichlorovinyl isomers also produced.

Compounds that are recovered in urine are shown in boxes while chemically unstable or reactive compounds are enclosed in brackets. $\mathrm{CGDP}=$ cysteinylglycine dipeptidases; $\mathrm{CTAC}=$ chlorothionoacetyl chloride; $\mathrm{CTK}=$ chlorothioketene; $\mathrm{DCVC}=S$ dichlorovinyl-L-cysteine; DCVG $=S$-dichlorovinyl-glutathione; DCVCS $=$ DCVC sulfoxide; DCVT $=S$-dichlorovinyl thiol; $\mathrm{FMO}=$ flavin monooxygenase; GGT $=\gamma$-glutamyl transpeptidase; GST $=$ glutathione-S-transferase; $\mathrm{NAcDCVC}=N$-acetyl DCVC; NAcDCVCS $=N$-acetyl DCVCS; NAT $=$ N-acetyltransferase.

Adapted from: Chiu et al. (2006), Dekant et al. (1988), EPA (2011a), Irving and Elfarra (2012).

The initial GSH-conjugation step occurs primarily in the liver and involves GSH displacement of a chloride ion from trichloroethylene via a nucleophilic substitution reaction. Products of this reaction include $S$-(1,2-dichlorovinyl)glutathione and its isomer $S$-(2,2-dichlorovinyl)glutathione 
(DCVG) (Lash et al. 2014; USEPA 2011a). Subsequent metabolism through the GSH conjugation pathway occurs primarily in the kidneys (Lash et al. 2014; USEPA 2011a). DCVG, whether it is formed in the liver or within the kidneys, is converted to its corresponding cysteine conjugate, $S$-dichlorovinyl-L-cysteine (DCVC), by hydrolytic reactions with $\gamma$ glutamyltransferase (GGT) and cysteinylglycine dipeptidases (CGDP) in the proximal tubular brush-border membrane. GGT and CGDP activity is much higher in the kidney than the liver in rodents and humans. These reactions also may take place in the bile or gut during enterohepatic circulation where DCVG and DCVC may be reabsorbed and further metabolized in the liver. DCVG and DCVC have been detected in blood, serum, and tissues of rodents, and DCVG has been detected in the blood of humans exposed to trichloroethylene (Lash et al. 2014). In vitro studies using rodent and human liver and kidney cellular and subcellular fractions of DCVG formation from trichloroethylene show considerable differences (USEPA 2011a). DCVC is a major branch point in the metabolism of trichloroethylene leading to three possible metabolites via reactions with $\mathrm{N}$-acetyltransferase, cysteine conjugate $\beta$-lyase, or flavin-containing monooxygenase 3 (FMO3) and are briefly described below.

$\mathrm{N}$-Acetylation of DCVC to $\mathrm{N}$-acetyl- $S$-dichlorovinyl-L-cysteine (NAcDCVC) can occur in the liver or kidney, thus, concentrations of the acetylated metabolite can exceed that which the kidney is capable of producing on its own (USEPA 2011a). NAcDCVC can be deacetylated to reform DCVC, oxidized by CYP3A to form the corresponding sulfoxide, or excreted in the urine. CYP3A expression is highly polymorphic in humans. NAcDCVC has been detected in urine samples from mice, rats, and humans, which indicates that $\mathrm{N}$-acetylation of DCVC is a common metabolic pathway among these species. In vitro studies of DCVC metabolism indicate that $\mathrm{N}$-acetylation to NAcDCVC is greater in rats than in mice or humans. Only NAcDCVC has been detected in the urine of experimental animals or humans, which might be due to the reactive nature of other metabolites generated from the GSH pathway.

Renal cysteine conjugate $\beta$-lyase catalyzes the formation of an unstable thiolate metabolite, $S$ dichlorovinyl-thiol (DCVT) from DCVC. This reaction has been demonstrated in vitro in rodents and humans with greater activity reported in rats compared with mice or humans (Green 1997). DCVT spontaneously rearranges to form two chemically reactive and unstable compounds, chlorothioketene and chlorothionoacetyl chloride (Dekant et al. 1988; Goeptar et al. 1995; Irving and Elfarra 2012).

Finally, DCVC also is a substrate for FMO3-catalyzed sulfoxidation (USEPA 2011a). Although the human kidney expresses relatively low levels of FMO3 (see Section 1.3.2), the available data suggest that FMO may play a more prominent role relative to $\beta$-lyase in human kidney while the reverse occurs in rat kidney (Lash et al. 2014). However, none of the possible sulfoxidation products of trichloroethylene metabolism have been reported in tissues or urine in vivo in rodents or humans.

Strain, species, and sex differences in GSH-conjugation have been reported (Lash et al. 2014; USEPA 2011a). Lash et al. (1999b) reported markedly higher amounts of DCVG formation in healthy male volunteers exposed to trichloroethylene vapors compared with females (see Section 1.3.4). In general, in vitro DCVG formation rates by liver and kidney subcellular fractions were higher in male rats and mice compared with females of the same species. Lash et al. (2006) reported that male rats formed more DCVC (considered the nephrotoxic precursor metabolite) than females. Hepatic concentrations of GSH also were reduced in male but not 
female rats exposed to higher doses of trichloroethylene. In mice exposed to trichloroethylene, Bradford et al. (2011) showed that the levels of DCVG and DCVC were much lower than oxidative metabolites and varied considerably with strain.

\subsubsection{Trichloroethylene Metabolites}

A summary of trichloroethylene metabolite formation and their systemic availability is shown in Table 1-2. Systemic availability depends on the chemical stability or reactivity of the metabolite. Metabolites that are chemically unstable or reactive are likely to spontaneously generate other molecules through non-enzymatic rearrangement or bind with cellular proteins, lipids, and DNA near their site of formation rather than distributing via the systemic circulation.

Table 1-2. Trichloroethylene Metabolite Formation and Systemic Availability

\begin{tabular}{|c|c|c|c|c|}
\hline Pathway/Metabolite & $\begin{array}{l}\text { Tissues Where } \\
\text { Formed }\end{array}$ & Human & Rodent & $\begin{array}{c}\text { Systemic Availability } \\
\text { (Rodents and Humans) }\end{array}$ \\
\hline \multicolumn{5}{|l|}{ P450 oxidation } \\
\hline TCE-O, DCAC & $\begin{array}{l}\text { liver } \\
\text { lung } \\
\text { testes }\end{array}$ & $\begin{array}{l}\text { yes } \\
\text { yes } \\
\text { yes }\end{array}$ & $\begin{array}{l}\text { yes } \\
\text { yes } \\
\text { yes }\end{array}$ & no \\
\hline $\mathrm{CH} / \mathrm{CHL}$ & $\begin{array}{l}\text { liver } \\
\text { lung } \\
\text { testes }\end{array}$ & $\begin{array}{l}\text { yes } \\
\text { yes } \\
\text { yes }\end{array}$ & $\begin{array}{l}\text { yes } \\
\text { yes } \\
\text { yes }\end{array}$ & yes \\
\hline $\mathrm{TCOH}$ & $\begin{array}{l}\text { liver } \\
\text { lung } \\
\text { GI } \\
\text { testes }\end{array}$ & $\begin{array}{c}\text { yes } \\
- \\
\text { yes } \\
\text { yes }\end{array}$ & $\begin{array}{l}\text { yes } \\
\text { yes } \\
\text { yes } \\
\text { yes }\end{array}$ & yes \\
\hline TCA & $\begin{array}{l}\text { liver } \\
\text { lung } \\
\text { testes }\end{array}$ & $\begin{array}{l}\text { yes } \\
\text { yes } \\
\text { yes }\end{array}$ & $\begin{array}{c}\text { yes } \\
\text { yes } \\
-\end{array}$ & yes \\
\hline TCOG & liver & yes & yes & yes \\
\hline DCA & $\begin{array}{l}\text { liver } \\
\text { lung } \\
\text { testes }\end{array}$ & $\begin{array}{c}- \\
- \\
\text { yes }\end{array}$ & $\begin{array}{c}\text { yes } \\
\text { yes } \\
-\end{array}$ & $\begin{array}{c}\text { yes } \\
\text { (low amount) }\end{array}$ \\
\hline \multicolumn{5}{|l|}{ GSH-conjugation } \\
\hline DCVG, DCVC & $\begin{array}{l}\text { liver } \\
\text { kidney }\end{array}$ & $\begin{array}{l}\text { yes } \\
\text { yes }\end{array}$ & $\begin{array}{l}\text { yes } \\
\text { yes }\end{array}$ & yes \\
\hline DCVT, DCVCS, CTK/CTAC & $\begin{array}{l}\text { kidney } \\
\text { hematopoietic }\end{array}$ & $\begin{array}{c}\text { yes } \\
-\end{array}$ & $\begin{array}{l}\text { yes } \\
\text { yes }\end{array}$ & no \\
\hline NAcDCVC, NAcDCVS & $\begin{array}{l}\text { liver } \\
\text { kidney }\end{array}$ & $\begin{array}{l}\text { yes } \\
\text { yes }\end{array}$ & $\begin{array}{l}\text { yes } \\
\text { yes }\end{array}$ & yes \\
\hline
\end{tabular}

Source: Lash et al. (2014).

- = no data; $\mathrm{CH} / \mathrm{CHL}=$ chloral/chloral hydrate; $\mathrm{CTK} / \mathrm{CTAC}=$ chlorothioketene/chlorothionoacetyl chloride;

$\mathrm{DCA}=$ dichloroacetic acid; DCAC $=$ dichloroacetylchloride; DCVC $=S$-dichlorovinyl-L-cysteine; DCVG $=S$-dichlorovinylglutathione; DCVCS = DCVC sulfoxide; DCVT $=S$-dichlorovinyl thiol; NAcDCVC $=N$-acetyl DCVC; NAcDCVCS $=N$-acetyl DCVCS; TCA = trichloroacetic acid; TCE-O = trichloroethylene oxide; TCOG = trichloroethanol-glucuronide conjugate;

$\mathrm{TCOH}=$ trichloroethanol. 


\subsection{Toxicokinetic Data}

The kinetics of trichloroethylene metabolism for the oxidative and GSH conjugation pathways and elimination of metabolites are described below. Since reactive metabolites are responsible for trichloroethylene toxicity, especially for the liver and kidney (USEPA 2011a), it is important to understand the factors that affect the flux through each metabolic pathway.

\subsubsection{Oxidative Metabolism}

The oxidative metabolites of trichloroethylene proposed to contribute to liver carcinogenicity are chloral hydrate, TCA, TCOH, and DCA (see Figure 1-1 and Section 6.2). The initial oxidative step that produces chloral hydrate is critical because this is the rate-limiting step in formation of TCA and DCA, which are the putative toxic metabolites (USEPA 2011a). Mice have a greater oxidative metabolic capacity for trichloroethylene (i.e., higher Vmax) than either rats or humans (see Table B-1). However, human liver microsomes generally showed a higher affinity (i.e., lower $\mathrm{K}_{\mathrm{m}}$ ) than rat or mouse microsomes. Thus, the lower apparent $\mathrm{K}_{\mathrm{m}}$ in humans may partially offset the lower Vmax resulting in similar clearance efficiencies $\left(\mathrm{Vmax} / \mathrm{K}_{\mathrm{m}}\right)$ compared with rodents. Rat kidney microsomes also had a much lower affinity for trichloroethylene than rat liver microsomes. $\mathrm{K}_{\mathrm{m}}$ values for $\mathrm{TCOH}$ formation were much lower than for TCA formation and are consistent with TCOH formation predominating over TCA formation in all three species (see Table B-2). Since the metabolism of chloral hydrate to TCA and TCOH involves several enzymes and cofactors, changes in the cellular cofactor ratio or redox status in the liver could impact the relative amounts of TCOH and TCA produced. In humans, the total amount of TCA excreted may be similar to the amount of TCOH because TCA has a much longer urinary half-life.

Lipscomb et al. (1997) reported that $K_{m}$ values were not normally distributed and could be separated into three statistically distinct populations among 23 human hepatic microsomal samples (see Table B-1). $\mathrm{K}_{\mathrm{m}}$ values were significantly higher $(33.1, \mathrm{~N}=13)$ in males than in females $(21.9, \mathrm{~N}=10)$ but Vmax values were not significantly different. Vmax values were normally distributed and generally correlated with increasing $K_{m}$ values. Lipscomb et al. (1998b) compared the metabolism of trichloroethylene in pooled human, mouse, and rat liver microsomes at different concentration ranges. $K_{m}$ values in rats showed marked differences at different concentration ranges while those for mice and humans were constant. These data indicate that several CYP isoforms with different $\mathrm{K}_{\mathrm{m}}$ values (high-, medium-, and low-affinity forms) metabolize trichloroethylene in the rat. High concentrations of trichloroethylene (1,000 ppm) inhibited CYP2E1 activity but increased CYP1A1/1A2 activity in all three species. Elfarra et al. (1998) reported species- and sex-related differences in kinetics of trichloroethylene metabolism. Vmax and $V \max / K_{m}$ values from female mouse liver microsomes were consistently higher than values from the corresponding male mouse liver microsomes or rat and human liver microsomes. There were no sex-related differences in the rates of metabolism with rat or human microsomes. Rat and human microsomes exhibited biphasic kinetics consistent with the involvement of both low-affinity and high-affinity enzymes while mouse liver microsome kinetics were described by single values for $K_{m}$ and $V \max$. 


\subsubsection{GSH Conjugation}

The GSH-conjugation pathway results in formation of reactive species several steps downstream from the initial conjugation, and some of these metabolites (particularly DCVC) are nephrotoxic (see Figure 1-2 and Section 4.2) (USEPA 2011a). In vitro studies of trichloroethylene conjugation show considerable intra- and interspecies differences and, in some cases, contradictory results. For example, conjugation rates reported by Green et al. (1997) and Dekant et al. (1990) were orders of magnitude lower than those reported by Lash et al. (1999a) and Lash et al. (1998). Green et al. (1997) also reported some DCVG formation in rat liver cytosol while Dekant et al. (1990) did not. The reasons for the discrepancies are not completely understood but may be explained in part by different analytical methods (Lash et al. 1999a; Lash et al. 2000a; USEPA 2011a). However, serum concentrations of DCVG and DCVC collected from rats exposed to an oral dose of $1,970 \mathrm{mg} / \mathrm{kg}$ (Lash et al. 2006) were comparable (i.e., within an order of magnitude) to those obtained in mice exposed to an oral dose of $2,140 \mathrm{mg} / \mathrm{kg}$ in a more recent study (Kim et al. 2009a; Kim et al. 2009b).

DCVG formation was significantly higher in liver cells from male rats compared with female rats while the rates in kidney cells and subcellular fractions were comparable for both sexes. Rates of DCVG formation were significantly higher in male mouse liver microsomes and kidney cytosol compared with females, but female mice had higher rates in kidney microsomes. Overall, DCVG formation was unexpectedly higher in mice than in rats. There were no significant sexrelated differences in DCVG formation in humans; however, the rate of GSH conjugation in human liver spanned a range of 2.4-fold in cytosol and 6.5-fold in microsomes (Lash et al. 1999a). Although the data show that rates of trichloroethylene conjugation are higher in human liver and kidney subcellular fractions [with the exception of Green et al. (1997)] than in rats or mice (Table B-3), there is significant uncertainty in the quantitative estimation of DCVG formation from trichloroethylene.

Reported $\mathrm{K}_{\mathrm{m}}$ constants and Vmax values of GSH conjugation from pooled human kidney and liver cells and subcellular fractions and rat kidney proximal tubular cells (Table B-4) show that the liver is the primary site of GSH conjugation; however, the kidney also has significant capacity to catalyze DCVG formation. Further, conjugation of trichloroethylene in all systems, with the exception of human hepatocytes and kidney subcellular fractions, included two kinetically distinct processes (high affinity and low affinity). In human hepatocytes, DCVG formation exhibited time-, trichloroethylene concentration-, and cell concentration-dependent formation (Lash et al. 1999a). Maximum formation occurred with 500-ppm trichloroethylene but decreased at concentrations of 1,000 ppm and above. DCVG formation in liver and kidney subcellular fractions exhibited time-, protein concentration-, and both trichloroethylene and GSH concentration-dependent formation.

Most DCVG is converted to DCVC in a two-step process involving GGT and CGDP (see Figure 1-2). GGT activity is concentrated in the microsomal fraction of the cell and is much higher in the kidney than the liver in rodents and humans (USEPA 2011a). GGT activity in rat kidney microsomes were about two-fold greater than in humans and about 20-fold greater than in mice (Lash et al. 1998; Lash et al. 1999a). Whole organ CGDP activity also was higher in the kidney than liver in all mammalian species tested (Hinchman and Ballatori 1990). 
As mentioned above, three potential bioactivating pathways for DCVC are cysteine conjugate $\beta$ lyase, FMO3, and CYP3A. Limited data were available describing species differences in the activities of these metabolic enzymes. Lash et al. (2000a) compiled $\beta$-lyase activity and kinetic parameters ( $\mathrm{K}_{\mathrm{m}}$ and $\mathrm{Vmax}$ ) in kidney cytosol from rats, mice, and humans for several cysteine conjugates (Table B-5). These data show that $\beta$-lyase activity varies with substrate and laboratory but is higher in rats compared with humans or mice.

FMO3 is the predominant FMO isoform in the adult human liver and orthologues from various species were catalytically similar (Ripp et al. 1999). Sulfoxide formation (nmol sulfoxide/min/mg protein) was sex-dependent in mice and dogs (higher in females), but not in humans, rats, or rabbits. Sulfoxide formation was highest in rabbit liver microsomes followed by humans and rats. Data for kidney microsomes were highest for rats and were similar to values derived from rat liver microsomes. S-Oxidase activity in mouse kidney microsomes was lower than observed in mouse liver microsomes and did not show sex-dependence. No data were available for human kidney microsomes in this study. $\mathrm{K}_{\mathrm{m}}$ and Vmax values obtained from incubating DCVC with membrane fractions of bacteria expressing human or rabbit FMO3 cDNA in the presence of NADPH were similar. In another study, DCVC sulfoxidation was detected with FMO3 but not with other isoforms (Krause et al. 2003). Incubations of DCVC with human liver microsomes resulted in detection of the corresponding sulfoxide but not when incubated with kidney microsomes. Expression levels of FMO1 (3.2 to $11.5 \mathrm{pmol} / \mathrm{mg}$ protein) and FMO5 (trace to $5.8 \mathrm{pmol} / \mathrm{mg}$ protein) were higher than FMO3 levels (trace to $1.3 \mathrm{pmol} / \mathrm{mg}$ protein) in human kidney samples. There were no data on species differences in CYP3A-mediated sulfoxidation of NAcDCVC (USEPA 2011a).

\subsubsection{Comparative Elimination Half-lives}

Reported plasma half-lives of trichloroethylene metabolites were much shorter in rodents than in humans (Lash et al. 2000a). Plasma half-lives of trichloroacetic acid in humans ranged from 51 to 99 hours compared with 3 to 16 hours in rodents. The plasma half-lives of trichloroethanol were about 12 hours in humans and 3 hours in mice. Reported half-lives for chloral hydrate and trichloroethanol glucuronide were 3 and 5 hours, respectively, in mice but these metabolites were not detected in humans exposed to 100 ppm for 4 hours. Lash et al. (1999b) reported that the elimination half-life of DCVG in blood of human volunteers was 0.74 hours in males and 0.94 hours in females. Several studies have investigated urinary elimination half-lives of trichloroacetic acid and trichloroethanol in workers exposed to trichloroethylene (reviewed by U.S. EPA (2011a)). Urinary trichloroacetic acid levels exhibited marked saturation at exposure $>50$ ppm while trichloroethanol did not. Reported half-lives for trichloroethanol ranged from about 15 to 43 hours compared with 40 to 58 hours for trichloroacetic acid. The elimination halflives for both metabolites were higher in females than in males. Urinary elimination kinetics also were faster in rodents than in humans with some studies reporting complete elimination within 1 to 2 days.

\subsubsection{Relative Roles of the CYP and GSH Pathways}

Comprehensive mass-balance studies are unavailable in humans, but studies in rodents given 2 to $2,000 \mathrm{mg} / \mathrm{kg}\left[{ }^{14} \mathrm{C}\right]$-trichloroethylene reported that $95 \%$ to $99 \%$ of radioactivity excreted in urine was attributed to oxidative metabolites (USEPA 2011a). Genetic polymorphisms or exposure to CYP inducers or inhibitors can alter the balance between oxidation and GSH conjugation of 
trichloroethylene (Lash et al. 2014). Impacts may be more substantial at higher substrate concentrations where the Vmax may become more limiting than hepatic blood flow. Reported ratios of primary oxidative metabolites to NAcDCVC in urine ranged from 986 to 2,562:1 in rats and 3,292 to 7,163:1 in humans. Although NAcDCVC is a useful indicator of GSH conjugation, it likely represents only a small fraction of trichloroethylene flux through this pathway. The range of kinetic data for oxidation and conjugation of trichloroethylene derived from in vitro studies show substantial overlap (Table B-6) and suggest that the total flux through the GSH pathway is much more substantial than estimates derived from urinary mercapturates $(<0.1 \%)$ alone would suggest. Lash et al. (1999b) also reported that maximum blood concentrations of DCVG in human volunteers exposed to trichloroethylene vapors (50 or $100 \mathrm{ppm})$ were similar to those of TCA and TCOH in the same subjects; however, the area under the curve (AUC) values for the oxidative metabolites were much higher than those for DCVG. DCVG blood concentrations were higher in males $(46.1 \pm 14.2 \mathrm{nmol} / \mathrm{mL})$ than in females $(13.4 \pm 6.6 \mathrm{nmol} / \mathrm{mL})$ in this study but elimination half-lives were similar. Based on an analysis of the distribution of individual values for DCVG in blood the results could indicate the existence of two subpopulations of individuals with a genetic polymorphism rather than a true gender difference. Lash et al. (1999a) also noted that GSH conjugation of trichloroethylene in vitro was inhibited by about $50 \%$ in the presence of the oxidative pathway while the addition of GSH had no effect on CYP-catalyzed formation of chloral hydrate.

EPA (2011a) developed an updated physiologically based pharmacokinetic model (PBPK) for trichloroethylene and its metabolites. A hierarchical Bayesian population analysis using Markov chain Monte Carlo sampling was performed to evaluate uncertainty in population parameters and variability within a population. Simulations for a number of representative dose-metrics across species were conducted to predict the fraction of trichloroethylene metabolized by oxidative or GSH-conjugation pathways (liver and kidney) under conditions of continuous inhalation or oral exposure. Results from these simulations for humans show that the fraction metabolized by oxidation decreases at higher doses while the fraction metabolized by GSH-conjugation increases with dose (Figure 1-3 and Figure 1-4). 


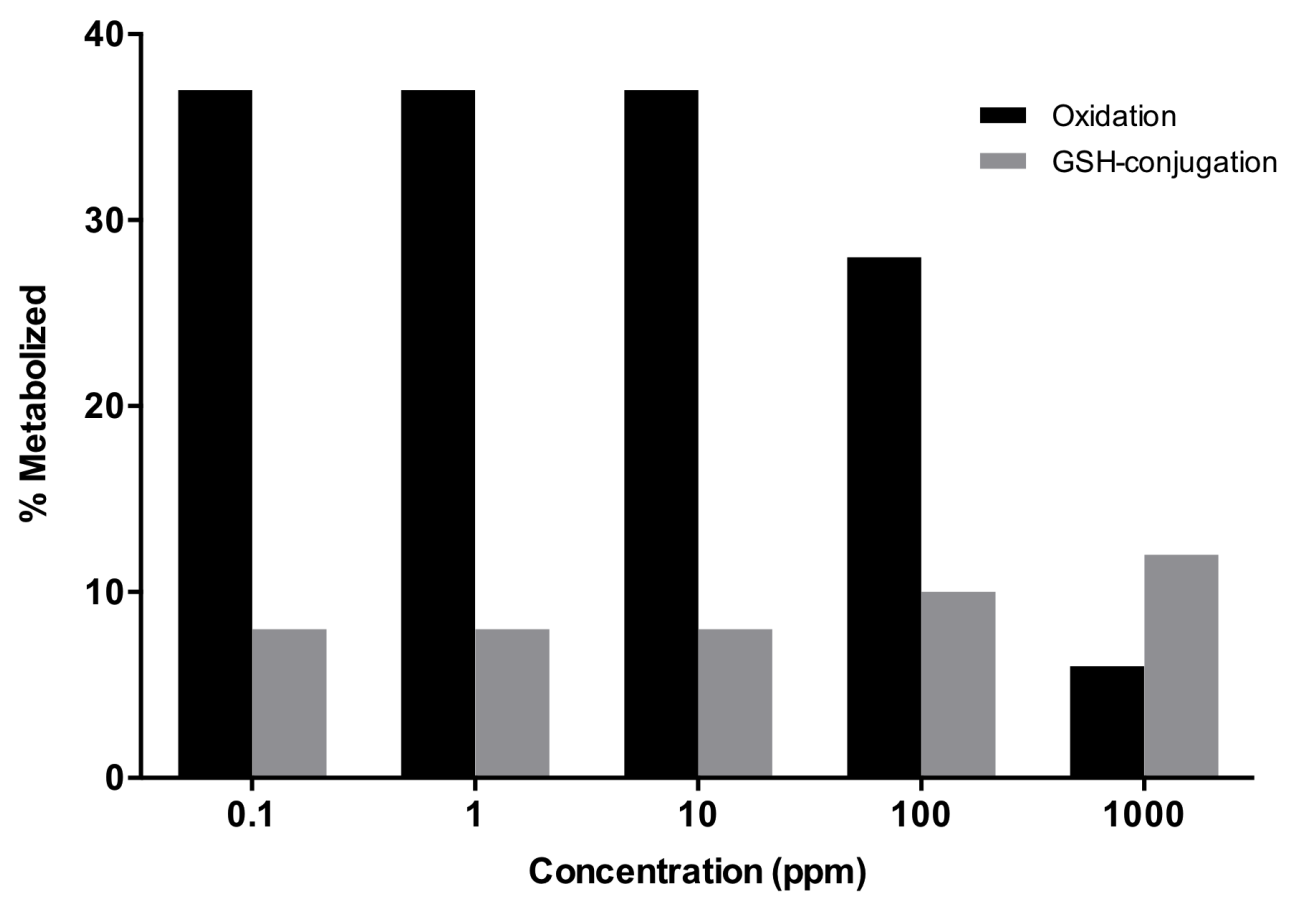

Figure 1-3. PBPK Model Predictions for the Fraction of Trichloroethylene Intake That Is Metabolized under Continuous Inhalation Exposure in Humans

Source: EPA (2011a).

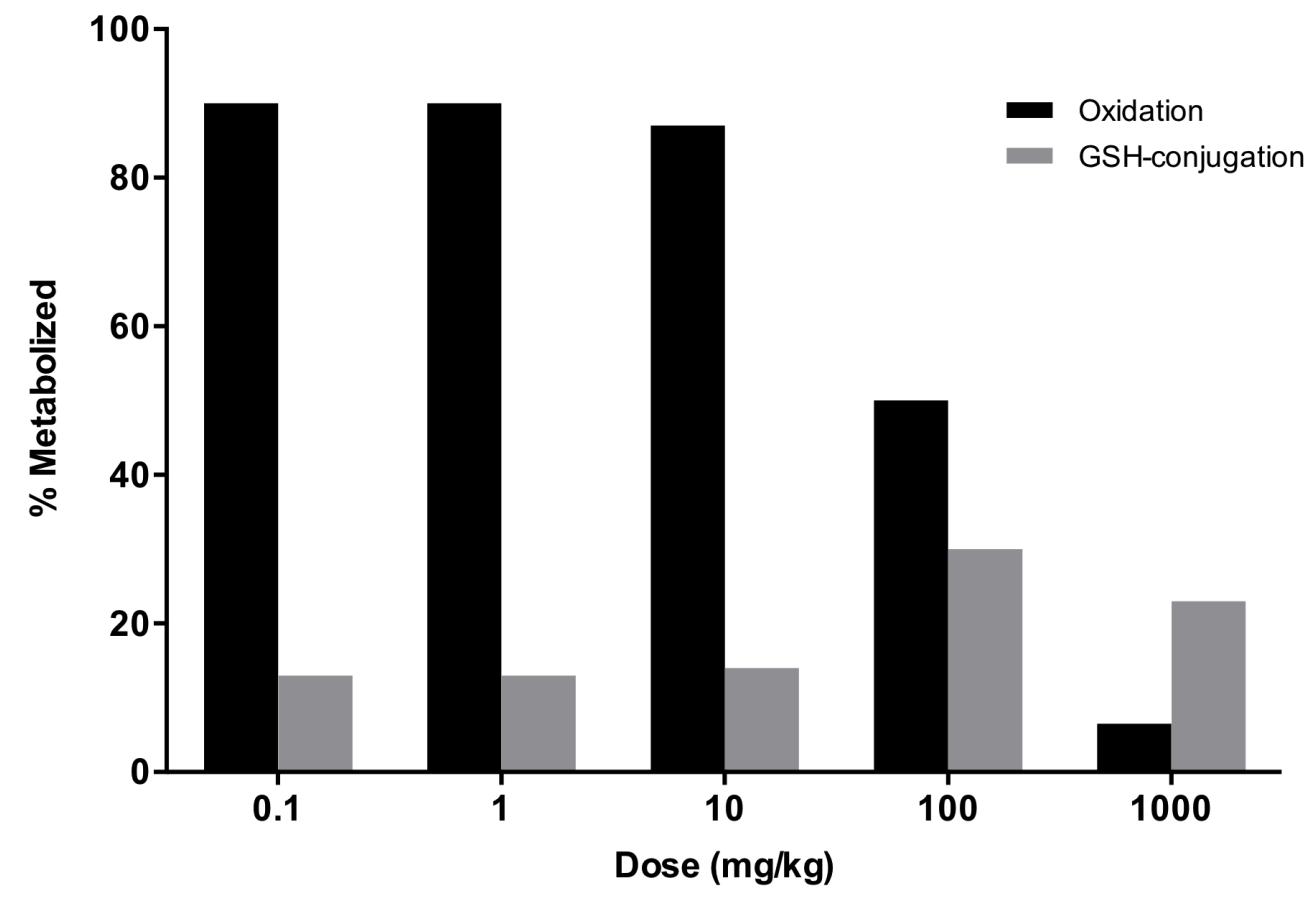

Figure 1-4. PBPK Model Predictions for the Fraction of Trichloroethylene Intake That Is Metabolized under Continuous Oral Exposure in Humans 


\subsection{Synthesis and Summary}

Trichloroethylene is a small, lipophilic compound that readily crosses biological membranes. Studies in humans and experimental animals confirm that trichloroethylene is rapidly and efficiently absorbed following oral, inhalation, or dermal exposure. Distribution from blood to tissues is determined by the blood:tissue partition coefficients, which are largely related to tissue lipid content. High concentrations, relative to blood, occur in the kidney, liver, brain, and fat. Adipose tissue may serve as a reservoir for trichloroethylene, thus prolonging internal exposure. Metabolism is complex; however, two distinct metabolic pathways have been identified that are common to all mammalian species studied: CYP oxidation and GSH conjugation. These pathways operate in parallel. Important sites of metabolism include the liver, kidneys, lungs, blood, and male reproductive system. Oxidation is the predominant pathway and CYP2E1 is the primary isoform involved. Trichloroethanol, trichloroethanol-glucuronide, and trichloroacetic acid are the primary oxidative metabolites detected in blood and urine of humans and laboratory animals. Chloral and chloral hydrate also are formed but are rapidly metabolized. The GSH conjugation pathway produces several metabolites (DCVG, DCVC, DCVCS, DCVT, NAcDCVC, NAcDCVCS, chlorothioketene, and chlorothionoacetyl chloride); however, only NAcDCVC is stable enough to be detected in urine. Trichloroethylene is eliminated unchanged or as carbon dioxide in expired breath. Metabolites are primarily eliminated in the urine with generally smaller amounts eliminated in feces. Conjugated metabolites may be excreted in the bile and reabsorbed from the gastrointestinal tract via enterohepatic recirculation. Although metabolic pathways and metabolites detected in humans and laboratory animals are qualitatively similar, the data show substantial quantitative intra- and interspecies variability that may explain differences in susceptibility to toxic effects. Much of the variability is due to gender-, species-, and individual-dependent differences in content and activity of key metabolic enzymes (CYP2E1 and GSTs).

In vitro data indicate that mice have a higher oxidative metabolic capacity for trichloroethylene than rats or humans, but the variability within species can be 2- to 10 -fold. However, $\mathrm{K}_{\mathrm{m}}$ values derived from liver microsomal metabolism of trichloroethylene indicate that affinity is higher in humans than rodents. Thus, the clearance efficiency of oxidative metabolites $\left(\mathrm{Vmax} / \mathrm{K}_{\mathrm{m}}\right)$ is similar among these species when exposed to low concentrations. There is evidence that humans can be divided into statistically distinct populations based on $\mathrm{K}_{\mathrm{m}}$ values. Overall, females have a significantly higher affinity (lower $\mathrm{K}_{\mathrm{m}}$ ) than males. Rat and human liver microsomes exhibited biphasic kinetics (consistent with the involvement of low-affinity and high-affinity enzymes) while incubations with mouse liver microsomes were monophasic. Reported plasma and urinary elimination half-lives of oxidative metabolites were shorter in rodents than in humans.

As with oxidative metabolism, in vitro studies of GSH conjugation of trichloroethylene in mice, rats, and humans show considerable intra- and interspecies variability. Conjugation rates also differed by several orders of magnitude between laboratories. The reasons for the discrepancies have not been fully resolved, thus, there is considerable uncertainty in quantitative estimates associated with this pathway. Most in vitro studies with subcellular fractions from the liver and kidney showed that two kinetically distinct processes (high affinity and low affinity) were involved in GSH conjugation of trichloroethylene. One study reported that the rate of GSH conjugation spanned a range of 2.4-fold in human liver cytosol and 6.5-fold in microsomes. The activities of two important enzymes in this pathway (GGT and $\beta$-lyase) were higher in rat kidney 


\section{RoC Monograph on Trichloroethylene}

cytosol than in mice or humans; however, the rate of DCVG formation was higher in mouse liver and kidney subcellular fractions than in rats. Although oxidation clearly is the predominant metabolic pathway, the range of in vitro kinetic estimates for the two pathways showed substantial overlap and indicated that the total flux through the GSH pathway was higher than estimates derived solely from urinary metabolites. 


\section{Genotoxicity and Related Effects}

This section addresses genotoxicity and related biological adverse effects that are possibly involved in the mode of action of trichloroethylene-induced carcinogenicity. Genotoxicity is well recognized as a characteristic of many carcinogenic chemicals and a key event for many malignant diseases. The mechanistic implications of these genotoxic effects are discussed in subsequent sections. Related effects, such as cell transformation and DNA and protein binding, are included in the review when data were available.

Trichloroethylene has been tested in short-term assays to evaluate mutagenicity and other potential genotoxic effects. The data presented in Section 2 are a compilation of evidence in studies available from authoritative reviews (IARC 1995; NRC 2006; USEPA 2011a) as well as a few recently published primary peer-reviewed articles. Trichloroethylene is often stabilized using a number of different chemicals, such as epichlorohydrin or 1,2-epoxybutane (both of which are potent mutagens); the presence of these stabilizers and/or the purity of trichloroethylene test substance are noted if that information is available.

While many variables in experimental design can affect the results of studies and create apparent discrepancies in responses for the same endpoint, two in particular are pertinent to trichloroethylene exposures, chemical volatility and the choice of solvent used for test agents. Another potential cause for differences in results between studies includes cytotoxicity or other physiological changes to the test organism, which can affect results but is not always measured and/or reported. Finally, consideration of the positive or negative result should be informed by the study design and reporting (e.g., it is possible that an impure test sample could result in a 'positive' result that is actually due to a contaminant). For example, when epichlorohydrin or 1,2-epoxybutane is present as a stabilizer in the test sample of trichloroethylene, an observed mutagenic response could actually be due to those chemicals rather than to the trichloroethylene. Conversely, false 'negative' results could occur if the study design is not optimal; the use of inappropriate treatment methods or assay type could compromise the results. For example, a volatile chemical may test "negative" in a standard mutagenicity assay but "positive" when the assay is modified for testing vapor phase samples. In addition, solvents such as DMSO can react chemically with some test chemicals, including raising the $\mathrm{pH}$, which could result in effects that would not otherwise be observed; thus, careful consideration should be made of assays in which reactive solvents are used.

Results from studies on the genotoxic effects of trichloroethylene are summarized in tables in Appendix $C$ and an overall summary call is provided by endpoint in Table 2-1, based on the integration of the evidence from authoritative reviews (IARC 2014) and any additional studies identified.

\subsection{In Vitro Mutagenicity Studies of Trichloroethylene in Bacteria}

Trichloroethylene exposure induced mutants in Salmonella typhimurium tester strain TA100 in several, but not all, studies that tested pure (no stabilizer) samples of trichlorethylene. Although results in other strains (TA97, TA98, and TA1537) were negative, the positive results in stain TA100 are attributed to base-pair substitution and thus provide some evidence for mutagenicity 
of trichloroethylene in the presence of metabolic activation (IARC 2014). Results from these studies are discussed below and summarized in Table C-1.

Trichloroethylene without stabilizers (high purity) induced a slight, but reproducible, response in most, but not all, studies using Salmonella strain TA100, with the addition of exogenous metabolic activation (S9). Of the five positive studies in TA100 that tested samples without stabilizers, only one used DMSO as a solvent (see Section 2.7.1 for a discussion of the potential interaction between DMSO and TCA), suggesting that the solvent used did not affect the results. Trichloroethylene was weakly positive in one study with strain TA1535, tested without S9. A negative response was noted for all other strains, either with or without S9. Different tester strains of Salmonella are designed to detect the type of mutation that is induced. Negative results in TA97, TA98, and TA1537 suggest that trichloroethylene does not induce frameshift mutations while the positive results observed for strains TA1535 and TA100 are attributed to base-pair substitution. In addition, strain TA100 was derived from TA1535 with the addition of plasmid pKM101, which makes it more sensitive and could explain the results observed with these two strains. Some mutagenic activity was reported in multiple Salmonella strains when impure trichloroethylene or trichloroethylene with stabilizers was used as the test agent. One study reported a mutagenic response but only at high levels of toxicity (McGregor et al. 1989). A study utilizing a Salmonella strain competent in CYP2E1 metabolism (Emmert et al. 2006) reported mutagenic effects and there was a low-level (two-fold) response at a single locus ( $\arg 56)$ observed in a reversion assay using $E$. coli $\mathrm{K} 12$, but only with the addition of metabolic activation; however, DMSO was used in this study. Furthermore, the use of certain solvents (e.g., DMSO, ethanol) can be a concern if they chemically interact with the test compound (see discussion in Section 2.7.1) or affect key metabolizing enzymes such as CYP2E1, which can lead to false negative results.

Mutagenicity studies of trichloroethylene in wastewater suggest that the parent compound or its metabolites interact with other chemicals present in the water to enhance the genotoxicity of complex mixtures, based on the results from tests with trichloroethylene alone or in the wastewater. In a study by Tabrez and Ahmad (2012), wastewater samples contaminated with trichloroethylene (determined by gas chromatography analysis to be 28.4 and $8.97 \mathrm{mg} / \mathrm{L}$ were mutagenic in the Ames fluctuation assay using S. typhimurium strains TA98 and TA100. The authors reported that exposure to trichloroethylene alone at concentrations up to $1,000 \mathrm{mg} / \mathrm{L}$ did not induce mutations in the assay. However, there was a significant increase in mutant induction when the wastewater samples plus $100 \mathrm{mg} / \mathrm{L}$ trichloroethylene (purity not reported) were tested, both with and without S9 activation. No determination of cytotoxicity was reported in this study.

\subsection{In Vitro Genotoxicity Studies of Trichloroethylene in Non- mammalian Eukaryotes}

Results of in vitro genotoxicity studies of trichloroethylene in non-mammalian eukaryotes are summarized in Table C-2. Positive effects were observed in several studies, for both pure (no stabilizers) test samples and those of unknown purity; none of these studies used DMSO as a solvent. Overall, there is limited evidence for genotoxic activity of trichloroethylene in fungi, and possibly plants, and this activity is most likely mediated by its metabolites.

In fungi, trichloroethylene has been evaluated for gene mutation, conversion, and recombination, as well as mitotic segregation and aneuploidy. There is some evidence that trichloroethylene 
causes gene conversion and gene mutation in Saccharomyces cerevisiae D7 in the presence of metabolic activation. Evidence for gene conversion comes from positive findings in two of three studies (Bronzetti et al. 1978; Callen et al. 1980), one of which used trichloroethylene that did not contain stabilizers (Bronzetti et al. 1978); findings were negative in strain D4, which has a lower activity of CYP than strain D7. Trichloroethylene exposure caused gene mutations in all three studies in S. cerevisiae D7 including one study using a preparation that did not use stabilizers, and in actively growing (not quiescent) cultures of the mold Aspergillus nidulans (Crebelli et al. 1985). However, trichloroethylene was not mutagenic in the yeast Schizosaccharomyces pombe, either with or without S9 activation (Rossi et al. 1983). Trichloroethylene also caused aneuploidy in S. cerevisiae D7 (with and without activation) and recombination and mitotic crossover in $S$. cerevisiae D7 (with metabolic activation) but not in quiescent or growing $A$. nidulans cells. Interpretation of these endpoints is limited because purity of trichloroethylene is not known in any of the studies.

In the study of wastewater genotoxicity described above, wastewater samples alone (which were contaminated with trichloroethylene) also induced a significant rise in chromosomal aberrations in the Allium cepa (onion) bulb genotoxicity test. Wastewater samples spiked with $100 \mathrm{mg} / \mathrm{L}$ trichloroethylene (purity not reported) increased the frequency of chromosomal aberrations. Since there was no effect of trichloroethylene exposure alone at up to $1,000 \mathrm{mg} / \mathrm{L}$, this suggests that trichloroethylene and/or its metabolites might have interacted with chemicals present in the wastewater to enhance the genotoxicity. No determination of cytotoxicity was reported in this study (Tabrez and Ahmad 2012).

\subsection{In Vitro Studies of Genotoxicity and Related Effects of Trichloroethylene in Mammalian Cells}

Several studies have examined the potential for trichloroethylene-induced genotoxicity in mammalian systems in vitro; findings are discussed below and summarized in Table C-3. These studies suggest that trichloroethylene causes genotoxicity in vitro, specifically DNA strand breaks, micronucleus formation, and sister chromatid exchanges in vitro. Importantly, some of these effects (DNA strand breaks and micronuclei) were observed in the kidney. A limitation of these studies is that, for many of them, the purity of trichloroethylene is unknown. Regarding the use of DMSO as a solvent in these studies, it does not appear to be a confounding issue. Very few studies included exogenous metabolic activation and the only two studies that reported positive results apparently used DMSO as a solvent. However, several assays reporting positive results were conducted using primary cells, which presumably have retained endogenous metabolic capability, and most of these studies did not use DMSO as a solvent. In addition, trichloroethylene also caused cell transformation, which can arise from genotoxic and nongenotoxic mechanisms.

Trichloroethylene exposure induced dose-dependent increases in micronucleus formation and DNA strand breaks in primary cultures of rat and human kidney cells and in the human hepatoma HepG2 cell line (Hu et al. 2008; Robbiano et al. 2004; Wang et al. 2001); these results may be due to trichloroethylene metabolites since cultured primary cells generally retain endogenous metabolic activation capabilities. However, there was also a significant increase in micronuclei

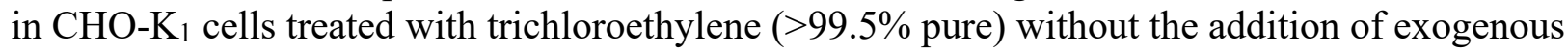
S9, suggesting metabolism was not needed for the observed effect (Wang et al. 2001) but not in 
human lymphocytes (Kumar et al. 2009). In vitro trichloroethylene exposure increased the frequency of sister chromatid exchange (SCE) in mammalian cells in two studies using pure samples (Galloway et al. 1987; Gu et al. 1981; White et al. 1979); a short exposure time, limited dose levels, and lack of a positive control limit the interpretation of the results of the third study (White et al. 1979). Trichloroethylene exposure did not induce chromosomal aberrations in Chinese hamster ovary or lung cells or in human lymphocytes (Galloway et al. 1987; Kumar et al. 2009; Sofuni et al. 1985).

Findings were inclusive for gene mutation; increased mutations were observed in mouse lymphoma cells treated with trichloroethylene (in the presence, but not absence, of exogenous metabolic activation S9); however, mutation was not reported in treated human TK6 cells, with or without S9 (Caspary et al. 1988). Results for trichloroethylene induction of unscheduled DNA synthesis (UDS) were negative in rat and mouse hepatocytes when pure samples were tested (Shimada et al. 1985; Williams et al. 1989), but results were mixed when test samples of trichloroethylene contained stabilizers or were of unknown purity (Costa and Ivanetich 1984; Milman et al. 1988; Shimada et al. 1985; Williams et al. 1989). A study in human lymphocytes showed a weak response for UDS induction after exposure to trichloroethylene; although the test sample presumably did not contain stabilizers and the DMSO concentration was only $1 \%$ (IARC 2014).

Cell transformation was induced by trichloroethylene in BALB/c-3T3, rat embryo cells, and Syrian hamster embryo cells (Amacher and Zelljadt 1983; Price et al. 1978; Tu et al. 1985). Cell transformation assays may not indicate a genotoxic mechanism.

\subsection{Studies of Nucleic Acid and Protein Binding of Trichloroethylene}

Binding of trichloroethylene to nucleic acids and proteins has been studied in cell-free systems and in vivo in rodents and are discussed below and summarized in Table C-4. The available evidence shows that trichloroethylene can bind both DNA and protein. None of the reviewed studies reported using DMSO as a solvent.

In vitro trichloroethylene exposure results in binding to nucleic acids and protein and is likely dependent on metabolite formation, with mouse microsomes inducing a higher level of binding than rat microsomes. Incubation with ${ }^{14} \mathrm{C}$-labeled trichloroethylene resulted in covalent binding to salmon sperm DNA (Banerjee and Van Duuren 1978), calf thymus DNA (DiRenzo et al. 1982), and rat and mouse hepatocyte DNA (Miller and Guengerich 1983). Binding was observed in microsomal proteins from mouse and rat liver, lung, stomach, and kidney (Banerjee and Van Duuren 1978; Miller and Guengerich 1983) and human liver (Miller and Guengerich 1983). All but one of these in vitro studies used test samples that did not contain stabilizers; Mazzullo et al. (1992) used $98.9 \%$ pure trichloroethylene, which may have contained stabilizers or impurities (IARC 2014). Studies showing significant binding of trichloroethylene metabolites to DNA and protein postulated that trichloroethylene oxide, which is formed as an oxidative intermediate in trichloroethylene metabolism in rodent microsomes, is the form that binds most readily to protein (Cai and Guengerich 2001) and, to a lesser extent, DNA (Miller and Guengerich 1983).

Phenobarbital pretreatment increased the formation of the trichloroethylene metabolites chloral hydrate $(\mathrm{CH})$ and trichloroethylene oxide and increased the formation of DNA and protein adducts (Miller and Guengerich 1983). 
Studies in vivo provide evidence of binding to protein and DNA in both mice and rats following trichloroethylene administration. Protein binding was observed in both liver and kidney from $\mathrm{B}_{6} \mathrm{C}_{3} \mathrm{~F}_{1}$ mice but not Osborne-Mendel rats exposed to trichloroethylene by inhalation (Stott et al. 1982). In the same study, results for DNA binding in the liver for mice treated orally were inconclusive. However, a second study reported weak DNA binding in the liver, kidney, lung, and stomach of both the BALB/c mouse and the Wistar rat exposed to trichloroethylene by i.p. injection; test sample purity was 98.9\% (Mazzullo et al. 1992). NMRI mice treated i.p. with trichloroethylene ( $\geq 99 \%$ pure) showed increased radioactivity in nucleic acids for several tissues (spleen, lung, kidney, pancreas, testis, and brain), this effect was due to the metabolic incorporation of ${ }^{14} \mathrm{C}$-labeled nucleotides directly into DNA and RNA, especially guanine and adenine, rather than adduct formation (Bergman 1983).

\subsection{In Vivo Genotoxicity Studies in Rodents}

Trichloroethylene has been tested for genotoxicity in vivo and study results are discussed below and summarized in Table C-5. Overall, there is some evidence that trichloroethylene can induce DNA strand breaks and micronucleus formation, depending on the tissue, in rodents. These two endpoints are consistent with in vitro studies, and similar to in vitro studies, positive findings were observed in the kidney. Studies on the effects of trichloroethylene exposure at other endpoints, including gene mutation, chromosomal aberrations, SCE, and UDS, were all negative (see Table C-5 and IARC (2014)). DMSO was probably not used in any of the studies (there were only two studies where its use was unknown).

Trichloroethylene caused DNA strand breaks in liver in a study in rats (Nelson and Bull 1988) and in two of three studies in mice (Nelson and Bull 1988; Parchman and Magee 1982; Robbiano et al. 2004). Findings in kidney were positive in the mouse (Walles 1986) but inconsistent in the rat. Robbiano et al. (2004) reported positive findings in the rat kidney after a single exposure to $3,591 \mathrm{mg} / \mathrm{kg}$ trichloroethylene (reagent grade purity) by oral administration, while a 5-day 2,000ppm inhalation study ( $99.5 \%$ pure test sample, no information on stabilizers) yielded negative results (Clay 2008). Differences do not seem to be explained by dose because the dose from the inhalation exposure may have been higher than the oral dose. Estimated inhalation exposure is $10,800 \mathrm{mg} / \mathrm{kg}$ /day assuming $100 \%$ absorption, which is most likely lower at high exposures, such as 2,000 ppm, and thus would result in a lower estimated $\mathrm{mg} / \mathrm{kg} / \mathrm{day}$ dose.

Trichloroethylene exposure in vivo induced micronucleus formation in kidney cells of rats treated orally (Robbiano et al. 2004). For rats treated by inhalation, one study reported doserelated micronucleus induction in bone marrow erythrocytes after a single inhalation exposure; the authors replicated the findings in a subsequent one-dose experiment (Kligerman et al. 1994). No increase in micronucleus formation was observed in a 4-day inhalation exposure by the same authors; however, the authors noted that the micronucleus formation in the concurrent controls was unusually high. A negative finding was reported in a single inhalation exposure study by a different author (Wilmer et al. 2014). All of the studies used trichloroethylene exposure without stabilizers. No increase in micronucleus formation was observed in peripheral blood lymphocytes after inhalation exposure (Kligerman et al. 1994). In studies in the mouse, there was micronucleus induction in the bone-marrow erythrocytes of exposed animals in two of four studies (Duprat and Gradiski 1980; Hrelia et al. 1994; Kligerman et al. 1994; Shelby et al. 1993), which used different routes of exposure (inhalation, i.p. and p.o.) and strains of mice. One study reported a correlation with urinary $\mathrm{TCOH}$, which strengthens the findings (Hrelia et al. 1994). 
No increase in micronuclei was observed in either splenocytes or spermatocytes from mice exposed to trichloroethylene by inhalation (Allen et al. 1994; Kligerman et al. 1994).

\subsection{Studies of Genotoxicity in Humans Exposed to Trichloroethylene}

A few studies have examined cytogenetic endpoints in peripheral blood lymphocytes of trichloroethylene-exposed workers, including one that evaluated chromosomal aberrations and three that measured SCEs. Findings from these studies are discussed below and summarized in Table C-6.

In addition, several case-control studies of renal-cell cancer evaluated mutations in the von Hippel-Lindau $(V H L)$ gene of trichloroethylene-exposed workers (see Section 4.2.2.1 and the associated table in that section).

The available database on cytogenetic studies is inadequate to evaluate conclusively because it is limited by small numbers of exposed workers in a few studies. In a group of Danish workers, Rasmussen et al. (1988) found statistically significant increases in chromosomal aberrations among 15 metal degreasers exposed to trichloroethylene for $>20$ hours per week. Conflicting findings were described for SCE induction. Although Gu et al. (1981) measured a statistically significant increase in SCE in 6 exposed workers, no increase was reported in a somewhat larger study of trichloroethylene-exposed workers (22) from Japan (Nagaya et al. 1989). Another study in Japan found statistically significant increases in SCE among male smokers but not among male or female non-smokers; smoking was not independently related to SCE in the study (Seiji et al. 1990).

\subsection{Genotoxic and Related Effects of the Metabolites of Trichloroethylene}

The metabolites of trichloroethylene have been tested in short-term assays to evaluate mutagenicity and other potential genotoxic effects. This section provides a summary of the available information from authoritative reviews (IARC 2014; USEPA 2011a) on several metabolites, including trichloroacetic acid (TCA), trichloroethanol (TCOH), dichloroacetic acid (DCA), chloral hydrate (CH), $S$-(1,2-dichlorovinyl)-L-cysteine (DCVC), $S$-(1,2dichlorovinyl)glutathione (DCVG) and $N$-acetyl DCVC (NAcDCVC). Information is available for all of these metabolites, but is limited to a few studies for TCOH, DCVG, and NAcDCVC. Results on the genotoxic effects of trichloroethylene metabolites are summarized in Table 2-1 and are based on the integration of the evidence provided from the authoritative reviews (IARC 2014), as well as any additional studies identified. A 2000 review of genotoxicity information for trichloroethylene and its metabolites discussed the mutagenic potency of trichloroethylene metabolites and reported that the oxidative metabolites required very high doses to induce an effect (Moore and Harrington-Brock 2000). In their evaluation, DCVC was the most potent mutagen while TCA was the least potent mutagen.

\subsubsection{Trichloroacetic Acid (TCA)}

Overall, there is weak evidence for the genotoxicity of TCA based on a recent study reporting it caused chromosomal aberrations in vivo; however, there is limited or no evidence for other 
genotoxicity endpoints. TCA was reported as non-mutagenic in almost all bacterial assays, both with and without exogenous metabolic activation (S9). Considering both in vitro and in vivo studies, findings for DNA strand breaks were mostly negative, and were mixed for micronucleus formation. Methodological concerns in the in vitro studies limited the interpretation of the evidence for other endpoints. Table 2-1 summarizes the conclusions for each genotoxic endpoint across studies) and details of the study findings are discussed below (as cited in IARC (2014) TCA Monograph, pp. 413-437).

TCA was tested for mutation in bacterial systems by numerous investigators (see IARC (2014)), with only two studies reporting a positive response. TCA induced mutation in assays using $S$. typhimurium TA1535 with metabolic activation in an SOS DNA repair assay and in strain TA100, both with and without metabolic activation, using a fluctuation assay (Giller et al. 1997; Ono et al. 1991). However, TCA did not induce mutations (with or without S9) in several $S$. typhimurium reverse mutation assays, using standard or special tester strains or protocols, nor in a lambda prophage assay in E. coli (IARC 2014). One of two studies of gene mutation in cultured mammalian cells reported a weak mutagenic effect (with the addition of S9) but both studies reported high cytotoxicity (Harrington-Brock et al. 1998; Zhang et al. 2010).

The acidity of TCA is an important consideration in evaluating in vitro test results. An earlier study by Nestmann et al. (1980) showed that TCA was mutagenic in S. typhimurium bacteria only when dissolved in DMSO; results were negative when water was used as the solvent. Their observations suggested that a short-lived mutagen was formed when the test compound TCA was dissolved in DMSO. In another experiment in the same study, the findings for mutagenicity were negative when water was used as the solvent. Decarboxylation of TCA in DMSO was described in a study by Laque and Ronneberg (1970) and showed a first order reaction that was ratedependent on the concentration of DMSO and availability of trichloroacetate ion. A report of increased chromosomal aberrations in cultured human peripheral lymphocytes exposed to TCA was considered by the authors (Mackay et al. 1995) to be related to a treatment-induced reduction in $\mathrm{pH}$, rather than due to direct genotoxic action of the TCA. Recent studies in cultured human peripheral blood lymphocytes exposed to TCA in vitro by Varshney et al. reported that TCA ( $0.3 \%$ DMSO in culture) increased micronucleus frequency (2013a) and chromosomal aberrations (2013b).

TCA also reportedly induced dose-related increases in DNA strand breaks as measured by the comet assay in human HepG2 liver carcinoma cells (Zhang et al. 2012) but not in CHO cells (Plewa et al. 2002; Plewa et al. 2010); no studies used DMSO as a solvent.

In vivo studies of TCA reported chromosomal aberrations in bone marrow cells of Swiss mice (Bhunya and Behera 1987) and chickens (Bhunya and Jena 1996). TCA induced micronucleus formation in the peripheral erythrocytes of newt larvae (Giller et al. 1997) and bone marrow erythrocytes of Swiss mice (Bhunya and Behera 1987) but not in C57BL/6JfBL10/Alpk mice (Bhunya and Behera 1987; Bhunya and Jena 1996; Mackay et al. 1995). Dose-dependent increases in DNA single-strand breaks were induced by TCA in studies in B6C3F 1 mouse liver (Hassoun et al. 2010a; Nelson and Bull 1988; Nelson et al. 1989) However, some subsequent studies by the same authors failed to confirm the original finding (Nelson et al. 1989) even in the presence of liver growth induction (Styles et al. 1991). In addition, oral treatment by TCA did not induce DNA single-strand breaks in liver or epithelial cells from the stomach or duodenum 
of $\mathrm{B} 6 \mathrm{C}_{3} \mathrm{~F}_{1}$ mice, nor in $\mathrm{F} 344$ rats following a single treatment by oral gavage (Chang et al. 1992).

\subsubsection{Trichloroethanol (TCOH)}

$\mathrm{TCOH}$ was negative in all bacterial mutagenicity tests without exogenous metabolic activation S9 (IARC 2014), but it did increase mutant frequency in the presence of S9 at a dose $>2,500 \mu \mathrm{g} /$ plate (NTP 1999). It also induced formation of micronuclei in vitro in cultured human peripheral lymphocytes (Varshney et al. 2013a) (see Table 2-1 for conclusions of the evidence across studies).

\subsubsection{Dichloroacetic Acid (DCA)}

There is some evidence for genotoxicity of DCA. Overall results for DCA in vitro show some evidence for mutagenicity both in vivo and in vitro and for DNA strand breaks in vivo but not in vitro. Mixed results were observed for chromosomal aberrations (in vitro only) and micronucleus induction (in vitro and in vivo). Table 2-1 summarizes the conclusions for each genotoxic endpoint across studies and details of the findings are discussed below (as cited in IARC (2014) Monograph of DCA, pp. 368-375).]

DCA was mutagenic in the bacteria S. typhimurium tester strains TA98 and TA100 in some studies, both with and without the addition of metabolic activation, but was not mutagenic in all other strains or in E. coli WP2 uvrA (DeMarini et al. 1994; Giller et al. 1997; Kargalioglu et al. 2002). Analysis of mutation spectra in TA100 indicates that DCA induces primarily GC-AT transitions in this strain. DCA also induced prophage (DeMarini et al. 1994) and weakly induced SOS repair (Giller et al. 1997) in E. coli. Exposure to DCA in vitro resulted in statistically significant increases in HGPRT mutant frequency in $\mathrm{CHOK}_{1}$ cells at a single concentration $(1,000 \mu \mathrm{M})$; it was cytotoxic at higher doses (Zhang et al. 2010). Harrington-Brock et al. (1998) reported dose-related increases in TK locus mutations and chromosomal aberrations in mouse lymphoma L5178Y/TK $K^{ \pm}-3.7 .2 \mathrm{C}$ cells treated with DCA. In contrast, Fox et al. (1996) found no evidence for elevated mutation levels in mouse lymphoma cells nor increased chromosomal aberrations in $\mathrm{CHO}$ cells after exposure to DCA.

Results were conflicting for DCA-induced micronucleus formation in vitro; a significant increase in micronuclei was reported in human peripheral blood lymphocytes (Varshney et al. 2013a) but not in L5178Y/Tk+/- mouse lymphoma cells (Harrington-Brock et al. 1998). Zhang et al. (2012) reported that DCA induced a dose-related increase in DNA damage by the comet assay after 4 hours of exposure in human HepG2 cells. However, treatment with DCA did not induce DNA strand breaks in several other cell types, including cultured primary rat or mouse hepatocytes, or in human CCRF-CEM lymphoblastoid cells in the DNA unwinding assay (Chang et al. 1992) nor in CHO or CHO-AS52 cells in the comet assay (Plewa et al. 2002; Plewa et al. 2010).

In vivo, DCA administered in drinking water induced lacI mutations in $\mathrm{B}_{6} \mathrm{C}_{3} \mathrm{~F}_{1}$ transgenic mice in a dose-related manner at 60 weeks; the induced mutations were $33 \%$ GC-AT transitions and 21\% GC-TA transversions (Leavitt et al. 1997). Fuscoe et al. (1996) reported increased micronucleus frequency in peripheral PCEs of male $\mathrm{B}_{6 \mathrm{C}} 3 \mathrm{~F}_{1}$ mice following subchronic (9 days) or chronic (>10 weeks) exposure to DCA, but not after a 28-day exposure; studies were negative in rat and newt larvae peripheral erythrocytes. However, two other studies reported negative results for micronuclei in bone-marrow erythrocytes of male and female Sprague-Dawley rats 
(Fox et al. 1996) and newt larvae peripheral erythrocytes (Giller et al. 1997). There is evidence that DCA induces single-strand breaks. Nelson and Bull (1988) and Nelson et al. (1989) reported increased DNA strand breaks in livers of $\mathrm{B}_{6} \mathrm{C} 3 \mathrm{~F}_{1}$ mice and Sprague-Dawley rats exposed to DCA orally. DNA strand breaks, alkali-labile sites, and crosslinking were also induced in blood leukocytes of male B6C3F 1 mice treated in drinking water (Fuscoe et al. 1996). However, there was no evidence of DNA strand breaks in the liver of male F344 rats, nor in the liver, spleen, or intestinal epithelium of male $\mathrm{B} 6 \mathrm{C}_{3} \mathrm{~F}_{1}$ mouse after oral or drinking-water treatment with DCA (Chang et al. 1992). Study results on DCA induction of micronuclei were also somewhat conflicting.

\subsubsection{Chloral Hydrate (CH)}

In vitro tests showed $\mathrm{CH}$ to be a direct mutagen and genotoxic for most of the endpoints tested, including the induction of DNA damage, chromosomal aberrations, and micronuclei. In other assays, $\mathrm{CH}$ caused non-disjunction and aneuploidy/polyploidy as well as transformed cells. A limited number of studies were conducted in vivo, and test results for many of these were inconsistent although there was some evidence suggesting that $\mathrm{CH}$ causes micronuclei (similar to the in vitro studies) in mouse bone marrow erythrocytes and spermatids, and mixed findings for DNA strand breaks, aneuploidy, and hyperploidy. Table 2-1 summaries the conclusions for each genotoxic endpoint across studies, and details of the findings are described below (as cited in IARC (2014) CH Monograph, pp. 452-462).

In several experiments in bacteria, $\mathrm{CH}$ exposure induced mutants in Salmonella tester strains TA100 and TA104, both with and without S9 metabolic activation; results in other strains were negative. Different tester strains of Salmonella are designed to detect different types of mutagenicity; positive results in TA100 are attributed to base-pair substitution so the overall response is considered positive for mutation. In the fungi Aspergillus nidulans, $\mathrm{CH}$ exposure caused aneuploidy and nondisjunction but not mitotic crossover (Crebelli et al. 1991; Kafer 1986; Kappas 1989). CH induced disomy and mitotic malsegregation in the yeast $S$. cerevisiae and was positive for wing-spot mutations, but negative for sex-linked lethal mutations, in Drosophila melanogaster (Albertini 1990; NTP 1999; Sora and Agostini Carbone 1987; Zordan et al. 1994). There were no increases in mutations in L5178Y/TK $-3.7 .2 \mathrm{C}$ cells (Liviac et al. 2011).

In vitro exposure to $\mathrm{CH}$ in mammalian cells, both with and without $\mathrm{S} 9$, resulted in increased SCEs and chromosomal aberrations. It also induced micronuclei and aneuploidy, as well as cell transformation in Syrian hamster cells (IARC 2014). Several studies reported positive results for micronucleus formation; the micronuclei were consistently kinetochore positive, indicating that they formed from whole chromosomes or larger chromosome segments rather than from chromosome fragments (Degrassi and Tanzarella 1988; Lynch and Parry 1993; Parry et al. 1990). Only one study evaluated micronucleus formation with the addition of exogenous metabolic activation S9; in that study, micronuclei were induced in lymphocytes in the absence, but not presence, of S9. An increase in cytotoxicity, but not micronucleus induction, was noted in human peripheral lymphocytes after a 48 -hour exposure to 25,50 , or $100 \mu \mathrm{g} / \mathrm{mL}$ of CH (Varshney et al. 2013a). There were no increases in micronucleus formation in peripheral human lymphocytes or TK6 cells (Liviac et al. 2010) or mutations in L5178Y/TK ${ }^{ \pm}-3.7 .2 \mathrm{C}$ cells (Liviac et al. 2011). 
Three studies reported that $\mathrm{CH}$ caused aneuploidy induction without exogenous metabolic activation in Chinese hamster cells; one study in mouse lymphoma cells reported it negative. $\mathrm{CH}$ exposure did not cause the formation of DNA-protein crosslinks in rat liver nuclei nor induce DNA single-strand breaks in rat primary hepatocytes (Chang et al. 1992; Keller and Heck 1988). A few studies have examined DNA binding of $\mathrm{CH}$ and adduct formation in $\mathrm{CH}$-exposed tissues and DNA. Keller and Heck (1988) demonstrated that protein from $\left[{ }^{14} \mathrm{C}\right]$ chloral-treated rat liver nuclei had a concentration-related binding of $\left[{ }^{14} \mathrm{C}\right]$ but did not observe DNA adducts. Other studies demonstrated an increase in malondialdehyde-derived DNA adducts and an increase in the levels of 8-oxoguanine adducts in livers of $\mathrm{CH}$-exposed mice, as well as increased $\mathrm{CH}$ adducts in calf thymus DNA (Ni et al. 1995; Von Tungeln et al. 2002).

There are a few in vitro studies of $\mathrm{CH}$ exposure in human cells. DNA single-strand breaks were induced after $\mathrm{CH}$ exposure in vitro in human lymphoblast TK6 cells (Liviac et al. 2010), but not HepG2 cells (Zhang et al. 2012), as measured by the comet assay.

Results of in vivo studies of genotoxicity following exposure to $\mathrm{CH}$ were limited by few studies for some endpoints and somewhat inconsistent results for others. $\mathrm{CH}$ induced DNA single-strand breaks in both mouse and rat liver in one study (Nelson and Bull 1988), but not in another (Chang et al. 1992). CH exposure (i.p.) in mouse strains C57B1, B6C3F 1 , and BALB/c (early spermatids only) resulted in increased frequency of micronucleus formation in spermatids (Allen et al. 1994; Nutley et al. 1996; Russo and Levis 1992). Studies of micronucleus induction in bone-marrow erythrocytes reported positive effects in several strains of male mice, but not others. Positive results were reported for BALB/c, Swiss CD-1 and B6C3F 1 mice (Marrazzini et al. 1994; NTP 1999; Russo and Levis 1992; Russo et al. 1992) but not NMRI (Leuschner and Leuschner 1991) or (C57BL/Cne x C3H/Cne) $\mathrm{F}_{1}$ mice (Leopardi et al. 1993). Results were negative for micronucleus induction in BALB/c mouse peripheral blood lymphocytes (Grawe et al. 1997).

Significant increases in both micronuclei and SCE frequencies in peripheral blood lymphocytes from human infants were found following administration of $\mathrm{CH}$ as a sedative prior to a hearing test (Ikbal et al. 2004). CH induced chromosomal aberrations in sperm cells in one study (Russo et al. 1984), but was negative for other studies (IARC 2014). Aneuploidy was observed after exposure by i.p. injection in one of two studies in mouse secondary spermatocytes (Leopardi et al. 1993; Miller and Adler 1992) and hyperploidy, but not polyploidy, was reported for mouse bone-marrow erythrocytes (Marrazzini et al. 1994; Xu and Adler 1990).

\subsubsection{S-(1,2-dichlorovinyl)-L-cysteine (DCVC), S-(1,2- dichlorovinyl)glutathione (DCVG), and NAcDCVC}

The available studies on GSH-conjugation pathway metabolites of trichloroethylene suggested that they are genotoxic, however, there are few in vivo studies. More genotoxicity studies were available for DCVC than for DCVG or NAcDCVC. Almost all of the genotoxic endpoints evaluated in vitro were positive for DCVC, including mutation, DNA strand breaks, UDS (DNA repair), cell transformation, gene expression, and DNA and protein binding. Tests for micronucleus induction were negative. In vivo studies were limited to two endpoints, DNA strand breaks and protein binding, but both were positive. Table 2-1 summarizes the conclusions of the evidence for each genotoxic endpoint and details of the findings are discussed below (as cited in IARC (2014) TCE Monograph, pp. 145-149). 
DCVC and DCVG are cysteine intermediates of trichloroethylene formed during metabolic conjugation by glutathione-S-transferase; NAcDCVC has also been identified as another metabolite of trichloroethylene. DCVC has consistently shown genotoxic effects but there are very few studies on the genotoxicity of DCVG or NAcDCVC (IARC 2014).

Both DCVC and DCVG were positive for mutation induction in bacterial assays; both metabolites were direct acting, i.e., induced mutations without the addition of metabolic activation, but the response was increased with the addition of kidney-derived activation systems (S9) (Dekant et al. 1986; Vamvakas et al. 1988a). Additionally, this response was diminished by addition of a beta-lyase inhibitor, suggesting that beta-lyase bioactivation plays a role in the genotoxicity (Irving and Elfarra 2013). DCVC induced DNA strand breaks in male rabbits in vivo and in perfused kidneys and proximal tubules in vitro (Jaffe et al. 1985). Clay (2008) observed a significant increase in DNA strand breaks 2 hours after a single oral dose of trichloroethylene (purity 99.5\%) but reported no effect 16 hours after treatment.

In vitro exposure to DCVC resulted in increased UDS in Syrian hamster embryo fibroblasts and in a porcine kidney epithelial cell line (Vamvakas et al. 1988b; Vamvakas et al. 1989) and cell transformation in rat kidney epithelial cells (Mally et al. 2006; Vamvakas et al. 1996). Gene expression was also increased in a kidney tubular epithelial cell line after treatment with DCVC (Vamvakas et al. 1996). Studies have shown that DCVC forms covalent adducts in vitro with DNA (Muller et al. 1998) and protein adducts in vitro and in vivo, (Eyre et al. 1995; Hayden et al. 1992). NAcDCVC was a direct-acting mutagen in a study in S. typhimurium strain TA2638; the effects were enhanced when kidney metabolic activation was included (Vamvakas et al. 1987).

\subsection{Summary of Genotoxicity and Related Effects of Trichloroethylene and Its Metabolites}

A table of summary assessments of the genotoxicity studies for trichloroethylene and its metabolites (primarily from authoritative reviews by IARC (2014) and EPA (2011a) and as discussed in this document) is provided in Table 2-1. The assessment for each endpoint in the table takes into account all of the information currently available, including consideration of any methodological and/or purity issues, to provide an overall evaluation. For example, positive findings for trichloroethylene might have been due to impurities or chemical stabilizers present in the test sample. Other issues considered that might have caused mixed findings are the use of DMSO as a solvent; whether trichloroethylene, which is a volatile liquid, was tested in liquid solution or in the vapor phase; and the metabolic activation system used in the assay.

\subsubsection{Trichloroethylene}

Overall, there is some evidence that trichloroethylene is genotoxic, which is likely caused by its metabolites. Some of these metabolites have been shown to be direct mutagens (see Section 2.8.2). In most in vitro studies of rodent and human cells and in in vivo studies, exposure to trichloroethylene caused DNA strand breaks and micronucleus formation. Importantly, trichloroethylene was shown to cause some types of genotoxicity in kidney cells or tissue from exposed animals. It also increased SCE in studies in vitro but not in vivo. There is little evidence that trichloroethylene is a direct mutagen; however, there is some evidence that trichloroethylene is mutagenic in bacteria (strain TA100, which detects base-pairing changes) and in yeast in the 
presence of metabolic activation. Evidence in other bacteria strains was weak and most positive findings in other bacteria strains were only observed in the presence of mutagenic stabilizers. Findings for mutagenicity in mammalian cells were mixed (based on only two studies) and negative in in vivo rodent studies. In vivo studies in rodents evaluating chromosomal aberrations, increased sister chromatid exchange, and UDS were negative.

Although not necessarily a genotoxic effect, trichloroethylene was reported to covalently bind mammalian DNA and protein from several tissues in rodents and humans in most in vitro and in vivo studies. Binding to DNA and protein was enhanced by metabolic activation.

Trichloroethylene also was shown to transform cells. DNA and protein binding and cell transformation were included in this section as relevant effects; however, positive results do not necessarily imply that the test agent is genotoxic. Cell transformation assays measure the phenotypic conversion from normal to malignant characteristics in mammalian cells and are capable of detecting both genotoxic and non-genotoxic carcinogens.

Trichloroethylene is highly metabolized, and trichloroethylene metabolites, as previously noted, appear to be responsible for many of the biological effects reported. A wide variety of activation systems were used in the reviewed studies, including exogenous liver microsome preparations, metabolically competent cell lines, and induced and uninduced primary cells from liver, kidney, blood, and embryos. Mixed results may be a consequence of incomplete metabolic activation in some of the systems used. Moreover, in a few cases, a requirement for metabolic activation was not observed, as trichloroethylene induced micronuclei and sister chromatid exchange in cultured Chinese hamster ovary $(\mathrm{CHO})$ cells without the addition of exogenous activation.

Another possible cause to consider for confounding results is the use of solvents such as DMSO to solubilize the test chemical for treatment. Since trichloroethylene is not very water soluble, some in vitro assays (discussed above) utilized DMSO as the test chemical solvent; however, none of the in vivo assays identified reported using DMSO as a vehicle control. As discussed for the trichloroethylene metabolite TCA, there is a concern for $\mathrm{pH}$ effects when using solvents such as DMSO to prepare the test chemical. However, the reaction rate was dependent on the concentration of DMSO; the reaction-rate constants increased by a factor of 6 to 7 with a change in DMSO concentration from $50 \%$ to $86 \%$. When trichloroethylene is tested in vitro with metabolic activation, either endogenous (e.g., primary cells) or exogenous (addition of S9), it can be metabolized to TCA. However, DMSO can be ruled out as an alternative explanation for explaining the positive findings of trichloroethylene. DMSO was not used as a solvent in the majority of the genotoxicity studies reporting positive finding. In the few studies where it was used (e.g., two in vitro studies in mammalian cells and in some studies in bacteria), positive findings for the specific endpoint (e.g., mutagenicity) were also found in studies not using DMSO as a solvent. Moreover, the DMSO used was usually at $0.3 \%$ to $1 \%$ final volume; only one study exceeded that at $2 \%$, and thus it is unlikely that the few positive findings were due to DMSO interacting with the trichloroethylene metabolite, TCA, and some studies using DMSO as a solvent were negative. Finally, the other trichloroethylene metabolites are more likely to contribute to trichloroethylene genotoxicity, and no evidence was identified to suggest that they would interact with DMSO to cause a false positive. 


\subsubsection{Trichloroethylene Metabolites}

Metabolites of trichloroethylene resulting from both the GSH conjugation and oxidative pathways have been shown to induce genotoxic effects. The strongest evidence for genotoxicity is for DCVC and DCVG, followed by $\mathrm{CH}$. There is some evidence for the genotoxicity of DCA and weak evidence for TCA. The GSH conjugation pathway metabolite DCVG may be formed in the kidney as well as the liver; DCVC is formed in the kidney. Both are mutagenic in bacterial assays (with and without metabolic activation); notably there was an increased mutagenic response with the addition of kidney-derived microsomal metabolic activation. In vitro, DCVC induced UDS, and increased cell transformation in a variety of cell types, including rodent kidney cells; DCVC induced DNA strand breaks both in vitro and in vivo and showed protein binding. The evidence is not strong for genotoxicity for the oxidative metabolites $(\mathrm{CH}, \mathrm{DCA}$, TCA, TCOH) and there are only a few available studies for some. The most active metabolite of these is $\mathrm{CH}$, which induced sister chromatid exchange, chromosomal aberrations, and cell transformation in vitro. Test results showed that $\mathrm{CH}$ is direct acting, i.e., similar effects were observed in the in vitro assays with or without the addition of metabolic activation. DCA was mutagenic and there was limited evidence that it induced DNA strand breaks and micronuclei, and possibly chromosomal aberrations. TCA is the least genotoxic metabolite; it was not mutagenic, and the results in vitro may have been a $\mathrm{pH}$-effect and/or due to the use of DMSO as a solvent. Results in vivo were mixed except for increases in chromosomal aberrations.

Table 2-1. Summary Assessment of Genotoxicity and Related Effects for Trichloroethylene and Its Metabolites

\begin{tabular}{|c|c|c|c|}
\hline \multirow{3}{*}{$\begin{array}{c}\text { TCE or Metabolite } \\
\text { Endpoint }\end{array}$} & \multicolumn{3}{|c|}{ Summary of Findings across Studies } \\
\hline & \multicolumn{2}{|c|}{ In Vitro } & \multirow[t]{2}{*}{ In Vivo (Animal) } \\
\hline & $(-\mathrm{S} 9)$ & $(+\mathrm{S} 9)$ & \\
\hline \multicolumn{4}{|l|}{ TCE } \\
\hline Gene mutation (bacteria and yeast) & - & $(+)$ & NT \\
\hline Gene mutation (mammalian) & - & $\pm^{\mathrm{a}}$ & - \\
\hline Gene conversion & - & $(+)$ & NT \\
\hline Aneuploidy & + & $(+)$ & NT \\
\hline Recombination/gene crossover & - & $(+)$ & NT \\
\hline DNA strand break & + & NT & $(+)$ \\
\hline UDS (DNA repair) & $(-)$ & NT & - \\
\hline Chromosomal aberrations & - & - & - \\
\hline Sister chromatid exchange & + & $(+)$ & - \\
\hline Micronucleus induction & + & NT & $(+)$ \\
\hline DNA binding & \pm & + & $(+)$ \\
\hline Protein binding & + & NT & + \\
\hline \multicolumn{4}{|l|}{ TCA } \\
\hline Gene mutation & - & $(-)$ & NT \\
\hline DNA damage/strand breaks & \pm & NT & $(-)$ \\
\hline Chromosomal aberrations & $(?)^{\mathrm{b}}$ & NT & + \\
\hline Micronucleus induction & $(+)^{\mathrm{c}}$ & NT & \pm \\
\hline
\end{tabular}


TCE or Metabolite

Endpoint
Summary of Findings across Studies

In Vitro (+S9)

\section{TCOH}

Gene mutation

Micronucleus induction

$(-\mathrm{S} 9$

DCA

Gene mutation

Aneuploidy

DNA strand break

Chromosomal aberrations

Micronucleus induction

CH

Gene mutation

Non-disjunction

Aneuploidy/polyploidy

Gene crossover

DNA strand break (liver)

DNA damage (human lymphoblast)

Chromosomal aberrations

Sister chromatid exchange

Micronucleus induction

$\begin{array}{ccc}- & + & \text { NT } \\ + & \text { NT } & \text { NT }\end{array}$

DNA binding

DNA-protein crosslinks

$(+)$

$-$

$-$

$\pm$

$\pm$

$+$

$+$

$+$

$-$

$-$

$+$

$+$

$+$

$(+)$

$(+)$

$-$
In Vivo (Animal)

\section{DCVC/DCVG}

Gene mutation

Mutation (loss of heterozygosity)

DNA strand break

UDS (DNA repair)

Micronucleus induction

Cell transformation

Gene expression

DNA binding

Protein binding

$\begin{array}{lcc}+ & + & \text { NT } \\ - & \text { NT } & \text { NT } \\ + & + & + \\ + & \text { NT } & \text { NT } \\ - & \text { NT } & \text { NT } \\ + & \text { NT } & \text { NT } \\ + & \text { NT } & \text { NT } \\ + & \text { NT } & \text { NT } \\ + & \text { NT } & + \\ + & & \\ + & + & \text { NT }\end{array}$

Gene mutation

Sources: IARC (2014) and EPA (2011a), also Tabrez and Ahmad (2012), Varshney et al. (2013a; 2013b)), and Zhang et al. (2012), as described in the text.

Summary calls for all of the endpoints in this table were determined by integrating the findings across all available studies, with consideration of methodological and/or purity issues.

$+=$ positive; $(+)=$ mostly positive evidence; $\pm=$ mixed results; $(-)=$ mostly negative evidence; $-=$ negative; $\mathrm{NT}=$ not tested. ${ }^{\text {aBacteria }}$ results are based on positive findings in TA 100 studies not using stabilizers.

${ }^{b}$ Methodological concerns limit interpretation of the evidence across studies and positive findings may be due to a pH effect.

'Based on one study using $0.3 \%$ DMSO (see text). 


\section{Human Cancer Studies}

\section{Introduction}

As discussed in the "Introduction" and "Methods" sections, the cancer hazard evaluation of trichloroethylene focuses on three specific cancers: kidney (see Section 4.1), NHL and its histological subtypes and related cancers (see Section 5.1), and liver (see Section 6.1). Because many studies (primarily the cohort studies) are common for all these cancer sites, this section provides information on the studies that are relevant for cancer hazard evaluation, including an overview of the studies' methods and characteristics and an assessment of the studies' ability to inform the cancer evaluation. The steps in the cancer hazard evaluation, including the location of the discussion of these steps, are listed below.

(1) Selection of the relevant literature included in the cancer evaluation (Section 3.1 and Trichloroethylene Protocol, http://ntp.niehs.nih.gov/NTP/roc/thirteenth/Protocols/TCE Protocol12-3113 508.pdf).

(2) Description of the study methods and characteristics and evaluation of study quality and other elements related to the utility of the studies to inform the cancer hazard evaluation: Cohort studies (Section 3.2, Table D-1, Table D-4, and Table D-5), kidney and liver case-control studies (Section 3.3, Table D-2, Table D-6 and Table D-7), and NHL case-control studies (Section 3.4, Table D-3, Table D-8, and Table D-9).

(3) Cancer assessment: Kidney (Section 4.1), NHL and its subtypes (Section 5.1), and liver (Section 6.1).

(4) NTP recommendation for the level of evidence of carcinogenicity (sufficient, limited, or inadequate) of trichloroethylene from human studies (Section 7).

\subsection{Literature Search Strategy and Selection of the Relevant Literature}

The literature search strategy (including the databases and search terms, and other sources for identifying literature) and procedures for selecting the literature (systematic screening procedures and inclusion/exclusion criteria) are described in detail in the Protocol. Primary epidemiological studies were considered for the cancer evaluation if the study was peer reviewed, provided risk estimates (or information to calculate risk estimates) for trichloroethylene and human cancer (kidney, liver, or NHL), and provided information specific for trichloroethylene exposure at the individual level or an estimate of the proportion of exposed subjects in defined exposure groups. Studies of dry cleaners and laundry workers were excluded, as the extent of exposure to trichloroethylene is often unclear and indistinguishable from tetrachloroethylene, or other petroleum-derived solvents such as carbon tetrachloride (NRC 2006). In general, cohort or casecontrol studies of populations with jobs, workplaces or environmental exposures in which trichloroethylene exposure may have occurred (e.g., studies of grouped chlorinated or organic solvents, degreasing agents, metal cleaners, or jobs and occupations such as degreasing, metalworking, painting, electronics manufacturing, aircraft manufacturing) were excluded if a specific risk estimate for trichloroethylene exposure was not reported as noted above, although 
several were included in one or more reviews or meta-analyses of trichloroethylene (Asal et al. 1988; Chang et al. 2003; Costa et al. 1989; Garabrant et al. 1988; Harrington et al. 1989; Seldén and Ahlborg 1991; Sinks et al. 1992). In addition, descriptive studies (with no risk estimate) and geographic studies were also excluded, again because these studies were unlikely to be specific for trichloroethylene exposure, with the exception of one drinking water study (Bove et al. 2014). This study was included because it identified an exposed cohort and assigned individual exposure based on the household drinking water level of trichloroethylene (rather than the township level), which increased the likelihood for ever exposure to trichloroethylene.

\subsection{Cohort Studies}

\subsubsection{Overview of the Methodologies and Study Characteristics}

Table 3-1 lists the 16 occupational cohort studies, nested case-control studies, or pooled analyses that satisfied the inclusion criteria. In general, the list includes only the latest update of the study or the most comprehensive report on a population; however, additional relevant analyses or information from previous publications are considered in the evaluation. Studies of overlapping publications are included if the overlap is not known and there are differences in methodologies (such as exposure assessment). For each of the reviewed studies, detailed data on study design, methods, and findings were systematically extracted from relevant publications, as described in the study protocol, into Table D-1 and Table D-2. The cohort and nested case-control studies can be divided into several broad occupational groups related to the exposure scenarios or occupations. Within each of these groups, studies are organized by descending publication date.

\subsubsection{Nordic Studies: Three Incidence Studies}

Several cohort studies reporting on cancer incidence were published among workers in Nordic countries. These studies include subjects with occupational exposure to trichloroethylene from diverse industries, and workers and exposed subjects were identified from broad occupational or population-based databases. The largest study (Vlaanderen et al. 2013) includes

trichloroethylene-exposed workers in the Nordic Occupational Cancer (NOCCA) study, which links occupational data reported in censuses (Sweden, Finland, Denmark, Iceland, and Norway) with their national cancer registries. The census occupational history data were linked to the NOCCA job exposure matrix (JEM) to develop semi-quantitative estimates of exposure to trichloroethylene. This large study may have included some of the same subjects as the other studies, but these were likely a very small percentage. Three cohort studies reported on cancer findings among workers who had urinary trichloroacetic acid (U-TCA) measurements as part of trichloroethylene monitoring programs in Sweden (Axelson et al. 1978; Axelson et al. 1994), Finland (Anttila et al. 1995), and Denmark (Hansen et al. 2001). These workers were included in a pooled analysis reported by Hansen et al. (2013), which is reviewed rather than the individual studies because it includes all the workers from the constituent studies and uses a similar exposure assessment (although any additional information from the individual studies will be brought forward). The third cohort study is of blue-collar Danish workers at companies using trichloroethylene (Raaschou-Nielsen et al. 2003). Although this study may include a small proportion of workers from the Danish component of the pooled analysis, it is included in the review because the extent of the overlap is unknown, and the exposure assessment is different. 


\subsubsection{Aerospace (Rocket Engine) and Aircraft Manufacturing Workers: Five Incidence or Mortality Studies}

Two cohort studies evaluated risk among rocket engine workers with potential exposure to trichloroethylene at the Santa Susana Field Laboratory (SSFL) in California; these studies are part of the Rocketdyne Aerospace workers cohort (Boice et al. 2006; Zhao et al. 2005). Although there is likely to be considerable overlap between the two studies, both studies are reviewed (noting potential overlap) because of differences in exposure and disease assessments and numbers of exposed workers. Boice et al. (2006) reported mortality findings based on a qualitative exposure assessment and Zhao et al. (2005) reported both mortality and incidence findings for trichloroethylene-exposed workers (intensity score $>3$ ) based on a semi-quantitative JEM. Among the three cohort studies of U.S. aircraft manufacturing workers with potential exposure to trichloroethylene, two cohort studies reported mortality findings (Lipworth et al. 2011; Morgan et al. 1998) and the third (Blair et al. 1998; Radican et al. 2008) reported both incidence and mortality. Morgan et al. (1998) and Radican et al. (2008) used a semi-quantitative exposure assessment and Lipworth et al. (2011) used a qualitative exposure assessment, all of which assigned exposure using individual work history information and expert-assigned JEMs. All studies conducted both internal and external analyses.

\subsubsection{Other Studies of Specific Industries: Seven Incidence or Mortality Studies}

Two cohort studies (Bahr et al. 2011; Ritz et al. 1999) and one nested case-control study (Yiin et al. 2009) of uranium processing or enrichment workers, which used a JEM and/or individual work histories to classify workers according to ranked exposure levels or probability of exposure, were identified that met the inclusion criteria. Bahr et al. (2011) evaluated cancer incidence and mortality among Kentucky uranium enrichment workers, and Ritz (1999) and Yiin et al. (2009) evaluated mortality among Ohio and Tennessee uranium processing workers, respectively. The nested case-control study by Yiin et al. (2009) of multiple myeloma evaluated trichloroethylene as a potential confounder for uranium exposure, which was the major focus of the study. The remaining studies consisted of one study in each of several different manufacturing industries using trichloroethylene as a degreaser or solvent. Silver et al. (2014) conducted a cohort mortality analysis of New York electronics workers, based on ranked exposure scores. A small cohort study of German cardboard manufacturing workers focusing on renal-cell carcinoma (Henschler et al. 1995) assessed exposure using job location at the plant and surveys of plant conditions. Greenland et al. (1994) conducted a nested case-control study of lymphoma, and kidney and liver cancer among a cohort of workers at a Massachusetts electrical transformer manufacturing plant, using a qualitative JEM to assess exposure. Finally, Wilcosky et al. (1984) reported on a small nested case-control study of NHL and other cancers among a cohort of rubber manufacturing workers in which potential exposure to trichloroethylene was assessed by work in an area where trichloroethylene was authorized for use.

\subsubsection{Environmental Exposure: One Mortality Study}

In the drinking water study, exposure to trichloroethylene was based on duration at a residence and modeled trichloroethylene concentration levels from the water supply system (Bove et al. 2014). 
Table 3-1. Cohort and Nested Case-control Studies of Trichloroethylene Exposure

\begin{tabular}{|c|c|c|c|}
\hline Reference & Population & $\begin{array}{l}\text { Exposure Assessment } \\
\text { Exposure Metric }\end{array}$ & $\begin{array}{c}\text { Cancer Assessment } \\
\text { Endpoints }^{\mathrm{a}}\end{array}$ \\
\hline \multicolumn{4}{|l|}{ Nordic studies } \\
\hline $\begin{array}{l}\text { Vlaanderen et al. } \\
\text { (2013) }\end{array}$ & $\begin{array}{l}\text { NOCCA study } \\
\text { Population-based cancer } \\
\text { registry and occupational } \\
\text { database linkage } \\
\text { Kidney }(\mathrm{N}=76,130) \text {, } \\
\text { liver }(\mathrm{N}=896) \\
\text { NHL }(\mathrm{N}=69,254) \\
\mathrm{MM}(\mathrm{N}=35,534)\end{array}$ & $\begin{array}{l}\text { Linkage of historical job } \\
\text { information from census with } \\
\text { national JEMs constructed from } \\
\text { occupation data } \\
\text { Cumulative exposure } \\
\text { (incorporates exposure } \\
\text { prevalence) }\end{array}$ & $\begin{array}{l}\text { Incidence } \\
\text { Internal analysis } \\
\text { Kidney, liver, NHL, MM }\end{array}$ \\
\hline Hansen et al. (2013) & $\begin{array}{l}\text { Pooled Nordic } \\
\text { biomonitored cohort: } \\
\text { diverse occupations } \\
\mathrm{N}=5,553 \text { workers }\end{array}$ & $\begin{array}{l}\text { Urine TCA surveillance } \\
\text { U-TCA }(\mathrm{mg} / \mathrm{L})\end{array}$ & $\begin{array}{l}\text { Incidence } \\
\text { External and internal analyses } \\
\text { Kidney, liver, NHL, MM }\end{array}$ \\
\hline $\begin{array}{l}\text { Raaschou-Nielsen et } \\
\text { al. (2003) }\end{array}$ & $\begin{array}{l}\text { Danish TCE-exposed } \\
\text { blue-collar workers } \\
\text { cohort: diverse } \\
\text { occupations } \\
\mathrm{N}=40,049\end{array}$ & $\begin{array}{l}\text { Blue-collar workers in TCE- } \\
\text { using companies with potential } \\
\text { exposure to TCE } \\
\text { Employment duration } \\
\text { Year of first employment (crude } \\
\text { surrogate for exposure level) }\end{array}$ & $\begin{array}{l}\text { Incidence } \\
\text { External analysis } \\
\text { Kidney, liver, NHL, MM }\end{array}$ \\
\hline \multicolumn{4}{|c|}{ Aerospace and aircraft manufacturing workers } \\
\hline $\begin{array}{l}\text { Lipworth et al. } \\
\text { (2011) }\end{array}$ & $\begin{array}{l}\text { Burbank, CA (USA) } \\
\text { aircraft manufacturing } \\
\text { workers cohort } \\
\mathrm{N}=5,443\end{array}$ & $\begin{array}{l}\text { Qualitative JEM } \\
\text { Employment duration }\end{array}$ & $\begin{array}{l}\text { Mortality } \\
\text { External and internal analyses } \\
\text { Kidney, liver, NHL, MM }\end{array}$ \\
\hline $\begin{array}{l}\text { Radican et al. } \\
(2008) / \text { Blair et al. } \\
(1998)\end{array}$ & $\begin{array}{l}\text { Utah (USA) aircraft } \\
\text { maintenance workers } \\
\text { cohort } \\
\mathrm{N}=7,204\end{array}$ & $\begin{array}{l}\text { Semi-quantitative JEM } \\
\text { Cumulative exposure (units/yr) } \\
\text { Exposure pattern (continuous, } \\
\text { intermittent, peaks) }\end{array}$ & $\begin{array}{l}\text { Mortality (Radican)/ incidence } \\
\text { (Blair) } \\
\text { External and internal analyses } \\
\text { Kidney, liver, NHL, MM }\end{array}$ \\
\hline $\begin{array}{l}\text { Boice et al. (2006) } \\
\text { (overlaps with Zhao } \\
\text { et al. }(2005))\end{array}$ & $\begin{array}{l}\text { Los Angeles (USA) } \\
\text { rocket engine testing } \\
\text { workers cohort } \\
\mathrm{N}=1,111\end{array}$ & $\begin{array}{l}\text { Qualitative JEM } \\
\text { Ever exposure } \\
\text { Exposure duration (kidney only) }\end{array}$ & $\begin{array}{l}\text { Mortality } \\
\text { External and internal analyses } \\
\text { Kidney, liver, NHL, MM, CLL }\end{array}$ \\
\hline $\begin{array}{l}\text { Zhao et al. (2005) } \\
\text { (overlaps with } \\
\text { Boice et al. (2006)) }\end{array}$ & $\begin{array}{l}\text { Los Angeles (USA) } \\
\text { aerospace workers cohort } \\
\mathrm{N}=6,044\end{array}$ & $\begin{array}{l}\text { Semi-quantitative JEM } \\
\text { Cumulative exposure score }\end{array}$ & $\begin{array}{l}\text { Mortality/incidence } \\
\text { External and internal analyses } \\
\text { Kidney, liver, } \\
\text { NHL + leukemia combined }\end{array}$ \\
\hline $\begin{array}{l}\text { Morgan et al. } \\
(1998)\end{array}$ & $\begin{array}{l}\text { Arizona (USA) aircraft } \\
\text { manufacturing workers } \\
\text { cohort } \\
\mathrm{N}=4,733\end{array}$ & $\begin{array}{l}\text { Semi-quantitative JEM } \\
\text { Cumulative exposure score }\end{array}$ & $\begin{array}{l}\text { Mortality } \\
\text { External and internal analyses } \\
\text { NHL, kidney, liver }\end{array}$ \\
\hline
\end{tabular}




\begin{tabular}{|c|c|c|c|}
\hline Reference & Population & $\begin{array}{l}\text { Exposure Assessment } \\
\text { Exposure Metric }\end{array}$ & $\begin{array}{l}\text { Cancer Assessment } \\
\text { Endpoints }^{\mathrm{a}}\end{array}$ \\
\hline \multicolumn{4}{|c|}{ Other studies of specific industries } \\
\hline Silver et al. (2014) & $\begin{array}{l}\text { New York (USA) micro- } \\
\text { electronics manufacturing } \\
\text { cohort } \\
\mathrm{N}=34,494\end{array}$ & $\begin{array}{l}\text { Department-year exposure } \\
\text { matrix } \\
\text { Cumulative exposure ranking }\end{array}$ & $\begin{array}{l}\text { Mortality } \\
\text { Internal analyses } \\
\text { Kidney, NHL, multiple } \\
\text { myeloma, liver, biliary and } \\
\text { gallbladder combined }\end{array}$ \\
\hline Bahr et al. (2011) & $\begin{array}{l}\text { Kentucky (USA) uranium } \\
\text { enrichment workers } \\
\text { cohort } \\
\mathrm{N}=4,792\end{array}$ & $\begin{array}{l}\text { JEM } \\
\text { Exposure level (ranked order) }\end{array}$ & $\begin{array}{l}\text { Mortality } \\
\text { External and internal analyses } \\
\text { NHL, liver }\end{array}$ \\
\hline Yiin et al. (2009) & $\begin{array}{l}\text { Tennessee (USA) nested } \\
\text { case-control study of } \\
\text { uranium enrichment } \\
\text { workers } \\
\mathrm{N}=47,941 \text { cohort } \\
98 \mathrm{MM} \text { cases, } 483 \\
\text { controls }\end{array}$ & $\begin{array}{l}\text { Modified semi-quantitative } \\
\text { JEM } \\
\text { TCE evaluated as a potential } \\
\text { confounder; major focus: } \\
\text { uranium exposure } \\
\text { Average cumulative exposure } \\
\text { score }\end{array}$ & $\begin{array}{l}\text { Mortality } \\
\text { Internal analysis } \\
\text { MM }\end{array}$ \\
\hline Ritz (1999) & $\begin{array}{l}\text { Ohio (USA) uranium } \\
\text { processing workers cohort } \\
\mathrm{N}=3,184\end{array}$ & $\begin{array}{l}\text { Semi-quantitative JEM } \\
\text { Exposure level (low, moderate) } \\
\text { Exposure duration }\end{array}$ & $\begin{array}{l}\text { Mortality } \\
\text { Internal analysis } \\
\text { Liver }\end{array}$ \\
\hline $\begin{array}{l}\text { Henschler et al. } \\
\text { (1995) }\end{array}$ & $\begin{array}{l}\text { German cardboard } \\
\text { manufacturers cohort } \\
\mathrm{N}=169\end{array}$ & $\begin{array}{l}\text { Job location from individual } \\
\text { work histories and knowledge } \\
\text { of plant conditions. } \\
\text { Ever exposed }\end{array}$ & $\begin{array}{l}\text { Incidence } \\
\text { External and internal analyses } \\
\text { Kidney }\end{array}$ \\
\hline $\begin{array}{l}\text { Greenland et al. } \\
\text { (1994) }\end{array}$ & $\begin{array}{l}\text { Massachusetts (USA) } \\
\text { nested case-control study } \\
\text { of electrical } \\
\text { manufacturers cohort } \\
\mathrm{N}=1,821 \text { cohort; } 512 \\
\text { cancer deaths; } 1,202 \text { non- } \\
\text { cancer deaths (controls) }\end{array}$ & $\begin{array}{l}\text { Qualitative JEM } \\
\text { Ever exposed }\end{array}$ & $\begin{array}{l}\text { Mortality } \\
\text { Kidney }(\mathrm{N}=12), \\
\text { liver }(\mathrm{N}=9) \\
\text { lymphoma }(\mathrm{N}=15)\end{array}$ \\
\hline $\begin{array}{l}\text { Wilcosky et al. } \\
\text { (1984) }\end{array}$ & $\begin{array}{l}\text { Ohio (USA) nested case- } \\
\text { control study of rubber } \\
\text { manufacturing workers } \\
\text { cohort } \\
\mathrm{N}=6,678 \text { cohort } \\
\text { (controls } 20 \% \text { sampling) }\end{array}$ & $\begin{array}{l}\text { Working in area of authorized } \\
\text { use of specific solvents } \\
\text { Ever exposed (potential) }\end{array}$ & $\begin{array}{l}\text { Mortality } \\
\text { NHL }(\mathrm{N}=9)\end{array}$ \\
\hline \multicolumn{4}{|c|}{ Environmental exposure } \\
\hline Bove et al. (2014) & $\begin{array}{l}\text { North Carolina (USA) } \\
\text { military cohort } \\
\text { Drinking water study } \\
\mathrm{N}=154,932\end{array}$ & $\begin{array}{l}\text { Duration of residence and } \\
\text { modeled TCE concentration in } \\
\text { drinking water and cumulative } \\
\text { exposure } \\
\text { TCE ( } \mu \mathrm{g} / \mathrm{L}-\text { month) }\end{array}$ & $\begin{array}{l}\text { Mortality } \\
\text { External and internal analyses } \\
\text { NHL, kidney, liver }\end{array}$ \\
\hline
\end{tabular}

$\overline{\mathrm{CLL}}=$ chronic lymphocytic leukemia; JEM = job-exposure matrix; $\mathrm{MM}=$ multiple myeloma; NHL = non-Hodgkin lymphoma. ${ }^{\mathrm{a} C a n c e r}$ endpoints of a priori interest only (kidney cancer, liver cancer, and NHL and its subtypes). 


\subsubsection{Evaluation of Study Quality and Other Elements Related to the Utility of the Studies to Inform the Cancer Hazard Evaluation}

This section discusses the assessment of study quality and other elements related to the utility of the individual studies to inform the cancer hazard evaluation. Each study was assessed (prior to evaluating the findings) for the potential for biases and other factors related to informing the cancer hazard evaluation according to the approach described in the protocol. (See Table D-4 and Table D-5 for a study-by-study assessment of potential for biases, study quality, and study sensitivity.) The impact of these factors, for example the analysis of cancer incidence vs. mortality, the length of follow-up, the potential for disease misclassification, and the statistical power of the study, may differ according to the specific cancer endpoint being evaluated (kidney cancer, liver cancer, and NHL and its subtypes), and will be discussed separately where relevant.

\subsubsection{Selection Bias}

The potential for selection bias was considered unlikely in the majority of cohort or nested casecontrol studies (Blair et al. 1998; Bove et al. 2014; Hansen et al. 2013; Lipworth et al. 2011; Morgan et al. 1998; Radican et al. 2008; Vlaanderen et al. 2013; Wilcosky et al. 1984; Zhao et al. 2005). There was the potential for bias in the following studies. The German cardboard manufacturing cohort (Henschler et al. 1995) was initiated because of a cluster of renal cancers and included the index cases in their cohort analysis (Bloemen and Tomenson 1995; IARC 2014), which would result in an over-estimate of the risk estimate. In two of the uranium workers cohorts (Ritz 1999; Yiin et al. 2009), workers were selected based on having radiation monitoring data, which may result in selection bias (if trichloroethylene-exposed workers without monitoring data were excluded) and potential confounding. In the nested case-control study of electrical workers by Greenland et al. (1994), the cohort was selected from workers participating in the pension scheme, introducing potential selection bias. In addition, the bluecollar workers included in the Nordic study by Raaschou-Nielsen et al. (2003) differed with respect to socioeconomic status from the referent (general) population, which may result in an over- or underestimate of expected cases, depending on the endpoint. Little information was provided to evaluate how workers were selected or excluded in the study of Kentucky uranium enrichment workers (Bahr et al. 2011).

There was evidence of a possible healthy worker effect in five studies, based on statistically significant decreases in all-cause mortality rates: the aerospace worker study reported by Boice et al. (2006), two aircraft manufacturing studies (Lipworth et al. 2011; Morgan et al. 1998), and two studies of uranium workers (Bahr et al. 2011; Ritz 1999); and the micro-electronic study (Silver et al. 2014) and the study of cardboard manufacturing workers (Henschler et al. 1995). A healthy worker effect would bias the findings of an external analysis towards the null. The study by Silver et al. (2014) only conducted internal analyses. There was also evidence for a healthy worker survival effect in the study by Bahr et al. (2011), which would also bias internal analyses. In addition, three of the cohorts are relatively young (Bove et al. 2014; Raaschou-Nielsen et al. 2003; Silver et al. 2014), suggesting that further follow-up would be informative.

There was generally insufficient information to evaluate the possibility of systematic selection out of the cohorts once established with the possible exception of Bahr et al. (2011) as noted above. The loss to follow-up was minimal in the studies that reported it, but it is not reported in several studies. Internal analyses, conducted in addition to external (SMR, SIR) analyses in the majority of cohort studies (Boice et al. 2006; Bove et al. 2014; Hansen et al. 2013; Henschler et 
al. 1995; Lipworth et al. 2011; Morgan et al. 1998; Radican et al. 2008; Ritz 1999; Vlaanderen et al. 2013; Zhao et al. 2005), also indirectly address the potential for selection bias.

\subsubsection{Information Bias: Exposure Assessment}

The quality of the exposure assessment and the potential for exposure misclassification were systematically evaluated for each study. In general, the evaluation of the exposure assessment refers to the quality of the expert assessment and/or JEM used to evaluate the frequency, confidence, and probability of exposure to trichloroethylene from specific jobs or tasks. The evaluation of the potential for exposure misclassification integrates the quality of the exposure assessment with other exposure information such as the exposure setting. The majority of studies used qualitative exposure assessments or semi-quantitative categories of exposure based on jobexposure or job-task exposure matrices and/or estimates of exposure ranks or levels; quantitative historical exposure monitoring data, if available, were limited. The quality of job-exposure matrices and similar methods of estimating exposure varies considerably; for example, generic JEMs based on broad occupational or industry classifications (e.g., occupational titles or standardized industrial classification codes) may be insufficiently detailed for specific workplaces, jobs or tasks compared with those developed specifically for the study and validated or reviewed using, for example, expert assessment or veteran workers.

In the most recent population-based Nordic study (Vlaanderen et al. 2013), exposure was assessed by linking generic country-specific JEMs to job titles reported on census data. Individuals were assumed to have the same job between censuses. Cumulative exposure was characterized as the product of the average exposure intensity and prevalence of exposure. For jobs with low exposure prevalence, this approach would underestimate exposure intensity and classify unexposed workers with these jobs as exposed. Misclassification of exposure for individual participants was likely to be considerable because of the lack of detailed occupational information (tasks and working conditions), heterogeneity of exposure levels within and across jobs with the same job title, and overtime.

The pooled and updated Nordic study of Hansen et al. (2013) was based on biomonitoring data (urinary U-TCA measurements), together with some ambient air monitoring data. This study most likely had high sensitivity for identifying exposed workers; however, specificity may be a concern because some workers were exposed to other chlorinated solvents that are metabolized to TCA (Anttila et al. 1995). In addition, because large numbers of workers may have only had one to three U-TCA measurements, and the half-life of U-TCA is approximately 100 hours, the available U-TCA measurements may not represent a worker's past or future exposure to trichloroethylene. Individuals classified as unexposed workers could in fact be exposed and misclassification of intensity of exposure is possible. Furthermore, this study did not provide information on lifetime or cumulative exposure, thus limiting the analysis of exposure-response relationships.

Non-differential misclassification of exposure was a concern in the Danish blue-collar workers study (Raaschou-Nielsen et al. 2003), in which an estimate of the proportion of blue-collar workers in companies using trichloroethylene was used as a surrogate for trichloroethylene exposure; only an estimated $41 \%$ of workers included in the analysis were probably exposed to trichloroethylene. Air and urine monitoring data were available for only a small proportion of workers. Although these measurements were not used in the exposure assessment, they provided information on the estimated level of exposure for different calendar periods, which was used in 
the analysis as a surrogate for exposure intensity. There was greater confidence in exposure classification for the analyses of a subcohort considered to have higher exposure than for the entire cohort.

Among the five U.S. aerospace or aircraft manufacturing cohorts, the studies that used semiquantitative job-exposure matrices based on detailed job tasks and work histories to classify exposure among individual workers by ever vs. never, and/or by categories of exposure level or duration of employment (Blair et al. 1998; Morgan et al. 1998; Radican et al. 2008; Zhao et al. 2005) were the most informative with respect to the overall quality of the exposure assessment; Zhao et al. (2005) classified aerospace workers as exposed if they had a trichloroethylene exposure score $>3$, which reduced the potential for exposure misclassification. Although the quality of the exposure assessment of the Utah aircraft-manufacturing workers by Radican et al. (2008) was considered to be adequate, exposure assessment for some subjects with missing exposure records was based on position description, which increases the potential for exposure misclassification. A limitation of the study of Arizona aircraft manufacturing workers (Morgan et al. 1998) was that the exposure assessment does not appear to be calendar specific; however, there was greater confidence of actual exposure among the highest exposed workers in this study. The exposure assessments of the other two studies (Boice et al. 2006; Lipworth et al. 2011), especially the study of California aircraft manufacturing workers, were considered to be more limited because they provided little information on exposure intensity. In the study of aerospace workers by Boice et al. (2006), non-differential exposure misclassification was a concern in the analysis of any exposure to trichloroethylene, (which included test stand mechanics using trichloroethylene as a general utility cleaning agent) although there was greater confidence in actual exposure in the analysis restricted to exposure duration of workers engaged in test engine flushing, a task in which exposure intensity is suspected to be high. No information or analysis of exposure intensity was available for the California trichloroethylene-exposed aerospace workers studied by Lipworth et al. (2011); evaluation of exposure-response relationships was based only on duration, i.e., length of time in jobs with potential exposure to trichloroethylene; no data were reported for exposure intensity or cumulative exposure.

In general, exposure misclassification was a concern in the studies of other specific industries because of low-quality exposure assessments with the possible exception of the German study of cardboard manufacturing workers (Henschler et al. 1995). Although the exposure assessment in this study was based on job location in the plant as well as a detailed description of the work environment and considered to be of limited quality, exposure misclassification is unlikely because high levels of exposure in an open system in small work environments were likely to have occurred in the past, based on job task descriptions of and reports of illness and the odor or taste of trichloroethylene by workers. Wilcosky et al. (1984) classified workers based on ever working in an area of authorized use of trichloroethylene in the nested case-control study of rubber workers; however, actual use of trichloroethylene was not reported, and thus the exposure assessment was considered to be inadequate to inform the hazard evaluation.

The remaining studies used JEMs of varying quality to estimate ranked exposure level (Bove et al. 2014; Ritz 1999), duration (Ritz 1999), probability of exposure (Bahr et al. 2011), a cumulative exposure score (Silver et al. 2014; Yiin et al. 2009) or ever exposure (Greenland et al. 1994). The quality of the exposure assessment in two studies of uranium enrichment or processing workers using semi-quantitative assessments (Ritz 1999; Yiin et al. 2009) were considered to be somewhat better than the other studies. Ritz (1999) used a semi-quantitative 
exposure assessment, although not calendar-year specific, to assign uranium-processing workers to two exposure categories. A modified job-exposure matrix was employed in the Tennessee uranium workers nested case-control study (Yiin et al. 2009). Exposure assessment in the electronics worker cohort (Silver et al. 2014) used a relative cumulative exposure score, based on department-year level use of trichloroethylene and employment duration. Non-differential exposure misclassification was a concern in this study due to lack of information on job tasks, exposure conditions, levels of use and incomplete records. Electronic workers in the nested casecontrol study by Greenland et al. (1994) were classified as ever exposed to trichloroethylene based on a generic JEM. It is difficult to evaluate the quality of the exposure assessment including the basis for the exposure probabilities categories in the study of Kentucky uranium processing workers (Bahr et al. 2011) because of inadequate information provided in the publication. Finally, in the cohort study of drinking water contamination (Bove et al. 2014), exposure misclassification for both ever exposure and exposure category (based on modeled trichloroethylene concentration by residence) was a concern, although less so for participants estimated to have higher cumulative exposure.

In all the studies, the potential for exposure misclassification was generally considered to be nondifferential and would most likely bias towards the null. In subgroup analyses, exposure misclassification between exposure groups would most likely attenuate any exposure-response relationships.

\subsubsection{Information Bias: Cancer Ascertainment and Disease Misclassification}

Studies evaluating cancer incidence (or incidence and mortality) (Bove et al. 2014; Hansen et al. 2013; Henschler et al. 1995; Raaschou-Nielsen et al. 2003; Radican et al. 2008; Vlaanderen et al. 2013; Zhao et al. 2005) Mortality-only analyses (Bahr et al. 2011; Boice et al. 2006; Greenland et al. 1994; Lipworth et al. 2011; Morgan et al. 1998; Ritz 1999) Disease misclassification was unlikely for kidney cancers (and for liver cancers, either in the mortality-only studies or the incidence analyses. Mortality analyses are less informative for kidney cancer due to high 5-year survival rates. Disease assessment was considered to be limited in the German study of cardboard manufacturing workers because different methods for disease diagnosis may have been used for the exposed cohort (physicians' records, abdominal sonogram) than for the general population, which could potentially bias external analyses towards an over-estimate of the risk estimate. This bias should not affect internal analyses. The quality of disease ascertainment of the Kentucky uranium enrichment workers (Bahr et al. 2011) could not be evaluated because of inadequate information on the source and completeness of vital status and cause of death data.

In the case of NHL and its subtypes, however, changes in classification systems, together with differences between studies with respect to groupings of lymphohematopoietic cancer endpoints used in analyses, were of greater concern. Considerable changes in the classification systems used for these lymphomas have been made. Starting with the Revised European American Classification of Lymphoid Neoplasms (REAL) in 1994 (Harris et al. 1994), which was partly incorporated into the ICD Oncology Second Revision (ICD-O-2), recent substantial revisions in the classification of NHL and its subtypes were made by the WHO in 2001 (Morton et al. 2007) (and again in 2008) and used in the ICD Oncology Third Revision (ICD-O-3). The 2001 and 2008 revisions are the most informative for the classification of NHL and its subtypes. The ICD7 NHL classifications used in the Nordic studies (Hansen et al. 2013; Raaschou-Nielsen et al. 2003; Vlaanderen et al. 2013) and to a lesser extent the older classifications used by Greenland et 
al. (1994), Morgan et al. (1998), Blair et al. (1998), Ritz (1999), Boice et al. (2006), Lipworth et al. (2011), Bahr et al. (2011), Silver et al. (2014) and Yiin et al. (2009) were somewhat broader and less informative than more recent systems, which were applied in only two studies (Radican et al. 2008; Zhao et al. 2005).

Finally, death certificate data used in mortality studies (which also use underlying cause of death only, with the exception of Zhao et al. (2005)), may be more likely to result in both missing cases and NHL misclassification than cancer registry data used in incidence studies.

\subsubsection{Study Sensitivity and Exposure-response Relationships}

In addition to the analyses of biases and confounding, study sensitivity and analyses of exposureresponse relationships also impacts the ability of a study to inform the cancer evaluation. Study sensitivity (or the ability to detect an effect), is dependent on the numbers of exposed subjects or cases and controls (which is related to the sample size and exposure prevalence), exposure level (intensity and/or duration), and the length of follow-up, which is of particular concern for longer latency cancers such as liver and kidney cancer. True relative risks will usually be lower among study populations with lower exposure (NRC 2006) and are also dependent on the biological properties of the agent. The evaluation of exposure-response relationships depends on an adequate range of exposure (in intensity or duration) among the study participants, adequate numbers of subjects in each exposure category and the confidence with which exposure groups are correctly classified.

A strength of the database is that all the studies had relatively long overall follow-up periods, although the average length of follow-up is not always clear. Three cohorts (Bove et al. 2014; Raaschou-Nielsen et al. 2003; Silver et al. 2014) were relatively young, however, suggesting additional follow-up may be informative, particularly for kidney and liver cancer.

Without considering exposure levels or exposure misclassification, only the largest cohort studies (Bove et al. 2014; Hansen et al. 2013; Lipworth et al. 2011; Raaschou-Nielsen et al. 2003; Radican et al. 2008; Vlaanderen et al. 2013) probably had adequate statistical power to observe a two-fold relative risk (see calculations by EPA (2011a) and NRC (2006) for some of these studies) for ever vs. never exposed analyses, and only the two largest Nordic cohorts (Raaschou-Nielsen et al. 2003; Vlaanderen et al. 2013) had adequate numbers of cases in subgroup analyses, specifically for the highest trichloroethylene-exposed workers in the cohort. Most studies may not have had sufficient power to detect lower risk estimates (e.g., 1.3) for ever vs. never exposure. Some studies did not report the number of trichloroethylene-exposed cases for the cancer sites of interest (Silver et al. 2014; Yiin et al. 2009).

Although overall there are limited quantitative ambient or personal air monitoring data in the body of studies, there were reported levels of exposure for some of the Nordic studies and estimated levels of exposure for other populations. Moreover, different studies used different exposure metrics (see Table 3-1), which complicates comparisons of exposure levels across studies. Biomonitoring data from individual studies in the pooled Nordic cohort (Hansen et al. 2013) indicated that exposure levels were relatively low in this study (median equivalent ambient trichloroethylene levels probably ranged between 4 and $12 \mathrm{ppm}$ based on the individual studies) and only $20 \%$ of the subjects had U-TCA levels $>50 \mathrm{mg} / \mathrm{L}$, which is equivalent to approximately $20 \mathrm{ppm}$ ambient air trichloroethylene, in the pooled analyses. Ambient air monitoring data relevant to Raaschou-Nielsen et al. (2003) (see Raaschou-Nielsen et al. (2002)) indicate that 
exposures were higher prior to 1970 (40 to $60 \mathrm{ppm}$ ), 10 to $20 \mathrm{ppm}$ between 1970 and 1979 and 4 ppm after 1980. Thus, analyses of the subcohort of presumably higher exposed workers with employment before 1980 are considered to be more informative than analyses for the total cohort. Exposure levels, although not measured, were estimated by the JEM to be low in the large study reported by Vlaanderen et al. (2013). Estimated median exposure (units-yr [approximately equivalent to ppm]) for the cumulative exposure categories were 0.01 to 0.04 for the first tertile (depending on the endpoint), 0.12 to 0.13 for the second tertile, and 0.72 to 0.77 for the third tertile of cumulative exposure. However, the use of prevalence to calculate cumulative exposure complicates the interpretation of these levels. High exposure in this study was assigned to laundry workers, shoe and leather workers, or mechanics.

There were few data on exposure levels among the aerospace and aircraft cohorts. Most of the available data were estimated levels for Radican et al. (2008). Exposure intensity from degreasing was most likely high (ranging from 200 to $600 \mathrm{ppm}$ depending on time period) and estimated cumulative exposure was likely to range from 8 to $38 \mathrm{ppm}-\mathrm{yr}$ for use as a degreasing agent and 5 to 15 ppm-yr for benchwork (personal communication from Dr. Patricia Stewart to Dr. Ruth Lunn [June 23, 2014]). The National Academy of Sciences (NRC 2006) concluded that the cohort had modest numbers of highly exposed workers but most workers were exposed to approximately $10 \mathrm{ppm}$. There were few exposed cases $(<5)$ for kidney or liver cancer and 12 cases of NHL in the highest exposed group, and thus the study had limited statistical power to evaluate effects from high exposure to trichloroethylene. Little information is available on the other cohorts, although exposures among the highest exposure group in the study by Morgan et al. (1998) were estimated to be $>50 \mathrm{ppm}$. Exposure intensity was likely high among test mechanics in the aerospace worker cohort especially in the earlier time periods (Boice et al. 2006; Zhao et al. 2005). The study by Lipworth et al. (2006) was considered to have limited ability to detect an effect because exposure duration can be an insensitive metric for cumulative exposure and was likely to be low. The cohort enrolled workers employed at three facilities on or after 1960; however, trichloroethylene use ceased in 1966, and an unknown proportion of the cohort was exposed for shorter periods, although they were followed for long periods of time. Years exposed would include individuals with low and high cumulative and intensity of exposure.

In the German study of cardboard manufacturing workers, estimated peak exposure was $>2,000 \mathrm{ppm}$ and long-term exposure exceeded $100 \mathrm{ppm}$ (Cherrie et al. 2001); in addition, the workers were exposed for long periods (average 17.8 months). Thus, despite the low numbers of exposed cases, statistical power was probably adequate to detect the effect of high exposure. Exposure levels were not measured or estimated in the other studies (Fleming et al. 2014; Silver et al. 2014; Yiin et al. 2009). In other studies, exposure level (Ritz 1999), probability (Wilcosky et al. 1984), or few workers appear to be exposed to trichloroethylene by indirect means (Greenland et al. 1994). With respect to the drinking water study (Bove et al. 2014), the authors estimated that maximum consumption could be $3.6 \mathrm{mg}$ /day from water, based on measured trichloroethylene levels (combining ingestion, dermal, and inhalation exposure from showering), which would be the equivalent of approximately $0.07 \mathrm{ppm}$ as an 8-hour TWA (assuming 100\% intestinal absorption) and potentially as high as $25 \mathrm{ppm}$-year. It is more difficult to assess levels of exposure due to unknown actual individual consumption patterns and compare with studies in which ambient exposure occurred, due to uncertainty as to whether biological effects would 
differ by route of exposure. In addition, the number of exposed cases in subgroup analyses was not reported.

The ability of a study to evaluate exposure-response relationships depends on the adequacy of the exposure assessment, statistical power, and range of exposure levels included in the exposure-response analysis. Of the 16 identified studies, 10 reported risk estimates for 2 or more categories of exposure (Bahr et al. 2011; Boice et al. 2006; Bove et al. 2014; Hansen et al. 2013; Lipworth et al. 2011; Morgan et al. 1998; Raaschou-Nielsen et al. 2003; Radican et al. 2008; Ritz 1999; Vlaanderen et al. 2013; Zhao et al. 2005). However, most studies had limited ability to evaluate exposure-response relationships because of (1) lack of information on lifetime exposure (Hansen et al. 2013), (2) substantial concerns for exposure misclassification (Bove et al. 2014; Vlaanderen et al. 2013), (3) limited range of exposure levels because of overall low exposure levels or only two exposure categories (Boice et al. 2006; Morgan et al. 1998; Ritz 1999; Vlaanderen et al. 2013), (4) adequacy of the exposure surrogate for evaluating exposure, i.e., time since first exposure (Raaschou-Nielsen et al. 2003), exposure probability (Bahr et al. 2011), and exposure duration (Boice et al. 2006; Lipworth et al. 2011), or (5) limited statistical power because of few subjects in each exposure subgroup (most studies except for Vlaanderen et al. (2013)). The remaining studies only reported risk estimates for one category of exposure (Greenland et al. 1994; Henschler et al. 1995; Silver et al. 2014; Wilcosky et al. 1984; Yiin et al. 2009).

\subsubsection{Methods for Evaluating Confounding}

The majority of cohort and nested case-control studies conducted age-, sex-, race- and calendar year- or period-standardized comparisons in external analyses (SMR or SIR) where appropriate and age-, sex-, race- and in some cases calendar-period-adjusted comparisons in internal analyses. Most studies did not collect information on lifestyle factors, although internal analyses were conducted in a number of studies, which can reduce the potential for confounding as well as selection bias. However, confounding is generally unlikely to strongly influence risk estimates unless there is a strong association between the potential confounder to both exposure and cancer endpoint, which has not been demonstrated for most lifestyle or demographic factors or many of the identified co-exposures. The most informative study for considering confounding was the study of aircraft workers by Zhao et al. (2005), which conducted analyses controlling for coexposures. Some studies conducted separate analyses of major co-exposures (Boice et al. 2006; Bove et al. 2014; Zhao et al. 2005). Yiin et al. (2009) conducted multivariate analyses including trichloroethylene, nickel, mercury, and radiation dose, although the latter was the primary focus of the study and thus detailed exposure data on trichloroethylene exposure was lacking. A discussion of confounding is presented in the cancer evaluation of each cancer endpoint, as their potential impact differs depending on the specific cancer endpoint.

\subsubsection{Summary}

The database of cohort studies consisted of a large number of studies, many of which were considered to have adequate methodologies for evaluating potential cancer hazards. Although many of the cohorts were relatively large, most studies were still underpowered because of few exposed cases or deaths, especially in subgroups with higher exposure to trichloroethylene, to evaluate risks from the cancer sites of a priori interest, i.e., kidney cancer, liver cancer, and NHL, which are relatively uncommon. In addition, for some studies with adequate numbers of observed cases or deaths for kidney cancer, NHL, or liver cancer, exposure levels for 
trichloroethylene were low and/or exposure misclassification was a concern. Figure 3-1 depicts the overall assessment of the ability to inform the cancer evaluation based on the overall utility of the studies, including potential for biases and study sensitivity. The most informative studies (moderate- or high-utility studies) include the Nordic study of biomonitored workers (Hansen et al. 2013), and the aerospace and aircraft worker cohorts by Zhao et al. (2005), Morgan et al. (1998), and Radican et al. (2008)/Blair et al. (1998). Overall, these studies had adequate methods to assess exposure, little evidence of differential biases, and evaluated risks among subjects exposed to moderate to high levels of trichloroethylene. The study of aerospace workers (Zhao et al. 2005) was considered to be the most informative study because it evaluated cancer incidence, conducted detailed analysis of exposure-response relationships, and adjusted for potential confounding from co-exposures. Although the biomonitoring study was relatively good for identifying individuals who were ever exposed to trichloroethylene, U-TCA may not be a good marker for lifetime exposure or exposure intensity. The study by Morgan et al. (1998) was ranked lower for evaluating NHL compared with kidney and liver cancer because of fewer NHL cases compared with cases for the other endpoints.

Studies considered to have low/moderate ability to inform the cancer hazard evaluation, primarily because of more limited (mainly qualitative) exposure assessments and/or lower sensitivity, include the Nordic blue-collar worker study by Raaschou-Nielsen et al. (2003), aerospace workers by Boice et al. (2006), California aircraft manufacturing workers (Lipworth et al. 2011), and the nested case-control study of multiple myeloma among Tennessee uranium enrichment workers (Yiin et al. 2009). In the blue-collar worker study, the analysis of the subcohort of workers employed before 1980, when exposure levels were high, was considered to be more informative than analysis of the entire cohort. Exposure levels were presumably moderate to high in these studies; however, the study by Lipworth et al. (2011) only evaluated exposure duration, which most likely included workers with low levels of exposure. In addition, exposure duration was likely short in this study, which limited its sensitivity to detect an effect. In the Nordic study of blue-collar workers, there was the potential for confounding by, e.g., smoking, due to the differences in socioeconomic status between the cohort and the referent population; potential residual confounding from radiation exposure was also considered possible in the study by Yiin et al. (2009). Overall, however, the other limitations in all the studies (e.g., study sensitivity) were primarily toward the null.

The population-based Nordic study (Vlaanderen et al. 2013), the study of micro-electronic workers (Silver et al. 2014), the drinking water study (Bove et al. 2014), and the nested casecontrol study of electrical workers (Greenland et al. 1994) were considered to be of limited ability to inform the cancer hazard evaluation primarily because of low study sensitivity (e.g., lower levels of exposure) or potential for exposure misclassification. Studies having potential differential biases (away from the null) or potential confounding from occupational co-exposures include the German cardboard manufacturers cohort study (Henschler et al. 1995) and the uranium enrichment workers study (Ritz 1999). The study by Bahr et al. (2011) had limited documentation on the selection of the cohort and exposure and disease assessments, which complicate the evaluation of its quality. Finally, there was low confidence as to whether exposure to trichloroethylene occurred in the nested case-control study by Wilcosky et al. (1984) in addition to other methodological concerns and it was considered to have inadequate utility and was not brought forward in the hazard evaluations for specific cancers (Sections 4, 5, and 6). 


\section{High}

Zhao 2005

Selection bias unlikely

Little concern for misclassification or disease

misclassification

Adequate evaluation of $E / R$

Limited study sensitivity but moderate to high exposure levels

Adequate consideration of potential confounding

\section{Moderate}

Hansen 2013

Selection bias unlikely for internal analyses

Some concern for exposure misclassification

Mortality or older classification systems

Evaluation of E/R

Limited study sensitivity; moderate to high exposure level

Limited consideration of potential confounders

\section{Low/moderate}

Selection bias possible (except for internal analysis)

Higher concerns for exposure misclassification

Mortality or older classification systems

Limited or no evaluation of E/R

Low study sensitivity (except for R-N)

Limited consideration of potential confounders

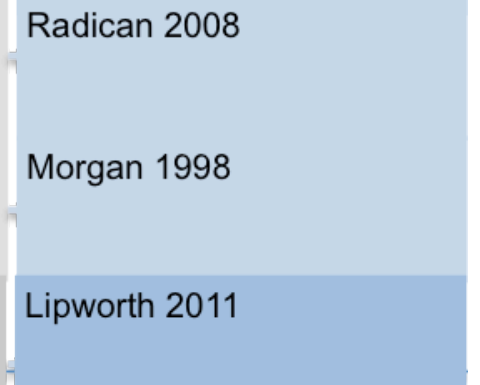

Yiin 2009

Boice 2006

Raaschou-Nielsen (R-N)

2003

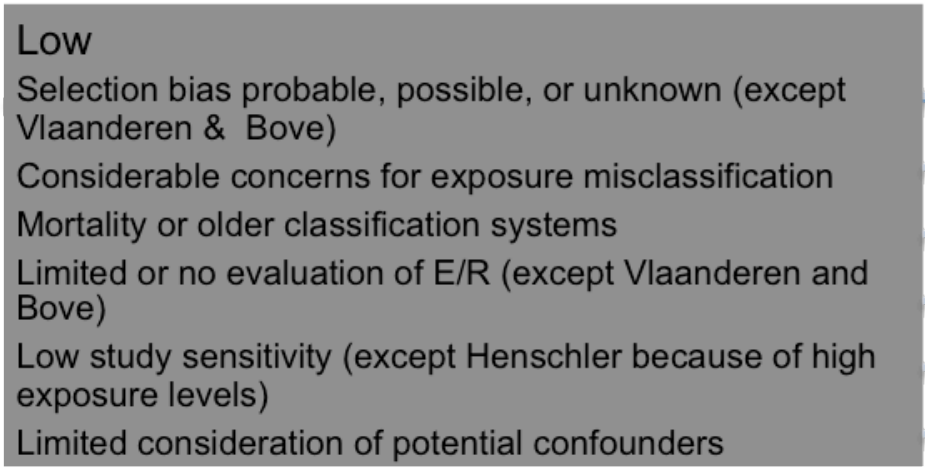

\begin{tabular}{|l|}
\hline Silver 2014 \\
\hline Bove 2014 \\
\hline Vlaanderen 2013
\end{tabular}

Bahr 2011a

Henschler $1995^{\text {b }}$

Ritz 1999b

Greenland 1994

\section{Figure 3-1. Study Utility Ranking: Cohort Studies}

\section{$\mathrm{E} / \mathrm{R}=$ exposure response.}

Gray shading (left-hand column): Utility to inform hazard evaluation; light shading = highest utility; dark shading = lowest utility. Blue shading (right-hand column): Potential bias and study sensitivity; light shading = least biased or most sensitive; dark shading $=$ overall potential biases towards the null or lower sensitivity.

a(Tan shading): Multiple limitations; overall direction of potential biases is unknown.

b(Peach shading): Most potential biases away from the null. 


\subsection{Kidney or Liver Cancer Case-control Studies}

\subsubsection{Overview of the Methodologies and Study Characteristics}

Table 3-2 lists seven kidney case-control studies that satisfied the inclusion criteria; one of these studies also reported on liver cancer (Christensen et al. 2013) (see Section 6). These include four studies conducted in areas with presumably higher levels and prevalence of trichloroethylene exposure using experts with knowledge of the local industry, and three studies of more widespread populations with more varying exposure potential for trichloroethylene, resulting in an overall lower average exposure levels in these populations. A multi-center study of renal-cell cancer was conducted in four countries of Central and Eastern Europe, in which occupational exposure to trichloroethylene was thought to be higher and more prevalent than in other industrial areas. Exposure was assessed in each of the different countries by a team of experts with knowledge of industries in their area (Moore et al. 2010). Charbotel et al. (2006); (2009) conducted a case-control study of kidney cases in the Arve Valley, France, which is an area with a widespread screw-cutting industry that used trichloroethylene as a degreaser. Although population based, the French and German studies had a higher prevalence of subjects with presumably higher levels of trichloroethylene and from more homogenous industries. Two studies (Brüning et al. 2003; Vamvakas et al. 1998) were conducted on a non-overlapping consecutive series of cases and controls among the same general population in the town and immediate surrounds of Arnsberg, Germany (Vamvakas et al. 1998) or the town and a somewhat wider surrounding area (Brüning et al. 2003), which has a large number of companies doing metal and electronics work. Trichloroethylene use was widespread and one of only two principal solvents (the other was carbon tetrachloride) used in the industry. Neither study included renalcell carcinoma (RCC) cases from the cohort study of workers at a cardboard factory from the same region (Henschler et al. 1995). Although population based, the French and German studies had a higher prevalence of subjects with presumably higher levels of trichloroethylene and from more homogenous industries. The remaining studies included population-based case-control studies in Minnesota (Dosemeci et al. 1999) and Germany (Pesch et al. 2000b) and a populationbased study using both population and hospital controls in Montreal, Quebec (Christensen et al. 2013). The population-based study by Pesch et al. (2000b) included five German regions, one of which (Leverkusen) was, like Arnsberg, in North Rhine-Westphalia.

For each of the reviewed studies, detailed data on study design, methods, and findings were systematically extracted from relevant publications, as described in the study protocol, into Table D-1 and Table D-2. 
Table 3-2. Case-control Studies of Trichloroethylene Exposure: Kidney or Liver Cancer

\begin{tabular}{|c|c|c|c|}
\hline Primary Reference & $\begin{array}{c}\text { Study Population } \\
\text { Total \# Cases/Controls }\end{array}$ & $\begin{array}{l}\text { Exposure Assessment } \\
\text { Exposure Metric }\end{array}$ & Cancer Assessment \\
\hline \multicolumn{4}{|c|}{ Studies in specific areas with knowledge of local industries } \\
\hline Moore et al. (2010) & $\begin{array}{l}\text { Multi-center, Central and } \\
\text { Eastern Europe, hospital- } \\
\text { based } \\
1,097 / 1,476\end{array}$ & $\begin{array}{l}\text { In-person interviews using } \\
\text { structured questionnaire, expert } \\
\text { assessment } \\
\text { Exposure duration (years, hours) } \\
\text { Cumulative exposure (ppm-yr) } \\
\text { Average exposure (ppm) }\end{array}$ & Incident $\mathrm{RCC}$ cases \\
\hline $\begin{array}{l}\text { Charbotel et al. } \\
(2006) ; \text { Charbotel et } \\
\text { al. (2009) }\end{array}$ & $\begin{array}{l}\text { Arve Valley, France, } \\
\text { hospital-based } \\
86 / 326\end{array}$ & $\begin{array}{l}\text { Telephone interviews using } \\
\text { structured questionnaire, expert } \\
\text { assessment, semi-quantitative } \\
\text { JEM } \\
\text { Cumulative exposure (ranked) }\end{array}$ & $\begin{array}{l}\text { Incident and deceased RCC } \\
\text { cases }\end{array}$ \\
\hline Brüning et al. (2003) & $\begin{array}{l}\text { Arnsberg, Germany, } \\
\text { hospital-based } \\
134 / 401\end{array}$ & $\begin{array}{l}\text { In-person or proxy interviews } \\
\text { using structured questionnaire, } \\
\text { self- and expert assessment (JEM) } \\
\text { Exposure + narcotic symptoms } \\
\text { Exposure duration (yr) } \\
\text { Longest job using TCE, metal } \\
\text { degreasing }\end{array}$ & $\begin{array}{l}\text { Incident and deceased RCC } \\
\text { cases }\end{array}$ \\
\hline $\begin{array}{l}\text { Vamvakas et al. } \\
\text { (1998) }\end{array}$ & $\begin{array}{l}\text { Arnsberg, Germany, } \\
\text { hospital-based 58/84 }\end{array}$ & $\begin{array}{l}\text { In-person (case or proxy) } \\
\text { interviews using structured } \\
\text { questionnaire, expert assessment } \\
\text { Ever exposed } \\
\text { Exposure category (ranked) }\end{array}$ & $\begin{array}{l}\text { Incident and deceased RCC } \\
\text { cases }\end{array}$ \\
\hline \multicolumn{4}{|l|}{ Other studies } \\
\hline $\begin{array}{l}\text { Christensen et al. } \\
(2013)\end{array}$ & $\begin{array}{l}\text { Montreal, Quebec } \\
\text { (Canada), hospital- and } \\
\text { population-based } \\
177 / 533\end{array}$ & $\begin{array}{l}\text { In-person interviews using } \\
\text { structured questionnaire, expert } \\
\text { assessment } \\
\text { Ever and substantial exposure } \\
\text { (includes probability) }\end{array}$ & $\begin{array}{l}\text { Incident RCC and liver } \\
\text { cancer cases }\end{array}$ \\
\hline Pesch et al. (2000b) & $\begin{array}{l}\text { Multi-center, Germany, } \\
\text { population-based } \\
935 / 4,298\end{array}$ & $\begin{array}{l}\text { In-person interviews using } \\
\text { structured questionnaire, expert } \\
\text { assessment, JTEM } \\
\text { Median, high \& substantial } \\
\text { exposure (includes probability) }\end{array}$ & Incident RCC cases \\
\hline $\begin{array}{l}\text { Dosemeci et al. } \\
\text { (1999) }\end{array}$ & $\begin{array}{l}\text { Minnesota, (USA) } \\
\text { population-based } \\
438 / 687\end{array}$ & $\begin{array}{l}\text { In-person interviews using } \\
\text { structured questionnaire } \\
\text { (occupation, exposures), JEM } \\
\text { Ever exposed }\end{array}$ & Incident RCC cases \\
\hline
\end{tabular}

$\mathrm{JEM}=$ job-exposure matrix; JTEM = job-task exposure matrix; RCC = renal-cell carcinoma. 


\subsubsection{Evaluation of Study Quality and Other Elements Related to the Utility of the Studies to Inform the Cancer Hazard Evaluation}

The methods for evaluation of study quality and other relevant study elements of the kidney and liver cancer case-control studies were similar to those described for cohort studies (see Section 3.2.2). Details of the systematic assessment of potential bias, study quality, and factors related to study sensitivity and assessment of exposure-response relationships for each study are available in Table D-6 and Table D-7.

\subsubsection{Selection and Participation Bias}

Selection bias was not a concern in the population-based case-control studies conducted in Montreal (which also used cancer controls) (Christensen et al. 2013), Minnesota (Dosemeci et al. 1999), and Germany (multi-center) (Pesch et al. 2000b) and the hospital-based case-control study in France (Charbotel et al. 2006; Charbotel et al. 2009). These studies selected cases and controls from the same population using similar inclusion criteria; controls were randomly selected and matched to the cases on age, sex, or location, if relevant. Although hospital controls may introduce selection bias if the diseases observed among controls are related to trichloroethylene exposure, several hospital-based case-control studies minimized this impact by excluding diseases related to kidney cancer (Charbotel et al. 2006; Charbotel et al. 2009; Moore et al. 2010), or restricted the inclusion of any specific disease (Moore et al. 2010) or cancer (Christensen et al. 2013) to $<20 \%$ of the total number of diseases/tumor sites. Persons with tobacco-related diseases were excluded for controls in the multicenter European study (Moore et al. 2010), because the potential for selection bias could be increased if smoking or other (lifestyle or co-exposure) risk factors related to smoking are related to kidney cancer risk and to exposure to trichloroethylene.

In the later German case-control study (Brüning et al. 2003), there was the potential for selection bias (possible) because of the use of prevalent cases (selected from1992 to 2000) and residual non-cases as controls (1999 to 2000). Controls were matched to cases on sex and age and were selected from surgery and geriatric departments from the same region as cases (selected from the urology department).

The study with the most concern for potential selection bias (probable) was the earlier Arnsberg study (Vamvakas et al. 1998). Cases (prevalent) were not interviewed until after the selection period, and cases who died in the interim were excluded from that analysis. In contrast, unmatched controls were recruited from hospitals adjacent to Arnsberg and selected at the end of the study. Controls were also younger than cases, but age was adjusted for in the analysis. This could potentially bias the findings away from the null and towards an overestimate of the risk estimate if exposure prevalence varies by geographical location and within the time period of the study. In addition, the study was conducted during a time period of legal proceedings.

Similar to many case-control studies, participation rates were somewhat higher among cases (>80\%) than controls (Charbotel et al. 2006; Charbotel et al. 2009; Christensen et al. 2013; Pesch et al. 2000b; Vamvakas et al. 1998). Participation rates were lower among controls in the Minnesota case-control study (Dosemeci et al. 1999), which could reduce precision. Rates were not reported for controls and/or cases in two of the hospital-based studies (Brüning et al. 2003; Moore et al. 2010). 


\subsubsection{Information Bias: Exposure Assessment and Misclassification}

The adequacy of the exposure assessment and the potential for exposure misclassification were considered, both with respect to whether cases or controls were ever exposed, and, if exposure ranks, categories, or levels were assigned, the degree to which misclassification among exposed subjects may have occurred within those categories. Misclassification of exposure category, low levels of exposure, or similar exposure levels across exposure groups can attenuate exposureresponse relationships.

Case-control studies typically rely on questionnaire data and recall of past occupational histories to assign exposure in the absence of quantitative exposure data. The assignment of exposure to trichloroethylene thus depends on detailed job or job-task description data or recall of actual exposures, which depending also on the availability of industrial hygiene data and the type and quality of the expert review or job exposure or job-task exposure matrix used, might limit exposure characterization and introduce the probability of exposure misclassification for ever/never exposure or within categories of exposure. Exposure misclassification was likely to be non-differential and to bias towards the null.

These studies used self- and/or proxy-report of work histories, jobs, or tasks using structured questionnaires and interviews, combined with expert assessment and/or JEM/job-task exposure assessment (JTEM) to estimate exposure probability, frequency, or level of potential exposure to trichloroethylene. However, the quality of the assessment varied depending on the available information. The studies in France (Charbotel et al. 2006; Charbotel et al. 2009), the Montreal study (Christensen et al. 2013), and the multi-center European study (Moore et al. 2010) were considered to have high-quality assessments because they collected detailed information on job tasks, considered calendar year and personal protective equipment, and provided semiquantitative estimates of trichloroethylene exposure. In the French (Charbotel et al. 2006; Charbotel et al. 2009) study, these estimates were based on ambient trichloroethylene levels for different jobs and tasks reported or estimated by other investigators in other studies. The likelihood of exposure, especially among individuals with higher exposure levels, is probably greater in the French (Charbotel et al. 2006; Charbotel et al. 2009) and European (Moore et al. 2010) studies than in the Montreal study (Christensen et al. 2013). In contrast to Christensen et al. (2013), in which the subjects were employed in diverse industries and jobs, the French study was conducted in an area with more homogeneous industries and with a high prevalence of exposure to high levels of trichloroethylene. In addition, the study had a good exposure assessment so that misclassification of workers was not a concern. Although the Central and East European study was conducted in several different areas, researchers chose the study subjects from industrial areas thought to have higher exposure to trichloroethylene, used experts from the region for the exposure assessment, and validated the assessment at a later time period (with $83 \%$ agreement in one country and $100 \%$ agreement in two countries). Additionally, investigators presented separate analyses among individuals with high confidence of exposure as an attempt to reduce the potential for exposure misclassification bias. Although the quality of the exposure assessment was considered to be adequate in the Montreal study, misclassification of exposure is still possible because of the lower likelihood of exposure in a population-based study.

The analysis by Vamvakas et al. (1998), and to a lesser extent by Brüning et al. (2003), relied more heavily on self-reported "pre-narcotic symptoms" (dizziness, nausea, headaches, and drowsiness) to indirectly characterize exposure to trichloroethylene (and tetrachloroethylene). In 
Vamvakas et al. (1998), experts integrated this information with exposure duration to assign workers to different exposure categories, whereas in Brüning et al. (2003), there was no expert assessment of the self-reported symptoms or exposure information. However, although selfreported exposure is usually considered to be less reliable than semi-quantitative or quantitative assessments and is subject to recall bias, exposure misclassification was not a serious concern in these studies, because of the frequency and severity of symptoms among the majority of cases in both studies as well as detailed descriptions of working conditions in the local industries, all of which suggest that most subjects were exposed to substantial levels of trichloroethylene. Interviewers were not blinded in the Vamvakas et al. (1998) study, and it is not clear whether the exposure assessment experts were blinded to disease status, which would have greater impact on misclassification than lack of blinding among the interviewers. In both cases, potential for differential misclassification is a concern and could lead to an overestimation of risk. Brüning et al. (2003) also assessed exposure to trichloroethylene using a JEM (see below).

Three studies used less detailed work information with respect to job task or a more generic JEM to assess exposure to trichloroethylene: the larger multi-center German study (Pesch et al. 2000b), the later Arnsberg study (Brüning et al. 2003) and the Minnesota study (Dosemeci et al. 1999). In the Pesch et al. study, the JTEM was considered to be more informative than the JEM exposure assessment, although detailed information on job tasks was limited. The JEM used in the other two studies was based on broad occupation groups, was not calendar specific or area specific; a U.S.-wide JEM was used in the Minnesota study (Dosemeci et al. 1999) and a British JEM was used in the German study by (Brüning et al. 2003). These JEMs were considered to be more limited in quality than the JTEM used by Pesch et al. (2000b), which was developed by the study investigators although little information was provided on job tasks. Misclassification of exposure (or the use of the JEM analysis to classify exposure in the Brüning et al. (2003) study) is a concern in these three studies.

\subsubsection{Information Bias: Disease Assessment}

Renal-cell carcinomas were identified from hospital files or cancer registries and appear to have been histologically or sonographically confirmed in each study, and thus there is little concern about misclassification of disease. Three studies (Brüning et al. 2003; Charbotel et al. 2006; Charbotel et al. 2009; Vamvakas et al. 1998) also included a small number of deceased cases, but as the sources for both the living and deceased cases were the same, it is unlikely that any misclassification would have occurred among the latter cases. In the single study that reported on liver cancer (Christensen et al. 2013), incident cases (identified via hospitals) were also histologically confirmed.

\subsubsection{Study Sensitivity and Exposure-response Relationships}

In addition to the analysis of biases and confounding, study sensitivity and the ability to analyze exposure-response relationships also impact the ability of a study to inform the cancer evaluation. As noted in the discussion of the cohort studies, study sensitivity (i.e., the ability to detect an effect) is a function of exposure prevalence and levels, sample size, and length of follow-up. Subgroup analyses that examine risks among individuals with higher exposure or higher probability of exposure were considered to be more informative for cancer hazard evaluation. 
Few quantitative exposure data were available for the reviewed studies although estimated exposure levels are available for several studies. Moreover, different metrics were used in the different studies, which complicates comparisons of exposure levels across studies (see Table 32). In the studies conducted in the industrial regions of the Arve Valley in France (Charbotel et al. 2006; Charbotel et al. 2009) and the Arnsberg region of Germany (Brüning et al. 2003; Vamvakas et al. 1998), the study authors or other reviewers (Cherrie et al. 2001; NRC 2006) have provided estimates of exposure intensity that indicate that the study participants were exposed to high levels of trichloroethylene in both regions. In the German study, peak exposure was estimated to range from 400 to $600 \mathrm{ppm}$ and overall exposure was $>100 \mathrm{ppm}$ (Cherrie et al. 2001). In the French study, exposures of 300 to $600 \mathrm{ppm}$ were estimated for some tasks. In contrast, the NRC (2006) estimated that most subjects in the multicenter study in Germany (Pesch et al. 2000a) had minimal contact with trichloroethylene, with concentrations up to $10 \mathrm{ppm}$.

The French (Charbotel et al. 2006; Charbotel et al. 2009) and German (Brüning et al. 2003; Vamvakas et al. 1998) studies conducted in small industrial areas had adequate sensitivity to detect an effect (if one exists) because of the higher levels of exposure and/or prevalence in these regions. These studies appear to have had adequate overall numbers of workers and had higher estimated levels of exposure than the population-based studies. The statistical power for subgroup analyses conducted by Charbotel et al. (2006); Charbotel et al. (2009) and Vamvakas et al. (1998) was more limited, however. A further strength of the French study was analyses of exposure-response relationships with cumulative exposure that included an adequate range of exposure levels for trend analyses and greater confidence in the exposure assessment. The Eastern and Central European study (Moore et al. 2010) had adequate study sensitivity due to its large size and estimated moderate exposure (among the highest exposed subjects), although there were fewer subjects with both high exposure and high-confidence exposure assessments. Another strength of the study was that it conducted analyses of several metrics of exposures (duration, cumulative, and intensity). The two studies of the Arnsberg area workers probably had limited ability to look at exposure-response relationships because of a presumed narrow range due to widespread high exposure; Vamvakas et al. (1998) reported risk estimates for ranked exposure category and Brüning et al. (2003) evaluated categories of severity of symptoms (surrogates for exposure intensity and exposure duration).

Study sensitivity in the Montreal study (Christensen et al. 2013), reporting on kidney and liver cancer, was limited by few cases (two for kidney and one for liver) with substantial exposure to trichloroethylene (levels unknown), and that in the German multicenter study (Pesch et al. 2000b) was limited by lower levels of exposure. The studies by Pesch et al. (2000b) and Christensen et al. (2013) combined confidence or probability as part of their exposure categories, which complicated the evaluation of exposure-response relationships. The study by Dosemeci et al. (1999) appeared to have sufficient statistical power (based on numbers of exposed subjects) to evaluate ever-exposed cases, but it did not report data on levels or duration of exposure.

\subsubsection{Confounding}

All of the studies adjusted for (or considered) age, sex, and smoking, and all except Vamvakas et al. (1998) and Christensen et al. (2013) adjusted for body mass index for renal-cell carcinoma. Some studies considered socioeconomic factors and, for risk estimates for liver cancer, alcohol consumption (Christensen et al. 2013), medical history or conditions (Dosemeci et al. 1999; 
Moore et al. 2010; Pesch et al. 2000b; Vamvakas et al. 1998), or other lifestyle factors (Christensen et al. 2013). Only the French study (Charbotel et al. 2006; Charbotel et al. 2009) presented additional analyses adjusting for co-exposures to other occupational carcinogens.

\subsubsection{Summary}

The major strength of the database of case-control studies was the inclusion of studies that selected populations with higher likelihood of exposure to trichloroethylene, more homogeneous industries, and use of experts with knowledge of the local industries. In addition, most of the studies were able to adjust or consider potential confounding from lifestyle habits or medical history. Most studies had limited statistical power due to small numbers of subjects exposed to high levels of trichloroethylene. The studies by Charbotel et al. (2006); Charbotel et al. (2009) and Moore et al. (2010) were considered to be the most informative for the cancer evaluation because of greater confidence that most of the subjects classified as exposed were most likely exposed to substantial levels of trichloroethylene (Charbotel et al. 2006; Charbotel et al. 2009); in the study by Moore et al. (2010), analyses focused on the highest exposed individuals with high probability of exposure. Other strengths of the Charbotel et al. study were controlling for potential confounding from co-exposures in the analysis and evaluating exposure-response relationships for cumulative and peak exposure. The study by Brüning et al. (2003) was considered to be of moderate ability to inform the cancer hazard evaluation. Although the exposure assessment relied primarily on self-assessed exposure to identify workers exposed to trichloroethylene, study sensitivity was high because the presence of symptoms and qualitative job description data strongly suggest that these workers experienced high levels of exposure. The remaining studies were considered to have low to low/moderate utility. Although the study by Christensen et al. (2013) also evaluated risks among individuals with substantial exposure (integration of intensity, duration, and frequency), there were few exposed cases (two cases for kidney, and one for liver cancer), which limited its ability to inform the cancer hazard evaluation. No other case-control study reported on liver cancer. The study by Pesch et al. (2000b), and, to a greater degree, the study by Dosemeci et al. (1999), were considered to be more limited because of concerns of exposure misclassification, which would most likely bias towards the null, and limit the ability to detect an effect (if present). Finally, the study by Vamvakas et al. (1998) should be viewed with some caution because of the potential for selection bias, which would most likely lead to an overestimate of the risk estimate. However, the likelihood of exposure to substantial levels of trichloroethylene in this study should also be considered in evaluating the degree of distortion due to selection bias on the findings in this study.

The findings from these studies and the cohort studies reporting risk estimates for kidney cancer are discussed in the cancer hazard evaluation for kidney cancer, which will integrate the study quality assessment and other elements related to its utility to inform the cancer evaluation, discuss whether chance, bias, or confounding can be ruled out for studies with positive findings, discuss other studies and integrate the findings from meta-analyses of these studies (see Section 4.1). 


\subsection{Case-control Studies of NHL and Related Subtypes}

\subsubsection{Overview of the Methodologies and Study Characteristics}

Table 3-3 lists six case-control studies of NHL (some of which also evaluated several subtypes, and one study specific for hairy-cell leukemia [HCL], a type of NHL), and two studies specific for multiple myeloma (which is considered a type of B-cell lymphoma) that met the inclusion criteria. The studies include the International Lymphoma Epidemiology Consortium study (InterLymph) pooled case-control study (Cocco et al. 2013), and population-based studies in Montreal, Quebec, Canada (Christensen et al. 2013), Connecticut, USA (Deng et al. 2013; Wang et al. 2009b), and Sweden (Hardell et al. 1994); a pooled analysis of two studies from Sweden (Persson and Fredrikson 1999); and a study of HCL in Sweden (Nordström et al. 1998). The InterLymph study (Cocco et al. 2013) includes pooled cases and controls from four large multicenter studies: the EPILYMPH study in Europe (Cocco et al. 2010), the ENGELA study in France (Orsi et al. 2010), the Multicentre Italian Study (MIS) (Miligi et al. 2006), and the NCISEER study in the United States (Purdue et al. 2011a). Because the InterLymph pooled analysis included all the subjects of the four constituent studies and harmonized the exposure and disease assessment, this evaluation primarily reviews the pooled analysis. Information (e.g., analyses of different exposure metrics) from the individual studies that was not incorporated in the pooled analysis was considered in the cancer hazard evaluation. An additional study in Germany by Seidler et al. (2007) was also identified, but was not reviewed because its population was included in the EPILYMPH multi-center study (Cocco et al. 2010), which was then included in the InterLymph pooled analysis.

For multiple myeloma, two additional studies were identified, one in Italy (Costantini et al. 2008), using the same population as the MIS study) and one in the United States (two of the SEER registries) (Gold et al. 2011), as well as the InterLymph study pooled analysis (Cocco et al. 2013) and the Montreal study (Christensen et al. 2013). The InterLymph pooled analysis and the Italian study (Costantini et al. 2008) also reported findings for chronic lymphocytic leukemia (CLL).

For each of the reviewed studies, detailed data on study design, methods, and findings were systematically extracted from relevant publications, as described in the study protocol, into Table D-3. Studies are organized by lymphoma type and then by chronological order.

Table 3-3. Case-control Studies of Trichloroethylene Exposure and NHL and Its Subtypes

\begin{tabular}{llll}
\hline $\begin{array}{c}\text { Primary } \\
\text { Reference } \\
\text { Years }\end{array}$ & \multicolumn{1}{c}{$\begin{array}{c}\text { Study Population } \\
\text { Cases/Controls }\end{array}$} & \multicolumn{1}{c}{$\begin{array}{c}\text { Exposure Classification } \\
\text { Exposure Metric }\end{array}$} & Cancer Assessment \\
\hline $\begin{array}{l}\text { Christensen et al. } \\
(2013)\end{array}$ & $\begin{array}{l}\text { Montreal, Quebec } \\
\text { (Canada) }\end{array}$ & $\begin{array}{l}\text { In-person interview using structured } \\
\text { questionnaire, expert assessment }\end{array}$ & ICD-9: 200+202 (NHL) \\
$1979-1985$ & $215 / 533$ & $\begin{array}{l}\text { Ever and substantial exposure } \\
\text { (includes probability) }\end{array}$ & Hospital; histologically confirmed \\
& & & \\
\hline
\end{tabular}




\begin{tabular}{|c|c|c|c|}
\hline $\begin{array}{c}\text { Primary } \\
\text { Reference } \\
\text { Years }\end{array}$ & $\begin{array}{l}\text { Study Population } \\
\text { Cases/Controls }\end{array}$ & $\begin{array}{l}\text { Exposure Classification } \\
\text { Exposure Metric }\end{array}$ & Cancer Assessment \\
\hline $\begin{array}{l}\text { Cocco et al. } \\
(2013) \\
1991-2004\end{array}$ & $\begin{array}{l}4 \text { pooled studies Cocco } \\
\text { et al. (2010); Miligi et } \\
\text { al. (2006); Orsi et al. } \\
\text { (2010); Purdue et al. } \\
\text { (2011a) } \\
3,788 / 4,279\end{array}$ & $\begin{array}{l}\text { Questionnaire, expert assessment } \\
\text { Exposure probability } \\
\text { Exposure duration (yr) } \\
\text { Exposure frequency (\% work time) } \\
\text { Exposure intensity (ppm) }\end{array}$ & $\begin{array}{l}\text { NHL and subtypes } \\
\text { WHO InterLymph consortium } \\
\text { classification } \\
\text { Histologically confirmed }\end{array}$ \\
\hline $\begin{array}{l}\text { Deng et al. } \\
(2013) / \text { Wang et al. } \\
(2009 b) \\
1996-2000\end{array}$ & $\begin{array}{l}\text { Connecticut (USA) } \\
601 / 7,171\end{array}$ & $\begin{array}{l}\text { Questionnaire, JEM } \\
\text { Exposure probability } \\
\text { Exposure intensity (ranked) }\end{array}$ & $\begin{array}{l}\text { NHL and subtypes } \\
\text { ICD-O-2, codes M-9590-9642, } \\
9690-9701,9740-9750 \\
\text { Histologically confirmed }\end{array}$ \\
\hline $\begin{array}{l}\text { Gold et al. (2011) } \\
2000-2002\end{array}$ & $\begin{array}{l}\text { SEER registries, } \\
\text { Seattle Detroit } \\
9,731 / 9,732\end{array}$ & $\begin{array}{l}\text { In-person interviews using } \\
\text { structured questionnaire, expert } \\
\text { assessment } \\
\text { Exposure duration (yr) } \\
\text { Cumulative exposure (ppm-hr) }\end{array}$ & $\begin{array}{l}\text { MM } \\
\text { ICD-O-2/3 } \\
\text { SEER cancer registry; histologically } \\
\text { confirmed }\end{array}$ \\
\hline $\begin{array}{l}\text { Costantini et al. } \\
(2008) \\
1991-1993\end{array}$ & $\begin{array}{l}\text { Regional Italy } \\
263 / 1,100 \mathrm{MM} \\
586 / 1,278 \text { (all } \\
\text { leukemia; subtype } \\
\text { totals NR) }\end{array}$ & $\begin{array}{l}\text { In-person interviews using } \\
\text { structured questionnaire, expert } \\
\text { assessment } \\
\text { Exposure intensity (ranked) } \\
\text { Exposure duration (yr) }\end{array}$ & $\begin{array}{l}\text { MM, CL } \\
\text { ICD-9: } 203 \text { (MM), } 204.1 \text { (CLL) } \\
\text { Hospitals; histological confirmation } \\
\text { NR }\end{array}$ \\
\hline $\begin{array}{l}\text { Persson and } \\
\text { Fredrikson (1999) } \\
\text { 1964-1986 }\end{array}$ & $\begin{array}{l}\text { Sweden } \\
\text { Pooled analysis of } 2 \\
\text { studies (1983 and } \\
1989) \\
199 / 479\end{array}$ & $\begin{array}{l}\text { Self-reported ranked exposure } \\
\text { Ever exposed }\end{array}$ & $\begin{array}{l}\text { NHL } \\
\text { 2nd (1989) study: ICD-8: } 200+202 \\
\text { NR in } 1989 \text { study } \\
\text { Hospital; histologically confirmed }\end{array}$ \\
\hline $\begin{array}{l}\text { Nordström et al. } \\
\text { (1998) } \\
1987-1992\end{array}$ & $\begin{array}{l}\text { Sweden } \\
121 / 484\end{array}$ & $\begin{array}{l}\text { Self-reported occupational history } \\
\text { Ever exposed }\end{array}$ & $\begin{array}{l}\text { HCL } \\
\text { Cancer registry; classification and } \\
\text { histological confirmation NR }\end{array}$ \\
\hline $\begin{array}{l}\text { Hardell et al. } \\
(1994) \\
1974-1978\end{array}$ & Umeå Region Sweden & $\begin{array}{l}\text { Self-reported occupational history } \\
\text { Ever exposed }\end{array}$ & $\begin{array}{l}\text { NHL } \\
\text { Hospital histologically verified; } \\
\text { Rappaport classification; stages and } \\
\text { anatomical sites }\end{array}$ \\
\hline
\end{tabular}

Other lymphohematopoietic endpoints, including all leukemia combined (ICD-9 204-208), are not included in the table. $\mathrm{CLL}=$ chronic lymphocytic leukemia; ICD = International Classification of Diseases; HCL = hairy-cell leukemia; JEM = jobexposure matrix; NHL = non-Hodgkin lymphoma; $\mathrm{MM}=$ multiple myeloma; NR = not reported; SEER = Surveillance, Epidemiology and End Results program (U.S. National Cancer Institute); WHO = World Health Organization.

\subsubsection{Evaluation of Study Quality and Other Elements Related to the Utility of the Studies to Inform the Cancer Hazard Evaluation}

The methods for evaluation of study quality of the NHL case-control studies were similar to that described for cohort studies (see Section 3.2.2). Details of the systematic review of bias and factors and study sensitivity for each study are available in Table D-8 and Table D-9.

\subsubsection{Selection and Participation Bias}

Selection bias was considered unlikely in these studies. In general, cases and controls were selected from the same underlying population using similar inclusion criteria; controls were 
randomly selected and age matched (and sex matched where both sexes were included) to the controls. Most studies had participation rates $>80 \%$ for cases and $>70 \%$ for controls. The Swedish studies had high participation rates (83\% to approximately 96\%) Studies having lower participation rates among cases and controls were the Connecticut population-based case-control study of NHL (Deng et al. 2013; Wang et al. 2009b), the SEER study (Seattle, Washington and Detroit Michigan) of multiple myeloma, and one of the component studies (Purdue et al. (2011a), Cocco et al. (2010) for population controls) of the pooled InterLymph case-control study (Cocco et al. 2013).

\subsubsection{Information Bias: Exposure Assessment and Misclassification}

The exposure assessments in the InterLymph pooled case-control study (Cocco et al. 2013), the Montreal study (Christensen et al. 2013), the SEER study of multiple myeloma (Gold et al. 2011), and the Italian study of multiple myeloma and chronic lymphocytic leukemia (Costantini et al. 2008) used experts to rate frequency, confidence, intensity, and duration of exposure to trichloroethylene for each job (or task) reported in the questionnaire data, taking into consideration changes in trichloroethylene exposure over calendar periods. The InterLymph (Cocco et al. 2013) and Seattle and Detroit-SEER (Gold et al. 2011) studies provided quantitative ratings, and the exposure assessment approaches were considered as high quality. The Detroit-SEER study used the same methods to assess exposure as the NCI-SEER study by Purdue et al. (2011a), one of the studies in the pooled analysis in the InterLymph analysis. An advantage of these two studies was that they conducted separate analyses of individuals with high probability of exposure, which helped to mitigate concerns of exposure misclassification, especially among subjects with higher levels of exposure. The Montreal study (Christensen et al. 2013) and the Italian study (Costantini et al. 2008) provided semi-quantitative ratings of exposure.

The Connecticut study (Deng et al. 2013; Wang et al. 2009b) used a JEM to provide semiquantitative ratings, and exposure ranks were based on broad occupational groups rather than job tasks. The quality of the exposure assessment is considered to be more limited than in studies using job and task rankings and exposure misclassification was a concern, although to a lesser degree among individuals in the categories of higher probability or higher intensity of exposure. The exposure assessments of the three Swedish studies were primarily based on self-reported job titles and exposures to a specific set of chemicals with either a minimum (1 day) criterion for the exposed group (Hardell et al. 1994; Nordström et al. 1998) or five categories of ranked exposure with a minimum of 1 year of exposure (Persson and Fredrikson 1999) and thus were considered to be of lower quality. Misclassification of exposure is likely to be substantial and is a concern. The direction of the bias is not known since self-reported data may differ by disease status; however, there is also the potential for non-differential misclassification.

As noted in the discussion for kidney cancer, misclassification of exposure in these studies was most likely non-differential and would bias results towards the null. This type of misclassification would most likely attenuate the ability to observe an exposure-response relationship. There was generally greater confidence that individuals in the highest exposure categories were actually exposed to trichloroethylene than in the lower categories, although there may be misclassification with respect to the intensity of exposure. 


\subsubsection{Information Bias: Disease Endpoints}

Histological confirmation of cases was conducted on all studies with the possible exception of the study of HCL (Nordström et al. 1998) and the Italian study of multiple myeloma (Costantini et al. 2008), neither of which reported whether the cases were confirmed. As noted in the discussion of cohort studies, changes have been made in the classification systems used for these lymphomas. The WHO REAL classification (used from 2001 on; see e.g., Morton et al. (2007)) used in the ICD Oncology Second and Third Revisions is the most recent and most informative for the revised classification of B- and T-cell lymphomas (including NHL and its subtypes). This classification system was used in the InterLymph pooled analysis (Cocco et al. 2013), the Connecticut study (Deng et al. 2013; Wang et al. 2009b), and the SEER study of multiple myeloma (Gold et al. 2011) (see table in Section 4.1.2. Meta-analyses: Kidney Cancer). Older classifications (ICD-9 and earlier) were used in the Swedish studies (Hardell et al. 1994; Nordström et al. 1998; Persson and Fredrikson 1999) and the Montreal study (Christensen et al. 2013). Costantini et al. (2008) use a broader grouped classification for NHL, together with MM and CLL, from ICD-9. Overall, changes in the classification systems used would be expected to introduce heterogeneity in study comparisons because of differences in lymphoma groupings between the systems.

\subsubsection{Study Sensitivity and Exposure-response Relationships}

In addition to the analysis of biases and confounding, study sensitivity (the ability to detect an effect from exposure) and the ability to analyze exposure-response relationships also impact the ability of a study to inform cancer evaluation. Population- or hospital-based case-control studies often lack adequate power to detect an effect for NHL, as reflected by the low numbers of exposed cases (Table D-9). Actual exposure levels were not reported for any studies. Some studies (Cocco et al. 2013; Gold et al. 2011; Purdue et al. 2011a) reported estimates in their exposure-response analysis. Estimated exposure levels in the highest exposure categories were $>75$ or 150 ppm (Cocco et al. 2013) for average exposure intensity, $>200,000$ ppm-hr (Purdue et al. 2011a) or up to 50,000 ppm-hr (Gold et al. 2011) for cumulative exposure. Purdue et al. (2011a) was a component study of the InterLymph pooled analysis.) These estimates suggest that exposure level among the highest exposed may be in the range of estimated levels reported in the cohort studies, although these should be interpreted with caution because actual ambient trichloroethylene levels were generally not available.

Although the available database included one very large study, and several medium to large studies, exposure prevalence was low to relatively low in most of the studies, ranging from $<1 \%$ to $11 \%$ for most studies with the exception of the multiple myeloma study by Gold et al. (2011), which had an exposure prevalence of close to $30 \%$. The prevalence of subjects with higher probability of exposure was even lower. In the InterLymph pooled case-control study of over 35,000 cases (Cocco et al. 2013), 7\% of the workers were exposed to trichloroethylene, but only 1\% were classified as definitely exposed. Two studies (Cocco et al. 2013; Deng et al. 2013; Wang et al. 2009b) had relatively large numbers of exposed cases and controls and most likely had adequate statistical power, although average exposure levels were not reported. A strength of both studies was that they stratified by both probability of exposure and exposure intensity level; however, in the study by Deng et al. (2013)/Wang et al. (2009b), no subjects had high probability and medium or high intensity of exposure. In the InterLymph study, statistical power for NHL subtypes appeared to be good in evaluating risks for high exposure among all subjects although there were fewer subjects in the analyses of subjects with high probability of exposure. Finally, 
in the smaller studies conducted in Sweden (Hardell et al. 1994; Nordström et al. 1998; Persson and Fredrikson 1999) the observed prevalence of trichloroethylene exposure was $<5 \%$ among referents, and these studies did not present analyses by exposure categories. In addition, these studies had low minimal criteria to be considered as ever exposed, based on either 1-week or 1day duration of exposure, and thus some exposed individuals would typically be considered as unexposed by other investigators.

Studies evaluating exposure-response relationships (or looking at different levels of exposure) using multiple metrics of exposure (Cocco et al. 2013; Deng et al. 2013; Gold et al. 2011; Wang et al. 2009b) were considered to be more informative for the cancer hazard evaluation. Although the exposure range was adequate in these studies and exposure levels were high in some of the studies, they had limited statistical power because of small numbers of cases and controls in each level of exposure.

\subsubsection{Confounding}

Each of the studies matched or adjusted for age, sex, birth year, or race, using conditional or unconditional logistic regression, as appropriate. Some studies (Christensen et al. 2013; Costantini et al. 2008; Deng et al. 2013; Wang et al. 2009b), and some of the component studies of the pooled analysis (Cocco et al. 2010; Miligi et al. 2006; Purdue et al. 2011a) also considered or adjusted for smoking, other lifestyle factors, and surrogates of socioeconomic status. Little information was available on potential occupational co-exposures, with the partial exception of Gold et al. (2011) who reported modest correlations (16\% or less) between trichloroethylene, carbon tetrachloride, methylene chloride, and 1,1,1-trichloroethane among controls. In the study by Hardell et al. (1994), subjects may have been exposed to phenoxyacetic acids, chlorophenols or other organic solvents. None of the other studies adjusted for co-exposures in their analysis, although the InterLymph study (Cocco et al. 2013) conducted a sensitivity analysis excluding subjects exposed to benzene. Study participants in these population-based studies of NHL most likely came from diverse industries and thus it was not clear whether any specific co-exposures (other than perhaps other chlorinated or other organic solvents) would likely be correlated with trichloroethylene exposure.

\subsubsection{Summary}

Overall, the strengths of the NHL case-control study database are two studies of large populations, high-quality exposure assessment, evaluation of NHL subtypes and consideration or adjustment for potential confounding from life-style habits. The pooled analysis (Cocco et al. 2013) and the SEER study on multiple myeloma (Gold et al. 2011) were considered to be the most informative studies because of the quality of the exposure and disease assessments, evaluation of multiple metrics of exposure, and larger numbers of exposed cases and controls, especially among individuals with higher probability or intensity of exposure. Studies by Christensen et al. (2013), Costantini et al. (2008) and Deng et al. (2013)/Wang et al. (2009b) were considered to be of low to moderate utility for the cancer hazard evaluation and were limited by one or more factors: limited statistical power, lower quality exposure assessment, or use of older disease classifications. The three Swedish case-control studies (Hardell et al. 1994; Nordström et al. 1998; Persson and Fredrikson 1999) were considered to be of low utility because of concerns for substantial misclassification of exposure (self-reported), use of older disease classification systems, and relatively small numbers of exposed subjects. 


\section{Kidney Cancer}

The previous sections of the cancer evaluation component contain relevant information-ADME (Section 1), genetic and related effects (Section 2), and overview and assessment of the quality of the human cancer studies (Section 3) - that are important for several of the three cancer endpoints of interest. This section builds on that information and evaluates the human cancer studies (Section 4.1) and mechanistic data (Section 4.2) specifically for kidney cancer.

\subsection{Human Cancer Studies}

Kidney cancer is considered to be uncommon; the age-adjusted annual kidney cancer (kidney and renal pelvis) rates (per 100,000 males or females) in the United States from 2007 to 2011 (SEER 2014d) were approximately 21.2 (male) and 10.6 (female) for incidence and 5.8 (male) and 2.6 (female) for mortality, with a five-year survival rate of approximately $70 \%$, suggesting that incidence data may be more informative than mortality data. The incidence rate of kidney cancer has been increasing for several decades in the United States from an incidence rate of approximately 7 per 100,000 in 1975 at a rate of approximately $2 \%$ per year over the past decade, while death rates have declined slightly by approximately $0.6 \%$ per year. Incidence rates and trends in the European countries (Ferlay et al. 2013; Ferlay et al. 2014) in which the remainder of the studies were conducted are broadly similar to those in the United States. For example, age-standardized incidence rates in the United Kingdom in 2011 (Cancer Research U.K. 2014b) were approximately 16 per 100,000 (male) and 9 per 100,000 (female), with an increase in the combined U.K. male and female incidence rate from approximately 5 per 100,000 in 1975 to 12.3 per 100,00 in 2011 , representing a rate of increase of approximately $3 \%$ per year over the decade from 2001 to 2011. Latencies for solid tumors such as kidney cancer are generally estimated to exceed approximately 20 years but may vary considerably. Incidence rates generally increase sharply after approximately 50 years of age. Case-control studies of trichloroethylene and kidney cancer are of renal-cell carcinoma, which makes up approximately $90 \%$ of all kidney cancers, whereas most of the cohort studies are of combined (renal, pelvis and/or ureter) kidney cancer.

For each of the reviewed studies, summary data on study design, methods, and findings, systematically extracted from relevant publications as described in the study protocol, are presented in Table D-2. The evaluation of study quality, including methods of exposure and cancer endpoint assessment, and other elements related to the ability to inform the cancer hazard assessment (such as study sensitivity) is reported in Table D-4, Table D-5, Table D-6, and Table D-7. Section 3 provided an overview of the study population characteristics and methods and a discussion of the utility of the studies. Figure 4-1 (below) provides an overview of the conclusions from that evaluation and identifies the most informative studies based on the overall utility of the study.

This section summarizes and interprets the findings for kidney cancer from the individual epidemiological studies brought forward for evaluation and integrates the evidence across studies. The key questions for evaluating the level of evidence across the body of studies is whether there is credible evidence of an association between trichloroethylene exposure and kidney cancer, and if so, could it be explained by chance, bias, or confounding. Several of the considerations developed by Austin Bradford Hill (Hill 1965) are relevant to the evaluation of 
the level of evidence for human carcinogenicity, including the magnitude (strength) and consistency of any observed associations across studies; evidence for exposure-response relationships and associations with appropriate latency; and the degree to which chance, bias, and confounding could plausibly explain observed associations. The level of evidence conclusion for the carcinogenicity of trichloroethylene from studies in humans is provided in Section 7.

\subsubsection{Study Findings}

This cancer hazard evaluation reports on the latest update of a cohort or case-control study and meta-analyses and includes any additional relevant data (e.g., analyses or exposure information) from previous publications. The available studies that reported on kidney cancer and trichloroethylene exposure and were considered to be adequate for inclusion in the evaluation include 12 cohort or nested case-control studies and 7 population-based case-control studies. (Two cohort studies of uranium processing workers (Bahr et al. 2011; Ritz 1999) did not report on kidney cancer and exposure specifically to trichloroethylene.) In addition, three recent metaanalyses were identified and are included in the evaluation. The findings of the individual studies are discussed below and presented in Table 4-1 and Table 4-2. Although the database consists of many reasonably well-conducted studies, some of which are large, in the majority of studies few workers were exposed to high levels of trichloroethylene with reasonable confidence of exposure. Thus, most studies had limited statistical power to evaluate a modest risk of kidney cancer (if it exists) from exposure to trichloroethylene and exposure-response relationships. Statistical power was limited in the cohort studies, in part, because kidney cancer is uncommon or exposure levels were low in the larger studies, and in case-control studies because trichloroethylene exposure prevalence was low and exposure levels in the general population studies were most likely lower than the cohort studies. These studies may not be sensitive to detect effects that are due to high exposures. In addition, a major limitation of the studies was non-differential exposure misclassification, which would most likely bias the findings toward the null. The case-control study by Vamvakas et al. (1998) and the cohort study by Henschler et al. (1995) had methodological concerns that may potentially bias the findings away from the null.

The findings of the individual studies are discussed below and presented in Table 4-1 and Table 4-2. 


\begin{tabular}{|c|c|}
\hline High & Moore 2010 \\
\hline Selection bias unlikely (except Moore) & \\
\hline Little concern for exposure or disease misclassification & \\
\hline Adequate evaluation of $E / R$ & Charbotel 2006 \\
\hline $\begin{array}{l}\text { Adequate or good study sensitivity (limited for Zhao, but } \\
\text { moderate to high exposure levels) }\end{array}$ & \\
\hline Adequate consideration of potential confounders & Zhao 2005 \\
\hline $\begin{array}{l}\text { Moderate } \\
\text { Selection bias unlikely (except Brüning, Morgan*) }\end{array}$ & Hansen 2013 \\
\hline $\begin{array}{l}\text { Some concern for exposure misclassification (except } \\
\text { Brüning) }\end{array}$ & Radican 2008 \\
\hline Mortality analysis (Radican, Morgan) & Brüning 2003a \\
\hline $\begin{array}{l}\text { Evaluation of E/R (limited for Brüning) } \\
\text { Limited study sensitivity (except Brüning) }\end{array}$ & \\
\hline $\begin{array}{l}\text { Limited methods to consider potential confounding (except } \\
\text { Brüning) }\end{array}$ & Morgan 1998 \\
\hline Low/moderate & $\begin{array}{l}\text { Raaschou-Nielsen (R-N) } \\
2003\end{array}$ \\
\hline $\begin{array}{l}\text { Selection bias unlikely (except R-N and Lipworth) } \\
\text { Some concern for exposure misclassification }\end{array}$ & Lipworth 2011 \\
\hline $\begin{array}{l}\text { Good outcome assessment (except Lipworth) } \\
\text { Limited or no evaluation of E/R }\end{array}$ & Pesch 2000 \\
\hline Lower study sensitivity (except R-N) & Christensen 2013 \\
\hline & Dosemeci 1999 \\
\hline Low & Silver 2014 \\
\hline Selection bias a concern (Vamvakas, Henschler, Greenland) & Bove 2014 \\
\hline (except Vamvakas, Henschler) & Vlaanderen 2013 \\
\hline $\begin{array}{l}\text { Mortality (except Vamvakas) } \\
\text { Limited or no evaluation of E/R (except Vlaanderen, Bove) }\end{array}$ & Greenland 1994 \\
\hline Limited methods to consider potential confounding (except & Vamvakas $1998^{\mathrm{b}}$ \\
\hline & Henschler $1995^{\mathrm{b}}$ \\
\hline
\end{tabular}

\section{Figure 4-1. Study Utility Ranking: Kidney Cancer}

$\mathrm{E} / \mathrm{R}=$ exposure response.

Gray shading (left-hand column): Utility to inform hazard evaluation; light shading = highest utility; dark shading = lowest utility. Blue shading (right-hand column): Potential bias and study sensitivity; light shading = least biased or most sensitive; dark shading $=$ overall potential biases towards the null or lower sensitivity.

${ }^{a}$ (Tan shading): Multiple limitations; overall direction of potential biases is unknown.

b(Peach shading): Most potential biases away from the null. 


\subsubsection{Cohort and Nested Case-control Studies}

The available cohort studies include three studies conducted in Nordic countries, five cohorts of aerospace and aircraft workers, a cohort study of cardboard manufacturing workers, a nested case-control study of electronic workers and a cohort of military personnel exposed to trichloroethylene in drinking water. An overview of the ability of the studies to inform the cancer hazard evaluation is shown in Figure 4-1 and details are presented in Section 3 and Appendix D.

\section{Nordic Studies}

These studies consist of a pooled analysis of biomonitored workers (Hansen et al. 2013), a cohort study of blue-collar workers at companies using trichloroethylene (Raaschou-Nielsen et al. 2003), and a large population-based cancer registry study (Vlaanderen et al. 2013). The studies included subjects with occupational exposure to trichloroethylene from diverse industries, and workers and exposed subjects who were identified from broad occupational or population-based databases. Both the cohort study of blue-collar workers (Raaschou-Nielsen et al. 2003) and the updated and pooled analysis of three cohort studies of biomonitored workers in Sweden, Finland, and Norway (Hansen et al. 2013) provide some evidence of an association of exposure to trichloroethylene and kidney cancer. In the former study, statistically significant (or approaching significance) increased risks of renal-cell cancer incidence were found among all workers $(\mathrm{SIR}=1.4,95 \% \mathrm{CI}=1.0$ to $1.8,53$ exposed cases), workers with longer lag time (SIR $=1.6$, $95 \% \mathrm{CI}=1.0$ to $2.3,25$ exposed cases), and workers employed before 1970 ( $\mathrm{SIR}=1.9,95 \%$ $\mathrm{CI}=1.4$ to $2.6,41$ exposed cases). Air monitoring data indicated that trichloroethylene levels were much higher (40 to $60 \mathrm{ppm}$ ) prior to 1970 . Strengths of this study were its large size and analysis of long-term exposure using duration of exposure and calendar period as surrogates. Although the study was limited by its use of crude exposure surrogates (blue-collar workers, duration of employment), exposure misclassification was probably lower among the higher exposed subcohort than the total cohort. In the pooled analysis of biomonitored workers (Hansen et al. 2013), a statistically non-significant increase in risk of kidney cancer (hazard rate ratio $[\mathrm{HRR}]=2.04,95 \% \mathrm{CI}=0.81$ to 5.17 ; 9 exposed cases) was found among the highest exposed workers with urinary trichloroacetic acid (U-TCA) levels $>50 \mathrm{mg} / \mathrm{L}$ (estimated as equivalent to $20 \mathrm{ppm}$ ) but not among ever-exposed workers. Although this study was a large, well-conducted study, only $20 \%$ of the workers were exposed to levels $>20$ ppm and estimated exposures for most of the workers were between 4 and $12 \mathrm{ppm}$. There was also a lack of specificity and possible misclassification of exposure, in part because some of the population was exposed to tetrachloroethylene, which is also metabolized to trichloroacetic acid (Anttila et al. 1995). In addition, most workers only had one to three U-TCA measurements over their entire work history and no information was available on lifetime cumulative exposure (Hansen et al. 2013).

No association between trichloroethylene exposure and kidney cancer was found in the large population-based study by Vlaanderen et al. (2013). Exposure to trichloroethylene was likely low in the study, and exposure misclassification (non-differential) was considered to be substantial because of lack of detailed occupational information (tasks, working conditions), heterogeneity of exposure levels within and across jobs with the same job title, and overtime, and use of a JEM that may not be country specific. These limitations would bias the findings towards the null.

\section{Aerospace or Aircraft Manufacturing Workers}

These studies include two overlapping, but with different exposure assessments, cohorts of rocket engine workers (Boice et al. 2006; Zhao et al. 2005) and three studies of aircraft 
manufacturing workers in Burbank, California (Lipworth et al. 2011), Utah (Blair et al. 1998; Radican et al. 2008), and Arizona (Morgan et al. 1998). Taken together, the studies of the rocket engine workers provide evidence of an association between trichloroethylene exposure and renal-cell cancer, with the strongest evidence coming from the Zhao et al. study, which was considered to be a highly informative study based on a semi-quantitative exposure assessment and evaluation of exposure-response relationships for both cancer incidence and mortality in models that adjusted for co-exposure to other chemicals. In this study, the risk of kidney cancer increased with increasing cumulative exposure in both adjusted and unadjusted models (although the trend was only statistically significant in the unadjusted model $(\mathrm{p}=0.023)$ with risks ranging from 5-fold (unadjusted) to 7-fold (adjusted) in the highest exposure category. Statistical power was most likely reduced in the adjusted models. Similar patterns of increasing risks were also observed for kidney cancer mortality, although the magnitudes of the risk estimates were lower as might be expected since mortality is a less informative outcome measure than incidence. The study by Boice et al. (2006) (using a qualitative JEM) found a three-fold, statistically nonsignificant increased risk among workers with the longest exposure to trichloroethylene during engine flush and support the findings by Zhao et al. (2005), although this may not be independent evidence. Although exposure levels were not reported, the potential for high exposure to trichloroethylene during this task was much higher than during other tasks, such as the use of trichloroethylene as a utility solvent, according to the authors.

Among the studies of aircraft manufacturing workers, the mortality study of Arizona workers (Morgan et al. 1998) found statistically non-significant increased risks for kidney cancer among workers with the highest cumulative exposure $(\mathrm{RR}=1.59,95 \% \mathrm{CI}=0.68$ to $3.71,7$ exposed deaths) and with high peak exposure ( $\mathrm{RR}=1.89 ; 95 \% \mathrm{CI}=0.85$ to $4.23,8$ exposed deaths) with some evidence of increasing risks with increasing exposure. Statistical power was limited due to few exposed subjects in the high-exposure categories. Exposure intensity for the highest exposed workers was estimated to be $\geq 50 \mathrm{ppm}$. Findings were null in the other two studies. Radican et al. (2008) found small non-statistically significant elevated risks in some subgroup analyses of the Utah workers but no evidence of an exposure-response gradient. Risks were less than unity in the internal and external analyses in the study of California workers by Lipworth et al. (2011). These studies had limited statistical power to detect a small excess in risk based on few workers with higher or longer exposure, and the study by Lipworth et al. (2011) had a higher potential for nondifferential exposure misclassification. Although exposure levels were not reported, the NRC (2006) estimated that a modest number of the Utah workers (Radican et al. 2008) were exposed to higher levels (approximately $100 \mathrm{ppm}$ ) but that most workers were exposed to low levels of trichloroethylene. There was evidence of a healthy worker effect in two of the aircraft manufacturing studies (Lipworth et al. 2011; Radican et al. 2008) and the aerospace worker study by Boice et al. (2006), which would bias external analyses towards the null. In addition, exposure duration for some workers in the Lipworth et al. (2011) study may have been relatively short for some workers because use of trichloroethylene was discontinued in 1966.

\section{Other Mortality Cohort Studies}

These studies were generally considered to be less informative (i.e., lower utility to inform the cancer hazard evaluation) than most of the cohort studies of aerospace workers or the Nordic studies. A statistically significant high risk estimate (approximately 8- to 13-fold depending on reference population rates) was observed in the study of cardboard manufacturing workers (Henschler et al. 1995), which may in part reflect selection and diagnostic biases because the 
study was designed around a cluster and cases of kidney cancer were identified using sonography (the latter would bias the external but probably not the internal analyses). However, the NRC (2006) estimated that the SMR would be approximately 3.2 if the three cases diagnosed in 1990 that represented the original cluster were excluded from the analyses. There was also qualitative evidence that high exposures (estimated peak exposure $>2,000 \mathrm{ppm}$ and long-term exposure of $>100 \mathrm{ppm}$ ) (Cherrie et al. 2001) occurred in this cohort. Silver et al. (2014) reported a hazard ratio [HR] of 1.24 (95\% CI $=0.87$ to $1.77,56$ exposed deaths) among U.S. microelectronics workers; the cohort was relatively young, with only $17 \%$ deaths in the total cohort, and the exposure assessment was limited. No excess risk was found in the nested case-control study of electrical workers (Greenland et al. 1994) which had several methodological limitations and low probability of exposure; only $10 \%$ of jobs had exposure to trichloroethylene, most of which was from indirect exposure. Finally, Bove et al. (2014) reported an HR of $1.52(95 \% \mathrm{CI}=0.64$ to 3.61, 11 exposed deaths) among U.S. military personnel exposed to the highest level of trichloroethylene in their drinking water. The exposure assessment was based on modeled levels and duration at residence and no information on individual water consumption was available. Although follow-up was long, the cohort was relatively young, suggesting additional follow-up might increase statistical power.

\subsubsection{Population-based Case-control Studies}

The case-control studies include four studies conducted in areas with presumably higher levels and prevalence of trichloroethylene exposure using experts with knowledge of the local industry, and three studies of more widespread populations.

\section{Studies in Specific Areas with Knowledge of Local Industries}

As mentioned in Section 3, two non-overlapping case-control studies (Brüning et al. 2003; Vamvakas et al. 1998) were conducted in Arnsberg, Germany, which is a small geographical area with a large number of companies engaged in metal and electronics work. Trichloroethylene use was widespread and reportedly one of only two solvents (the other was carbon tetrachloride) used in the industry. This is the same geographical area as the German cardboard manufacturing cohort study of renal cancer, although cases do not overlap. A third case-control study (Charbotel et al. 2006; Charbotel et al. 2009) was conducted in the Arve Valley in France, which is an area with a widespread screw-cutting industry that used trichloroethylene as a degreaser. Although population based, the French and German studies have a higher prevalence of subjects with presumably higher levels of trichloroethylene and from more homogenous industries. The fourth study was a multi-center study of renal-cell cancer conducted in four countries in central and Eastern Europe, in regions in which occupational exposure to trichloroethylene was thought to be higher and more prevalent than other industrial areas. Exposure was assessed in each of the different countries by a team of experts with knowledge of industries in their area (Moore et al. 2010). The studies by Brüning et al., Charbotel et al., and Moore et al. are considered to have a greater ability to detect an effect because of greater confidence that most of the subjects classified as exposed were most likely exposed to substantial levels of trichloroethylene (Brüning et al. 2003; Charbotel et al. 2006; Charbotel et al. 2009) or, in the study by Moore et al. (2010), analyses focused on the highest exposed individuals with high probability of exposure. The studies by Charbotel et al. (2006) and Moore et al. (2010) were considered to have the best methodologies. 
The study by Charbotel et al. (2006); (2009) is considered to be the most informative because in addition to the advantages stated above, it also evaluated exposure-response relationships and controlled for potential confounding from lifestyle factors and mineral oils, the major coexposure in this industry, and conducted separate analyses among workers with high confidence of exposure. Kidney cancer risk increased with increasing exposure (no trend reported) with statistically significant risks (approximately 2- to 3-fold) observed among individuals with the highest cumulative exposure and high cumulative exposure together with peak exposure. Exposure levels were considered to be high in this study, ranging up to 300 to $600 \mathrm{ppm}$ for highexposure jobs.

Strengths of the multi-center study in Central/Eastern Europe (Moore et al. 2010) were its large size and good exposure assessment. In this study, statistically significant risks were found among trichloroethylene-exposed individuals with high confidence exposure assessments $(\mathrm{OR}=2.05$, $95 \% \mathrm{CI}=1.13$ to $3.73,29 \mathrm{cases} / 19$ controls). Risk estimates were higher among individuals with longer or higher levels of exposure (both average intensity and cumulative exposure). The authors also evaluated exposure to trichloroethylene and kidney cancer risk stratified by GSTT1 genotypes; a significant increase in risk was observed among those with at least one active allele of the GSTT1 genotype but not among individuals with GSTT1-null genotypes (see Section 4.2). Although there was potential selection bias due to the exclusion of controls with tobacco-related diseases, it can reasonably be ruled out since initial regression analyses of exposure-response relationships examining smoking did not alter the ORs.

Statistically significant high risks were found for exposure to trichloroethylene and renal-cell cancer in the two German studies (Brüning et al. 2003; Vamvakas et al. 1998). Although the exposure assessments were rather limited in both studies and relied on self-reported exposure, there is reasonable confidence that most workers were exposed to trichloroethylene based on detailed information on the exposure settings suggesting high exposure, the presence of narcotic symptoms and use of expert assessment (integrating frequency and severity of symptoms with exposure duration) (see Section 3). The earlier study by Vamvakas et al. reported a much higher risk estimate for any exposure $(\mathrm{OR}=10.80,95 \% \mathrm{CI}=3.36$ to $34.75,19$ cases and 7 controls $)$ than the later study by Brüning et al. $(\mathrm{OR}=2.47,95 \% \mathrm{CI}=1.36$ to $4.49,25$ cases and 38 controls). The higher risk estimate in the earlier study should be viewed with some caution because of the potential for selection and other biases (see Section 3), which would most likely bias towards an overestimation of the risk. However, given the high levels of exposure to trichloroethylene, it seems unlikely that the distortion of the potential biases would nullify the observed positive association. Estimated levels of exposure were high; peak exposures were estimated to be 400 to $600 \mathrm{ppm}$ and long-term exposure to be $>100 \mathrm{ppm}$ (Cherrie et al. 2001; NRC 2006). The later study by Brüning et al. (2003) minimized some of the methodological concerns of the Vamvakas et al. study and thus is given greater weight in this evaluation. In the Vamvakas et al. study, there was little evidence of a linear exposure-response relationship, although risks were higher in both the moderate and high exposure categories compared with the lowest exposure category; exposure levels may have been somewhat homogeneous due to exposure from open systems in small spaces. In the Brüning et al. study, a higher risk (compared with any exposure) was found among individuals with daily narcotic symptoms (OR $=5.91,95 \%$ $\mathrm{CI}=1.46$ to $23.99,5$ exposed cases and 4 controls), which may be a surrogate for exposure intensity. Brüning et al. also used a crude JEM from the United Kingdom to assess exposures in German industries, likely introducing misclassification bias, and found a two-fold increase 
among workers who held a job with trichloroethylene exposure compared with those who did not.

\section{Other Studies}

These included population-based case-control studies in Minnesota (Dosemeci et al. 1999) and Germany (five regions) (Pesch et al. 2000b) and a study using both population and hospital controls in Montreal (Christensen et al. 2013). These studies are considered to have more limited ability to inform hazard identification because of limited statistical power (inadequate numbers of exposed subjects), low overall exposure or exposure misclassification. In the Minnesota study, risk approached statistical significance $(\mathrm{OR}=1.96,95 \% \mathrm{CI}=1.0$ to $4.4,22$ cases) among women ever exposed to trichloroethylene but risks were close to unity among men. A small, nonstatistically elevated risk was reported in the multi-center German study (Pesch et al. 2000b) and no increase in risk was found among subjects with substantial exposure in the Canadian study, but there were only two exposed cases (Christensen et al. 2013). 
Table 4-1. Trichloroethylene Cohort and Nested Case-control Studies: Findings for Kidney Cancer ${ }^{\mathrm{a}}$

\begin{tabular}{|c|c|c|c|c|c|}
\hline Reference & $\begin{array}{c}\text { Study } \\
\text { Size (N) } \\
\text { Exposure } \\
\text { Assessment }\end{array}$ & Exposure Groups & $\begin{array}{c}\text { External Analysis } \\
\text { SMR, SIR (95\% CI) } \\
\text { \# Cases/Deaths }\end{array}$ & $\begin{array}{c}\text { Internal Analysis: } \\
\text { HR, RR, SRR, or OR } \\
\text { (95\% CI) \# Exposed } \\
\text { Cases/Deaths }\end{array}$ & Interpretation \\
\hline \multicolumn{6}{|l|}{ Nordic studies } \\
\hline $\begin{array}{l}\text { Vlaanderen et } \\
\text { al. (2013) }\end{array}$ & $\begin{array}{l}5 \text { Nordic countries } \\
\text { Record linkage of } \\
\text { cancer registry with } \\
\text { census questionnaire } \\
\text { M: } 44,708 \text { cases, } \\
223,540 \text { controls } \\
\text { F: } 31,422 \text { cases, } \\
157,110 \text { controls } \\
\text { Semi-quantitative } \\
\text { JEM }\end{array}$ & 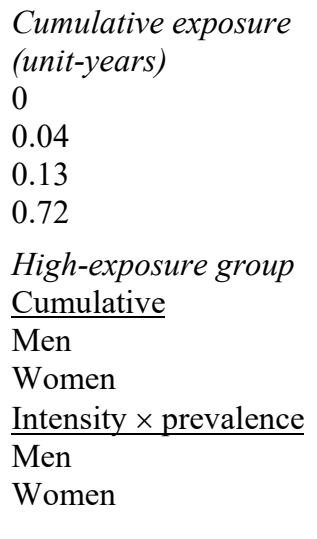 & & $\begin{array}{l}H R \text { (incidence) } \\
1.00 \\
1.01(0.95-1.07) ; 1,217 \\
1.02(0.97-1.08) ; 1,556 \\
1.00(0.95-1.07) ; 1,372\end{array}$ & $\begin{array}{l}\text { Low prevalence of exposure (TCE) and exposure } \\
\text { levels likely to be low } \\
\text { Matching criteria: Age, country, sex } \\
\text { Strengths: Long follow-up, large numbers of cases } \\
\text { Limitations: Misclassification of exposure likely; } \\
\text { JEM had poor sensitivity and did not account for } \\
\text { heterogeneity within jobs and over time } \\
\text { Null: No evidence for a positive association but } \\
\text { limited utility due to low exposure levels and } \\
\text { exposure misclassification }\end{array}$ \\
\hline $\begin{array}{l}\text { Hansen et al. } \\
(2013) \\
\text { (Potential } \\
\text { overlap with } \\
\text { Raaschou- } \\
\text { Nielsen et al. } \\
(2003))\end{array}$ & $\begin{array}{l}\text { Pooled and updated } \\
\text { Nordic cohorts } \\
\text { Axelson et al. (1994), } \\
\text { Anttila et al. (1995), } \\
\text { Hansen et al. (2001) } \\
5,553 \text { (3,776 M, } \\
\text { 1,777 F) } \\
\text { Biomonitoring (U- } \\
\text { TCA) }\end{array}$ & $\begin{array}{l}\text { All exposed subjects } \\
0-\mathrm{yr} \text { lag } \\
10-\mathrm{yr} \text { lag } \\
20-\mathrm{yr} \text { lag } \\
U-T C A(\mathrm{mg} / \mathrm{L}) \\
<5 \\
5-25 \\
25-50 \\
>50 \\
\mathrm{p}_{\text {trend }}\end{array}$ & $\begin{array}{l}\text { SIR } \\
1.01(0.70-1.42) ; 32 \\
1.04(0.71-1.50) ; 30 \\
1.11(0.67-1.73) ; 19\end{array}$ & $\begin{array}{l}H R R \text { (no lag); incidence } \\
1.0 \text { (Ref); } 9 \\
1.12(0.46-2.70) ; 11 \\
0.81(0.21-2.97) ; 3 \\
2.04(0.81-5.17) ; 9 \\
0.19\end{array}$ & $\begin{array}{l}\text { Low exposure levels for most workers (only } 20 \% \\
\text { exposed to } \geq 20 \mathrm{ppm} \text { ) and short duration of } \\
\text { employment } \\
\text { Covariates: Age, sex, calendar period, country; } \\
\text { indirect consideration of smoking and alcohol } \\
\text { consumption } \\
\text { Strengths: Biomonitoring data; large numbers of } \\
\text { workers ever exposed } \\
\text { Limitations: Only } 2 \text { or } 3 \text { U-TCA measurements per } \\
\text { individual and unlikely to estimate lifetime or } \\
\text { cumulative exposure; low statistical power for } \\
\text { evaluating modest risks; limited ability to evaluate } \\
\text { exposure-response relationship } \\
\text { Limited evidence for a positive association: } \\
\text { Statistically non-significant, moderately elevated } \\
\text { effect estimate in the highest exposed group }\end{array}$ \\
\hline
\end{tabular}


RoC Monograph on Trichloroethylene

\begin{tabular}{|c|c|c|c|c|c|}
\hline Reference & $\begin{array}{c}\text { Study } \\
\text { Size (N) } \\
\text { Exposure } \\
\text { Assessment }\end{array}$ & Exposure Groups & $\begin{array}{l}\text { External Analysis } \\
\text { SMR, SIR (95\% CI) } \\
\text { \# Cases/Deaths }\end{array}$ & $\begin{array}{l}\text { Internal Analysis: } \\
\text { HR, RR, SRR, or OR } \\
\text { (95\% CI) \# Exposed } \\
\text { Cases/Deaths }\end{array}$ & Interpretation \\
\hline \multirow{5}{*}{$\begin{array}{l}\text { Raaschou- } \\
\text { Nielsen et al. } \\
\text { (2003) } \\
\text { (Potential } \\
\text { overlap with } \\
\text { Hansen et al. } \\
(2013) \text { ) }\end{array}$} & \multirow{2}{*}{$\begin{array}{l}\text { Danish blue-collar } \\
\text { workers } \\
40,049 \mathrm{M}+\mathrm{F} \text { (approx. } \\
70 \% \mathrm{M} \text { ) }\end{array}$} & $\begin{array}{l}\text { Subcohort: higher } \\
\text { exposed }\end{array}$ & \multicolumn{2}{|l|}{$\begin{array}{l}\text { SIR } \\
1.4(1.0-1.8) ; 53\end{array}$} & $\begin{array}{l}\text { Higher levels of TCE prior to } 1970(40-60 \mathrm{ppm}) \text {; } \\
\text { low levels of exposure after that time }\end{array}$ \\
\hline & & \multirow{2}{*}{$\begin{array}{l}\underline{\text { Lag time }(\mathrm{yr})} \\
0-9 \\
10-19 \\
\geq 20\end{array}$} & & Covariates: Age, sex, calendar year \\
\hline & \multirow{3}{*}{$\begin{array}{l}\text { Working at TCE } \\
\text { company; size of } \\
\text { company surrogate } \\
\text { for TCE exposure } \\
\text { prevalence }\end{array}$} & & $\begin{array}{l}1.5(0.9-2.2) ; 22 \\
1.6(1.0-2.3) ; 25\end{array}$ & & $\begin{array}{l}\text { Strengths: Large numbers of exposed cases; } \\
\text { subcohort of subjects with higher exposure } \\
\text { potential }\end{array}$ \\
\hline & & $\begin{array}{l}\text { Duration employment } \\
\text { (yr) } \\
1-4 \\
\geq 5\end{array}$ & $\begin{array}{l}1.1(0.7-1.7) ; 23 \\
1.7(1.1-2.4) ; 30\end{array}$ & & $\begin{array}{l}\text { Limitations: Young cohort, possible selection bias } \\
\text { for difference in SES, external analysis only; } \\
\text { possible exposure misclassification }\end{array}$ \\
\hline & & $\begin{array}{l}1-4 \\
\geq 5 \\
\text { Yr. of 1st employment } \\
\text { Before 1970 } \\
1970-1979\end{array}$ & $\begin{array}{l}1.9(1.4-2.6) ; 41 \\
0.7(0.4-1.2) ; 12\end{array}$ & & $\begin{array}{l}\text { Evidence for a positive association: Statistically } \\
\text { significant, moderately elevated effect estimates; } \\
\text { some evidence for exposure-response relationship } \\
\text { unlikely to be explained by confounding by } \\
\text { smoking or differences in SES }\end{array}$ \\
\hline \multicolumn{6}{|c|}{ Aerospace and aircraft workers } \\
\hline \multirow{5}{*}{$\begin{array}{l}\text { Lipworth et al. } \\
\text { (2011) (update } \\
\text { of Boice et al. } \\
(1999) \text { ) }\end{array}$} & \multirow{2}{*}{$\begin{array}{l}\text { Burbank, CA (USA) } \\
\text { aircraft } \\
\text { manufacturing } \\
\text { workers }\end{array}$} & & \multirow[t]{5}{*}{$\begin{array}{l}S M R \\
0.66(0.38-1.07) ; 16\end{array}$} & \multirow{5}{*}{$\begin{array}{l}R R \text { (mortality) } \\
1.00 ; 33 \\
0.52(0.21-1.30) ; 6 \\
0.42(0.13-1.42) ; 3 \\
0.85(0.33-2.19) ; 6 \\
0.20\end{array}$} & $\begin{array}{l}\text { Exposure levels not reported; exposure duration } \\
\text { likely to be short }\end{array}$ \\
\hline & & \multirow{4}{*}{$\begin{array}{l}\text { TCE: years exposed } \\
0 \\
<1 \\
1-4 \\
5+ \\
\text { ptrend }\end{array}$} & & & $\begin{array}{l}\text { Covariates: Age, date of birth, date of hire, } \\
\text { termination date, sex, and race }\end{array}$ \\
\hline & $\begin{array}{l}5,443 \text { (approx. } 80 \% \\
\text { M) }\end{array}$ & & & & $\begin{array}{l}\text { Strengths: Long follow up, adequate number of } \\
\text { deaths for ever exposure }\end{array}$ \\
\hline & \multirow[t]{2}{*}{$\begin{array}{l}\text { Qualitative JEM } \\
\text { Individual work } \\
\text { histories }\end{array}$} & & & & $\begin{array}{l}\text { Limitations: Evidence of HWE, few exposed } \\
\text { deaths in subgroup analysis; exposure } \\
\text { misclassification is a concern; no evaluation of } \\
\text { exposure intensity, } 70 \% \text { had exposure to mixed } \\
\text { solvents }\end{array}$ \\
\hline & & & & & $\begin{array}{l}\text { Null: No evidence for a positive association but } \\
\text { limited utility (limitations are mainly towards the } \\
\text { null) }\end{array}$ \\
\hline
\end{tabular}


RoC Monograph on Trichloroethylene

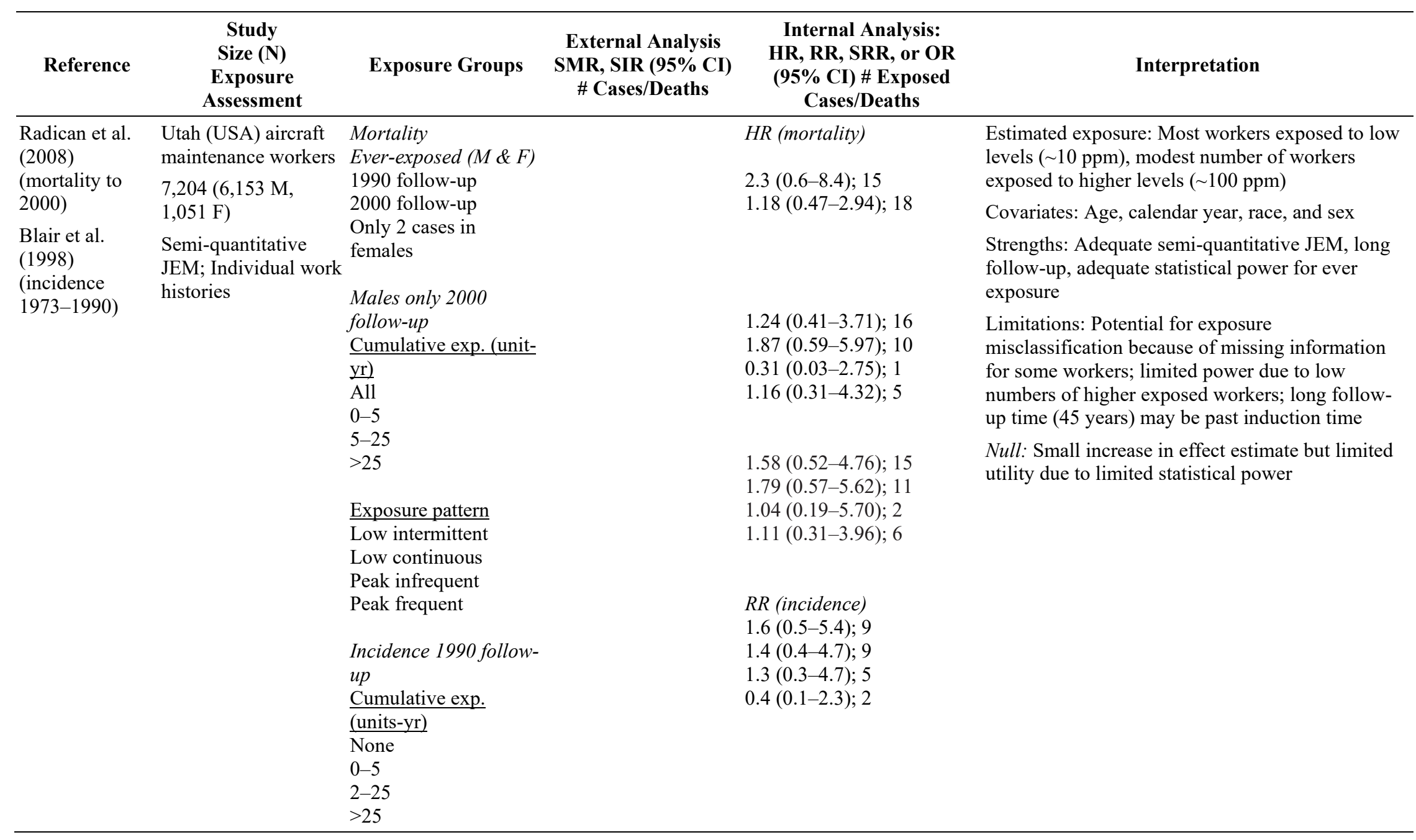


RoC Monograph on Trichloroethylene

\begin{tabular}{|c|c|c|c|c|c|}
\hline Reference & $\begin{array}{c}\text { Study } \\
\text { Size (N) } \\
\text { Exposure } \\
\text { Assessment }\end{array}$ & Exposure Groups & $\begin{array}{l}\text { External Analysis } \\
\text { SMR, SIR (95\% CI) } \\
\text { \# Cases/Deaths }\end{array}$ & $\begin{array}{l}\text { Internal Analysis: } \\
\text { HR, RR, SRR, or OR } \\
\text { (95\% CI) \# Exposed } \\
\text { Cases/Deaths }\end{array}$ & Interpretation \\
\hline \multirow{5}{*}{$\begin{array}{l}\text { Boice et al. } \\
(2006) \\
\text { (Overlaps with } \\
\text { Zhao et al. } \\
(2005))\end{array}$} & $\begin{array}{l}\text { Los Angeles, CA } \\
\text { (USA) }\end{array}$ & Ever exposed & $\begin{array}{l}\text { SMR } \\
2.22(0.89-4.57) ; 7\end{array}$ & \multirow{5}{*}{$\begin{array}{l}R R \text { (mortality) } \\
1.00 ; 28 \\
1.21(0.33-4.35) ; 3 \\
2.51(0.27-23.5) ; 1 \\
3.13(0.74-13.2) ; 3 \\
0.59\end{array}$} & $\begin{array}{l}\text { Exposure occurs during test engine flush, which is } \\
\text { likely to be high }\end{array}$ \\
\hline & $\begin{array}{l}\text { Rocket engine testing } \\
\text { workers }\end{array}$ & \multirow{4}{*}{$\begin{array}{l}\text { Exposure to TCE } \\
\text { during engine flush } \\
\text { (test-yr) } \\
\text { Referent (other depts.) } \\
0 \\
<4 \\
\geq 4 \\
\text { p }_{\text {trend }}\end{array}$} & & & $\begin{array}{l}\text { Covariates: Date of birth, year of hire, pay type } \\
\text { (surrogate for SES), and exposure to hydrazine }\end{array}$ \\
\hline & 1,111 Men & & & & Strengths: Adequate follow-up \\
\hline & $\begin{array}{l}\text { Qualitative JEM; } \\
\text { Individual work }\end{array}$ & & & & $\begin{array}{l}\text { Limitations: Qualitative exposure assessment; few } \\
\text { exposed deaths }\end{array}$ \\
\hline & histories & & & & $\begin{array}{l}\text { Limited evidence for a positive association: } \\
\text { Statistically non-significant elevated effect estimate } \\
\text { among highest exposed group }\end{array}$ \\
\hline
\end{tabular}


RoC Monograph on Trichloroethylene

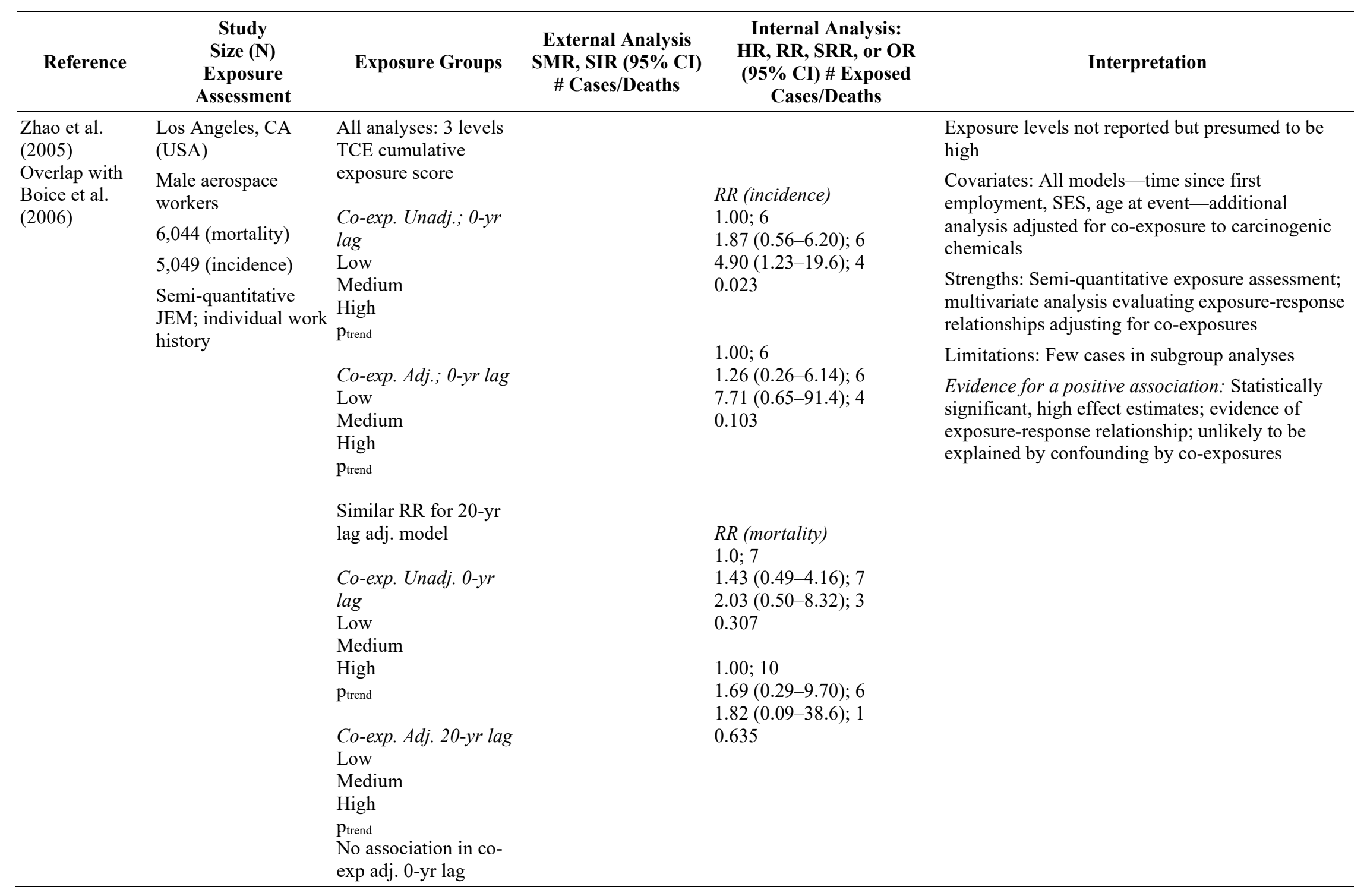


RoC Monograph on Trichloroethylene

\begin{tabular}{|c|c|c|c|c|c|}
\hline Reference & $\begin{array}{c}\text { Study } \\
\text { Size (N) } \\
\text { Exposure } \\
\text { Assessment }\end{array}$ & Exposure Groups & $\begin{array}{l}\text { External Analysis } \\
\text { SMR, SIR (95\% CI) } \\
\text { \# Cases/Deaths }\end{array}$ & $\begin{array}{l}\text { Internal Analysis: } \\
\text { HR, RR, SRR, or OR } \\
\text { (95\% CI) \# Exposed } \\
\text { Cases/Deaths }\end{array}$ & Interpretation \\
\hline \multirow{8}{*}{$\begin{array}{l}\text { Morgan et al. } \\
\text { (1998) }\end{array}$} & Arizona (USA) & \multirow{8}{*}{$\begin{array}{l}\text { All TCE-exposed } \\
\text { workers } \\
\text { Cumulative exp. score } \\
\text { Low }(2,357) \\
\text { High }(2,376) \\
\text { Peak (med/high) vs. } \\
\text { low/no }\end{array}$} & $\operatorname{SMR}($ All $)$ & \multirow{2}{*}{$\begin{array}{l}R R(\text { mortality) } \\
1.14(0.51-2.58) ; 8^{\mathrm{b}}\end{array}$} & High-exposure jobs were considered to be $\geq 50 \mathrm{ppm}$ \\
\hline & \multirow{3}{*}{$\begin{array}{l}\text { Aircraft } \\
\text { manufacturing } \\
\text { workers }\end{array}$} & & $1.32(0.57-2.60) ; 8$ & & \multirow{4}{*}{$\begin{array}{l}\text { Covariates: Age at hire, gender (decade of hire } \\
\text { considered but no effect) } \\
\text { Strengths: Long follow-up and semi-quantitative } \\
\text { exposure }\end{array}$} \\
\hline & & & $0.47(0.01-2.62) ; 1$ & $0.31(0.04-2.36) ; 1$ & \\
\hline & & & $1.78(0.72-3.66) ; 7$ & $1.59(0.68-3.71) ; 7$ & \\
\hline & \multirow{2}{*}{$\begin{array}{l}4,733(2,555 \mathrm{M} \\
2,178 \mathrm{~F})\end{array}$} & & & $1.89(0.85-4.23) ; 8$ & \\
\hline & & & & & \multirow{2}{*}{$\begin{array}{l}\text { Limitations: Evidence of an HWE; potential } \\
\text { exposure misclassification among low/medium } \\
\text { exposure groups; mortality analysis and few } \\
\text { exposed deaths }\end{array}$} \\
\hline & \multirow[t]{2}{*}{$\begin{array}{l}\text { Semi-quantitative } \\
\text { JEM; individual work } \\
\text { history }\end{array}$} & & & & \\
\hline & & & & & $\begin{array}{l}\text { Limited evidence for a positive association: } \\
\text { Statistically non-significant elevated effect estimate } \\
\text { among the highest exposed group }\end{array}$ \\
\hline \multicolumn{6}{|c|}{ Other occupational studies } \\
\hline \multirow{4}{*}{$\begin{array}{l}\text { Silver et al. } \\
(2014)\end{array}$} & \multirow{4}{*}{$\begin{array}{l}\text { New York State } \\
\text { (USA) micro- } \\
\text { electronics } \\
\text { manufacturing } \\
\text { workers cohort } \\
\text { mortality } \\
\text { 3,113 TCE exposed } \\
\text { Semi-qualitative JEM }\end{array}$} & \multirow{4}{*}{$\begin{array}{l}5 \text { modified exposure } \\
\text { years (exposure } \\
\text { duration modified by } \\
\text { exposure potential); } \\
10 \text {-yr lag }\end{array}$} & & \multirow{4}{*}{1.24 (0.87-1.77); NR } & Exposure levels NR; only $13.9 \%$ of cohort exposed \\
\hline & & & & & $\begin{array}{l}\text { Covariates: Paycode and sex, age. Variables } \\
\text { considered in analyses but which did not change } \\
\text { risk estimate were birth cohort, time since last } \\
\text { exposure (healthy worker survival), hire era, and } \\
\text { employment duration prior to } 1966\end{array}$ \\
\hline & & & & & $\begin{array}{l}\text { Limitations: Evidence of HWE, Exposure } \\
\text { classification based on potential exposure and } \\
\text { duration; only one cumulative exposure variable } \\
\text { reported in analysis. Young cohort with only } 17 \% \\
\text { deaths }\end{array}$ \\
\hline & & & & & $\begin{array}{l}\text { Limited evidence for a positive association: Non- } \\
\text { statistically significant elevated effect estimate }\end{array}$ \\
\hline
\end{tabular}


RoC Monograph on Trichloroethylene

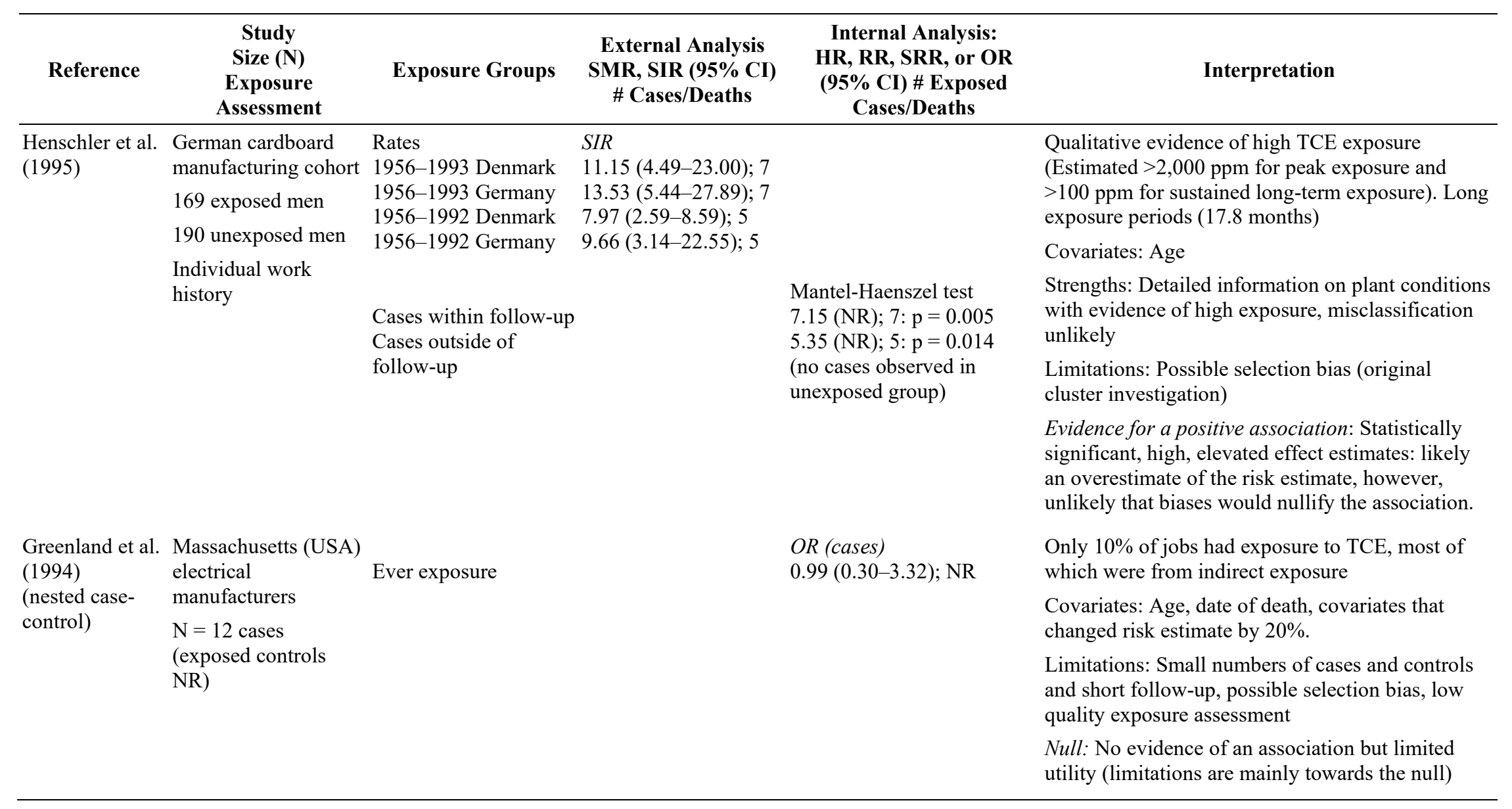


RoC Monograph on Trichloroethylene

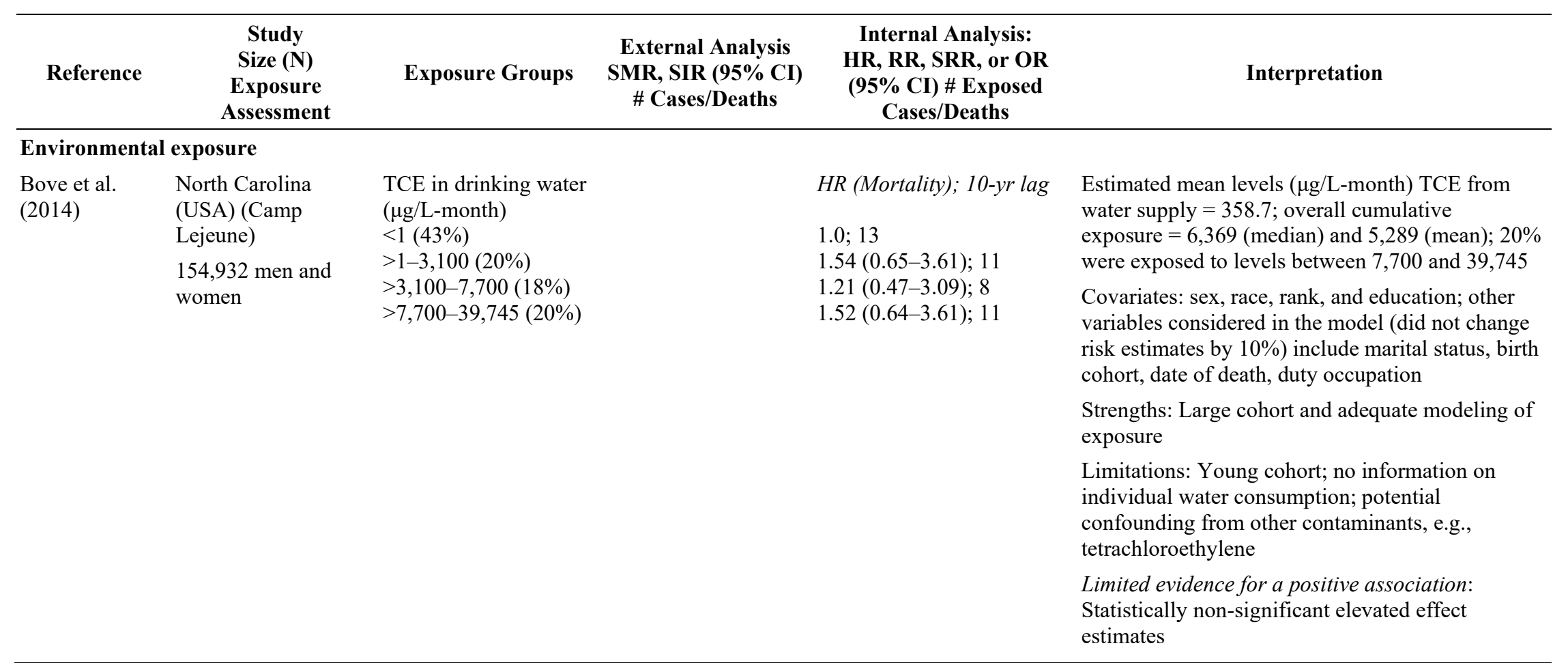

$\mathrm{CI}=$ confidence interval $\mathrm{F}=$ female $\mathrm{HR}=$ hazard ratio; $\mathrm{JEM}=$ job-exposure matrix $; \mathrm{M}=$ male $; \mathrm{NR}=$ not reported; $\mathrm{OR}=$ odds ratio; $\mathrm{RR}=$ relative risk $; \mathrm{SIR}=$ standardized incidence ratio; $\mathrm{SMR}=$ standardized mortality ratio; $\mathrm{SRR}=$ standardized rate ratio; TCA $=$ trichloroacetic acid; TCE $=$ trichloroethylene; $\mathrm{U}-\mathrm{TCA}=$ urine trichloroacetic acid.

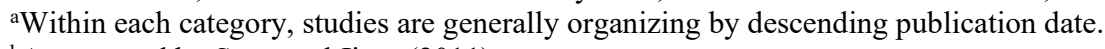

bAs reported by Scott and Jinot (2011). 
Table 4-2. Case-control Studies of Trichloroethylene Exposure: Findings for Kidney Cancer

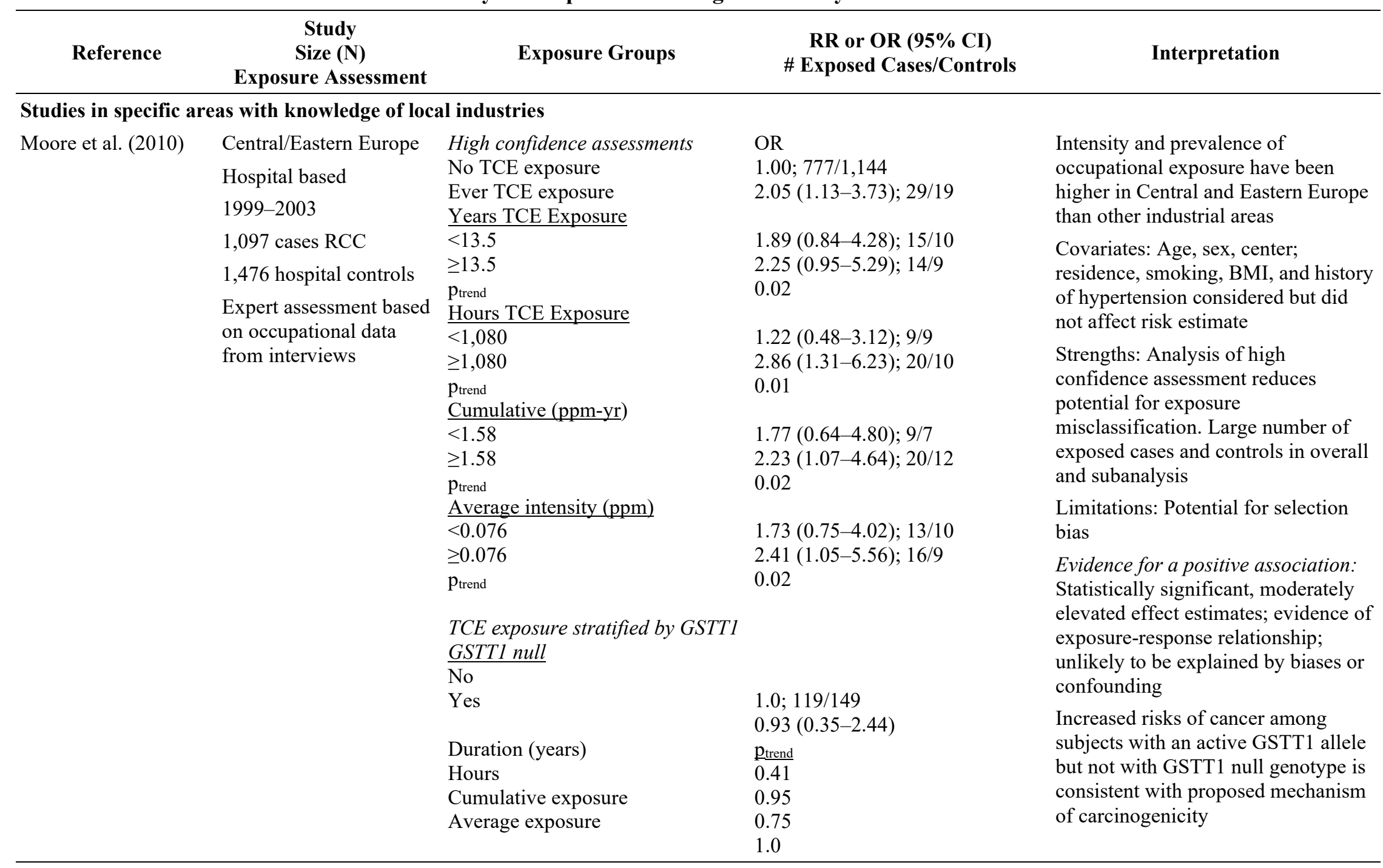


RoC Monograph on Trichloroethylene

\begin{tabular}{|c|c|c|c|c|}
\hline Reference & $\begin{array}{c}\text { Study } \\
\text { Size (N) } \\
\text { Exposure Assessment } \\
\end{array}$ & Exposure Groups & $\begin{array}{c}\text { RR or OR }(95 \% \text { CI }) \\
\text { \# Exposed Cases/Controls }\end{array}$ & Interpretation \\
\hline & & GSTT1 Active & & \\
\hline & & $\overline{\text { No }}$ & $1.0 ; 466 / 729$ & \\
\hline & & Yes & $1.88(1.06-3.33) ; 23$ & \\
\hline & & & $\mathrm{p}_{\text {trend }}$ & \\
\hline & & Duration (years) & 0.03 & \\
\hline & & Hours & 0.02 & \\
\hline & & Cumulative exposure & 0.01 & \\
\hline & & Average exposure & 0.02 & \\
\hline
\end{tabular}




\begin{tabular}{|c|c|c|c|c|}
\hline Reference & $\begin{array}{c}\text { Study } \\
\text { Size (N) } \\
\text { Exposure Assessment }\end{array}$ & Exposure Groups & $\begin{array}{c}\text { RR or OR }(95 \% \text { CI }) \\
\text { \# Exposed Cases/Controls }\end{array}$ & Interpretation \\
\hline $\begin{array}{l}\text { Charbotel et al. } \\
(2006) \text {; Charbotel et } \\
\text { al. (2009) }\end{array}$ & $\begin{array}{l}\text { Arve Valley, France } \\
86 \text { RCC cases } \\
326 \text { hospital controls } \\
\text { Expert assessment, semi- } \\
\text { quantitative JEM }\end{array}$ & $\begin{array}{l}2006 \text { analysis } \\
\text { Non-exposed (ever) } \\
\text { Ever exposed } \\
\text { High confidence (Model 1) } \\
\text { Cumulative dose } \\
\text { Non-exposed } \\
\text { Low } \\
\text { Medium } \\
\text { High } \\
\text { Cumulative exp. }+ \text { peaks } \\
\text { Non-exposed } \\
\text { Low/medium no peaks } \\
\text { Low/medium }+ \text { peaks } \\
\text { High no peaks } \\
\text { High }+ \text { peaks } \\
\text { All workers } \\
\text { High cum. dose (Model 1) } \\
\text { High cum. dose (Model 2) } \\
\text { High }+ \text { peaks (Model 1) } \\
\text { High }+ \text { peaks (Model 2) } \\
2009 \text { analysis: combined effects } \\
\text { TCE \& cutting oil (Model 2) } \\
\text { Cutting oil/TCE } \\
\text { No/No } \\
\text { Yes/No } \\
\text { No/Yes } \\
\text { Yes/ } / 50 \text { ppm } \\
\text { Yes/ } \geq 50 \text { ppm }\end{array}$ & $\begin{array}{l}1.00 ; 46 / 200 \\
2.39(0.52-11.03) ; 3 / 6 \\
1.62(0.76-3.44) ; 15 / 46 \\
1.14(0.49-2.66) ; 12 / 47 \\
2.70(1.02-7.17) ; 10 / 17\end{array}$ & $\begin{array}{l}\text { High intensity of exposure and high } \\
\text { exposure prevalence. Screw cutting } \\
\text { industry. Estimated TCE intensities } \\
\text { for high exposure jobs were } 300- \\
600 \text { ppm } \\
\text { Covariates: (Model 1) Sex, age, } \\
\text { smoking, BMI; (Model 2) sex, age, } \\
\text { cutting oils, petroleum oils, and/or } \\
\text { other mineral oils. No significant } \\
\text { difference between cases and controls } \\
\text { in a number of medical history- } \\
\text { related factors } \\
\text { Strengths: Good exposure assessment } \\
\text { and consideration of co-exposures } \\
\text { Limitations: Small number of } \\
\text { exposed cases and controls in } \\
\text { subgroup analyses } \\
\text { Evidence for a positive association } \\
\text { Statistically significant, moderately } \\
\text { elevated effect estimates; evidence of } \\
\text { exposure-response relationship; } \\
\text { unlikely to be explained by } \\
\text { confounding }\end{array}$ \\
\hline
\end{tabular}




\begin{tabular}{|c|c|c|c|c|}
\hline Reference & $\begin{array}{c}\text { Study } \\
\text { Size (N) } \\
\text { Exposure Assessment }\end{array}$ & Exposure Groups & $\begin{array}{c}\text { RR or OR }(95 \% \text { CI }) \\
\text { \# Exposed Cases/Controls }\end{array}$ & Interpretation \\
\hline $\begin{array}{l}\text { Brüning et al. (2003) } \\
\text { (no overlap with } \\
\text { Vamvakas et al. } \\
(1998) \text { ) }\end{array}$ & $\begin{array}{l}\text { Germany regional } \\
\text { (Arnsberg) hospital- } \\
\text { based } \\
134 \text { cases ( } 83 \mathrm{M}, 51 \mathrm{~F}) \\
401 \text { hospital controls } \\
(225 \mathrm{M}, 176 \mathrm{~F}) \\
\text { Self-assessed exposure } \\
\text { including self-reported } \\
\text { narcotic symptoms, JEM } \\
\text { based on CAREX } \\
\text { database (job titles) or } \\
\text { British JEM for grouped } \\
\text { solvents }\end{array}$ & $\begin{array}{l}\text { CAREX Database } \\
\text { Longest held job with TCE/Perc } \\
\text { exposure (compared with no TCE) } \\
\text { Any metal greasing/degreasing } \\
\text { Self-assessed TCE exposure } \\
\text { Ever } \\
\text { Exposure + Narcotic symptoms } \\
\text { Any } \\
\text { Non-daily occurrence } \\
\text { Daily occurrence } \\
\text { Duration exposure (yr) } \\
\text { No exposure } \\
<10 \\
10-19 \\
20+ \\
\text { Time since } 1 \text { st exp (yr) } \\
\text { No exposure } \\
5-9 \\
10-19 \\
20+ \\
\text { No increasing risks with time since } \\
\text { last exposure }\end{array}$ & $\begin{array}{l}1.00 ; 109 / 363 \\
3.78(1.54-9.28) ; 11 / 14 \\
1.80(0.67-4.79) ; 7 / 13 \\
2.69(0.84-8.66) ; 6 / 7 \\
\\
1.00 ; 109 / 363 \\
3.21(0.28-37.38) ; 1 / 2 \\
1.50(0.28-8.10) ; 2 / 6 \\
2.86(1.49-5.49) ; 22 / 27\end{array}$ & $\begin{array}{l}\text { Very high exposure and long } \\
\text { exposures; estimated exposure } 400 \text { to } \\
600 \text { ppm during peak (hot dipping) } \\
\text { and }>100 \text { ppm overall (Cherrie et al. } \\
2001 \text { ). Approx. } 50 \% \text { cases }>10 \text { years' } \\
\text { exposure } \\
\text { Covariates: Sex, age, smoking; cases } \\
\text { and controls had similar BMI } \\
\text { Strengths: Appears reasonable that } \\
\text { workers with self-reported exposure } \\
\text { had high levels of exposure and } \\
\text { exposure to other chlorinated } \\
\text { solvents was unlikely } \\
\text { Limitations: Qualitative exposure } \\
\text { assessment; possible selection bias } \\
\text { Evidence for a positive association: } \\
\text { Statistically significant, high effect } \\
\text { estimates; unlikely to be explained by } \\
\text { confounding }\end{array}$ \\
\hline
\end{tabular}


RoC Monograph on Trichloroethylene

\begin{tabular}{|c|c|c|c|c|}
\hline Reference & $\begin{array}{c}\text { Study } \\
\text { Size (N) } \\
\text { Exposure Assessment }\end{array}$ & Exposure Groups & $\begin{array}{c}\text { RR or OR }(95 \% \text { CI }) \\
\text { \# Exposed Cases/Controls }\end{array}$ & Interpretation \\
\hline $\begin{array}{l}\text { Vamvakas et al. } \\
\text { (1998) }\end{array}$ & $\begin{array}{l}\text { Germany regional } \\
\text { hospital-based } \\
58 \mathrm{RCC} \text { cases } \\
84 \text { hospital controls } \\
\text { Expert assessment based } \\
\text { on severity of pre- } \\
\text { narcotic symptom and } \\
\text { exposure duration using } \\
\text { occupational history data } \\
\text { from interviews }\end{array}$ & $\begin{array}{l}\text { Ever TCE exposure } \\
\text { TCE exposure categories } \\
\text { No TCE exposure } \\
\text { Low TCE exposure } \\
\text { Medium TCE exposure } \\
\text { High TCE exposure }\end{array}$ & $\begin{array}{l}\text { OR } \\
10.80(3.36-34.75) ; 19 / 7 \\
1.00 ; 39 / 77 \\
6.61(0.50-87.76) ; 2 / 2 \\
11.92(2.55-55.60) ; 9 / 3 \\
11.42(1.96-66.79) ; 8 / 2\end{array}$ & $\begin{array}{l}\text { High level of exposure [see Brüning } \\
\text { et al. (2003)] Mean duration of } \\
\text { exposure: } 16 \text { years cases, } 7 \text { years } \\
\text { controls } \\
\text { Covariates: Age, sex, smoking, BMI, } \\
\text { blood pressure, and diuretic intake } \\
\text { Strengths: High level of confidence } \\
\text { that workers had high level of } \\
\text { exposure and exposure to other } \\
\text { chlorinated solvents was unlikely } \\
\text { Limitations: Potential selection bias } \\
\text { (differential) away from the null } \\
\text { Evidence for a positive association: } \\
\text { Statistically significant, high effect } \\
\text { estimates; unlikely to be explained by } \\
\text { confounding or co-exposures; } \\
\text { potential for biases would lead to an } \\
\text { over-estimate of the risk estimate }\end{array}$ \\
\hline
\end{tabular}




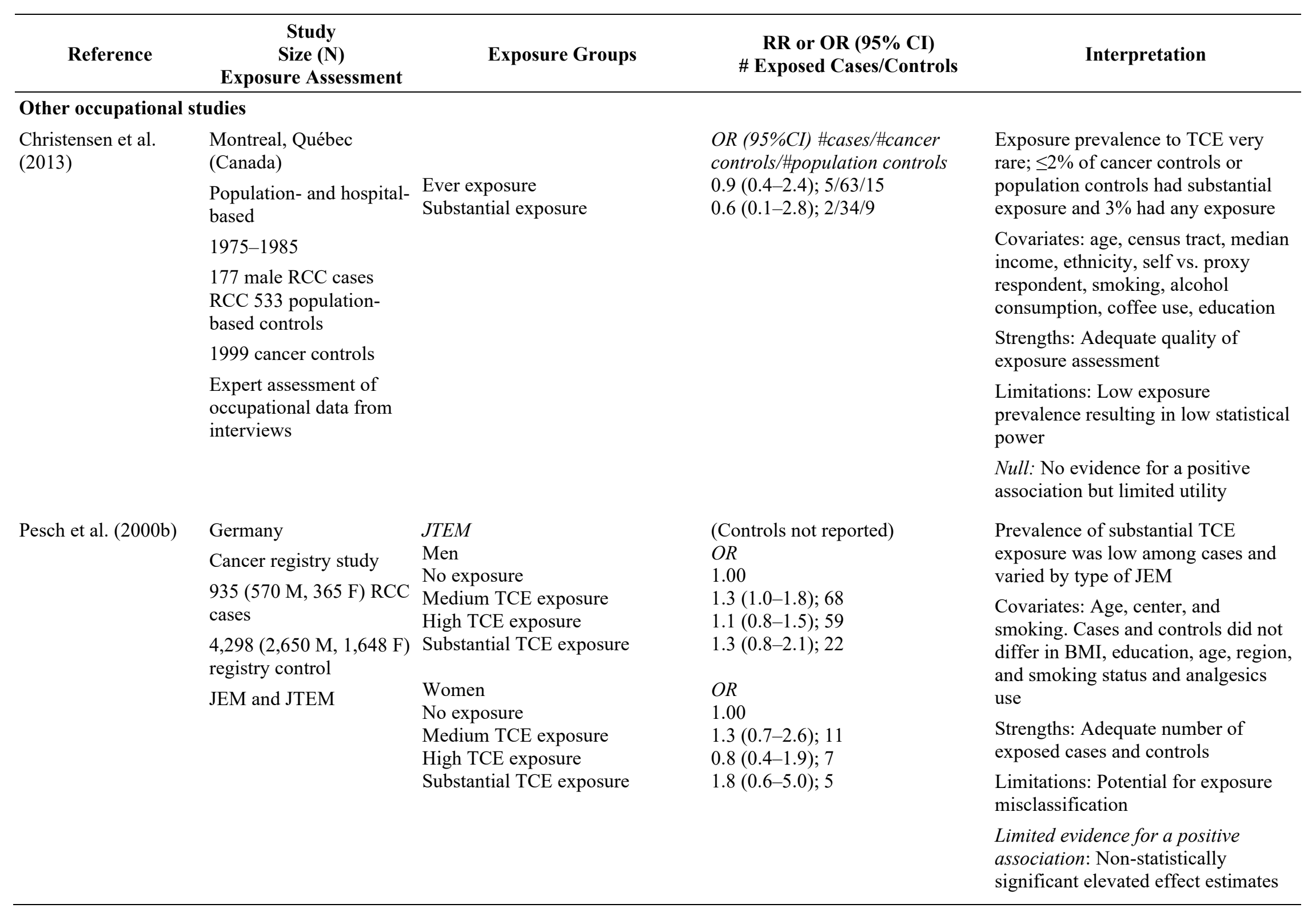


RoC Monograph on Trichloroethylene

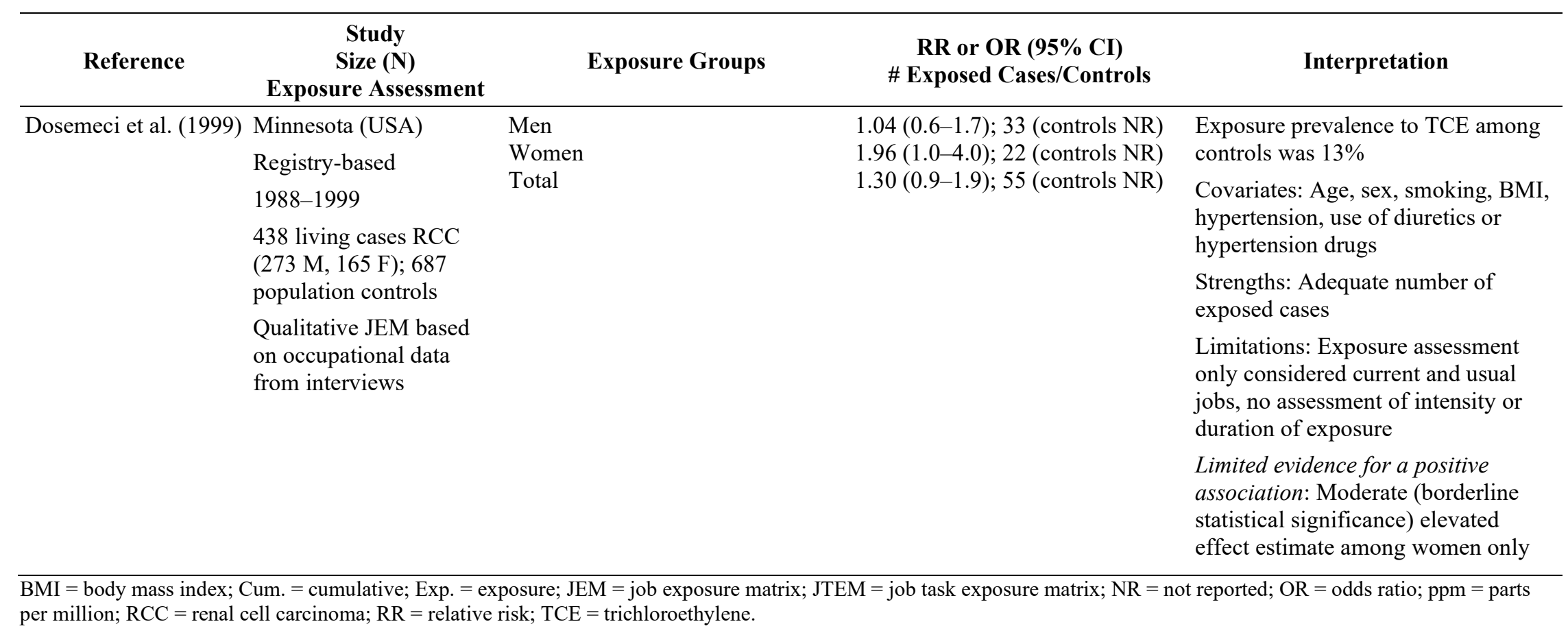




\subsubsection{Meta-analyses: Kidney Cancer}

Meta-analyses have been recommended as an approach to analyze the body of epidemiological studies of trichloroethylene (NRC 2006) in order to provide a synthesis of data and to partly overcome the limitations of individual studies due to low statistical power. Several metaanalyses of kidney cancer and trichloroethylene exposure have been conducted (Karami et al. 2012; Kelsh et al. 2010; Scott and Jinot 2011; USEPA 2011a; Wartenberg and Reyner 2000). This evaluation is limited to the recent meta-analyses by the EPA (2011a), Scott and Jinot (2011), Karami et al. (2012) and Kelsh et al. (2010) because many studies have been published since the older evaluations. The EPA and Karami et al. meta-analyses primarily analyzed cohort and case-control studies with specific exposure to trichloroethylene reviewed in this monograph although Karami et al. (2012) also included two studies (Asal et al. 1988; Harrington et al. 1989) that were not considered to be specific for trichloroethylene and thus were excluded from this evaluation. The meta-analysis by Kelsh et al. also included a larger number of studies not specific for trichloroethylene that were excluded from this monograph (see Table 4-3). Both the EPA (Scott and Jinot 2011) and Karami et al. (2012) meta-analyses pre-dated the pooled and updated Nordic cohort study (Hansen et al. 2013), the population-based Nordic study by Vlaanderen et al. (2013), the Montreal case-control study by Christensen et al. (2013), and the drinking water study by Bove et al. (2014). The EPA meta-analysis (Scott and Jinot 2011) included an earlier update (Boice et al. 1999) of the cohort study of aircraft manufacturing workers in Burbank, CA, whereas the later update by Lipworth et al. (2011) was included in the analysis by Karami et al. (2012). An earlier update of the Montreal Canadian study or the component studies (in the case of the pooled analysis) were included in both meta-analyses, and the only new study populations are those reported by Vlaanderen et al. (2013) and Bove et al. (2014).

The EPA meta-analyses (Scott and Jinot 2011; USEPA 2011a) included systematic data extraction of eight cohort and seven case-control studies (including one nested case-control study) in which potential trichloroethylene exposure was documented and risk estimates for kidney cancer and trichloroethylene exposure were calculated (Table 4-3). Studies with evidence of a low potential for exposure to trichloroethylene were excluded. Fixed and random effects models, tests for heterogeneity and publication bias, and sensitivity analyses (to examine the impact of individual studies and selection of alternative relative risk selections on meta-relative risk estimates) were used to calculate summary meta-relative risks using, where provided, adjusted or crude risk estimates from internal analyses rather than external (SMR or SIR) estimates. In addition, separate meta-analyses were conducted for the highest exposure groups (either by duration and/or intensity) within trichloroethylene-exposed populations (reported in 13 of the 15 constituent studies). In these highest exposure subgroups, non-differential misclassification of exposure would be expected to be less than among the whole group, bearing in mind that actual levels and lengths of exposure might have differed considerably across studies.

Karami et al. (2012) used similar inclusion/exclusion criteria to the EPA and considered an overlapping body of studies, but with the inclusion of cohort studies by Boice et al. (2006) (rather than Zhao et al. (2005), with which it overlaps) and Lipworth et al. (2011) and, as noted, the case-control studies by Asal et al. (1988) and Harrington et al. (1989). Initial examination of the cohort study of German cardboard manufacturers Henschler et al. (1995) and the case-control 
study by Vamvakas et al. (1998) introduced the greatest heterogeneity and so were excluded from some analyses. Only the data for analyses excluding these studies, which are more closely comparable with the EPA analysis, are reported in Table 4-3 below. Since Kelsh et al. (2010) included a number of studies that were considered non-specific for trichloroethylene exposure, the results should be interpreted with caution.

Table 4-3. Meta-analyses of Kidney Cancer and Trichloroethylene Exposure

\begin{tabular}{|c|c|c|c|c|}
\hline Reference & $\begin{array}{l}\text { Study Design (\# } \\
\text { Studies) }\end{array}$ & $\begin{array}{c}\text { mRR }(95 \% \mathrm{CI}) \\
\text { All }\end{array}$ & $\begin{array}{c}\text { mRR (95\% CI) } \\
\text { Highest Exposure }\end{array}$ & Comments \\
\hline \multirow{3}{*}{$\begin{array}{l}\text { EPA } \\
(2011 \mathrm{a}) / \mathrm{Scott} \\
\text { and Jinot }(2011)\end{array}$} & \multirow{3}{*}{$\begin{array}{l}\text { Combined cohort and } \\
\text { case-control studies } \\
\text { (15 for any exposure, } \\
13 \text { for high exposure) }\end{array}$} & \multirow[t]{3}{*}{$1.27(1.13-1.43)$} & \multirow[t]{3}{*}{$1.58(1.28-1.96)$} & Random effects model \\
\hline & & & & $\begin{array}{l}\text { Low sensitivity to removal } \\
\text { of individual studies or } \\
\text { selection of alternative RRs }\end{array}$ \\
\hline & & & & $\begin{array}{l}\text { Little evidence of } \\
\text { heterogeneity or } \\
\text { publication bias }\end{array}$ \\
\hline $\begin{array}{l}\text { EPA } \\
(2011 \mathrm{a}) / \mathrm{Scott} \\
\text { and Jinot }(2011)\end{array}$ & Cohorts (8) & $1.16(0.96-1.40)$ & NR & $\begin{array}{l}\text { No sig. diff. between } \\
\text { cohort and case-control } \\
\text { mRRs }\end{array}$ \\
\hline $\begin{array}{l}\text { EPA } \\
(2011 \mathrm{a}) / \mathrm{Scott} \\
\text { and Jinot }(2011)\end{array}$ & Case-control (7) & $1.48(1.15-1.91)$ & NR & $\begin{array}{l}\text { No heterogeneity in } \\
\text { cohorts, low to moderate } \\
\text { heterogeneity in case- } \\
\text { control studies }\end{array}$ \\
\hline \multirow{3}{*}{$\begin{array}{l}\text { Karami et al. } \\
(2012)\end{array}$} & \multirow{3}{*}{$\begin{array}{l}\text { TCE-exposed } \\
\text { cohort }+ \text { case-control } \\
\text { studies (18) }\end{array}$} & \multirow[t]{3}{*}{$1.32(1.17-1.50)^{\mathrm{a}}$} & \multirow[t]{3}{*}{ NR } & Random effects model \\
\hline & & & & $\begin{array}{l}\text { Little evidence of } \\
\text { heterogeneity and } \\
\text { publication bias }\end{array}$ \\
\hline & & & & $\begin{array}{l}\text { Higher mRR among } \\
\text { incidence vs. mortality } \\
\text { studies }\end{array}$ \\
\hline \multirow[t]{5}{*}{$\begin{array}{l}\text { Karami et al. } \\
\text { (2012) }\end{array}$} & $\begin{array}{l}\text { TCE-exposed cohorts } \\
\text { (9) }\end{array}$ & $1.26(1.02-1.56)^{\mathrm{a}}$ & & $\begin{array}{l}\text { Little evidence of } \\
\text { heterogeneity or } \\
\text { publication bias }\end{array}$ \\
\hline & Exp.-Response & & & \\
\hline & Long duration vs. & & $1.52(1.08-2.13)$ & \\
\hline & & & & \\
\hline & $\begin{array}{l}\text { Subset of U-TCA } \\
\text { studies (3) }\end{array}$ & $1.03(0.59-1.78)$ & & \\
\hline \multirow[t]{4}{*}{$\begin{array}{l}\text { Karami et al. } \\
\text { (2012) }\end{array}$} & $\begin{array}{l}\text { TCE-exposed case- } \\
\text { control studies (9) }\end{array}$ & $1.35(1.17-1.57)^{\mathrm{a}}$ & & $\begin{array}{l}\text { Little evidence of } \\
\text { heterogeneity or } \\
\text { publication bias }\end{array}$ \\
\hline & Exp.-Response & & & \\
\hline & High intensity vs. & & $1.68(1.23-2.30)$ & \\
\hline & low intensity (6) & & $1.49(1.02-2.17)^{\mathrm{a}}$ & \\
\hline
\end{tabular}




\begin{tabular}{llccl}
\hline \multicolumn{1}{c}{ Reference } & \multicolumn{1}{c}{$\begin{array}{c}\text { Study Design (\# } \\
\text { Studies) }\end{array}$} & $\begin{array}{c}\text { mRR (95\% CI) } \\
\text { All }\end{array}$ & $\begin{array}{c}\text { mRR (95\% CI) } \\
\text { Highest Exposure }\end{array}$ & \multicolumn{1}{c}{ Comments } \\
\hline $\begin{array}{l}\text { Kelsh et al. } \\
(2010)\end{array}$ & $\begin{array}{l}\text { TCE-exposed cohorts } \\
(8)\end{array}$ & $1.34(1.07-1.67)^{\mathrm{a}}$ & & $\begin{array}{l}\text { Little evidence of } \\
\text { heterogeneity or } \\
\text { publication bias }\end{array}$ \\
& $\begin{array}{l}\text { Exp.-Response } \\
\text { Long duration vs. }\end{array}$ & & & \\
& short duration ${ }^{\mathrm{b}}(7)$ & & $1.24(0.69-2.23)$ & \\
& High cum. exp. vs. & & $1.50(0.89-2.26)$ & \\
& low cum. exp. (3) & & $1.39(0.75-2.59)$ & \\
Kelsh et al. & TCE-exposed case- & $1.33(1.02-1.73)$ & See above & Little evidence of \\
$(2010)$ & control (6) & & & $\begin{array}{l}\text { heterogeneity or } \\
\text { publication bias }\end{array}$ \\
\hline
\end{tabular}

See Appendix D for a list of the studies included in the meta-analyses.

$\mathrm{mRR}=$ meta-relative risk; $\mathrm{NR}=$ not reported; $\mathrm{RR}=$ relative risk; $\mathrm{U}-\mathrm{TCA}=$ urine trichloroacetic acid.

${ }^{a}$ Excluding studies by Henschler et al. (1995) and/or Vamvakas et al. (1998).

${ }^{\mathrm{b}}$ Combined cohort and case-control studies.

The overall results of the three meta-analyses were broadly comparable, with some variation partly depending on which specific studies were included. Both cohort and case-control studies, separately and combined, yield robust and statistically significant but modest increases in metarelative risks (mRRs) for kidney cancer in the two most recent and comparable meta-analyses, approximately 1.3 (for case-control and cohort combined), with little evidence of heterogeneity and publication bias, and with slightly higher statistically significant mRRs among the casecontrol studies than the cohort studies. Importantly, the mRR was robust and not sensitive to removal of individual studies or selection of alternative RRs. Investigation of the highest exposure groups in the EPA analysis of the combined cohort and case-control studies (Scott and Jinot 2011; USEPA 2011a), yielding a statistically significant mRR of 1.58, provides some evidence of higher risk among more highly exposed workers. This was similar to the mRRs for higher exposure calculated in the separate cohort and case-control analyses by Karami et al. (2012), but slightly higher than those reported by Kelsh et al. (2010) (which, as noted, included some different studies). However, the data were insufficient to distinguish which metric of exposure (among the studies categorized as "high" exposure) is more clearly associated with an increase in the risk of kidney cancer mRRs.

\subsubsection{Evaluation of Potential Confounding by Occupational Co-exposures or Other Risk Factors}

Section 3 discussed the adequacy of the methods used in the cohort (Section 3.2) and casecontrol studies (Sections 3.3 and 3.4) for evaluating potential confounding from occupational coexposures and non-occupational factors. However, that assessment was not specific for kidney cancer. This section builds on that assessment, integrating it with other relevant information and evaluating whether confounding can explain the increased risks of kidney cancer observed in many of the studies.

\subsubsection{Occupational Co-exposures}

With respect to occupational agents, IARC (Cogliano et al. 2011) and/or the Report on Carcinogens (NTP 2011) have identified X-radiation as a known kidney carcinogen in humans and concluded that there was limited evidence of carcinogenicity for arsenic, cadmium, and 
printing processes. Few of the cohort and nested case-control studies provided qualitative or quantitative data on potential co-exposures or adjusted for (or considered) them in statistical analyses. The potential co-exposures include a wide range of other chemical or physical agents, principally the chlorinated solvents tetrachloroethylene and 1,1,1-trichloroethane in both the Nordic and aerospace and aircraft studies, and cutting fluids such as mineral and petroleum oils, hydrazine, benzene, chromates, and PAHs in the aerospace and aircraft industries, although the most common co-exposures among the group of studies are probably chlorinated solvents and cutting oils such as mineral and petroleum oils. The workers in the Nordic studies had diverse occupations, and thus the types, patterns, and levels of co-exposures to other agents are likely to vary across the different industries and time periods. In most studies, it is not clear if or how strongly exposures to other occupational agents were correlated with exposure to trichloroethylene. Moreover, none of these substances has been identified as a known or suspected kidney carcinogen in humans to date although some are carcinogenic in animals. IARC (2014) recently concluded that there was little overall evidence of an association of exposure to tetrachloroethylene with kidney cancer in humans. No independent epidemiological data on 1,1,1-trichoroethane and kidney cancer were identified.

The two studies of aerospace workers, which found a positive association between trichloroethylene exposure and kidney cancer, adjusted for exposure to known co-exposures. Zhao et al. (2005) directly adjusted for co-exposures (mineral or petroleum oils) in their internal analysis of trichloroethylene and kidney cancer risk, and Boice et al. (2006) adjusted for hydrazine exposure.

Case-control studies were more limited on information for potential occupational co-exposures. However, as in the Nordic studies, workers were from diverse industries, with varying types and patterns of co-exposures. Only one study (Charbotel et al. 2006; Charbotel et al. 2009) adjusted for co-exposures (to petroleum and cutting oils); risks for trichloroethylene exposure were still elevated but slightly attenuated (from 2.23 to 1.96) and an elevated risk (although not statistically significant) was observed among workers without exposure to mineral oils in combined analyses. There is no independent evidence that mineral oils are associated with kidney cancer.

\subsubsection{Lifestyle and Other Potential Confounders}

Non-occupational risk factors for kidney cancer include tobacco smoking, obesity (BMI), diabetes, hypertension (diuretics), and X-radiation (see e.g., Chow et al. (2010), Cogliano et al. (2011)). It is not clear whether any of these would be associated with trichloroethylene exposure, but tobacco smoking may be the most likely risk factor.

The majority of cohort and nested case-control studies conducted age-, sex-, race- and calendaryear or period-standardized comparisons in external analyses (SMR or SIR) where appropriate and age-, sex-, race- and in some cases calendar-period-adjusted comparisons in internal analyses. In addition, all of the studies, except for the Danish blue-collar worker study, conducted internal analyses, which would mitigate potential confounding from lifestyle factors. Each of the case-control studies, in addition to matching or adjusting for demographic variables including age, sex, and residential location, examined or adjusted for BMI and/or measures of hypertension, with the exception of Christensen et al. (2013) and Vamvakas et al. (1998). There was no clear evidence of confounding by these variables in the studies that examined or adjusted for them. Some studies also considered socioeconomic factors (Christensen et al. 2013), medical 
history or conditions (Dosemeci et al. 1999; Moore et al. 2010; Pesch et al. 2000b; Vamvakas et al. 1998), or other lifestyle factors (Christensen et al. 2013).

Potential confounding from smoking can reasonably be ruled out. Smoking is a relatively weak risk factor for kidney cancer (approximately 1.4 for current smoking in meta-analyses data), and the NRC (2006) estimated that it most likely would only account for approximately $10 \%$ increase in risk if smoking differences were $20 \%$ higher among trichloroethylene-exposed populations. Increased risks of kidney cancer were observed in several case-control studies that adjusted for or considered smoking habits (Brüning et al. 2003; Charbotel et al. 2006; Charbotel et al. 2009; Dosemeci et al. 1999; Moore et al. 2010; Pesch et al. 2000b; Vamvakas et al. 1998). Although most of the cohort studies did not adjust for smoking, lung cancer rates among the trichloroethylene-exposed workers appear to be unremarkable, with the exception of significantly elevated risks (approximately 40\%) for men and women in the Danish blue-collar cohort (Raaschou-Nielsen et al. 2003); however, this likely explains $<6 \%$ of the excess risk from trichloroethylene (USEPA 2011a). The EPA (2011a) also found no association with lung cancer and trichloroethylene in a meta-analysis of studies (OR approximately 1 for all studies and also for high trichloroethylene exposure).

Overall, there was little evidence to suggest that confounding by occupational co-exposures explains the observed increases in kidney cancer, which have been reported in populations with different industries and lifestyle factors and in different regions.

\subsubsection{Forest Plot Methods}

Forest plots were constructed using risk estimates for kidney cancer and ever exposure (Figure 42) or the highest exposure category (Figure 4-3) and grouping the studies by ranking of the utility of the studies to inform the cancer hazard evaluation or by broad group of estimated exposure (high exposure risk estimates only) (Figure 4-4). Cohort and case-control studies were presented together because the meta-analyses did not report statistically significant differences for the meta-relative risks between the two study designs.

High-exposure category: For each study, risk estimates (SMR, SIR, RR, HR, or OR) were extracted for the highest estimated exposure group (intensity or cumulative exposure), if reported. In the cohort study by Lipworth et al. (2011), duration was used because risk by exposure level was not reported. In some cases, surrogates for exposure intensity were used. Calendar year was used as a surrogate in one cohort study (Raaschou-Nielsen et al. 2003) because additional data suggested that average exposures were highest in the earliest calendar period (prior to 1970). In the case-control study of renal-cell carcinoma by Brüning et al. (2003), prevalence of narcotic symptoms was used as a surrogate by the authors to indicate the highest exposed workers.

Utility of the studies to inform the cancer hazard evaluation: Studies were ranked into categories of utility: high; moderate; low with potential bias most likely towards the null; and low with potential bias most likely towards a positive effect (overestimate of the risk estimate). Studies with low or low/moderate utility in Figure 4-2 were combined into one category. This broad ranking was based on consideration of selection bias and information bias (quality of exposure and disease characterization and likely degree of exposure or disease misclassification), and on study sensitivity (as a function of statistical power, estimated exposure levels, and length of 
follow-up). (See Sections 3.2.2.6, 3.3.2.6, and 3.4.2.6; Appendix D; and Figure 4-1) for detailed summaries of these elements.)

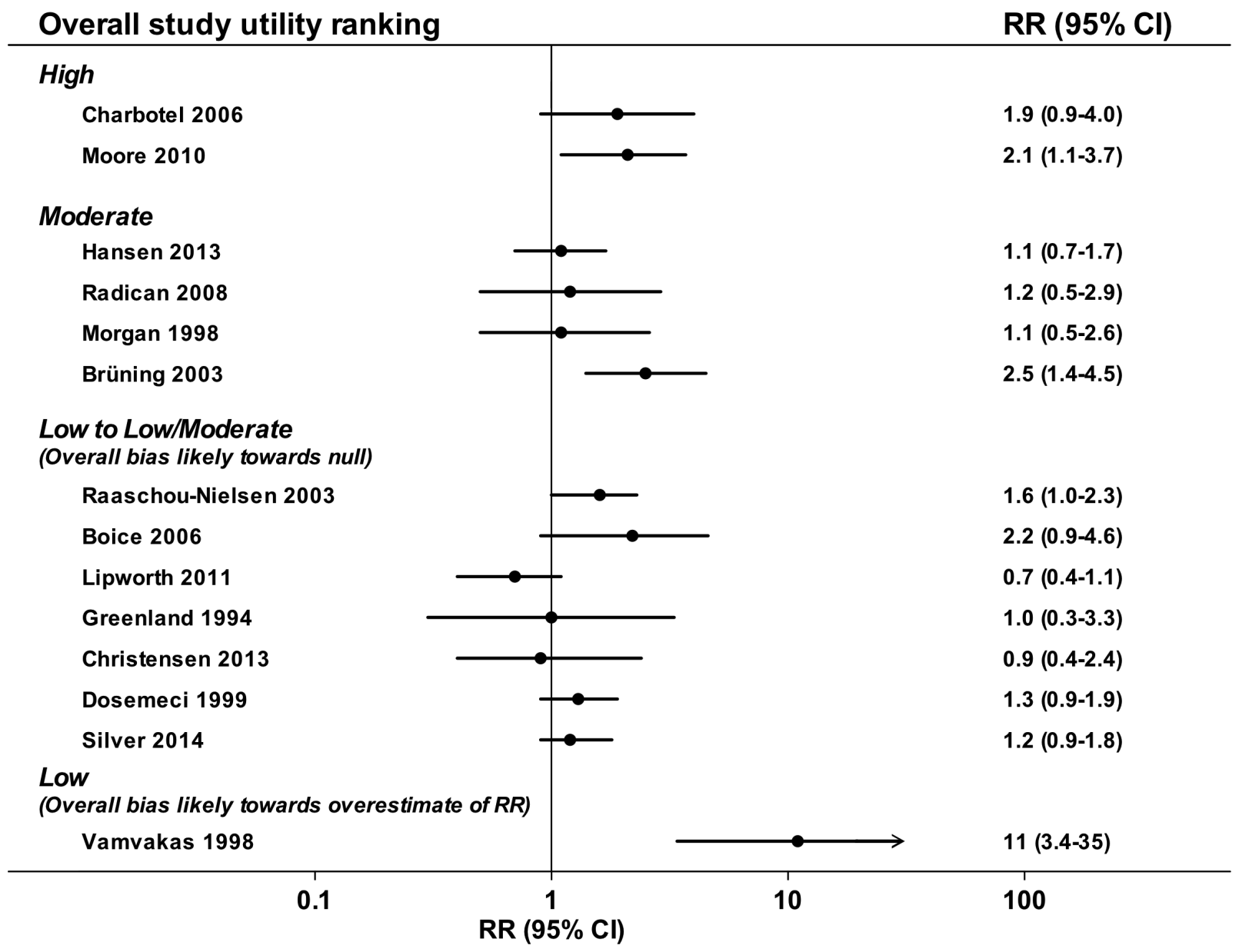

Figure 4-2. Forest Plot-1: Kidney Cancer and Ever Exposure to Trichloroethylene

Effect estimate and 95\% CI for ever exposure to trichloroethylene and kidney cancer by study utility category (see Figure 4-1) and overall prediction of direction of any bias for low utility studies as described in Section 4.1.3. Studies by Bove et al. (2014) and Vlaanderen et al. (2013) are not graphed because they did not report relative risk for ever exposure but they are reported in Figure 4-3 and Table 4-1. Effect estimates are also reported in Table 4-1. For studies reporting multiple effect estimate, preference is given to analysis for longer lags (Hansen et al. (2013) [20 years], Raaschou-Nielsen et al. (2003) [ $\geq 20$ years]) and internal analysis; RR for Morgan et al. (1998) was reported by Scott and Jinot (2011).

Ranked estimated exposure: For each study the effect estimate and 95\% CI for the highest exposure level was plotted as described above. The studies reported different metrics of exposure, including intensity (or surrogate for intensity), cumulative exposure, and duration. Some studies used an exposure category that integrated confidence or probability with intensity or duration (Christensen et al. 2013; Pesch et al. 2000b). Although there were very few data on actual exposure levels, some authors or reviewers have estimated exposure for either jobs or cumulative exposure or intensity for individuals, and this information was used to group the studies in three broad exposure level groups. (See Table D-4, and Table D-6 for estimated exposure level and ranked exposure group.) The exposure group (high to very high, moderate to high, low) is for the estimated exposure level for the exposure metric reported in that study- 
e.g., studies reporting effect estimates for exposure for cumulative exposure are ranked according to the estimated cumulative exposure for that study.

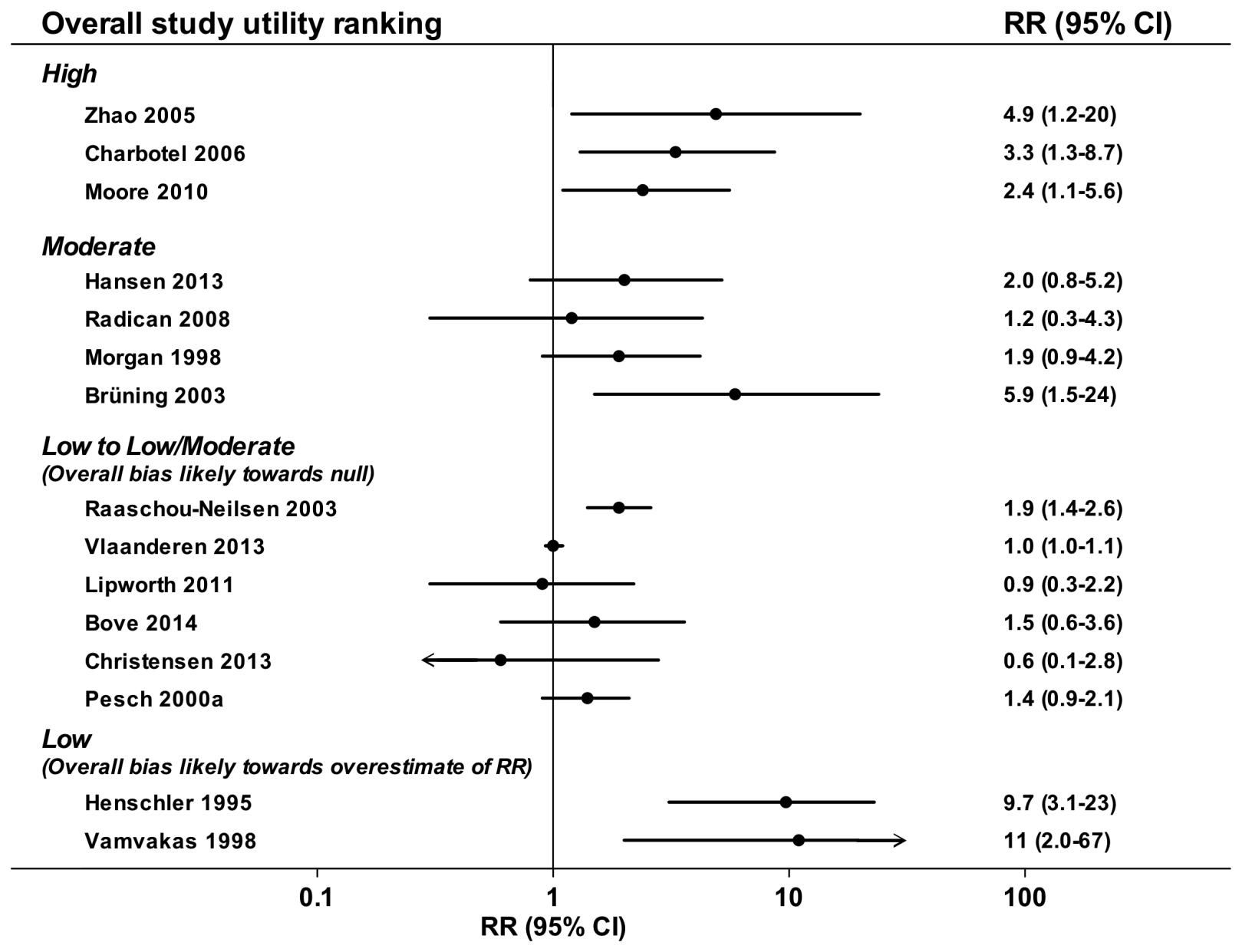

Figure 4-3. Forest Plot-2: Kidney Cancer and High Exposure to Trichloroethylene

Effect estimate and 95\% CI for high exposure to trichloroethylene and kidney cancer by study utility category (see Figure 4-1) and overall prediction of direction of any bias for low utility studies as described in Section 4.1.3. Studies by Greenland et al. (1994), Dosemeci et al. (1999), and Silver et al. (2014) are not graphed because they did not report a risk estimate for high exposure. Findings for these studies are reported in Table 4-1 and in Figure 4-2. Effect estimates are also reported in Table 4-1; the effect estimate for men and women combined in Pesch et al. (2000b) was reported by Scott and Jinot (2011).

\subsubsection{Integration Across Studies}

There is credible evidence of an association between exposure to trichloroethylene and kidney cancer risk based on consistent findings of increased risks of kidney cancer across studies of different designs, different geographical areas, and different occupational settings (see Figure 4-2 and Figure 4-3) and evidence of exposure-response relationships. The most convincing evidence for an association between kidney cancer incidence and exposure to trichloroethylene comes from the three most informative (high utility) studies (Charbotel et al. 2006; Charbotel et al. 2009; Moore et al. 2010; Zhao et al. 2005), and two studies with moderate or moderate to low utility, a Nordic cohort of blue-collar workers in companies using trichloroethylene (RaaschouNielsen et al. 2003), and a case-control study from an area in Germany with known 
trichloroethylene exposure (Brüning et al. 2003), all of which found statistically significant elevated risks of kidney cancer among workers with the highest exposure to trichloroethylene (see Figure 4-3). These findings are supported by weaker associations (in analyses of high or ever exposure to trichloroethylene) found in most of the other studies considered to be of moderate utility (Hansen et al. 2013; Morgan et al. 1998), and some studies considered to have low to moderate utility (Dosemeci et al. 1999; Pesch et al. 2000b) or low utility (Bove et al. 2014; Silver et al. 2014). Limitations in most of these studies would most likely bias towards the null, and the fact that the studies were considered to be of lower utility does not detract from the positive evidence. Statistically significant increased risks were also found in two German studies located in a geographical area known to have industries with exposure to high levels of trichloroethylene: a cohort study of cardboard manufacturing workers (Henschler et al. 1995), and a case-control study by Vamvakas et al. (1998). Both of these studies have potential biases that would most likely lead to an overestimate of the risk estimate although it is unlikely that the biases nullify the large excess risk found in these studies. Figure 4-3 plots the risk estimate for the high-exposure group from each study and groups the studies according to broad groups of estimated exposure. The highest risks were found among studies with very high or high to moderate exposure to trichloroethylene, and findings were more heterogeneous among studies with low estimated exposure.

Meta-analyses are useful for evaluating potential heterogeneity between studies or types of studies and also for summarizing the results of studies. The most recent meta-analyses (Karami et al. 2012; Scott and Jinot 2011) provide strong evidence for an association between trichloroethylene exposure and kidney cancer. Both analyses found statistically significant metarelative risks of similar magnitudes, i.e., $1.27(95 \% \mathrm{CI}=1.13$ to 1.43$)$ by Scott and Jinot (2011) and $1.32(95 \% \mathrm{CI}=1.17$ to 1.50$)$ by Karami et al. (2012). Importantly, the mRR was robust and not sensitive to removal of individual studies or selection of alternative RRs. There was no evidence of publication bias or heterogeneity across studies (which did not include the studies by Vamvakas et al. and Henschler et al., which have the high-risk estimates) or publication bias in both meta-analyses. Although a lower mRR was found for cohort studies than case-control studies, the subgroup risk estimates for case-control and cohort studies did not significantly differ from each other.

There was evidence for positive exposure-response relationships or higher risks in more highly or longer exposed groups in both cohort and case-control studies with several exposure metrics. Risks increased with increasing exposure intensity or cumulative exposure in the cohort study of aerospace workers (Zhao et al. 2005), the French case-control study, which was primarily of workers in the screw-cutting industries (Charbotel et al. 2006; Charbotel et al. 2009), the European study (Moore et al. 2010), and the Nordic study of blue-collar workers (RaaschouNielsen et al. 2003), using calendar year of first exposure as a surrogate for exposure level. Other studies found higher risk among individuals with longer exposure to trichloroethylene (Moore et al. 2010) or employment duration (Raaschou-Nielsen et al. 2003). The meta-analyses also provide evidence for exposure-response relationships between trichloroethylene exposure and kidney cancer. The EPA meta-analysis found a higher mRR for higher-exposure groups (1.6) across studies compared with the risk for ever exposure across studies (1.3) (Scott and Jinot 2011). Karami et al. (2012) found higher mRRs for high (vs. low) intensity exposure and long (vs. short) duration of exposure in separate analyses of cohort and case-control studies. 


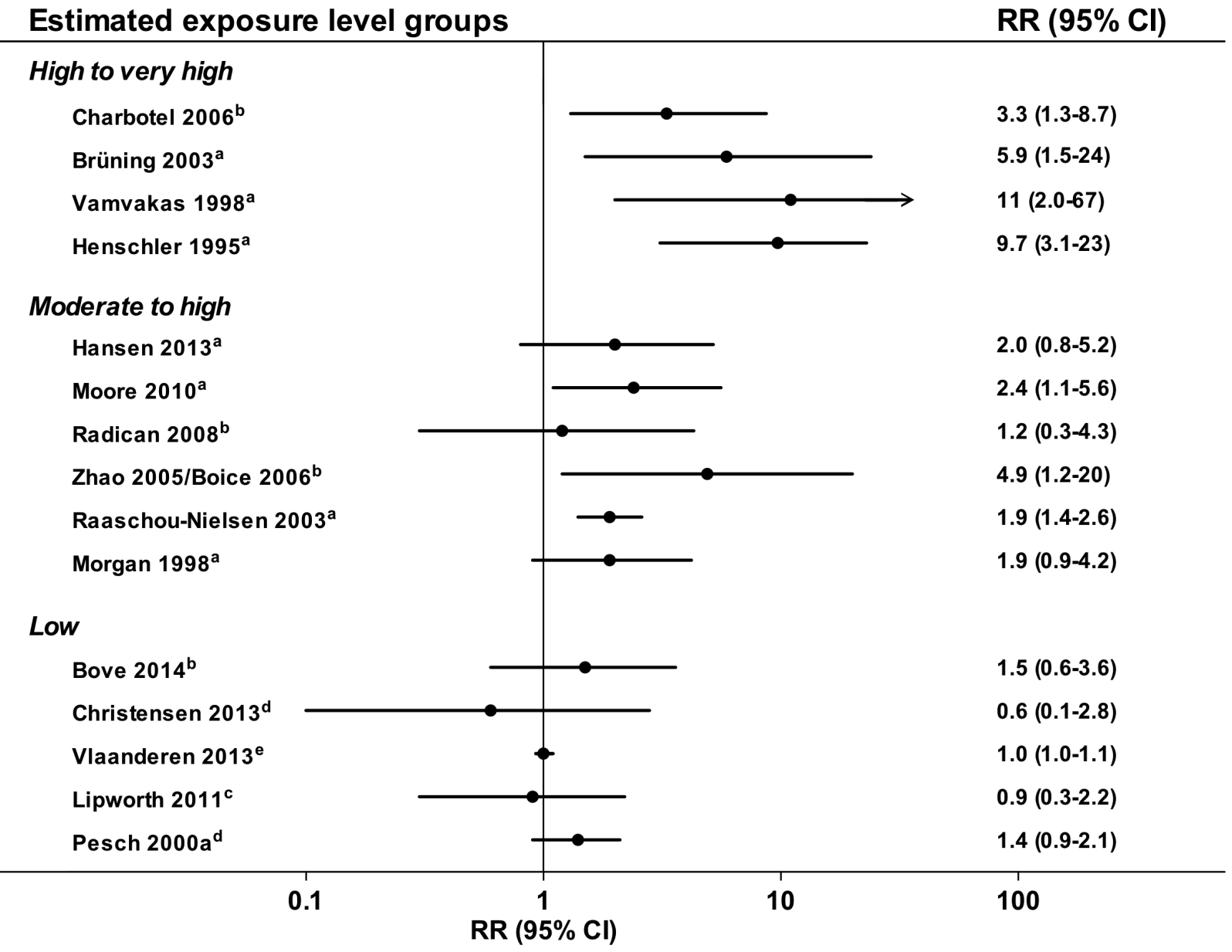

Figure 4-4. Forest Plot-3: Kidney Cancer and Estimated Exposure Level for Trichloroethylene

Effect estimate and 95\% CI for high exposure to trichloroethylene and kidney cancer and estimated exposure level (see Table D-5 and Table D-7) as described in Section 4.1.3. Studies by Greenland et al. (1994), Dosemeci et al. (1999), and Silver et al. (2014) are not graphed because they did not report a risk estimate for high exposure. Findings for these studies are reported in Table 4-1 and Figure 4-2. The effect estimate for Pesch et al. (2000b) was reported by Scott and Jinot (2011).

Different metrics of exposure were graphed and are as follows:

${ }^{a}$ Exposure intensity.

${ }^{\mathrm{b}}$ Cumulative exposure.

${ }^{\mathrm{c}}$ Exposure duration.

${ }^{\mathrm{d} C a t e g o r i e s ~ i n c l u d i n g ~ c o n f i d e n c e ~ o f ~ p r o b a b i l i t y ~ o f ~ e x p o s u r e ~ w i t h ~ l e v e l ~ a n d / o r ~ d u r a t i o n . ~}$

${ }^{\mathrm{e}} \mathrm{Cumulative}$ exposure measures that included exposure prevalence.

The database was inadequate to evaluate the effect of latency, as few cohort or case-control studies conducted lagged vs. unlagged or time since first exposure analyses and data are generally sparse due to limited numbers of cases. SIRs for kidney cancer increased with increasing lagging time in the study of blue-collar workers (Raaschou-Nielsen et al. 2003) and were slightly higher $(10 \%)$ in the 20-year lagged analysis compared with the 10-year lagged and unlagged analyses in the study of biomonitored workers (Hansen et al. 2013). However, no differences in effect estimates after lagging by varying periods of between approximately 0 and $\geq 20$ years were reported in other studies (Brüning et al. 2003; Moore et al. 2010; Vlaanderen et al. 2013; Zhao et al. 2005). The rest of the studies did not conduct lagged analysis (Boice et al. 2006; Charbotel et al. 2006; Charbotel et al. 2009; Christensen et al. 2013; Dosemeci et al. 1999; 
Henschler et al. 1995; Lipworth et al. 2011; Morgan et al. 1998; Pesch et al. 2000b; Radican et al. 2008) or only reported effect estimates for one lagging period (Bove et al. 2014; Greenland et al. 1994) and Silver et al. (2014).

The findings across studies are unlikely to be explained by biases. Although selection bias cannot be ruled out in the studies by Henschler et al. (1995) and Vamvakas et al. (1998), these studies were not included in the meta-analyses, and thus do not affect the overall conclusion. Confounding from smoking and other lifestyle factors can also be reasonably ruled out across studies. Increased risks were found in case-control studies, which adjusted for these factors. Almost all the cohort studies conducted internal analyses, which can mitigate concerns about lifestyle factors, and the lack of an association with exposure to trichloroethylene and lung cancer in these studies argues against confounding by smoking, which is not strongly associated with renal cancer. Potential selection bias and confounding from smoking in the study of bluecollar workers does not explain all of the excess risk of kidney cancer associated with trichloroethylene exposure. Although information on occupational co-exposures is missing in most of the studies, the identified co-exposures were neither known nor suspected renal carcinogens. In addition, some of the positive studies found increased risks after controlling for (primarily mineral oils) or considering exposure to known occupational co-exposures; coexposures were not likely to confound the German studies (Brüning et al. 2003; Henschler et al. 1995; Vamvakas et al. 1998). Most of the other positive studies were from diverse industries with varying levels and patterns of co-exposures. Exposure to chlorinated solvents other than trichloroethylene and mineral oils may be the most common exposures across industries, and these are not known or suspected renal carcinogens. Thus, no identified risk factors for renal cancer are likely to explain the increased risks found in these studies.

\subsection{Mechanistic Data for Kidney Carcinogenicity}

EPA (2011a; 2011b) and IARC (2014) recently reviewed the mechanistic data for trichloroethylene. The findings from these reviews and other mechanistic data are presented here. Relevant primary literature is cited if the study was not included in these reviews, or if specific data or further details of the study were needed for clarification.

\subsubsection{Hypothesized Modes of Action}

Hypothesized modes of action for trichloroethylene-induced kidney carcinogenicity include key events attributed to GSH-conjugation-derived metabolites (genotoxicity and cytotoxicity) and those attributed to oxidative metabolites (peroxisome proliferation activated receptor $\alpha(\operatorname{PPAR} \alpha)$ activation, $\alpha_{2 u}$-globulin-related nephropathy, and formic acid-related nephrotoxicity) (USEPA 2011a). The key events associated with each of these hypothesized modes of action are listed in Table 4-4. Modes of action associated with GSH-derived metabolites are discussed in Section 4.2.2 while those associated with oxidative metabolites are discussed in Section 4.2.3. 
Table 4-4. Hypothesized Modes of Action and Key Events for Kidney Tumors

\begin{tabular}{|c|c|}
\hline Mode of Action & Key Events \\
\hline Genotoxicity & $\begin{array}{l}\text { 1. GSH-conjugation-derived metabolites produced in situ or delivered systemically to } \\
\text { kidney } \\
\text { 2. Genotoxic effects induced by metabolites in kidney (e.g., mutations, DNA damage, } \\
\text { DNA strand breaks, micronuclei) that advance acquisition of multiple critical traits } \\
\text { contributing to carcinogenesis }\end{array}$ \\
\hline $\begin{array}{l}\text { Cytotoxicity and } \\
\text { regenerative } \\
\text { proliferation }\end{array}$ & $\begin{array}{l}\text { 1. GSH-conjugation-derived metabolites produced in situ or delivered systemically to } \\
\text { kidney } \\
\text { 2. Cytotoxicity and compensatory cell proliferation } \\
\text { 3. Clonal expansion of initiated cells }\end{array}$ \\
\hline $\operatorname{PPAR} \alpha$ activation & $\begin{array}{l}\text { 1. Oxidative metabolites produced in the liver activate PPAR } \alpha \text { in the kidney } \\
\text { 2. Alterations in cell proliferation and apoptosis } \\
\text { 3. Clonal expansion of initiated cells }\end{array}$ \\
\hline $\begin{array}{l}\alpha_{2 u}-\text { Globulin-related } \\
\text { nephropathy (relevant } \\
\text { only in male rats) }\end{array}$ & $\begin{array}{l}\text { 1. Oxidative metabolites cause hyaline droplet accumulation and an increase in } \alpha_{2 u^{-}} \\
\text {globulin resulting in nephrotoxicity } \\
\text { 2. Subsequent cytotoxicity, necrosis, and sustained regenerative tubule-cell } \\
\text { proliferation } \\
\text { 3. Development of intraluminal granular casts from sloughed cellular debris associated } \\
\text { with tubule dilation and papillary mineralization } \\
\text { 4. Foci of tubule hyperplasia in the convoluted proximal tubules } \\
\text { 5. Renal tubule tumors }\end{array}$ \\
\hline $\begin{array}{l}\text { Formic acid-related } \\
\text { nephropathy }\end{array}$ & $\begin{array}{l}\text { 1. Oxidative metabolites produced in the liver lead to increased formation and urinary } \\
\text { 2. Incretion of formic acid } \\
\text { 3. Compensatory cell proliferation } \\
\text { 4. Clonal expansion of initiated cells }\end{array}$ \\
\hline
\end{tabular}

Source: Adapted from EPA (2011a).

\subsubsection{The Proposed Role of GSH-conjugation-derived Metabolites in Kidney Carcinogenicity}

The toxicology of trichloroethylene has been extensively studied, and the data indicate that metabolites are responsible for most of the toxic effects. As discussed in Section 1.2, trichloroethylene is metabolized in the liver by two separate pathways, cytochrome P450dependent oxidation (Figure 1-1) and GSH conjugation (Figure 1-2). These metabolic pathways act in parallel and may compete for trichloroethylene as a substrate, thus, factors that affect the relative flux of trichloroethylene through each pathway (e.g., metabolic saturation, polymorphisms, enzyme induction/inhibition) can alter the toxic response. Both oxidative (trichloroethanol and trichloroacetic acid) and GSH-conjugation-derived metabolites (DCVG, DCVC, and related metabolites) have been associated with various nephrotoxic effects; however, the experimental data indicate that metabolites derived from the GSH-conjugation pathway are more important for nephrotoxicity. The role of oxidative metabolites, if any, is comparatively small.

The proposed key events for kidney carcinogenicity include (1) GSH-conjugation-derived metabolites produced in situ or delivered systemically to the kidneys, and (2) mutagenic, genotoxic (see Section 4.2.2.1) and cytotoxic effects (see Section 4.2.2.2) induced by these metabolites in the kidneys advance the acquisition of multiple critical traits contributing to carcinogenesis (USEPA 2011a). 
Disposition and toxicokinetic data (reviewed in Section 1) show that metabolites from the GSHconjugation pathway are formed in the liver and kidneys and that flux through the GSH pathway is more substantial than previous estimates based on urinary metabolites indicated. Metabolites formed in the liver are delivered to the kidneys through the systemic circulation. In vitro studies using liver and kidney cells from humans and rodents and subcellular fractions incubated with trichloroethylene also have shown formation of GSH-derived metabolites. These metabolites include DCVG, DCVC, NAcDCVC, and other metabolites derived from subsequent $\beta$-lyase, flavin-containing monooxygenase 3 (FMO3), or CYP3A metabolism within the liver or kidneys (see Section 1.2.2, Figure 1-2). The data also show that levels of some of the metabolites (e.g., NAcDCVC) may accumulate in the kidney due to both in situ production and systemic delivery from the liver.

Studies in humans provide support for the importance of the GSH-conjugation pathway in renal cancer development. Three studies, using different types of analyses (or statistical analyses), specifically investigated GST polymorphisms and renal-cell cancer among humans exposed to trichloroethylene (Brüning et al. 1997a; Moore et al. 2010; Wiesenhutter et al. 2007). The Central and Eastern European case-control study by Moore et al. (2010) (see Sections 3 and 4.1) was considered to be the most informative study to evaluate potential effect modification of GST genotypes and trichloroethylene exposure because of a large number of exposed kidney cancer cases $(1,097)$ and controls $(1,476)$, study design (calculated ORs for trichloroethylene exposure stratified by GSTT1 genotype), and evaluation of exposure-response relationships. Positive associations $\left(p_{\text {trend }}<0.05\right)$ with kidney cancer were found for all trichloroethylene exposure metrics (any, duration, average, and cumulative exposure) among subjects with GSTT1 active genotypes but not among subjects with two deleted alleles (null genotype) (see Table 4-2). Moore et al. also found statistically significant interaction between trichloroethylene exposure (ever versus never) and minor alleles in single nucleotide polymorphisms (SNPs) spanning the renal cysteine $\beta$-lyase $(C C B L 1)$ gene region. Thus, the findings of Moore et al. (2010) are consistent with the hypothesis that genes involved in the GSH-conjugation pathway are involved in trichloroethylene-induced renal cancer.

The other two studies had limited methods for evaluating potential effect modification. Brüning et al. (1997a) reported that having a GSTT1 or GSTM1 active genotype increased the risk of renal cancer in a small study of cases and controls, all of whom had been exposed to high concentrations of trichloroethylene, from a highly industrial region in Germany (see Section 3 for a description of occupational exposure in the Arnsberg area of Germany). EPA (2011a) noted that the frequency of GSTM1 in the controls was lower than that of background European populations. In a later study, using cases and controls from the hospital-based case-control study by Brüning et al. (2003) (see Sections 3 and 4.1), and an additional control group, Wiesenhutter et al. (2007) reported that frequency of GSTT1, GSTM1, and NAT1 polymorphisms was similar among cases and controls, and among trichloroethylene-exposed cases and non-exposed cases. Genotype distribution in exposed controls versus non-exposed controls was not reported and there appear to be some errors in the reporting of the frequency of the GSTT1 genotypes of exposed and non-exposed cases.

\subsubsection{Genotoxicity}

Genotoxicity is a well-established cause of carcinogenicity. Although trichloroethylene was not mutagenic without metabolic activation in most standard bacterial assays, GSH-conjugation 
pathway-derived metabolites are genotoxic (see Section 2). Although there are some data limitations, the available evidence indicates that DCVC is a more potent mutagen than any of the oxidative metabolites (Moore and Harrington-Brock 2000). Positive genotoxicity data for GSHderived metabolites were reported (primarily from in vitro assays). DCVG, DCVC, and NAcDCVC were mutagenic in the Ames test, and kidney-specific genotoxic effects also were reported (IARC 2014; USEPA 2011a). DCVC and DCVG were direct-acting mutagens in some strains of $S$. typhimurium. Furthermore, the use of $\beta$-lyase inhibitors or kidney subcellular fractions for metabolic activation supported the importance of in situ metabolism in the genotoxicity of these metabolites in the kidney. DCVC induced dose-dependent increases in unscheduled DNA synthesis in porcine kidney tubular epithelial cells and Syrian hamster embryo fibroblasts. Other genotoxic effects of DCVC included DNA strand breaks in the kidneys of rats and rabbits (oral exposure), and micronuclei in primary kidney cells from humans and rats. A single study in Eker rats, which are prone to the development of renal tumors, showed no increase in tumor incidence or in VHL mutations in trichloroethylene-exposed animals compared with controls (Mally et al. 2006).

Doses used in the in vitro assays were generally in the sub-nmol to nmol range for the Ames assay and in the $\mu \mathrm{M}$ to $\mathrm{mM}$ range with mammalian cells. Thus, many of the in vitro assays used concentrations higher than those observed in vivo. Lash et al. (1999b) reported maximum DCVG levels of approximately $110 \mathrm{nmol} / \mathrm{mL}(0.11 \mu \mathrm{M})$ in the blood of human volunteers exposed to trichloroethylene vapors $(100 \mathrm{ppm})$ for 4 hours. DCVG concentrations were not measured in tissues but would likely have been higher in the kidney due to in situ metabolism and a trichloroethylene tissue:blood partition coefficient $>1$ (see Section 1.1.1). The available in vivo data do show some genotoxic effects in target tissues (likely resulting from GSH-conjugationderived metabolites and including micronuclei and DNA single-strand breaks in the kidney) in rodents exposed to trichloroethylene. Other studies in rodents show that sufficient DCVC is formed in vivo from trichloroethylene metabolism to account for histological changes in the renal tubules (USEPA 2011a).

Only one study was identified that investigated the genotoxic effects of trichloroethylene in the mouse kidney (Douglas et al. 1999). Mutations were not increased in the kidney of lacZ transgenic mice exposed to trichloroethylene vapors for 12 days (USEPA 2011a). However, these results are not highly informative as to the role of mutagenicity in trichloroethyleneinduced kidney tumors given the uncertainties of the mouse model in the production of genotoxic GSH-conjugation-derived metabolites and the low carcinogenic potency of trichloroethylene in the mouse kidney. Although renal tumors were not increased in mice, this is not an unusual finding compared with results for other genotoxic kidney carcinogens. Five of seven directacting genotoxic carcinogens also induced kidney tumors in rats but not in mice. Since kidney tumors are rare in rodents and given that the incidence of kidney tumors was low in rats, it is not unreasonable that a small difference in potency in mice compared with rats would not be detected in chronic bioassays. Toxicokinetic data (see Section 1.3.2 and Appendix B) did not indicate that GSH conjugation and subsequent renal metabolism were lower in mice compared with rats; however, there is substantial uncertainty in the total flux through this pathway. Therefore, the lack of a detectable response in mice does not rule out a genotoxic mode of action.

Inactivation of the $V H L$ tumor suppressor gene from base-change mutations, silencing, or small deletions is thought to be an early and causative event in human renal clear-cell carcinomas (USEPA 2011a). Mutations in the VHL gene from exposure to trichloroethylene were evaluated 
in four case-control studies (Brauch et al. 1999; Brauch et al. 2004; Brüning et al. 1997b; Charbotel et al. 2007) and one case-series study (Moore et al. 2011) of renal-cell carcinomas (Table 4-5). Moore et al. (2011) reported that VHL inactivation, either through genetic alterations or promoter methylation in tumor DNA, occurred in more than $86 \%$ of the 470 sporadic clearcell renal cancer cases examined. In addition, some researchers have reported differences between trichloroethylene-exposed and nonexposed renal-cell carcinoma patients in the frequency of somatic mutations in the VHL gene (Brauch et al. 1999; Brauch et al. 2004; Brüning et al. 1997b). The two studies by Brauch et al. reported multiple mutations in the VHL gene and increased frequencies with trichloroethylene exposure. Additionally, Brauch et al. (2004) reported that trichloroethylene-exposed patients were diagnosed with renal-cell carcinoma at a younger age than non-exposed patients. Brauch et al. (1999) reported that 39\% of clear-cell renal carcinomas from trichloroethylene-exposed individuals contained a hot-spot mutation $(\mathrm{C}$ to $\mathrm{T}$ transition) in the $V H L$ gene at nucleotide 463 that caused a substitution of serine for proline at amino acid 81 (P81S). Overall, VHL mutations were found in about $75 \%$ of the exposed patients and there was an association between the number of mutations and the severity of trichloroethylene exposure.

$V H L$ mutations also were frequently accompanied by loss of heterozygosity. However, other researchers have not found a higher incidence of $V H L$ mutations in trichloroethylene-exposed patients with renal clear-cell carcinomas compared with nonexposed patients (Charbotel et al. 2007). The Moore et al. (2011) study reported that most of the renal-cell carcinomas were clearcell renal carcinoma, while $<10 \%$ of the cancers were non-clear-cell renal carcinoma. One study was unable to assess a change in mutations from trichloroethylene exposure, because no unexposed control was included (Brüning et al. 1997b). Of those cases with mutations in the VHL gene, mutations occurred more frequently in exon 1 (Brauch et al. 1999; Moore et al. 2011), exon 2 (Brüning et al. 1997b), and nucleotide 454, which is considered a hotspot (Brauch et al. 1999; Brauch et al. 2004). DeSimone et al. (2013) compared the activity of the trichloroethyleneassociated P81S VHL mutation with cells expressing normal VHL and another VHL mutant (R167Q). Their data indicated that the P81S VHL mutation initiated pleiotropic effects that selectively influenced tumor behavior in a mutation-specific manner. These effects provided a selective growth advantage through metabolic pathway diversification, suppression of apoptosis, and alteration of DNA damage response.

Further data are needed to determine the validity of $V H L$ mutations as a legitimate biomarker for trichloroethylene-induced renal tumors (USEPA 2011a). If valid, these data suggest that a specific mutational spectrum might be associated with trichloroethylene-induced kidney tumors and adds biological plausibility for a mutagenic mode of action. There are currently no data to determine if there is a possible link between trichloroethylene metabolites and these events. Recent studies do suggest that multiple genes are involved in renal clear-cell carcinoma; therefore, the inconsistent results with respect to $V H L$ mutations do not constitute negative evidence for a mutagenic mode of action. Overall, the data clearly show that human and rodent kidneys are exposed to GSH-conjugation-derived metabolites following exposure to trichloroethylene and that these metabolites are capable of causing genetic damage. Thus, the data are sufficient to conclude that a mutagenic mode of action is likely operative in trichloroethylene-induced kidney tumors. 
Table 4-5. Studies of $V H L$ Mutation in Trichloroethylene-exposed Human Subjects with Renal-cell Carcinoma

\begin{tabular}{|c|c|c|c|c|}
\hline $\begin{array}{l}\text { Reference } \\
\text { Country }\end{array}$ & $\begin{array}{l}\text { Study Type } \\
\text { Population }\end{array}$ & $\begin{array}{c}\text { Exposure Estimate } \\
\text { Method }\end{array}$ & $\begin{array}{c}\text { Findings } \\
\text { Incidences }\end{array}$ & Comments \\
\hline $\begin{array}{l}\text { Brüning et al. } \\
\text { (1997a) } \\
\text { Germany }\end{array}$ & $\begin{array}{l}\text { Case-control } \\
23 \text { exposed }\end{array}$ & $\begin{array}{l}\text { Semi-quantitative } \\
\text { Work history and acute exposure } \\
\text { symptoms }\end{array}$ & $\begin{array}{l}\text { Unable to assess mutation differences } \\
23 / 23 \text { mutated in exposed } \\
30 \% \text { in exon } 1 \\
44 \% \text { in exon } 2 \\
26 \% \text { in exon } 3\end{array}$ & $\begin{array}{l}\text { No unexposed controls or } \\
\text { increase in mutations with higher } \\
\text { exposure }\end{array}$ \\
\hline $\begin{array}{l}\text { Brauch et al. (1999) } \\
\text { Germany }\end{array}$ & $\begin{array}{l}\text { Case-control } \\
44 \text { exposed } \\
107 \text { controls }\end{array}$ & $\begin{array}{l}\text { Low/medium/high } \\
\text { Occupational hygienist }\end{array}$ & $\begin{array}{l}\text { Increased mutations } \\
33 / 44 \text { mutated in exposed } \\
54 \% \text { in exon } 1 \\
39 \% \text { at nucleotide } 454 \\
32 \% \text { of mutations were multiple mutations } \\
42 / 73 \text { mutated in controls } \\
0 \% \text { of mutations were multiple mutations }\end{array}$ & $\begin{array}{l}\text { Number of mutations increased } \\
\text { with higher levels of estimated } \\
\text { exposure }\end{array}$ \\
\hline $\begin{array}{l}\text { Brauch et al. (2004) } \\
\text { Germany }\end{array}$ & $\begin{array}{l}\text { Case-control } \\
17 \text { (exposed) } \\
21 \text { (unexposed) }\end{array}$ & $\begin{array}{l}\text { Low/medium/high } \\
\text { Occupational hygienist }\end{array}$ & $\begin{array}{l}\text { Increased mutations } \\
14 / 17 \text { mutated in exposed } \\
39 \% \text { at nucleotide } 454 \\
50 \% \text { of mutations were multiple mutations } \\
2 / 21 \text { mutated in controls }\end{array}$ & $\begin{array}{l}\text { RCC cases exposed or } \\
\text { unexposed to trichloroethylene } \\
\text { Exposure decreased the age of } \\
\text { diagnosis }\end{array}$ \\
\hline $\begin{array}{l}\text { Charbotel et al. } \\
(2007) \\
\text { France }\end{array}$ & $\begin{array}{l}\text { Case-control } \\
69 \text { cases of RCC }\end{array}$ & $\begin{array}{l}\text { Low/medium/high } \\
\text { Expert-evaluated questionnaire }\end{array}$ & $\begin{array}{l}\text { No mutation differences } \\
2 / 23 \text { mutated in exposed } \\
2 / 25 \text { mutated in controls }\end{array}$ & $\begin{array}{l}\text { Low rate of mutation and no } \\
\text { difference in mutations with } \\
\text { exposure } \\
\text { Potential for exposure } \\
\text { misclassification }\end{array}$ \\
\hline $\begin{array}{l}\text { Moore et al. (2011) } \\
\text { Europe }\end{array}$ & $\begin{array}{l}\text { Case-series } \\
470 \text { sporadic clear } \\
\text { cell RCC cases }\end{array}$ & $\begin{array}{l}\text { Levels of exposure not reported } \\
\text { Expert interview }\end{array}$ & $\begin{array}{l}\text { No mutation differences } \\
415 / 470 \text { mutated in clear-cell renal carcinoma } \\
37 \% \text { in exon } 1 \\
30 \% \text { in exon } 2 \\
26 \% \text { in exon } 3\end{array}$ & $\begin{array}{l}\text { Level of exposure not reported. } \\
\text { Only } 1 \text { unexposed case had } \\
\text { mutation at nucleotide } 454 \\
\text { Non-clear-cell renal carcinoma } \\
\text { were }<10 \% \text { of RCC }\end{array}$ \\
\hline
\end{tabular}

Source: IARC (2014).

$\mathrm{RCC}=$ renal-cell carcinoma . 


\subsubsection{Cytotoxicity and Regenerative Proliferation}

The key events for cytotoxicity and regenerative proliferation are: (1) formation of cytotoxic GSH-conjugated metabolites of trichloroethylene, which are either formed within the kidney or delivered systemically to the kidney, (2) nephrotoxicity leading to compensatory cellular proliferation and an increased mutation rate, and (3) tumor formation through clonal expansion of initiated cells. Although the available data currently are insufficient to establish a causal link between trichloroethylene-induced nephrotoxicity and sustained regenerative cellular proliferation and carcinogenicity, there is substantial evidence that trichloroethylene and/or its metabolites are nephrotoxic (USEPA 2011a). The experimental evidence includes the following: (1) increased urinary excretion of nephrotoxicity markers in humans (especially evident from chronic occupational exposure to high concentrations), (2) high incidences of proximal tubule cytomegaly and toxic nephropathy only in dosed male and female rats from five strains in chronic bioassays, (3) high incidences of proximal tubule cytomegaly only in dosed male and female mice in a chronic bioassay, (4) kidney toxicity in rodents exposed to DCVC and other GSH-conjugation-derived metabolites, (5) toxicokinetic data showing that DCVC is formed in the kidney following exposure to trichloroethylene, and (6) data that demonstrate that nephrotoxic metabolites formed in the liver are delivered through the systemic circulation to the kidney.

Nephrotoxic trichloroethylene metabolites derived from the GSH-conjugation pathway are formed in the kidney and also are delivered from the liver to the kidney via the systemic circulation (Irving and Elfarra 2012). Some nephrotoxic effects also have been reported for trichloroethanol and trichloroacetic acid (oxidative metabolites of trichloroethylene) in rats. Chronic exposure to trichloroethanol caused tubular degeneration in rats but there was no evidence of karyomegaly or cytomegaly (Green et al. 2003; USEPA 2011a). Overall, trichloroethanol did not induce the same pathology as trichloroethylene or DCVC.

Trichloroacetic acid administered to rats caused an increase in the kidney-weight to body-weight ratio but did not cause histopathologic changes in the kidney. However, trichloroacetic acid has been associated with peroxisomal proliferation in the kidney (discussed below). Both trichloroethanol and trichloroacetic acid may contribute to trichloroethylene-induced nephrotoxicity through formic acid formation (discussed below), but the overall contribution is likely very small compared with the GSH-conjugation-derived metabolites (USEPA 2011a).

Urinary biomarkers of early renal dysfunction include glutathione-S-transferase $\alpha$, glutathione-Stransferase $\pi, \beta_{2}$-microglobulin, $\alpha_{1}$-microglobulin, retinol binding protein, $\mathrm{N}$ acetylglucosaminidase (NAG), kidney injury molecule-1, albumin, and total protein (Green et al. 2004; Vermeulen et al. 2012). Several studies have reported an increase in urinary markers of proximal tubule injury in workers repeatedly exposed to high concentrations of trichloroethylene over an extended period (Bolt et al. 2004; Brüning et al. 1999a; Brüning et al. 1999b) or following acute intoxication (Brüning et al. 1998). Peak exposures were estimated to have frequently exceeded $500 \mathrm{ppm}$ based on reported narcotic symptoms (drunkenness, dizziness, headache, and drowsiness). The workers also reported that they frequently had to leave the work area to recover in fresh air. Two of these studies also reported that there were significantly more cases of tubular damage (measured by increased $\alpha_{1}$-microglobulin in the urine) among renal-cell carcinoma patients exposed to high levels of trichloroethylene over many years compared with nonexposed patients with renal-cell carcinoma or exposed controls (Bolt et al. 2004; Brüning et al. 1999a). Vermeulen et al. (2012) investigated nephrotoxicity among 80 Chinese factory 
workers (mean duration of employment 2 years) exposed to trichloroethylene concentrations (22.2 ppm \pm 35.9$)$ below the Occupational Safety and Health Administration permissible exposure limit of 100 ppm (8-h TWA). Urinary levels of kidney injury molecule-1 were significantly elevated in exposed workers compared with controls and an increase in glutathioneS-transferase $\pi$ was borderline statistically significant. Other markers of kidney toxicity (NAG and glutathione-S-transferase $\alpha$ ) were not significantly different. This was the first study to show that relatively low occupational exposures to trichloroethylene could induce kidney toxicity.

An earlier study by Green et al. (2004) did not find evidence of exposure-related kidney damage in 70 workers exposed to relatively low trichloroethylene concentrations (mean $=32 \mathrm{ppm}$, range $=0.5$ to $252 \mathrm{ppm}$ ). There was a significant dose-dependent increase in urinary glutathioneS-transferase $\alpha$ activity; however, the levels were not significantly increased compared with controls. Although NAG and albumin levels were significantly higher in the exposed workers compared with controls, the levels of these markers were not correlated with either the magnitude or duration of exposure and could be explained by chance or by exposure to some unidentified agent. In vitro studies with primary cultures of human proximal tubular cells show that DCVC caused necrosis at high concentrations $(>100 \mu \mathrm{M})$ and increased cell proliferation and apoptosis at lower concentrations (Lash et al. 2005). These effects were associated with changes in expression of proteins that regulate apoptosis, cellular growth, differentiation, and stress response. A study by $\mathrm{Xu}$ et al. (2008) indicated that mitochondrial dysfunction was an early, obligatory step in DCVC-induced cytotoxicity in cultured human proximal tubular cells. Overall, the data support the hypothesis that chronic tubular damage is a precondition for the nephrocarcinogenic effects of trichloroethylene in humans.

DCVC was nephrotoxic in rats, mice, guinea pigs, rabbits, cats, and dogs (USEPA 2011a). DCVC may be metabolized to other nephrotoxic metabolites by FMO3, $\beta$-lyase, or NAT (see Figure 1-2). Mice appear to be more sensitive to the acute nephrotoxic effects than rats but are less susceptible to renal carcinogenesis. Studies reviewed by EPA (2011a) reported that mice administered a single dose of $1 \mathrm{mg} / \mathrm{kg}$ DCVC developed proximal tubule cell damage, and karyomegaly was noted following repeat doses of $1 \mathrm{mg} / \mathrm{kg} /$ day for 10 days. Higher doses in mice resulted in more severe damage including desquamation and necrosis of the tubular epithelium. In rats, no histological changes were observed following single doses up to $10 \mathrm{mg} / \mathrm{kg}$ or 10 daily doses of 0.5 to $5 \mathrm{mg} / \mathrm{kg}$. Single doses in rats of $25 \mathrm{mg} / \mathrm{kg}$ or $50 \mathrm{mg} / \mathrm{kg}$ resulted in cellular debris in the tubular lumen and slight degeneration and necrosis, respectively. Irving et al. (2013) investigated the nephrotoxicity of NAcDCVCS, NAcDCVC, and DCVCS in male SpragueDawley rats following a single i.p. injection $(230 \mu \mathrm{mol} / \mathrm{kg}$ b.w.). Nephrotoxic effects occurred at 24 hours post treatment for all three compounds. NAcDCVCS and NAcDCVC had similar effects causing necrosis in the proximal tubules in the outer medulla and adjacent inner cortex but were less nephrotoxic than DCVCS on an equimolar basis. DCVCS caused acute proximal tubular necrosis in the cortex but not in the medulla. Based on a comparison of kidney lesions of rats dosed with trichloroethylene or DCVC, the data suggest that these compounds also may play a role in trichloroethylene-induced nephrotoxicity.

Subchronic and chronic studies in rats and mice exposed to DCVC via drinking water consistently report pathological and histological effects in the kidney and show a progression from tubular necrosis and shedding of pyknotic cells into the lumen during the first few days to increased prominence of karyomegaly and cytomegaly in tubular cells after several weeks of exposure (USEPA 2011a). Effects were noted at doses as low as 1 to $2 \mathrm{mg} / \mathrm{kg} / \mathrm{day}$ and did not 
show a difference in sensitivity between rats and mice. In a recent study, Shirai et al. (2012) administered DCVC to male BALB/c mice orally or by i.p. injection for 13 weeks at 1,10 , and $30 \mathrm{mg} / \mathrm{kg} /$ day. Dose-related effects in the kidney were reported that progressed from weak tubular dilation, but no necrosis or fibrosis, at the low dose to renal tubular degeneration characterized by moderate tubular necrosis and marked interstitial fibrosis at the high dose.

The histological and morphological changes in the tubular cells observed in studies with DCVC were similar to those reported in chronic studies with trichloroethylene (NTP 1988; 1990). The NTP studies were conducted with five rat strains and one mouse strain and reported high incidences of cytomegaly of the proximal tubules (82\% to $100 \%)$ in dosed groups of males and females of all strains and species. Cytomegaly was more severe in male rats than female rats and more severe in rats than in mice, but it was not observed in the unexposed control or vehicle control groups. In addition, NTP (1988) reported high incidences of toxic nephropathy (17\% to $80 \%$ ) only in dosed rats from four strains that was not related to the common spontaneous nephropathy of aging rats. Lash et al. (1998) reported that the greater sensitivity of trichloroethylene-induced kidney toxicity in male rats compared with females was correlated with the rate of DCVG formation. However, species-dependent differences in nephrotoxicity and carcinogenicity between rats and mice were not correlated with rates of DCVG formation and suggested that other enzymes (e.g., $\beta$-lyase, NAT, GGT, or deacetylase) may be responsible for the lower susceptibility in mice.

Cytotoxicity alone is insufficient for tumor formation because all cytotoxins clearly are not carcinogenic. Further, nephrotoxicity occurred at much lower doses and was observed at near $100 \%$ incidences in all dose groups while renal tumors occurred only in rats in the high-dose group (NTP 1988; 1990). Multiple factors may contribute to cytotoxicity including oxidative stress, alterations in calcium ion homeostasis, mitochondrial dysfunction, protein alkylation, cellular repair processes, and alterations in gene expression (Lash et al. 2000b). Each of these factors may have ancillary consequences related to tumor induction that are independent of cytotoxicity per se (USEPA 2011a). El Arem et al. (2014a); El Arem et al. (2014b) reported that dichloroacetic acid and trichloroacetic acid were nephrotoxic in rats and that the kidney damage could be prevented by antioxidants. However, it is not known whether cytotoxicity is causally related to carcinogenesis or is merely a marker for a different, key causal event. Although experimental data currently do not demonstrate a causal link between nephrotoxicity/sustained cellular proliferation and renal tumors, the data are consistent with the hypothesis that cytotoxicity and regenerative proliferation contribute to trichloroethylene-induced kidney tumors, either independently or in combination with a mutagenic mode of action. The more biologically plausible mode of action likely involves a combination of mutagenicity and cytotoxicity. That is, DNA adducts in combination with toxic doses of trichloroethylene could lead to sustained regenerative cellular proliferation that promotes the selection, survival, and clonal expansion of mutated cells in the tubular epithelium.

\subsubsection{Proposed Modes of Action Associated with Oxidative Metabolites}

As mentioned above, several modes of action associated with oxidative metabolites have been proposed (PPAR $\alpha$ activation, $\alpha_{2 u}$-globulin-related nephropathy, and formic acid-related nephrotoxicity). These modes of action have little to no experimental support and are briefly reviewed below. 


\subsubsection{PPARa Activation}

Tubular epithelial cells are relatively rich in peroxisomes and trichloroacetic acid and dichloroacetic acid, oxidative metabolites of trichloroethylene, are PPAR $\alpha$ agonists (USEPA Lash et al. 2000b; Rusyn et al. 2014; 2011a). However, renal peroxisomes are generally less responsive than hepatic peroxisomes to peroxisome proliferators and humans are markedly less responsive to peroxisome proliferation than rodents. Only one study was identified that investigated peroxisome proliferation in kidneys of rats and mice exposed to trichloroethylene (Goldsworthy and Popp 1987). Trichloroethylene induced peroxisome proliferation in the liver and kidneys of rats and mice; however, similar levels were observed in both species. Thus, there was no correlation between induction of peroxisome proliferation in the kidneys and speciesspecific renal carcinogenicity. Another study investigated the role of trichloroacetic acid in carcinogenesis and peroxisome proliferation in liver and kidneys of rats and mice exposed to tetrachloroethylene, which can be metabolized to trichloroacetic acid (Odum et al. 1988). Due to differences in toxicokinetics, male mice were exposed to higher levels of trichloroacetic acid than male rats, and peroxisome proliferation was observed only in male mouse liver. The data did not support a role of trichloroacetic acid and peroxisome proliferation in the carcinogenicity of tetrachloroethylene in the male rat kidney. Although some metabolites of trichloroethylene are peroxisome proliferators, the available data are insufficient to support peroxisome proliferation as a mode of action for trichloroethylene-induced kidney tumors (USEPA 2011a).

\subsubsection{2. $\alpha_{2 u}$-Globulin-related Nephropathy}

$\alpha_{2 u}$-Globulin-related nephropathy is characterized by the rapid accumulation of protein droplets containing $\alpha_{2 u}$-globulin (hyaline droplets) in lysosomes in the P2 segment of the proximal tubule and is specific to male rats (IARC 1999; Lash et al. 2000b; Swenberg and Lehman-McKeeman 1999). A number of chemicals, including several halogenated organic solvents, are known to cause hyaline droplet nephropathy. Goldsworthy et al. (1988) investigated $\alpha_{2 u}$-globulin nephropathy in both male and female F344 rats exposed to trichloroethylene, tetrachloroethylene, or pentachloroethane to determine if the effects were male rat specific. There was no increase in renal $\alpha_{2 u}$-globulin concentrations or cell replication in male or female rats exposed to trichloroethylene but some effects were consistent with $\alpha_{2 u}$-globulin nephropathy in male rats for the other compounds tested. Trichloroethylene nephrotoxicity has been observed in rats and mice of both sexes and in humans, and kidney tumor incidences were elevated (although not always statistically significant) in both male and female rats. Thus, the data do not support the hypothesis that $\alpha_{2 u}$-globulin nephropathy is a factor in trichloroethylene-induced kidney carcinogenesis in rats.

\subsubsection{Formic Acid-related Nephrotoxicity}

Some investigators have suggested that since the nephrotoxic metabolite DCVC is formed in very small amounts it fails to explain the male rat specific renal carcinogenicity of trichloroethylene and have proposed that trichloroethylene nephrotoxicity may be caused by formic acid (Green et al. 1998; Green et al. 2003). The sequence of events for formic acid-related nephropathy is the same as for cytotoxicity induced by GSH-conjugation-derived metabolites discussed above but is related to oxidative metabolites (trichloroacetic acid and trichloroethanol). Formic acid is not a metabolite of trichloroethylene but may accumulate as an indirect consequence of vitamin $\mathrm{B}_{12}$ and folate depletion caused by trichloroethylene exposure (Dow and Green 2000). Male Fischer rats exposed to trichloroethylene, trichloroacetic acid, or trichloroethanol via gavage, drinking water, or inhalation for 1 day to 1 year excreted large 
amounts of formic acid in urine (Dow and Green 2000; Green et al. 1998; Green et al. 2003). No kidney damage was reported in rats following acute exposures (1 to 5 daily doses) or subacute exposures of 15 to 28 days (oral and inhalation). In contrast, male rats exposed to trichloroethanol at 500 to $1,000 \mathrm{mg} / \mathrm{L}$ for 52 weeks developed kidney damage characterized by increased urinary NAG, protein excretion, basophilic tubules, tubular damage, increased cell replication, and focal proliferation of abnormal tubules (Green et al. 2003). However, the characteristics of trichloroethanol-induced nephrotoxicity did not account for the full range of effects observed after exposure to trichloroethylene or DCVC (USEPA 2011a). Studies with trichloroacetic acid did not report histopathologic changes in the rat kidney. Yaqoob et al. (2013) also reported that male and female rats exposed to low doses of trichloroethylene for 3 days excreted formic acid in the urine but did not develop nephropathy. The induced formic aciduria was less pronounced in female rats and was less in male Wistar rats compared with male F344 rats. Yaqoob et al. (2014) compared the renal toxicity of trichloroethylene and trichloroethanol administered to male F-344 rats for 12 weeks to determine whether the GSH pathway or formic aciduria were responsible for nephrotoxicity. Although their findings did not clearly identify the pathway responsible for renal toxicity, the data provided some support for the GSH conjugation pathway.

Although rats chronically exposed to trichloroethanol excreted significantly larger amounts of formic acid and developed tubular degeneration, there were important dissimilarities in the characteristics of the nephrotoxicity compared with rats exposed to trichloroethylene or DCVC (USEPA 2011a). Histological changes associated with trichloroethylene and DCVC included karyomegaly, cytomegaly, and flattening and dilation of the tubular epithelium. These effects did not occur in rats exposed to trichloroethanol. Furthermore, no specific evidence links the particular nephrotoxic effects caused by trichloroethanol/formic acid to carcinogenesis. Thus, the data do not support the hypothesis that cytotoxicity mediated by oxidative metabolites via increased formic acid production is a major contributor to trichloroethylene-induced kidney carcinogenesis.

\subsubsection{Summary}

The mode of action for trichloroethylene-induced kidney cancer is not completely understood but the available data provide support for a mutagenic and cytotoxic mode of action mediated by GSH-conjugation-derived metabolites. There is experimental evidence that GSH metabolites (particularly DCVC) are genotoxic and nephrotoxic and are both formed in and delivered to the kidney following exposure to trichloroethylene. Factors that increase the proportion of trichloroethylene undergoing GSH conjugation (e.g., CYP enzyme inhibition or saturation, polymorphic expression of metabolizing enzymes) would be expected to increase kidney toxicity. Although there is some evidence that chronic tubular damage might be a precondition for the nephrocarcinogenic effects of trichloroethylene in humans, tubular toxicity has not been established as a necessary precursor or causal event for carcinogenesis. However, the data are consistent with the hypothesis that cytotoxicity and regenerative proliferation contribute to trichloroethylene-induced kidney tumors, most likely in combination with a mutagenic mode of action. Mutagenic and cytotoxic modes of action are relevant to humans. Other hypothesized modes of action for kidney carcinogenicity have inadequate or limited experimental support. 


\section{Non-Hodgkin Lymphoma (NHL)}

Previous sections of the cancer hazard evaluation component contain relevant informationADME (Section 1), genetic and related effects (Section 2), and overview and assessment of the quality of the human cancer studies (Section 3) - that are important for several of the three cancer endpoints of interest. This section builds on that information and evaluates the human cancer studies (Section 5.1) and mechanistic data (Section 5.2) specifically for non-Hodgkin lymphoma (NHL) and other related B-cell lymphohematopoietic cancers.

\subsection{Human Cancer Studies}

This review of NHL includes other B-cell lymphohematopoietic cancers thought to be related to NHL, including multiple myeloma (now renamed plasma-cell lymphoma), chronic lymphocytic leukemia (CLL), and hairy-cell leukemia (HCL). Other subtypes, such as diffuse large B-cell lymphoma (DLBCL) and follicular lymphoma, have also been studied in large cohorts.

NHL and its related subtypes are relatively uncommon, with NHL constituting about $4.3 \%$ of all new cancer cases per year in the United States. The U.S. age-adjusted incidence rate for NHL is approximately 24 and 16 cases per 100,000 per year in men and women, respectively (2007 to 2011 rates; SEER (2014b)) compared with approximately 8 and 5 per 100,000 deaths per year in men and women, respectively, due to a 70\% 5-year survival rate, an increase from approximately $46 \%$ in 1975. NHL rates in other European countries (see e.g., Clarke and Glaser (2002), Muller et al. (2005), Adamson et al. (2007), Ferlay et al. (2013); Ferlay et al. (2014)), from which the studies included in the evaluation are drawn, appear to be broadly similar, but with some variations. For example, U.K. age-standardized incidence rates (2011) are approximately 18 and 13 per 100,000 per year in men and women, respectively, with a similar 5-year survival rate of approximately 63\% (Cancer Research U.K. 2014a) although diagnosed incidence was approximately half that of the United States in 1975. Studies reporting incidence are generally more informative than mortality studies. The latencies of lymphohematopoietic cancers such as NHL are generally less than for solid tumors, but vary widely; they may be as low as 1 or 2 years in association with some exposures (Howard 2013). Incidence rates generally increase steeply after approximately 50 years of age.

Multiple myeloma is a rare cancer, constituting approximately $0.8 \%$ of all cancers. U.S. incidence and mortality rates for multiple myeloma are approximately 6 per 100,000 and 3.4 per 100,000 per year (2007 to 2011), respectively (SEER 2014a), again suggesting that the studies reporting incidence rates are more informative than those reporting only mortality rates. For CLL, incidence and mortality are approximately 16,000 cases and 4,600 deaths per year, respectively, in the United States, and the onset of disease increases markedly with age, with an average age at diagnosis of 72 years. (No comparable data for these specific subtypes was identified for the United Kingdom and other European countries.)

The incidence rate for NHL in Europe and the Nordic countries has roughly quadrupled from the 1950s to the late 1990s and doubled from the 1970s to the 1990s (Adamson et al. 2007) but has then stabilized in the past decade or more. A broadly similar pattern has been observed in the United States (Clarke and Glaser 2002), with increases in incidence in the United States now slowing to approximately $0.5 \%$ per year over the past decade (see U.S. SEER rates). However, 
no study has yet adequately examined to what extent observed changes in temporal trends are attributable to changes in classification systems, or diagnostic improvements or changes in registration methods rather than true changes in incidence (Adamson et al. 2007), particularly as variations in these trends are observed among different age and racial subgroups (e.g., Clarke and Glaser (2002)). As noted in Section 3, classification and coding systems for NHL and its subtypes have changed considerably over the past 20 years, so that comparisons of incidence rates across different studies conducted over different calendar periods should be interpreted with caution. In addition, earlier studies of NHL generally do not report subtypes, which do not reflect the histological and possibly biologically distinct heterogeneity of the disease (Clarke and Glaser 2002) and differences in rates and trends for subtypes, e.g., follicular lymphoma. In the available studies in the present evaluation, NHL was classified by ICD-7, 8, 9 or 10, ICD-O-2 or 3, or the InterLymph classification (Cocco et al. 2013), with some studies, using more recent classifications, reporting on B-cell lymphoma subtypes, primarily DLBCL, follicular lymphoma, multiple myeloma, CLL, or HCL.

Details on the study design, methods, and findings are available in Table D-1 and Table D-3. The evaluation of study quality, including study design, methods of exposure and cancer endpoint assessment, analyses and other elements related to the utility to inform the hazard evaluation (such as study sensitivity) is reported in Table D-4, Table D-5, Table D-8, and Table D-9) and discussed in Section 3. Figure 5-1 provides an overview of the conclusions from that evaluation and identifies the most informative studies based on the overall utility of the study. This section summarizes and interprets the findings for NHL and related B-cell lymphomas from the individual epidemiological studies brought forward for evaluation, and integrates the evidence across studies, applies the RoC listing criteria to the body of evidence, and reaches an NTP recommendation for the level of evidence for NHL and related B-cell lymphomas using the same criteria as described for the evaluation of kidney cancer in Section 4. 
High

Cocco 2013

Selection bias unlikely

Little concern for exposure or disease misclassification

Adequate evaluation of $\mathrm{E} / \mathrm{R}$

Adequate study sensitivity

Limited methods to consider potential confounding

Moderate
Selection bias unlikely
Some concern for exposure classification
Mortality or older disease classification systems
Evaluation of E/R
Limited study sensitivity
Limited methods to consider potential confounding
Low/moderate
Section bias unlikely or possible
Some concern for exposure misclassification
Mortality or older classifications system (except Wang)
Lower study sensitivity (except R-N)
Limited evaluation of E/R
Limited methods to consider potential confounders
(cohorts)
Low
Selection bias unlikely (except Bahr, Boice*)
Limited exposure assessment with considerable
concerns for exposure misclassification
Mortality or older classification systems
No evaluation of E/R (exceptVlaanderen, Bahr, Bove)
Low study sensitivity
Limited methods to consider potential confounders

Christensen 2013

Wang 2009

Raaschou-Nielsen (R-N) 2003

Lipworth 2011

Morgan 1998

\begin{tabular}{l} 
Silver 2014 \\
\hline Bove 2014 \\
Vlaanderen 2013 \\
\hline
\end{tabular}

Bahr 2011a

Persson \& Fredrikson 1999a

Boice 2006

Hardell 1994a

Figure 5-1. Study Utility Ranking: NHL

$\mathrm{E} / \mathrm{R}=$ exposure response.

Gray shading (left-hand column): Utility to inform hazard evaluation; light shading = highest utility; dark shading = lowest utility. Blue shading (right-hand column): Potential bias and study sensitivity; light shading = least biased or most sensitive; dark shading $=$ overall potential biases towards the null or lower sensitivity. Morgan et al. (1998) was rated somewhat lower for NHL than for kidney or liver cancer because of fewer expected and exposed cases.

a(Tan shading): Multiple limitations; overall direction of potential biases is unknown.

*Selection bias possible for external but not internal analysis.

\subsubsection{Study Findings}

The available studies reporting on trichloroethylene exposure in association with NHL and related cancers that were considered for inclusion in the cancer evaluation include 11 cohort or 
nested case-control studies and 7 case-control studies (of independent populations). Two metaanalyses were also identified and contributed to the evaluation.

The database consists of many reasonably well-conducted studies; however, similar to kidney cancer, NHL is a relatively uncommon cancer, and the majority of the cohort studies had limited statistical power to evaluate a modest risk from exposure to trichloroethylene and to evaluate exposure-response relationships.

The findings of the individual studies are discussed below and presented in Table 5-1, Table 5-2, and Table 5-3.

\subsubsection{Cohort and Nested Case-control Studies}

The available cohort studies and nested case-control studies reporting on NHL, multiple myeloma, or CLL include the following:

- Three studies conducted in Nordic countries (Hansen et al. 2013; Raaschou-Nielsen et al. 2003; Vlaanderen et al. 2013),

- Four studies of U.S. aircraft workers (Blair et al. 1998; Boice et al. 2006; Lipworth et al. 2011; Morgan et al. 1998; Radican et al. 2008),

- Two studies of uranium processing workers (Bahr et al. 2011; Yiin et al. 2009),

- One study of micro-electronic workers (Silver et al. 2014), and

- One study of U.S. military personnel exposed to trichloroethylene in drinking water (Bove et al. 2014).

Several of these studies also reported data for multiple myeloma (Blair et al. 1998; Boice et al. 2006; Hansen et al. 2013; Lipworth et al. 2011; Raaschou-Nielsen et al. 2003; Radican et al. 2008; Silver et al. 2014) or specifically for CLL (Boice et al. 2006; Lipworth et al. 2011).

Several studies reported only on combined categories of NHL and leukemia (Zhao et al. 2005) or lymphoma (Greenland et al. 1994), and Ritz (1999) only reported data for all

lymphohematopoietic cancers combined, and so these studies are excluded from this section.

\subsubsection{Nordic Studies}

As discussed previously, workers and exposed subjects in these incidence studies (Hansen et al. 2013; Raaschou-Nielsen et al. 2003; Vlaanderen et al. 2013) were identified from broad occupational or population-based databases and with a wide range of occupations and likely coexposures. All of these studies reported cancer incidence. Modest increases in overall risk for NHL in external analyses were observed among men $(\mathrm{SIR}=1.55,95 \% \mathrm{CI}=1.06$ to $2.20 ; 32$ cases) in the biomonitoring study (Hansen et al. 2013) and among those considered to have higher exposure in the blue-collar workers study $(\mathrm{SIR}=1.7,95 \% \mathrm{CI}=1.1$ to $2.4,31$ exposed cases, 20-year lagged) (Raaschou-Nielsen et al. 2003). In internal analyses by Hansen et al. (2013), the highest risk was found among workers in the second highest exposure group but the risk estimate was lower in the highest exposure group. This study had limited ability to evaluate exposure-response relationships because of lack of information on lifetime exposures and possible misclassification of exposure intensity. Among blue-collar workers (Raaschou-Nielsen et al. 2003), risks did not increase by employment duration or presumed exposure level (as assessed by date of first employment). No increases in NHL risk were observed in the 
population-based study by Vlaanderen et al. (2013), which might have included subjects with a broader range of exposures and with a greater probability of exposure misclassification. It is not clear whether a linear exposure-response pattern would be predicted if the proposed mechanism involves immunomodulation.

No increases in the risks of multiple myeloma were observed in all three Nordic studies (Hansen et al. 2013; Raaschou-Nielsen et al. 2003; Vlaanderen et al. 2013).

\subsubsection{Aircraft Manufacturing Workers}

Four mortality studies of aircraft manufacturing workers (Boice et al. 2006; Lipworth et al. 2011; Morgan et al. 1998; Radican et al. 2008) reported findings for NHL. The study of Utah aircraft workers (Radican et al. 2008) also included incidence data in an earlier update (Blair et al. 1998). The mortality study by Radican et al. (2008) had a 10-year longer follow-up and reported about two times as many NHL deaths as cases reported in the incidence study. There is some evidence of modest statistically non-significant increases in mortality for NHL (approximately $30 \%$ ) and multiple myeloma among workers ever exposed to trichloroethylene in two studies (Lipworth et al. 2011; Radican et al. 2008); however, no clear patterns of increasing risk with cumulative exposure or exposure pattern (Radican et al. 2008) or exposure duration (Lipworth et al. 2011) were reported. Most of the exposed cases were in the low-exposure group in the latter study. No association between trichloroethylene exposure and NHL was found in the smaller study by Morgan et al. (1998) based on three deaths, in the study of aerospace workers (Boice et al. 2006) based on only one death, or with cancer incidence in the earlier update of the Utah aircraft manufacturing worker cohort (Blair et al. 1998).

\subsubsection{Other Studies}

The remaining four studies had more limited sensitivity for informing these endpoints. Bahr et al. (2011) reported a statistically significant increase in mortality in external analyses but these were inversely related to higher exposure categories in internal analyses. In the study of microelectronic workers, hazard ratios (HRs) for 5-modified exposure years were $0.87(95 \% \mathrm{CI}=0.57$ to 1.35$)$ for NHL and $1.18(95 \% \mathrm{CI}=0.70$ to 1.99$)$ for multiple myeloma (Silver et al. 2014), but this study had a limited exposure assessment and was a relatively young cohort. No association was found for multiple myeloma and trichloroethylene exposure in the nested case-control study of Tennessee uranium enrichment workers (Yiin et al. 2009). Lastly, small increases in NHL and multiple myeloma were observed in some exposure categories in the drinking water mortality study (Bove et al. 2014), which was also a young cohort and was limited by indirect measures of trichloroethylene exposure.

\subsubsection{Population-based Case-control Studies}

Table 5-2 reports findings for NHL and Table 5-3 reports findings on NHL-related subtypes. Four population-based case-control studies in different geographical locations, including one each in Montreal (Christensen et al. 2013) and Connecticut (Deng et al. 2013; Wang et al. 2009b), and two in Sweden (Hardell et al. 1994; Persson and Fredrikson 1999), and one pooled analysis, the InterLymph study (Cocco et al. 2013), reported data on NHL. Two of these studies (Cocco et al. 2013; Deng et al. 2013; Wang et al. 2009b) also reported on the NHL subtypes DLBCL and follicular lymphoma. Three other case-control studies reported on multiple myeloma (Gold et al. (2011), Costantini et al. (2008), and Cocco et al. (2010), one of the 
constituent studies of the pooled analysis), and two studies reported on CLL (Cocco et al. 2013; Costantini et al. 2008). One study reported on the NHL subtype HCL (Nordström et al. 1998).

The InterLymph study included pooled cases and controls from four large multi-center studies: the EPILYMPH study in Europe (Cocco et al. 2010), the ENGELA study in France (Orsi et al. 2010), the MIS study in Italy (Miligi et al. 2006), and the NCI-SEER study in the United States (Purdue et al. 2011a). Because the pooled analysis included all the subjects of the individual studies and harmonized the exposure and disease assessment, this evaluation primarily reviews the pooled analysis. Importantly, the authors did not observe between-study heterogeneity. Findings from analyses on different exposure metrics from the NCI-SEER study (Purdue et al. 2011a), as well as findings for multiple myeloma from the EPILYMPH study (Cocco et al. 2010), are also included in the evaluation.

The major advantage of the recent case-control studies was greater statistical power, especially for evaluating NHL histological subtypes. The pooled InterLymph study (Cocco et al. 2013) and the SEER study on multiple myeloma (Gold et al. 2011) were considered to be the most informative studies because of the quality of the exposure and disease assessments, evaluation of multiple metrics of exposure, and larger numbers of exposed cases and controls, especially among individuals with higher probability or intensity of exposure. The other studies were more limited in their ability to inform cancer evaluation (Christensen et al. 2013; Costantini et al. 2008; Deng et al. 2013; Wang et al. 2009b), especially the three Swedish case-control studies (Hardell et al. 1994; Nordström et al. 1998; Persson and Fredrikson 1999) because of small numbers of exposed cases and controls, lower quality exposure assessments, and concerns for exposure misclassification or the use of older disease classifications (see Figure 5-1, Section 3, and Appendix D).

\section{NHL}

The InterLymph pooled analyses (Cocco et al. 2013) found a moderate increase in NHL risk for all exposed subjects $(\mathrm{OR}=1.4,95 \% \mathrm{CI}=0.9$ to $2.1,50$ exposed cases vs. 38 exposed controls); Fisher combined probability test, $\mathrm{p}=0.004$. Among subjects with a high probability of exposure, there was evidence of an exposure-response relationship with duration ( $p_{\text {trend }}=0.009$ ) and intensity $\left(\mathrm{p}_{\text {trend }}=0.059\right.$ ) of trichloroethylene exposure; risk estimates in the highest exposed categories were approximately two- to three-fold higher than in the lowest categories but were not statistically significant. The most informative of the constituent studies, the U.S. SEER analysis (Purdue et al. 2011a), had the advantage of a high-quality and detailed exposure assessment and reported on additional exposure metrics. Increased risks were observed with multiple metrics, but most notably a positive trend with average exposure $\left(\mathrm{p}_{\text {trend }}=0.02\right.$, $\mathrm{OR}=1.1,95 \% \mathrm{CI}=1.02$ to 1.21 for each $99 \mathrm{ppm}-\mathrm{hr}$ per week increase, and $7.9,95 \% \mathrm{CI}=1.8$ to 34.3 for $>360$ ppm-hour per week) and for cumulative exposure $\left(\mathrm{p}_{\text {trend }}=0.08, \mathrm{OR}=1.10,95 \%\right.$ $\mathrm{CI}=0.99$ to 1.22 per each 65,520 ppm-hour and $3.3,95 \% \mathrm{CI}=1.1$ to 10.01 for $>234,000 \mathrm{ppm}$ hour). Estimated (not measured) exposures for a proportion of the workers were high ( $>234,000$ ppm-hour cumulative exposure and 99-ppm average intensity of exposure), which increased the ability of the study to detect an effect.

Findings in the remaining case-control studies were somewhat inconsistent. The U.S. study of women (Deng et al. 2013; Wang et al. 2009b) reported increases in NHL risk for women with medium or high intensity of exposure $(\mathrm{OR}=2.2,95 \% \mathrm{CI}=0.9$ to $5.4,13$ exposed cases $)$. In addition, the risk associated with trichloroethylene exposure was higher (and statistically 
significant) among women with the AT or AA polymorphism of the IL2A_07 genotype than the TT polymorphism; most of this difference was observed in the DLBCL subtype rather than the follicular lymphoma subtype. Hardell et al. (1994) reported a high risk for NHL among trichloroethylene-exposed subjects ( $\mathrm{OR}=7.2,95 \% \mathrm{CI}=1.3$ to 42,4 exposed cases); however, this study's limitations include potential for exposure misclassification, including recall bias, use of proxy as controls, the minimum requirement to be classified as exposed was $<1$ week of continuous exposure in this study, potential confounding from exposure to other agents including other organic solvents, and small numbers of cases and controls. The other Swedish study by Persson and Fredrikson (1999) found an OR of $1.2(95 \% \mathrm{CI}=0.5$ to $2.4,16$ exposed cases) for ever exposure to trichloroethylene. In the Montreal study (Christensen et al. 2013), ORs were 1.0 ( $95 \% \mathrm{CI}=0.3$ to $3.5,3$ exposed cases) for substantial exposure and $1.2(95 \% \mathrm{CI}=0.5$ to $2.9,7$ exposed cases) for ever exposed.

\section{Multiple Myeloma, Follicular-cell Lymphoma, Chronic Lymphocytic Leukemia, Diffuse Large B-cell Lymphoma, and Hairy-cell Leukemia}

The most informative of the three studies reporting on multiple myeloma, a case-control study using SEER cancer registry data (Gold et al. 2011), found a statistically significant exposureresponse relationship for multiple myeloma ( $\left.p_{\text {trend }}=0.02\right)$, with a risk of $2.3(95 \% \mathrm{CI}=1.1$ to $5.0,18$ exposed cases) in the highest cumulative exposure category. The estimated highest cumulative exposure category was 6,593 to $49,500 \mathrm{ppm}$-hour. This study used the same detailed exposure assessment as Purdue et al. (2011a). There was little evidence of an association with multiple myeloma in the other two studies, the EPILYMPH study (Cocco et al. 2010), and the Italian multi-center study by Costantini et al. (2008).

The InterLymph analysis found evidence of statistically significant association with two NHL subtypes, follicular lymphoma and CLL; Fisher combined probability tests were 0.015 for follicular cell lymphoma and 0.005 for CLL. No association was found for any NHL subtypes in the EPILYMPH study (Cocco et al. 2010) but positive associations were found for CLL in the NCI-SEER study (Purdue et al. 2011a). In the case-control study among Connecticut women (Deng et al. 2013; Wang et al. 2009b), elevated risks were found for both DLBCL among genetically susceptible women, and an exposure-response relationship was found for DLBCL but not follicular-cell lymphoma. The Swedish study of HCL (Nordström et al. 1998) using similar methodologies as Hardell et al. (1994) observed a small (1.5) increase in this endpoint, based on 9 cases. 
Table 5-1. Cohort and Nested Case-control Studies and Trichloroethylene Exposure: Findings for NHL ${ }^{\mathrm{a}}$

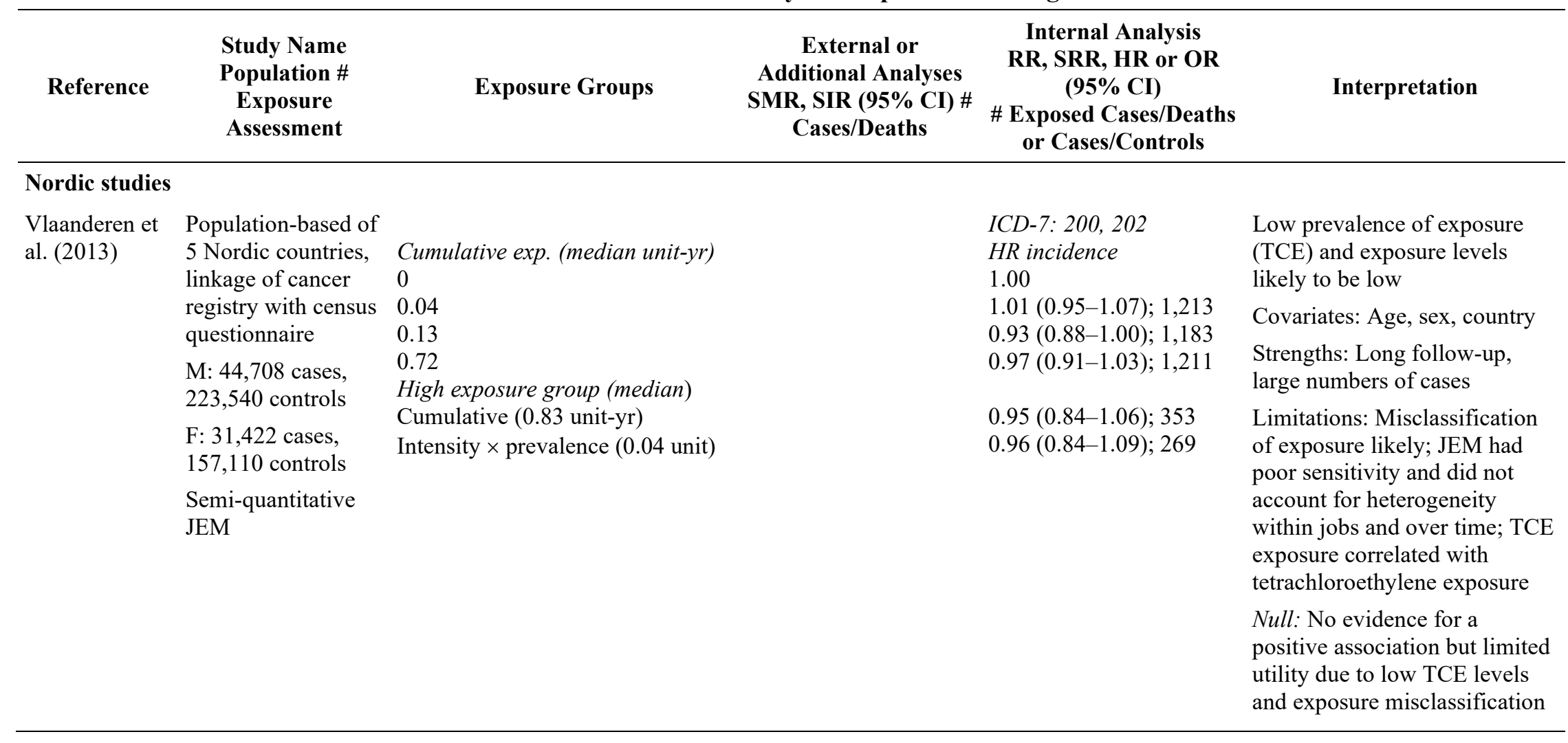




\begin{tabular}{|c|c|c|c|c|c|}
\hline Reference & $\begin{array}{c}\text { Study Name } \\
\text { Population \# } \\
\text { Exposure } \\
\text { Assessment }\end{array}$ & Exposure Groups & $\begin{array}{c}\text { External or } \\
\text { Additional Analyses } \\
\text { SMR, SIR (95\% CI) \# } \\
\text { Cases/Deaths }\end{array}$ & $\begin{array}{c}\text { Internal Analysis } \\
\text { RR, SRR, HR or OR } \\
\text { (95\% CI) } \\
\text { \# Exposed Cases/Deaths } \\
\text { or Cases/Controls }\end{array}$ & Interpretation \\
\hline $\begin{array}{l}\text { Hansen et al. } \\
(2013) \\
\text { (Potential } \\
\text { overlap with } \\
\text { Raaschou- } \\
\text { Nielsen et al. } \\
(2003))\end{array}$ & $\begin{array}{l}\text { Pooled and updated } \\
\text { Nordic cohorts } \\
\text { Axelson et al. } \\
\text { (1994), Anttila et al. } \\
(1995) \text {, Hansen et } \\
\text { al. (2001) } \\
5,553(3,776 \mathrm{M} \text {, } \\
\text { 1,777 F) } \\
\text { Biomonitoring (U- } \\
\text { TCA) }\end{array}$ & $\begin{array}{l}\text { Hansen et al. (2013) } \\
\text { Men } \\
\text { Women } \\
\text { Men \& women } \\
\text { Lag analysis (yr) } \\
0 \\
10 \\
20 \\
\underline{\mathrm{U}-\mathrm{TCA}(\mathrm{mg} / \mathrm{L})} \\
<5 \\
5-25 \\
25-50 \\
>50 \\
\mathrm{p}_{\text {trend }}\end{array}$ & $\begin{array}{l}I C D-7: 200,202 \\
\text { SIR } \\
1.55(1.06-2.20) ; 32 \\
0.63(0.23-1.37) ; 6 \\
\\
\\
1.26(0.89-1.73) ; 38 \\
1.21(0.83-1.71) ; 32 \\
1.11(0.68-1.72) ; 20\end{array}$ & $\begin{array}{l}\text { ICD-7: 200, } 202 \\
\text { HRR incidence (no lag) } \\
1.0 ; 12 \\
1.16(0.53-3.09) ; 14 \\
1.56(0.63-3.81) ; 8 \\
0.66(0.21-2.03) ; 4 \\
0.79\end{array}$ & $\begin{array}{l}\text { Low exposure levels (only } 20 \% \\
\text { exposed to } \geq 20 \text { ppm) and short } \\
\text { duration of employment } \\
\text { Covariates: Age, sex, calendar } \\
\text { period; indirect consideration } \\
\text { of smoking and alcohol } \\
\text { consumption } \\
\text { Strengths: Biomonitoring data; } \\
\text { large numbers of workers ever } \\
\text { exposed } \\
\text { Limitations: Only } 2 \text { or } 3 \text { U- } \\
\text { TCA measurements per } \\
\text { individual and unlikely to } \\
\text { estimate lifetime or cumulative } \\
\text { exposure; low statistical power } \\
\text { for evaluating modest risks; } \\
\text { limited ability to evaluate } \\
\text { exposure-response relationship } \\
\text { Limited evidence for a positive } \\
\text { association: Statistically } \\
\text { significant, moderately elevated } \\
\text { effect estimate among men } \\
\text { only; decreased risk with } \\
\text { increasing exposure }\end{array}$ \\
\hline
\end{tabular}


RoC Monograph on Trichloroethylene

\begin{tabular}{|c|c|c|c|c|c|}
\hline Reference & $\begin{array}{c}\text { Study Name } \\
\text { Population \# } \\
\text { Exposure } \\
\text { Assessment }\end{array}$ & Exposure Groups & $\begin{array}{c}\text { External or } \\
\text { Additional Analyses } \\
\text { SMR, SIR (95\% CI) \# } \\
\text { Cases/Deaths }\end{array}$ & $\begin{array}{c}\text { Internal Analysis } \\
\text { RR, SRR, HR or OR } \\
\text { (95\% CI) } \\
\text { \# Exposed Cases/Deaths } \\
\text { or Cases/Controls }\end{array}$ & Interpretation \\
\hline $\begin{array}{l}\text { Raaschou- } \\
\text { Nielsen et al. } \\
\text { (2003) } \\
\text { (Potential } \\
\text { overlap with } \\
\text { Hansen et al. } \\
(2013) \text { ) }\end{array}$ & $\begin{array}{l}\text { Danish blue-collar } \\
\text { workers } \\
40,049 \mathrm{M}+\mathrm{F} \\
\text { (approx. } 70 \% \mathrm{M} \text { ) } \\
\text { Working at a } \\
\text { company using TCE }\end{array}$ & $\begin{array}{l}\text { Higher TCE exposure subcohort } \\
\text { Ever exposed } \\
\text { Lag time (yr) } \\
0-9 \\
10-19 \\
\geq 20 \\
\text { Duration employment (yr) } \\
1-4 \\
\geq 5 \\
\text { Year of 1st employment } \\
\text { Before } 1970 \\
1970-1979\end{array}$ & $\begin{array}{l}\text { ICD-7: 200, } 202 \\
\text { SIR } \\
1.5(1.0-2.0) 65 \\
\\
1.8(0.9-3.1) ; 12 \\
1.3(0.8-2.0) ; 22 \\
1.7(1.1-2.4) ; 31 \\
\\
1.5(1.1-2.1) ; 35 \\
1.6(1.1-2.2) ; 30 \\
\\
1.6(1.1-2.3) ; 35 \\
1.5(1.0-2.1) ; 30\end{array}$ & NR & $\begin{array}{l}\text { Higher levels of TCE prior to } \\
1970 \text { ( } 40-60 \text { ppm); low levels } \\
\text { of exposure after that time } \\
\text { Covariates: Age, sex, calendar } \\
\text { year } \\
\text { Strengths: Large numbers of } \\
\text { exposed cases; subcohort of } \\
\text { subjects with higher exposure } \\
\text { potential } \\
\text { Limitations: Young cohort; } \\
\text { possible selection bias for } \\
\text { difference in SES; external } \\
\text { analysis only; possible } \\
\text { exposure misclassification } \\
\text { Evidence for an association: } \\
\text { Statistically significant, } \\
\text { moderate elevated effect } \\
\text { estimates but little evidence of } \\
\text { an exposure response }\end{array}$ \\
\hline
\end{tabular}




\begin{tabular}{|c|c|c|c|c|c|}
\hline Reference & $\begin{array}{l}\text { Study Name } \\
\text { Population \# } \\
\text { Exposure } \\
\text { Assessment }\end{array}$ & Exposure Groups & $\begin{array}{c}\text { External or } \\
\text { Additional Analyses } \\
\text { SMR, SIR (95\% CI) \# } \\
\text { Cases/Deaths }\end{array}$ & $\begin{array}{c}\text { Internal Analysis } \\
\text { RR, SRR, HR or OR } \\
\text { (95\% CI) } \\
\text { \# Exposed Cases/Deaths } \\
\text { or Cases/Controls }\end{array}$ & Interpretation \\
\hline \multicolumn{6}{|c|}{ Aerospace and aircraft workers } \\
\hline $\begin{array}{l}\text { Lipworth et al. } \\
\text { (2011) (update } \\
\text { Boice et al. } \\
(1999))\end{array}$ & $\begin{array}{l}\text { Burbank, CA (USA) } \\
\text { aircraft } \\
\text { manufacturing } \\
\text { workers } \\
\mathrm{N}=5,443 \text { (approx. } \\
80 \% \mathrm{M} \text { ) } \\
\text { Individual work } \\
\text { histories (JEM) }\end{array}$ & $\begin{array}{l}\text { TCE cohort (ever exposed) } \\
\text { TCE: years exposed } \\
0 \\
<1 \\
1-4 \\
5+ \\
\text { p }_{\text {trend }}\end{array}$ & $\begin{array}{l}\text { ICD (time of death) } \\
\text { SMR } \\
1.31(0.97-1.73) ; 50\end{array}$ & $\begin{array}{l}\text { ICD (time of death) } \\
\text { RR mortality } \\
1.00 ; 50 \\
0.84(0.48-1.47) ; 18 \\
1.10(0.59-2.04) ; 14 \\
1.02(0.55-1.90) ; 15 \\
>0.20\end{array}$ & $\begin{array}{l}\text { Exposure levels not reported; } \\
\text { short exposure duration } \\
\text { Covariates: Age, date of birth, } \\
\text { date of hire, termination date, } \\
\text { sex, and race } \\
\text { Strengths: Long follow-up, } \\
\text { adequate number of cases and } \\
\text { controls for ever exposure } \\
\text { Limitations: Evidence of HWE, } \\
\text { few exposed deaths in } \\
\text { subgroup analysis; exposure } \\
\text { misclassification is a concern; } \\
\text { no evaluation of exposure } \\
\text { intensity, } 70 \% \text { had exposure to } \\
\text { mixed solvents } \\
\text { Limited evidence for a positive } \\
\text { association: Elevated effect } \\
\text { estimate (approaching } \\
\text { statistical significance) for ever } \\
\text { exposure; no evidence of an } \\
\text { exposure-response relationship }\end{array}$ \\
\hline
\end{tabular}




\begin{tabular}{|c|c|c|c|c|c|}
\hline Reference & $\begin{array}{c}\text { Study Name } \\
\text { Population \# } \\
\text { Exposure } \\
\text { Assessment }\end{array}$ & Exposure Groups & $\begin{array}{c}\text { External or } \\
\text { Additional Analyses } \\
\text { SMR, SIR (95\% CI) \# } \\
\text { Cases/Deaths }\end{array}$ & $\begin{array}{c}\text { Internal Analysis } \\
\text { RR, SRR, HR or OR } \\
\text { (95\% CI) } \\
\text { \# Exposed Cases/Deaths } \\
\text { or Cases/Controls }\end{array}$ & Interpretation \\
\hline $\begin{array}{l}\text { Radican et al. } \\
\text { (2008) } \\
\text { (mortality to } \\
2000) \\
\text { Blair et al. } \\
\text { (1998) } \\
\text { (incidence } \\
\text { 1973-1990) } \\
\text { Note: mortality } \\
\text { only updated } \\
\text { by Radican) }\end{array}$ & $\begin{array}{l}\text { Utah (USA) aircraft } \\
\text { maintenance } \\
\text { workers } \\
\mathrm{N}=7,204(5,153 \mathrm{M} \text {, } \\
1,051 \mathrm{~F}) \\
\text { Individual work } \\
\text { histories (JEM) }\end{array}$ & $\begin{array}{l}\text { Mortality; } 2000 \text { follow-up } \\
\text { Cumulative exp. (unit-yr) } \\
\text { All } \\
0-5 \\
2-25 \\
>25 \\
\text { Exposure category } \\
\text { Low intermittent } \\
\text { Low continuous } \\
\text { Peak infrequent } \\
\text { Peak frequent } \\
\text { Incidence (1990) follow-up } \\
\text { Cumulative exp (unit-yr) } \\
\text { None } \\
0-5 \\
2-25 \\
>25\end{array}$ & $\begin{array}{l}\text { Internal analysis } \\
\text { HR mortality } \\
\frac{\text { Women }}{1.18(0.49-2.85) ; 9} \\
1.48(0.47-4.66) ; 4 \\
0 \\
1.30(0.45-3.77) ; 5\end{array}$ & $\begin{array}{l}\text { ICDA-8, ICD-9, } 10: 200 \text {, } \\
\text { 202, or C82-85 } \\
\text { HR mortality } \\
2.0(0.9-4.5) ; 28 \\
1.36(0.77-2.39) ; 46 \\
\text { Internal analysis } \\
\text { HR mortality } \\
\text { Men } \\
1.56(0.72-3.35) ; 37 \\
1.83(0.79-4.21) ; 18 \\
1.17(0.42-3.24) ; 7 \\
1.50(0.61-3.69) ; 12\end{array}$ & $\begin{array}{l}\text { Estimated exposure: Most } \\
\text { workers exposed to low levels } \\
\text { ( } \sim 10 \text { ppm), modest number of } \\
\text { workers exposed to higher } \\
\text { levels ( } \sim 100 \text { ppm) } \\
\text { Covariates: Age, calendar year } \\
\text { and sex } \\
\text { Strengths: Adequate semi- } \\
\text { quantitative JEM, long follow- } \\
\text { up, adequate statistical power } \\
\text { for ever exposure } \\
\text { Limitations: Potential for } \\
\text { exposure misclassification } \\
\text { because of missing information } \\
\text { for some workers; limited } \\
\text { power due to low numbers of } \\
\text { higher exposed workers; long } \\
\text { follow-up time (45 years) may } \\
\text { be past induction time; cannot } \\
\text { rule out confounding from } \\
\text { other co-exposures } \\
\text { Limited evidence for a positive } \\
\text { association: Statistically non- } \\
\text { significant elevated effect } \\
\text { estimates for ever exposure and } \\
\text { some subgroup analyses }\end{array}$ \\
\hline
\end{tabular}


RoC Monograph on Trichloroethylene

\begin{tabular}{|c|c|c|c|c|c|}
\hline Reference & $\begin{array}{c}\text { Study Name } \\
\text { Population \# } \\
\text { Exposure } \\
\text { Assessment }\end{array}$ & Exposure Groups & $\begin{array}{c}\text { External or } \\
\text { Additional Analyses } \\
\text { SMR, SIR (95\% CI) \# } \\
\text { Cases/Deaths }\end{array}$ & $\begin{array}{c}\text { Internal Analysis } \\
\text { RR, SRR, HR or OR } \\
\text { (95\% CI) } \\
\text { \# Exposed Cases/Deaths } \\
\text { or Cases/Controls }\end{array}$ & Interpretation \\
\hline \multirow{7}{*}{$\begin{array}{l}\text { Boice et al. } \\
(2006) \\
\text { (Overlaps with } \\
\text { Zhao et al. } \\
(2005))\end{array}$} & Los Angeles (USA) & \multirow{7}{*}{ Ever exposed to TCE } & $I C D-9 ; 200-2010$ & & \multirow{2}{*}{$\begin{array}{l}\text { Exposure occurs during text } \\
\text { engine flush, which is likely to } \\
\text { be high }\end{array}$} \\
\hline & $\begin{array}{l}\text { Rocket engine } \\
\text { testing workers }\end{array}$ & & $\begin{array}{l}S M R \\
0.21(0.01-1.18) ; 1\end{array}$ & & \\
\hline & 1,111 Men & & & & \multirow{2}{*}{$\begin{array}{l}\text { Covariates: Date of birth, year } \\
\text { of hire, pay type (surrogate fo } \\
\text { SES) and exposure to } \\
\text { hydrazine }\end{array}$} \\
\hline & \multirow{4}{*}{$\begin{array}{l}\text { Qualitative JEM; } \\
\text { Individual work } \\
\text { histories }\end{array}$} & & & & \\
\hline & & & & & Strengths: Adequate follow up \\
\hline & & & & & $\begin{array}{l}\text { Limitations: Qualitative } \\
\text { exposure assessment; one } \\
\text { exposed death }\end{array}$ \\
\hline & & & & & $\begin{array}{l}\text { Null: Limited utility (only } 1 \\
\text { exposed death) }\end{array}$ \\
\hline
\end{tabular}


RoC Monograph on Trichloroethylene

\begin{tabular}{|c|c|c|c|c|c|}
\hline Reference & $\begin{array}{c}\text { Study Name } \\
\text { Population \# } \\
\text { Exposure } \\
\text { Assessment }\end{array}$ & Exposure Groups & $\begin{array}{c}\text { External or } \\
\text { Additional Analyses } \\
\text { SMR, SIR }(95 \% \text { CI) \# } \\
\text { Cases/Deaths }\end{array}$ & $\begin{array}{c}\text { Internal Analysis } \\
\text { RR, SRR, HR or OR } \\
\text { (95\% CI) } \\
\text { \# Exposed Cases/Deaths } \\
\text { or Cases/Controls }\end{array}$ & Interpretation \\
\hline \multirow[t]{4}{*}{$\begin{array}{l}\text { Morgan et al. } \\
\text { (1998) }\end{array}$} & $\begin{array}{l}\text { Arizona aircraft } \\
\text { manufacturing }\end{array}$ & \multirow{4}{*}{$\begin{array}{l}\text { All TCE exposed workers } \\
\text { Cumulative exp. score } \\
\text { Low }(2,357) \\
\text { High }(2,376) \\
\text { Peak (med/high) vs. low/no }\end{array}$} & $\begin{array}{l}\text { ICD 7-9: } 200 \\
\text { SMR } \\
0.96(0.20-2.81) ; 3\end{array}$ & $\begin{array}{l}\text { ICD 7-9: } 200 \\
R R \text { mortality } \\
1.36(0.35-5.21) 3\end{array}$ & $\begin{array}{l}\text { High-exposure jobs were } \\
\text { considered to be } \geq 50 \mathrm{ppm}\end{array}$ \\
\hline & $\begin{array}{l}\text { workers } \\
\mathrm{N}=4,733(2,555 \mathrm{M}, \\
2,178 \mathrm{~F})\end{array}$ & & \multirow{3}{*}{$\begin{array}{l}1.79(0.22-6.46) ; 2 \\
0.50(0.01-2.79) ; 1\end{array}$} & \multirow{3}{*}{$\begin{array}{l}2.25(0.46-11.09) ; 2 \\
0.81(0.10-6.49) ; 1 \\
1.31(0.28-6.08) ; 2\end{array}$} & $\begin{array}{l}\text { Covariates: age at hire, gender } \\
\text { (decade at high exposure } \\
\text { considered but no effect) }\end{array}$ \\
\hline & \multirow[t]{2}{*}{$\begin{array}{l}\text { Semi-quantitative } \\
\text { JEM; individual } \\
\text { work history }\end{array}$} & & & & $\begin{array}{l}\text { Limitations: Evidence of an } \\
\text { HWE; potential exposure } \\
\text { misclassification among } \\
\text { low/medium exposure groups; } \\
\text { mortality analysis and few } \\
\text { exposed cases; inadequate } \\
\text { statistical power because of few } \\
\text { cases and ICD for NHL does } \\
\text { not include } 202\end{array}$ \\
\hline & & & & & $\begin{array}{l}\text { Limited evidence for a positive } \\
\text { association: Elevated, but } \\
\text { imprecise, effect estimate based } \\
\text { on few cases and no consistent } \\
\text { patterns }\end{array}$ \\
\hline
\end{tabular}




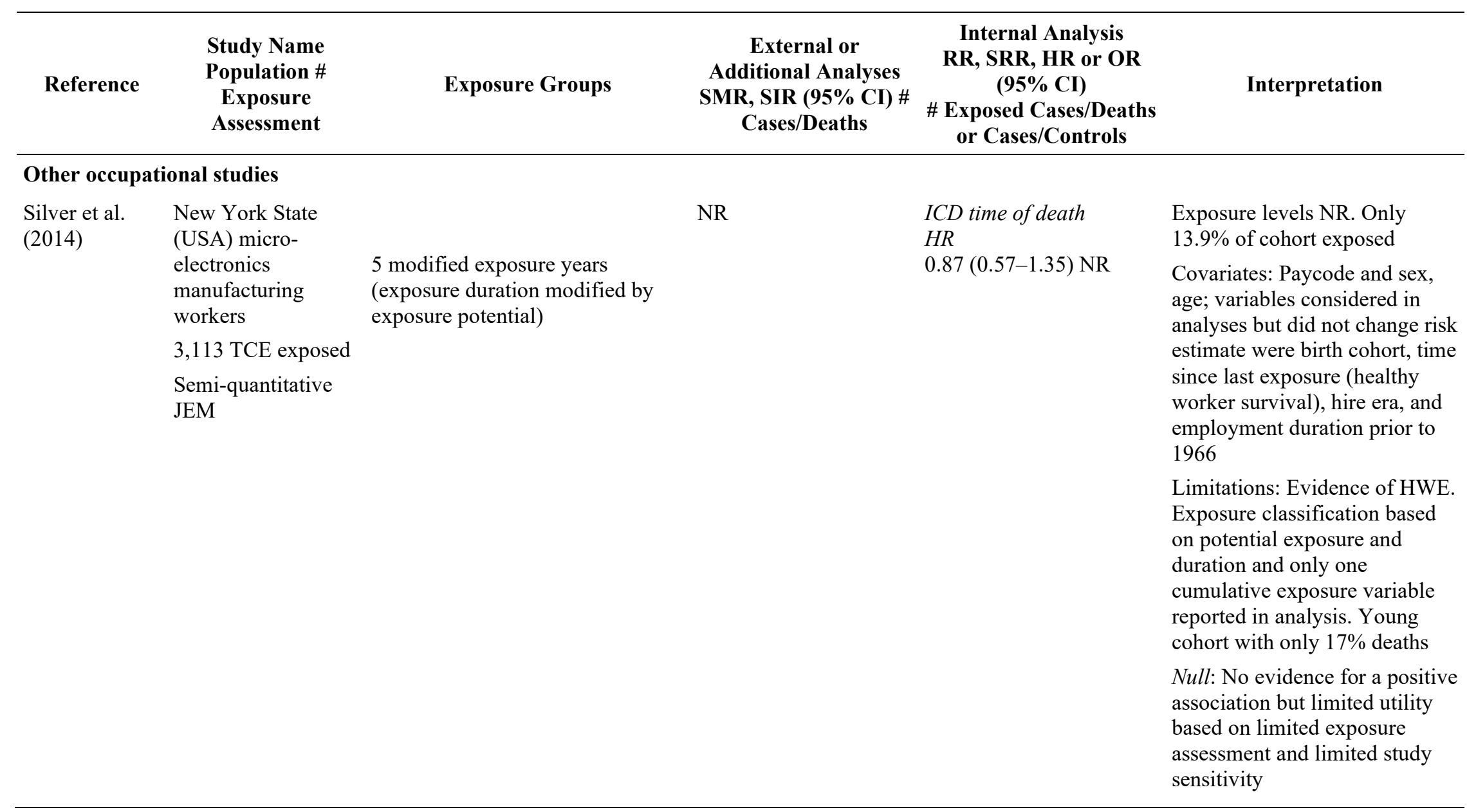


RoC Monograph on Trichloroethylene

\begin{tabular}{|c|c|c|c|c|c|}
\hline Reference & $\begin{array}{c}\text { Study Name } \\
\text { Population \# } \\
\text { Exposure } \\
\text { Assessment }\end{array}$ & Exposure Groups & $\begin{array}{c}\text { External or } \\
\text { Additional Analyses } \\
\text { SMR, SIR (95\% CI) \# } \\
\text { Cases/Deaths }\end{array}$ & $\begin{array}{c}\text { Internal Analysis } \\
\text { RR, SRR, HR or OR } \\
\text { (95\% CI) } \\
\text { \# Exposed Cases/Deaths } \\
\text { or Cases/Controls }\end{array}$ & Interpretation \\
\hline $\begin{array}{l}\text { Bahr et al. } \\
\text { (2011) }\end{array}$ & $\begin{array}{l}\text { Kentucky (USA) } \\
\text { Uranium } \\
\text { enrichment workers } \\
5,535(\mathrm{M}) \\
\text { Generic JEM }\end{array}$ & $\begin{array}{l}\text { TCE exposure probability } \\
\text { category } \\
0 \\
0-1 \\
2-3 \\
0-3 \\
4-5 \\
\text { Total } \\
\text { TCE exposure category } \\
1 \\
2 \\
3 \\
\text { Total }\end{array}$ & $\begin{array}{l}\text { ICD NR } \\
\text { SMR } \\
3.20(0.39-11.57) ; 2 \\
1.85(0.85-3.52) ; 9 \\
1.70(0.88-2.97) ; 12 \\
1.76(1.09-2.69) ; 21 \\
1.05(0.52-1.88) ; 11 \\
1.49(1.02-2.10) ; 32\end{array}$ & $\begin{array}{l}\text { SRR mortality } \\
1.0 \\
1.31(0.47-3.65) \\
0.75(0.27-2.12) \\
0.99(0.40-2.46)\end{array}$ & $\begin{array}{l}\text { No information on exposure } \\
\text { levels } \\
\text { Covariates: Age, sex, race } \\
\text { (unclear) } \\
\text { Limitations: Unclear } \\
\text { descriptions of methods and } \\
\text { findings; limited statistical } \\
\text { power; evidence of HWE hire } \\
\text { and survival bias } \\
\text { Null: No evidence for an } \\
\text { association (internal analysis) } \\
\text { but limited utility }\end{array}$ \\
\hline
\end{tabular}




\begin{tabular}{|c|c|c|c|c|c|}
\hline Reference & $\begin{array}{c}\text { Study Name } \\
\text { Population \# } \\
\text { Exposure } \\
\text { Assessment }\end{array}$ & Exposure Groups & $\begin{array}{c}\text { External or } \\
\text { Additional Analyses } \\
\text { SMR, SIR (95\% CI) \# } \\
\text { Cases/Deaths }\end{array}$ & $\begin{array}{c}\text { Internal Analysis } \\
\text { RR, SRR, HR or OR } \\
\text { (95\% CI) } \\
\text { \# Exposed Cases/Deaths } \\
\text { or Cases/Controls }\end{array}$ & Interpretation \\
\hline
\end{tabular}

\section{Environmental exposure}

$\begin{array}{lll}\begin{array}{l}\text { Bove et al. } \\ \text { (2014) }\end{array} & \begin{array}{l}\text { North Carolina } \\ \text { (USA) (Camp }\end{array} & \text { Cumulative TCE }(\mu \mathrm{g} / \text { L-months }) \\ \text { Lejeune) } & \leq 1 \\ & 154,932 & >1-3,100 \\ & \text { Drinking water } & >3,100-7,700 \\ \text { contamination } & \\ \text { Ecological } & \\ \text { assessment } & \end{array}$

\section{ICD NR}

HR mortality; 10-yr lag

$1.0(27)$

$0.90(0.42-1.92) ; 10$

$0.75(0.33-1.70) ; 8$

$1.15(0.56-2.34) ; 13$
Estimated mean levels $(\mu \mathrm{g} / \mathrm{L}-$ month) TCE from water supply = 358.7; overall cumulative exposure $=6,369$

(median) and 5,289 (mean); $20 \%$ were exposed to levels between 7,700 and 39,745

Covariates: Sex, race, rank, and education; other variables considered in the model (did not change risk estimates by $10 \%$ ) include marital status, birth cohort, date of death, duty occupation

Strengths: Large cohort; adequate modeling of exposures

Limitations: Young cohort; no information on individual water consumption; potential confounding from other contaminants e.g., tetrachloroethylene

Null: Small increase in effect estimate but limited utility based on limited study sensitivity and exposure assessment

$\mathrm{HR}=$ hazard ratio; ICD = International Classification of Diseases; JEM = job-exposure matrix; NHL = non-Hodgkin lymphoma; NR = not reported; OR = odds ratio; ppm = parts per million; $\mathrm{RR}=$ relative risk; $\mathrm{SIR}=$ standardized incidence ratio; $\mathrm{SMR}=$ standardized mortality ratio; $\mathrm{SRR}=$ standardized rate ratio; $\mathrm{TCE}=$ trichloroethylene.

aSee Table 5-3 for NHL subtypes and related cancers. 
RoC Monograph on Trichloroethylene

Table 5-2. Case-control Studies of Trichloroethylene Exposure: Findings for NHL ${ }^{\mathrm{a}}$

\begin{tabular}{|c|c|c|c|c|}
\hline Reference & $\begin{array}{c}\text { Study } \\
\text { Size (N) } \\
\text { Exposure Assessment }\end{array}$ & Exposure Groups & $\begin{array}{c}\text { RR or OR }(95 \% \text { CI }) \\
\text { \# Exposed } \\
\text { Cases/Controls }\end{array}$ & Interpretation \\
\hline $\begin{array}{l}\text { Christensen et } \\
\text { al. (2013) }\end{array}$ & $\begin{array}{l}\text { Montreal (Canada) } \\
\text { Population- and hospital-based } \\
\text { 1975-1985 } \\
\text { NHL: } 215 \text { cases, } 2,341 \text { cancer } \\
\text { controls } \\
\text { Expert assessment of } \\
\text { occupational data from } \\
\text { interviews }\end{array}$ & $\begin{array}{l}\text { Ever exposed } \\
\text { Substantial exposure }\end{array}$ & $\begin{array}{l}\text { ICD-9 200, } 202 \\
1.2(0.5-2.9) ; 7 / 65^{\mathrm{b}} \\
1.0(0.3-3.5) ; 3 / 30^{\mathrm{b}}\end{array}$ & $\begin{array}{l}\text { Exposure prevalence to TCE was very rare; } \leq 2 \% \text { of } \\
\text { cancer controls or population controls had } \\
\text { substantial exposure and } 3 \% \text { had any exposure } \\
\text { Covariates: age, census tract, median income, } \\
\text { ethnicity, education, self vs. proxy respondent, } \\
\text { smoking, alcohol assumption, coffee use } \\
\text { Strengths: Adequate quality of exposure assessment } \\
\text { Limitations: Low statistical power } \\
\text { Null: Small increased risk for ever-exposed but } \\
\text { limited utility based on low statistical power }\end{array}$ \\
\hline $\begin{array}{l}\text { Cocco et al. } \\
(2013)\end{array}$ & $\begin{array}{l}4 \text { pooled multi-center studies } \\
\text { (Italy, France, Europe } \\
\text { "EPILYMPH" multi-center } \\
\text { study, U.S. region } 4 \text { SEER } \\
\text { study) } \\
\text { Population-based } \\
\text { NHL: } 3,788 \text { cases, } 4,279 \\
\text { controls } \\
\text { Questionnaires on occupational } \\
\text { history, industrial hygiene } \\
\text { reports, expert assessments }\end{array}$ & $\begin{array}{l}\text { All exposed } \\
\text { Duration }(y r) \\
\text { No exposure } \\
1-14 \\
15-29 \\
30-39 \\
40+ \\
p_{\text {trend }} \\
\text { Intensity }(\text { ppm) } \\
\leq 5 \\
5-75 \\
>75 \\
p_{\text {trend }}\end{array}$ & $\begin{array}{l}1.0 ; 3,453 / 3,903 \\
0.7(0.4-1.5) ; 15 / 23 \\
1.9(0.8-4.3) ; 17 / 9 \\
2.8(1.0-7.8) ; 15 / 5 \\
3.3(0.3-33) ; 3 / 1 \\
0.009\end{array}$ & $\begin{array}{l}\text { Exposure prevalence: } 9 \% \text { ever exposed; } 1 \% \text { high } \\
\text { probability of exposure. Exposure levels not } \\
\text { measured but high exposure categories are }>75 \mathrm{ppm} \\
\text { Covariates: age, sex, study location } \\
\text { Strengths: Good exposure and disease assessment; } \\
\text { analysis of NHL subtypes } \\
\text { Limitations: Reduced statistical power in NHL } \\
\text { subtype analysis, no adjustment for lifestyle factors } \\
\text { or co-exposures } \\
\text { Evidence for a positive association: Statistically } \\
\text { significant association with NHL; Evidence for } \\
\text { exposure-response relationship }\end{array}$ \\
\hline
\end{tabular}


RoC Monograph on Trichloroethylene

\begin{tabular}{|c|c|c|c|c|}
\hline Reference & $\begin{array}{c}\text { Study } \\
\text { Size (N) } \\
\text { Exposure Assessment }\end{array}$ & Exposure Groups & $\begin{array}{c}\text { RR or OR }(95 \% \text { CI }) \\
\text { \# Exposed } \\
\text { Cases/Controls }\end{array}$ & Interpretation \\
\hline \multirow{8}{*}{$\begin{array}{l}\text { Deng et al. } \\
(2013) \\
\text { Wang et al. } \\
(2009 b)\end{array}$} & \multirow{4}{*}{$\begin{array}{l}\text { Connecticut (USA) } \\
\text { All NHL: } 601 \text { cases, } 717 \\
\text { controls } \\
\text { Questionnaire on occupational } \\
\text { history. Linkage of occupation } \\
\text { code to JEM }\end{array}$} & \multirow{4}{*}{$\begin{array}{l}\text { Wang et al. }(2009 b) \\
\text { Ever exposed } \\
\text { Exposure intensity } \\
\text { Low intensity } \\
\text { Medium/high intensity } \\
\text { p trend }\end{array}$} & \multirow{2}{*}{$\begin{array}{l}\text { ICD-O-2; OR } \\
1.2(0.9-1.8) ; 77 / 79\end{array}$} & \multirow{2}{*}{$\begin{array}{l}\text { No cases or controls with high probability of } \\
\text { exposure and medium/high exposure. Exposure } \\
\text { prevalence } 8 \%\end{array}$} \\
\hline & & & & \\
\hline & & & \multirow{2}{*}{$\begin{array}{l}1.1(0.8-1.6) ; 64 / 71 \\
2.2(0.9-5.4) ; 13 / 8 \\
0.06\end{array}$} & \multirow{2}{*}{$\begin{array}{l}\text { Covariates: Age, history of hematopoietic cancer, } \\
\text { race, and alcohol consumption. Smoking, medical } \\
\text { history of immune diseases, income, education level } \\
\text { did not affect OR }\end{array}$} \\
\hline & & & & \\
\hline & & \multirow{4}{*}{$\begin{array}{l}\text { Deng et al. (2013) } \\
\text { polymorphism } \\
\text { Ever exposed } \\
\text { IL12A_07 genotype } \\
\text { TT } \\
\text { AA } \\
\text { p interaction }\end{array}$} & $N H L(I C D-O-2)$ & $\begin{array}{l}\text { Strengths: Consideration of potential confounding } \\
\text { from lifestyle factors, analyses by genotype \& NHL } \\
\text { subtype }\end{array}$ \\
\hline & & & $0.70(0.34-1.42) ; 14 / 26$ & Limitations: Limited JEM (not calendar-year \\
\hline & & & $\begin{array}{l}2.09(1.28-3.42) ; 51 / 31 \\
0.009\end{array}$ & $\begin{array}{l}\text { specific and based on occupations not job tasks), no } \\
\text { control for co-exposures }\end{array}$ \\
\hline & & & & $\begin{array}{l}\text { Evidence for a positive association: Statistically } \\
\text { significant, moderate elevated effect estimate in } \\
\text { genotype analysis; some evidence for an exposure- } \\
\text { response relationship }\end{array}$ \\
\hline \multirow{5}{*}{$\begin{array}{l}\text { Persson and } \\
\text { Fredrikson } \\
\text { (1999) }\end{array}$} & \multirow{5}{*}{$\begin{array}{l}\text { Sweden } \\
\text { Population-based } \\
\text { NHL: } 199 \text { cases, } 479 \text { controls } \\
\text { Questionnaire on occupational } \\
\text { history }\end{array}$} & \multirow{5}{*}{ Ever exposed } & \multirow{5}{*}{$\begin{array}{l}\text { ICD-8 used in } 2 \text { nd study, } \\
\text { NR 1st study } \\
1.2(0.5-2.4) ; 16 / 32\end{array}$} & Exposure prevalence $1 \%$ in controls \\
\hline & & & & Covariates: Age, sex \\
\hline & & & & $\begin{array}{l}\text { Limitations: Limited exposure assessment, potential } \\
\text { for exposure misclassification is substantial }\end{array}$ \\
\hline & & & & 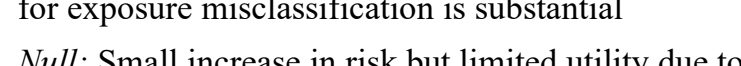 \\
\hline & & & & concern about exposure misclassification \\
\hline
\end{tabular}


RoC Monograph on Trichloroethylene

\begin{tabular}{|c|c|c|c|c|}
\hline Reference & $\begin{array}{c}\text { Study } \\
\text { Size (N) } \\
\text { Exposure Assessment }\end{array}$ & Exposure Groups & $\begin{array}{c}\text { RR or OR }(95 \% \text { CI }) \\
\text { \# Exposed } \\
\text { Cases/Controls }\end{array}$ & Interpretation \\
\hline $\begin{array}{l}\text { Hardell et al. } \\
\text { (1994) }\end{array}$ & $\begin{array}{l}\text { Sweden } \\
\text { Population-based } \\
\text { NHL: } 105 \text { cases, } 335 \text { controls } \\
\text { Questionnaire on occupational } \\
\text { history and leisure activities }\end{array}$ & Ever exposed & $\begin{array}{l}\text { Rappaport classification } \\
7.2(1.3-42) ; 4 / 4\end{array}$ & $\begin{array}{l}\text { Exposure prevalence: } 1 \% \text { in controls } \\
\text { Covariates: Age, vital status } \\
\text { Limitations: Limited exposure assessment, and } \\
\text { potential for exposure misclassification is } \\
\text { substantial } \\
\text { Limited evidence for an association: Statistically } \\
\text { significant, high elevated effect estimate; } \\
\text { methodological concerns and small numbers of } \\
\text { exposed cases/controls may bias towards an } \\
\text { overestimate of the risk estimate }\end{array}$ \\
\hline $\begin{array}{l}\text { Purdue et al. } \\
(2011 \mathrm{a})^{\mathrm{d}} \\
\text { incorporated in } \\
\text { pooled analysis } \\
\text { (Cocco et al. } \\
\text { 2013) }\end{array}$ & $\begin{array}{l}\text { U.S. SEER registry } \\
\text { Population-based } \\
\text { NHL: } 1,189 \text { cases, } 982 \text { controls } \\
\text { Interviews on occupational } \\
\text { histories and exposures; expert } \\
\text { assessment by industrial } \\
\text { hygienists based on } \\
\text { questionnaire data and } \\
\text { systematic industrial hygiene } \\
\text { literature review }\end{array}$ & $\begin{array}{l}\text { Exposure-response analyses } \\
\text { Average exposure } \\
\text { Per } 90 \mathrm{ppm}-\mathrm{hr} / \mathrm{wk}^{\mathrm{e}} \\
\mathrm{p}_{\text {trend }} \\
>360 \mathrm{ppm}-\mathrm{hr} / \mathrm{wk} \\
\text { Average exp. intensity } \\
\text { Per estimated } 99 \mathrm{ppm} \\
\mathrm{p}_{\text {trend }} \\
\text { Exposure duration (yr) } \\
\text { Per } 10 \text { yr } \\
\mathrm{p}_{\text {trend }} \\
\text { Cumulative exposure } \\
\text { Per } 65,520 \mathrm{ppm}-\mathrm{hr} \\
\mathrm{p}_{\text {trend }} \\
>234,000 \mathrm{ppm}-\mathrm{hr}\end{array}$ & $\begin{array}{l}I C D-O-2 \\
\text { OR (\# cases NR) } \\
1.11(1.02-1.21) \\
0.02 \\
7.9(1.8-34.3)\end{array}$ & $\begin{array}{l}\text { Exposure not measured but high-exposure } \\
\text { categories are }>99 \text { ppm, } 360 \text { ppm-hr-wk and } \\
234,000 \text { ppm-hr } \\
\text { Covariates: Age, sex, race, education level, and } \\
\text { study area } \\
\text { Strengths: Good exposure and disease assessment, } \\
\text { detailed analyses using multiple exposure metrics } \\
\text { including analyses per estimated quantitative } \\
\text { exposure, by intra-category high exposure and NHL } \\
\text { subtype } \\
\text { Limitations: Reduced statistical power in NHL } \\
\text { subtype analysis, no adjustment for lifestyle factors } \\
\text { or co-exposures } \\
\text { Evidence for a positive association: Evidence for } \\
\text { exposure-response relationship with multiple } \\
\text { exposure metrics }\end{array}$ \\
\hline
\end{tabular}

ICD = International Classification of Diseases; JEM = job-exposure matrix; NHL = non-Hodgkin lymphoma; NR = not reported; OR = odds ratio; ppm = parts per million; $\mathrm{RR}=$ relative risk; SEER $=$ Surveillance, Epidemiology and End Results program.

a See Table 5-3 for findings on NHL subtypes and related cancers.

${ }^{b}$ Cancer controls only reported.

'The InterLymph Consortium classification (see Morton et al. (2007)) was harmonized with earlier WHO lymphoid neoplasms classification and the ICD-O-3.

${ }^{\mathrm{d}}$ Study findings presented that provide additional informative analyses that are not available in the pooled analyses. Findings from other studies are not presented in the tables since they did not have additional information thought to be informative.

Intracategory based on mean among controls. 
RoC Monograph on Trichloroethylene

Table 5-3. Cohort, Nested Case-control, and Population-based Case-control Studies of Trichloroethylene Exposure and NHL Subtypes

\begin{tabular}{|c|c|c|c|c|c|}
\hline Reference & Exposure Group & DLBCL & Follicular Lymphoma & CLL & Multiple Myeloma \\
\hline \multicolumn{6}{|c|}{ Cohort and nested case-control studies } \\
\hline $\begin{array}{l}\text { Vlaanderen et al. } \\
\text { (2013) }\end{array}$ & $\begin{array}{l}\text { Cumulative exp. (unit-yr) } \\
0 \\
0.04 \\
0.13 \\
0.74 \\
\text { High-exposure group } \\
\text { Cumulative ( } 0.83 \text { unit-yr) } \\
\text { Intensity } \times \text { prevalence } \\
(0.04 \text { unit) }\end{array}$ & NR & NR & NR & $\begin{array}{l}\text { ICD-7; HR (incidence) } \\
1.00 \\
0.93(0.84-1.03) ; 468 \\
0.92(0.84-1.01) ; 574 \\
0.96(0.88-1.06) ; 541 \\
\\
1.01(0.84-1.22) ; 132 \\
1.03(0.88-1.22) ; 134\end{array}$ \\
\hline Hansen et al. (2013) & $\begin{array}{l}\text { Men } \\
\text { Women } \\
\text { Men \& women }\end{array}$ & NR & NR & NR & $\begin{array}{l}I C D-7 ; S I R \\
0.47(0.13-1.20) ; 4 \\
1.04(0.29-2.67) ; 4 \\
0.65(0.28-1.27) ; 8\end{array}$ \\
\hline $\begin{array}{l}\text { Raaschou-Nielsen et } \\
\text { al. (2003) }\end{array}$ & $\begin{array}{l}\text { Entire cohort } \\
\text { Men }(588,047 \text { p-yr }) \\
\text { Women }(118,270 \text { p-yr })\end{array}$ & NR & NR & NR & $\begin{array}{l}I C D-7 ; S I R \\
1.1(0.70-1.52) ; 28 \\
0.90(0.18-2.56) ; 3\end{array}$ \\
\hline Lipworth et al. (2011) & $\begin{array}{l}\text { Ever exposed } \\
\text { TCE: years exposed } \\
0 \\
<1 \\
1-4 \\
5+ \\
p_{\text {trend }}\end{array}$ & NR & NR & $0.93(0.40-1.83) ; 8$ & $\begin{array}{l}\text { ICD time of death: SMR } \\
1.21(0.76-1.81) ; 23 \\
R R \text { mortality } \\
1.00 \\
0.70(0.31-1.58) ; 8 \\
1.45(0.68-3.09) ; 10 \\
0.67(0.25-1.83) ; 5 \\
>0.20\end{array}$ \\
\hline $\begin{array}{l}\text { Radican et al. (2008) } \\
\text { Mortality }\end{array}$ & $\begin{array}{l}\text { Ever exposed } M \& W \\
\text { Cumulative exp. (unit-yr) } \\
\frac{\text { Men }^{\mathrm{a}}}{\text { All }} \\
0-5 \\
2-25 \\
>25\end{array}$ & NR & NR & NR & $\begin{array}{l}1.08(0.43-2.71) ; 19 \\
0.69(0.21-2.27) ; 5 \\
1.58(0.53-4.71) ; 7 \\
1.19(0.40-3.54) ; 7\end{array}$ \\
\hline
\end{tabular}


RoC Monograph on Trichloroethylene

\begin{tabular}{|c|c|c|c|c|c|}
\hline Reference & Exposure Group & DLBCL & Follicular Lymphoma & CLL & Multiple Myeloma \\
\hline $\begin{array}{l}\text { Blair et al. (1998) } \\
\text { Incidence (RR) } \\
\text { Same population as } \\
\text { Radican et al. (2008) }\end{array}$ & $\begin{array}{l}\text { Ever exposed } M \& W \\
\text { Cumulative exposure } \text { Men }^{b} \\
\text { No exposure } \\
<5 \\
5-25 \\
>25\end{array}$ & NR & NR & NR & $\begin{array}{l}1.7(0.5-5.5) ; 10 \\
1.0(0.2-4.2) ; 4 \\
0.8(0.1-4.4) ; 2 \\
1.2(0.3-4.7) ; 4\end{array}$ \\
\hline Boice et al. (2006) & & & & $\begin{array}{l}\text { ICD-9; SMR } \\
0.21(0.01-1.18) 1\end{array}$ & $\begin{array}{l}I C D-9 ; S M R \\
0.50(0.01-2.77) 1\end{array}$ \\
\hline Silver et al. (2014) & $\begin{array}{l}5 \text { modified exposure duration } \\
\text { yr (exposure duration } \\
\text { modified by exposure } \\
\text { potential) }\end{array}$ & & & & $\begin{array}{l}\text { ICD time of death } \\
\text { (HR mortality) } \\
1.18(0.70-1.99) \mathrm{NR}\end{array}$ \\
\hline $\begin{array}{l}\text { Yiin et al. (2009) } \\
\text { Nested case-control } \\
\text { study }\end{array}$ & $\begin{array}{l}\text { Average cumulative TCE } \\
\text { exposure score } / 100\end{array}$ & & & & $\begin{array}{l}\text { OR }(I C D-8) \\
1.02(0.98-1.05) \mathrm{NR}\end{array}$ \\
\hline Bove et al. (2014) & $\begin{array}{l}\text { Cumulative }(\mu \mathrm{g} / \mathrm{L}-\mathrm{mo}) \\
\leq 1 \\
>1-155 \\
>155-380 \\
>380-8,585\end{array}$ & NR & NR & NR & $\begin{array}{l}\mathrm{HR}(\mathrm{ICD} N \mathrm{NR}) \\
1.0 ; 6 \\
2.09(0.66-6.62) ; 7 \\
1.29(0.34-4.88) ; 4 \\
0 \text { cases }\end{array}$ \\
\hline \multicolumn{6}{|c|}{ Case-control studies (OR) } \\
\hline $\begin{array}{l}\text { Cocco et al. (2013) } \\
\text { Pooled analysis }\end{array}$ & $\begin{array}{l}\text { Intensity }(\mathrm{ppm}) \\
\leq 5 \\
5-75 \\
>75 \\
p_{\text {trend }}\end{array}$ & $\begin{array}{l}\text { InterLymph } \\
\text { classification }^{a} ; \text { OR } \\
1.2(0.3-4.4) ; 3 / 8 \\
0.6(0.2-1.6) ; 5 / 25 \\
2.0(0.5-8.7) ; 3 / 5 \\
0.114\end{array}$ & $\begin{array}{l}\text { InterLymph } \\
\text { classification }^{a} ; \text { OR } \\
1.1(0.1-9.2) ; 1 / 8 \\
1.7(0.7-4.1) ; 7 / 25 \\
1.5(0.2-13) ; 1 / 5 \\
0.10\end{array}$ & $\begin{array}{l}\text { InterLymph } \\
\text { classification }^{a} ; \text { OR } \\
1.4(0.3-7.0) ; 2 / 8 \\
1.7(0.7-4.0) ; 7 / 25 \\
3.2(0.6-18) ; 2 / 5 \\
0.055\end{array}$ & NR \\
\hline $\begin{array}{l}\text { Purdue et al. (2011a) } \\
\text { Incorporated into the } \\
\text { pooled analysis }\end{array}$ & $\begin{array}{l}\text { Average exposure } \\
\text { Per } 90 \mathrm{ppm}-\mathrm{hr} / \mathrm{week} \\
\mathrm{p}_{\text {trend }} \\
\text { Cumulative exposure } \\
\text { Per } 65,520 \mathrm{ppm}-\mathrm{hr} \\
\text { ptrend }\end{array}$ & $\begin{array}{l}\text { ICD-O-2; OR } \\
1.11(1.01-1.23) \\
0.03 \\
1.07(0.94-1.22) \\
0.29\end{array}$ & $\begin{array}{l}\text { ICD-O-2; OR } \\
1.15(1.04-1.28) \\
0.005 \\
1.17(1.04-1.32) \\
0.01\end{array}$ & $\begin{array}{l}\text { ICD-O-2; OR } \\
1.09(0.96-1.24) \\
0.16 \\
1.11(0.96-1.27) \\
0.16\end{array}$ & NR \\
\hline
\end{tabular}


RoC Monograph on Trichloroethylene

\begin{tabular}{|c|c|c|c|c|c|}
\hline Reference & Exposure Group & DLBCL & Follicular Lymphoma & CLL & Multiple Myeloma \\
\hline $\begin{array}{l}\text { Cocco et al. (2010) } \\
\text { Incorporated into } \\
\text { pooled analysis }\end{array}$ & $\begin{array}{l}\text { Ever exposed } \\
\text { Cumulative exposure } \\
\text { Low exposure } \\
\text { Medium exposure } \\
\text { High exposure } \\
\text { ptrend }\end{array}$ & $\begin{array}{l}2001 \text { WHO REAL } \\
\text { classification; OR } \\
0.7 \text { (0.4-1.1); } 17 \\
\\
0.7 \text { (CI NR); } 6 / 37 \\
0.4 \text { (CI NR); } 4 / 43 \\
0.9 \text { (CI NR); } 7 / 37 \\
0.16\end{array}$ & $\begin{array}{l}2001 \text { WHO REAL } \\
\text { classification; OR } \\
1.2 \text { (0.6-2.3); } 11 \\
\\
2.4 \text { (CI NR); } 7 / 37 \\
0.3 \text { (CI NR); } 1 / 43 \\
1.0 \text { (CI NR); } 3 / 37 \\
0.65\end{array}$ & $\begin{array}{l}2001 \text { WHO REAL } \\
\text { classification; OR } \\
0.9 \text { (0.5-1.5); } 18 \\
\\
1.0 \text { (CI NR); } 6 / 37 \\
0.4 \text { (CI NR); } 3 / 43 \\
1.2 \text { (CI NR); } 9 / 37 \\
0.94\end{array}$ & $\begin{array}{l}\text { 2001 WHO REAL } \\
\text { classification; OR } \\
0.6(0.3-1) \\
\\
0.2 \text { (CI NR); } 1 / 37 \\
0.7 \text { (CI NR); } 4 / 43 \\
0.8 \text { (CI NR); } 4 / 37 \\
0.22\end{array}$ \\
\hline $\begin{array}{l}\text { Deng et al. } \\
\text { (2013)/Wang et al. } \\
(2009 \text { b) }\end{array}$ & $\begin{array}{l}\text { Ever exposed } \\
\text { IL12A_07 genotype } \\
\text { TT } \\
\text { AA } \\
\text { p interaction }\end{array}$ & $\begin{array}{l}2001 \text { WHO REAL } \\
\text { classification; OR } \\
0.59(0.19-1.85) ; 4 \\
2.66(1.42-4.96) ; 21 \\
0.0119\end{array}$ & $\begin{array}{l}2001 \text { WHO REAL } \\
\text { classification; OR } \\
0.82(0.25-2.72) ; 4 \\
1.71(0.78-3.77) ; 10 \\
0.3498\end{array}$ & NR & NR \\
\hline $\begin{array}{l}\text { Gold et al. (2011) } \\
\text { Seattle Region (SEER) }\end{array}$ & $\begin{array}{l}\text { High confidence } 10 \text {-yr lag } \\
\text { Cumulative exposure } \\
\text { No exposure } \\
1-415 \\
416-3,000 \\
3,001-6,592 \\
6,593-49,500 \\
p_{\text {trend }}\end{array}$ & NR & NR & NR & $\begin{array}{l}I C D-O-2 / 3 ; O R \\
1.0 ; 139 / 409 \\
1.1(0.4-2.9) ; 6 / 18 \\
1.6(0.7-3.5) ; 11 / 20 \\
1.4(0.5-3.8) ; 6 / 16 \\
2.3(1.1-5.0) ; 18 / 18 \\
0.02\end{array}$ \\
\hline $\begin{array}{l}\text { Costantini et al. (2008) } \\
\text { Italy }\end{array}$ & $\begin{array}{l}\text { Exposure intensity } \\
\text { Very low/low } \\
\text { Medium/high } \\
\leq 15 \text { years exposure } \\
>15 \text { years exposure } \\
\text { p trend }^{\text {tron }}\end{array}$ & & & $\begin{array}{l}\text { ICD-9; OR } \\
1.2(0.5-2.7) ; 8 / 47 \\
0.9(0.3-2.6) ; 4 / 35 \\
0.7(0.1-3.4) ; 2 / 24 \\
1.2(0.2-6.2) ; 2 / 11\end{array}$ & $\begin{array}{l}\text { ICD-9; OR } \\
1.5(0.7-3.5) ; 9 / 28 \\
0.9(0.3-2.4) ; 5 / 27 \\
0.5(0.1-2.3) ; 2 / 19 \\
1.3(0.3-5.9) ; 8 / 13 \\
0.82\end{array}$ \\
\hline $\begin{array}{l}\text { Nordström et al. } \\
\text { (1998) }\end{array}$ & Ever exposed & & & $\begin{array}{l}H C L(I C D N R) ; O R \\
1.5(0.7-3.3) ; 9 / 26\end{array}$ & \\
\hline
\end{tabular}

DLBCL = diffuse large B-cell lymphoma; CLL = chronic lymphocytic leukemia; HR = hazard ratio; ICD = International Classification of Diseases; JEM = job-exposure matrix; $\mathrm{HCL}=$ hairy cell leukemia; NHL = non-Hodgkin lymphoma; NR = not reported; OR = odds ratio; ppm = parts per million; RR = relative risk; SIR = standardized incidence ratio; $\mathrm{SMR}=$ standardized mortality ratio; $\mathrm{SRR}=$ standardized rate ratio; TCE = trichloroethylene; WHO REAL = World Health Organization Revised European American Classification of Lymphoid Neoplasms.

${ }^{a}$ The InterLymph Consortium classification (see Morton et al. (2007)) was harmonized with the earlier WHO lymphoid neoplasms classification and the ICD-O-3. It combines chronic lymphocytic leukemia (CLL) with small lymphocytic lymphoma (SLL). 


\subsubsection{Meta-analyses of Trichloroethylene Exposure and NHL and Related Subtypes}

Several recent meta-analyses of NHL and trichloroethylene exposure have been conducted (Karami et al. 2013; Mandel et al. 2006; Scott and Jinot 2011; USEPA 2011a; Wartenberg et al. 2000). This evaluation is limited to the recent meta-analyses by the EPA (Scott and Jinot 2011; USEPA 2011a), Karami et al. (2012) and Kelsh et al. (2010) because many studies have been published since the older evaluations. Both meta-analyses were conducted prior to the publication of studies by Hansen et al. (2013), Vlaanderen et al. (2013), Christensen et al. (2013) and Bove et al. (2014) or the InterLymph study by Cocco et al. (2013)), all of which are included in our review. The individual studies contributing to the two pooled studies (Cocco et al. 2013; Hansen et al. 2013) were included in the meta-analysis; however, the pooled study by Hansen also updated their constituent cohorts.

The EPA meta-analyses included systematic data extraction of nine cohort and eight case-control studies in which potential trichloroethylene exposure was documented and risk estimates for NHL and trichloroethylene exposure were calculated. Studies with evidence of a low potential for exposure to trichloroethylene were excluded. Fixed and random-effects models, tests for heterogeneity and publication bias, and sensitivity analyses (to examine the impact of individual studies and selection of alternative relative risk selections on meta-relative risk estimates) were used to calculate summary meta-relative risks, using, where provided, adjusted or crude risk estimates from internal analyses rather than external (SMR or SIR) estimates. In addition, separate meta-analyses were conducted for the highest exposure groups (either by duration and/or intensity) within trichloroethylene-exposed populations (reported in 17 of the 19 constituent studies). Low to moderate heterogeneity among risk estimates was observed and there was some evidence of publication bias.

Karami et al. (2013) used similar exclusion criteria and methods of analysis to the EPA analysis and considered a closely overlapping body of 10 cohort and 9 case-control studies (see Table B-1 and Table D-10). Low to moderate heterogeneity but little evidence of publication bias was observed. Slightly higher $\mathrm{mRRs}$ were observed among the four European cohorts $(\mathrm{mRR}=1.66$, $95 \% \mathrm{CI}=1.29$ to 2.14$)$ than among the 6 U.S. cohorts $(\mathrm{mRR}=1.41,95 \% \mathrm{CI}=1.11$ to 1.78$)$, and among studies reporting NHL incidence compared with combined incidence and mortality.

In the EPA analysis, mRRs for the highest exposure groups within studies (where reported) were used to calculate mRRs for the highest exposure (intensity and/or duration) group(s) within studies. In contrast, Karami et al. calculated mRRs by high or low intensity of exposure and separately by high and low duration of exposure, based on a subset of studies that reported these metrics, thus yielding somewhat different mRR estimates from those in the EPA analysis. The summary mRRs for NHL are given in Table 5-4 below. 
Table 5-4. Meta-analyses of Trichloroethylene Exposure and NHL and Related Subtypesa

\begin{tabular}{|c|c|c|c|c|}
\hline Reference & $\begin{array}{l}\text { Study Design } \\
\text { (Number of } \\
\text { Studies) }\end{array}$ & $\begin{array}{c}\text { mRR }(95 \% \text { CI }) \\
\text { All }\end{array}$ & $\begin{array}{c}\text { mRR (95\% CI) } \\
\text { Highest Exposure }\end{array}$ & Comments \\
\hline \multirow{4}{*}{$\begin{array}{l}\text { EPA (2011a)/Scott } \\
\text { and Jinot (2011) }\end{array}$} & \multirow{2}{*}{$\begin{array}{l}\text { Combined cohort } \\
\text { and case-control } \\
\text { studies }\end{array}$} & \multirow[t]{4}{*}{$1.23(1.07-1.42)^{\mathrm{b}}$} & \multirow[t]{4}{*}{$1.43(1.13-1.82)$} & \multirow{3}{*}{$\begin{array}{l}\text { Random-effects model } \\
\text { Low sensitivity to removal } \\
\text { of individual studies or } \\
\text { selection of alternative RRs }\end{array}$} \\
\hline & & & & \\
\hline & Any exposure (17) & & & \\
\hline & High exposure (13) & & & $\begin{array}{l}\text { Low to moderate } \\
\text { heterogeneity; some } \\
\text { evidence of publication } \\
\text { bias }\end{array}$ \\
\hline $\begin{array}{l}\text { EPA (2011a)/Scott } \\
\text { and Jinot (2011) }\end{array}$ & Cohorts (9) & $1.33(1.13-1.58)$ & $1.60(1.24-2.08)$ & \multirow{2}{*}{$\begin{array}{l}\text { No sig. diff. between } \\
\text { cohort and case-control } \\
\text { mRRs (any or highest } \\
\text { exposure); lower } \\
\text { heterogeneity for highest } \\
\text { exposure groups }\end{array}$} \\
\hline $\begin{array}{l}\text { EPA (2011a)/Scott } \\
\text { and Jinot (2011) }\end{array}$ & Case-control (8) & $1.11(0.89-1.38)$ & $1.29(0.76-2.20)$ & \\
\hline \multirow[t]{2}{*}{ Karami et al. (2013) } & \multirow{2}{*}{$\begin{array}{l}\text { TCE-exposed } \\
\text { cohort + case- } \\
\text { control studies (19) }\end{array}$} & \multirow[t]{2}{*}{$1.32(1.14-1.54)$} & \multirow[t]{2}{*}{ NR } & Random-effects model \\
\hline & & & & $\begin{array}{l}\text { Little evidence of } \\
\text { heterogeneity and } \\
\text { publication bias }\end{array}$ \\
\hline \multirow[t]{7}{*}{ Karami et al. (2013) } & $\begin{array}{l}\text { TCE-exposed } \\
\text { cohorts }(10)\end{array}$ & \multirow[t]{6}{*}{$1.52(1.29-1.79)$} & & $\begin{array}{l}\text { Some evidence of positive } \\
\text { exposure response among a } \\
\text { total of } 4 \text { studies using }\end{array}$ \\
\hline & Exp-response: & & & measures of duration \\
\hline & Long duration & & $1.56(1.02-2.40)$ & \multirow{5}{*}{$\begin{array}{l}\text { Negative exposure } \\
\text { response observed among } \\
5^{\text {b }} \text { studies using measures } \\
\text { of intensity (excluding } 3 \\
\text { Nordic studies of U-TCA) }\end{array}$} \\
\hline & Short duration & & $1.30(0.92-1.84)$ & \\
\hline & High intensity $^{\mathrm{c}}$ & & $1.27(0.83-1.96)$ & \\
\hline & Low intensity & & $1.68(1.14-2.46)$ & \\
\hline & $\begin{array}{l}\text { Subset of U-TCA } \\
\text { studies (3) }\end{array}$ & $2.15(1.34-3.45)$ & & \\
\hline \multirow[t]{6}{*}{ Karami et al. (2013) } & $\begin{array}{l}\text { TCE-exposed case- } \\
\text { control (9) }\end{array}$ & $1.14(0.93-1.40)$ & & $\begin{array}{l}\text { Some evidence of } \\
\text { publication bias }\end{array}$ \\
\hline & Exp-response: & & & \multirow{5}{*}{$\begin{array}{l}\text { No association between } \\
\text { exposure duration among } 2 \\
\text { studies or intensity among } \\
3 \text { studies }\end{array}$} \\
\hline & Long duration & & $1.18(0.60-2.34)$ & \\
\hline & Short duration & & $1.46(0.78-2.73)$ & \\
\hline & High intensity & & $1.42(0.86-2.33)$ & \\
\hline & Low intensity & & $1.06(0.79-1.42)$ & \\
\hline
\end{tabular}

$\mathrm{mRR}=$ meta-relative risk; $\mathrm{NR}=$ not reported; $\mathrm{RR}=$ relative risk; $\mathrm{U}-\mathrm{TCA}=$ urine trichloroacetic acid.

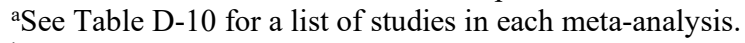

${ }^{\mathrm{b}}$ Adjustment for publication bias yielded $\mathrm{mRR}=1.15(95 \% \mathrm{CI}=0.97-1.36)$.

${ }^{\mathrm{c}} 6$ studies cited in text, 5 in table.

The overall results of both meta-analyses (Karami et al. 2013; Scott and Jinot 2011; USEPA 2011a), are broadly comparable. Both show statistically significant mRRs for cohort and casecontrol studies combined and the body of cohort studies. The mRR for case-control studies was 
lower, but not significantly different, than the mRR for cohort studies (Scott and Jinot 2011). Importantly, the mRR was robust and not sensitive to the removal of individual studies or selection of alternative RRs. Overall, there was evidence from the EPA meta-analysis, among the body of cohort studies, and to a somewhat lesser extent among the case-control studies, that the risk of NHL is greater in the subgroups with the highest exposure compared with the overall exposure groups. In the analysis by Karami et al. (2013), associations between intensity or duration of exposure were less clear; the differences may be attributable to the smaller number of studies, the use of separate analyses of intensity and duration, less comparability between highand low-exposure groups, or some differences in the included studies. The highest mRR was observed among the three Nordic studies using biomonitoring of urine TCA (Karami et al. 2013), which was not observed in the later pooled and updated study by Hansen et al. (2013). Neither meta-analysis included the InterLymph pooled analyses, although they included three of the component studies, two of which were null. Substitution of the component studies with the InterLymph study could possibly strengthen the association with trichloroethylene exposure in the meta-analysis of case-control studies (higher risk, less heterogeneity). The more recent metaanalysis (Karami et al. 2013) found a stronger association among studies that specifically assessed trichloroethylene than among studies of broadly assessed chlorinated solvents, in which effects from trichloroethylene would be diluted. They also calculated meta-relative risks among studies conducted across two different calendar time periods (divided by the median year of publication) and observed no differences for cohort studies of NHL and kidney cancer (but an increase in $\mathrm{mRR}$ for case-control studies of kidney cancer conducted since 1995). The latter finding, according to the authors, suggested "possible improvements" in the validity and reliability of exposure assessment methods in case-control studies (Karami et al. 2013).

Meta-analyses for other NHL subtypes have been largely inconclusive and were based on a small number of studies. Karami et al. (2013) conducted an analysis of multiple myeloma and CLL among the studies reporting for these endpoints that were included in their NHL meta-analysis and found no significant increases in risk. However, this meta-analysis did not include recent studies reporting on one or other of these endpoints (Bove et al. 2014; Cocco et al. 2013; Costantini et al. 2008; Gold et al. 2011; Hansen et al. 2013; Vlaanderen et al. 2013).

\subsubsection{Evaluation of Potential Confounding by Occupational Co-exposures or Other Risk Factors}

Section 3 discussed the adequacy of the methods used in the cohort (Section 3.2) and casecontrol studies (Sections 3.3 and 3.4) for evaluating potential confounding from occupational coexposures and non-occupational factors. However, that assessment was not specific for NHL. This section builds on that assessment, integrating it with other relevant information and evaluating whether confounding can explain the increased risks of NHL and its related subtypes observed in a number of the studies.

\subsubsection{Occupational Co-exposures}

The major occupational risk factors that have been associated with NHL (with limited evidence) include benzene, ethylene oxide, 2,3,7,8-TCDD, polychlorinated biphenyls, phenoxy herbicides, styrene, and ionizing radiation by IARC and the Report on Carcinogens (Cogliano et al. 2011; NTP 2011). Organic solvents may be potential risk factors and have been the focus of the recent EPILYMPH study (Cocco et al. 2010). The most common co-exposures in the cohort studies are 
the chlorinated and possible other organic solvents and cutting oils such as mineral and petroleum oils. Radiation is a possible co-exposure in the two studies of uranium workers (Bahr et al. 2011; Yiin et al. 2009). Other organic solvents, and possibly other exposures, may be coexposures in two of the Swedish studies (Hardell et al. 1994; Nordström et al. 1998). Benzene was also a potential co-exposure in the InterLymph study (Cocco et al. 2013).

Tetrachloroethylene exposure was correlated with trichloroethylene exposure in the Nordic population-based cohort; exposure to tetrachloroethylene but not to trichloroethylene was associated with increased risks of NHL.

None of the cohort and most of the case-control studies that reported NHL findings attempted to examine or control for potential confounding by co-exposures. The InterLymph study conducted sensitivity analyses that excluded subjects with benzene exposure; risks were elevated for both high probability $(\mathrm{OR}=1.4,95 \% \mathrm{CI}=0.8$ to 2.6$)$ and high intensity of exposure $(\mathrm{OR}=1.9,95 \%$ $\mathrm{CI}=1.2$ to 3.0$)$ in the total cohort and analyses restricted to subjects with high probability of exposure (OR not reported), but trends were no longer apparent.

Several lines of evidence argue against a major impact from confounding of potential coexposures across studies. None of the documented co-exposures are identified risk factors for NHL and the types and co-exposures of other agents are likely to vary in the patterns and levels across the various industries and time periods, especially in the studies of mixed occupation groups. In addition, an exposure-response relationship for NHL and exposure intensity was observed in the InterLymph study (considered to be the most informative study) and there was no evidence that other potential co-exposures were highly correlated with trichloroethylene exposure in that study. However, potential confounding by other solvents or chlorinated solvents may be possible, especially in the aircraft-manufacturing studies.

\subsubsection{Lifestyle and Other Potential Confounders}

Of the non-occupational risk factors for NHL, which include viral infections, immunosuppressive disorders, autoimmune diseases, and exposure to immunosuppressive drugs or chemotherapy drugs (Cogliano et al. 2011; Hardell and Axelson 1998), there is little a priori reason to suspect that most of these would vary by trichloroethylene-exposure status in the cohort or nested case-control studies. Smoking is not considered to be a risk factor for NHL but may be a risk factor for follicular lymphoma. Each case-control study matched or adjusted for age, sex, birth year, or race, using conditional or unconditional logistic regression, as appropriate. Some studies (Christensen et al. 2013; Costantini et al. 2008; Deng et al. 2013; Wang et al. 2009b), and some of the constituent studies of the pooled analysis (Cocco et al. 2010; Miligi et al. 2006; Purdue et al. 2011a) also considered or adjusted for smoking, other lifestyle factors, and surrogates of socioeconomic status. Thus, confounding by these factors across studies seems unlikely.

\subsubsection{Integration across Studies}

Overall, there is some evidence of an association between exposure to trichloroethylene and NHL based on findings of a modest increase in risk of NHL in several studies with different study designs and in different populations, although the strength of the evidence varied (see Figure 5-1). The strongest evidence of an association between exposure to trichloroethylene and NHL comes from the InterLymph pooled analysis ( $p$ for Fisher combined probability $=0.004$ ), which was considered to be the most informative study and is supported by findings of relatively 
small, mostly statistically non-significant increases $(>20 \%)$ in NHL risk among workers exposed to trichloroethylene in most studies of moderate (Hansen et al. 2013; Radican et al. 2008) or low to moderate utility (Deng et al. 2013; Lipworth et al. 2011; Morgan et al. 1998; RaaschouNielsen et al. 2003; Wang et al. 2009b). Limitations in studies would primarily bias findings toward the null. The high increased risk reported by Hardell et al. (1994) should be viewed with caution because it was based on small numbers of exposed cases and controls and this study had several methodological limitations. There was little evidence $(\leq 20 \%)$ (Bove et al. 2014; Persson and Fredrikson 1999) to no evidence (OR $\leq 1.0)$ (Bahr et al. 2011; Silver et al. 2014; Vlaanderen et al. 2013) for an association in most studies considered to be of lower utility, primarily because of low sensitivity to detect an effect. (Only 1 case of NHL was reported by Boice et al. (2006).)

Statistically significant increases in NHL risk were found in two recent meta-analyses $(\mathrm{mRR}=1.23,95 \% \mathrm{CI}=1.07$ to 1.42 , Scott and $\operatorname{Jinot}(2011) ; \mathrm{mRR}=1.23,95 \% \mathrm{CI}=1.07$ to 1.42, (Karami et al. 2013) for combined cohort and case-control studies). In the meta-analysis by Scott and Jinot, the mRR was robust and not sensitive to removal of individual studies or use of alternative risk estimates. In the most recent meta-analysis, there was little evidence of heterogeneity or publication bias (for the analysis of the combined cohort and case-control studies); however, there was some evidence for both publication bias and low to moderate heterogeneity in the EPA meta-analysis.

Some, but not all, studies found evidence for exposure-response relationships. The InterLymph study (Cocco et al. 2013) found that the risk of NHL increased with longer duration and higher intensity of exposure, and its constituent study by Purdue et al. (2011a) also found exposureresponse relationships with other exposure metrics, including average weekly exposure and cumulative exposure. The study of Connecticut women (Deng et al. 2013; Wang et al. 2009b) found higher risks among women with the medium-high exposure intensity compared with women with low exposure. However, evidence for an exposure-response relationship was lacking among cohort studies (Hansen et al. 2013; Lipworth et al. 2011; Raaschou-Nielsen et al. 2003; Radican et al. 2008) and in some cases risks were lower among the higher exposed compared with the lowest exposed. These studies had limited ability to evaluate exposureresponse relationships because of low statistical power or concerns about exposure misclassification. The EPA meta-analyses found a somewhat higher risk in analyses of high exposure than ever exposure; however, the latest meta-analysis found some evidence for exposure response with duration but not intensity. This pattern could possibly change with the inclusion of the InterLymph study.

No biases (such as selection) were identified that would bias towards a positive association, although the direction of the biases were not known in the studies by Hardell et al. (1994) and Persson and Fredrikson (1999) (see Figure 5-2). Confounding by other co-exposures can be ruled out reasonably in most of the large case-control studies and the Nordic studies of workers in diverse industries because the co-exposures to other agents are likely to vary in pattern and levels across the various industries and different time periods. Confounding by other solvents or chlorinated solvents may be possible in the aircraft-manufacturing studies. No evidence for confounding by lifestyle factors was found. 


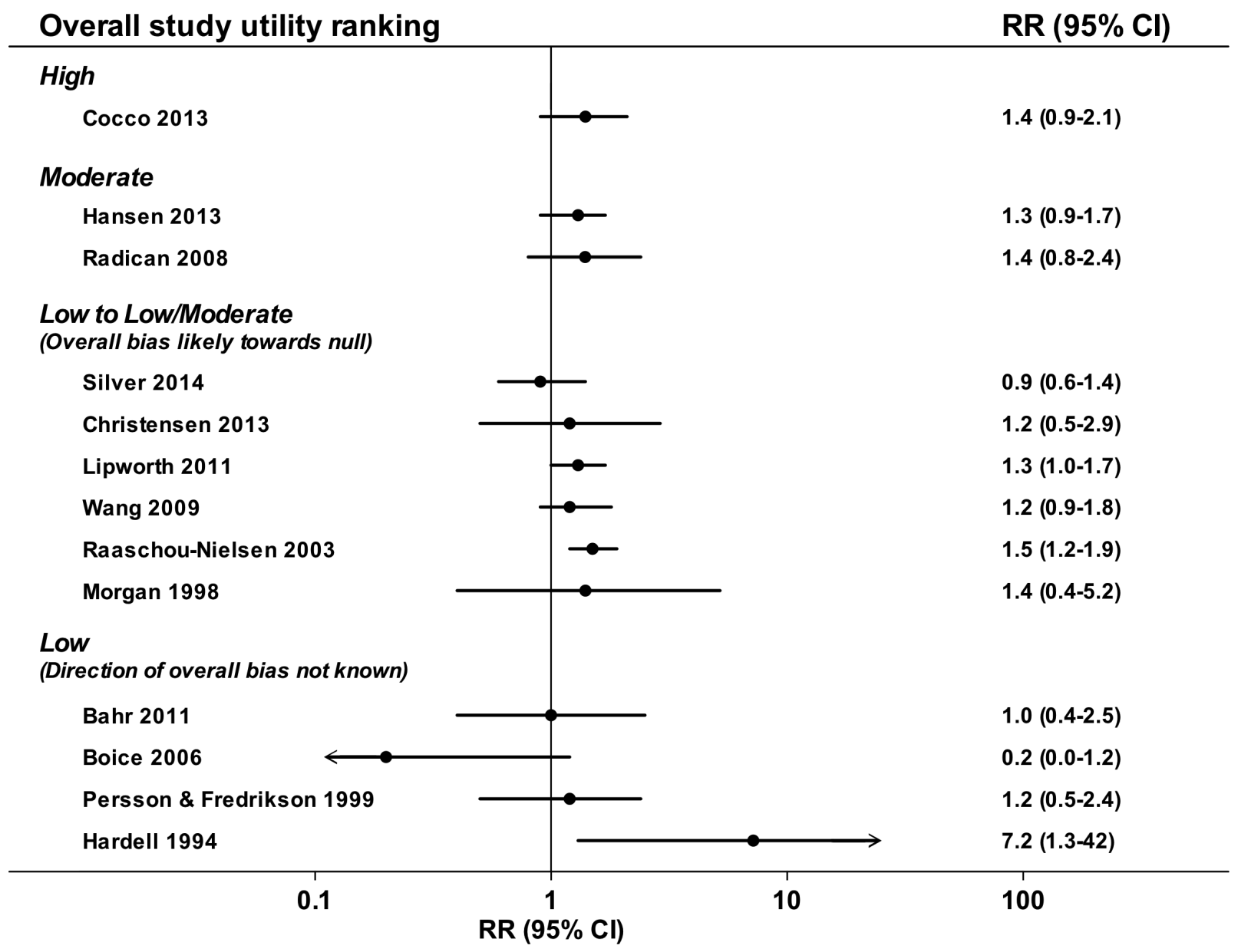

Figure 5-2. Forest Plot: NHL and Ever Exposure to Trichloroethylene

Effect estimate and 95\% CI for ever exposure to trichloroethylene and NHL (see Figure 5-1) and overall prediction of direction of any bias (if possible) for low utility studies as described in Section 4.1.3 according to utility of the studies to inform the cancer hazard evaluation (see Section 3, Appendix D, and Figure 5-1). The effect estimate for Boice et al. (2006) is based on one NHL case. Studies by Bove et al. (2014) and Vlaanderen et al. (2013) are not graphed because they did not report relative risk for ever exposure. Findings for these studies are reported in Table 5-1. For studies reporting multiple effect estimates, a preference was given to internal analysis; the effect estimate using internal analysis (RR) for Morgan et al. (1998) was reported by Scott and Jinot (2011).

No association between NHL and exposure was found in some cohort studies with more limited designs or limited statistical power (Bahr et al. 2011; Bove et al. 2014; Morgan et al. 1998) and the recent, large population-based Nordic cohort study (Vlaanderen et al. 2013) in which exposures were likely low and non-differential exposure misclassification was likely. The studies by Vlaanderen et al. and Bove et al. were not included in the most recent meta-analyses.

With respect to related subtypes of NHL, the strongest evidence of an association for follicular lymphoma and CLL is based on positive associations in the InterLymph study (Cocco et al. 2013) and its constituent study, the NCI-SEER study by Purdue et al. (2011a). The most informative study on multiple myeloma (Gold et al. 2011) reported a statistically significant increase in incidence with increasing cumulative exposure. Weaker associations were found in some of the cohort studies. The meta-analysis on trichloroethylene exposure and NHL and 
related subtypes by Karami et al. (2013) also reported a meta-risk of $1.05(95 \% \mathrm{CI}=0.88$ to 1.27) for multiple myeloma and $0.98(95 \% \mathrm{CI}=0.69$ to 1.41$)$ for combined chronic/small cell leukemia.

\subsection{Mechanistic Data for NHL and Related Neoplasms}

An increased risk of NHL and related neoplasms (e.g., follicular lymphoma, multiple myeloma, chronic lymphocytic leukemia) was identified in some epidemiological studies of humans exposed to trichloroethylene (see Section 5.1). In addition, increased incidences of lymphoma (female mice) and leukemia (female rats) were reported in experimental animals exposed to trichloroethylene (NTP 2011). Although the modes of action of trichloroethylene-induced NHL and related neoplasms are unknown, the key events may be related to effects of trichloroethylene on the immune system. There are also studies in humans and experimental animals that have evaluated the relationship between trichloroethylene and immunotoxicity or markers of immunomodulation. The following sections include a brief review of risk factors for NHL (Section 5.2.1) the immune effects of trichloroethylene in humans and experimental animals (Section 5.2.2), possible modes of action for trichloroethylene-induced immune modulation and NHL (Section 5.2.3). Section 5.2.4 summarizes the information.

\subsubsection{Risk Factors for NHL}

Many known risk factors for NHL are related to mechanisms involving chronic antigenic stimulation due to immunomodulation, including autoimmunity and/or immunosupression (Grulich et al. 2007; Hardell et al. 1998; Ponce et al. 2014). Both clinical and experimental data clearly show that chronic inflammation mediated by immunoglobulins and immune complexes contributes to cancer development (Balkwill et al. 2005; Coussens and Werb 2002; de Visser et al. 2006; Tan and Coussens 2007). Increased incidences of NHL have been reported among patients with congenital immune deficiency, autoimmune disease, or virus infection (e.g., HIV, human T-cell leukemia/lymphoma virus, Epstein-Barr virus); patients receiving immunosuppressive therapy following bone marrow or organ transplants; or as a late complication of certain chemotherapy and radiotherapy regimens for Hodgkin lymphoma (Aligo et al. 2014; Bernatsky et al. 2006; Besson et al. 2006; Hardell et al. 1998; Ponce et al. 2014).

Autoimmune disorders associated with an increased risk of NHL and related neoplasms include rheumatoid arthritis, systemic lupus erythematosus, Sjögren syndrome, sarcoidosis, and systemic sclerosis (scleroderma) (Ponce et al. 2014). NHL (predominantly B cell) accounts for about half the cancers observed in patients with primary immunodeficiencies and increases to about $75 \%$ in patients diagnosed with severe combined immunodeficiency (Ponce et al. 2014). Lymphoma risk also appears to increase with autoimmune disease severity. In addition, exposure to various immunotoxic industrial chemicals and pesticides (e.g., phenoxyacetic acids, chlorophenols, dioxins, organic solvents, DDT, PCBs, toxaphene, and chlordane) are recognized risk factors for NHL (Cantor et al. 1992; Hardell et al. 1998).

Although NHL includes many subtypes (e.g., diffuse large B cell lymphoma, T cell NHL, follicular lymphoma, chronic lymphocytic leukemia, and others), diffuse large B cell lymphoma is the most common among patients with autoimmune disorders. Autoimmune disorders are characterized by B cell hyperactivity and chronic inflammation. Lymphomas can develop from errors arising during the hypermutable stages of B cell development and can arise from either 
chronic antigenic stimulation (inflammation or autoimmunity) or from impaired pathogen control (immunosuppression). B cells initiate autoimmunity through several mechanisms including enhanced production of autoantibodies and immune complexes, dendritic and $\mathrm{T}$ cell activation, and cytokine production (Tan and Coussens 2007). The susceptibility of mature B cells to oncogenic transformation is due to DNA hypermutation and recombination during immunogeninduced activation and differentiation and results from the increased risk of genetic damage (e.g., double-strand breaks and chromosomal translocations) resulting from these processes during $\mathrm{B}$ cell maturation (Baecklund et al. 2014; Ponce et al. 2014).

Markers that may be important indicators for NHL risk include autoantibodies, lymphocyte subsets and activated lymphocytes, immunoglobulins, serum cytokines, and natural killer (NK) cell cytotoxicity. Several studies have reported an association between immune biomarkers and risk of NHL. Case-control studies using pre-diagnostic blood or serum and cohort studies of several immune biomarkers have reported predictive value for some lymphocyte subsets or immune markers and NHL. Several biomarkers or cytokines (such as sCD27, sCD30, sCD44, CXCL13, CD30, TNF-R1, sTNF2, BCA-1, vascular endothelial growth factor receptor, intercellular adhesion molecule (ICAM), IL-2, IL-10) are associated with NHL (Conroy et al. 2013; De Roos et al. 2012; Hosnijeh et al. 2010; Purdue et al. 2011b; Vermeulen et al. 2011).

Most malignancies, as well as pre-malignant tissues associated with chronic inflammatory diseases, have an altered immune cell status (Dalgleish and O'Byrne 2002; Tan and Coussens 2007). These alterations frequently include suppressed cell-mediated immunity and enhanced humoral immunity marked by a decrease in Th1 T helper cells and an increase in Th2 Thelper cells (Tan and Coussens 2007) and the cytokines, i.e., interleukins or interferon, that they produce. A number of case-control studies have reported that polymorphisms in genes coding for immunoregulatory cytokines that mediate inflammation, apoptosis, and Th1/Th2 balance influence susceptibility to NHL (Bel Hadj Jrad et al. 2006; Deng et al. 2013; Hosnijeh et al. 2010; Lan et al. 2006; Purdue et al. 2007; Rothman et al. 2006; Wang et al. 2007c).

\subsubsection{Immune Effects of Trichloroethylene}

The effects of trichloroethylene on the immune system have been investigated in humans (Section 5.2.2.1) and experimental animals (Section 5.2.2.2). In addition, some studies have looked at biomarkers for immunomodulation. Of interest is whether changes in these biomarkers are consistent with proposed pathways for lymphoma development.

\subsubsection{Studies of Immunomodulation in Humans}

This section summarizes the findings of studies (1) reporting risk estimates for autoimmune diseases and trichloroethylene exposure, (2) of trichloroethylene-induced skin hypersensitivity and (3) evaluating the relationship of trichloroethylene and biomarkers of immunomodulation. None of the studies evaluated phenotypic markers that would directly demonstrate immune suppression. The major limitation is they did not examine NHL or other disease.

Studies of trichloroethylene exposure and autoimmune diseases consisted of four case-control studies of systemic sclerosis (sclerodoma) (Diot et al. 2002; Garabrant et al. 2003; Nietert et al. 1998), including one pooled analysis of these studies (Cooper et al. 2009), and one case-control study of undifferentiated connective tissue disease (Lacey et al. 1999). Results from these studies 
are summarized in Table 5-5. No epidemiological studies of trichloroethylene exposure and rheumatoid arthritis or other autoimmune diseases were identified.

There is consistent evidence from the body of studies that trichloroethylene exposure is associated with scleroderma for men, but not consistently for women (Diot et al. 2002; Garabrant et al. 2003; Marie et al. 2014; Nietert et al. 1998); see Table 5-5. One study found a stronger association of cumulative and maximum intensity exposure to TCE and systemic sclerosis among both men and women who tested positive for anti-Scl-70 autoantibody compared with those who tested negative for the antibody (Nietert et al. 1998). The studies have somewhat limited exposure assessments and statistical power due to small numbers of exposed cases to detect an effect of exposure, however. A strength of the studies was that they considered potential demographic or lifestyle confounders. In a pooled analysis of three of the four casecontrol studies of scleroderma, combined ORs of $2.5(95 \% \mathrm{CI}=1.1$ to 5.4$)$ among men and 1.2 $(95 \% \mathrm{CI}=0.6$ to 2.6$)$ among women were reported (Cooper et al. 2009). The data were insufficient to evaluate the findings for undifferentiated connective tissue disease since there was only one exposed case in the only study reporting on this disease (Lacey et al. 1999).

Cases of severe generalized dermatitis (i.e., hypersensitivity skin disorders) also were reported among workers in China (Dai et al. 2004; Dai et al. 2009; Huang et al. 2006; Huang et al. 2012; Kamijima et al. 2008; Kamijima et al. 2013; Liu et al. 2009) and in Japan, the United States, Canada, and Spain (see also review by Watanabe 2011). Disease onset usually occurs within 2 to 5 weeks of exposure, resembles severe drug-induced hypersensitivity syndrome, and is associated with elevated inflammatory responses, oxidative stress, and reactivation of latent human herpesvirus 6 (Huang et al. 2006; Huang et al. 2012; Kamijima et al. 2013). The cases of hypersensitivity skin disorders are frequently accompanied by immune-mediated (toxic) hepatitis and liver dysfunction (Huang et al. 2006; Kamijima et al. 2013; see also review by Watanabe 2011). Cases of idiosyncratic toxic hepatitis have also been reported in Korean workers occupationally exposed to trichloroethylene (see review by Kim and Kim 2010). An immunologic-type reaction was thought to be responsible because disease onset was sporadic, generally not dose related, and usually occurred after 30 days of exposure. Although these reports do indicate that exposure to trichloroethylene could be related to the observed immunomodulation, no studies evaluated whether these effects could be linked to NHL.

Studies of trichloroethylene exposure and biomarkers of immunomodulation (e.g., lymphocyte subset populations, antibodies, or other biomarkers of immune function) included both occupational and population-based studies. The occupational studies consisted of a series of studies of trichloroethylene-exposed workers in metalworking and electronic factories in Guangdong province, China (Bassig et al. 2013; Dai et al. 2004; Hosgood et al. 2012; Huang et al. 2006; Huang et al. 2012; Kamijima et al. 2008; Lan et al. 2010; Liu et al. 2009; Zhang et al. $2013 b$ ) and a study in the Italian printing industry (Iavicoli et al. 2005). The population studies included two prospective studies of immune markers among a birth cohort exposed pre- and postnatally to trichloroethylene in Leipzig, Germany (Lehmann et al. 2001; Lehmann et al. 2002). Further details of these studies are provided in Table 5-6.

The series of cross-sectional studies of metal and electronics workers in Guangdong province, China, and the Italian study of printing workers conducted the most extensive exposure assessments and provided clear evidence that subjects were exposed to moderate to high levels of trichloroethylene. Studies by Lan et al. (2010), Hosgood et al. (2012), Bassig et al. (2013), and 
Zhang et al. (2013b) were conducted on total lymphocyte and specific subsets. Lan et al. (2010) reported that workers exposed to trichloroethylene had dose-related statistically significant lower counts of total lymphocytes, B cells and specific subsets of T lymphocytes (CD4+, CD8+) and natural killer cells in peripheral blood compared with unexposed controls. A further analysis found significant decreases in CD4+ and CD8 naïve and CD4+ effector memory cells but not other types of CD4+ (central memory) and CD8 (memory) subsets or T cell regulation subsets among trichloroethylene workers compared with controls (Hosgood et al. 2012). Trichloroethylene-exposed workers had lower serum levels of IgG, IgM, and lower levels of $\mathrm{CD} 27$, and sCD30 cells (members of the TNF receptor family that help regulate cellular activity of T, B and natural killer cells) (Lan et al. 2010) suggesting that trichloroethylene impairs B cell stimulation. Bassig et al. (2013) also reported lower serum levels of IL-10 among exposed workers, which may indicate chemically induced alterations in Th1/Th2 balance. Iavicoli et al. (2005) also reported lower serum levels of the Th2 cytokine, IL-4, and increased levels of the Th1 cytokines, IL-2 and interferon-gamma, among trichloroethylene-exposed workers. Overall, these studies provide some evidence of immune modulation associated with trichloroethylene exposure, and possibly with measures of precursors of autoimmunity (e.g., IFN- $\gamma$ ).

Two studies of possible immune effects of trichloroethylene in children and infants were identified but they are of limited utility, in part due to the limited exposure assessment of maternal and child exposure and low reported overall levels of trichloroethylene. The German birth cohort studies of children with atopy (Lehmann et al. 2001) potentially exposed to trichloroethylene and other volatile organic compounds reported no association for trichloroethylene exposure and allergies and IL-4 and IFN- $\gamma$-producing T cells or cytokines. In the study of infants (Lehmann et al. 2002), increasing trichloroethylene exposure was associated with a statistically significant decrease in IL-4-producing $\mathrm{T}$ cells and non-statistically significant increase in IFN- $\gamma$-producing T cells but not with IL-2- (in multivariate analysis) or TNF- $\alpha$ producing T cells. An immunosuppressive effect of trichloroethylene is suggested by the significant reduction reported for IL-2-producing T cells in cord blood. However, due to the limited exposure noted above and the correlation of trichloroethylene with other volatile organic compounds and the small number of subjects available for cytokine analysis (in the children's study), no conclusions can be drawn from these studies. 
Table 5-5. Case-control Studies of Trichloroethylene Exposure and Autoimmune Diseases in Humans ${ }^{\mathrm{a}}$

\begin{tabular}{|c|c|c|c|c|}
\hline Reference & $\begin{array}{l}\text { Study Population } \\
\text { \# Cases \& Controls }\end{array}$ & Exposure Assessment & $\begin{array}{c}\text { OR (95\% CI); \# of Exposed } \\
\text { Cases }\end{array}$ & Comments \\
\hline \multicolumn{5}{|c|}{ Systemic sclerosis (SSc) } \\
\hline $\begin{array}{l}\text { Nietert et al. } \\
(1998) \\
\text { South Carolina } \\
\text { (USA) }\end{array}$ & $\begin{array}{l}\text { Hospital-based case- } \\
\text { control study } \\
178 \text { cases of SSc } \\
200 \text { unmatched clinic } \\
\text { controls free of } \\
\text { autoimmune and } \\
\text { connective tissue disease }\end{array}$ & $\begin{array}{l}\text { Structured interview of self- } \\
\text { reported job history (titles, } \\
\text { industry, task) } \\
\text { Semi-quantitative JEM with expert } \\
\text { review to assign scores of intensity } \\
\text { and probability of solvents for } \\
\text { each job. Cumulative exposure } \\
\text { intensity also factored in duration, } \\
\text { frequency, and calendar year }\end{array}$ & $\begin{array}{l}\text { Total cases } \\
\text { Cumulative intensity } \\
2.0(0.7-5.3) ; 32 \mathrm{M} \\
1.2(0.5-2.6) ; 10 \mathrm{~W} \\
\text { Maximum intensity } \\
3.3(1.0-10.3) ; 30 \mathrm{M} \\
0.9(0.3-2.3) ; 6 \mathrm{~W} \\
\text { Anti-Scl-70 } \mathrm{SSc} \text { cases } \\
\text { Cumulative intensity } \\
2.6^{*} \mathrm{M} ; 4.0^{*} \mathrm{~W} \\
\text { Maximum intensity } \\
4.8^{*} \mathrm{M} ; 0.9 \mathrm{~W}\end{array}$ & $\begin{array}{l}\text { Covariates/consideration of confounding: } \\
\text { Adjusted for age at disease onset; findings } \\
\text { stratified by sex. On average, cases were } \\
\text { younger than controls. A greater proportion } \\
\text { of cases were women than controls, but } \\
\text { racial distribution was similar } \\
\text { Strengths: Relatively large study; analysis } \\
\text { by multiple matrices of exposure and disease } \\
\text { subtypes } \\
\text { Limitations: Limited exposure assessment; } \\
\text { no control for possible co-exposures to other } \\
\text { solvents and other potential confounders }\end{array}$ \\
\hline $\begin{array}{l}\text { Diot et al. (2002) } \\
\text { France }\end{array}$ & $\begin{array}{l}\text { Hospital-based case- } \\
\text { control study } \\
80 \text { cases of SSc } \\
160 \text { matched (age, } \\
\text { gender, smoking habits) } \\
\text { controls without known } \\
\text { autoimmune or chronic } \\
\text { interstitial lung disease }\end{array}$ & $\begin{array}{l}\text { Structured interview of self- } \\
\text { reported job history (appears self- } \\
\text { reported solvent exposures) } \\
\text { Semi-quantitative/expert } \\
\text { assessment to assign scores for } \\
\text { probability, intensity, frequency, } \\
\text { and duration of exposure for each } \\
\text { employment period; cumulative } \\
\text { exposure sum of exposure scores } \\
\text { for all employment periods }\end{array}$ & $\begin{array}{l}\text { Ever vs. never exposure } \\
4.7(1.0-21.9) ; 7 \mathrm{M} \\
2.1(0.7-6.8) ; 6 \mathrm{~W} \\
\text { High cumulative exposure } \\
7.6(1.5-37.4) ; 7 \mathrm{M}+\mathrm{W}\end{array}$ & $\begin{array}{l}\text { Consideration of confounding: } \\
\text { Socioeconomic level, professional status, } \\
\text { age, sex, and smoking habits similar } \\
\text { between cases and controls. No subjects } \\
\text { reported history of silicone implants, } \\
\text { cosmetic surgery, frequency of hair dyes or } \\
\text { drug use (which may be associated with } \\
\text { SSc) } \\
\text { Strengths: Analysis of high cumulative } \\
\text { exposure reduces potential for exposure } \\
\text { misclassification. Consideration of potential } \\
\text { confounding } \\
\text { Limitations: Limited statistical power for } \\
\text { TCE exposure, no adjustment for possible } \\
\text { co-exposures to other solvents }\end{array}$ \\
\hline
\end{tabular}




\begin{tabular}{|c|c|c|c|c|}
\hline Reference & $\begin{array}{l}\text { Study Population } \\
\text { \# Cases \& Controls }\end{array}$ & Exposure Assessment & $\begin{array}{c}\text { OR (95\% CI); \# of Exposed } \\
\text { Cases }\end{array}$ & Comments \\
\hline $\begin{array}{l}\text { Garabrant et al. } \\
\text { (2003) } \\
\text { Michigan \& } \\
\text { Ohio (USA) }\end{array}$ & $\begin{array}{l}\text { Population-based case- } \\
\text { control study } \\
660 \text { cases } \\
2,227 \text { matched (race, age, } \\
\text { and geographical region) } \\
\text { controls without SSc } \\
\text { identified by RDD }\end{array}$ & $\begin{array}{l}\text { Structured interview of self- } \\
\text { reported job and hobby exposure } \\
\text { to } 9 \text { solvents and PPE } \\
\text { Self-reported exposure to solvent } \\
\text { confirmed by expert review of job } \\
\text { history }\end{array}$ & $\begin{array}{l}\text { Women only } \\
\text { Self-reported } \\
2.0(0.8-4.8) ; 8 \\
\text { Confirmed by expert review } \\
1.9(0.6-6.6) ; 4\end{array}$ & $\begin{array}{l}\text { Covariates/consideration of confounding: } \\
\text { Adjusted for age, race, region, and year of } \\
\text { birth. Race/ethnicity, education, marital } \\
\text { status, frequency of smoking, and alcohol } \\
\text { consumption were similar among cases and } \\
\text { controls. Current smoking more common in } \\
\text { controls and annual income higher in } \\
\text { controls } \\
\text { Limitations: Potential for exposure } \\
\text { misclassification because of limited } \\
\text { exposure assessment; small number of } \\
\text { exposed cases and controls no control for } \\
\text { co-exposures to solvents }\end{array}$ \\
\hline $\begin{array}{l}\text { Cooper et al. } \\
(2009)\end{array}$ & $\begin{array}{l}\text { Pooled case-control } \\
\text { analysis (Diot et al. 2002; } \\
\text { Garabrant et al. 2003; } \\
\text { Nietert et al. 1998) }\end{array}$ & See individual studies in this table & $\begin{array}{l}2.5(1.1-5.4) \mathrm{M} \\
1.2(0.6-2.6) \mathrm{W}\end{array}$ & $\begin{array}{l}\text { Evidence of association with TCE exposure, } \\
\text { mostly in men }\end{array}$ \\
\hline $\begin{array}{l}\text { Marie et al. } \\
(2014) \\
\text { France }\end{array}$ & $\begin{array}{l}\text { Population-based case- } \\
\text { control study } \\
100 \text { cases } \\
300 \text { controls matched (for } \\
\text { age, gender, and smoking } \\
\text { habits) without history of } \\
\text { connective tissue disease, } \\
\text { systemic vasculitis, other } \\
\text { autoimmune systemic } \\
\text { disease, cancer, or } \\
\text { chronic interstitial lung } \\
\text { disease }\end{array}$ & $\begin{array}{l}\text { Structured interview of self- } \\
\text { reported job history } \\
\text { Semi-quantitative/expert } \\
\text { assessment assignment of } \\
\text { probability, intensity, frequency, } \\
\text { and duration of exposure for each } \\
\text { employment period; cumulative } \\
\text { exposure based on sum of } \\
\text { exposure scores for all } \\
\text { employment periods }\end{array}$ & $\begin{array}{l}\text { Ever exposure } \\
2.8(0.8-9.4) ; 8 \mathrm{M} \\
1.4(0.3-5.4) ; 4 \mathrm{~W} \\
\text { High cumulative exposure } \\
(M+W) \\
3.6(1.2-12.09) ; 8\end{array}$ & $\begin{array}{l}\text { Consideration of confounding: No } \\
\text { differences between cases and controls for } \\
\text { age, sex, geographical region, smoking } \\
\text { habits, socioeconomic and medical history, } \\
\text { including surgeries and drug use, which may } \\
\text { be related to autoimmune disease, and hair } \\
\text { dying } \\
\text { Strengths: Analysis of high cumulative } \\
\text { exposure reduces potential for exposure } \\
\text { misclassification } \\
\text { Limitations: Limited statistical power for } \\
\text { TCE exposure, no adjustment for possible } \\
\text { co-exposures and other confounders }\end{array}$ \\
\hline
\end{tabular}


RoC Monograph on Trichloroethylene

\begin{tabular}{|c|c|c|c|c|}
\hline Reference & $\begin{array}{l}\text { Study Population } \\
\text { \# Cases \& Controls }\end{array}$ & Exposure Assessment & $\begin{array}{c}\text { OR (95\% CI); \# of Exposed } \\
\text { Cases }\end{array}$ & Comments \\
\hline \multicolumn{5}{|c|}{ Undifferentiated connective tissue disease } \\
\hline $\begin{array}{l}\text { Lacey et al. } \\
\text { (1999) }\end{array}$ & $\begin{array}{l}\text { Population-based case- } \\
\text { control study }\end{array}$ & Same as Garabrant et al. (2003) & \multirow{4}{*}{$\begin{array}{l}\text { Women only } \\
\text { Self-reported } \\
0.9(0.1-7.0) 1 \text { case } \\
\text { Confirmed by expert review } \\
1.7(0.2-15.0) 1 \text { case }\end{array}$} & \multirow{4}{*}{$\begin{array}{l}\text { Covariates/consideration of confounding: } \\
\text { Adjusted for age, year of birth. Ethnicity, } \\
\text { annual household income, education, marital } \\
\text { status, and smoking frequencies and alcohol } \\
\text { use were similar between cases and controls } \\
\text { Limitations: Potential for exposure and } \\
\text { disease misclassification. Only one exposed } \\
\text { case }\end{array}$} \\
\hline Michigan \& & Women only & & & \\
\hline Ohio (USA) & 205 cases & & & \\
\hline $\begin{array}{l}\text { Same design as } \\
\text { Garabrant et al. } \\
\text { (2003) }\end{array}$ & $\begin{array}{l}2,079 \text { matched (race, age, } \\
\text { and geographical region) } \\
\text { controls without a } \\
\text { medical history of other } \\
\text { connective tissue diseases } \\
\text { identified by RDD }\end{array}$ & & & \\
\hline
\end{tabular}

$* \mathrm{p}<0.05$.

$\mathrm{JEM}=$ job exposure matrix; $\mathrm{PPE}=$ personal protective equipment $\mathrm{RRD}=$ random digit dialing.

${ }^{a}$ Auto-antibody. 
Table 5-6. Studies of Trichloroethylene Exposure and Lymphocytes, and Immune Markers in Humans ${ }^{\mathrm{a}}$

\begin{tabular}{|c|c|c|c|c|c|}
\hline Reference & Study Population & $\begin{array}{l}\text { Exposure Assessment } \\
\text { Exposure Levels }\end{array}$ & \multicolumn{2}{|l|}{ Findings } & Comments \\
\hline \multicolumn{6}{|c|}{ Series of studies of Chinese workers from 6 factories in Guangdong } \\
\hline $\begin{array}{l}\text { Lan et al. (2010) } \\
\text { Hosgood et al. } \\
(2012) \\
\text { China }\end{array}$ & $\begin{array}{l}\text { Metal/electronics } \\
\text { factory workers } \\
\text { Cross-sectional study of } \\
\text { healthy workers } \\
\text { (excluded those with } \\
\text { history of cancer, } \\
\text { chemotherapy, and } \\
\text { radiotherapy) } \\
80 \text { exposed } \\
96 \text { unexposed } \\
\text { (frequency matched by } \\
\text { age, sex, and region) }\end{array}$ & $\begin{array}{l}\text { Personal air samples } 3 \text { weeks } \\
\text { prior to blood and urine } \\
\text { collection } \\
\text { Mean air (SD) } \\
\text { All: } 22.5(35.9) \\
\text { Low (<12 ppm): } 5.2(3.5) \\
\text { High (>12 ppm) } 38.4(44.6)\end{array}$ & $\begin{array}{l}\text { Exposed vs. non-expos } \\
\text { Lan et al. (2010) } \\
\text { sCD27 : } \\
\text { sCD30 } \text { : } \\
\text { Lymphocytes: } \\
\text { NK cells: } \\
\text { B cells: } \\
\text { Total T cells: } \\
\text { CD4 T cells: } \\
\text { CD8 T cells: } \\
\text { No differences: WBC, } \\
\text { granulocytes, monocyte } \\
\text { Hosgood et al. (2012) } \\
\text { CD4 and CD8 Subsets } \\
\text { CD4 naïve } \\
\text { CD4 effector memory } \\
\text { CD8 naïve } \\
\text { T subsets: P > 0.05: } \\
\text { CD4 central memory } \\
\text { CD8 central and effecto } \\
\text { Regulatory }\end{array}$ & $\begin{array}{l}\text { ed } \\
\downarrow, \mathrm{E} / \mathrm{R} \\
\downarrow, \mathrm{E} / \mathrm{R} \\
\downarrow, \mathrm{E} / \mathrm{R} \\
\downarrow, \mathrm{E} / \mathrm{R} \\
\downarrow, \mathrm{E} / \mathrm{R} \\
\downarrow, \mathrm{E} / \mathrm{R} \\
\downarrow, \mathrm{E} / \mathrm{R} \\
\downarrow, \mathrm{E} / \mathrm{R} \\
\mathrm{s} \\
\downarrow, \mathrm{E} / \mathrm{R}\end{array}$ & $\begin{array}{l}\text { Covariates/consideration of confounding: } \\
\text { Adjusted for age, sex; (B cells also adjusted } \\
\text { for smoking status); smoking status, alcohol } \\
\text { consumption, recent infection, and BMI } \\
\text { considered in analysis. No differences } \\
\text { between exposed and unexposed in smoking } \\
\text { status, sex distribution, recent infection, and } \\
\text { BMI } \\
\text { Strengths: Exposure misclassification unlikely } \\
\text { because of good exposure assessment; } \\
\text { negligible co-exposures to e.g., benzene, } \\
\text { styrene, formaldehyde, chlorinated solvents; } \\
\text { ability to evaluate exposure-response } \\
\text { relationships; healthy participants with no } \\
\text { previous cancer, chemotherapy, radiation } \\
\text { Limitations: Small study population, cross- } \\
\text { sectional design }\end{array}$ \\
\hline
\end{tabular}


RoC Monograph on Trichloroethylene

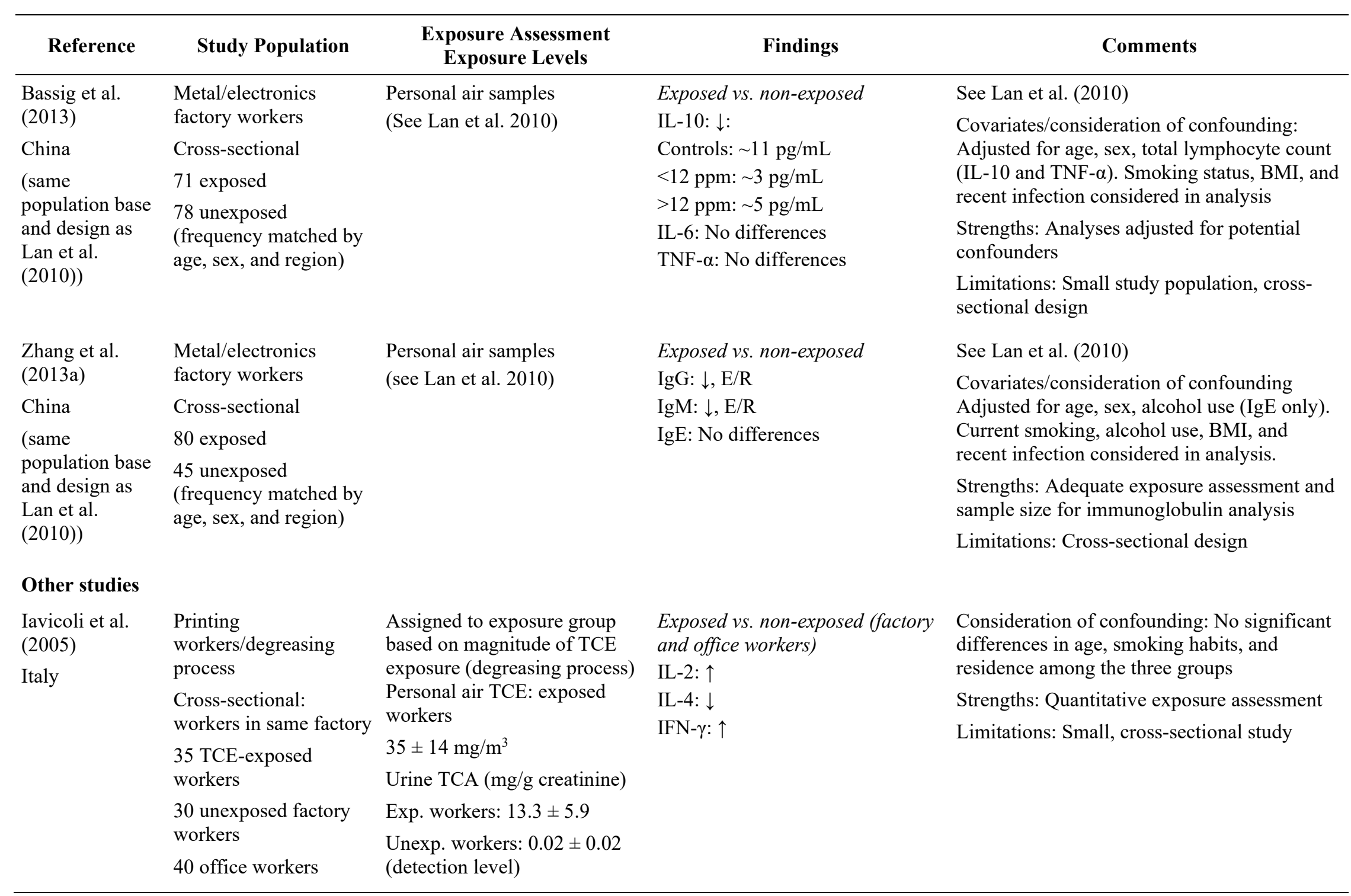




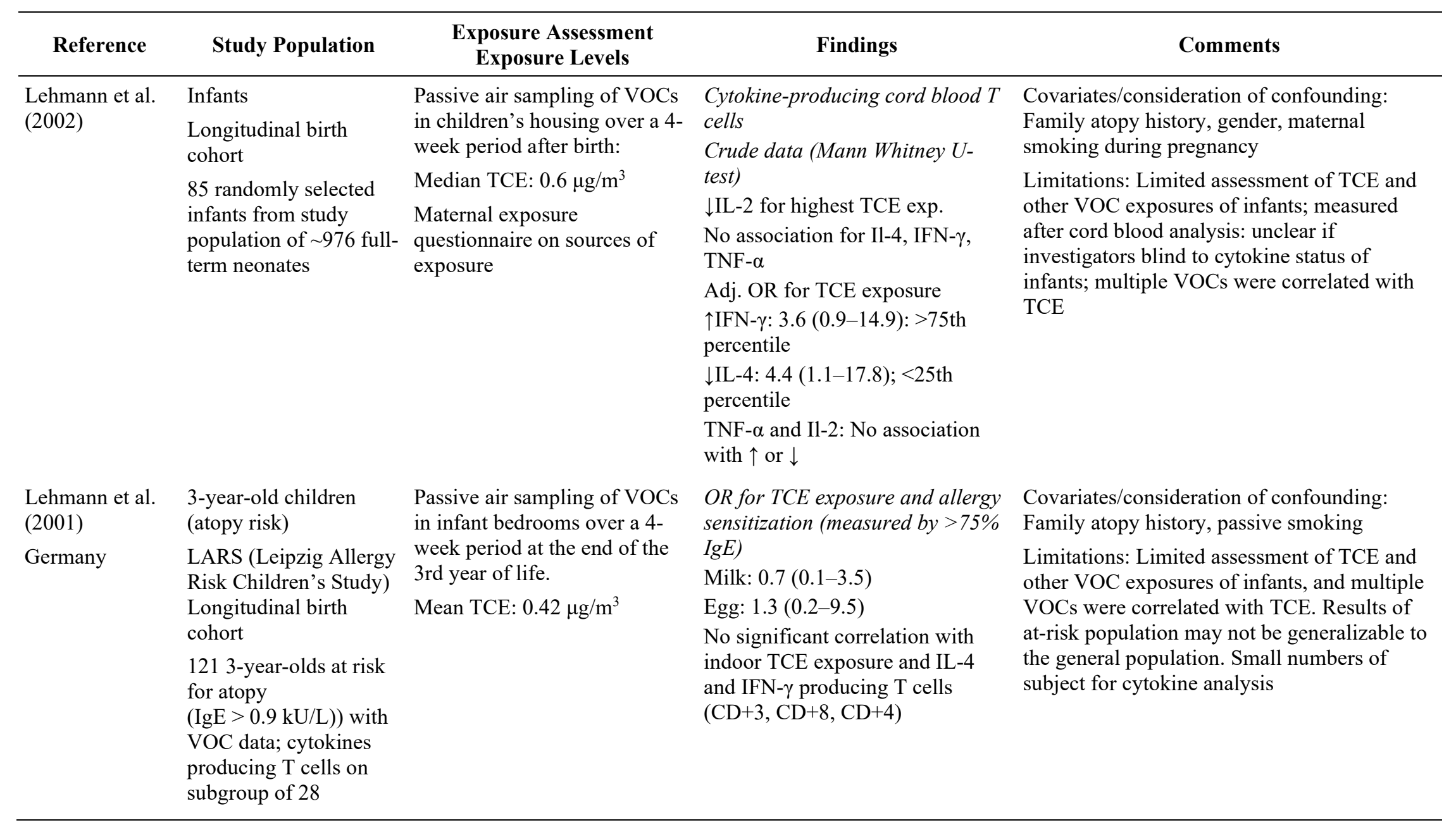

$\mathrm{ANCA}=$ antinuclear antibodies; $\mathrm{CD}=$ cluster of differentiation (T cell types); $\mathrm{E} / \mathrm{R}=$ exposure-response relationship; IFN = interferon; IgG, $\mathrm{E}, \mathrm{M}=$ immunoglobulin $\mathrm{G}, \mathrm{E}, \mathrm{M}$; $\mathrm{NK}=$ natural killer cells; IL = interleukin; TNF = tumor necrosis factor; VOC $=$ volatile organic compounds; $\downarrow=$ statistically significant decrease; $\uparrow=$ statistically significant increase.

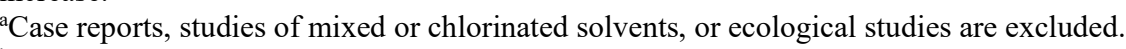

${ }^{\mathrm{b}} \mathrm{TNF}$ receptor markers. 


\subsubsection{Studies of Immunomodulation in Experimental Animals}

Overall, evidence suggests that exposure to trichloroethylene or its metabolites causes alterations in the immune system, including autoimmune disease, in experimental animals based on studies showing signs of autoimmune disease and/or changes in leukocyte numbers, proliferation, activation, and function (see Table E-4). As explained in more detail in Section 5.2.1, immunomodulation resulting from autoimmunity or immunosuppression leading to continual B cell activation are linked to NHL and could possibly be involved in the mode of action for trichloroethylene-induced lymphoma.

Many studies were identified that examined the immunological effects of trichloroethylene in experimental animals. The results for the same endpoint often varied between studies, but these differences might be explained by differences in exposure or by intra- or interspecies variation (e.g., strain of mice, use of rats or dogs). Differences in species, strain, and exposure were considered and are noted in the text below when results differ between studies. Many of the relevant studies were conducted in MRL+/+ mice, which spontaneously develop a systemic lupus erythematosus-like autoimmunity. The various study designs and immunomodulatory endpoints are presented in Table E-1 and Table E-2. Results from these studies are summarized by endpoint in Table E-3 (blood - adducts and leukocyte numbers), Table E-4 (blood antibodies), Table E-5 (spleen), Table E-6 (liver and kidney), and Table E-7 (splenic ex vivo cytokines, lymph nodes, and anti-bacterial response).

While experimental animal models do not exist for all human autoimmune diseases (see Section 5.2.2.1), the autoimmune-prone MRL+/+ mice develop many of the features of systemic lupus erythematosus. General signs of autoimmune disease were suggested by changes in antibodies, immune cell activities, and autoimmune hepatitis in MRL+/+ mice and other species and strains of experimental animals exposed to trichloroethylene or its metabolites (see Table F-1, Table F-2, Table F-3, and Table F-4). Exposure-related effects included increased IgG and autoantibody formation (anti-nuclear, anti-DNA, anti-albumin, and anti-liver) (Blossom et al. 2004; Cai et al. 2006; Cai et al. 2007b; Gilbert et al. 2009; Griffin et al. 2000a; Griffin et al. 2000c; Keil et al. 2009; Khan et al. 1995; Wang et al. 2007a; Wang et al. 2007b; Wang et al. 2008; Wang et al. 2012b; Wang et al. 2013). The presence of autoantibodies indicated that selfantigens were recognized by the immune system as "foreign" which can provide persistent antigen stimulation and B cell activation. However, cell surface markers for B cell activation (MHC II) and B cell proliferation were not consistently altered and other markers of B cell activation were not examined. In addition to autoantibodies, lymphocyte numbers (especially CD4 T cells) were increased in the spleen as well as lymphocyte proliferation (Cai et al. 2006; Griffin et al. 2000b; Sanders et al. 1982; Wang et al. 2008). These general signs support the idea that autoimmunity is induced by trichloroethylene or its metabolites and that continual B cell activation may be occurring in target organs such as the liver.

The initiation of autoimmune disease from exposure to trichloroethylene or its metabolites may have been caused by the formation of protein adducts with metabolites (dichloroacetyl-protein) and, through increased oxidative stress, with products of lipid peroxidation (malondialdehydeprotein, hydroxynonenal-protein) (Cai et al. 2006; Cai et al. 2007b; Griffin et al. 2000a; Griffin et al. 2000b; Griffin et al. 2000c; Halmes et al. 1997; Khan et al. 1995; Khan et al. 2001; Wang et al. 2007a; Wang et al. 2008; Wang et al. 2012b; Wang et al. 2013). Protein adducts were found in the serum and liver, and antibodies against these adducts were found in the serum. A 
role for formation of trichloroethylene metabolites in these effects is indicated by the finding that inhibition of CYP2E1 by co-exposure with diallyl sulfide prevented the formation of dichloroacetyl-protein adducts and its specific antibodies (Griffin et al. 2000b). In addition, decreasing oxidative stress by the enhancement of the antioxidant activity of glutathione, by coexposure to $\mathrm{N}$-acetylcysteine, prevented the formation of malondialdehyde-protein and hydroxynonenal-protein adducts and their specific antibodies (Wang et al. 2013). Splenocytes from trichloroethylene-exposed mice produced Th1 cytokines (IFN-gamma, IL-2) when stimulated with preformed lipid peroxidation product-albumin adducts (malondialdehydealbumin, hydroxynonenal-albumin) (Cai et al. 2006; Wang et al. 2008; Wang et al. 2012b). These studies show that trichloroethylene induced neoimmunogenic protein adducts in the serum and liver by both CYP2E1-mediated metabolic activation and increased oxidative stress. In addition to antibodies against the protein adducts found in the liver, antibodies against normal, non-adducted, liver proteins were formed (Gilbert et al. 2009). Similarly, exposure to preformed trichloroethylene-albumin adducts not only induced the formation of antibodies against the albumin adducts (formyl-albumin, trichloroethene oxide-albumin, and dichloroacetyl-albumin), but also to the non-adducted albumin (Cai et al. 2007b). These results indicate that trichloroethylene is inducing autoimmunity toward "self" proteins found in the blood and liver. Besides autoimmunity, other trichloroethylene-induced immune effects were reported.

Immunomodulatory findings were reported for a number of different organs and endpoints. While immunomodulatory effects can include immunosuppression, direct evidence for this effect was not generally available. Some evidence for a systemic immunomodulatory effect of trichloroethylene was reported from studies showing increased mortality in mice following a bacterial challenge; however, effects on alveolar macrophage phagocytic activity and phagocytic clearance of bacteria were not entirely consistent with the mortality data (Aranyi et al. 1986; Selgrade and Gilmour 2010). Immunomodulation was suggested by a decrease in various peripheral blood leukocytes in studies in different species by different routes of administration. These included decreased numbers of leukocytes, lymphocytes, CD4 T cells, CD8 T cells, and B cells in NOD mice exposed via drinking water (Ravel et al. 2004), but the effects were observed only at 23 weeks of exposure and no treatment-related functional effects were observed for serum cytokine levels. Decreased CD4 T cell numbers were also reported in rats exposed by intradermal injection (Chen et al. 2006), but no differences in cytokines (IL-4 and IFN $\gamma$ ) were found. Decreases in both leukocytes and neutrophils were reported in dogs exposed by intratracheal instillation or intravenous injection (Chen et al. 2006; Hobara et al. 1984; Ravel et al. 2004); however, the leukocyte count in the latter study reached a minimum 30 minutes after injection and gradually returned toward normal. The only endpoints that decreased in more than one study were the CD4 T-cell numbers and leukocyte numbers. No effect on peripheral blood leukocyte populations was seen in one study testing chloral hydrate in mice.

Possible signs of immunomodulation were observed in specific organs. In the liver, the cytolytic activity of NK cells was decreased (Wright et al. 1991); however, mixed results were seen in the spleen and lymph nodes. For the spleen, most studies found no differences with exposure to trichloroethylene or its metabolites, and no differences in experimental design variables of species, strain, or route of exposure were identified that could explain the mixed results in the spleen. Immune effects observed in some of these studies included decreases in the number of CD4 T cells (2/4 studies), CD8 T cells ( $1 / 7$ studies), and B cells (2/7 studies) and increases in lymphocyte number ( $2 / 2$ studies), lymphocyte proliferation (1/1 study), and CD4 T cell 
proliferation (2/3 studies) (Blossom et al. 2004; Blossom and Gilbert 2006; Blossom and Doss 2007; Blossom et al. 2007; Cai et al. 2006; Gilbert et al. 2011; Griffin et al. 2000a; Griffin et al. 2000b; Kauffmann et al. 1982; Keil et al. 2009; Peden-Adams et al. 2006; Peden-Adams et al. 2008; Wang et al. 2008). Initial B cell activation against sheep red blood cells in the spleen was increased in one study and decreased in two studies (Kauffmann et al. 1982; Peden-Adams et al. 2006; Sanders et al. 1982). The numbers of CD4 T cells, CD8 T cells, and B cells in lymph nodes and activation of those B cells were unaffected (Blossom et al. 2004; Blossom et al. 2006; Gilbert et al. 2011; Gilbert et al. 2012).

The mixed results in the spleen and lack of effect in the lymph nodes suggest that trichloroethylene does not affect the amount or activity of immune cells in those organs. Since there were decreases in leukocytes in the peripheral blood, but no consistent results in the spleen or lymph nodes, it is possible that the effects seen in the blood were the result of leukocyte extravasation into tissue. The data from studies in mice (Ravel et al. 2004) and rats (Chen et al. 2006) do not give support for either extravasation or decreases in leukocyte numbers since they did not also look at leukocyte numbers in the spleen or lymph nodes. Changes in cytokines, however, did not similarly decrease along with CD4 and CD8 T cells (Chen et al. 2006; Ravel et al. 2004). Blood leukocyte numbers in the dog studies (Hobara et al. 1984) suggest extravasation might be the explanation since the decreases were temporary and occurred for only $\leq 2$ hours before increasing back towards pre-dose levels.

\subsubsection{Possible Modes of Action for Trichloroethylene-induced Immune Modulation and NHL}

As discussed above, trichloroethylene induces immune modulation in humans and laboratory animals with the strongest evidence for autoimmune effects (Boverhof et al. 2013; Cooper et al. 2009; Rusyn et al. 2014; Weinhold 2009). Immune modulation and autoimmunity can lead to chronic inflammation and antigenic stimulation. Only a few studies in humans examined the immunomodulatory effects of trichloroethylene. Most studies in experimental animals used mouse strains that spontaneously develop conditions resembling systemic lupus erythematosus. Since immunomodulation and autoimmune diseases are known risk factors for NHL, altered immunity and chronic inflammation may be involved in trichloroethylene-induced NHL.

The relationship between immune status and cancer risk is complex. It is well accepted that chronic inflammation plays an essential role in tumorigenesis; however, the underlying molecular mechanisms linking inflammation and cancer are not completely understood (Wu et al. 2013). The normal physiological response to infection or tissue damage is acute inflammation. Cases of unresolved inflammation, as occurs with immune suppression or autoimmune disease, evoke chronic inflammation and antigenic stimulation. Chronic inflammation predisposes the host to cancer by inducing DNA damage and chromosomal instability and promoting tumor development. Possible modes of action include the following: (1) immunosuppression of tumor surveillance activity, (2) effects from oncogenic viruses (and, for NHL, Epstein-Barr virus [EBV] reactivation in particular) due to impaired viral surveillance and clearance, and (3) chronic antigenic stimulation due to an unchecked inflammatory response to foreign triggers (infections, allograft) or autoimmunity (Ponce et al. 2014). Chronic antigenic stimulation leads to a state of sustained B cell hyperstimulation and the potential for oncogenic transformation (see Section 5.2.2). 
Findings from the series of Chinese studies generally suggest that trichloroethylene exerts immunomodulatory effects; however, lower serum levels of IgG, IgM, sCD27, and sCD30 suggest that trichloroethylene impairs B cell stimulation and is counter to the proposed mechanism of B cell activation. Trichloroethylene-exposed workers generally had lower levels of B and T lymphocytes but not of granulocytes, platelets, or monocytes. These data suggest that trichloroethylene exerts a specific effect on lymphoid progenitor cell division or maturation. However, reduced CD4 T cells reflect immunosuppressive effects that could impair control over inflammation and increase B cell activation. Autoimmune effects in humans, in particular, are consistent with the hypothesized mechanisms of action linking severe immune dysregulation and NHL. It is quite possible that the observed immunologic effects of trichloroethylene are reflective of other poorly understood mechanisms that increase the risk of malignant transformation of B cells.

The data show that trichloroethylene is immunomodulatory in rodents (see Section 5.2.2.2). Several studies in MRL+/+ mice suggested that oxidative and nitrosative stress from reactive oxygen and nitrogen species may contribute to the autoimmune response (Khan et al. 2001; Wang et al. 2007a; Wang et al. 2007b; Wang et al. 2008; Wang et al. 2009a; Wang et al. 2012b; Wang et al. 2013). Reactive oxygen and nitrogen species have been implicated in the pathogenesis of several autoimmune diseases including systemic lupus erythematosus (Wang et al. 2007b). Other studies with MRL+/+ mice demonstrated that trichloroethylene metabolites also formed immunoreactive protein adducts resulting in antibody formation (Cai et al. 2006; Cai et al. 2007a; Cai et al. 2007b; Griffin et al. 2000a; Griffin et al. 2000b; Griffin et al. 2000c; Halmes et al. 1996; Halmes et al. 1997; Khan et al. 1995). Metabolic activation by CYP2E1 was at least partially responsible for the autoimmune response (Griffin et al. 2000b).

\subsubsection{Summary}

Severe immune dysregulation, whether from immunosuppression, inflammation, or autoimmune disease, is associated with an increased risk of NHL. Thus, it is biologically plausible that the mode of action of trichloroethylene-induced NHL could involve altered immunity. However, no human or animal studies directly investigated the possible relationship between trichloroethylene exposure, immunomodulation, and lymphoproliferative disorders and some of the data were not consistent with the proposed mechanisms. Use of other activation markers (e.g., CD23, CD27, CD30, CD44, and CXCL13) might have allowed for a more complete assessment of B cell response. Although few applicable studies were conducted in humans, the available data provide evidence that trichloroethylene can alter the immune system based on some studies finding an association between markers of immune modulation and other studies showing an association with autoimmune disease (e.g., systemic sclerosis). Studies in MRL+/+ mice show that trichloroethylene accelerates lupus conditions. Possible key events demonstrated in mice (mostly in strains predisposed to autoimmune disease) include lipid peroxidation, CYP2E1 metabolism to reactive metabolites, formation of immunoreactive protein adducts, formation of antibodies against the adducted proteins, autoimmune response via cross-reaction of antibodies to nonadducted (normal "self") proteins, and chronic inflammation. Chronic inflammation is a known risk factor for tumor promotion and progression. However, the available data are insufficient to demonstrate that immunomodulation is operant as a mode of action for trichloroethyleneinduced NHL. 


\section{Liver Cancer}

The previous sections of the cancer evaluation component contain relevant information-ADME (Section 1), genetic and related effects (Section 2), and overview and assessment of the quality of the human cancer studies - that are important for several of the three cancer endpoints of interest (Section 3). This section builds on that information and evaluates the human cancer studies (Section 6.1), mechanistic data, including immune effects of trichloroethylene (Section 6.2), specifically for liver cancer.

\subsection{Human Cancer Studies}

Liver cancer is considered to be rare, with higher rates observed among males; the U.S. ageadjusted rates per 100,000 per year (2007 to 2011) are 12.4 (male) and 4.1 (female) for incidence and 8.5 (male) and 3.4 (female) for mortality (SEER 2014c). The 5-year survival rate is 16.6\%, suggesting that mortality and incidence data are likely to be broadly comparable, at least for recent years. U.S. rates have been increasing at about $4 \%$ per year for the past decade with an overall incidence rate in 1975 of approximately 2.8 per 100,000. Incidence rates in European countries, from which the remaining studies in the evaluation are drawn, appear to be broadly comparable: for example, age-standardized U.K. incidence rates per 100,000 (2011) are approximately 7.0 (male) and 3.1 (female). The overall rate was 1.5 in 1975, with an increase of approximately 4\% per year over the past decade (Cancer Research U.K. 2014b). As noted, latencies of solid tumors such as liver cancer are generally considered to be longer than for most lymphohematopoietic cancers (e.g., >20 years), although a shorter latency has been reported in association with some exposures (see Howard 2013). Incidence rates start to increase steeply at a somewhat earlier age (40 to 44 years) than for kidney cancer, particularly among men. Liver cancer is reported in some studies as cancer of the liver and intrahepatic bile ducts (ICD-9 155, ICD-10 22) and in others as combined cancers of the liver and intrahepatic and extrahepatic bile ducts and gallbladder (ICD-9 155+156, ICD-10 22-24); some papers report primary liver cancer (ICD-9 155.1) separately. Approximately $75 \%$ of primary liver cancers are hepatocellular carcinomas, with cholangiocarcinomas forming the bulk of the remainder.

For each of the reviewed studies, summary data on study design, methods, and findings, systematically extracted from relevant publications as described in the study protocol, are presented in Table D-1. The evaluation of study quality, including study design, methods of exposure and cancer endpoint assessment, analysis and other relevant elements (such as study sensitivity) data, is reported in Table D-4 and Table D-5. Section 3 provided an overview of the study population characteristics and methods and a discussion of study quality and other elements across studies. Figure 6-1 (below) provides an overview of the studies broadly grouped according to their utility to inform the cancer evaluation.

\subsubsection{Study Findings}

This section summarizes and interprets the findings for liver cancer from the individual epidemiological studies brought forward for evaluation (see Table 6-1), and integrates the evidence across studies, applies the RoC listing criteria to the body of evidence, and reaches an NTP recommendation for the level of evidence for liver cancer using the same criteria as described for the evaluation of kidney cancer in Section 4 and NHL in Section 5. 
The cancer evaluation reports on the latest update of a cohort study unless there are additional relevant data (e.g., analyses or exposure information) in previous publications. The available studies that reported on liver cancer and trichloroethylene exposure and were considered for inclusion in the cancer evaluation include 12 cohort or nested case-control studies and 1 population-based case-control study (Christensen et al. 2013). The cohort studies include three studies of occupationally exposed subjects from Nordic countries (Hansen et al. 2013; RaaschouNielsen et al. 2003; Vlaanderen et al. 2013), four studies of U.S. aerospace or aircraft manufacturing workers (Blair et al. 1998; Boice et al. 2006; Lipworth et al. 2011; Morgan et al. 1998; Radican et al. 2008), two studies of U.S. uranium processing workers (Bahr et al. 2011; Ritz 1999), one nested case-control study (Greenland et al. 1994) and one cohort study (Silver et al. 2014) of U.S. electronic workers, and a cohort of military personnel exposed to trichloroethylene in drinking water (Bove et al. 2014). Two meta-analyses were also identified that contributed to the evaluation.

Although the available database consists of several well-conducted studies, liver cancer is rare, and few workers were exposed to high levels of trichloroethylene with reasonable confidence of exposure. Thus, the major overall limitation across studies is low statistical power to evaluate a modest risk of liver cancer from exposure to trichloroethylene and exposure-response relationships. In addition, some of the studies report findings for both liver and biliary cancer combined and others for primary liver cancer only, making cross comparisons more difficult. Similar to kidney cancer, meta-analyses may be informative, although heterogeneity of findings, if considerable, can reduce their utility and should be noted. The findings of the individual studies are discussed below and presented in Figure 6-1. 


\begin{tabular}{|c|c|}
\hline Moderate & Hansen 2013 \\
\hline Selection bias unlikely for internal analyses & \\
\hline Some concern for exposure misclassification & \\
\hline Evaluation of $E / R$ & Radican 2008 \\
\hline $\begin{array}{l}\text { Limited study sensitivity; moderate to high } \\
\text { exposure level }\end{array}$ & \\
\hline Limited consideration of potential confounders & Morgan 1998 \\
\hline Low/moderate & Lipworth 2011 \\
\hline $\begin{array}{l}\text { Selection bias possible (except for internal } \\
\text { analyses) }\end{array}$ & \\
\hline Some concern for exposure misclassification & Boice 2006 \\
\hline Limited evaluation of $E / R$ & \\
\hline Low study sensitivity (except for R-N) & \\
\hline Limited consideration of potential confounders & Raaschou-Nielsen (R-N) 2003 \\
\hline Low & Bove 2014 \\
\hline Selection bias possible, probable or unknown & Silver 2014 \\
\hline Considerable concern for exposure & Vlaanderen 2013 \\
\hline misclassification & Christensen $2013^{a}$ \\
\hline $\begin{array}{l}\text { No evaluation of E/R (except Vlaanderen and } \\
\text { Bove) }\end{array}$ & Bahr 2011a \\
\hline Low study sensitivity & Ritz 1999b \\
\hline Limited consideration of potential confounders & Greenland 1994 \\
\hline
\end{tabular}

Figure 6-1. Study Utility Ranking: Liver Cancer

$\mathrm{E} / \mathrm{R}=$ exposure response.

Gray shading (left-hand column): Utility to inform hazard evaluation; light shading = highest utility; dark shading = lowest utility. Blue shading (right-hand column): Potential bias and study sensitivity; light shading = least biased or most sensitive; dark shading $=$ overall potential biases towards the null or lower sensitivity.

a(Tan shading): Multiple limitations; overall direction of potential biases is unknown or 1 exposed case [for Christensen et al. (2013)].

b(Peach shading): Most potential biases away from the null.

\subsubsection{Nordic Studies}

These three studies include subjects with occupational exposure to trichloroethylene from diverse industries and workers identified from a broad occupational or a broad population-based database. Two studies reported an association with potential trichloroethylene exposure and liver cancer, with the strongest evidence from the pooled analyses of biomonitored workers (Hansen et al. 2013), in which a statistically significant increase in risk was observed among men and women combined ( $\mathrm{SIR}=1.77,95 \% \mathrm{CI}=1.24$ to $2.45,36$ cases). Risks increased with increasing latency (as assessed by lag time), which partly reflect the longer average latencies of liver cancer (Manton et al. 2009), and provides support for an association between trichloroethylene exposure 
and liver cancer in this population. However, in internal analyses, which examined exposureresponse relationships, risks were less than one and the highest risk (with the largest number of cases) was in the lowest exposure group, the referent group in this analysis, which complicates the interpretation of the study. Few U-TCA samples were available for each subject, and thus UTCA, which is a measure of short-term exposure, may not have accurately captured exposure intensity from the past or in the future.

Increases in liver cancer risk were observed among women (total trichloroethylene-exposed cohort) in the Danish study of Raaschou-Nielsen et al. (2003) ( $\mathrm{SIR}=2.8,95 \% \mathrm{CI}=1.13$ to 5.80, 7 cases for ever exposed) and a SIR of $4.1(95 \% \mathrm{CI}=1.1$ to $10.5,4$ cases $)$ was observed among workers with 1 to 4 years employment duration. A higher risk occurred among women with later years of first employment, when exposures were reportedly lower than the earlier years; however, the number of cases is small. In contrast, among men (with more overall cases than women), the highest risk was found among men employed before $1970(\mathrm{SIR}=1.5,95 \% \mathrm{CI}=0.9$ to $2.4,17$ cases). It is important to note that the authors only conducted analyses for liver in the total cohort and not among the subcohort of workers considered to have higher exposure (as they did for kidney cancer). Some misclassification of exposure is likely as only a portion of the cohort was exposed to trichloroethylene. There was little evidence of an association with liver cancer in the large population-based study (Vlaanderen et al. 2013), although, as noted previously, exposure misclassification is likely to be substantial and estimated exposures were low.

\subsubsection{Aerospace and Aircraft Workers}

The evidence for an increase in liver cancer risk among the group of U.S. studies of aerospace and aircraft workers (Blair et al. 1998; Boice et al. 2006; Lipworth et al. 2011; Morgan et al. 1998; Radican et al. 2008) is limited. In most of the studies, there were few exposed cases, especially in subgroup analyses, if reported, and the studies had limited ability to evaluate exposure-response relationships. In the Radican et al. (2008) cohort, which evaluated primary liver separately from liver and biliary combined, non-statistically significant increases were observed for both cancer categories in analyses of cumulative exposure for all workers and workers with the highest exposure. There is some evidence of a weak exposure response for cumulative exposure and primary liver cancer among male workers, but confidence intervals are wide. Findings from the other studies were null. Two mortality studies (Boice et al. 2006; Morgan et al. 1998) observed non-statistically significant, small increases in liver cancer, but were based on small numbers of exposed cases; no exposure gradient was observed in the Morgan study. The mortality study by Lipworth et al. (2011) observed a decrease in liver cancer by employment duration, a poor surrogate for cumulative exposure. It is not clear how many workers were exposed to trichloroethylene in the different categories of exposure duration, since exposure duration was short, so that exposure misclassification is likely. In addition, there is evidence of a healthy worker effect in this study, all of which limit the study's ability to inform the cancer hazard evaluation.

\subsubsection{Other Studies}

The remaining studies are of more limited utility for informing the cancer hazard evaluation. Ritz (1999) found an increased risk of liver cancer among uranium processing workers; risks increased with increasing lag time, exposure duration, and exposure level in analyses controlling for radiation exposure, which suggests a positive relationship between trichloroethylene 
exposure and cancer risk; however, the numbers are based on small numbers of workers, most with low exposure to trichloroethylene. In addition, there is the potential for selection bias and residual confounding, possibly by radiation exposure. The electronics worker cohort (Silver et al. 2014) and the drinking water study (Bove et al. 2014) reported no increases in risk but both studies had limited exposure assessment, and were relatively young cohorts. Overall, these limitations would tend to bias findings towards the null. The cohort study of uranium workers by Bahr et al. (2011) and the nested case-control study (Greenland et al. 1994), both of which have a number of methodological limitations, report decreases in risk, and neither study reported numbers of deaths. Finally, while the Montreal study (Christensen et al. 2013) had adequate exposure assessment and analytical methods, only one case of liver cancer was observed in the substantially exposed group, so this study is uninformative for this endpoint. 
Table 6-1. Findings for Trichloroethylene and Cancers of the Liver, Biliary Tract, or Gallbladder

\begin{tabular}{|c|c|c|c|c|c|}
\hline Reference & $\begin{array}{c}\text { Study } \\
\text { Size (N) } \\
\text { Exposure } \\
\text { Assessment }\end{array}$ & Exposure Groups & $\begin{array}{l}\text { External Analysis } \\
\text { SMR, SIR (95\% CI) \# } \\
\text { Exposed Cases/Deaths }\end{array}$ & $\begin{array}{c}\text { Internal Analysis } \\
\text { RR }(95 \% \text { CI) } \\
\text { \# Exposed Cases/Deaths } \\
\text { or Cases/Controls }\end{array}$ & Interpretation \\
\hline \multicolumn{6}{|l|}{ Nordic studies } \\
\hline \multirow[t]{6}{*}{$\begin{array}{l}\text { Vlaanderen et } \\
\text { al. (2013) }\end{array}$} & 5 Nordic countries & Cumulative exp.(unit-yr) & & $\begin{array}{l}\text { ICD-7 } 155 \\
\text { HR (Incidence) }\end{array}$ & $\begin{array}{l}\text { Low prevalence of exposure (TCE) } \\
\text { and exposure levels likely to be low }\end{array}$ \\
\hline & $\begin{array}{l}\text { Record linkage of } \\
\text { cancer registry with } \\
\text { census questionnaire }\end{array}$ & $\begin{array}{l}0 \\
0.04\end{array}$ & & $\begin{array}{l}1.00 \\
1.03(0.91-1.16) ; 340\end{array}$ & $\begin{array}{l}\text { Strengths; long follow-up, large } \\
\text { numbers of cases }\end{array}$ \\
\hline & $\begin{array}{l}\text { Semi-quantitative } \\
\text { JEM }\end{array}$ & $\begin{array}{l}0.13 \\
0.72 \\
\text { High-exnosure oroun }\end{array}$ & & $\begin{array}{l}0.99(0.90-1.09) ; 508 \\
1.00(0.90-1.11) ; 422\end{array}$ & $\begin{array}{l}\text { Limitations: Misclassification of } \\
\text { exposure likely; JEM had poor }\end{array}$ \\
\hline & $\begin{array}{l}\text { M: } 14,702 \text { cases, } \\
73,510 \text { controls }\end{array}$ & $\frac{\text { Cumulative }}{\text { Men }}$ & & $1.01(0.78-1.31) ; 69$ & $\begin{array}{l}\text { sensitivity and did not account for } \\
\text { heterogeneity within jobs and over }\end{array}$ \\
\hline & F: 9,194 cases, & $\begin{array}{l}\text { Women } \\
\text { Intensity } \times \text { prevalence }\end{array}$ & & $1.02(0.72-1.46) ; 37$ & $\begin{array}{l}\text { time; TCE exposure correlated with } \\
\text { tetrachloroethylene exposure }\end{array}$ \\
\hline & & $\begin{array}{l}\text { Men } \\
\text { Women }\end{array}$ & & $\begin{array}{l}1.07(0.86-1.33) ; 99 \\
1.12(0.79-1.59) ; 38\end{array}$ & $\begin{array}{l}\text { Null: No evidence for an } \\
\text { association but limited utility }\end{array}$ \\
\hline
\end{tabular}


RoC Monograph on Trichloroethylene

\begin{tabular}{|c|c|c|c|c|c|}
\hline Reference & $\begin{array}{c}\text { Study } \\
\text { Size (N) } \\
\text { Exposure } \\
\text { Assessment }\end{array}$ & Exposure Groups & $\begin{array}{c}\text { External Analysis } \\
\text { SMR, SIR (95\% CI) \# } \\
\text { Exposed Cases/Deaths }\end{array}$ & $\begin{array}{c}\text { Internal Analysis } \\
\text { RR }(95 \% \text { CI })^{\mathbf{a}} \\
\text { \# Exposed Cases/Deaths } \\
\text { or Cases/Controls }\end{array}$ & Interpretation \\
\hline $\begin{array}{l}\text { Hansen et al. } \\
(2013) \\
\text { (potential } \\
\text { overlap with } \\
\text { Raaschou- } \\
\text { Nielsen et al. } \\
(2003))\end{array}$ & $\begin{array}{l}\text { Pooled and updated } \\
\text { Nordic cohorts } \\
\text { Axelson et al. (1994), } \\
\text { Anttila et al. (1995), } \\
\text { Hansen et al. (2001) } \\
5,553 \\
(3,776 \mathrm{M}, 1,777 \mathrm{~F}) \\
\text { Biomonitoring } \\
\text { (U-TCA) }\end{array}$ & $\begin{array}{l}\text { All exposed subjects } \\
\text { 0-yr lag } \\
10-y r \text { lag } \\
20-y r \text { lag } \\
U-T C A(m g / L) \\
<5 \\
5-25 \\
25-50 \\
>50 \\
p_{\text {trend }}\end{array}$ & $\begin{array}{l}\text { ICD-7 155: } \\
\text { liver + biliary } \\
\text { SIR } \\
1.77(1.24-2.45) ; 36 \\
1.83(1.24-2.56) ; 32 \\
2.09(1.34-3.11) ; 24\end{array}$ & $\begin{array}{l}\text { HR incidence (no lag) } \\
1.00 ; 16 \\
0.66(0.31-1.42) ; 12 \\
0.45(0.13-1.54) ; 5 \\
0.63(0.22-1.68) ; 3 \\
0.20\end{array}$ & $\begin{array}{l}\text { Low exposure levels (only } 20 \% \\
\text { exposed to } \geq 20 \text { ppm) and short } \\
\text { duration of employment } \\
\text { Covariates: Age, sex, calendar } \\
\text { period; indirect consideration of } \\
\text { smoking and alcohol consumption } \\
\text { Strengths: Biomonitoring data; } \\
\text { large numbers of workers ever } \\
\text { exposed } \\
\text { Limitations: Only } 2 \text { or } 3 \text { U-TCA } \\
\text { measurements per individual and } \\
\text { unlikely to estimate lifetime or } \\
\text { cumulative exposure; low statistical } \\
\text { power for evaluating modest risks; } \\
\text { limited ability to evaluate exposure- } \\
\text { response relationship } \\
\text { Evidence for a positive association: } \\
\text { Statistically significant, moderately } \\
\text { elevated effect estimate for ever } \\
\text { exposure; risks increase with } \\
\text { increasing lag but not exposure } \\
\text { level }\end{array}$ \\
\hline
\end{tabular}




\begin{tabular}{|c|c|c|c|c|c|}
\hline Reference & $\begin{array}{c}\text { Study } \\
\text { Size (N) } \\
\text { Exposure } \\
\text { Assessment }\end{array}$ & Exposure Groups & $\begin{array}{c}\text { External Analysis } \\
\text { SMR, SIR (95\% CI) \# } \\
\text { Exposed Cases/Deaths }\end{array}$ & $\begin{array}{c}\text { Internal Analysis } \\
\text { RR }(95 \% \text { CI })^{\mathrm{a}} \\
\text { \# Exposed Cases/Deaths } \\
\text { or Cases/Controls }\end{array}$ & Interpretation \\
\hline $\begin{array}{l}\text { Raaschou- } \\
\text { Nielsen et al. } \\
(2003) \\
\text { (Potential } \\
\text { overlap with } \\
\text { Hansen et al. } \\
(2013))\end{array}$ & $\begin{array}{l}\text { Danish blue-collar } \\
\text { workers } \\
40,049 \mathrm{M}+\mathrm{F} \text { (approx. } \\
70 \% \mathrm{M} \text { ) } \\
\text { Working at TCE } \\
\text { company; size of } \\
\text { company surrogate } \\
\text { for TCE exposure } \\
\text { prevalence }\end{array}$ & $\begin{array}{l}\text { Men }(588,047 p-y r) \\
\text { Primary liver (ICD-7 155) } \\
\text { Other liver (ICD-7 156) } \\
\text { Women (118,270 p-yr) } \\
\text { Primary liver (ICD-7 155) } \\
\text { Other liver (ICD-7 156) } \\
\text { Men and women } \\
\text { Yr. of } 1 \text { st employment } \\
\text { Women } \\
\text { Before } 1970 \\
1970-1979 \\
1980 \text { and later } \\
\text { Men } \\
\text { Before } 1970 \\
1970-1979 \\
1980 \text { and later } \\
\text { Duration employment (yr) } \\
\text { Men } \\
<1 \\
1 \text { to } 4 \\
\geq 5 \\
\frac{\text { Women }}{<1} \\
1 \text { to } 4 \\
\geq 5 \\
\end{array}$ & $\begin{array}{l}\text { SIR (Total cohort) } \\
1.1(0.74-1.64) ; 27 \\
1.2(0.73-1.77) ; 22 \\
\\
2.8(1.13-5.80) ; 7 \\
1.1(0.22-3.23) ; 3 \\
\text { Primary liver } \\
1.28(0.89-1.8)^{c} \\
2.5(0.5-7.3) ; 3 \\
2.1(0.2-7.7) ; 2 \\
5.9(0.7-21.2) ; 2 \\
1.5(0.9-2.4) ; 17 \\
0.8(0.3-1.6) ; 7 \\
0.9(0.2-2.6) ; 3 \\
\\
\text { pattern for lagged } \\
\text { exposure, } \\
1.3(0.6-2.5) ; 9 \\
1.0(0.5-1.9) ; 9 \\
1.1(0.5-2.1) ; 9 \\
2.8(0.3-10.0) ; 2 \\
4.1(1.1-10.5) ; 4 \\
\text { No exposure-response } \\
\\
\end{array}$ & NR & $\begin{array}{l}\text { Higher levels of TCE prior to } 1970 \\
\text { ( } 40-60 \text { ppm); low levels of } \\
\text { exposure after that time } \\
\text { Covariates: age, sex, calendar year } \\
\text { Strengths: Large numbers of } \\
\text { exposed cases; subcohort of } \\
\text { subjects with higher exposure } \\
\text { potential } \\
\text { Limitations: Young cohort, possible } \\
\text { selection bias of difference in SES, } \\
\text { external analysis only } \\
\text { Potential for confounding by } \\
\text { smoking among women } \\
\text { Limited evidence for a positive } \\
\text { association: Statistically significant } \\
\text { elevated risk of primary liver } \\
\text { cancer among women; little } \\
\text { evidence of exposure-response } \\
\text { relationship }\end{array}$ \\
\hline
\end{tabular}


RoC Monograph on Trichloroethylene

\begin{tabular}{|c|c|c|c|c|c|}
\hline Reference & $\begin{array}{c}\text { Study } \\
\text { Size (N) } \\
\text { Exposure } \\
\text { Assessment }\end{array}$ & Exposure Groups & $\begin{array}{c}\text { External Analysis } \\
\text { SMR, SIR (95\% CI) \# } \\
\text { Exposed Cases/Deaths }\end{array}$ & $\begin{array}{c}\text { Internal Analysis } \\
\text { RR }(95 \% \text { CI })^{\mathrm{a}} \\
\text { \# Exposed Cases/Deaths } \\
\text { or Cases/Controls }\end{array}$ & Interpretation \\
\hline \multicolumn{6}{|c|}{ Aerospace and aircraft manufacturing workers } \\
\hline $\begin{array}{l}\text { Lipworth et al. } \\
\text { (2011) (update } \\
\text { of Boice et al. } \\
(1999))\end{array}$ & $\begin{array}{l}\text { Burbank (USA) } \\
\text { aircraft } \\
\text { manufacturing } \\
\text { workers } \\
\text { 5,443 (approx. 80\% } \\
\text { M) } \\
\text { Qualitative JEM } \\
\text { Individual work } \\
\text { histories }\end{array}$ & $\begin{array}{l}\text { TCE } \\
\text { Ever exposed } \\
\text { TCE: years exposed } \\
0 \\
<1 \\
1-4 \\
5+ \\
\text { p }_{\text {trend }}\end{array}$ & $\begin{array}{l}S M R \\
(I C D-9155+156) \\
0.89(0.57-1.33) ; 24\end{array}$ & $\begin{array}{l}R R \text { mortality } \\
1.00 ; 32 \\
0.67(0.32-1.42) ; 10 \\
0.69(0.28-1.71) ; 6 \\
0.83(0.36-1.91) ; 8 \\
0.20\end{array}$ & $\begin{array}{l}\text { Exposure levels not reported; short } \\
\text { exposure duration } \\
\text { Covariates: age, date of birth, date } \\
\text { of hire, termination date, sex, and } \\
\text { race } \\
\text { Strengths: Long follow-up } \\
\text { Limitations: Evidence of HWE, few } \\
\text { exposed deaths in subgroup } \\
\text { analysis; likely exposure } \\
\text { misclassification; no evaluation of } \\
\text { exposure intensity, } 70 \% \text { had } \\
\text { exposure to mixed solvents } \\
\text { Null: No evidence for a positive } \\
\text { association but limited utility } \\
\text { (limitations mainly towards the } \\
\text { null) }\end{array}$ \\
\hline
\end{tabular}


RoC Monograph on Trichloroethylene

\begin{tabular}{|c|c|c|c|c|c|}
\hline Reference & $\begin{array}{c}\text { Study } \\
\text { Size (N) } \\
\text { Exposure } \\
\text { Assessment }\end{array}$ & Exposure Groups & $\begin{array}{c}\text { External Analysis } \\
\text { SMR, SIR (95\% CI) \# } \\
\text { Exposed Cases/Deaths }\end{array}$ & $\begin{array}{c}\text { Internal Analysis } \\
\text { RR }(95 \% \text { CI })^{\mathbf{a}} \\
\text { \# Exposed Cases/Deaths } \\
\text { or Cases/Controls }\end{array}$ & Interpretation \\
\hline $\begin{array}{l}\text { Radican et al. } \\
\text { (2008) } \\
\text { (mortality to } \\
\text { 2000) } \\
\text { Blair et al. } \\
\text { (1998) } \\
\text { (incidence } \\
\text { 1973-1990) }\end{array}$ & $\begin{array}{l}\text { Utah (USA) aircraft } \\
\text { maintenance workers } \\
\mathrm{N}=7,204(5,153 \mathrm{M}, \\
1,051 \mathrm{~F}) \\
\text { Semi-quantitative } \\
\text { JEM, individual } \\
\text { work histories }\end{array}$ & $\begin{array}{l}\text { Radican et al.: } \\
\text { Ever-exposed } \\
155+156 \\
\text { Primary liver: } 155.0 \\
\text { Cum. exp. (unit-yr) } \text { Men }^{b} \\
\text { All } \\
0-5 \\
5-25 \\
>25 \\
\text { All } \\
0-5 \\
\text { 5-25 } \\
>25 \\
\text { Blair et al. (1998) } \\
\text { Cumulative Exp Men } \\
\text { No TCE exposure } \\
<5 \text { units-yr } \\
\text { 5-25 units-yr } \\
\geq 25 \text { units-yr }\end{array}$ & NR & $\begin{array}{l}\text { ICD-9 } \\
\text { HR mortality } \\
1.12(0.57-2.19) ; 31 \\
1.25(0.31-4.97) ; 8 \\
\text { ICD-9 155+156) } \\
1.36(0.59-3.11) ; 28 \\
1.17(0.45-3.09) ; 10 \\
1.16(0.39-3.46) ; 6 \\
1.72(0.68-4.38) ; 12 \\
\text { Primary liver } \\
2.72(0.34-21.88) ; 8 \\
3.28 \text { (0.37-29.45); } 4 \\
0 \\
4.05 \text { (0.45-36.41); } 4 \\
\text { RR (incidence) } \\
\text { (ICD-9 155+156) } \\
0.2 \text { (0.1-2.4); } 1 \\
0.6(0.1-3.1) ; 3 \\
0.6(0.1-3.8) ; 2 \\
1.1(0.2-4.8) ; 4\end{array}$ & $\begin{array}{l}\text { Estimated exposure: Most workers } \\
\text { exposed to low levels ( } 10 \mathrm{ppm}) \text {, } \\
\text { modest number of workers exposed } \\
\text { to higher levels ( } \sim 100 \mathrm{ppm}) \\
\text { Covariates: age, calendar year, and } \\
\text { sex } \\
\text { Strengths: Adequate semi- } \\
\text { quantitative JEM, long follow-up, } \\
\text { adequate statistical power for ever } \\
\text { exposure } \\
\text { Limitations: Potential for exposure } \\
\text { misclassification because of } \\
\text { missing information for some } \\
\text { workers; limited power due to low } \\
\text { numbers of higher exposed } \\
\text { workers; long follow-up time } \\
\text { (45 years) may be past induction } \\
\text { time; cannot rule out confounding } \\
\text { from other co-exposures } \\
\text { Limited evidence for a positive } \\
\text { association: Statistically non- } \\
\text { significant elevated effect estimates } \\
\text { for primary liver cancer; some } \\
\text { evidence (not significant) for an } \\
\text { exposure-response gradient }\end{array}$ \\
\hline
\end{tabular}


RoC Monograph on Trichloroethylene

\begin{tabular}{|c|c|c|c|c|c|}
\hline Reference & $\begin{array}{c}\text { Study } \\
\text { Size (N) } \\
\text { Exposure } \\
\text { Assessment }\end{array}$ & Exposure Groups & $\begin{array}{c}\text { External Analysis } \\
\text { SMR, SIR (95\% CI) \# } \\
\text { Exposed Cases/Deaths }\end{array}$ & $\begin{array}{c}\text { Internal Analysis } \\
\text { RR }(95 \% \text { CI })^{\mathbf{a}} \\
\text { \# Exposed Cases/Deaths } \\
\text { or Cases/Controls } \\
\end{array}$ & Interpretation \\
\hline \multirow{6}{*}{$\begin{array}{l}\text { Boice et al. } \\
(2006) \\
\text { (overlap with } \\
\text { Zhao et al. } \\
(2005) \text { ) }\end{array}$} & Los Angeles (USA) & \multirow{6}{*}{ Ever exposed } & \multirow{6}{*}{$\begin{array}{l}\text { SMR (ICD-9 155+156) } \\
1.28(0.35-3.27) ; 4\end{array}$} & & Exposure occurred during test \\
\hline & $\begin{array}{l}\text { Rocket engine testing } \\
\text { workers } 1.111 \mathrm{Men}\end{array}$ & & & & $\begin{array}{l}\text { engine flush, which is likely to be } \\
\text { high }\end{array}$ \\
\hline & \multirow[t]{4}{*}{$\begin{array}{l}\text { Qualitative JEM; } \\
\text { Individual work } \\
\text { histories }\end{array}$} & & & & $\begin{array}{l}\text { Covariates: Date of birth, year of } \\
\text { hire, pay type (surrogate for SES), } \\
\text { and exposure to hydrazine }\end{array}$ \\
\hline & & & & & Strengths: Adequate follow up \\
\hline & & & & & $\begin{array}{l}\text { Limitations: Qualitative exposure } \\
\text { assessment; few exposed cases }\end{array}$ \\
\hline & & & & & $\begin{array}{l}\text { Null: Small increase in risk but } \\
\text { limited utility }\end{array}$ \\
\hline \multirow[t]{6}{*}{$\begin{array}{l}\text { Morgan et al. } \\
(1998)\end{array}$} & \multirow{3}{*}{$\begin{array}{l}\text { Arizona (USA) } \\
\text { aircraft } \\
\text { manufacturing } \\
\text { workers } \\
\mathrm{N}=4,733(2,555 \mathrm{M}, \\
2,178 \mathrm{~F})\end{array}$} & \multirow{6}{*}{$\begin{array}{l}\text { Ever exposed } \\
\text { Cumulative exp. score } \\
\text { Low }(2,357) \\
\text { High }(2,376) \\
\text { Peak (med/high) vs. low/no }\end{array}$} & $\begin{array}{l}\text { SMR (liver \& biliary) } \\
0.98(0.36-2.13) ; 6\end{array}$ & $\begin{array}{l}R R(\text { mortality }) \\
1.48(0.56-3.91) ; 6^{c}\end{array}$ & $\begin{array}{l}\text { High-exposure jobs were } \\
\text { considered to be } \geq 50 \mathrm{ppm}\end{array}$ \\
\hline & & & $1.32(0.27-3.85) ; 3$ & $2.12(0.59-7.66) ; 3$ & Covariates: age at hire, gender \\
\hline & & & $0.78(0.16-2.28) ; 3$ & $1.19(0.34-4.16) ; 3$ & $\begin{array}{l}\text { (decade of hire considered but no } \\
\text { effect) }\end{array}$ \\
\hline & \multirow{3}{*}{$\begin{array}{l}\text { Semi-quantitative } \\
\text { JEM; individual } \\
\text { work history }\end{array}$} & & & & $\begin{array}{l}\text { Strengths: Long follow-up and } \\
\text { semi-quantitative exposure }\end{array}$ \\
\hline & & & & & $\begin{array}{l}\text { Limitations: Evidence of an HWE; } \\
\text { potential exposure misclassification } \\
\text { among low/medium exposure } \\
\text { groups; mortality analysis and few } \\
\text { exposed cases }\end{array}$ \\
\hline & & & & & $\begin{array}{l}\text { Limited evidence of a positive } \\
\text { association: Statistically non- } \\
\text { significant, elevated effect estimate } \\
\text { (internal analysis); no evidence for } \\
\text { exposure-response relationship; } \\
\text { based on few exposed subjects }\end{array}$ \\
\hline
\end{tabular}




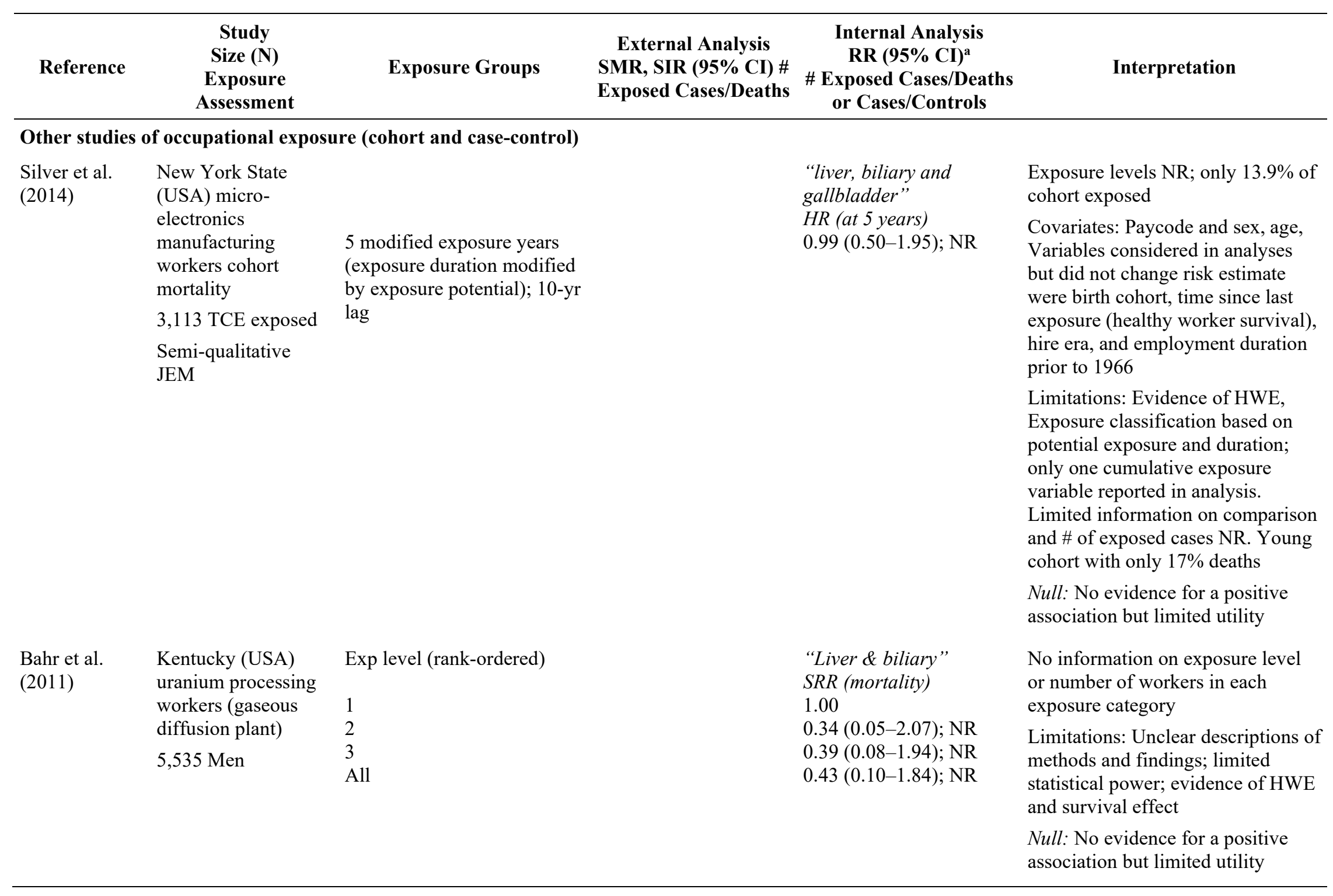


RoC Monograph on Trichloroethylene

\begin{tabular}{|c|c|c|c|c|c|}
\hline Reference & $\begin{array}{c}\text { Study } \\
\text { Size (N) } \\
\text { Exposure } \\
\text { Assessment }\end{array}$ & Exposure Groups & $\begin{array}{c}\text { External Analysis } \\
\text { SMR, SIR (95\% CI) \# } \\
\text { Exposed Cases/Deaths }\end{array}$ & $\begin{array}{c}\text { Internal Analysis } \\
\text { RR }(95 \% \text { CI })^{\mathrm{a}} \\
\text { \# Exposed Cases/Deaths } \\
\text { or Cases/Controls }\end{array}$ & Interpretation \\
\hline \multirow[t]{11}{*}{ Ritz (1999) } & \multirow{11}{*}{$\begin{array}{l}\text { Ohio (USA) uranium } \\
\text { processing workers } \\
2,971(\mathrm{M})\end{array}$} & \multirow{11}{*}{$\begin{array}{l}\text { Low exp. no lag } \\
>2 \text { years } \\
>5 \text { years } \\
\text { Moderate exp. no lag } \\
>2 \text { years } \\
>5 \text { years } \\
\text { Low exp. 15-yr lag } \\
>2 \text { years } \\
>5 \text { years } \\
\text { Moderate exp. 15-yr lag } \\
>2 \text { years } \\
>5 \text { years }\end{array}$} & NR & $I C D-9155+156$ & $96 \%$ workers with low exposure \\
\hline & & & & $R R$ (mortality) & Covariates: Time since 1 st hire, pay \\
\hline & & & & $0.93(0.19-4.53) ; 3$ & type, internal radiation, \& same \\
\hline & & & & $1.90(0.35-10.3) ; 3$ & chemical at a different level \\
\hline & & & & $4.97(0.48-51.1) ; 1$ & Strengths: Follow-up adequate \\
\hline & & & & $8.82(0.79-98.6) ; 1$ & Limitations: Low exposure, limited \\
\hline & & & & & power; selection bias possible \\
\hline & & & & $1.16(0.24-5.60) ; 3$ & Possible residual confounding by \\
\hline & & & & $2.86(0.48-17.3) ; 3$ & radiation \\
\hline & & & & $5.53(0.54-56.9) ; 1$ & Limited evidence for a positive \\
\hline & & & & $12.1(1.03-144) ; 1$ & $\begin{array}{l}\text { association: Pattern of increasing } \\
\text { risk with increasing exposure and } \\
\text { lag but based on small numbers }\end{array}$ \\
\hline \multirow{7}{*}{$\begin{array}{l}\text { Greenland et } \\
\text { al. (1994) } \\
\text { (nested case- } \\
\text { control study) }\end{array}$} & \multirow{7}{*}{$\begin{array}{l}\text { Massachusetts (USA) } \\
\text { electrical } \\
\text { manufacturers } \\
\mathrm{N}=12 \text { cases } \\
\text { (exposed controls } \\
\mathrm{NR} \text { ) }\end{array}$} & & & $I C D-8155+156$ & Limited statistical power; only $10 \%$ \\
\hline & & & & OR (mortality) & of jobs had exposure to TCE, most \\
\hline & & Ever exposure & & $0.54(0.11-2.63) ; \mathrm{NR}$ & of which were from indirect \\
\hline & & & & & exposure \\
\hline & & & & & $\begin{array}{l}\text { Covariates: Age, date of death, } \\
\text { covariates that changed risk } \\
\text { estimate by } 20 \%\end{array}$ \\
\hline & & & & & $\begin{array}{l}\text { Limitations: Small numbers of } \\
\text { cases and controls and short follow- } \\
\text { up, possible selection bias, low } \\
\text { quality exposure assessment }\end{array}$ \\
\hline & & & & & $\begin{array}{l}\text { Null: No evidence for a positive } \\
\text { association but limited utility }\end{array}$ \\
\hline \multirow{4}{*}{$\begin{array}{l}\text { Christensen et } \\
\text { al. (2013) } \\
\text { (case-control) }\end{array}$} & \multirow{4}{*}{$\begin{array}{l}\text { Montreal (Canada) } \\
\text { Population- and } \\
\text { hospital-exposure }\end{array}$} & & & Liver, presume ICD 155 & \multirow{4}{*}{$\begin{array}{l}\text { Number of cases inadequate for } \\
\text { evaluation }\end{array}$} \\
\hline & & & & OR (incidence) & \\
\hline & & Ever exposure & & $1.1(0.1-8.5) ; 1$ & \\
\hline & & Substantial exposure & & $2.1(0.2-18) ; 1$ & \\
\hline
\end{tabular}




\begin{tabular}{|c|c|c|c|c|c|}
\hline Reference & $\begin{array}{c}\text { Study } \\
\text { Size (N) } \\
\text { Exposure } \\
\text { Assessment }\end{array}$ & Exposure Groups & $\begin{array}{c}\text { External Analysis } \\
\text { SMR, SIR (95\% CI) \# } \\
\text { Exposed Cases/Deaths }\end{array}$ & $\begin{array}{c}\text { Internal Analysis } \\
\text { RR }(95 \% \text { CI })^{\mathrm{a}} \\
\text { \# Exposed Cases/Deaths } \\
\text { or Cases/Controls }\end{array}$ & Interpretation \\
\hline
\end{tabular}

\section{Environmental exposure}

Bove et al. North Carolina

(2014)

\section{(USA) (Camp \\ Lejeune)}

Drinking water

contamination

TCE in drinking water $(\mu g / L-$

\section{month)}

$\leq 1$

$>1-3,100$

$>3,100-7,700$

Ecological exposure

$$
>7,700-39,745
$$

assessment

154,932 men and

women
"Liver and biliary" HR (mortality); 10-yr lag $1.0(19)$

$1.02(0.48-2.15) ; 12$

$1.04(0.47-2.27) ; 11$

$0.86(0.37-1.97) ; 9$
Estimated mean levels $(\mu \mathrm{g} / \mathrm{L}$ -

month) TCE from water supply $=358.7$; overall cumulative exposure $=6,369$ (median) and 5,289 (mean); $20 \%$ were exposed to levels between 7,700 and 39,745

Covariates: sex, race, rank, and education; other variables considered in the model (did not change risk estimates by $10 \%$ ) include marital status, birth cohort, date of death, duty occupation.

Strengths: Large cohort and adequate modeling of exposure

Limitations: Young cohort; no information on individual water consumption; potential confounding from other contaminants e.g., tetrachloroethylene

Null: No evidence for a positive association but limited utility

Studies reported one or more of primary liver, liver plus intrahepatic biliary ducts, or liver, intrahepatic and extrahepatic biliary ducts and gallbladder combined (as noted). Not all studies reported ICD diagnostic codes used.

$\mathrm{CI}=$ confidence interval; $\mathrm{HR}=$ hazard ratio; $\mathrm{OR}=$ odds ratio, $\mathrm{p}-\mathrm{yr}=$ people years; $\mathrm{RR}=$ relative risk; $\mathrm{SIR}=$ standardized incidence ratio, $\mathrm{SMR}=$ standardized mortality ratio, $\mathrm{SRR}=$ standardized rate ratio.

a $\mathrm{HR}, \mathrm{OR}, \mathrm{RR}$, or SRR.

${ }^{b}$ Study also report risk by exposure patterns (continuous and peak). Among men, HR $>1$ for both primary liver and liver + biliary cancer in all exposure categories with no clear exposure-response relationships. Few cases of liver + biliary cancer were reported for women: $\mathrm{HR}<1.0$ for all cumulative exposure and exposure pattern categories except for peak, infrequent, $\mathrm{HR}=4.30(0.87-21.33) ; 2$.

'Reported by Scott and Jinot (2011): combined risk for men and women in Raaschou-Nielsen et al. (2003), and RR adjusted for age and sex for Morgan et al. (1998). 


\subsubsection{Meta-analyses}

Two meta-analyses have been conducted on the cohort studies of liver cancer, by the EPA (Scott and Jinot 2011; USEPA 2011a) and by Alexander et al. (2007) (see Table 6-2). The inclusion and exclusion criteria, systematic data extraction, and methods of analysis used in the EPA metaanalysis were identical to those used for meta-analyses of kidney cancer and NHL and have been described in Sections 4 and 5, respectively. (See Table D-10 for a list of the studies included in these meta-analyses.) Studies included in this review that are not part of the meta-analyses include the pooled analyses by Hansen et al. (2013) (which includes the populations reported on by Axelson et al. (1994), Anttila et al. (1995), and Hansen et al. (2001), the population-based cancer registry study of Nordic countries by Vlaanderen et al. (2013), two studies of uranium processing workers (Bahr et al. 2011; Ritz 1999), the microelectronics workers study by Silver et al. (2014), and the drinking water study by Bove et al. (2014).

Table 6-2. Meta-analyses of Liver Cancer (Including Gall Bladder and Biliary Passages) and Trichloroethylene Exposure

\begin{tabular}{llccl}
\hline \multicolumn{1}{c}{ Reference } & $\begin{array}{c}\text { Study Design } \\
\text { (Number of } \\
\text { Studies) }\end{array}$ & $\begin{array}{c}\text { mRR (95\% CI) } \\
\text { All }\end{array}$ & $\begin{array}{c}\text { mRR (95\% CI) } \\
\text { Highest Exposure }\end{array}$ & \multicolumn{1}{c}{ Comments } \\
\hline $\begin{array}{l}\text { EPA (2011a)/Scott } \\
\text { and Jinot (2011) }\end{array}$ & $\begin{array}{l}\text { Cohort studies (8) } \\
\text { and nested case- } \\
\text { control study (1) }\end{array}$ & $1.29(1.07-1.56)$ & $1.28(0.93-1.77)$ & $\begin{array}{l}\text { Random and fixed effects } \\
\text { models; little evidence of } \\
\text { heterogeneity or } \\
\text { publication bias } \\
\text { Random effects model; } \\
\text { some evidence of } \\
\text { heterogeneity }\end{array}$ \\
$\begin{array}{l}\text { Alexander et al. } \\
(2007)\end{array}$ & Cohort studies (8) & $1.30(1.09-1.55)$ & NR & \\
\hline
\end{tabular}

$\mathrm{mRR}=$ meta-relative risk; $\mathrm{NR}=$ not reported; $\mathrm{RR}=$ relative risk.

The two meta-analyses are broadly comparable in terms of the studies included and the method of analysis. Scott and Jinot (2011) considered each of the studies up to and including 2011 listed in Table 6-1, with the exception of Bahr et al. (2011), Lipworth et al. (2011), and Ritz (1999). They did, however, include Boice et al. (1999), the earlier study followed up by Lipworth et al. (2011). Alexander et al. (2007) included each of the studies listed in Table 6-1 up to 2007 except for Zhao et al. (2005). The meta-analysis by Scott and Jinot (2011) suggests an overall statistically significant increase in the mRR for combined liver and biliary cancers, but a slight decrease in the mRR for the highest exposed groups was observed. Alexander et al. (2007) reported a comparable mRR. These authors also calculated mRRs for studies that reported primary liver cancer and biliary tract cancers separately and reported closely comparable risk estimates.

In the EPA meta-analysis, the mRR was elevated but less precise and no longer statistically significant $(\mathrm{OR}=1.22,95 \% \mathrm{CI}=0.93$ to 1.61$)$ with the removal of Raaschou-Nielsen et al. (2003), which was the largest study in that analysis, contributing $53 \%$ of the weight, and reporting twice the number of events as the other contributing studies. Differences in exposure metrics used in the component studies, and small numbers of cases or deaths place limitations on analyses by exposure intensity or duration. In the EPA analysis, the lower mRR observed among the highest exposed groups primarily reflects the inverse exposure-duration response relationship for exposure duration reported in the largest study by Raaschou-Nielsen et al. (2003) (Scott and 
Jinot 2011). Studies conducted since 2011 have not observed overall increases in risks or are of limited utility due to limited statistical power or exposure assessments, or other concerns.

\subsubsection{Occupational Co-exposures}

With respect to occupational carcinogens, IARC and/or the Report on Carcinogens (Cogliano et al. 2011; NTP 2011) have identified some types of radiation (plutonium, thorium and its decay products), vinyl chloride and polychlorinated biphenyls as known human liver carcinogens, and concluded that there was limited evidence of human carcinogenicity for inorganic arsenic, and $\mathrm{X}$ - and gamma-radiation. Trichloroethylene-exposed workers in some studies may have been exposed to a range of other chemical or physical agents, primarily (1) chlorinated solvents (primarily tetrachloroethylene and 1,1,1-trichloroethane) in the Nordic studies, the studies of aircraft manufacturing and aerospace workers, and the drinking water study, (2) cutting fluids such as mineral and petroleum oils, organic solvents, hydrazine, benzene, chromates, and PAHs in the aerospace and aircraft industries, and (3) radiation (Ritz 1999), or cutting oils and metals in the studies of uranium processing workers (Bahr et al. 2011), and (4) vinyl chloride in the drinking water study (Bove et al. 2014). The co-exposure with the strongest potential for confounding is ionizing radiation in the study of uranium workers by Ritz (1999). A positive association was observed for liver cancer in this study after adjusting for exposure to radiation, which helps to reduce concern that confounding occurred, although residual confounding cannot be ruled out. In addition, there is limited evidence of exposure-response patterns with trichloroethylene intensity and duration in this study; however, few workers were exposed to moderate levels of trichloroethylene. Vinyl chloride is not a concern because no association with trichloroethylene was found in the drinking water study.

The other principal co-exposures identified in these studies have not been classified as known or suspected liver carcinogens in humans; however, there is some or sufficient evidence in animal studies for the liver carcinogenicity of several chlorinated and non-chlorinated solvents, including tetrachloroethylene, 1,1,2,2- and 1,1,1,2-tetrachloroethane, carbon tetrachloride, methylene chloride, and hydrazine. The chlorinated solvents tetrachloroethylene and 1,1,1trichloroethane are probably common co-exposures in the aircraft manufacturing studies and possibly in the Nordic studies. In addition, the strength of the association with trichloroethylene was limited in these studies. Thus, confounding, especially in the aircraft manufacturing studies, cannot be reasonably ruled out.

\subsubsection{Lifestyle and Other Potential Confounders}

Non-occupational risk factors include alcohol consumption, aflatoxins, estrogen-progestogen contraceptives, tobacco smoking, betel quid use without tobacco, viral infections (hepatitis B and $\mathrm{C}$ and human immunodeficiency virus type 1), parasites (liver flukes and Schistosoma), longterm use of anabolic steroids, and ionizing radiation (Cogliano et al. 2011; NTP 2011). Some of these factors, such as smoking, alcohol consumption, and possibly biological infections, may be related to socioeconomic status and could possibly vary by trichloroethylene exposure status.

The majority of cohort and nested case-control studies conducted age-, sex-, race- and calendaryear or period-standardized comparisons in external analyses (SMR or SIR) where appropriate and age-, sex-, race- and in some cases calendar period-adjusted comparisons in internal analyses. In addition, all of the studies, except for the Danish blue-collar worker study, conducted internal analyses, which would mitigate potential confounding from lifestyle factors. 
Although none of the cohort studies adjusted for smoking, tobacco smoking is a weaker risk factor for liver cancer than other cancers (meta-risk estimate approximately 1.5, (Lee et al. 2009)) and as noted in Section 4 for kidney cancer, there was little evidence for an association of trichloroethylene and lung cancer, which suggests that confounding from smoking is not a concern.

While none of the studies directly addressed alcohol consumption, incidence rates of cancers of the oral cavity, pharynx, or esophagus, or of cirrhosis (where reported) may provide indirect evidence of alcohol consumption relative to the reference population. While these rates are unremarkable in most of the studies, approximately 2-fold, statistically non-significant increases in incidence rates were observed for oropharyngeal and esophageal cancers among women in the Danish blue-collar workers cohort (Raaschou-Nielsen et al. 2003). Smoking- and alcohol-related diseases were not statistically significantly increased in the pooled analysis reported by Hansen et al. (2013). Overall, there is no strong indirect evidence for potential confounding by alcohol use in most of the cohorts, with the possible exception of the Danish cohort of trichloroethyleneexposed women workers (Raaschou-Nielsen et al. 2003).

\subsubsection{Integration}

Several individual cohort studies with moderate or low to moderate utility found modest increases in risk of liver cancer (Hansen et al. 2013; Morgan et al. 1998; Raaschou-Nielsen et al. 2003; Radican et al. 2008); the strongest evidence was from the external analysis in the updated and pooled analysis of biomonitored workers in Nordic countries (Hansen et al. 2013). (See Figure 6-2 for a plot of risk estimates for groups ever exposed to trichloroethylene grouped according to study utility.) However, no exposure-response relationship was observed in the internal analysis in this study and there was little evidence of an exposure-response relationship in any study with the possible exception of the Utah aircraft-manufacturing workers in analyses specific for primary liver cancer (Radican et al. 2008).

The database is inadequate to evaluate the effect of latency, as few cohort studies conducted lagged vs. unlagged or time since first exposure analyses and data are generally sparse due to limited numbers of cases. SIRs increased with increasing lagged time (no lagged, 10 year lagged, and 20-year lagged) in the Nordic study of biomonitored workers and (Hansen et al. 2013). However, no relationship between risk of liver cancer and lagging was observed in two other studies (Raaschou-Nielsen et al. 2003; Vlaanderen et al. 2013). The rest of the studies did not conduct lagged analyses (Bahr et al. 2011; Boice et al. 2006; Christensen et al. 2013; Lipworth et al. 2011; Morgan et al. 1998; Radican et al. 2008) or only reported effect estimates for one lagging period (Bove et al. 2014; Greenland et al. 1994; Silver et al. 2014).

No or little evidence of an association of trichloroethylene exposure (for either ever exposed groups or among the highest exposed) and liver cancer risk was found in other studies, most of which were considered to be of low to low/moderate utility because of inadequate sensitivity to detect rare cancers such as liver cancer, concerns about non-differential exposure misclassification (Boice et al. 2006; Bove et al. 2014; Greenland et al. 1994; Lipworth et al. 2011; Silver et al. 2014; Vlaanderen et al. 2013) and/or other methodological concerns (Bahr et al. 2011). The only case-control study (Christensen et al. 2013) had too few exposed cases (one) to be informative. Ritz (1999) reported a positive association among uranium processing workers; however, this should be viewed with some caution because of potential residual 
confounding from exposure to radiation and small numbers of exposed cases. Two meta-analyses based on either nine or eight studies suggest a modest but statistically significant increase in liver cancer risk (Alexander et al. 2007; Scott and Jinot 2011), although they did not include some recent studies. Confounding by one or more of the common co-exposures, or chance, cannot be completely ruled out in some studies.

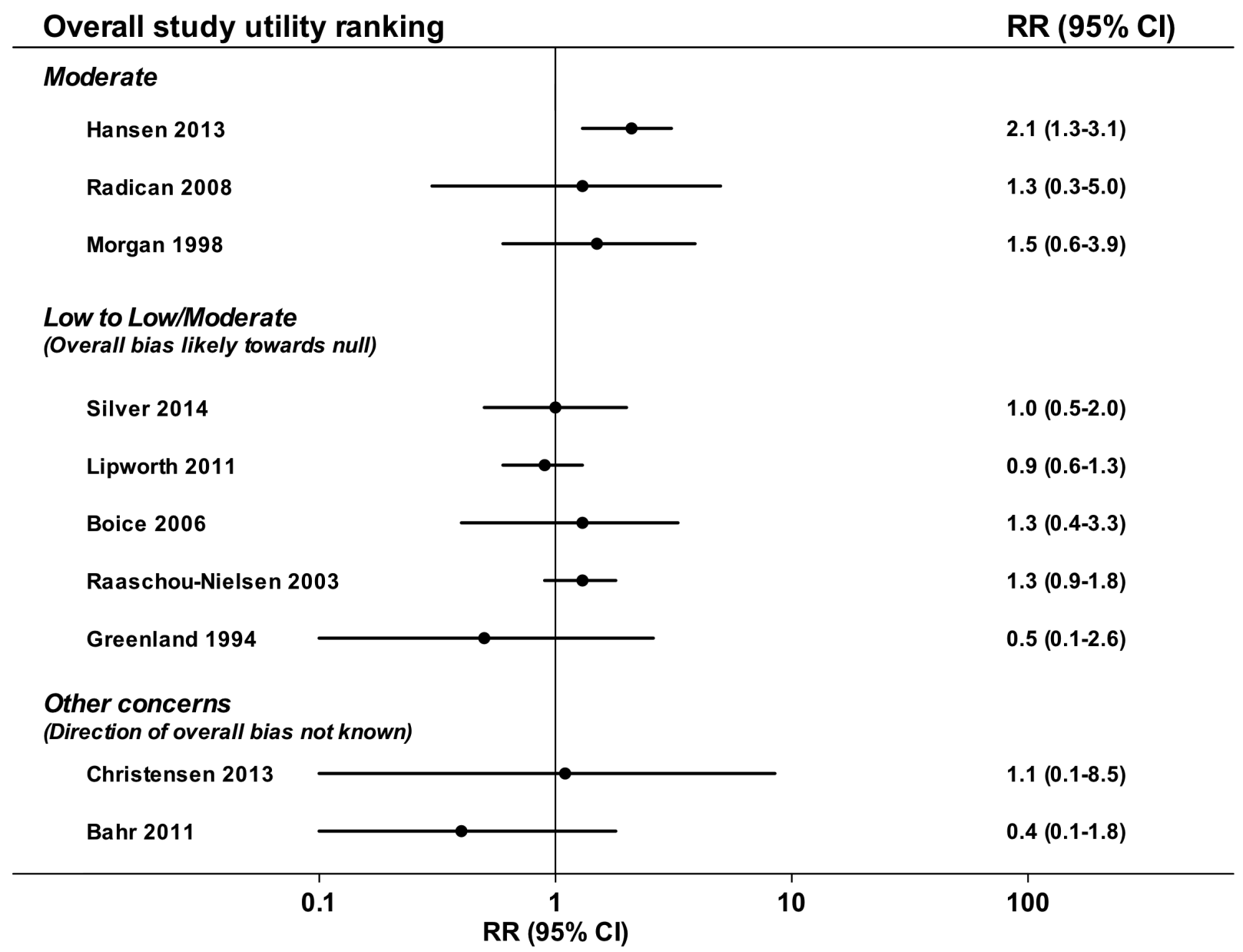

Figure 6-2. Forest Plot: Liver Cancer and Ever Exposure to Trichloroethylene

Relative risk and 95\% CI for ever exposure to trichloroethylene and liver cancer according to study utility category (see Figure 61) and overall prediction of direction of any bias for low utility studies as described in Section 4.1.3. The effect estimate for Christensen et al. (2013) is based on only one liver case observed. Studies by Bove et al. (2014), Vlaanderen et al. (2013), and Ritz et al. (1999) are not graphed because they did not report relative risk for ever exposure. Findings for these studies are reported in Table 6-1. For studies reporting multiple risk estimates, preference was given to studies with longer lag (Hansen et al. (2013) [20 years]) and internal analysis. Risk estimates for Raaschou-Nielsen et al. (2003) (combined men and women) and internal analysis for Morgan et al. (1998) were reported by Scott and Jinot (2011).

\subsection{Mechanistic Data for Liver Carcinogenicity}

Trichloroethylene metabolites produced by P450 oxidation, primarily CYP2E1, (see Section 1.3) are most likely responsible for liver toxicity and cancer (USEPA 2011a). Support for this hypothesis includes the following: trichloroethylene and its oxidative metabolites have similar hepatotoxic and hepatocarcinogenic effects, pretreatment with CYP inducers enhances hepatotoxicity, and treatment with CYP inhibitors decreases hepatotoxicity. In addition, liver 
tumor analyses based on immunostaining for c-Jun show that neither trichloroacetic acid nor dichloroacetic acid alone can account for the full characteristics of trichloroethylene-induced liver tumors (Bull et al. 2002).

This section reviews the hypothesized modes of action for liver carcinogenicity and is divided into two subsections: modes of action with limited experimental support and modes of action that are inadequately defined or have little to no experimental support. As with the previous mechanistic sections for kidney cancer and non-Hodgkin lymphoma, the discussion relies on recent comprehensive reviews by EPA (2011a; 2011b) and IARC (2014). The findings from these reviews are supplemented with primary literature that was not included in the reviews or as needed for clarity.

\subsubsection{Hypothesized Modes of Action with Limited Experimental Support}

Modes of action proposed for trichloroethylene-induced liver cancer that are perhaps the most biologically plausible include the following: genotoxicity from oxidative metabolites, PPAR $\alpha$ activation, oxidative stress, and hypomethylation and gene expression changes (IARC 2014; USEPA 2011a; 2011b). Another possible mode of action is autoimmune hepatitis (Czaja 2013; Wang et al. 2013).

There are several similarities between the hypothesized modes of action in trichloroethyleneinduced liver tumors in mice and some of the known characteristics of human hepatocellular carcinoma (USEPA 2011a). The mode of action for trichloroethylene-induced liver tumors is complex and likely involves key events from several pathways. Overall, a role for many of the key events could not be ruled out. Although the level of evidence varied for the different modes of action, the data were inadequate to support a definite conclusion that any of the proposed modes of action is operant. The key events associated with the proposed modes of action with the most experimental support are listed in Table 6-3 and are discussed below.

Table 6-3. Possible Modes of Action and Key Events for Trichlorethylene-induced Liver Cancer

\begin{tabular}{|c|c|}
\hline Mode of Action & Key Events \\
\hline Genotoxicity & $\begin{array}{l}\text { 1. One or more oxidative metabolites are produced in situ or delivered systemically } \\
\text { to the liver } \\
\text { 2. Genotoxicity induced by oxidative metabolites advances acquisition of the } \\
\text { multiple critical traits contributing to carcinogenesis }\end{array}$ \\
\hline PPAR $\alpha$ activation & $\begin{array}{l}\text { 1. Oxidative metabolites activate PPAR } \alpha \text { in the liver } \\
\text { 2. PPAR } \alpha \text { activation leads to alterations in cell proliferation and apoptosis } \\
\text { 3. Alterations in cell proliferation and apoptosis cause clonal expansion of initiated } \\
\text { cells } \\
\text { 4. Clonal expansion of initiated cells leads to tumor formation }\end{array}$ \\
\hline Oxidative stress & $\begin{array}{l}\text { 1. Trichloroethylene or its metabolites induce oxidative stress } \\
\text { 2. Oxidative stress leads to chronic inflammation, mutations, and damage to } \\
\text { proteins, lipids, and DNA } \\
\text { 3. Mutations and damage to macromolecules activates cell-signaling pathways, } \\
\text { induces genomic instability and cell transformation, and leads to cancer }\end{array}$ \\
\hline Epigenetic changes & $\begin{array}{l}\text { 1. Epigenetic changes, particularly DNA methylation, are induced by one or more } \\
\text { metabolites } \\
\text { 2. These changes advance acquisition of multiple critical traits contributing to } \\
\text { carcinogenesis }\end{array}$ \\
\hline
\end{tabular}




\begin{tabular}{|c|c|}
\hline Mode of Action & Key Events \\
\hline Autoimmune hepatitis & $\begin{array}{l}\text { 1. Reactive metabolites form protein adducts and/or induce oxidative stress leading } \\
\text { to lipid peroxidation and oxidative modifications to proteins in the liver } \\
\text { (neoantigens) } \\
\text { 2. Activation and hepatic infiltration of } \mathrm{CD} 4^{+} \mathrm{T} \text { cells and secretion of inflammatory } \\
\text { cytokines } \\
\text { 3. Inhibition of apoptosis in self-reactive } \mathrm{CD} 4^{+} \mathrm{T} \text { cells } \\
\text { 4. Formation of anti-malondialdehyde- and anti-hydroxynonenal-protein adduct } \\
\text { antibodies in association with increases in anti-nuclear antibodies } \\
\text { 5. Hepatocyte damage/autoimmune hepatitis } \\
\text { 6. Autoimmune hepatitis/cirrhosis contributes to hepatocarcinogenesis }\end{array}$ \\
\hline
\end{tabular}

Sources: Czaja (2013), EPA (2011a). Gilbert et al. (2006), Griffin et al. (2000c), Wang et al. (2013).

\subsubsection{Genotoxicity}

Since genotoxicity is a well-established cause of carcinogenicity, one hypothesis is that trichloroethylene causes liver cancer by a genotoxic/mutagenic mode of action, presumably through formation of reactive oxidative metabolites that cause direct alterations in hepatocyte DNA (e.g., mutations, DNA damage, and/or clastogenic effects) (USEPA 2011a). The genotoxic effects of trichloroethylene and its metabolites were presented in Section 2. Chloral hydrate appears to have the greatest genotoxic potential among the oxidative metabolites. Genotoxic effects associated with chloral hydrate included mutagenicity in the Ames test; micronucleus formation, chromosome aberrations, aneuploidy, and cell transformation in mammalian cell cultures; and in vivo studies reported DNA single-strand breaks and micronucleus induction in mice. Some have argued that chloral hydrate is unlikely to be the cause of trichloroethylene carcinogenicity because it is a short-lived intermediate metabolite that is rapidly converted to trichloroacetic acid and trichloroethanol in the liver. Furthermore, doses used in the in vitro genotoxic studies were generally much higher than the reported peak concentrations achieved in the liver of rodents administered hepatocarcinogenic doses of trichloroethylene. However, it is uncertain if a direct comparison between concentrations in culture media used in genotoxicity assays in vitro and concentrations in whole-liver homogenates achieved in vivo is appropriate. Furthermore, some in vivo genotoxicity assays with chloral hydrate reported positive results at doses similar to those that induced a carcinogenic response in chronic bioassays.

Several studies investigated the frequency and spectra of H-ras mutations in liver tumors induced by trichloroethylene, trichloroacetic acid, or dichloroacetic acid (Bull 2000; Bull et al. 2002). Although there were some differences in the H-ras mutation frequencies reported among the studies, the data indicate that trichloroethylene, dichloroacetic acid, and trichloroacetic acid activate mutations in codon 61 of the $\mathrm{H}$-ras protooncogene in liver carcinomas of male $\mathrm{B}_{6} \mathrm{C}_{3} \mathrm{~F}_{1}$ mice at a frequency similar to that observed in spontaneous liver tumors. Trichloroacetic acidinduced tumors showed the same mutational spectrum as spontaneous liver tumors; however, trichloroethylene- and dichloroacetic acid-induced tumors had a significant decrease in AAA mutations and a significant increase in CTA mutations compared to spontaneous- or trichloroacetic acid-induced liver tumors. The similarity in frequency and types of H-ras mutations in liver tumors induced by trichloroacetic acid compared with spontaneous tumors suggests that trichloroacetic acid may act as a promoter of spontaneous tumors (Eastmond 2012). $\mathrm{H}$-ras mutations appeared to be a late event because the frequency of $\mathrm{H}$-ras mutations increased with time and was higher in hepatocellular carcinomas compared with adenomas (Bull et al. 2002). The effects of dichloroacetic acid and trichloroacetic acid were not typical of genotoxic 
agents and suggested that these compounds promoted clonal expansion of initiated cells while DNA damage accumulated with tumor growth. The data also suggest that both trichloroacetic acid and dichloroacetic acid may be involved in trichloroethylene-induced liver tumors through activation of the H-ras protooncogene. However, the mechanisms do not appear to be the same for dichloroacetic acid and trichloroacetic acid (Ferreira-Gonzalez et al. 1995).

It is clear that human and rodent livers are exposed to the oxidative metabolites of trichloroethylene. Chloral hydrate is the most genotoxic oxidative metabolite but is rapidly converted to trichloroacetic acid and trichloroethanol. The data are insufficient to assess the genotoxic contributions from the nongenotoxic contributions of chloral hydrate or other oxidative metabolites. Although the data are inadequate to conclude that a genotoxic mode of action is responsible for trichloroethylene-induced liver tumors, a genotoxic mode of action mediated by the oxidative metabolites is biologically plausible and cannot be ruled out.

\subsubsection{PPARa Activation}

Trichloroethylene, trichloroacetic acid, and dichloroacetic acid induce peroxisome proliferation in mice but are relatively weak PPAR $\alpha$ agonists requiring $\mathrm{mM}$ concentrations (Corton 2008; Keshava and Caldwell 2006). The peroxisome-related effects of trichloroethylene are most likely mediated through trichloroacetic acid because it is a primary oxidative metabolite of trichloroethylene and is a stronger PPAR $\alpha$ agonist than dichloroacetic acid. The data linking trichloroethylene-induced liver tumors to a PPAR $\alpha$-dependent mechanism include the following: (1) there is a relatively good correlation between trichloroethylene- and trichloroacetic acidinduced liver tumors and induction of markers of PPAR $\alpha$ activation in the mouse but not in the rat, (2) transactivation assays show that trichloroacetic acid activates mouse and human $\operatorname{PPAR} \alpha$, (3) markers of PPAR $\alpha$ activation are elevated at trichloroethylene or trichloroacetic acid doses below or coincident with doses that induce mouse liver tumors in a manner similar to other peroxisome proliferators, (4) trichloroethylene increases hepatocyte proliferation and peroxisome proliferator-associated genes in wild-type but not PPAR $\alpha$-null mice $(93 \%$ of the altered genes in wild-type mice were PPAR $\alpha$ dependent), and (5) trichloroacetic acid-induced mouse liver tumors have properties similar to those induced by classic peroxisome proliferators in rat liver (Corton 2008; Laughter et al. 2004).

However, it is unlikely that trichloroethylene induces liver tumors solely through metabolism to trichloroacetic acid and PPAR $\alpha$ activation. The dose-response for liver weight increases were different for the two compounds, and liver weight increases did not correlate with peroxisomal enzyme activity or changes in peroxisomal number or volume (USEPA 2011a). Bull et al. (2002) also reported differences in tumor phenotypes (based on c-Jun expression) between trichloroethylene and trichloroacetic-acid-induced liver tumors. The H-ras mutation frequency in trichloroethylene-induced liver tumors was more similar to spontaneous or dichloroacetic acidinduced tumors than to trichloroacetic acid-induced tumors (discussed above in the Genotoxicity subsection) (Bull 2000; Bull et al. 2002). The H-ras mutation frequency pattern in trichloroacetic acid-induced liver tumors also was opposite that observed with other peroxisome proliferators. Furthermore, recent studies have demonstrated that PPAR $\alpha$ activation is not the sole mode-ofaction of hepatocarcinogenesis for known PPAR $\alpha$ agonists (Guyton et al. 2009; USEPA 2011a).

Although trichloroethylene activates PPAR $\alpha$ and other key events in the hypothesized mode of action, most of the proposed key events are nonspecific and may be caused by multiple mechanisms. A causal linkage between trichloroethylene exposure and alterations in gene 
expression and DNA synthesis with PPAR $\alpha$ has not been established. Together, these data suggest that multiple mechanisms and cell types are likely involved in the hepatocarcinogenicity of PPAR $\alpha$ agonists. It is biologically plausible that PPAR $\alpha$ agonism mediated by trichloroacetic acid is operant; however, it is unlikely that it is the sole or predominant mode of action for trichloroethylene-induced hepatocarcinogenicity in mice.

\subsubsection{Oxidative Stress}

Oxidative stress is an important factor in a number of human diseases, including cancer, and occurs when the concentration of reactive oxygen species (ROS) generated exceeds the antioxidant capacity of the cell (Klaunig et al. 1998). It can be induced by exposure to drugs or other chemicals, but also is part of normal cellular respiration and cell signaling. The consequences of oxidative stress may include damage to critical cellular macromolecules including DNA, lipids, and proteins. One of the most common forms of damage is the generation of 8-hydroxy-2'-deoxyguanosine (8-OHdG), a highly mutagenic adduct capable of causing cellular DNA damage. Other common biomarkers of oxidative stress include thiobarbituric acidreactive substances (TBARS, an index of lipid peroxidation) and superoxide anion production.

Several studies reported evidence of oxidative stress in the liver of mice or rats following acute, subacute, or subchronic exposure to trichloroethylene, trichloroacetic acid, or dichloroacetic acid (Austin et al. 1996; Channel et al. 1998; Larson and Bull 1992; Parrish et al. 1996; Tabrez and Ahmad 2009; Toraason et al. 1999). EPA (2011a; 2011b) identified several issues in most of these studies (i.e., lack of appropriate controls, incomplete reporting, marked toxicity, and possible confounding by vehicle or route of administration effects) that limited interpretation of the data. However, a series of more recent studies show that dichloroacetic acid and trichloroacetic acid induce oxidative stress and macrophage activation in $\mathrm{B}_{6} \mathrm{C} 3 \mathrm{~F}_{1}$ mice (Hassoun and Ray 2003; Hassoun and Dey 2008; Hassoun et al. 2010a; Hassoun et al. 2010b; Hassoun and Cearfoss 2011; Hassoun et al. 2013). These studies were not reviewed by EPA (2011a; 2011b) and are briefly reviewed below.

In vitro studies using murine macrophage J774A.1 cells exposed to dichloroacetic acid or trichloroacetic acid showed dose- and time-dependent increases in superoxide anion production, cellular death, and lactate dehydrogenase (LDH) release (a marker of cell death) (Hassoun and Ray 2003). In contrast to in vivo studies (discussed below), there were no significant differences in the effects of these two compounds.

A series of in vivo studies were conducted to investigate oxidative stress in male B6C3F $\mathrm{F}_{1}$ mice exposed to dichloroacetic acid and trichloroacetic acid (Cearfoss and Hassoun 2012; Hassoun and Dey 2008; Hassoun et al. 2010a; Hassoun et al. 2010b; Hassoun and Cearfoss 2011; Hassoun et al. 2013). Data from these studies are summarized in Appendix F and include the following: superoxide anion production in liver and peritoneal lavage cells (Table F-1), lipid peroxidation and DNA single-strand breaks in liver cells (Table F-2), phagocyte activation and superoxide dismutase (SOD) in peritoneal lavage cells (Table F-3), and antioxidant enzymes in liver cells (Table F-4).

Overall, these data show that both dichloroacetic acid and trichloroacetic acid induced dose- and time-dependent increases in superoxide anion production, lipid peroxidation, and DNA singlestrand breaks. The data also indicated that antioxidant enzymes (e.g., SOD, catalase, and glutathione peroxidase) were involved in cellular resistance to oxidative stress. In most cases, 
dichloroacetic acid had a greater effect than trichloroacetic acid. These data suggest that superoxide anion production contributes to lipid peroxidation and DNA damage in the liver. There also was a strong correlation between superoxide anion production in peritoneal lavage cells (considered as a surrogate for Kupffer cells) and hepatic tissues that suggested phagocytic activation may contribute to oxidative stress in the liver.

Hepatocyte oxidative stress also was identified as a key event associated with other modes of action. These include PPAR $\alpha$ activation (Klaunig et al. 2003), GST-zeta inhibition (Blackburn et al. 2006), and autoimmunity (see Section 2.2) (Wang et al. 2007a; Wang et al. 2007b; Wang et al. 2009a; Wang et al. 2012b; Wang et al. 2013; Wang et al. 2012c). Parrish et al. (1996) showed that markers of peroxisome proliferation and $8-\mathrm{OHdG}$ levels were not significantly different from controls in mice exposed to dichloroacetic acid and concluded that oxidative damage did not play an important role in the chronic hepatotoxicity of peroxisome proliferators. Blackburn et al. (2006) reported that GST-zeta deficiency results in a constant level of oxidative stress due to the accumulation of maleylacetone and maleylacetoacetate. As discussed below, dichloroacetic acid is an inhibitor of GST-zeta, thus, dichloroacetic acid could cause oxidative stress by diminishing GST-zeta levels. Finally, Wang et al. (2013) reported that $N$-acetylcysteine supplementation protected against trichloroethylene-induced autoimmunity by attenuating oxidative stress.

There is evidence that oxidative metabolites of trichloroethylene can cause oxidative stress in the liver and it is biologically plausible that oxidative stress can contribute to hepatotoxicity and hepatocarcinogenicity. However, the key events for this mode of action have not been fully specified and the data are insufficient to determine the necessity or sufficiency of oxidative stress in trichloroethylene-induced hepatocarcinogenicity.

\subsubsection{Epigenetic Changes (Altered Gene Expression/Hypomethylation)}

Altered gene expression, whether through global DNA hypomethylation or other mechanisms, can contribute to carcinogenesis by affecting genes identified with cell growth and differentiation, tissue remodeling, signal transduction, metabolism, apoptosis, cancer progression, and other processes (Caldwell and Keshava 2006; USEPA 2011a). Genetic expression studies and studies of changes in methylation status induced by trichloroethylene and its metabolites are reviewed below.

A limited number of in vitro and in vivo studies in experimental animals have investigated gene expression changes in liver induced by trichloroethylene or its oxidative metabolites (Caldwell and Keshava 2006; USEPA 2011a; 2011b). These studies reported that trichloroethylene alters expression of various stress-response, xenobiotic metabolizing, and homeostatic genes. Mice exposed to dichloroacetic acid also showed altered expression patterns in genes associated with cell growth, tissue remodeling, apoptosis, cancer progression, and xenobiotic metabolism in normal liver tissue and liver tumors.

Sano et al. (2009) investigated differences in gene expression profiles of liver in mice and rats exposed to acute and subacute oral doses of trichloroethylene. These differences included suppression of TGF- $\beta$ signaling, activation of MAPK signaling, and alteration of the ubiquitinproteasome system in mice but not rats and may play a role in the species-specific biochemical effects of trichloroethylene-induced liver carcinogenesis. Bradford et al. (2011) analyzed whole liver gene expression profiles in male mice from 15 inbred strains exposed to a single oral dose 
of trichloroethylene. PPAR $\alpha$-mediated molecular networks, primarily consisting of upregulation of lipid and drug metabolism genes, were the most pronounced effects that were dependent on genetic background. Gene expression changes that were significantly affected by treatment but not genotype included cell death, liver necrosis, and inflammatory-mediated response networks; however, there was little observable liver toxicity in this study. Transcription factor analysis of these genes revealed several inflammation-related regulatory proteins that are associated with activation of macrophages and lymphocytes and suggested that trichloroethylene may affect Kupffer cells. Recent in vitro studies using human hepatic L-02 cells reported that exposure to trichloroethylene induced alterations in the expression, distribution, and interactions of SETassociated proteins (Hong et al. 2012; Hong et al. 2013). SET (also known as protein phosphatase 2A inhibitor, I2PP2A, or template-activating factor-1, TAF-1) is a nuclear protein with roles in histone modification, gene transcription, DNA replication, nucleosome assembly, phosphatase activity, and kinase activity. Trichloroethylene also induced over-expression of several SET-binding proteins, including eukaryotic translation elongation factor 1 alpha 1 and 1 alpha 2 (eEF1A1 and eEF1A2), in a dose-dependent manner. Over-expression of eEF1A1 and eEF1A2 are associated with a variety of human tumors. Endogenous SET is known to decrease in the nucleus and increase in the cytoplasm upon cell death induced by toxic stress. eEF1A is primarily localized in the cytoplasm, but redistribution to the nucleus has been associated with cell proliferation and tumor development. Cytoplasmic translocation of SET and nuclear translocation of eEF1A also were observed in L-02 cells exposed to trichloroethylene. These results suggest that the translocation and over-expression of SET and eEF1A1/eEF1A2 are involved in trichloroethylene-induced liver cancer.

EPA (2011a; 2011b) also reviewed several studies that investigated the effects of trichloroethylene, trichloroacetic acid, and dichloroacetic acid on DNA methylation status in mice. Aberrant DNA methylation is a common hallmark of all types of cancer; however, it has not yet been determined whether altered DNA methylation is a consequence or cause of cancer. Rats and mice fed diets that induce hypomethylation (deficient in choline and methionine) develop liver tumors. A high dose of methionine $(8 \mathrm{~g} / \mathrm{kg})$ was reported to decrease the number of dichloroacetic acid-induced liver foci and adenomas; however, mice fed a lower level of methionine $(4 \mathrm{~g} / \mathrm{kg})$ had a higher incidence of foci. Although the authors believed their data indicated that methionine supplementation slowed the progression of tumors, the study did not demonstrate that enhanced tumor progression is a key event for the mode of action for dichloroacetic acid-induced liver carcinogenicity. Other studies reported that female $\mathrm{B}_{6} \mathrm{C}_{3} \mathrm{~F}_{1}$ mice administered subacute oral doses of trichloroethylene $(1,000 \mathrm{mg} / \mathrm{kg})$, trichloroacetic acid $(500 \mathrm{mg} / \mathrm{kg})$, or dichloroacetic acid $(500 \mathrm{mg} / \mathrm{kg})$ had increased cell proliferation and hypomethylation of the promoter regions of $c-J u n$ and $c-M y c$ in the liver. Methionine treatment was reported to eliminate this effect in one study, but low doses of methionine had no effect. Hypomethylation also was reported in total liver and liver tumor DNA in mice initiated with $\mathrm{N}$ methyl- $N$-nitrosourea and exposed to trichloroacetic acid or dichloroacetic acid for 46 weeks.

A few studies have reported altered gene expression and/or hypomethylation of liver DNA in mice exposed to high doses of trichloroethylene, trichloroacetic acid, and dichloroacetic acid; thus, it is biologically plausible that these events could contribute to trichloroethylene-induced liver carcinogenesis. Although there is evidence that hypomethylation is sufficient for carcinogenesis, it is uncertain if it is necessary for trichloroethylene-induced liver carcinogenesis. The doses of trichloroacetic acid and dichloroacetic acid tested for 
hypomethylation were higher than those used to induce liver tumors in mice. It is not known if hypomethylation also occurs at doses relevant to trichloroethylene carcinogenicity.

\subsubsection{Autoimmune Hepatitis}

Hepatocellular carcinoma is recognized as a life-threatening complication in the course of autoimmune hepatitis in humans, (Arslan et al. 2011; Czaja 2013; Nishiyama et al. 2004; Watanabe et al. 2009). The primary risk factors for malignant transformation include the presence of cirrhosis at presentation or during treatment and long-term immunosuppressive therapy (Czaja 2013). Although the overall frequency of hepatocellular carcinoma in patients with autoimmune hepatitis and cirrhosis ranges from about $1 \%$ to $9 \%$, recent clinical data in the United States suggests that the frequency of malignancy in autoimmune hepatitis is comparable with that reported for other types of cirrhosis. Wang and Czaja (1988) reported that the probability of hepatocellular carcinoma in corticosteroid-treated cases of severe autoimmune hepatitis with cirrhosis was $29 \%$ after 13 years.

Trichloroethylene exposure had been linked to non-viral (toxic) hepatitis (usually in association with idiosyncratic generalized hypersensitivity skin diseases), liver dysfunction, and liver cancer in humans (Hansen et al. 2013; Kamijima et al. 2007; Kamijima et al. 2008; Kim and Kim 2010) and autoimmune hepatitis in MRL+/+ mice (Gilbert et al. 2006; Gilbert et al. 2009; Griffin et al. 2000c). Autoimmune hepatitis in mice exposed to trichloroethylene was indicated by immune responses to protein adducts and liver inflammation. Inflammation of the liver in mice was induced by exposure to trichloroethylene and preformed metabolite protein adducts (Cai et al. 2007b; Cai et al. 2008; Gilbert et al. 2009; Kaneko et al. 2000; Kondraganti et al. 2012; Ramdhan et al. 2010; Tang et al. 2008). Autoantibodies were formed against "self" antigens of normal tissue, neoimmunogens of trichloroethylene-induced protein adducts, and the concomitant non-adducted proteins. Liver cirrhosis was not reported; however, cirrhosis, like cancer, takes time to develop (Meza-Junco et al. 2007). The longest study that looked at liver inflammation was 48 weeks, which might not have been long enough to allow for cirrhosis to develop. Nevertheless, these studies suggest that trichloroethylene exposure can initiate an autoimmune response, possibly resulting in B-cell activation and autoimmune hepatitis.

The underlying mechanisms of trichloroethylene-induced autoimmunity are not completely understood; however, the studies in MRL+/+ mice suggest that oxidative stress, formation of protein adducts, stimulation of $\mathrm{CD}^{+} \mathrm{T}$ cells and release of inflammatory cytokines, and autoantibody formation may be involved (Gilbert et al. 2006; Gilbert et al. 2009; Gilbert et al. 2012; Griffin et al. 2000a; Griffin et al. 2000c; Khan et al. 1995; Khan et al. 2001; Wang et al. 2007a; Wang et al. 2007b; Wang et al. 2013). None of the MRL+/+ mice developed hepatocellular carcinoma; however, as noted above, the maximum study duration was 48 weeks with most studies lasting only 4 to 32 weeks. There also was no evidence of hepatitis or liver cirrhosis in exposed B6C3F 1 mice in the NTP (1990) study. The cases of non-viral hepatitis in humans were not evaluated for an immune component and the cases of liver cancer in humans did not report on hepatitis. Although the epidemiological and experimental data are not inconsistent with trichloroethylene promoting liver cancer via autoimmune hepatitis, the data are insufficient to determine if this proposed mode of action is operative. 


\subsubsection{Hypothesized Modes of Action with Inadequate Support}

Several other modes of action have been proposed for trichloroethylene-induced liver cancer that are incompletely defined or have inadequate experimental support. These include increased liver weight or liver/body weight ratios, negative selection, glycogen accumulation, inactivation of GST-zeta, and cytotoxicity and regenerative hyperplasia (IARC 2014; USEPA 2011a; 2011b). These modes of action are briefly discussed below.

Increased liver weight: Although increased liver weight or increased liver/body weight ratios are associated with an increased liver cancer risk, these effects are nonspecific and may be caused by a number of factors (USEPA 2011a). Liver weight increases have been reported in rodents exposed to trichloroethylene, trichloroacetic acid, or dichloroacetic acid. However, no studies have evaluated the necessity of liver weight changes in trichloroethylene-induced liver carcinogenesis. Further, a mode of action hypothesis based on liver weight changes has not been adequately characterized and cannot be fully evaluated.

Negative selection: Negative selection refers to circumstances that convey a growth advantage to initiated cells relative to normal hepatocytes. This hypothesis suggests that the oxidative metabolites of trichloroethylene may contribute to liver tumor formation by several processes including the following: (1) downregulation of mitogenic stimulation in normal hepatocytes while initiated cells are refractory to this downregulation, (2) direct growth enhancement of certain populations of initiated cells, or (3) altered apoptosis (Bull 2000; USEPA 2011a). Bull (2000) suggested that data showing that trichloroethylene and its oxidative metabolites induced a transient increase in DNA synthesis in the liver of mice were consistent with a "negative selection" mode of action. However, the transient increases in cellular proliferation were confined to small populations of hepatocytes, and liver weight changes were associated with hypertrophy from increased glycogen storage and polyploidy rather than hyperplasia. Thus, mitogenic stimulation does not appear to play a significant role in trichloroethylene-induced liver cancer, and a mechanism for downregulation of mitogenic stimulation in normal hepatocytes has not been identified. Selective clonal expansion of initiated cells is a general feature of carcinogenesis and is not specific to trichloroethylene or its oxidative metabolites. Finally, trichloroethylene either does not affect apoptosis or causes only a slight increase at high doses. Although dichloroacetic acid has been reported to decrease apoptosis in mice, the data are inadequate to determine its relevance to liver cancer considering that mice have a very low background rate of apoptosis (Carter et al. 1995; USEPA 2011a). Therefore, the data are currently inadequate to properly define a mode of action based on negative selection. In addition, some of the data are inconsistent with this hypothesis.

Polyploidization: Tetraploidy has been associated with chromosome instability (CIN) that might persist or give way to a stably propagating aneuploid karyotype (Ganem et al. 2007). Both CIN and stable aneuploidy are common features of neoplasms, and tetraploidy is known to promote chromosomal aberrations and tumorigenesis in vivo. There is considerable experimental evidence that supports the theory that tetraploid cells are an important intermediate in the route to aneuploidy and cancer (Storchova and Kuffer 2008). Several chemicals, including trichloroethylene and dichloroacetic acid, that induce liver cancer in experimental animals also shift the hepatocyte ploidy distribution toward a greater percentage of diploid or polyploid cells (USEPA 2011a). Although polyploidization may be an important key event in tumor induction, the mechanisms are not well understood. Although it is biologically plausible that polyploidy can 
contribute to hepatocarcinogenicity, it is not known if polyploidization is necessary for trichloroethylene-induced liver tumors. Therefore, the data are inadequate to support polyploidization as operant in trichloroethylene-induced mouse liver tumors.

Glycogen storage: Several studies reviewed by EPA (2011a) reported that mice and rats exposed to dichloroacetic acid developed hepatomegaly that was partially attributable to accumulation of glycogen. Glycogen accumulation was observed as early as 1 week in normal liver while liver tumors were consistently glycogen-poor. However, rodent studies with trichloroethylene or trichloroacetic acid have reported either no change or a slight decrease in liver glycogen content or have not addressed this endpoint. Several studies have shown that glycogen accumulation can be pathogenic and that glycogen storage disease or poorly controlled diabetes is associated with an increased risk of liver cancer in humans (Lingohr et al. 2002; USEPA 2011a). Although it is biologically plausible that hepatocyte glycogen content may be affected by the apparent opposing actions of the trichloroethylene metabolites, dichloroacetic acid and trichloroacetic acid, the effects on glycogen content due to trichloroethylene exposure have not been adequately studied. Therefore, the data are inadequate to determine if this hypothesized mode of action contributes to trichloroethylene-induced hepatocarcinogenesis.

Inactivation of GST-zeta: Dichloroacetic acid inhibits its own metabolism through inactivation of GST-zeta (Board and Anders 2005; 2011). Successive doses of dichloroacetic acid have been reported to increase its plasma half-life in humans and mice and reduce its biotransformation in rat liver (Board and Anders 2005; Schultz et al. 2002). Several polymorphic variants of GST-zeta also have been identified that differ in their susceptibility to inactivation (Board and Anders 2011; Fang et al. 2006; Li et al. 2012). GST-zeta also is known as maleylacetoacetate isomerase (MMAI) and is part of the tyrosine catabolism pathway and metabolizes maleylacetoacetate and maleylacetone to fumarylacetoacetate and fumarylacetone, respectively (Board and Anders 2011; Stacpoole et al. 2008). Inhibition of GST-zeta by exposure to dichloroacetic acid results in the accumulation of maleylacetoacetate, maleylacetone, and succinylacetone and lower concentrations of fumarylacetoacetate (Blackburn et al. 2006; USEPA 2011a). Hereditary tyrosinemia type 1 is a metabolic disease caused by a deficiency of an enzyme involved in the last step of tyrosine catabolism. Individuals with this disease develop hepatocellular carcinoma at a young age (Stacpoole 2011; Tanguay et al. 1996). The increased cancer risk may be caused by the accumulation of one or more reactive tyrosine metabolites; however, it is not known which of these metabolites poses the greatest risk. Schultz et al. (2002) concluded that reduced MMAI activity is unlikely to be the sole carcinogenic mode of action for dichloroacetic acid and may be important only during the early stages of exposure. This conclusion is further supported by observations that GST-zeta knockout mice do not spontaneously develop hepatocellular carcinoma. Thus, the available data are insufficient to fully define the key events associated with this mode of action or to determine their necessity or sufficiency for carcinogenicity.

Cytotoxicity and regenerative hyperplasia: Cytotoxicity and regenerative hyperplasia have been recognized as key events in the mode of action of some chlorinated solvents (e.g., carbon tetrachloride, chloroform); however, trichloroethylene, trichloroacetic acid, and dichloroacetic acid induce liver carcinogenicity at doses that do not produce cytotoxicity (Bull et al. 2004; NTP 1976; 1990; USEPA 2011a). Further, there is no evidence that the transient increases in DNA synthesis in mouse liver are related to reparative hyperplasia. Thus, it is unlikely that cytotoxicity and reparative hyperplasia play a significant role in trichloroethylene-induced liver carcinogenicity. 


\subsubsection{Summary}

Although species differences in sensitivity to the proposed modes of action are likely, no data suggest that trichloroethylene causes liver tumors in mice by mechanisms that are irrelevant to humans. Most of the hypothesized modes of action for liver tumors have some experimental support and are biologically plausible in humans and rodents. However, the data currently are inadequate to support the conclusion that any of the particular mode-of-action hypotheses are operant because a collection of key events sufficient to induce liver tumors has not been identified or demonstrated. It is likely that the oxidative metabolites (e.g., trichloroacetic acid, dichloroacetic acid, chloral hydrate) are involved in liver carcinogenicity because they induce hepatotoxic and hepatocarcinogenic effects that are similar to trichloroethylene. Liver tumor phenotype (e.g., immunostaining for c-Jun) and genotype (e.g., H-ras mutation frequency and spectrum) analyses support a role for both dichloroacetic acid and trichloroacetic acid and show that neither metabolite alone can account for the full characteristics of trichloroethylene-induced liver tumors. The data suggest that the mode of action is complex and likely involves key events from several pathways. 


\section{NTP Listing Recommendation}

Trichloroethylene is currently listed in the $\mathrm{RoC}$ as reasonably anticipated to be a human carcinogen. Since it was first listed in the RoC, additional cancer studies have been published. This monograph focuses on the potential for trichloroethylene exposure to cause kidney cancer, non-Hodgkin lymphoma (NHL), or liver cancer. This section brings forward and integrates the evaluations of the human and mechanistic data for each of these cancers (Sections 4, 5, and 6), other relevant data (Sections 1 and 2), and the level of evidence for carcinogenicity in experimental animals (current listing in the RoC), and reaches an NTP listing recommendation for trichloroethylene. The conclusions are based on applying the RoC listing criteria to the evidence across studies.

\section{Overall Listing Recommendation}

Trichloroethylene is known to be a human carcinogen based on sufficient evidence of carcinogenicity from humans. This conclusion is based on epidemiological studies showing that it causes kidney cancer in humans, together with supporting evidence from toxicological, toxicokinetic, and mechanistic studies demonstrating the biological plausibility of its carcinogenicity in humans. Epidemiological studies also provide limited evidence for a causal association for non-Hodgkin lymphoma (NHL) in humans. Supporting evidence is provided by studies in experimental animals demonstrating that trichloroethylene causes cancer at several tissue sites, including some of the same sites as seen in humans-kidney tumors in male rats, liver tumors in mice of both sexes, and lymphoma in female mice - as well as tumors at other sites, including testicular tumors in male rats and lung tumors in mice of both sexes.

The epidemiological, toxicological, toxicokinetic, and mechanistic evidence for kidney cancer, NHL and related cancers, and liver cancer is summarized below.

\subsection{Kidney Cancer}

Epidemiological studies have demonstrated a causal relationship between trichloroethylene exposure and kidney cancer based on consistent evidence of increased risk across studies with different study designs, in different geographical areas, and in different occupational settings; evidence of increasing cancer risk with increasing level or duration of exposure; and statistically significant increased risks of kidney cancer across studies combined in two meta-analyses.

Overall, increased risks of kidney cancer were found among individuals with the highest exposure in the most informative studies (i.e., studies with higher levels of exposure to trichloroethylene and better assessments of exposure and disease; see Figure 4-2 and Figure 4-3). Although several studies did not find an association between kidney cancer and trichloroethylene exposure, non-differential misclassification and lower sensitivity to detect an association (e.g., because of low exposure levels or small numbers of subjects) were concerns in these studies. The meta-analyses also provide strong evidence for an association with kidney cancer. A sensitivity analysis of one meta-analysis found that the meta-relative risk was robust and not sensitive to removal of individual studies or use of alternative risk estimates. Finally, biases or confounding by known or suspected occupational co-exposures, smoking, or other lifestyle factors are unlikely to explain the positive findings across studies (see Section 4.1.4 for a detailed discussion of the evidence). 
Toxicokinetic and mechanistic data in both humans and animals provide credible evidence for the biological plausibility of the proposed mechanisms of trichloroethylene's carcinogenicity in humans. The key events most likely contributing to tumorigenicity include (1) GSH-conjugationderived metabolites produced in situ or delivered systemically to the kidneys and (2) mutagenic, genotoxic, and cytotoxic effects induced by these metabolites in the kidneys. Metabolism of trichloroethylene is qualitatively similar in humans and experimental animals. In vitro studies in kidney and liver cells from humans and animals have demonstrated the formation of several GSH-conjugation-derived metabolites, some of which (NAcDCVC and DCVG) have been detected in the urine or blood of trichloroethylene-exposed humans and experimental animals. The finding of a significantly elevated risk of renal-cell cancer among trichloroethylene-exposed individuals with a functionally active GSTT1 genotype but not among subjects with a GST-null genotype provides support for the importance of the GSH-conjugation pathway in the carcinogenicity of trichloroethylene in humans.

The available mechanistic data support a mutagenic and cytogenetic mode of action mediated by GSH-conjugation-derived metabolites. These metabolites have been shown to be mutagenic in vitro and genotoxic both in vitro and in vivo, most notably causing damage to human and animal kidney cells in vitro, cellular transformation of rat kidney cells in vitro, and DNA damage and micronucleus formation in kidney cells from rats exposed in vivo. A mechanism potentially contributing to trichloroethylene's carcinogenicity is cytotoxicity and associated regenerative proliferation. Studies in humans also provide evidence that trichloroethylene causes nephrotoxicity, supporting the role of this mechanism in humans. Thus, the mode of action for kidney carcinogenicity may involve a combination of mutagenicity and cytotoxicity.

\subsection{NHL and Related Cancers}

Epidemiological studies provide limited evidence for a causal association between trichloroethylene exposure and NHL, based on positive associations in several studies and evidence for increased risk of NHL across studies combined in two meta-analyses. The evidence across studies is less consistent than for kidney cancer, and alternative explanations such as chance or confounding cannot reasonably be ruled out.

The strongest evidence for an association between trichloroethylene exposure and NHL comes from the InterLymph pooled analysis ( $\mathrm{p}$ for Fisher's combined probability $=0.004$ ), supported by modest increases in risk in several cohort and case-control studies. The risk of NHL increased with increasing level or duration of exposure in the pooled InterLymph study, one of its component studies, and another case-control study, but evidence for an exposure-response relationship was lacking in several cohort studies. No evidence was found for confounding by lifestyle factors; however, potential confounding by exposure to other solvents, including chlorinated solvents, may have been possible in the aircraft-manufacturing studies.

The mechanisms by which trichloroethylene could cause lymphoma are largely unknown. Immunomodulation, including autoimmunity and immunosuppression, are strongly linked to NHL. There is evidence that trichloroethylene causes immunomodulation in both people and animals, suggesting a biologically plausible role for immunomodulation in induction of NHL by trichloroethylene. It has been proposed that lymphomas can develop from errors arising during the somatic hypermutation phase of B-cell activation, resulting from either chronic antigenic stimulation (autoimmunity) or from impaired pathogen control (immunosuppression). However, 
the results of some studies in humans and animals that measured immune biomarkers (such as those for B-cell activation) were not entirely consistent with this model. The mechanisms of immunomodulation and lymphomagenesis are not completely understood, and neither the proposed model nor the potential association between trichloroethylene-induced immune effects and lymphoma has been directly tested in either humans or animals.

\subsection{Liver Cancer}

The data available from studies in humans are inadequate to evaluate the relationship between trichloroethylene exposure and liver cancer. A few studies, including two meta-analyses, found modest increases in the risk of liver cancer; however, the findings are inconsistent across studies, and there was little evidence for exposure-response relationships in the individual studies or the meta-analyses. The evidence from recent studies, published since the latest meta-analysis (USEPA 2011a), appears to be weaker. Most of the studies (both recent and older) had limited ability to detect an association between trichloroethylene exposure and rare cancers such as liver cancer. In addition, the role of chance or confounding by one or more of the common occupational co-exposures or lifestyle factors cannot be completely ruled out.

The mode of action for trichloroethylene-induced liver cancer in mice is unknown but likely is complex, involving key events in several pathways. Studies in experimental animals provide evidence for several potential modes of action resulting primarily from oxidative stress, such as genotoxicity, oxidative damage, peroxisome proliferation, epigenetic events, and autoimmunity (hepatitis). Oxidative metabolites are considered to be more important than GSH-pathway metabolites in liver carcinogenicity because trichloroethylene, trichloroacetic acid, dichloroacetic acid, and chloral hydrate have similar hepatotoxic and hepatocarcinogenic effects. These metabolites are found in humans, and chloral or chloral hydrate is genotoxic in several in vitro and in vivo test systems. Although species differences in sensitivity to the proposed modes of action are likely, no data suggest that trichloroethylene causes liver tumors in mice solely by mechanisms that are not relevant to humans.

\subsection{Other Cancer Sites}

Although this evaluation focused on kidney cancer, NHL, and liver cancer, authoritative evaluations of the carcinogenicity of trichloroethylene are available for other tissue sites. IARC concluded that although cancer incidence was increased at several other tissue sites, the data were insufficient for an evaluation. Of some interest is cervical cancer, for which statistically significant increased risks were found among women in two of the Nordic cohort studies (the pooled biomonitoring study and the study of blue-collar workers). Excesses of cervical cancer, though not statistically significant, were also observed in the Utah aircraft-manufacturing study and in a case-control study in the Arve Valley area of France, where the screw-cutting industry was prevalent (Charbotel et al. 2013). However, the latter study found no association of cervical cancer with cumulative trichloroethylene exposure level or exposure duration. The database for this tissue site is limited by the small number of studies reporting on cervical cancer and the potential for confounding by smoking or human papilloma virus infection. 


\subsection{Toxicological Considerations across End Points}

The available evidence indicates that trichloroethylene causes genotoxicity, toxicity, and cancer via its metabolic activation to reactive metabolites. Two distinct metabolic pathways for trichloroethylene have been identified that are common to all mammalian species studied: CYP oxidation and GSH conjugation. As discussed above, kidney cancer is most likely mediated through the GSH-conjugation pathway, whereas liver cancer (and toxicity) is thought to be mediated through the CYP-oxidation pathway. The oxidative pathway, primarily through CYP2E1, predominates in all species studied. However, the balance between oxidation and GSH conjugation of trichloroethylene can be altered by genetic polymorphisms or exposure to CYP inducers, and the impacts may be more substantial at higher substrate concentrations; this is consistent with the findings of increased risk of kidney cancer primarily among workers with high exposure to trichloroethylene. Differences among study populations in co-exposures or genetic susceptibility factors, both of which could affect the flux through the two metabolic pathways, may explain some of the heterogeneity across studies and cancer end points. Potentially sensitive subpopulations include individuals with GST, CYP2E1, or alcohol dehydrogenase polymorphisms. The frequencies of GSTT1 and GSTM1 polymorphisms vary among ethnic groups, with $40 \%$ to $85 \%$ of the population having GSTM1- or GSTT1-active genotypes and thus possibly a higher risk of developing cancer from trichloroethylene exposure. (A higher percentage and larger range of GST polymorphisms are found in African populations.) In addition, sex differences in human cancer risk are unclear. Only a few human cancer studies reported risk estimates for specific tissue sites separately for men and women, and several studies included fewer women than men (see Sections 4, 5, and 6), limiting the evaluation of potential patterns of sex differences in cancer risk. 


\section{References}

Adamson P, Bray F, Costantini AS, Tao MH, Weiderpass E, Roman E. 2007. Time trends in the registration of Hodgkin and non-Hodgkin lymphomas in Europe. Eur J Cancer. 43(2):391-401. http://dx.doi.org/10.1016/j.ejca.2006.10.008

Agency for Toxic Substances Disease Registry (ATSDR). 1997. Toxicological profile for trichloroethylene. Atlanta, GA: Agency for Toxic Substances and Disease Registry.

Agency for Toxic Substances Disease Registry (ATSDR). 2013. Addendum to the toxicological profile for trichloroethylene. Atlanta, GA: Agency for Toxic Substances and Disease Registry. https://www.atsdr.cdc.gov/toxprofiles/tce addendum.pdf.

Albertini S. 1990. Analysis of nine known or suspected spindle poisons for mitotic chromosome malsegregation using Saccharomyces cerevisiae D61.M. Mutagenesis. 5(5):453-459. http://dx.doi.org/10.1093/mutage/5.5.453

Alexander DD, Kelsh MA, Mink PJ, Mandel JH, Basu R, Weingart M. 2007. A meta-analysis of occupational trichloroethylene exposure and liver cancer. Int Arch Occup Environ Health.

81(2):127-143. http://dx.doi.org/10.1007/s00420-007-0201-4

Aligo J, Walker M, Bugelski P, Weinstock D. 2014. Is murine gammaherpesvirus-68 (MHV-68) a suitable immunotoxicological model for examining immunomodulatory drug-associated viral recrudescence? J Immunotoxicol.

Allen JW, Collins BW, Evansky PA. 1994. Spermatid micronucleus analyses of trichloroethylene and chloral hydrate effects in mice. Mutat Res. 323(1-2):81-88. http://dx.doi.org/10.1016/0165-7992(94)90049-3

Amacher DE, Zelljadt I. 1983. The morphological transformation of Syrian hamster embryo cells by chemicals reportedly nonmutagenic to Salmonella typhimurium. Carcinogenesis. 4(3):291295. http://dx.doi.org/10.1093/carcin/4.3.291

Anttila A, Pukkala E, Sallmén M, Hernberg S, Hemminki K. 1995. Cancer incidence among Finnish workers exposed to halogenated hydrocarbons. J Occup Environ Med. 37(7):797-806. http://dx.doi.org/10.1097/00043764-199507000-00008

Aranyi C, O'Shea WJ, Graham JA, Miller FJ. 1986. The effects of inhalation of organic chemical air contaminants on murine lung host defenses. Fundam Appl Toxicol. 6(4):713-720.

http://dx.doi.org/10.1016/0272-0590(86)90184-3

Arp EW, Jr., Wolf PH, Checkoway H. 1983. Lymphocytic leukemia and exposures to benzene and other solvents in the rubber industry. J Occup Med. 25(8):598-602.

Arslan C, Kiliçkap S, Yalçin S. 2011. Gastric cancer after cadaveric liver transplantation in a patient with autoimmune hepatitis: A case report and review of the literature. Turk $\mathrm{J}$

Gastroenterol. 22(1):73-76. http://dx.doi.org/10.4318/tjg.2011.0160 
Asal NR, Geyer JR, Risser DR, Lee ET, Kadamani S, Cherng N. 1988. Risk factors in renal cell carcinoma. II. Medical history, occupation, multivariate analysis, and conclusions. Cancer Detect Prev. 13(3-4):263-279.

Austin EW, Parrish JM, Kinder DH, Bull RJ. 1996. Lipid peroxidation and formation of 8hydroxydeoxyguanosine from acute doses of halogenated acetic acids. Fundam Appl Toxicol. 31(1):77-82. http://dx.doi.org/10.1006/faat.1996.0078

Axelson O, Andersson K, Hogstedt C, Holmberg B, Molina G, de Verdier A. 1978. A cohort study on trichloroethylene exposure and cancer mortality. J Occup Med. 20(3):194-196. http://dx.doi.org/10.1097/00043764-197803000-00008

Axelson O, Selden A, Andersson K, Hogstedt C. 1994. Updated and expanded Swedish cohort study on trichloroethylene and cancer risk. J Occup Med. 36(5):556-562.

Baden JM, Kelley M, Mazze RI, Simmon VF. 1979. Mutagenicity of inhalation anaesthetics: Trichloroethylene, divinyl ether, nitrous oxide and cyclopropane. Br J Anaesth. 51(5):417-421. http://dx.doi.org/10.1093/bja/51.5.417

Baecklund E, Smedby KE, Sutton LA, Askling J, Rosenquist R. 2014. Lymphoma development in patients with autoimmune and inflammatory disorders--what are the driving forces? Semin Cancer Biol. 24:61-70. http://dx.doi.org/10.1016/j.semcancer.2013.12.001

Bahr DE, Aldrich TE, Seidu D, Brion GM, Tollerud DJ, Muldoon S, Reinhart N, Youseefagha A, McKinney P, Hughes T et al. 2011. Occupational exposure to trichloroethylene and cancer risk for workers at the Paducah Gaseous Diffusion Plant. Int J Occup Med Environ Health. 24(1):67-77. http://dx.doi.org/10.2478/s13382-011-0007-1

Bakke B, Stewart PA, Waters MA. 2007. Uses of and exposure to trichloroethylene in US industry: A systematic literature review. J Occup Environ Hyg. 4(5):375-390. http://dx.doi.org/10.1080/15459620701301763

Balkwill F, Charles KA, Mantovani A. 2005. Smoldering and polarized inflammation in the initiation and promotion of malignant disease. Cancer Cell. 7(3):211-217. http://dx.doi.org/10.1016/j.ccr.2005.02.013

Banerjee S, Van Duuren BL. 1978. Covalent binding of the carcinogen trichloroethylene to hepatic microsomal proteins and to exogenous DNA in vitro. Cancer Res. 38(3):776-780.

Bartoníček V. 1962. Metabolism and excretion of trichloroethylene after inhalation by human subjects. Br J Ind Med. 19:134-141. http://dx.doi.org/10.1136/oem.19.2.134

Bartsch H, Malaveille C, Barbin A, Planche G. 1979. Mutagenic and alkylating metabolites of halo-ethylenes, chlorobutadienes and dichlorobutenes produced by rodent or human liver tissues. Arch Toxicol. 41(4):249-277. http://dx.doi.org/10.1007/BF00296896

Bassig BA, Zhang L, Tang X, Vermeulen R, Shen M, Smith MT, Qiu C, Ge Y, Ji Z, Reiss B et al. 2013. Occupational exposure to trichloroethylene and serum concentrations of IL-6, IL-10, and TNF-alpha. Environ Mol Mutagen. 54(6):450-454. http://dx.doi.org/10.1002/em.21789 
Bel Hadj Jrad B, Chatti A, Laatiri A, Ahmed SB, Romdhane A, Ajimi S, Chouchane L. 2006. Tumor necrosis factor promoter gene polymorphism associated with increased susceptibility to non-Hodgkin's lymphomas. Eur J Haematol. 78(2):117-122. http://dx.doi.org/10.1111/j.16000609.2006.00784.x

Bergman K. 1983. Interactions of trichloroethylene with DNA in vitro and with RNA and DNA of various mouse tissues in vivo. Arch Toxicol. 54(3):181-193.

http://dx.doi.org/10.1007/BF01239202

Bernatsky S, Ramsey-Goldman R, Clarke A. 2006. Malignancy and autoimmunity. Curr Opin Rheumatol. 18(2):129-134. http://dx.doi.org/10.1097/01.bor.0000209423.39033.94

Besson H, Brennan P, Becker N, Nieters A, De Sanjosé S, Font R, Maynadie M, Foretova L, Cocco PL, Staines A et al. 2006. Tobacco smoking, alcohol drinking and non-Hodgkin's lymphoma: A European multicenter case-control study (Epilymph). Int J Cancer. 119(4):901908. http://dx.doi.org/10.1002/ijc.21913

Bhunya SP, Behera BC. 1987. Relative genotoxicity of trichloroacetic acid (TCA) as revealed by different cytogenetic assays: Bone marrow chromosome aberration, micronucleus and spermhead abnormality in the mouse. Mutat Res. 188(3):215-221. http://dx.doi.org/10.1016/0165$\underline{1218(87) 90092-9}$

Bhunya SP, Jena GB. 1996. The evaluation of clastogenic potential of trichloroacetic acid (TCA) in chick in vivo test system. Mutat Res. 367(4):254-259. http://dx.doi.org/10.1016/S0165$\underline{1218(96) 90085-3}$

Blackburn AC, Matthaei KI, Lim C, Taylor MC, Cappello JY, Hayes JD, Anders MW, Board PG. 2006. Deficiency of glutathione transferase zeta causes oxidative stress and activation of antioxidant response pathways. Mol Pharmacol. 69(2):650-657.

http://dx.doi.org/10.1124/mol.105.018911

Blair A, Hartge P, Stewart PA, McAdams M, Lubin J. 1998. Mortality and cancer incidence of aircraft maintenance workers exposed to trichloroethylene and other organic solvents and chemicals: Extended follow up. Occup Environ Med. 55(3):161-171. http://dx.doi.org/10.1136/oem.55.3.161

Bloemen LJ, Tomenson J. 1995. Increased incidence of renal cell tumours in a cohort of cardboard workers exposed to trichloroethylene. Arch Toxicol. 70(2):129-133.

http://dx.doi.org/10.1007/BF02733675

Blossom SJ, Pumford NR, Gilbert KM. 2004. Activation and attenuation of apoptosis of CD4+ T cells following in vivo exposure to two common environmental toxicants, trichloroacetaldehyde hydrate and trichloroacetic acid. J Autoimmun. 23(3):211-220.

http://dx.doi.org/10.1016/j.jaut.2004.06.007

Blossom SJ, Doss JC, Gilbert KM. 2006. Ability of trichloroethylene metabolite to promote immune pathology is strain-specific. J Immunotoxicol. 3(4):179-187.

http://dx.doi.org/10.1080/15476910600978046 
Blossom SJ, Gilbert KM. 2006. Exposure to a metabolite of the environmental toxicant, trichloroethylene, attenuates CD4+ T cell activation-induced cell death by metalloproteinasedependent FasL shedding. Toxicol Sci. 92(1):103-114. http://dx.doi.org/10.1093/toxsci/kfj212

Blossom SJ, Doss JC. 2007. Trichloroethylene alters central and peripheral immune function in autoimmune-prone MRL(+/+) mice following continuous developmental and early life exposure. J Immunotoxicol. 4(2):129-141. http://dx.doi.org/10.1080/15476910701337035

Blossom SJ, Doss JC, Gilbert KM. 2007. Chronic exposure to a trichloroethylene metabolite in autoimmune-prone MRL+/+ mice promotes immune modulation and alopecia. Toxicol Sci. 95(2):401-411. http://dx.doi.org/10.1093/toxsci/kfl149

Board PG, Anders MW. 2005. Human glutathione transferase zeta. Methods Enzymol. 401:6177. http://dx.doi.org/10.1016/S0076-6879(05)01004-9

Board PG, Anders MW. 2011. Glutathione transferase zeta: Discovery, polymorphic variants, catalysis, inactivation, and properties of Gstz1 -/- mice. Drug Metab Rev. 43(2):215-225. http://dx.doi.org/10.3109/03602532.2010.549132

Bogen KT. 1988. Pharmacokinetics for regulatory risk analysis: The case of trichloroethylene. Regul Toxicol Pharmacol. 8(4):447-466. http://dx.doi.org/10.1016/0273-2300(88)90045-1

Bogen KT, Colston BW, Jr., Machicao LK. 1992. Dermal absorption of dilute aqueous chloroform, trichloroethylene, and tetrachloroethylene in hairless guinea pigs. Fundam Appl Toxicol. 18(1):30-39. http://dx.doi.org/10.1016/0272-0590(92)90192-K

Boice JD, Jr., Marano DE, Fryzek JP, Sadler CJ, McLaughlin JK. 1999. Mortality among aircraft manufacturing workers. Occup Environ Med. 56(9):581-597.

http://dx.doi.org/10.1136/oem.56.9.581

Boice JD, Jr., McLaughlin JK. 2001. Errors in TCE analysis. Environ Health Perspect.

109(3):A108. http://dx.doi.org/10.1289/ehp.109-a108a

Boice JD, Jr., Marano DE, Cohen SS, Mumma MT, Blot WJ, Brill AB, Fryzek JP, Henderson BE, McLaughlin JK. 2006. Mortality among Rocketdyne workers who tested rocket engines, 1948-1999. J Occup Environ Med. 48(10):1070-1092.

http://dx.doi.org/10.1097/01.jom.0000240661.33413.b5

Bolt HM, Lammert M, Selinski S, Brüning T. 2004. Urinary alpha(1)-microglobulin excretion as biomarker of renal toxicity in trichloroethylene-exposed persons. Int Arch Occup Environ Health. 77(3):186-190. http://dx.doi.org/10.1007/s00420-003-0500-3

Bove FJ, Ruckart PZ, Maslia M, Larson TC. 2014. Evaluation of mortality among marines and navy personnel exposed to contaminated drinking water at USMC base Camp Lejeune: A retrospective cohort study. Environ Health. 13(1):10. http://dx.doi.org/10.1186/1476-069X-13-10

Boverhof DR, Krieger SM, Hotchkiss JA, Stebbins KE, Thomas J, Woolhiser MR. 2013. Assessment of the immunotoxic potential of trichloroethylene and perchloroethylene in rats following inhalation exposure. J Immunotoxicol. 10(3):311-320. http://dx.doi.org/10.3109/1547691X.2012.735275 
Bradford BU, Lock EC, Kosyk O, Kim S, Uehara T, Harbourt D, DeSimone M, Threadgill DW, Tryndyak V, Pogribny IP et al. 2011. Interstrain differences in the liver effects of trichloroethylene in a multistrain panel of inbred mice. Toxicol Sci. 120(1):206-217. http://dx.doi.org/10.1093/toxsci/kfq362

Brauch H, Weirich G, Hornauer MA, Storkel S, Wohl T, Bruning T. 1999. Trichloroethylene exposure and specific somatic mutations in patients with renal cell carcinoma. J Natl Cancer Inst. 91(10):854-861. http://dx.doi.org/10.1093/jnci/91.10.854

Brauch H, Weirich G, Klein B, Rabstein S, Bolt HM, Bruning T. 2004. VHL mutations in renal cell cancer: Does occupational exposure to trichloroethylene make a difference? Toxicol Lett. 151(1):301-310. http://dx.doi.org/10.1016/j.toxlet.2003.12.074

Brennan P, van der Hel O, Moore LE, Zaridze D, Matveev V, Holcatova I, Janout V, Kollarova H, Foretova L, Szeszenia-Dabrowska N et al. 2008. Tobacco smoking, body mass index, hypertension, and kidney cancer risk in central and eastern Europe. Br J Cancer. 99(11):19121915. http://dx.doi.org/10.1038/sj.bjc.6604761

Bronzetti G, Zeiger E, Frezza D. 1978. Genetic activity of trichloroethylene in yeast. J Environ Pathol Toxicol. 1(4):411-418.

Brüning T, Lammert M, Kempkes M, Thier R, Golka K, Bolt HM. 1997a. Influence of polymorphisms of GSTM1 and GSTT1 for risk of renal cell cancer in workers with long-term high occupational exposure to trichloroethene. Arch Toxicol. 71(9):596-599.

http://dx.doi.org/10.1007/s002040050432

Brüning T, Weirich G, Hornauer MA, Höfler H, Brauch H. 1997b. Renal cell carcinomas in trichloroethene (TRI) exposed persons are associated with somatic mutations in the von HippelLindau (VHL) tumour suppressor gene. Arch Toxicol. 71(5):332-335.

http://dx.doi.org/10.1007/s002040050394

Brüning T, Vamvakas S, Makropoulos V, Birner G. 1998. Acute intoxication with trichloroethene: Clinical symptoms, toxicokinetics, metabolism, and development of biochemical parameters for renal damage. Toxicol Sci. 41(2):157-165.

http://dx.doi.org/10.1093/toxsci/41.2.157

Brüning T, Mann H, Melzer H, Sundberg AG, Bolt HM. 1999a. Pathological excretion patterns of urinary proteins in renal cell cancer patients exposed to trichloroethylene. Occup Med (Lond). 49(5):299-305. http://dx.doi.org/10.1093/occmed/49.5.299

Brüning T, Sundberg AG, Birner G, Lammert M, Bolt HM, Appelkvist EL, Nilsson R, Dallner G. 1999b. Glutathione transferase alpha as a marker for tubular damage after trichloroethylene exposure. Arch Toxicol. 73(4-5):246-254. http://dx.doi.org/10.1007/s002040050613

Brüning T, Pesch B, Wiesenhütter B, Rabstein S, Lammert M, Baumüller A, Bolt HM. 2003. Renal cell cancer risk and occupational exposure to trichloroethylene: Results of a consecutive case-control study in Arnsberg, Germany. Am J Ind Med. 43(3):274-285.

http://dx.doi.org/10.1002/ajim.10185 
Bull RJ. 2000. Mode of action of liver tumor induction by trichloroethylene and its metabolites, trichloroacetate and dichloroacetate. Environ Health Perspect. 108(Suppl 2):241-259. http://dx.doi.org/10.1289/ehp.00108s2241

Bull RJ, Orner GA, Cheng RS, Stillwell L, Stauber AJ, Sasser LB, Lingohr MK, Thrall BD. 2002. Contribution of dichloroacetate and trichloroacetate to liver tumor induction in mice by trichloroethylene. Toxicol Appl Pharmacol. 182(1):55-65.

http://dx.doi.org/10.1006/taap.2002.9427

Bull RJ, Sasser LB, Lei XC. 2004. Interactions in the tumor-promoting activity of carbon tetrachloride, trichloroacetate, and dichloroacetate in the liver of male B6C3F1 mice.

Toxicology. 199(2-3):169-183. http://dx.doi.org/10.1016/j.tox.2004.02.018

Buzio L, De, Palma G, Mozzoni P, Tondel M, Buzio C, Franchini I, Axelson O, Mutti A. 2003. Glutathione S-transferases M1-1 and T1-1 as risk modifiers for renal cell cancer associated with occupational exposure to chemicals. Occup Environ Med. 60(10):789-793.

http://dx.doi.org/10.1136/oem.60.10.789

Cai H, Guengerich FP. 2001. Reaction of trichloroethylene oxide with proteins and DNA: Instability of adducts and modulation of functions. Chem Res Toxicol. 14(1):54-61. http://dx.doi.org/10.1021/tx000185n

Cai P, Konig R, Khan MF, Qiu S, Kaphalia BS, Ansari GA. 2006. Autoimmune response in MRL+/+ mice following treatment with dichloroacetyl chloride or dichloroacetic anhydride. Toxicol Appl Pharmacol. 216(2):248-255. http://dx.doi.org/10.1016/j.taap.2006.05.010

Cai P, Boor PJ, Khan MF, Kaphalia BS, Ansari GAS, Konig R. 2007a. Immuno- and hepatotoxicity of dichloroacetic acid in MRL+/+ and B6C3F1 mice. J Immunotoxicol. 4(2):107-115. http://dx.doi.org/10.1080/15476910701337225

Cai P, König R, Khan MF, Kaphalia BS, Ansari GA. 2007b. Differential immune responses to albumin adducts of reactive intermediates of trichloroethene in MRL+/+ mice. Toxicol Appl Pharmacol. 220(3):278-283. http://dx.doi.org/10.1016/j.taap.2007.01.020

Cai P, König R, Boor PJ, Kondraganti S, Kaphalia BS, Khan MF, Ansari GA. 2008. Chronic exposure to trichloroethene causes early onset of SLE-like disease in female MRL +/+ mice. Toxicol Appl Pharmacol. 228(1):68-75. http://dx.doi.org/10.1016/j.taap.2007.11.031

Caldwell JC, Keshava N. 2006. Key issues in the modes of action and effects of trichloroethylene metabolites for liver and kidney tumorigenesis. Environ Health Perspect. 114(9):1457-1463. http://dx.doi.org/10.1289/ehp.8692

Callen DF, Wolf CR, Philpot RM. 1980. Cytochrome P-450 mediated genetic activity and cytotoxicity of seven halogenated aliphatic hydrocarbons in Saccharomyces cerevisiae. Mutat Res. 77(1):55-63. http://dx.doi.org/10.1016/0165-1218(80)90120-2

Non-Hodgkin lymphoma incidence statistics. 2014a. Cancer Research UK; [accessed Accessed on 10/21/14]. http://www.cancerresearchuk.org/cancer-info/cancerstats/types/nhl/incidence/uknonhodgkin-lymphoma-incidence-statistics\#trends\%29 
Liver cancer incidence statistics. 2014b. Cancer Research UK; [accessed Accessed on 10/21/14]. http://www.cancerresearchuk.org/cancer-info/cancerstats/types/liver/incidence/uk-liver-cancerincidence-statistics

Cantor KP, Blair A, Everett G, Gibson R, Burmeister LF, Brown LM, Schuman L, Dick FR. 1992. Pesticides and other agricultural risk factors for non-Hodgkin's lymphoma among men in Iowa and Minnesota. Cancer Res. 52(9):2447-2455.

Carter JH, Carter HW, DeAngelo AB. 1995. Biochemical, pathologic and morphometric alterations induced in male $\mathrm{B} 6 \mathrm{C} 3 \mathrm{~F} 1$ mouse liver by short-term exposure to dichloroacetic acid. Toxicol Lett. 81(1):55-71. http://dx.doi.org/10.1016/0378-4274(95)03409-9

Caspary WJ, Langenbach R, Penman BW, Crespi C, Myhr BC, Mitchell AD. 1988. The mutagenic activity of selected compounds at the TK locus: Rodent vs. human cells. Mutat Res. 196(1):61-81. http://dx.doi.org/10.1016/0165-1110(88)90028-0

Cearfoss J, Hassoun E. 2012. The effects of a low vitamin E diet on dichloroacetate- and trichloroacetate-induced oxidative stress in the livers of mice. J Biochem Mol Toxicol. 26(4):147-154. http://dx.doi.org/10.1002/jbt.20421

Chang LW, Daniel FB, DeAngelo AB. 1992. Analysis of DNA strand breaks induced in rodent liver in vivo, hepatocytes in primary culture, and a human cell line by chlorinated acetic acids and chlorinated acetaldehydes. Environ Mol Mutagen. 20(4):277-288.

http://dx.doi.org/10.1002/em.2850200406

Chang YM, Tai CF, Yang SC, Chen CJ, Shih TS, Lin RS, Liou SH. 2003. A cohort mortality study of workers exposed to chlorinated organic solvents in Taiwan. Ann Epidemiol. 13(9):652660. http://dx.doi.org/10.1016/S1047-2797(03)00038-3

Channel SR, Latendresse JR, Kidney JK, Grabau JH, Lane JW, Steel-Goodwin L, Gothaus MC. 1998. A subchronic exposure to trichloroethylene causes lipid peroxidation and hepatocellular proliferation in male B6C3F1 mouse liver. Toxicol Sci. 43:145-154.

http://dx.doi.org/10.1093/toxsci/43.2.145

Charbotel B, Fevotte J, Hours M, Martin JL, Bergeret A. 2006. Case-control study on renal cell cancer and occupational exposure to trichloroethylene. Part II: Epidemiological aspects. Ann Occup Hyg. 50(8):777-787.

Charbotel B, Gad S, Caïola D, Béroud C, Fevotte J, Bergeret A, Ferlicot S, Richard S. 2007. Trichloroethylene exposure and somatic mutations of the VHL gene in patients with Renal Cell Carcinoma. J Occup Med Toxicol. 2:13. http://dx.doi.org/10.1186/1745-6673-2-13

Charbotel B, Fevotte J, Martin JL, Bergeret A. 2009. Renal cell carcinoma and exposure to trichloroethylene: Are French occupational exposure limits relevant? Rev Epidemiol Sante Publique. 57(1):41-47. http://dx.doi.org/10.1016/j.respe.2008.09.008

Charbotel B, Massardier-Pilonchery A, Fort E, Dananche B, Fevotte J, Confavreux-Romestaing C, Bergeret A. 2013. Occupational trichloroethylene exposure and cervical pathology: A casecontrol study. Ann Occup Hyg. 57(3):407-416. 
Chatterjee N, Hartge P, Cerhan JR, Cozen W, Davis S, Ishibe N, Colt J, Goldin L, Severson RK. 2004. Risk of non-Hodgkin's lymphoma and family history of lymphatic, hematologic, and other cancers. Cancer Epidemiol Biomarkers Prev. 13(9):1415-1421.

Chen XY, Zhuang ZX, Wang XH, Zhang JZ. 2006. Immune responses to trichloroethylene and skin gene expression profiles in Sprague Dawley rats. Biomed Environ Sci. 19(5):346-352.

Cheng HY, You HY, Zhou TB. 2012. Relationship between GSTM1/GSTT1 null genotypes and renal cell carcinoma risk: A meta-analysis. Ren Fail. 34(8):1052-1057.

http://dx.doi.org/10.3109/0886022X.2012.708380

Cherrie JW, Kromhout H, Semple S. 2001. The importance of reliable exposure estimates in deciding whether trichloroethylene can cause kidney cancer. J Cancer Res Clin Oncol.

127(6):400-402. http://dx.doi.org/10.1007/s004320000206

Chiu WA, Okino MS, Lipscomb JC, Evans MV. 2006. Issues in the pharmacokinetics of trichloroethylene and its metabolites. Environ Health Perspect. 114(9):1450-1456.

http://dx.doi.org/10.1289/ehp.8691

Chiu WA, Jinot J, Scott CS, Makris SL, Cooper GS, Dzubow RC, Bale AS, Evans MV, Guyton KZ, Keshava N et al. 2013. Human health effects of trichloroethylene: key findings and scientific issues. Environ Health Perspect. 121(3):303-311.

http://dx.doi.org/10.1289/ehp.1205879

Chow WH, Gridley G, McLaughlin JK, Mandel JS, Wacholder S, Blot WJ, Niwa S, Fraumeni JF, Jr. 1994. Protein intake and risk of renal cell cancer. J Natl Cancer Inst. 86(15):1131-1139. http://dx.doi.org/10.1093/jnci/86.15.1131

Chow WH, Dong LM, Devesa SS. 2010. Epidemiology and risk factors for kidney cancer. Nat Rev Urol. 7(5):245-257. http://dx.doi.org/10.1038/nrurol.2010.46

Christensen KY, Vizcaya D, Richardson H, Lavoué J, Aronson K, Siemiatycki J. 2013. Risk of selected cancers due to occupational exposure to chlorinated solvents in a case-control study in Montreal. J Occup Environ Med. 55(2):198-208.

http://dx.doi.org/10.1097/JOM.0b013e3182728eab

Clapp RW, Hoffman K. 2008. Cancer mortality in IBM Endicott plant workers, 1969-2001: An update on a NY production plant. Environ Health. 7:13. http://dx.doi.org/10.1186/1476-069X-7$\underline{13}$

Clarke CA, Glaser SL. 2002. Changing incidence of non-Hodgkin lymphomas in the United States. Cancer. 94(7):2015-2023. http://dx.doi.org/10.1002/cncr.10403

Clay P. 2008. Assessment of the genotoxicity of trichloroethylene and its metabolite, S-(1,2dichlorovinyl)-L-cysteine (DCVC), in the comet assay in rat kidney. Mutagenesis. 23(1):27-33. http://dx.doi.org/10.1093/mutage/gem034

Cocco P, t'Mannetje A, Fadda D, Melis M, Becker N, de, Sanjose S, Foretova L, Mareckova J, Staines A et al. 2010. Occupational exposure to solvents and risk of lymphoma subtypes: Results from the Epilymph case-control study. Occup Environ Med. 67(5):341-347.

http://dx.doi.org/10.1136/oem.2009.046839 
Cocco P, Vermeulen R, Flore V, Nonne T, Campagna M, Purdue M, Blair A, Monnereau A, Orsi $\mathrm{L}$, Clavel $\mathrm{J}$ et al. 2013. Occupational exposure to trichloroethylene and risk of non-Hodgkin lymphoma and its major subtypes: A pooled IinterLlymph analysis. Occup Environ Med. 70:795-802. http://dx.doi.org/10.1136/oemed-2013-101551

Cogliano VJ, Baan R, Straif K, Grosse Y, Lauby-Secretan B, El Ghissassi F, Bouvard V, Benbrahim-Tallaa L, Guha N, Freeman C et al. 2011. Preventable exposures associated with human cancers. J Natl Cancer Inst. 103(24):1827-1839. http://dx.doi.org/10.1093/jnci/djr483

Conroy SM, Maskarinec G, Morimoto Y, Franke AA, Cooney RV, Wilkens LR, Goodman MT, Hernadez BY, Le Marchand L, Henderson BE et al. 2013. Non-hodgkin lymphoma and circulating markers of inflammation and adiposity in a nested case-control study: The multiethnic cohort. Cancer Epidemiol Biomarkers Prev. 22(3):337-347.

http://dx.doi.org/10.1158/1055-9965.EPI-12-0947

Cooper GS, Makris SL, Nietert PJ, Jinot J. 2009. Evidence of autoimmune-related effects of trichloroethylene exposure from studies in mice and humans. Environ Health Perspect.

117(5):696-702. http://dx.doi.org/10.1289/ehp.11782

Corton JC. 2008. Evaluation of the role of peroxisome proliferator-activated receptor alpha (PPARalpha) in mouse liver tumor induction by trichloroethylene and metabolites. Crit Rev Toxicol. 38(10):857-875. http://dx.doi.org/10.1080/10408440802209796

Costa AK, Ivanetich KM. 1984. Chlorinated ethylenes: Their metabolism and effect on DNA repair in rat hepatocytes. Carcinogenesis. 5(12):1629-1636.

http://dx.doi.org/10.1093/carcin/5.12.1629

Costa G, Merletti F, Segnan N. 1989. A mortality cohort study in a north Italian aircraft factory. Br J Ind Med. 46(10):738-743. http://dx.doi.org/10.1136/oem.46.10.738

Costantini AS, Miligi L, Kriebel D, Ramazzotti V, Rodella S, Scarpi E, Stagnaro E, Tumino R, Fontana A, Masala G et al. 2001. A multicenter case-control study in Italy on hematolymphopoietic neoplasms and occupation. Epidemiology. 12(1):78-87. http://dx.doi.org/10.1097/00001648-200101000-00014

Costantini AS, Benvenuti A, Vineis P, Kriebel D, Tumino R, Ramazzotti V, Rodella S, Stagnaro E, Crosignani P, Amadori D et al. 2008. Risk of leukemia and multiple myeloma associated with exposure to benzene and other organic solvents: evidence from the Italian multicenter casecontrol study. Am J Ind Med. 51(11):803-811. http://dx.doi.org/10.1002/ajim.20592

Coussens LM, Werb Z. 2002. Inflammation and cancer. Nature. 420(6917):860-867. http://dx.doi.org/10.1038/nature01322

Crebelli R, Bignami M, Conti L, Carere A. 1982. Mutagenicity of trichloroethylene in Salmonella typhimurium TA100. Ann Ist Super Sanita. 18(1):117-121.

Crebelli R, Conti G, Conti L, Carere A. 1985. Mutagenicity of trichloroethylene, trichloroethanol and chloral hydrate in Aspergillus nidulans. Mutat Res. 155(3):105-111.

http://dx.doi.org/10.1016/0165-1218(85)90126-0 
Crebelli R, Conti G, Conti L, Carere A. 1991. In vitro studies with nine known or suspected spindle poisons: Results in tests for chromosome malsegregation in Aspergillus nidulans. Mutagenesis. 6(2):131-136. http://dx.doi.org/10.1093/mutage/6.2.131

Cummings BS, Lash LH. 2000. Metabolism and toxicity of trichloroethylene and S-(1,2dichlorovinyl)-L-cysteine in freshly isolated human proximal tubular cells. Toxicol Sci. 53(2):458-466. http://dx.doi.org/10.1093/toxsci/53.2.458

Cummings BS, Parker JC, Lash LH. 2000. Role of cytochrome P450 and glutathione Stransferase alpha in the metabolism and cytotoxicity of trichloroethylene in rat kidney. Biochem Pharmacol. 59(5):531-543. http://dx.doi.org/10.1016/S0006-2952(99)00374-3

Cummings BS, Parker JC, Lash LH. 2001. Cytochrome p450-dependent metabolism of trichloroethylene in rat kidney. Toxicol Sci. 60(1):11-19.

http://dx.doi.org/10.1093/toxsci/60.1.11

Czaja AJ. 2013. Hepatocellular carcinoma and other malignancies in autoimmune hepatitis. Dig Dis Sci. 58(6):1459-1476. http://dx.doi.org/10.1007/s10620-012-2525-5

Dai Y, Leng S, Li L, Niu Y, Huang H, Cheng J, Zheng Y. 2004. Genetic polymorphisms of cytokine genes and risk for trichloroethylene-induced severe generalized dermatitis: A casecontrol study. Biomarkers. 9(6):470-478. http://dx.doi.org/10.1080/13547500400026920

Dai Y, Leng S, Li L, Niu Y, Huang H, Liu Q, Duan H, Cheng J, Liu Q, Zheng Y. 2009. Effects of genetic polymorphisms of N-Acetyltransferase on trichloroethylene-induced hypersensitivity dermatitis among exposed workers. Ind Health. 47(5):479-486.

http://dx.doi.org/10.2486/indhealth.47.479

Dalgleish AG, O'Byrne KJ. 2002. Chronic immune activation and inflammation in the pathogenesis of AIDS and cancer. Adv Cancer Res. 84:231-276.

http://dx.doi.org/10.1016/S0065-230X(02)84008-8

De Roos AJ, Mirick DK, Edlefsen KL, LaCroix AZ, Kopecky KJ, Madeleine MM, Magpantay L, Martínez-Maza O. 2012. Markers of B-cell activation in relation to risk of non-Hodgkin lymphoma. Cancer Res. 72(18):4733-4743. http://dx.doi.org/10.1158/0008-5472.CAN-12-1639

de Visser KE, Eichten A, Coussens LM. 2006. Paradoxical roles of the immune system during cancer development. Nat Rev Cancer. 6(1):24-37. http://dx.doi.org/10.1038/nrc1782

Degrassi F, Tanzarella C. 1988. Immunofluorescent staining of kinetochores in micronuclei: A new assay for the detection of aneuploidy. Mutat Res. 203(5):339-345.

http://dx.doi.org/10.1016/0165-1161(88)90030-1

Dekant W, Schulz A, Metzler M, Henschler D. 1986. Absorption, elimination and metabolism of trichloroethylene: a quantitative comparison between rats and mice? Xenobiotica. 16(2):143-152. http://dx.doi.org/10.3109/00498258609043517

Dekant W, Berthold K, Vamvakas S, Henschler D, Anders MW. 1988. Thioacylating intermediates as metabolites of S-(1,2-dichlorovinyl)-L-cysteine and S-(1,2,2-trichlorovinyl)-Lcysteine formed by cysteine conjugate beta-lyase. Chem Res Toxicol. 1(3):175-178.

http://dx.doi.org/10.1021/tx00003a008 
Dekant W, Koob M, Henschler D. 1990. Metabolism of trichloroethene--in vivo and in vitro evidence for activation by glutathione conjugation. Chem Biol Interact. 73(1):89-101. http://dx.doi.org/10.1016/0009-2797(90)90110-9

DeMarini DM, Perry E, Shelton ML. 1994. Dichloroacetic acid and related compounds: Induction of prophage in E. coli and mutagenicity and mutation spectra in Salmonella TA100. Mutagenesis. 9(5):429-437. http://dx.doi.org/10.1093/mutage/9.5.429

Deng Q, Zheng T, Lan Q, Lan Y, Holford T, Chen Y, Dai M, Leaderer B, Boyle P, Chanock SJ et al. 2013. Occupational solvent exposure, genetic variation in immune genes, and the risk for non-Hodgkin lymphoma. Eur J Cancer Prev. 22(1):77-82.

http://dx.doi.org/10.1097/CEJ.0b013e328354d2c1

DeSimone MC, Rathmell WK, Threadgill DW. 2013. Pleiotropic effects of the trichloroethyleneassociated P81S VHL mutation on metabolism, apoptosis, and ATM-mediated DNA damage response. J Natl Cancer Inst. 105(18):1355-1364. http://dx.doi.org/10.1093/jnci/djt226

Diot E, Lesire V, Guilmot JL, Metzger MD, Pilore R, Rogier S, Stadler M, Diot P, Lemarie E, Lasfargues G. 2002. Systemic sclerosis and occupational risk factors: A case-control study. Occup Environ Med. 59(8):545-549. http://dx.doi.org/10.1136/oem.59.8.545

DiRenzo AB, Gandolfi AJ, Sipes IG. 1982. Microsomal bioactivation and covalent binding of aliphatic halides to DNA. Toxicol Lett. 11(3-4):243-252. http://dx.doi.org/10.1016/0378$\underline{4274(82) 90157-6}$

Doolittle DJ, Muller G, Scribner HE. 1987. The in vivo-in vitro hepatocyte assay for assessing DNA repair and DNA replication: Studies in the CD-1 mouse. Food Chem Toxicol. 25(5):399405. http://dx.doi.org/10.1016/0278-6915(87)90176-1

Dosemeci M, Cocco P, Chow WH. 1999. Gender differences in risk of renal cell carcinoma and occupational exposures to chlorinated aliphatic hydrocarbons. Am J Ind Med. 36(1):54-59. http://dx.doi.org/10.1002/(SICI)1097-0274(199907)36:1<54::AID-AJIM8>3.0.CO;2-0

Douglas GR, Gingerich JD, Soper LM, Potvin M, Bjarnason S. 1999. Evidence for the lack of base-change and small-deletion mutation induction by trichloroethylene in lacZ transgenic mice. Environ Mol Mutagen. 34(2-3):190-194. http://dx.doi.org/10.1002/(SICI)10982280(1999)34:2/3<190::AID-EM18>3.0.CO;2-\#

Dow. 2008. Product safety assessment: Trichloroethylene. Dow Chemical Company.

Dow JL, Green T. 2000. Trichloroethylene induced vitamin B(12) and folate deficiency leads to increased formic acid excretion in the rat. Toxicology. 146(2-3):123-136. http://dx.doi.org/10.1016/S0300-483X(00)00156-6

Duprat P, Gradiski D. 1980. Cytogenetic effect of trichloroethylene in the mouse as evaluated by the micronucleus test. IRCS Med Sci. 8:182.

Eastmond DA. 2012. Factors influencing mutagenic mode of action determinations of regulatory and advisory agencies. Mutat Res. 751:49-63. http://dx.doi.org/10.1016/j.mrrev.2012.04.001 
El Arem A, Saafi EB, Ghrairi F, Thouri A, Zekri M, Ayed A, Zakhama A, Achour L. 2014a. Aqueous date fruit extract protects against lipid peroxidation and improves antioxidant status in the liver of rats subchronically exposed to trichloroacetic acid. J Physiol Biochem. 70(2):451464. http://dx.doi.org/10.1007/s13105-014-0323-6

El Arem A, Thouri A, Zekri M, Saafi EB, Ghrairi F, Zakhama A, Achour L. 2014b. Nephroprotective effect of date fruit extract against dichloroacetic acid exposure in adult rats. Food Chem Toxicol. 65:177-184. http://dx.doi.org/10.1016/j.fct.2013.12.023

Elfarra AA, Krause RJ, Last AR, Lash LH, Parker JC. 1998. Species- and sex-related differences in metabolism of trichloroethylene to yield chloral and trichloroethanol in mouse, rat, and human liver microsomes. Drug Metab Dispos. 26(8):779-785.

Emmert B, Bünger J, Keuch K, Müller M, Emmert S, Hallier E, Westphal GA. 2006. Mutagenicity of cytochrome P450 2E1 substrates in the Ames test with the metabolic competent S. typhimurium strain YG7108pin3ERb5. Toxicology. 228(1):66-76. http://dx.doi.org/10.1016/j.tox.2006.08.013

Eyre RJ, Stevens DK, Parker JC, Bull RJ. 1995. Renal activation of trichloroethene and S-(1,2dichlorovinyl)-L-cysteine and cell proliferative responses in the kidneys of F344 rats and B6C3F1 mice. J Toxicol Environ Health. 46(4):465-481.

http://dx.doi.org/10.1080/15287399509532049

Fahrig R. 1977. The mammalian spot test (Fellfleckentest) with mice. Arch Toxicol. 38(1-2):8798. http://dx.doi.org/10.1007/BF00293666

Fang YY, Kashkarov U, Anders MW, Board PG. 2006. Polymorphisms in the human glutathione transferase zeta promoter. Pharmacogenet Genomics. 16(5):307-313.

http://dx.doi.org/10.1097/01.fpc.0000205000.07054.b3

Ferlay J, Steliarova-Foucher E, Lortet-Tieulent J, Rosso S, Coebergh JWW, Comber H, Forman D, Bray F. 2013. Cancer incidence and mortality patterns in Europe: Estimates for 40 countries in 2012. Eur J Cancer. 49:1374-1403. http://dx.doi.org/10.1016/j.ejca.2012.12.027

Ferlay J, Soerjomataram I, Dikshit R, Eser S, Mathers C, Rebelo M, Parkin DM, Forman D, Bray F. 2014. Cancer incidence and mortality worldwide: Sources, methods and major patterns in GLOBOCAN 2012. Int J Cancer.

Ferreira-Gonzalez A, DeAngelo AB, Nasim S, Garrett CT. 1995. Ras oncogene activation during hepatocarcinogenesis in $\mathrm{B} 6 \mathrm{C} 3 \mathrm{~F} 1$ male mice by dichloroacetic and trichloroacetic acids. Carcinogenesis. 16(3):495-500. http://dx.doi.org/10.1093/carcin/16.3.495

Fevotte J, Charbotel B, Muller-Beauté P, Martin JL, Hours M, Bergeret A. 2006. Case-control study on renal cell cancer and occupational exposure to trichloroethylene. Part I: Exposure assessment. Ann Occup Hyg. 50(8):765-775.

Fleming DA, Woskie SR, Jones JH, Silver SR, Luo L, Bertke SJ. 2014. Retrospective assessment of exposure to chemicals for a microelectronics and business machine manufacturing facility. J Occup Environ Hyg. 11(5):292-305. http://dx.doi.org/10.1080/15459624.2013.862591 
Fox AW, Yang X, Murli H, Lawlor TE, Cifone MA, Reno FE. 1996. Absence of mutagenic effects of sodium dichloroacetate. Fundam Appl Toxicol. 32(1):87-95.

http://dx.doi.org/10.1006/faat.1996.0110

Fuscoe JC, Afshari AJ, George MH, DeAngelo AB, Tice RR, Salman T, Allen JW. 1996. In vivo genotoxicity of dichloroacetic acid: evaluation with the mouse peripheral blood micronucleus assay and the single cell gel assay. Environ Mol Mutagen. 27(1):1-9.

http://dx.doi.org/10.1002/(SICI)1098-2280(1996)27:1<1::AID-EM1>3.0.CO;2-L

Galloway SM, Armstrong MJ, Reuben C, Colman S, Brown B, Cannon C, Bloom AD, Nakamura F, Ahmed M, Duk S et al. 1987. Chromosome aberrations and sister chromatid exchanges in Chinese hamster ovary cells: Evaluations of 108 chemicals. Environ Mol Mutagen. 10 Suppl 10:1-175. http://dx.doi.org/10.1002/em.2850100502

Ganem NJ, Storchova Z, Pellman D. 2007. Tetraploidy, aneuploidy and cancer. Curr Opin Genet Dev. 17(2):157-162. http://dx.doi.org/10.1016/j.gde.2007.02.011

Garabrant DH, Held J, Langholz B, Bernstein L. 1988. Mortality of aircraft manufacturing workers in southern California. Am J Ind Med. 13(6):683-693.

http://dx.doi.org/10.1002/ajim.4700130607

Garabrant DH, Lacey, Jr JV, Laing TJ, Gillespie BW, Mayes MD, Cooper BC, Schottenfeld D. 2003. Scleroderma and solvent exposure among women. Am J Epidemiol. 157(6):493-500. http://dx.doi.org/10.1093/aje/kwf223

Ghanayem BI, Hoffler U. 2007. Investigation of xenobiotics metabolism, genotoxicity, and carcinogenicity using Cyp2e1(-/-) mice. Curr Drug Metab. 8(7):728-749.

http://dx.doi.org/10.2174/138920007782109760

Gilbert KM, Pumford NR, Blossom SJ. 2006. Environmental contaminant trichloroethylene promotes autoimmune disease and inhibits T-cell apoptosis in MRL(+/+) mice. J Immunotoxicol. 3(4):263-267. http://dx.doi.org/10.1080/15476910601023578

Gilbert KM, Przybyla B, Pumford NR, Han T, Fuscoe J, Schnackenberg LK, Holland RD, Doss JC, Macmillan-Crow LA, Blossom SJ. 2009. Delineating liver events in trichloroethyleneinduced autoimmune hepatitis. Chem Res Toxicol. 22(4):626-632.

http://dx.doi.org/10.1021/tx800409r

Gilbert KM, Rowley B, Gomez-Acevedo H, Blossom SJ. 2011. Coexposure to mercury increases immunotoxicity of trichloroethylene. Toxicol Sci. 119(2):281-292.

http://dx.doi.org/10.1093/toxsci/kfq345

Gilbert KM, Nelson AR, Cooney CA, Reisfeld B, Blossom SJ. 2012. Epigenetic alterations may regulate temporary reversal of $\mathrm{cd} 4+\mathrm{T}$ cell activation caused by trichloroethylene exposure.

Toxicol Sci. 127(1):169-178. http://dx.doi.org/10.1093/toxsci/kfs093

Giller S, Le Curieux F, Erb F, Marzin D. 1997. Comparative genotoxicity of halogenated acetic acids found in drinking water. Mutagenesis. 12(5):321-328.

http://dx.doi.org/10.1093/mutage/12.5.321 
Goeptar AR, Commandeur JN, van Ommen B, van Bladeren PJ, Vermeulen NP. 1995. Metabolism and kinetics of trichloroethylene in relation to toxicity and carcinogenicity. Relevance of the mercapturic acid pathway. Chem Res Toxicol. 8(1):3-21. http://dx.doi.org/10.1021/tx00043a001

Gold LS, Stewart PA, Milliken K, Purdue M, Severson R, Seixas N, Blair A, Hartge P, Davis S, De Roos AJ. 2011. The relationship between multiple myeloma and occupational exposure to six chlorinated solvents. Occup Environ Med. 68(6):391-399.

http://dx.doi.org/10.1136/oem.2009.054809

Goldsworthy TL, Popp JA. 1987. Chlorinated hydrocarbon-induced peroxisomal enzyme activity in relation to species and organ carcinogenicity. Toxicol Appl Pharmacol. 88(2):225-233. http://dx.doi.org/10.1016/0041-008X(87)90008-1

Goldsworthy TL, Lyght O, Burnett VL, Popp JA. 1988. Potential role of alpha-2 mu-globulin, protein droplet accumulation, and cell replication in the renal carcinogenicity of rats exposed to trichloroethylene, perchloroethylene, and pentachloroethane. Toxicol Appl Pharmacol. 96(2):367-379. http://dx.doi.org/10.1016/0041-008X(88)90095-6

Grawe J, Nusse M, Adler ID. 1997. Quantitative and qualitative studies of micronucleus induction in mouse erythrocytes using flow cytometry. I. Measurement of micronucleus induction in peripheral blood polychromatic erythrocytes by chemicals with known and suspected genotoxicity. Mutagenesis. 12(1):1-8. http://dx.doi.org/10.1093/mutage/12.1.1

Green T, Odum J, Nash JA, Foster JR. 1990. Perchloroethylene-induced rat kidney tumors: An investigation of the mechanisms involved and their relevance to humans. Toxicol Appl Pharmacol. 103(1):77-89. http://dx.doi.org/10.1016/0041-008X(90)90264-U

Green T. 1997. Trichloroethylene induced cancer in animals and its relevance to humans. J Occup Health. 39(4):261-273. http://dx.doi.org/10.1539/joh.39.261

Green T, Dow J, Ellis MK, Foster JR, Odum J. 1997. The role of glutathione conjugation in the development of kidney tumours in rats exposed to trichloroethylene. Chem Biol Interact. 105(2):99-117. http://dx.doi.org/10.1016/S0009-2797(97)00040-9

Green T, Dow J, Foster JR, Hext PM. 1998. Formic acid excretion in rats exposed to trichloroethylene: A possible explanation for renal toxicity in long-term studies. Toxicology. 127(1-3):39-47. http://dx.doi.org/10.1016/S0300-483X(98)00020-1

Green T, Dow J, Foster J. 2003. Increased formic acid excretion and the development of kidney toxicity in rats following chronic dosing with trichloroethanol, a major metabolite of trichloroethylene. Toxicology. 191(2-3):109-119. http://dx.doi.org/10.1016/S0300$\underline{483 X(03) 00206-3}$

Green T, Dow J, Ong CN, Ng V, Ong HY, Zhuang ZX, Yang XF, Bloemen L. 2004. Biological monitoring of kidney function among workers occupationally exposed to trichloroethylene. Occup Environ Med. 61(4):312-317. http://dx.doi.org/10.1136/oem.2003.007153 
Greenland S, Salvan A, Wegman DH, Hallock MF, Smith TJ. 1994. A case-control study of cancer mortality at a transformer-assembly facility. Int Arch Occup Environ Health. 66(1):49-54. http://dx.doi.org/10.1007/BF00386579

Greim H, Bonse G, Radwan Z, Reichert D, Henschler D. 1975. Mutagenicity in vitro and potential carcinogenicity of chlorinated ethylenes as a function of metabolic oxiran formation. Biochem Pharmacol. 24(21):2013-2017. http://dx.doi.org/10.1016/0006-2952(75)90396-2

Griffin JM, Blossom SJ, Jackson SK, Gilbert KM, Pumford NR. 2000a. Trichloroethylene accelerates an autoimmune response by Th1 T cell activation in MRL $+/+$ mice. Immunopharmacology. 46(2):123-137. http://dx.doi.org/10.1016/S0162-3109(99)00164-2

Griffin JM, Gilbert KM, Lamps LW, Pumford NR. 2000b. CD4(+) T-cell activation and induction of autoimmune hepatitis following trichloroethylene treatment in MRL+/+ mice. Toxicol Sci. 57(2):345-352. http://dx.doi.org/10.1093/toxsci/57.2.345

Griffin JM, Gilbert KM, Pumford NR. 2000c. Inhibition of CYP2E1 reverses CD4+ T-cell alterations in trichloroethylene-treated MRL+/+ mice. Toxicol Sci. 54(2):384-389. http://dx.doi.org/10.1093/toxsci/54.2.384

Grulich AE, Vajdic CM, Cozen W. 2007. Altered immunity as a risk factor for non-Hodgkin lymphoma. Cancer Epidemiol Biomarkers Prev. 16(3):405-408. http://dx.doi.org/10.1158/10559965.EPI-06-1070

Gu ZW, Sele B, Jalbert P, Vincent M, Vincent F, Marka C, Chmara D, Faure J. 1981. [Induction of sister chromatid exchange by trichloroethylene and its metabolites]. Toxicol Eur Res. 3(2):6367.

Guyton KZ, Chiu WA, Bateson TF, Jinot J, Scott CS, Brown RC, Caldwell JC. 2009. A reexamination of the PPAR-alpha activation mode of action as a basis for assessing human cancer risks of environmental contaminants. Environ Health Perspect. 117(11):1664-1672. http://dx.doi.org/10.1289/ehp.0900758

Halmes NC, McMillan DC, Oatis JE, Pumford NR. 1996. Immunochemical detection of protein adducts in mice treated with trichloroethylene. Chem Res Toxicol. 9(2):451-456.

http://dx.doi.org/10.1021/tx950171v

Halmes NC, Perkins EJ, McMillan DC, Pumford NR. 1997. Detection of trichloroethyleneprotein adducts in rat liver and plasma. Toxicol Lett. 92(3):187-194.

http://dx.doi.org/10.1016/S0378-4274(97)00053-2

Hansen J, Raaschou-Nielsen O, Christensen JM, Johansen I, McLaughlin JK, Lipworth L, Blot WJ, Olsen JH. 2001. Cancer incidence among Danish workers exposed to trichloroethylene. J Occup Environ Med. 43(2):133-139. http://dx.doi.org/10.1097/00043764-200102000-00012

Hansen J, Sallmén M, Seldén AI, Anttila A, Pukkala E, Andersson K, Bryngelsson IL, Raaschou-Nielsen O, Olsen JH, McLaughlin JK. 2013. Risk of cancer among workers exposed to trichloroethylene: Analysis of three Nordic cohort studies. J Natl Cancer Inst. 105(12):869877. http://dx.doi.org/10.1093/jnci/djt107 
Hardell L, Eriksson M, Lenner P, Lundgren E. 1981. Malignant lymphoma and exposure to chemicals, especially organic solvents, chlorophenols and phenoxy acids: A case-control study. Br J Cancer. 43(2):169-176. http://dx.doi.org/10.1038/bjc.1981.25

Hardell L, Eriksson M, Degerman A. 1994. Exposure to phenoxyacetic acids, chlorophenols, or organic solvents in relation to histopathology, stage, and anatomical localization of nonHodgkin's lymphoma. Cancer Res. 54(9):2386-2389.

Hardell L, Axelson O. 1998. Environmental and occupational aspects on the etiology of nonHodgkin's lymphoma. Oncol Res. 10(1):1-5.

Hardell L, Lindström G, van Bavel B, Fredrikson M, Liljegren G. 1998. Some aspects of the etiology of non-Hodgkin's lymphoma. Environ Health Perspect. 106 Suppl 2:679-681.

Harrington-Brock K, Doerr CL, Moore MM. 1998. Mutagenicity of three disinfection byproducts: di- and trichloroacetic acid and chloral hydrate in L5178Y/TK +/- (-)3.7.2C mouse lymphoma cells. Mutat Res. 413(3):265-276. http://dx.doi.org/10.1016/S1383-5718(98)00026-6

Harrington JM, Whitby H, Gray CN, Reid FJ, Aw TC, Waterhouse JA. 1989. Renal disease and occupational exposure to organic solvents: A case referent approach. Br J Ind Med. 46(9):643650. http://dx.doi.org/10.1136/oem.46.9.643

Harris NL, Jaffe ES, Stein H, Banks PM, Chan JK, Cleary ML, Delsol G, De Wolf-Peeters C, Falini B, Gatter KC et al. 1994. A revised European-American classification of lymphoid neoplasms: a proposal from the International Lymphoma Study Group. Blood. 84(5):1361-1392. http://dx.doi.org/10.1182/blood.V84.5.1361.1361

Hassoun E, Cearfoss J, Mamada S, Al-Hassan N, Brown M, Heimberger K, Liu MC. 2014. The effects of mixtures of dichloroacetate and trichloroacetate on induction of oxidative stress in livers of mice after subchronic exposure. J Toxicol Environ Health A. 77(6):313-323. http://dx.doi.org/10.1080/15287394.2013.864576

Hassoun EA, Ray S. 2003. The induction of oxidative stress and cellular death by the drinking water disinfection by-products, dichloroacetate and trichloroacetate in J774.A1 cells. Comp Biochem Physiol C Toxicol Pharmacol. 135(2):119-128. http://dx.doi.org/10.1016/S1532$\underline{0456(03) 00082-6}$

Hassoun EA, Dey S. 2008. Dichloroacetate- and trichloroacetate-induced phagocytic activation and production of oxidative stress in the hepatic tissues of mice after acute exposure. $\mathrm{J}$ Biochem Mol Toxicol. 22(1):27-34. http://dx.doi.org/10.1002/jbt.20210

Hassoun EA, Cearfoss J, Spildener J. 2010a. Dichloroacetate- and trichloroacetate-induced oxidative stress in the hepatic tissues of mice after long-term exposure. J Appl Toxicol.

30(5):450-456. http://dx.doi.org/10.1002/jat.1516

Hassoun EA, Spildener J, Cearfoss J. 2010b. The induction of tumor necrosis factor-alpha, superoxide anion, myeloperoxidase, and superoxide dismutase in the peritoneal lavage cells of mice after prolonged exposure to dichloroacetate and trichloroacetate. J Biochem Mol Toxicol. 24(2):136-144. http://dx.doi.org/10.1002/jbt.20322 
Hassoun EA, Cearfoss J. 2011. Dichloroacetate- and trichloroacetate-induced modulation of superoxide dismutase, catalase, and glutathione peroxidase activities and glutathione level in the livers of mice after subacute and subchronic exposure. Toxicol Environ Chem. 93(2):332-344. http://dx.doi.org/10.1080/02772248.2010.509602

Hassoun EA, Cearfoss J, Musser B, Krispinsky S, Al-Hassan N, Liu MC. 2013. The induction of phagocytic activation by mixtures of the water chlorination by-products, dichloroacetate- and trichloroacetate, in mice after subchronic exposure. J Biochem Mol Toxicol. 27(4):237-242. http://dx.doi.org/10.1002/jbt.21476

Hayden PJ, Welsh CJ, Yang Y, Schaefer WH, Ward AJ, Stevens JL. 1992. Formation of mitochondrial phospholipid adducts by nephrotoxic cysteine conjugate metabolites. Chem Res Toxicol. 5(2):232-237. http://dx.doi.org/10.1021/tx00026a013

Hazardous Substances Database. Trichloroethylene. 2012. National Library of Medicine; [accessed Accessed on 5/14/14]. http://toxnet.nlm.nih.gov/cgi-bin/sis/htmlgen?HSDB and search on CAS number or compound name

Henschler D, Eder E, Neudecker T, Metzler M. 1977. Carcinogenicity of trichloroethylene: Fact or artifact? Arch Toxicol. 37(3):233-236. http://dx.doi.org/10.1007/BF00355492

Henschler D, Vamvakas S, Lammert M, Dekant W, Kraus B, Thomas B, Ulm K. 1995. Increased incidence of renal cell tumors in a cohort of cardboard workers exposed to trichloroethene. Arch Toxicol. 69(5):291-299. http://dx.doi.org/10.1007/s002040050173

Hill AB. 1965. The environment and disease: Association or causation? Proc R Soc Med. 58:295-300. http://dx.doi.org/10.1177/003591576505800503

Hinchman CA, Ballatori N. 1990. Glutathione-degrading capacities of liver and kidney in different species. Biochem Pharmacol. 40(5):1131-1135. http://dx.doi.org/10.1016/0006$\underline{2952(90) 90503-\mathrm{D}}$

Hobara T, Kobayashi H, Higashihara E, Kawamoto T, Sakai T. 1984. Acute effects of 1,1,1trichloroethane, trichloroethylene, and toluene on the hematologic parameters in dogs. Arch Environ Contam Toxicol. 13(5):589-593. http://dx.doi.org/10.1007/BF01056337

Hong WX, Yang L, Chen M, Yang X, Ren X, Fang S, Ye J, Huang H, Peng C, Zhou L et al. 2012. Proteomic analysis of trichloroethylene-induced alterations in expression, distribution, and interactions of SET/TAF-Ialpha and two SET/TAF-Ialpha-binding proteins, eEF1A1 and eEF1A2, in hepatic L-02 cells. Toxicol Appl Pharmacol. 263(2):259-272.

http://dx.doi.org/10.1016/j.taap.2012.06.018

Hong WX, Ye JB, Chen MT, Yan Y, Zhou GF, Yang XF, Yang L, Ren XH, Huang HY, Zhou L et al. 2013. Trichloroethylene induces biphasic concentration-dependent changes in cell proliferation and the expression of SET-associated proteins in human hepatic L-02 Cells. Biomed Environ Sci. 26(7):618-621.

Hosgood HD, 3rd, Zhang L, Tang X, Vermeulen R, Qiu C, Shen M, Smith MT, Ge Y, Ji Z, Xiong J et al. 2012. Decreased numbers of CD4(+) naive and effector memory T cells, and 
CD8(+) naive T cells, are associated with trichloroethylene exposure. Front Oncol. 1:53. http://dx.doi.org/10.3389/fonc.2011.00053

Hosnijeh FS, Krop EJ, Scoccianti C, Krogh V, Palli D, Panico S, Tumino R, Sacredote C, Nawroly N, Portengen L et al. 2010. Plasma cytokines and future risk of non-Hodgkin lymphoma (NHL): A case-control study nested in the Italian European Prospective Investigation into Cancer and Nutrition. Cancer Epidemiol Biomarkers Prev. 19(6):1577-1584.

http://dx.doi.org/10.1158/1055-9965.EPI-09-1237

Howard J. 2013. Minimum Latency \& Types or Categories of Cancer. World Trade Center Health Program.

Hrelia P, Maffei F, Vigagni F, Fimognari C, Flori P, Stanzani R, Cantelli Forti G. 1994. Interactive effects between trichloroethylene and pesticides at metabolic and genetic level in mice. Environ Health Perspect. 102 Suppl 9:31-34. http://dx.doi.org/10.1289/ehp.94102s931

Hu C, Jiang L, Geng C, Zhang X, Cao J, Zhong L. 2008. Possible involvement of oxidative stress in trichloroethylene-induced genotoxicity in human HepG2 cells. Mutat Res. 652(1):88-94. http://dx.doi.org/10.1016/j.mrgentox.2008.01.002

Huang H, Kamijima M, Wang H, Li S, Yoshikawa T, Lai G, Huang Z, Liu H, Chen J, Takeuchi $Y$ et al. 2006. Human herpesvirus 6 reactivation in trichloroethylene-exposed workers suffering from generalized skin disorders accompanied by hepatic dysfunction. J Occup Health. 48(6):417423. http://dx.doi.org/10.1539/joh.48.417

Huang Z, Yue F, Yang X, Xia L, Chen C, Qiu X, Huang J, Li L, Kamijima M, Nakajima T et al. 2012. Upregulation of calprotectin and downregulation of retinol binding protein in the serum of workers with trichloroethylene-induced hypersensitivity dermatitis. J Occup Health. 54(4):299309. http://dx.doi.org/10.1539/joh.12-0012-OA

Hung RJ, Moore L, Boffetta P, Feng BJ, Toro JR, Rothman N, Zaridze D, Navratilova M, Bencko V, Janout V et al. 2007. Family history and the risk of kidney cancer: A multicenter case-control study in Central Europe. Cancer Epidemiol Biomarkers Prev. 16(6):1287-1290. http://dx.doi.org/10.1158/1055-9965.EPI-06-0963

Iavicoli I, Marinaccio A, Carelli G. 2005. Effects of occupational trichloroethylene exposure on cytokine levels in workers. J Occup Environ Med. 47(5):453-457.

http://dx.doi.org/10.1097/01.jom.0000161728.23285.66

Ikbal M, Tastekin A, Dogan H, Pirim I, Ors R. 2004. The assessment of genotoxic effects in lymphocyte cultures of infants treated with chloral hydrate. Mutat Res. 564(2):159-164. http://dx.doi.org/10.1016/j.mrgentox.2004.08.007

International Agency for Research on Cancer (IARC). 1976. Trichloroethylene. Cadmium, Nickel, Some Epoxides, Miscellaneous Industrial Chemicals and General Considerations on Volatile Anaesthetics. Lyon, France: International Agency for Research on Cancer. p. pp. 263276. 
International Agency for Research on Cancer (IARC). 1995. Trichloroethylene. Dry Cleaning, Some Chlorinated Solvents and Other Industrial Chemicals. Lyon, France: International Agency for Research on Cancer. p. 75-158.

International Agency for Research on Cancer (IARC). 1999. 1,1,1-Trichloroethane. Reevaluation of Some Organic Chemicals,Hydrazine and Hydrogen Peroxide. Lyon, France: International Agency for Research on Cancer. p. 881-903.

International Agency for Research on Cancer (IARC). 2014. Trichloroethylene, Tetrachloroethylene and Some Other Chlorinated Agents. Lyon, France: International Agency for Research on Cancer.

Irving RM, Elfarra AA. 2012. Role of reactive metabolites in the circulation in extrahepatic toxicity. Expert Opin Drug Metab Toxicol. 8(9):1157-1172. http://dx.doi.org/10.1517/17425255.2012.695347

Irving RM, Elfarra AA. 2013. Mutagenicity of the cysteine S-conjugate sulfoxides of trichloroethylene and tetrachloroethylene in the Ames test. Toxicology. 306:157-161. http://dx.doi.org/10.1016/j.tox.2013.02.003

Irving RM, Pinkerton ME, Elfarra AA. 2013. Characterization of the chemical reactivity and nephrotoxicity of N-acetyl-S-(1,2-dichlorovinyl)-L-cysteine sulfoxide, a potential reactive metabolite of trichloroethylene. Toxicol App Pharmacol. 267(1):1-10.

http://dx.doi.org/10.1016/j.taap.2012.12.002

Jaffe DR, Hassall CD, Gandolfi AJ, Brendel K. 1985. Production of DNA single strand breaks in rabbit renal tissue after exposure to 1,2-dichlorovinylcysteine. Toxicology. 35(1):25-33. http://dx.doi.org/10.1016/0300-483X(85)90129-5

Kafer E. 1986. Tests which distinguish induced crossing-over and aneuploidy from secondary segregation in Aspergillus treated with chloral hydrate or gamma-rays. Mutat Res. 164(3):145166. http://dx.doi.org/10.1016/0165-1161(86)90006-3

Kamijima M, Hisanaga N, Wang H, Nakajima T. 2007. Occupational trichloroethylene exposure as a cause of idiosyncratic generalized skin disorders and accompanying hepatitis similar to drug hypersensitivities. Int Arch Occup Environ Health. 80(5):357-370.

http://dx.doi.org/10.1007/s00420-006-0147-y

Kamijima M, Wang H, Huang H, Li L, Shibata E, Lin B, Sakai K, Liu H, Tsuchiyama F, Chen J et al. 2008. Trichloroethylene causes generalized hypersensitivity skin disorders complicated by hepatitis. J Occup Health. 50(4):328-338. http://dx.doi.org/10.1539/joh.L8013

Kamijima M, Wang H, Yamanoshita O, Ito Y, Xia L, Yanagiba Y, Chen C, Okamura A, Huang Z, Qiu X et al. 2013. Occupational trichloroethylene hypersensitivity syndrome: Human herpesvirus 6 reactivation and rash phenotypes. J Dermatol Sci. 72:218-224.

http://dx.doi.org/10.1016/j.jdermsci.2013.07.003

Kaneko T, Saegusa M, Tasaka K, Sato A. 2000. Immunotoxicity of trichloroethylene: A study with MRL-lpr/lpr mice. J Appl Toxicol. 20(6):471-475. http://dx.doi.org/10.1002/10991263(200011/12)20:6<471::AID-JAT716>3.0.CO;2-E 
Kappas A. 1989. On the mechanisms of induced aneuploidy in Aspergillus nidulans and validation of tests for genomic mutations. In: Resnick MA, Vig BK, editors. Mechanisms of Chromosome Distribution and Aneuploidy. New York, NY: Wiley. p. 377-384.

Karami S, Lan Q, Rothman N, Stewart PA, Lee KM, Vermeulen R, Moore LE. 2012. Occupational trichloroethylene exposure and kidney cancer risk: A meta-analysis. Occup Environ Med. 69(12):858-867. http://dx.doi.org/10.1136/oemed-2012-100932

Karami S, Bassig B, Stewart PA, Lee KM, Rothman N, Moore LE, Lan Q. 2013. Occupational trichloroethylene exposure and risk of lymphatic and haematopoietic cancers: A meta-analysis. Occup Environ Med. 70(8):591-599. http://dx.doi.org/10.1136/oemed-2012-101212

Kargalioglu Y, McMillan BJ, Minear RA, Plewa MJ. 2002. Analysis of the cytotoxicity and mutagenicity of drinking water disinfection by-products in Salmonella typhimurium. Teratog Carcinog Mutagen. 22(2):113-128. http://dx.doi.org/10.1002/tcm.10010

Kato I, Koenig KL, Watanabe-Meserve H, Baptiste MS, Lillquist PP, Frizzera G, Burke JS, Moseson M, Shore RE. 2005. Personal and occupational exposure to organic solvents and risk of non-Hodgkin's lymphoma (NHL) in women (United States). Cancer Causes Control. 16(10):1215-1224. http://dx.doi.org/10.1007/s10552-005-0385-4

Kauffmann BM, White KL, Jr., Sanders VM, Douglas KA, Sain LE, Borzelleca JF, Munson AE. 1982. Humoral and cell-mediated immune status in mice exposed to chloral hydrate. Environ Health Perspect. 44:147-151. http://dx.doi.org/10.1289/ehp.8244147

Kauppinen T, Heikkilä P, Plato N, Woldbæk T, Lenvik K, Hansen J, Kristjansson V, Pukkala E. 2009. Construction of job-exposure matrices for the Nordic Occupational Cancer Study (NOCCA). Acta Oncol. 48(5):791-800. http://dx.doi.org/10.1080/02841860902718747

Keil DE, Peden-Adams MM, Wallace S, Ruiz P, Gilkeson GS. 2009. Assessment of trichloroethylene (TCE) exposure in murine strains genetically-prone and non-prone to develop autoimmune disease. J Environ Sci Health A Tox Hazard Subst Environ Eng. 44(5):443-453. http://dx.doi.org/10.1080/10934520902719738

Keller DA, Heck HD. 1988. Mechanistic studies on chloral toxicity: Relationship to trichloroethylene carcinogenesis. Toxicol Lett. 42(2):183-191. http://dx.doi.org/10.1016/03784274(88)90076-8

Kelsh MA, Alexander DD, Mink PJ, Mandel JH. 2010. Occupational trichloroethylene exposure and kidney cancer: A meta-analysis. Epidemiology. 21(1):95-102.

http://dx.doi.org/10.1097/EDE.0b013e3181c30e92

Keshava N, Caldwell JC. 2006. Key issues in the role of peroxisome proliferator-activated receptor agonism and cell signaling in trichloroethylene toxicity. Environ Health Perspect. 114(9):1464-1470. http://dx.doi.org/10.1289/ehp.8693

Khan MF, Kaphalia BS, Prabhakar BS, Kanz MF, Ansari GAS. 1995. Trichloroethene-induced autoimmune response in female MRL +/+ mice. Toxicol App Pharmacol. 134:155-160.

http://dx.doi.org/10.1006/taap.1995.1179 
Khan MF, Wu X, Ansari GAS. 2001. Anti-malondialdehyde antibodies in mrl1/1 mice treated with trichloroethene and dichloroacetyl chloride: Possible role of lipid peroxidation in autoimmunity. Toxicol App Pharmacol. 170:88-92. http://dx.doi.org/10.1006/taap.2000.9086

Kim HR, Kim TW. 2010. Occupational hepatic disorders in Korea. J Korean Med Sci. 25(Suppl):S36-40. http://dx.doi.org/10.3346/jkms.2010.25.S.S36

Kim S, Collins LB, Boysen G, Swenberg JA, Gold A, Ball LM, Bradford BU, Rusyn I. 2009a. Liquid chromatography electrospray ionization tandem mass spectrometry analysis method for simultaneous detection of trichloroacetic acid, dichloroacetic acid, S-(1,2-

dichlorovinyl)glutathione and S-(1,2-dichlorovinyl)-L-cysteine. Toxicology. 262(3):230-238. http://dx.doi.org/10.1016/j.tox.2009.06.013

Kim S, Kim D, Pollack GM, Collins LB, Rusyn I. 2009b. Pharmacokinetic analysis of trichloroethylene metabolism in male B6C3F1 mice: Formation and disposition of trichloroacetic acid, dichloroacetic acid, S-(1,2-dichlorovinyl)glutathione and S-(1,2-dichlorovinyl)-L-cysteine. Toxicol Appl Pharmacol. 238(1):90-99. http://dx.doi.org/10.1016/j.taap.2009.04.019

Klaunig JE, Xu Y, Isenberg JS, Bachowski S, Kolaja KL, Jiang J, Stevenson DE, Walborg EF, Jr. 1998. The role of oxidative stress in chemical carcinogenesis. Environ Health Perspect. 106 Suppl 1:289-295.

Klaunig JE, Babich MA, Baetcke KP, Cook JC, Corton JC, David RM, DeLuca JG, Lai DY, McKee RH, Peters JM et al. 2003. PPARalpha agonist-induced rodent tumors: Modes of action and human relevance. Crit Rev Toxicol. 33(6):655-780. http://dx.doi.org/10.1080/713608372

Kligerman AD, Bryant MF, Doerr CL, Erexson GL, Evansky PA, Kwanyuen P, McGee JK. 1994. Inhalation studies of the genotoxicity of trichloroethylene to rodents. Mutat Res. 322(2):87-96. http://dx.doi.org/10.1016/0165-1218(94)00013-1

Knadle SA, Green CE, Baugh M, Vidensek M, Short SM, Partos X, Tyson CA. 1990. Trichloroethylene biotransformation in human and rat primary hepatocytes. Toxicol In Vitro. 4(4-5):537-541. http://dx.doi.org/10.1016/0887-2333(90)90114-9

Koch R, Schlegelmilch R, Wolf HU. 1988. Genetic effects of chlorinated ethylenes in the yeast Saccharomyces cerevisiae. Mutat Res. 206(2):209-216. http://dx.doi.org/10.1016/0165$\underline{1218(88) 90162-0}$

Kondraganti S, König R, Boor PJ, Khan S, Kaphalia BS, Firoze Khan M, Ansari GAS. 2012. Mechanistic evaluation of trichloroethene-mediated autoimmune hepatitis-like disease in female MRL+/+ Mice. Open Toxicol J. 5(1):1-10.

Krause RJ, Lash LH, Elfarra AA. 2003. Human kidney flavin-containing monooxygenases and their potential roles in cysteine s-conjugate metabolism and nephrotoxicity. J Pharmacol Exp Ther. 304(1):185-191. http://dx.doi.org/10.1124/jpet.102.042911

Kringstad KP, Ljungquist PO, De Sousa F, Stroemberg LM. 1981. Identification and mutagenic properties of some chlorinated aliphatic compounds in the spent liquor from kraft pulp chlorination. Environ Sci Technol. 15(5):562-566. http://dx.doi.org/10.1021/es00087a006 
Kumar M, Tewari S, Sharma P, Verma VK, Chauhan LK, Agarwal SK, Dwivedi UN, Goel SK. 2009. Study of genetic polymorphism in solvent exposed population and its correlation to in vitro effect of trichloroethylene on lymphocytes. J Environ Biol. 30(5):685-691.

Lacey JV, Jr., Garabrant DH, Laing TJ, Gillespie BW, Mayes MD, Cooper BC, Schottenfeld D. 1999. Petroleum distillate solvents as risk factors for undifferentiated connective tissue disease (UCTD). Am J Epidemiol. 149(8):761-770.

http://dx.doi.org/10.1093/oxfordjournals.aje.a009885

Lan Q, Zheng T, Rothman N, Zhang Y, Wang SS, Shen M, Berndt SI, Zahm SH, Holford TR, Leaderer B et al. 2006. Cytokine polymorphisms in the Th1/Th2 pathway and susceptibility to non-Hodgkin lymphoma. Blood. 107(10):4101-4108. http://dx.doi.org/10.1182/blood-2005-10$\underline{4160}$

Lan Q, Zhang L, Tang X, Shen M, Smith MT, Qiu C, Ge Y, Ji Z, Xiong J, He J et al. 2010. Occupational exposure to trichloroethylene is associated with a decline in lymphocyte subsets and soluble CD27 and CD30 markers. Carcinogenesis. 31(9):1592-1596.

http://dx.doi.org/10.1093/carcin/bgq121

Laque WE, Ronneberg CE. 1970. A study of the decarboxylation of trichloroacetic acid in solutions of water and dimethylsulfide. Ohio J Sci. 70(2):97-106.

Larson JL, Bull RJ. 1992. Metabolism and lipoperoxidative activity of trichloroacetate and dichloroacetate in rats and mice. Toxicol Appl Pharmacol. 115(2):268-277.

http://dx.doi.org/10.1016/0041-008X(92)90332-M

Lash LH, Elfarra AA, Anders MW. 1986. Renal cysteine conjugate beta-lyase. Bioactivation of nephrotoxic cysteine $\mathrm{S}$-conjugates in mitochondrial outer membrane. J Biol Chem. 261(13):5930-5935. http://dx.doi.org/10.1016/S0021-9258(17)38472-7

Lash LH, Qian W, Putt DA, Jacobs K, Elfarra AA, Krause RJ, Parker JC. 1998. Glutathione conjugation of trichloroethylene in rats and mice: sex-, species-, and tissue-dependent differences. Drug Metab Dispos. 26(1):12-19.

Lash LH, Lipscomb JC, Putt DA, Parker JC. 1999a. Glutathione conjugation of trichloroethylene in human liver and kidney: Kinetics and individual variation. Drug Metab Dispos. 27(3):351359.

Lash LH, Putt DA, Brashear WT, Abbas R, Parker JC, Fisher JW. 1999b. Identification of S(1,2-dichlorovinyl)glutathione in the blood of human volunteers exposed to trichloroethylene. $\mathrm{J}$ Toxicol Environ Health A. 56(1):1-21. http://dx.doi.org/10.1080/009841099158204

Lash LH, Fisher JW, Lipscomb JC, Parker JC. 2000a. Metabolism of trichloroethylene. Environ Health Perspect. 108(Suppl 2):177-200. http://dx.doi.org/10.1289/ehp.00108s2177

Lash LH, Parker JC, Scott CS. 2000b. Modes of action of trichloroethylene for kidney tumorigenesis. Environ Health Perspect. 108(Suppl 2):225-240.

http://dx.doi.org/10.1289/ehp.00108s2225 
Lash LH, Putt DA, Hueni SE, Horwitz BP. 2005. Molecular markers of trichloroethyleneinduced toxicity in human kidney cells. Toxicol Appl Pharmacol. 206(2):157-168.

http://dx.doi.org/10.1016/j.taap.2004.09.023

Lash LH, Putt DA, Parker JC. 2006. Metabolism and tissue distribution of orally administered trichloroethylene in male and female rats: Identification of glutathione- and cytochrome P-450derived metabolites in liver, kidney, blood, and urine. J Toxicol Environ Health A. 69(13):12851309. http://dx.doi.org/10.1080/15287390500360133

Lash LH, Chiu WA, Guyton KZ, Rusyn I. 2014. Trichloroethylene biotransformation and its role in mutagenicity, carcinogenicity and target organ toxicity. Mutat Res. (In Press).

http://dx.doi.org/10.1016/j.mrrev.2014.04.003

Laughter AR, Dunn CS, Swanson CL, Howroyd P, Cattley RC, Christopher Corton J. 2004. Role of the peroxisome proliferator-activated receptor $\alpha(\operatorname{PPAR} \alpha)$ in responses to trichloroethylene and metabolites, trichloroacetate and dichloroacetate in mouse liver. Toxicology. 203(1-3):8398. http://dx.doi.org/10.1016/j.tox.2004.06.014

Leavitt SA, DeAngelo AB, George MH, Ross JA. 1997. Assessment of the mutagenicity of dichloroacetic acid in lacI transgenic B6C3F1 mouse liver. Carcinogenesis. 18(11):2101-2106. http://dx.doi.org/10.1093/carcin/18.11.2101

Lee YC, Cohet C, Yang YC, Stayner L, Hashibe M, Straif K. 2009. Meta-analysis of epidemiologic studies on cigarette smoking and liver cancer. Int J Epidemiol. 38(6):1497-1511. http://dx.doi.org/10.1093/ije/dyp280

Lehmann I, Rehwagen M, Diez U, Seiffart A, Rolle-Kampczyk U, Richter M, Wetzig H, Borte M, Herbarth O, Leipzig Allergy Risk Children S. 2001. Enhanced in vivo IgE production and T cell polarization toward the type 2 phenotype in association with indoor exposure to VOC:

Results of the LARS study. Int J Hyg Environ Health. 204(4):211-221. http://dx.doi.org/10.1078/1438-4639-00100

Lehmann I, Thoelke A, Rehwagen M, Rolle-Kampczyk U, Schlink U, Schulz R, Borte M, Diez U, Herbarth O. 2002. The influence of maternal exposure to volatile organic compounds on the cytokine secretion profile of neonatal T cells. Environ Toxicol. 17(3):203-210.

http://dx.doi.org/10.1002/tox.10055

Leopardi P, Zijno A, Bassani B, Pacchierotti F. 1993. In vivo studies on chemically induced aneuploidy in mouse somatic and germinal cells. Mutat Res. 287(1):119-130. http://dx.doi.org/10.1016/0027-5107(93)90151-5

Leuschner J, Leuschner F. 1991. Evaluation of the mutagenicity of chloral hydrate in vitro and in vivo. Arzneimittelforschung. 41(10):1101-1103.

Li W, Gu Y, James MO, Hines RN, Simpson P, Langaee T, Stacpoole PW. 2012. Prenatal and postnatal expression of glutathione transferase $\zeta 1$ in human liver and the roles of haplotype and subject age in determining activity with dichloroacetate. Drug Metab Dispos. 40(2):232-239. http://dx.doi.org/10.1124/dmd.111.041533 
Lingohr MK, Bull RJ, Kato-Weinstein J, Thrall BD. 2002. Dichloroacetate stimulates glycogen accumulation in primary hepatocytes through an insulin-independent mechanism. Toxicol Sci. 68(2):508-515. http://dx.doi.org/10.1093/toxsci/68.2.508

Lipscomb JC, Mahle DA, Brashear WT, Garrett CM. 1996. A species comparison of chloral hydrate metabolism in blood and liver. Biochem Biophys Res Commun. 227(2):340-350. http://dx.doi.org/10.1006/bbrc.1996.1511

Lipscomb JC, Garrett CM, Snawder JE. 1997. Cytochrome P450-dependent metabolism of trichloroethylene: interindividual differences in humans. Toxicol Appl Pharmacol. 142(2):311318. http://dx.doi.org/10.1006/taap.1996.8040

Lipscomb JC, Fisher JW, Confer PD, Byczkowski JZ. 1998a. In vitro to in vivo extrapolation for trichloroethylene metabolism in humans. Toxicol Appl Pharmacol. 152(2):376-387.

http://dx.doi.org/10.1006/taap.1998.8485

Lipscomb JC, Garrett CM, Snawder JE. 1998b. Use of kinetic and mechanistic data un species extrapolation of bioactivation: Cytochrome P-540 dependent trichloroethylene metabolism at occupationally relevant concentrations. J Occup Health. 40:110-117.

http://dx.doi.org/10.1539/joh.40.110

Lipworth L, Tarone RE, McLaughlin JK. 2006. The epidemiology of renal cell carcinoma. J Urol. 176(6 Pt 1):2353-2358. http://dx.doi.org/10.1016/j.juro.2006.07.130

Lipworth L, Sonderman JS, Mumma MT, Tarone RE, Marano DE, Boice JD, Jr., McLaughlin JK. 2011. Cancer mortality among aircraft manufacturing workers: An extended follow-up. J Occup Environ Med. 53(9):992-1007. http://dx.doi.org/10.1097/JOM.0b013e31822e0940

Liu J, Xing X, Huang H, Jiang Y, He H, Xu X, Yuan J, Zhou L, Yang L, Zhuang Z. 2009. Identification of antigenic proteins associated with trichloroethylene-induced autoimmune disease by serological proteome analysis. Toxicol Appl Pharmacol. 240(3):393-400. http://dx.doi.org/10.1016/j.taap.2009.07.031

Liu R, Wang XH, Liu L, Zhou Q. 2012. No association between the GSTM1 null genotype and risk of renal cell carcinoma: A meta-analysis. Asian Pac J Cancer Prev. 13(7):3109-3112. http://dx.doi.org/10.7314/APJCP.2012.13.7.3109

Liviac D, Creus A, Marcos R. 2010. DNA damage induction by two halogenated acetaldehydes, byproducts of water disinfection. Water Res. 44(8):2638-2646.

http://dx.doi.org/10.1016/j.watres.2010.01.026

Liviac D, Creus A, Marcos R. 2011. Mutagenic analysis of six disinfection by-products in the Tk gene of mouse lymphoma cells. J Hazard Mater. 190(1-3):1045-1052.

http://dx.doi.org/10.1016/j.jhazmat.2011.04.062

Loprieno N, Abbondandolo A. 1980. Comparative mutagenic evaluation of some industrial compounds. In: Norpoth KH, Garner RC, editors. Short-term Test Systems for Detecting Carcinogens. Berlin, Germany: Springer-Verlag. p. 333-356. 
Lynch AM, Parry JM. 1993. The cytochalasin-B micronucleus/kinetochore assay in vitro: Studies with 10 suspected aneugens. Mutat Res. 287(1):71-86. http://dx.doi.org/10.1016/0027$\underline{5107(93) 90146-7}$

Mackay JM, Fox V, Griffiths K, Fox DA, Howard CA, Coutts C, Wyatt I, Styles JA. 1995. Trichloroacetic acid: Investigation into the mechanism of chromosomal damage in the in vitro human lymphocyte cytogenetic assay and the mouse bone marrow micronucleus test. Carcinogenesis. 16(5):1127-1133. http://dx.doi.org/10.1093/carcin/16.5.1127

Mally A, Walker CL, Everitt JI, Dekant W, Vamvakas S. 2006. Analysis of renal cell transformation following exposure to trichloroethene in vivo and its metabolite S(dichlorovinyl)-L-cysteine in vitro. Toxicology. 224(1-2):108-118. http://dx.doi.org/10.1016/j.tox.2006.04.036

Mandel JH, Kelsh MA, Mink PJ, Alexander DD, Kalmes RM, Weingart M, Yost L, Goodman M. 2006. Occupational trichloroethylene exposure and non-Hodgkin's lymphoma: a metaanalysis and review. Occup Environ Med. 63(9):597-607.

http://dx.doi.org/10.1136/oem.2005.022418

Manton KG, Akushevich I, Kravchenko J. 2009. Cancer Mortality and Morbidity Patterns in the U.S. Population. New York, NY: Springer Science+Business Media.

Marano DE, Boice JD, Jr., Fryzek JP, Morrison JA, Sadler CJ, McLaughlin JK. 2000. Exposure assessment for a large epidemiological study of aircraft manufacturing workers. Appl Occup Environ Hyg. 15(8):644-656. http://dx.doi.org/10.1080/10473220050075653

Marie I, Gehanno JF, Bubenheim M, Duval-Modeste AB, Joly P, Dominique S, Bravard P, Noël D, Cailleux AF, Weber J et al. 2014. Prospective study to evaluate the association between systemic sclerosis and occupational exposure and review of the literature. Autoimmun Rev. 13(2):151-156. http://dx.doi.org/10.1016/j.autrev.2013.10.002

Marrazzini A, Betti C, Bernacchi F, Barrai I, Barale R. 1994. Micronucleus test and metaphase analyses in mice exposed to known and suspected spindle poisons. Mutagenesis. 9(6):505-515. http://dx.doi.org/10.1093/mutage/9.6.505

Mazzullo M, Bartoli S, Bonora B, Colacci A, Lattanzi G, Niero A, Silingardi P, Grilli S. 1992. In vivo and in vitro interaction of trichloroethylene with macromolecules from various organs of rat and mouse. Res Comm Chem Pathol Pharmacol. 76:192-208.

McGregor DB, Reynolds DM, Zeiger E. 1989. Conditions affecting the mutagenicity of trichloroethylene in Salmonella. Environ Mol Mutagen. 13(3):197-202.

http://dx.doi.org/10.1002/em.2850130302

McMichael AJ, Spirtas R, Kupper LL. 1974. An epidemiologic study of mortality within a cohort of rubber workers, 1964-72. J Occup Med. 16(7):458-464.

McMichael AJ, Spirtas R, Gamble JF, Tousey PM. 1976. Mortality among rubber workers: Relationship to specific jobs. J Occup Med. 18(3):178-185. http://dx.doi.org/10.1097/00043764197603000-00012 
Mersch-Sundermann V, Muller G, Hofmeister J. 1989. [Examination of mutagenicity of organic microcontaminations of the environment. IV. Communication: The mutagenicity of halogenated aliphatic hydrocarbons with the SOS-chromotest]. Zbl Hyg. 189:266-271.

Meza-Junco J, Montaño-Loza AJ, Martínez-Benitez B, Kimura-Hayama E. 2007. Hepatocellular carcinoma in patients with autoimmune liver diseases: Two case reports and literature review. Ann Hepatol. 6(2):122-126. http://dx.doi.org/10.1016/S1665-2681(19)31945-3

Miligi L, Costantini AS, Benvenuti A, Kriebel D, Bolejack V, Tumino R, Ramazzotti V, Rodella S, Stagnaro E, Crosignani P et al. 2006. Occupational exposure to solvents and the risk of lymphomas. Epidemiology. 17(5):552-561.

http://dx.doi.org/10.1097/01.ede.0000231279.30988.4d

Miller BM, Adler ID. 1992. Aneuploidy induction in mouse spermatocytes. Mutagenesis. 7(1):69-76. http://dx.doi.org/10.1093/mutage/7.1.69

Miller RE, Guengerich FP. 1983. Metabolism of trichloroethylene in isolated hepatocytes, microsomes, and reconstituted enzyme systems containing cytochrome P-450. Cancer Res. 43(3):1145-1152.

Milman HA, Story DL, Riccio ES, Sivak A, Tu AS, Williams GM, Tong C, Tyson CA. 1988. Rat liver foci and in vitro assays to detect initiating and promoting effects of chlorinated ethanes and ethylenes. Ann N Y Acad Sci. 534:521-530. http://dx.doi.org/10.1111/j.17496632.1988.tb30143.x

Mirsalis JC, Tyson CK, Steinmetz KL, Loh EK, Hamilton CM, Bakke JP, Spalding JW. 1989. Measurement of unscheduled DNA synthesis and S-phase synthesis in rodent hepatocytes following in vivo treatment: Testing of 24 compounds. Environ Mol Mutagen. 14(3):155-164. http://dx.doi.org/10.1002/em.2850140305

Moore LE, Boffetta P, Karami S, Brennan P, Stewart PS, Hung R, Zaridze D, Matveev V, Janout $\mathrm{V}$, Kollarova $\mathrm{H}$ et al. 2010. Occupational trichloroethylene exposure and renal carcinoma risk: Evidence of genetic susceptibility by reductive metabolism gene variants. Cancer Res. 70(16):6527-6536. http://dx.doi.org/10.1158/0008-5472.CAN-09-4167

Moore LE, Nickerson ML, Brennan P, Toro JR, Jaeger E, Rinsky J, Han SS, Zaridze D, Matveev $\mathrm{V}$, Janout $\mathrm{V}$ et al. 2011. Von Hippel-Lindau (VHL) inactivation in sporadic clear cell renal cancer: Associations with germline VHL polymorphisms and etiologic risk factors. PLoS Genet. 7(10):e1002312. http://dx.doi.org/10.1371/journal.pgen.1002312

Moore MM, Harrington-Brock K. 2000. Mutagenicity of trichloroethylene and its metabolites: Implications for the risk assessment of trichloroethylene. Environ Health Perspect. 108 Suppl 2:215-223. http://dx.doi.org/10.1289/ehp.00108s2215

Morgan RW, Kelsh MA, Zhao K, Heringer S. 1998. Mortality of aerospace workers exposed to trichloroethylene. Epidemiology. 9(4):424-431. http://dx.doi.org/10.1097/00001648-199807000$\underline{00013}$ 
Morgenstern H, Froines J, Ritz B, Young B. 1997. Epidemiological study to determine possible adverse effects to rocketdyne/atomics international workers from exposure to ionizing radiation. Berkely, CA: Public Health Institute.

Mortelmans K, Haworth S, Lawlor T, Speck W, Tainer B, Zeiger E. 1986. Salmonella mutagenicity tests: II. Results from the testing of 270 chemicals. Environ Mutagen. 8 Suppl 7:1119. http://dx.doi.org/10.1002/em.2860080802

Morton LM, Turner JJ, Cerhan JR, Linet MS, Treseler PA, Clarke CA, Jack A, Cozen W, Maynadie M, Spinelli JJ et al. 2007. Proposed classification of lymphoid neoplasms for epidemiologic research from the Pathology Working Group of the International Lymphoma Epidemiology Consortium (InterLymph). Blood. 110(2):695-708.

http://dx.doi.org/10.1182/blood-2006-11-051672

Muller AM, Ihorst G, Mertelsmann R, Engelhardt M. 2005. Epidemiology of non-Hodgkin's lymphoma (NHL): Trends, geographic distribution, and etiology. Ann Hematol. 84(1):1-12. http://dx.doi.org/10.1007/s00277-004-0939-7

Muller M, Birner G, Sander M, Dekant W. 1998. Reactivity of haloketenes and halothioketenes with nucleobases: Reactions in vitro with DNA. Chem Res Toxicol. 11(5):464-470. http://dx.doi.org/10.1021/tx9701440

Nagaya T, Ishikawa N, Hata H. 1989. Sister-chromatid exchanges in lymphocytes of workers exposed to trichloroethylene. Mutat Res. 222(3):279-282. http://dx.doi.org/10.1016/0165$\underline{1218(89) 90144-4}$

Nakahama T, Maruyama I, Endo M, Inouye Y. 2001. Specificity in the metabolic activation of chlorinated ethylenes by cytochromes P450 in primary rat hepatocytes. J Health Sci. 47(1):3639. http://dx.doi.org/10.1248/jhs.47.36

National Research Council (NRC). 2006. Assessing the Human Health Risks of Trichloroethylene: Key Scientific Issues. Washington, D.C.: National Academies Press.

National Toxicology Program (NTP). 1976. Carcinogenesis bioassay of trichloroethylene. Bethesda, MD: National Institutes of Health. NTP TR-2. https://ntp.niehs.nih.gov/ntp/htdocs/lt rpts/tr002.pdf.

National Toxicology Program (NTP). 1988. Toxicology and carcinogenesis studies of trichloroethylene (CAS No. 79-01-6) in four strains of rats (ACI, August, Marshall, OsborneMendel) (gavage studies). Research Triangle Park, NC: National Toxicology Program. NTP TR273.

National Toxicology Program (NTP). 1990. Carcinogenesis studies of trichloroethylene (without epichlorohydrin) (CAS No. 79-01-6) in F344/N Rats and B6C3F1 mice (gavage studies). Research Triangle Park, NC: National Toxicology Program. NTP TR-243.

National Toxicology Program (NTP). 1999. NTP technical report on the toxicity and metabolism studies of chloral hydrate (CAS No. 302-17-0) administered by gavage to F344/N rats and B6C3F1 mice. Rockville, MD: U.S. Dept. of Health and Human Services, National Institutes of Health. https://ntp.niehs.nih.gov/ntp/htdocs/st_rpts/tox059.pdf. 
National Toxicology Program (NTP). 2011. Trichloroethylene. Rep Carcinog. 12th ed. Research Triangle Park, NC: National Toxicology Program. p. 420-423.

Nelson MA, Bull RJ. 1988. Induction of strand breaks in DNA by trichloroethylene and metabolites in rat and mouse liver in vivo. Toxicol Appl Pharmacol. 94(1):45-54.

http://dx.doi.org/10.1016/0041-008X(88)90335-3

Nelson MA, Lansing AJ, Sanchez IM, Bull RJ, Springer DL. 1989. Dichloroacetic acid and trichloroacetic acid-induced DNA strand breaks are independent of peroxisome proliferation. Toxicology. 58(3):239-248. http://dx.doi.org/10.1016/0300-483X(89)90139-X

Nestmann ER, Chu I, Kowbel DJ, Matula TI. 1980. Short-lived mutagen in Salmonella produced by reaction of trichloroacetic acid and dimethyl sulphoxide. Can J Genet Cytol. 22(1):35-40. http://dx.doi.org/10.1139/g80-006

Ni YC, Kadlubar FF, Fu PP. 1995. Formation of malondialdehyde-modified 2'-deoxyguanosinyl adduct from metabolism of chloral hydrate by mouse liver microsomes. Biochem Biophys Res Commun. 216(3):1110-1117. http://dx.doi.org/10.1006/bbrc.1995.2735

Nietert PJ, Sutherland SE, Silver RM, Pandey JP, Knapp RG, Hoel DG, Dosemeci M. 1998. Is occupational organic solvent exposure a risk factor for scleroderma? Arthritis Rheum. 41(6):1111-1118. http://dx.doi.org/10.1002/1529-0131(199806)41:6<1111::AIDART19>3.0.CO;2-J

Nishiyama R, Kanai T, Abe J, Hara R, Watahiki Y, Sakaguchi T, Nakamura S. 2004. Hepatocellular carcinoma associated with autoimmune hepatitis. J Hepatobiliary Pancreat Surg. 11(3):215-219. http://dx.doi.org/10.1007/s00534-003-0878-z

Nordström M, Hardell L, Magnuson A, Hagberg H, Rask-Andersen A. 1998. Occupational exposures, animal exposure and smoking as risk factors for hairy cell leukaemia evaluated in a case-control study. Br J Cancer. 77(11):2048-2052. http://dx.doi.org/10.1038/bjc.1998.341

Nutley EV, Tcheong AC, Allen JW, Collins BW, Ma M, Lowe XR, Bishop JB, Moore DH, 2nd, Wyrobek AJ. 1996. Micronuclei induced in round spermatids of mice after stem-cell treatment with chloral hydrate: Evaluations with centromeric DNA probes and kinetochore antibodies. Environ Mol Mutagen. 28(2):80-89. http://dx.doi.org/10.1002/(SICI)1098-

2280(1996)28:2<80::AID-EM3>3.0.CO;2-I

Odum J, Green T, Foster JR, Hext PM. 1988. The role of trichloroacetic acid and peroxisome profliferation in the differences in carcinogenicity of perchloroethylene in the mouse and rat. Toxicol Appl Pharmacol. 92:103-112. http://dx.doi.org/10.1016/0041-008X(88)90232-3

Ono Y, Somiya I, Kawamura M. 1991. The evaluation of genotoxicity using DNA repairing test for chemicals produced in chlorination and ozonation processes. Water Sci Technol. 23:329-338. http://dx.doi.org/10.2166/wst.1991.0431

Orsi L, Monnereau A, Dananche B, Berthou C, Fenaux P, Marit G, Soubeyran P, Huguet F, Milpied N, Leporrier M et al. 2010. Occupational exposure to organic solvents and lymphoid neoplasms in men: Results of a French case-control study. Occup Environ Med. 67(10):664-672. http://dx.doi.org/10.1136/oem.2009.049460 
Parchman LG, Magee PN. 1982. Metabolism of [14C]trichloroethylene to 14CO2 and interaction of a metabolite with liver DNA in rats and mice. J Toxicol Environ Health. 9(5-6):797-813. http://dx.doi.org/10.1080/15287398209530204

Parrish JM, Austin EW, Stevens DK, Kinder DH, Bull RJ. 1996. Haloacetate-induced oxidative damage to DNA in the liver of male B6C3F1 mice. Toxicology. 110(1-3):103-111. http://dx.doi.org/10.1016/0300-483X(96)03342-2

Parry EM, Hague A, Parry JM. 1990. A study of mitotic division fidelity and numerical chromosome changes in ageing Syrian hamster dermal cells. Mutat Res. 237(2):83-93. http://dx.doi.org/10.1016/0921-8734(90)90014-I

Peden-Adams MM, Eudaly JG, Heesemann LM, Smythe J, Miller J, Gilkeson GS, Keil DE. 2006. Developmental immunotoxicity of trichloroethylene (TCE): Studies in B6C3F1 mice. J Environ Sci Health A Tox Hazard Subst Environ Eng. 41(3):249-271.

http://dx.doi.org/10.1080/10934520500455289

Peden-Adams MM, Eudaly JG, Lee AM, Miller J, Keil DE, Gilkeson GS. 2008. Lifetime exposure to trichloroethylene (TCE) does not accelerate autoimmune disease in MRL +/+ mice. J Environ Sci Health A Tox Hazard Subst Environ Eng. 43(12):1402-1409. http://dx.doi.org/10.1080/10934520802232063

Perocco P, Prodi G. 1981. DNA damage by haloalkanes in human lymphocytes cultured in vitro. Cancer Lett. 13(3):213-218. http://dx.doi.org/10.1016/0304-3835(81)90020-3

Persson B, Dahlander AM, Fredriksson M, Brage HN, Ohlson CG, Axelson O. 1989. Malignant lymphomas and occupational exposures. Br J Ind Med. 46(8):516-520.

Persson B, Fredriksson M, Olsen K, Boeryd B, Axelson O. 1993. Some occupational exposures as risk factors for malignant lymphomas. Cancer. 72(5):1773-1778.

http://dx.doi.org/10.1002/1097-0142(19930901)72:5<1773::AID-CNCR2820720542>3.0.CO;26

Persson B, Fredrikson M. 1999. Some risk factors for non-Hodgkin's lymphoma. Int J Occup Med Environ Health. 12(2):135-142.

Pesch B, Haerting J, Ranft U, Klimpel A, Oelschlagel B, Schill W. 2000a. Occupational risk factors for urothelial carcinoma: Agent-specific results from a case-control study in Germany. MURC Study Group. Multicenter Urothelial and Renal Cancer. Int J Epidemiol. 29(2):238-247. http://dx.doi.org/10.1093/ije/29.2.238

Pesch B, Haerting J, Ranft U, Klimpel A, Oelschlagel B, Schill W, Barth W, Brettschneider U, Bronder E, Farker K et al. 2000b. Occupational risk factors for renal cell carcinoma: Agentspecific results from a case-control study in Germany. Int J Epidemiol. 29(6):1014-1024. http://dx.doi.org/10.1093/ije/29.6.1014

Plewa MJ, Kargalioglu Y, Vankerk D, Minear RA, Wagner ED. 2002. Mammalian cell cytotoxicity and genotoxicity analysis of drinking water disinfection by-products. Environ Mol Mutagen. 40(2):134-142. http://dx.doi.org/10.1002/em.10092 
Plewa MJ, Simmons JE, Richardson SD, Wagner ED. 2010. Mammalian cell cytotoxicity and genotoxicity of the haloacetic acids, a major class of drinking water disinfection by-products. Environ Mol Mutagen. 51(8-9):871-878. http://dx.doi.org/10.1002/em.20585

Poet TS, Corley RA, Thrall KD, Edwards JA, Tanojo H, Weitz KK, Hui X, Maibach HI, Wester RC. 2000. Assessment of the percutaneous absorption of trichloroethylene in rats and humans using MS/MS real-time breath analysis and physiologically based pharmacokinetic modeling. Toxicol Sci. 56(1):61-72. http://dx.doi.org/10.1093/toxsci/56.1.61

Ponce RA, Gelzleichter T, Haggerty HG, Heidel S, Holdren MS, Lebrec H, Mellon RD, Pallardy M. 2014. Immunomodulation and lymphoma in humans. J Immunotoxicol. 11(1):1-12. http://dx.doi.org/10.3109/1547691X.2013.798388

Price PJ, Hassett CM, Mansfield JI. 1978. Transforming activities of trichloroethylene and proposed industrial alternatives. In Vitro. 14(3):290-293. http://dx.doi.org/10.1007/BF02616038

Pukkala E, Martinsen JI, Lynge E, Gunnarsdottir HK, Sparén P, Tryggvadottir L, Weiderpass E, Kjaerheim K. 2009. Occupation and cancer - follow-up of 15 million people in five Nordic countries. Acta Oncol. 48(5):646-790. http://dx.doi.org/10.1080/02841860902913546

Purdue MP, Lan Q, Kricker A, Grulich AE, Vajdic CM, Turner J, Whitby D, Chanock S, Rothman N, Armstrong BK. 2007. Polymorphisms in immune function genes and risk of nonHodgkin lymphoma: Findings from the New South Wales non-Hodgkin Lymphoma Study. Carcinogenesis. 28(3):704-712. http://dx.doi.org/10.1093/carcin/bgl200

Purdue MP, Bakke B, Stewart P, De Roos AJ, Schenk M, Lynch CF, Bernstein L, Morton LM, Cerhan JR, Severson RK et al. 2011a. A case-control study of occupational exposure to trichloroethylene and non-Hodgkin lymphoma. Environ Health Perspect. 119(2):232-238. http://dx.doi.org/10.1289/ehp.1002106

Purdue MP, Lan Q, Bagni R, Hocking WG, Baris D, Reding DJ, Rothman N. 2011 b. Prediagnostic serum levels of cytokines and other immune markers and risk of non-hodgkin lymphoma. Cancer Res. 71(14):4898-4907. http://dx.doi.org/10.1158/0008-5472.CAN-11-0165

Raaschou-Nielsen O, Hansen J, Christensen JM, Blot WJ, McLaughlin JK, Olsen JH. 2001. Urinary concentrations of trichloroacetic acid in Danish workers exposed to trichloroethylene, 1947-1985. Am J Ind Med. 39(3):320-327. http://dx.doi.org/10.1002/10970274(200103)39:3<320::AID-AJIM1020>3.0.CO;2-8

Raaschou-Nielsen O, Hansen J, Thomsen BL, Johansen I, Lipworth L, McLaughlin JK, Olsen JH. 2002. Exposure of Danish workers to trichloroethylene, 1947-1989. Appl Occup Environ Hyg. 17(10):693-703. http://dx.doi.org/10.1080/10473220290096186

Raaschou-Nielsen O, Hansen J, McLaughlin JK, Kolstad H, Christensen JM, Tarone RE, Olsen JH. 2003. Cancer risk among workers at Danish companies using trichloroethylene: A cohort study. Am J Epidemiol. 158(12):1182-1192. http://dx.doi.org/10.1093/aje/kwg282

Radican L, Blair A, Stewart P, Wartenberg D. 2008. Mortality of aircraft maintenance workers exposed to trichloroethylene and other hydrocarbons and chemicals: Extended follow-up. J Occup Environ Med. 50(11):1306-1319. http://dx.doi.org/10.1097/JOM.0b013e3181845f7f 
Ramdhan DH, Kamijima M, Wang D, Ito Y, Naito H, Yanagiba Y, Hayashi Y, Tanaka N, Aoyama T, Gonzalez FJ et al. 2010. Differential response to trichloroethylene-induced hepatosteatosis in wild-type and PPARalpha-humanized mice. Environ Health Perspect. 118(11):1557-1563. http://dx.doi.org/10.1289/ehp.1001928

Rasmussen K, Sabroe S, Wohlert M, Ingerslev HJ, Kappel B, Nielsen J. 1988. A genotoxic study of metal workers exposed to trichloroethylene. Sperm parameters and chromosome aberrations in lymphocytes. Int Arch Occup Environ Health. 60(6):419-423.

http://dx.doi.org/10.1007/BF00381389

Ravel G, Christ M, Perron-Lepage MF, Condevaux F, Descotes J. 2004. Trichloroethylene does not Accelerate autoimmune diabetes in NOD mice. J Immunotoxicol. 1(3):141-148.

Ripp SL, Itagaki K, Philpot RM, Elfarra AA. 1999. Species and sex differences in expression of flavin-containing monooxygenase form 3 in liver and kidney microsomes. Drug Metab Dispos. 27(1):46-52.

Ritz B. 1999. Cancer mortality among workers exposed to chemicals during uranium processing. J Occup Environ Med. 41(7):556-566. http://dx.doi.org/10.1097/00043764-199907000-00004

Ritz B, Morgenstern H, Froines J, Moncau J. 1999. Chemical exposures of rocket-engine teststand personnel and cancer mortality in a cohort of aerospace workers. J Occup Environ Med. 41(10):903-910. http://dx.doi.org/10.1097/00043764-199910000-00011

Robbiano L, Baroni D, Carrozzino R, Mereto E, Brambilla G. 2004. DNA damage and micronuclei induced in rat and human kidney cells by six chemicals carcinogenic to the rat kidney. Toxicology. 204(2-3):187-195. http://dx.doi.org/10.1016/j.tox.2004.06.057

Roldan-Arjona T, Garcia-Pedrajas MD, Luque-Romero FL, Hera C, Pueyo C. 1991. An association between mutagenicity of the Ara test of Salmonella typhimurium and carcinogenicity in rodents for 16 halogenated aliphatic hydrocarbons. Mutagenesis. 6(3):199-205. http://dx.doi.org/10.1093/mutage/6.3.199

Rossi AM, Migliore L, Barale R, Loprieno N. 1983. In vivo and in vitro mutagenicity studies of a possible carcinogen, trichloroethylene, and its two stabilizers, epichlorohydrin and 1,2epoxybutane. Teratog Carcinog Mutagen. 3(1):75-87. http://dx.doi.org/10.1002/15206866(1990)3:1<75::AID-TCM1770030109>3.0.CO;2-5

Rothman N, Skibola CF, Wang SS, Morgan G, Lan Q, Smith MT, Spinelli JJ, Willett E, De Sanjose S, Cocco P et al. 2006. Genetic variation in TNF and IL10 and risk of non-Hodgkin lymphoma: a report from the InterLymph Consortium. Lancet Oncol. 7(1):27-38. http://dx.doi.org/10.1016/S1470-2045(05)70434-4

Russo A, Pacchierotti F, Metalli P. 1984. Nondisjunction induced in mouse spermatogenesis by chloral hydrate, a metabolite of trichloroethylene. Environ Mutagen. 6(5):695-703. http://dx.doi.org/10.1002/em.2860060507

Russo A, Levis AG. 1992. Further evidence for the aneuploidogenic properties of chelating agents: Induction of micronuclei in mouse male germ cells by EDTA. Environ Mol Mutagen. 19(2):125-131. http://dx.doi.org/10.1002/em.2850190206 
Russo A, Stocco A, Majone F. 1992. Identification of kinetochore-containing (CREST+) micronuclei in mouse bone marrow erythrocytes. Mutagenesis. 7(3):195-197. http://dx.doi.org/10.1093/mutage/7.3.195

Rusyn I, Chiu WA, Lash LH, Kromhout H, Hansen J, Guyton KZ. 2014. Trichloroethylene: Mechanistic, epidemiologic and other supporting evidence of carcinogenic hazard. Pharmacol Ther. 141(1):55-68. http://dx.doi.org/10.1016/j.pharmthera.2013.08.004

Sanders VM, Tucker AN, White KL, Kauffmann BM, Hallett P, Carchman RA, Borzelleca JF, Munson AE. 1982. Humoral and cell-mediated immune status in mice exposed to ttrichloroethylene in the drinking water. Toxicol Appl Pharmacol. 62:358-368. http://dx.doi.org/10.1016/0041-008X(82)90138-7

Sano Y, Nakashima H, Yoshioka N, Etho N, Nomiyama T, Nishiwaki Y, Takebayashi T, Oame K. 2009. Trichloroethylene liver toxicity in mouse and rat: Microarray analysis reveals species differences in gene expression. Arch Toxicol. 83(9):835-849. http://dx.doi.org/10.1007/s00204$\underline{009-0431-1}$

Schultz IR, Merdink JL, Gonzalez-Leon A, Bull RJ. 2002. Dichloroacetate toxicokinetics and disruption of tyrosine catabolism in B6C3F1 mice: Dose-response relationships and age as a modifying factor. Toxicology. 173(3):229-247. http://dx.doi.org/10.1016/S0300$\underline{483 X(02) 00034-3}$

Scott CS, Jinot J. 2011. Trichloroethylene and cancer: Systematic and quantitative review of epidemiologic evidence for identifying hazards. Int J Environ Res Public Health. 8(11):42384272. http://dx.doi.org/10.3390/ijerph8114238

SEER Stat Fact Sheets: Myeloma. 2014a. National Cancer Institute; [accessed Accessed on 10/21/14]. http://seer.cancer.gov/statfacts/html/mulmy.html

SEER Stat Fact Sheets: Non-Hodgkin Lymphoma. 2014b. National Cancer Institute; [accessed Accessed on 10/21/14]. http://seer.cancer.gov/statfacts/html/nhl.html

SEER Stat Fact Sheets: Liver and Intrahepatic Bile Duct Cancer. 2014c. National Cancer Institute; [accessed Accessed on 10/22/14]. http://seer.cancer.gov/statfacts/html/livibd.html

SEER Stat Fact Sheets: Kidney and Renal Pelvis Cancer. 2014d. National Cancer Institute; [accessed Accessed on 10/21/14]. http://seer.cancer.gov/statfacts/html/kidrp.html

Seidler A, Mohner M, Berger J, Mester B, Deeg E, Elsner G, Nieters A, Becker N. 2007. Solvent exposure and malignant lymphoma: A population-based case-control study in Germany. J Occup Med Toxicol. 2:1-11. http://dx.doi.org/10.1186/1745-6673-2-2

Seiji K, Jin C, Watanabe T, Nakatsuka H, Ikeda M. 1990. Sister chromatid exchanges in peripheral lymphocytes of workers exposed to benzene, trichloroethylene, or tetrachloroethylene, with reference to smoking habits. Int Arch Occup Environ Health. 62(2):171-176.

http://dx.doi.org/10.1007/BF00383594

Seldén A, Ahlborg G, Jr. 1991. Mortality and cancer morbidity after exposure to military aircraft fuel. Aviat Space Environ Med. 62(8):789-794. 
Selgrade MK, Gilmour MI. 2010. Suppression of pulmonary host defenses and enhanced susceptibility to respiratory bacterial infection in mice following inhalation exposure to trichloroethylene and chloroform. J Immunotoxicol. 7(4):350-356.

http://dx.doi.org/10.3109/1547691X.2010.520139

Shelby MD, Erexson GL, Hook GJ, Tice RR. 1993. Evaluation of a three-exposure mouse bone marrow micronucleus protocol: Results with 49 chemicals. Environ Mol Mutagen. 21(2):160179. http://dx.doi.org/10.1002/em.2850210210

Shimada T, Swanson AF, Leber P, Williams GM. 1985. Activities of chlorinated ethane and ethylene compounds in the Salmonella/rat microsome mutagenesis and rat hepatocyte/DNA repair assays under vapor phase exposure conditions. Cell Biol Toxicol. 1(3):159-179. http://dx.doi.org/10.1007/BF00120162

Shirai N, Ohtsuji M, Hagiwara K, Tomisawa H, Ohtsuji N, Hirose S, Hagiwara H. 2012. Nephrotoxic effect of subchronic exposure to S-(1,2-dichlorovinyl)-L-cysteine in mice. J Toxicol Sci. 37(5):871-878. http://dx.doi.org/10.2131/jts.37.871

Siemiatycki J. 1991. Risk Factors for Cancer in the Workplace. Boca Raton, FL: CRC Press.

Silver SR, Pinkerton LE, Fleming DA, Jones JH, Allee S, Luo L, Bertke SJ. 2014. Retrospective cohort study of a microelectronics and business machine facility. Am J Ind Med. 57(4):412-424. http://dx.doi.org/10.1002/ajim.22288

Simmon V, Kauhanen K, Tardiff R. 1977. Mutagenic activity of chemicals identified in drinking water. Progress in Genetic Toxicology. New York; Amsterdam: Elsevier/North Holland Press. p. 249-268.

Sinks T, Lushniak B, Haussler BJ, Sniezek J, Deng JF, Roper P, Dill P, Coates R. 1992. Renal cell cancer among paperboard printing workers. Epidemiology. 3(6):483-489.

http://dx.doi.org/10.1097/00001648-199211000-00004

Slacik-Erben R, Roll R, Franke G, Uehleke H. 1980. Trichloroethylene vapours do not produce dominant lethal mutations in male mice. Arch Toxicol. 45(1):37-44.

http://dx.doi.org/10.1007/BF00303293

Sofuni T, Hayashi M, Matsuoka A, Sawada M, Hatanaka M, Ishidate M, Jr. 1985. [Mutagenicity tests on organic chemical contaminants in city water and related compounds. II. Chromosome aberration tests in cultured mammalian cells]. Eisei Shikenjo Hokoku. (103):64-75.

Sora S, Agostini Carbone ML. 1987. Chloral hydrate, methylmercury hydroxide and ethidium bromide affect chromosomal segregation during meiosis of Saccharomyces cerevisiae. Mutat Res. 190(1):13-17. http://dx.doi.org/10.1016/0165-7992(87)90075-3

Souček B, Vlachová D. 1960. Excretion of trichloroethylene metabolites in human urine. Br J Ind Med. 17:60-64. http://dx.doi.org/10.1136/oem.17.1.60

Spirtas R, Stewart PA, Lee JS, Marano DE, Forbes CD, Grauman DJ, Pettigrew HM, Blair A, Hoover RN, Cohen JL. 1991. Retrospective cohort mortality study of workers at an aircraft maintenance facility. I. Epidemiological results. Br J Ind Med. 48(8):515-530.

http://dx.doi.org/10.1136/oem.48.8.515 
Stacpoole PW, Kurtz TL, Han Z, Langaee T. 2008. Role of dichloroacetate in the treatment of genetic mitochondrial diseases. Adv Drug Deliv Rev. 60(13-14):1478-1487.

http://dx.doi.org/10.1016/j.addr.2008.02.014

Stacpoole PW. 2011. The dichloroacetate dilemma: environmental hazard versus therapeutic goldmine--both or neither? Environ Health Perspect. 119(2):155-158.

http://dx.doi.org/10.1289/ehp.1002554

Stevens JL, Hatzinger PB, Hayden PJ. 1989. Quantitation of multiple pathways for the metabolism of nephrotoxic cysteine conjugates using selective inhibitors of L-alpha-hydroxy acid oxidase (L-amino acid oxidase) and cysteine conjugate beta-lyase. Drug Metab Dispos. 17(3):297-303.

Stewart PA, Lee JS, Marano DE, Spirtas R, Forbes CD, Blair A. 1991. Retrospective cohort mortality study of workers at an aircraft maintenance facility. II. Exposures and their assessment. Br J Ind Med. 48(8):531-537. http://dx.doi.org/10.1136/oem.48.8.531

Storchova Z, Kuffer C. 2008. The consequences of tetraploidy and aneuploidy. J Cell Sci. 121(Pt 23):3859-3866. http://dx.doi.org/10.1242/jcs.039537

Stott WT, Quast JF, Watanabe PG. 1982. The pharmacokinetics and macromolecular interactions of trichloroethylene in mice and rats. Toxicol Appl Pharmacol. 62(1):137-151. http://dx.doi.org/10.1016/0041-008X(82)90110-7

Styles JA, Wyatt I, Coutts C. 1991. Trichloroacetic acid: Studies on uptake and effects on hepatic DNA and liver growth in mouse. Carcinogenesis. 12(9):1715-1719.

http://dx.doi.org/10.1093/carcin/12.9.1715

Sweeney C, Farrow DC, Schwartz SM, Eaton DL, Checkoway H, Vaughan TL. 2000. Glutathione S-transferase M1, T1, and P1 polymorphisms as risk factors for renal cell carcinoma: A case-control study. Cancer Epidemiol Biomarkers Prev. 9(4):449-454.

Swenberg JA, Lehman-McKeeman LD. 1999. alpha 2-Urinary globulin-associated nephropathy as a mechanism of renal tubule cell carcinogenesis in male rats. IARC Sci Publ. (147):95-118.

Tabrez S, Ahmad M. 2009. Toxicity, biomarkers, genotoxicity, and carcinogenicity of trichloroethylene and its metabolites: A review. J Environ Sci Health C Environ Carcinog Ecotoxicol Rev. 27(3):178-196. http://dx.doi.org/10.1080/10590500903091340

Tabrez S, Ahmad M. 2012. Genotoxicity of trichloroethylene in the natural milieu. Int J Hyg Environ Health. 215(3):333-338. http://dx.doi.org/10.1016/j.ijheh.2011.08.004

Tabrez S, Ahmad M. 2013. Cytochrome P450 system as potential biomarkers of certain toxicants: Comparison between plant and animal models. Environ Monit Assess. 185(4):29772987. http://dx.doi.org/10.1007/s10661-012-2765-z

Tan TT, Coussens LM. 2007. Humoral immunity, inflammation and cancer. Curr Opin Immunol. 19(2):209-216. http://dx.doi.org/10.1016/j.coi.2007.01.001

Tang X, Que B, Song X, Li S, Yang X, Wang H, Huang H, Kamijima M, Nakajima T, Lin Y et al. 2008. Characterization of liver injury associated with hypersensitive skin reactions induced by 
trichloroethylene in the guinea pig maximization test. J Occup Health. 50(2):114-121. http://dx.doi.org/10.1539/joh.L7114

Tanguay RM, Jorquera R, Poudrier J, St-Louis M. 1996. Tyrosine and its catabolites: From disease to cancer. Acta Biochim Pol. 43(1):209-216. http://dx.doi.org/10.18388/abp.1996 4530

Tola S, Vilhunen R, Jarvinen E, Korkala ML. 1980. A cohort study on workers exposed to trichloroethylene. J Occup Med. 22(11):737-740.

Toraason M, Clark J, Dankovic D, Mathias P, Skaggs S, Walker C, Werren D. 1999. Oxidative stress and DNA damage in Fischer rats following acute exposure to trichloroethylene or perchloroethylene. Toxicology. 138(1):43-53. http://dx.doi.org/10.1016/S0300-483X(99)00083$\underline{9}$

Tu AS, Murray TA, Hatch KM, Sivak A, Milman HA. 1985. In vitro transformation of BALB/c3T3 cells by chlorinated ethanes and ethylenes. Cancer Lett. 28(1):85-92.

http://dx.doi.org/10.1016/0304-3835(85)90096-5

U.S. Environmental Protection Agency (USEPA). 2006. Approaches for the application of physiologically based pharmacokinetic (PBPK) models and supporting data in risk assessment. Washington, D.C.: U.S. Environmental Protection Agency. EPA/600/R-05/043F.

U.S. Environmental Protection Agency (USEPA). 2011a. Toxicological review of trichloroethylene (CAS No. 79-01-6) in support of summary information on the integrated risk information system (IRIS). U.S. Environmental Protection Agency. EPA/635/R-09/011F.

U.S. Environmental Protection Agency (USEPA). 2011b. Toxicological review of trichloroethylene appendices (CAS No. 79-01-6) in support of summary information on the integrated risk information system (IRIS). U.S. Environmental Protection Agency. EPA/635/R09/011F.

U.S. Environmental Protection Agency (USEPA). 2014. Trichloroethylene: Degreasing, spot cleaning and arts \& crafts uses. U.S. Environmental Protection Agency. EPA Document \#740R1-4002.

Vamvakas S, Dekant W, Berthold K, Schmidt S, Wild D, Henschler D. 1987. Enzymatic transformation of mercapturic acids derived from halogenated alkenes to reactive and mutagenic intermediates. Biochem Pharmacol. 36(17):2741-2748. http://dx.doi.org/10.1016/0006$\underline{2952(87) 90258-9}$

Vamvakas S, Dekant W, Schiffmann D, Henschler D. 1988a. Induction of unscheduled DNA synthesis and micronucleus formation in Syrian hamster embryo fibroblasts treated with cysteine S-conjugates of chlorinated hydrocarbons. Cell Biol Toxicol. 4(4):393-403. http://dx.doi.org/10.1007/BF00117768

Vamvakas S, Elfarra AA, Dekant W, Henschler D, Anders MW. 1988b. Mutagenicity of amino acid and glutathione S-conjugates in the Ames test. Mutat Res. 206(1):83-90.

http://dx.doi.org/10.1016/0165-1218(88)90144-9 
Vamvakas S, Dekant W, Henschler D. 1989. Assessment of unscheduled DNA synthesis in a cultured line of renal epithelial cells exposed to cysteine $\mathrm{S}$-conjugates of haloalkenes and haloalkanes. Mutat Res. 222(4):329-335. http://dx.doi.org/10.1016/0165-1218(89)90108-0

Vamvakas S, Richter H, Bittner D. 1996. Induction of dedifferentiated clones of LLC-PK1 cells upon long-term exposure to dichlorovinylcysteine. Toxicology. 106(1-3):65-74.

http://dx.doi.org/10.1016/0300-483X(95)03166-D

Vamvakas S, Brüning T, Thomasson B, Lammert M, Baumüller A, Bolt HM, Dekant W, Birner G, Henschler D, Ulm K. 1998. Renal cell cancer correlated with occupational exposure to trichloroethene. J Cancer Res Clin Oncol. 124(7):374-382.

http://dx.doi.org/10.1007/s004320050186

Varshney M, Chandra A, Chauhan LK, Goel SK. 2013a. Micronucleus induction by oxidative metabolites of trichloroethylene in cultured human peripheral blood lymphocytes: A comparative genotoxicity study. Environ Sci Pollut Res Int. http://dx.doi.org/10.1007/s11356-013-1806-7

Varshney M, Chandra A, Chauhan LK, Goel SK. 2013b. In vitro cytogenetic assessment of trichloroacetic acid in human peripheral blood lymphocytes. Environ Sci Pollut Res Int.

Vermeulen R, Hosnijeh FS, Portengen L, Krogh V, Palli D, Panico S, Tumino R, Sacredote C, Purdue M, Lan Q et al. 2011. Circulating soluble CD30 and future risk of lymphoma; evidence from two prospective studies in the general population. Cancer Epidemiol Biomarkers Prev. 20(9):1925-1927. http://dx.doi.org/10.1158/1055-9965.EPI-11-0396

Vermeulen R, Zhang L, Spierenburg A, Tang X, Bonventre JV, Reiss B, Shen M, Smith MT, Qiu C, Ge Y et al. 2012. Elevated urinary levels of kidney injury molecule-1 among Chinese factory workers exposed to trichloroethylene. Carcinogenesis. 33(8):1538-1541.

http://dx.doi.org/10.1093/carcin/bgs191

Vlaanderen J, Straif K, Pukkala E, Kauppinen T, Kyyrönen P, Martinsen JI, Kjaerheim K, Tryggvadottir L, Hansen J, Sparén P et al. 2013. Occupational exposure to trichloroethylene and perchloroethylene and the risk of lymphoma, liver, and kidney cancer in four Nordic countries. Occup Environ Med. 70(6):393-401. http://dx.doi.org/10.1136/oemed-2012-101188

von der Hude W, Behm C, Gurtler R, Basler A. 1988. Evaluation of the SOS chromotest. Mutat Res. 203(2):81-94. http://dx.doi.org/10.1016/0165-1161(88)90023-4

Von Tungeln LS, Yi P, Bucci TJ, Samokyszyn VM, Chou MW, Kadlubar FF, Fu PP. 2002. Tumorigenicity of chloral hydrate, trichloroacetic acid, trichloroethanol, malondialdehyde, 4 hydroxy-2-nonenal, crotonaldehyde, and acrolein in the B6C3F1 neonatal mouse. Cancer Lett. 185:13-19. http://dx.doi.org/10.1016/S0304-3835(02)00231-8

Walles SA. 1986. Induction of single-strand breaks in DNA of mice by trichloroethylene and tetrachloroethylene. Toxicol Lett. 31(1):31-35. http://dx.doi.org/10.1016/0378-4274(86)90191-8

Wang G, Ansari GA, Khan MF. 2007a. Involvement of lipid peroxidation-derived aldehydeprotein adducts in autoimmunity mediated by trichloroethene. J Toxicol Environ Health A. 70(23):1977-1985. http://dx.doi.org/10.1080/15287390701550888 
Wang G, Cai P, Ansari GA, Khan MF. 2007b. Oxidative and nitrosative stress in trichloroethene-mediated autoimmune response. Toxicology. 229(3):186-193. http://dx.doi.org/10.1016/j.tox.2006.10.014

Wang G, Konig R, Ansari GA, Khan MF. 2008. Lipid peroxidation-derived aldehyde-protein adducts contribute to trichloroethene-mediated autoimmunity via activation of CD4+ T cells. Free Radic Biol Med. 44(7):1475-1482. http://dx.doi.org/10.1016/j.freeradbiomed.2008.01.012

Wang G, Wang J, Ma H, Khan MF. 2009a. Increased nitration and carbonylation of proteins in MRL+/+ mice exposed to trichloroethene: Potential role of protein oxidation in autoimmunity. Toxicol Appl Pharmacol. 237(2):188-195. http://dx.doi.org/10.1016/j.taap.2009.03.010

Wang G, Hou J, Ma L, Xie J, Yin J, Xu D, Chang W, Tan X, Su T, Zhang H et al. 2012a. Risk factor for clear cell renal cell carcinoma in Chinese population: a case-control study. Cancer Epidemiol. 36(2):177-182. http://dx.doi.org/10.1016/j.canep.2011.09.006

Wang G, Wang J, Fan X, Ansari GA, Khan MF. 2012b. Protein adducts of malondialdehyde and 4-hydroxynonenal contribute to trichloroethene-mediated autoimmunity via activating Th17 cells: Dose- and time-response studies in female MRL+/+ mice. Toxicology. 292(2-3):113-122. http://dx.doi.org/10.1016/j.tox.2011.12.001

Wang G, Wang J, Ma H, Ansari GA, Khan MF. 2013. N-Acetylcysteine protects against trichloroethene-mediated autoimmunity by attenuating oxidative stress. Toxicol Appl Pharmacol. 273(1):189-195. http://dx.doi.org/10.1016/j.taap.2013.08.020

Wang GD, Li H, Khan MF. 2012c. Differential oxidative modification of proteins in MRL+/+ and MRL/lpr mice: Increased formation of lipid peroxidation-derived aldehyde-protein adducts may contribute to accelerated onset of autoimmune response. Free Radic Res. 46(12):1472-1481. http://dx.doi.org/10.3109/10715762.2012.727209

Wang JL, Chen WL, Tsai SY, Sung PY, Huang RN. 2001. An in vitro model for evaluation of vaporous toxicity of trichloroethylene and tetrachloroethylene to $\mathrm{CHO}-\mathrm{K} 1$ cells. Chem Biol Interact. 137(2):139-154. http://dx.doi.org/10.1016/S0009-2797(01)00226-5

Wang KK, Czaja AJ. 1988. Hepatocellular carcinoma in corticosteroid-treated severe autoimmune chronic active hepatitis. Hepatology. 8(6):1679-1683.

http://dx.doi.org/10.1002/hep.1840080635

Wang R, Zhang YW, Lan Q, Holford TR, Leaderer B, Zahm SH, Boyle P, Dosemeci M, Rothman N, Zhu Y et al. 2009b. Occupational exposure to solvents and risk of non-Hodgkin lymphoma in Connecticut women. Am J Epidemiol. 169(2):176-185.

http://dx.doi.org/10.1093/aje/kwn300

Wang SS, Cozen W, Cerhan JR, Colt JS, Morton LM, Engels EA, Davis S, Severson RK, Rothman N, Chanock SJ et al. 2007c. Immune mechanisms in non-Hodgkin lymphoma: Joint effects of the TNF G308A and IL10 T3575A polymorphisms with non-Hodgkin lymphoma risk factors. Cancer Res. 67(10):5042-5054. http://dx.doi.org/10.1158/0008-5472.CAN-06-4752

Wartenberg D, Reyner D. 2000. TCE Meta-Analyses: Wartenberg et al.'s Response. Environ Health Perspect. 108(12):A543-A544. http://dx.doi.org/10.1289/ehp.108-a543 
Wartenberg D, Reyner D, Scott CS. 2000. Trichloroethylene and cancer: Epidemiologic evidence. Environ Health Perspect. 108(Suppl 2):161-176.

http://dx.doi.org/10.1289/ehp.00108s2161

Waskell L. 1978. A study of the mutagenicity of anesthetics and their metabolites. Mutat Res. 57(2):141-153. http://dx.doi.org/10.1016/0027-5107(78)90261-0

Watanabe H. 2011. Hypersensitivity syndrome due to trichloroethylene exposure: A severe generalized skin reaction resembling drug-induced hypersensitivity syndrome. J Dermatol. 38(3):229-235. http://dx.doi.org/10.1111/j.1346-8138.2010.01155.x

Watanabe T, Soga K, Hirono H, Hasegawa K, Shibasaki K, Kawai H, Aoyagi Y. 2009. Features of hepatocellular carcinoma in cases with autoimmune hepatitis and primary biliary cirrhosis. World J Gastroenterol. 15(2):231-239. http://dx.doi.org/10.3748/wjg.15.231

Weinhold B. 2009. A clearer view of TCE: Evidence supports autoimmune link. Environ Health Perspect. 117(5):A210. http://dx.doi.org/10.1289/ehp.117-a210a

White AE, Takehisa S, Eger EI, 2nd, Wolff S, Stevens WC. 1979. Sister chromatid exchanges induced by inhaled anesthetics. Anesthesiology. 50(5):426-430.

http://dx.doi.org/10.1097/00000542-197905000-00010

Wiesenhutter B, Selinski S, Golka K, Bruning T, Bolt HM. 2007. Re-assessment of the influence of polymorphisms of phase-II metabolic enzymes on renal cell cancer risk of trichloroethyleneexposed workers. Int Arch Occup Environ Health. 81(2):247-251.

http://dx.doi.org/10.1007/s00420-007-0200-5

Wilcosky TC, Checkoway H, Marshall EG, Tyroler HA. 1984. Cancer mortality and solvent exposures in the rubber industry. Am Ind Hyg Assoc J. 45(12):809-811.

http://dx.doi.org/10.1080/15298668491400683

Williams GM, Mori H, McQueen CA. 1989. Structure-activity relationships in the rat hepatocyte DNA-repair test for 300 chemicals. Mutat Res. 221(3):263-286. http://dx.doi.org/10.1016/0165$\underline{1110(89) 90039-0}$

Wilmer JW, Spencer PJ, Ball N, Bus JS. 2014. Assessment of the genotoxicity of trichloroethylene in the in vivo micronucleus assay by inhalation exposure. Mutagenesis. 29(3):209-214. http://dx.doi.org/10.1093/mutage/geu006

Wong O, Morgan R. 1990. Final report: Historical prospective mortality study of Hughes Aircraft employment at Air Force Plant, no. 44. Alameda, CA: ENSR Health Sciences.

Wright PF, Thomas WD, Stacey NH. 1991. Effects of trichloroethylene on hepatic and splenic lymphocytotoxic activities in rodents. Toxicology. 70(2):231-242.

http://dx.doi.org/10.1016/0300-483X(91)90049-7

Wu Y, Antony S, Meitzler JL, Doroshow JH. 2013. Molecular mechanisms underlying chronic inflammation-associated cancers. Cancer Lett. 
Xu F, Papanayotou I, Putt DA, Wang J, Lash LH. 2008. Role of mitochondrial dysfunction in cellular responses to S-(1,2-dichlorovinyl)-L-cysteine in primary cultures of human proximal tubular cells. Biochem Pharmacol. 76(4):552-567. http://dx.doi.org/10.1016/j.bcp.2008.05.016

$\mathrm{Xu}$ W, Adler ID. 1990. Clastogenic effects of known and suspect spindle poisons studied by chromosome analysis in mouse bone marrow cells. Mutagenesis. 5(4):371-374.

http://dx.doi.org/10.1093/mutage/5.4.371

Yang X, Long S, Deng J, Deng T, Gong Z, Hao P. 2013. Glutathione S-transferase polymorphisms (GSTM1, GSTT1 and GSTP1) and their susceptibility to renal cell carcinoma: An evidence-based meta-analysis. PLoS One. 8(5):e63827.

http://dx.doi.org/10.1371/journal.pone.0063827

Yaqoob N, Evans AR, Lock EA. 2013. Trichloroethylene-induced formic aciduria: Effect of dose, sex and strain of rat. Toxicology. 304:49-56. http://dx.doi.org/10.1016/j.tox.2012.11.014

Yaqoob N, Evans A, Foster JR, Lock EA. 2014. Trichloroethylene and trichloroethanol-induced formic aciduria and renal injury in male F-344 rats following 12 weeks exposure. Toxicology. (In Press). http://dx.doi.org/10.1016/j.tox.2014.06.004

Yiin JH, Anderson JL, Daniels RD, Seel EA, Fleming DA, Waters KM, Chen PH. 2009. A nested case-control study of multiple myeloma risk and uranium exposure among workers at the Oak Ridge gaseous diffusion plant. Radiat Res. 171(6):637-645.

http://dx.doi.org/10.1667/RR1607.1

Zahm SH. 1992. Computerized Occupational Referent Population System (CORPS): Study documentation. Rockville, MD: National Cancer Institute, National Institute for Occupational Safety and Health.

Zhang JQ, Wan YN, Peng WJ, Yan JW, Li BZ, Mei B, Chen B, Yao H, Yang GJ, Tao JH et al. 2013a. The risk of cancer development in systemic sclerosis: a meta-analysis. Cancer Epidemiol. 37(5):523-527. http://dx.doi.org/10.1016/j.canep.2013.04.014

Zhang L, Xu L, Zeng Q, Zhang SH, Xie H, Liu AL, Lu WQ. 2012. Comparison of DNA damage in human-derived hepatoma line (HepG2) exposed to the fifteen drinking water disinfection byproducts using the single cell gel electrophoresis assay. Mutat Res. 741(1-2):89-94. http://dx.doi.org/10.1016/j.mrgentox.2011.11.004

Zhang L, Bassig BA, Mora JL, Vermeulen R, Ge Y, Curry JD, Hu W, Shen M, Qiu C, Ji Z et al. 2013b. Alterations in serum immunoglobulin levels in workers occupationally exposed to trichloroethylene. Carcinogenesis. 34(4):799-802. http://dx.doi.org/10.1093/carcin/bgs403

Zhang SH, Chen Z, Liao J, Wei W, Liu AL, Lu WQ. 2010. [Application of two assays for damage assessment of damage caused by drinking water disinfection by-products in HepG2 cells]. Zhongguo Huanjing Kexue. 30(2):275-278.

Zhao Y, Krishnadasan A, Kennedy N, Morgenstern H, Ritz B. 2005. Estimated effects of solvents and mineral oils on cancer incidence and mortality in a cohort of aerospace workers. Am J Ind Med. 48(4):249-258. http://dx.doi.org/10.1002/ajim.20216 
Zordan M, Osti M, Pesce M, Costa R. 1994. Chloral hydrate is recombinogenic in the wing spot test in Drosophila melanogaster. Mutat Res. 322(2):111-116. http://dx.doi.org/10.1016/0165$\underline{1218(94) 00017-4}$ 


\section{Abbreviations}

8-OHdG 8-hydroxy-2'-deoxyguanosine

ACGIH American Conference of Governmental Industrial Hygienists

$\mathrm{ADH} \quad$ alcohol dehydrogenase

ADME absorption, distribution, metabolism, and excretion

AF fraction of inhaled substance absorbed

ALDH aldehyde dehydrogenase

ANCA antinuclear antibodies

ATSDR Agency for Toxic Substances and Disease Registry

BMI body mass index

BTC $S$-(2-benzothiazolyl)-L-cysteine

BW body weight

CA chromosomal aberration

CAREX CARcinogen EXposure (Canada)

CASRN Chemical Abstracts Service registry number

CAT catalase

CD cluster of differentiation

CDC Centers for Disease Control and Prevention

CDR Chemical Data Reporting Rule

CERHR Center for the Evaluation of Risks to Human Reproduction

$\mathrm{CH} \quad$ chloral hydrate

CI confidence interval

CIN chromosomal instability

CLL chronic lymphocytic lymphoma

$\mathrm{cm}^{2} \quad$ square centimeter

cum. cumulative

CGDP cysteinylglycine dipeptidases

$\mathrm{CO}_{2} \quad$ carbon dioxide

CTAC chlorothionoacetyl chloride

CTFC $S$-(2-chloro-1,1,2-trifluoroethyl)-L-cysteine

CTK chlorothioketene

CYP cytochrome P450 


\begin{tabular}{|c|c|}
\hline D & day or days \\
\hline DIBCL & diffuse large B-cell lymphoma \\
\hline DCA & dichloroacetic acid \\
\hline DCAA & dichloroacetyl chloride \\
\hline DCAC & dichloroacetyl anhydride \\
\hline DCVC & $S$-(1,2-dichlorovinyl)-L-cysteine \\
\hline DCVG & $S$-(1,2-dichlorovinyl)glutathione \\
\hline DCVT & $S$-dichlorovinyl-thiol \\
\hline DLBCL & diffuse large B-cell lymphoma \\
\hline DMSO & dimethyl sulfoxide \\
\hline DMV & Department of Motor Vehicles \\
\hline DNA & deoxyribonucleic acid \\
\hline $\mathrm{E} / \mathrm{R}$ & exposure response \\
\hline EAC & equivalent airborne concentrations \\
\hline EBV & Epstein-Barr virus \\
\hline eEF1A1 & eukaryotic translation elongation factor 1 alpha 1 \\
\hline eEF1A2 & eukaryotic translation elongation factor 1 alpha 2 \\
\hline EHR & enterohepatic recirculation \\
\hline EL & exposure length (min) \\
\hline ENGELA & Environment and Adult Lymphoma Genetics \\
\hline EPA & Environmental Protection Agency \\
\hline EPILYMPH & Environmental exposures and lymphoid neoplasms \\
\hline Exp. & exposed \\
\hline $\mathrm{F}$ & female \\
\hline FDA & Food and Drug Administration \\
\hline FL & follicular lymphoma \\
\hline FMO & flavin monooxygenase \\
\hline FR & Federal Register \\
\hline $\mathrm{ft}$ & foot/feet \\
\hline G & guanine \\
\hline GGT & $\gamma$-glutamyltransferase \\
\hline GI & gastrointestinal \\
\hline GPO & glutathione peroxidase \\
\hline
\end{tabular}




$\begin{array}{ll}\text { GSH } & \text { glutathione } \\ \text { GST } & \text { glutathione-S-transferase } \\ \text { HCL } & \text { hairy-cell leukemia } \\ \text { Hg } & \text { mercury } \\ \text { HGPRT } & \text { hypoxanthine-guanine phosphoribosyltransferase } \\ \text { HHS } & \text { Department of Health and Human Services } \\ \text { HIC } & \text { highest ineffective concentration } \\ \text { HID } & \text { highest ineffective dose } \\ \text { HIV } & \text { human immunodeficiency virus } \\ \text { HL } & \text { Hodgkin lymphoma } \\ \text { HP } & \text { Hadnot Point (Camp Lejeune) } \\ \text { hr } & \text { hour or hours } \\ \text { HR } & \text { hazard ratio } \\ \text { HRR } & \text { hazard rate ratio } \\ \text { HWE } & \text { healthy worker (hire or survival) effect } \\ \text { I } & \text { inconclusive } \\ \text { I2PP2A } & \text { protein phosphatase 2A inhibitor } \\ \text { i.p. } & \text { intraperitoneal } \\ \text { IARC } & \text { International Agency for Research on Cancer } \\ \text { ICAM } & \text { intercellular adhesion molecule } \\ \text { ICD } & \text { International Classification of Diseases } \\ \text { ICD-O } & \text { International Classification of Diseases for Oncology } \\ \text { ICDA } & \text { International Classification of Diseases-Adjusted } \\ \text { ID } & \text { identity } \\ \text { IDLH } & \text { immediately dangerous to life and health } \\ \text { IFN } & \text { interferon } \\ \text { IgE } & \text { immunoglobulin E } \\ \text { IgG } & \text { immunoglobulin G } \\ \text { IgM } & \text { immunoglobulin M } \\ \text { IL } & \text { interleukin } \\ \text { in } & \text { inch or inches } \\ \text { inh } & \text { inhalation } \\ \text { INS } & \text { insignificant change compared to controls. } \\ & \end{array}$




\begin{tabular}{|c|c|}
\hline IQR & interquartile ratio \\
\hline IRIS & Integrated Risk Information System \\
\hline IUR & Inventory Update Rule \\
\hline JEM & job-exposure matrix \\
\hline JP4 & jet propellant-4 \\
\hline JTEM & job-task exposure matrix \\
\hline $\mathrm{kg}$ & kilogram \\
\hline $\mathrm{Km}$ & Michaelis-Menten constant \\
\hline $\mathrm{K}_{\mathrm{ow}}$ & Octanol-water partition coefficient \\
\hline $\mathrm{L}$ & liter \\
\hline LARS & Leipzig Allergy Risk Children's Study \\
\hline LDH & lactate dehydrogenase \\
\hline LEC & lowest effective concentration \\
\hline LED & lowest effective dose \\
\hline LH & lymphohematopoietic \\
\hline LHC & lymphohematopoietic cancer \\
\hline $\log K_{o w}$ & logarithm of octanol/water partition coefficient \\
\hline LP & lipid peroxidation \\
\hline M & male \\
\hline $\mathrm{m}^{3}$ & cubic meter \\
\hline MCA & monochloroacetic acid \\
\hline MCL & maximum contaminant level \\
\hline MG & methylguanine \\
\hline $\mathrm{mg}$ & milligram \\
\hline MHC II & major histocompatibility complex II \\
\hline MIS & Multicentre Italian Study \\
\hline $\mathrm{mL}$ & milliliter \\
\hline $\mathrm{mm}$ & millimeter \\
\hline MM & multiple myeloma \\
\hline MMAI & maleylacetoacetate isomerase \\
\hline $\mathrm{MN}$ & micronuclei \\
\hline mol & mole \\
\hline $\mathrm{mRR}$ & meta-relative risk \\
\hline
\end{tabular}




$\begin{array}{ll}\text { MV } & \text { minute volume } \\ \text { N } & \text { number } \\ \text { NA } & \text { not available; not applicable } \\ \text { NAcDCVC } & N \text {-acetyl-S-dichlorovinyl-L-cysteine } \\ \text { NAcDCVCS } & N \text {-acetyl DCVCS } \\ \text { NAS } & \text { National Academy of Science } \\ \text { NAT } & \text { N-acetyltransferase } \\ \text { NCI } & \text { National Cancer Institute } \\ \text { NCTR } & \text { National Center for Toxicological Research } \\ \text { ND } & \text { not detected; not determined; not done } \\ \text { NDI } & \text { National Death Index } \\ \text { ng } & \text { nanogram } \\ \text { NHANES } & \text { National Health and Nutrition Examination Survey } \\ \text { NHL } & \text { non-Hodgkin lymphoma } \\ \text { NIEHS } & \text { National Institute of Environmental Health Sciences } \\ \text { NIH } & \text { National Institutes of Health } \\ \text { NIOSH } & \text { National Institute for Occupational Safety and Health } \\ \text { NK } & \text { natural killer cells } \\ \text { NLM } & \text { National Library of Medicine } \\ \text { nmol } & \text { nanomole } \\ \text { NOCCA } & \text { Nordic Occupational Cancer } \\ \text { NOES } & \text { National Occupational Exposure Survey } \\ \text { NOS } & \text { not otherwise specified } \\ \text { NR } & \text { not reported; none reported } \\ \text { ns } & \text { not specified } \\ \text { NS } & \text { not significant } \\ \text { nt } & \text { nucleotides } \\ \text { NT } & \text { not tested } \\ \text { NTP } & \text { National Toxicology Program } \\ \text { OA } & \text { oxalic acid } \\ \text { OD } & \text { oral dose (mg/kg) } \\ \text { OR } & \text { odds ratio } \\ \text { OSHA } & \text { Occupational Safety and Health Administration } \\ & \end{array}$




$\begin{array}{ll}\text { p.o. } & \text { per os (oral administration) } \\ \text { PAH } & \text { polycyclic aromatic hydrocarbon } \\ \text { PBPK } & \text { physiologically based pharmacokinetic model } \\ \text { PCBs } & \text { polychlorinated biphenyls } \\ \text { PCE } & \text { polychromatic erythrocyte } \\ \text { PCNA } & \text { proliferating cell nuclear antigen } \\ \text { PEL } & \text { permissible exposure limit } \\ \text { Perc } & \text { perchloroethylene (tetrachloroethylene) } \\ \text { PGDP } & \text { Paduca Gaseous Diffusion Plant } \\ \text { PLC } & \text { peritoneal lavage cell } \\ \text { PPAR } & \text { peroxisome proliferation activated receptor } \alpha \\ \text { PPE } & \text { Personal Protective Equipment } \\ \text { ppm } & \text { parts per million } \\ \text { ppt } & \text { parts per trillion } \\ \text { ptrend } & \text { p value for trend } \\ \text { R } & \text { estimated daily production of adducts } \\ \text { r } & \text { correlation coefficient } \\ \text { RBC } & \text { red blood cell } \\ \text { RCC } & \text { renal-cell cancer } \\ \text { RDD } & \text { random-digit dialing } \\ \text { REAL } & \text { Revised European-American Lymphoma classification } \\ \text { REL } & \text { recommended exposure limit } \\ \text { RfC } & \text { reference dose } \\ \text { RLV } & \text { Rauscher-leukemia virus } \\ \text { R-N } & \text { Raaschou-Nielsen } \\ \text { RNA } & \text { ribonucleic acid } \\ \text { RoC } & \text { Report on Carcinogens } \\ \text { ROS } & \text { reactive oxygen species } \\ \text { RQ } & \text { reportable quantity } \\ \text { RR } & \text { relative risk } \\ \text { RRD } & \text { random digit dialing } \\ \text { SCE } & \text { sister-chromatid exchange } \\ \text { SD } & \text { standard deviation } \\ & \\ \text { RE } & \\ \text { RE } & \end{array}$


SEER Surveillance, Epidemiology and End Results Program (US National Cancer Institute)

SES Socioeconomic status

SIR standardized incidence ratio

SLL small cell lymphocytic lymphoma

SMR standardized mortality ratio

SOD superoxide dismutase

SRR standardized rate ratio, standardized relative risk

SSA Social Security Administration

SSB single strand break

SSc systemic sclerosis

SSFL Santa Susanna Field Laboratory

SSN Social Security number

TBARS thiobarbituric acid-reactive substances

TCA trichloroacetic acid

TCAH trichloroacetyl hydrate

TCDD 2,3,7,8-tetrachlorodibenzo- $p$-dioxin

TCE trichloroethylene

TCE-O Trichloroethylene oxide

TCOG trichloroethanol-glucuronide conjugate

$\mathrm{TCOH}$ trichloroethanol

TCVC $\quad S$-(1,2,2-trichlorovinyl)-L-cysteine

TLV-TWA threshold limit value time-weighted average

TLV-STEL Threshold limit value - short-term exposure limit

$t_{\max } \quad$ time to maximum concentration in plasma

TNF tumor necrosis factor

TRI Toxics Release Inventory

TSCA Toxic Substances Control Act

TT Tarawa Terrace (Camp Lejeune)

TWA time-weighted average

UDP uridine diphosphate

UDS unscheduled DNA synthesis

UGT UDP-glucuronosyltransferase 


$\begin{array}{ll}\text { UK } & \text { United Kingdom } \\ \text { U-TCA } & \text { urine trichloroacetic acid } \\ \text { VHL } & \text { von Hippel-Lindau } \\ \mathrm{V}_{\max } & \text { maximum reaction velocity } \\ \text { VOC } & \text { volatile organic compound } \\ \mathrm{W} & \text { women } \\ \mathrm{WBC} & \text { white blood cell } \\ \mathrm{WHO} & \text { World Health Organization } \\ \mathrm{wk} & \text { week or weeks } \\ \mathrm{wt} \% & \text { weight percent } \\ \mathrm{yr} & \text { year or years } \\ \mu \mathrm{g} & \text { microgram } \\ \mu \mathrm{g} / \mathrm{L} & \text { micrograms/liter } \\ \mu \mathrm{g} / \mathrm{L}-\mathrm{mo} & \text { micrograms/liter-months } \\ \mu \mathrm{M} & \text { micromolar }\end{array}$




\section{Glossary}

Alpha2u-globulin: $\alpha_{2 u}$-Globulin is a low molecular weight protein that is synthesized in the liver of male rats and is regulated by complex hormonal interactions. Androgens stimulate synthesis, whereas estrogens repress synthesis.

Ames assay: The Ames Salmonella/microsome mutagenicity assay is a short-term bacterial reverse mutation assay specifically designed to detect a wide range of chemical substances that can produce genetic damage that leads to gene mutations.

Aneuploidy: An abnormality involving a chromosome number that is not an exact multiple of the haploid number (one chromosome set is incomplete).

Apoptosis: Cell deletion by fragmentation into membrane-bound particles, which are phagocytosed by other cells.

Arabinose resistance: The L-arabinose resistance test with Salmonella typhimurium (Ara test) is a forward mutation assay that selects a single phenotypic change (from L-arabinose sensitivity to L-arabinose resistance) in a unique tester strain (an araD mutant).

Aroclor 1254-induced liver: Liver tissue treated with the polychlorinated biphenyl mixture Aroclor 1254 used as a source of S9 fraction for mutagenic and genotoxic effects testing.

Ascertainment bias: Systematic failure to represent equally all classes of cases or persons supposed to be represented in a sample.

Attrition bias: Systematic differences between comparison groups in withdrawals or exclusions of participants from the results of a study.

Biexponential process: A process of drug (or xenobiotic) clearance with two phases with different rates. The first phase often involves rapid distribution of a drug to peripheral tissues, while the second phase represents clearance mechanisms that eliminate the drug from the body. (See "Two-compartment pharmacokinetic model.")

Biodegradation: Biotransformation; the conversion within an organism of molecules from one form to another. A change often associated with change in pharmacologic activity.

Boiling point: The boiling point of the anhydrous substance at atmospheric pressure $(101.3 \mathrm{kPa})$ unless a different pressure is stated. If the substance decomposes below or at the boiling point, this is noted (dec). The temperature is rounded off to the nearest ${ }^{\circ} \mathrm{C}$.

CD8+ T-cell blast: An immature, undifferentiated lymphocyte that expresses the CD8 transmembrane glycoprotein.

Chemical Data Reporting Rule: Chemical Data Reporting (CDR) is the new name for Inventory Update Reporting (IUR). The purpose of Chemical Data Reporting is to collect quality screening-level, exposure-related information on chemical substances and to make that information available for use by the U.S. Environmental Protection Agency (EPA) and, to the extent possible, to the public. The IUR/CDR data are used to support risk screening, assessment, priority setting and management activities and constitute the most comprehensive source of basic 
screening-level, exposure-related information on chemicals available to EPA. The required frequency of reporting currently is once every 4 years.

Cochran-Armitage trend test: A statistical test used in categorical data analysis when the aim is to assess for the presence of an association between a variable with two categories and a variable with $k$ categories. It modifies the chi-square test to incorporate a suspected ordering in the effects of the $k$ categories of the second variable.

Comet assay: Single cell gel electrophoresis for assessment of DNA damage in presumptive target tissues.

Connexin proteins: A group of transmembrane proteins that form the intermembrane channels of gap junctions. They are used by inorganic ions and most small organic molecules to pass through cell interiors.

Conversion factor: A numerical factor used to multiply or divide a quantity when converting from one system of units to another.

Critical temperature: The temperature at and above which a gas cannot be liquefied, no matter how much pressure is applied.

Dehydrodehalogenation: An elimination reaction in which a halogen is removed from one carbon and a hydrogen is removed from an adjacent carbon.

Differential selection: Selective pressure for self-renewal. Gene mutations that confer a growth or survival advantage on the cells that express them will be selectively enriched in the genome of tumors.

Disposition: The description of absorption, distribution, metabolism, and excretion of a chemical in the body.

Dominant lethal mutation assay: The dominant lethal assay identifies germ cell mutagens by measuring the ability of a chemical to penetrate gonadal tissue and produce embryonic death due to chromosomal breakage in parent germ cells.

Double acid conjugate: A compound formed by the joining of two acids.

Ecological study: A study in which the units of analysis are populations or groups of people rather than individuals.

ELISA assay: Enzyme-linked immunosorbent assay; a sensitive immunoassay that uses an enzyme linked to an antibody or antigen as a marker for the detection of a specific protein, especially an antigen or antibody.

Epigenetic mechanisms: Changes in gene function that do not involve a change in DNA sequence but are nevertheless mitotically and/or meiotically heritable. Examples include DNA methylation, alternative splicing of gene transcripts, and assembly of immunoglobulin genes in cells of the immune system.

Fo generation: The initial parent generation in a multi-generation reproduction study. 
$F_{1}$ and $F_{2}$ offspring: $F_{1}$ offspring is the first filial generation, which comprises offspring resulting from a cross between strains of distinct genotypes. The $F_{1}$ generation is the generation resulting immediately from a cross of the first set of parents (parental generation, i.e., $\mathrm{F}_{0}$ generation). $\mathrm{F}_{2}$ offspring is the second filial generation, which comprises offspring resulting from a cross of the members of $F_{1}$ generation. The $F_{2}$ generation is the result of a cross between two $F_{1}$ individuals (from $F_{1}$ generation).

FDA Good Laboratory Practice Regulations: A quality system codified by the U.S. Food and Drug Administration that prescribes operating procedures for conducting nonclinical laboratory studies that support or are intended to support applications for research or marketing permits for products regulated by the Food and Drug Administration.

Fisher's exact test: The test for association in a two-by-two table that is based on the exact hypergeometric distribution of the frequencies within the table.

Follow-up: Observation over a period of time of a person, group, or initially defined population whose appropriate characteristics have been assessed to observe changes in health status or health-related variables.

Freund's adjuvant: A water-in-oil emulsion injected with immunogen (Freund's incomplete adjuvant) or with immunogen and killed mycobacteria (Freund's complete adjuvant) to enhance the immune response to the immunogen.

Genomic instability: An increased propensity for genomic alterations that often occurs in cancer cells. During the process of cell division (mitosis) the inaccurate duplication of the genome in parent cells or the improper distribution of genomic material between daughter cells can result from genomic instability.

Glioma: A cancer of the brain that begins in glial cells (cells that surround and support nerve cells).

Hairy-cell leukemia: A rare type of leukemia in which abnormal B-lymphocytes (a type of white blood cell) are present in the bone marrow, spleen, and peripheral blood. When viewed under a microscope, these cells appear to be covered with tiny hair-like projections.

Healthy worker hire effect: Initial selection of healthy individuals at time of hire so that their disease risks differ from the disease risks in the source (general) population.

Healthy worker survival effect: A continuing selection process such that those who remain employed tend to be healthier than those who leave employment.

Hemangiosarcoma: A type of cancer that begins in the cells that line blood vessels.

Henry's Law constant: The ratio of the aqueous-phase concentration of a chemical to its equilibrium partial pressure in the gas phase. The larger the Henry's law constant the less soluble it is (i.e., greater tendency for vapor phase). The relationship is defined for a constant temperature, e.g., $25^{\circ} \mathrm{C}$.

Hepatoma: A liver tumor. 
Host-mediated assay: This assay evaluates the genotoxicity of a substance to microbial cells introduced (e.g., by intravenous injection) into a host animal. The host animal receives the test compound orally, and therefore acts as a source of chemical metabolism, distribution, and excretion of the test compound.

Immersion cleaning: A process in which a tank containing cleaning solvent at a temperature below its boiling point is used for metal parts cleaning. To use the vapor degreaser, the operator places the parts to be cleaned in a metal wire basket, removes the cover, and lowers the basket of parts by hand into the cleaning solvent. After a brief period of time, the operator raises the basket and allows the parts to drip-dry inside the degreaser.

Keratosis: A localized horny overgrowth of the skin, such as a wart or callus.

Loss of heterozygosity: If there is one normal and one abnormal allele at a particular locus, as might be seen in an inherited autosomal dominant cancer susceptibility disorder, loss of the normal allele produces a locus with no normal function. When the loss of heterozygosity involves the normal allele, it creates a cell that is more likely to show malignant growth if the altered gene is a tumor suppressor gene.

Lymphokine-activated killer cell: Killer cell lymphocytes activated in the presence of interleukin-2 (IL-2). Lymphokine-activated killer cells (LAKs) are cytotoxic effector cells with an exceptionally wide target cell spectrum including normal and malignant cells of different origins. LAKs exhibit a profound heterogeneity with regard to phenotype surface marker expression; it remains to be determined if they represent a unique cell lineage.

Melting point: The melting point of the substance at atmospheric pressure $(101.3 \mathrm{kPa})$. When there is a significant difference between the melting point and the freezing point, a range is given. In case of hydrated substances (i.e., those with crystal water), the apparent melting point is given. If the substance decomposes at or below its melting point, this is noted (dec). The temperature is rounded off to the nearest ${ }^{\circ} \mathrm{C}$.

Metabolic activation: The chemical alteration of an exogenous substance by or in a biological system. The alteration may inactivate the compound, or it may result in the production of an active metabolite of an inactive parent compound.

Metaplasia: A change of cells to a form that does not normally occur in the tissue in which it is found.

Methemoglobin: A form of hemoglobin found in the blood in small amounts. Unlike normal hemoglobin, methemoglobin cannot carry oxygen. Injury or certain drugs, chemicals, or foods may cause a higher-than-normal amount of methemoglobin to be made. This causes a condition called methemoglobinemia.

Micronuclei: Small nuclei separate from, and additional to, the main nucleus of a cell, produced during the telophase of mitosis or meiosis by lagging chromosomes or chromosome fragments derived from spontaneous or experimentally induced chromosomal structural changes.

Miscible: A physical characteristic of a liquid that forms one liquid phase with another liquid (e.g., water) when they are mixed in any proportion. 
Molecular chaperone: Any of a diverse group of proteins that oversee the correct intracellular folding and assembly of polypeptides without being components of the final structure.

Molecular weight: The molecular weight of a substance is the weight in atomic mass units of all the atoms in a given formula. The value is rounded to the nearest tenth.

Multiple myeloma: A type of cancer that begins in plasma cells (white blood cells that produce antibodies). Also called Kahler disease, myelomatosis, and plasma cell myeloma.

Mutations: A change in the structure of a gene, resulting from the alteration of single base units in DNA, or the deletion, insertion, or rearrangement of larger sections of genes or chromosomes. The genetic variant can be transmitted to subsequent generations.

National Health and Nutrition Examination Survey: A program of studies designed to assess the health and nutritional status of adults and children in the United States. The survey is unique in that it combines interviews and physical examinations.

Natural killer cells: A type of white blood cell that contains granules with enzymes that can kill tumor cells or microbial cells. Also called large granular lymphocytes.

Non-differential misclassification: The probability of erroneous classification of an individual, a value, or an attribute into a category other than that to which it should be assigned is the same in all study groups.

Non-Hodgkin lymphoma: A heterogeneous group of malignant lymphomas; the only common feature being an absence of the giant Reed-Sternberg cells characteristic of Hodgkin disease.

Normochromatic erythrocyte: A mature erythrocyte that lacks ribosomes and can be distinguished from immature, polychromatic erythrocytes by stains selective for RNA.

Octanol/water partition coefficient $(\log K o w)$ : A measure of the equilibrium concentration of a compound between octanol and water.

One-compartment model: A pharmacokinetic modeling approach that models the entire body as a single compartment into which a drug is added by a rapid single dose, or bolus. It is assumed that the drug concentration is uniform in the body compartment at all times and is eliminated by a first order process that is described by a first order rate constant.

Ozone-depleting substance: A family of man-made compounds that includes, but are not limited to, chlorofluorocarbons (CFCs), bromofluorocarbons (halons), methyl chloroform, carbon tetrachloride, methyl bromide, and hydrochlorofluorocarbons (HCFCs). These compounds have been shown to deplete stratospheric ozone.

Papilloma: A small solid benign tumor with a clear-cut border that projects above the surrounding tissue.

Personal breathing zone: A sampling area as close as practical to an employee's nose and mouth, (i.e., in a hemisphere forward of the shoulders within a radius of approximately nine inches) so that it does not interfere with work performance or safety of the employee. 
Personal protective equipment: Specialized clothing or equipment, worn by an employee to minimize exposure to a variety of hazards. Examples of PPE include such items as gloves, foot and eye protection, protective hearing devices (earplugs, muffs), hard hats, respirators, and full body suits.

Phase I metabolism: Metabolism of drugs or other xenobiotic molecules, usually by oxidation or hydrolysis and involving a cytochrome P450 monooxygenase.

Phase II metabolism: A conjugation reaction that forms a covalent linkage between a functional group on a xenobiotic molecule and glucuronic acid, sulfate, glutathione, amino acid, or acetate.

Plaque assay: An assay for antibody production by single lymphocytes using cells isolated from the spleen or lymph nodes of animals injected with sheep red blood cells as an antigen. Incubation of the antibody-forming cells together with sheep red cells in an agar layer with exposure to guinea pig serum as complement results in formation of microscopic plaques (i.e., circular areas of hemolytic clearance around a lymphoid cell) due to release of hemolysin.

Plate incorporation: A commonly used procedure for performing a bacterial reverse mutation test. Suspensions of bacterial cells are exposed to the test substance in the presence and in the absence of an exogenous metabolic activation system. In the plate-incorporation method, these suspensions are mixed with an overlay agar and plated immediately onto minimal medium. After 2 or 3 days of incubation, revertant colonies are counted and compared with the number of spontaneous revertant colonies on solvent control plates.

Point emission: A release that can be identified with a single discharge source or attributed to a specific physical location.

Polychromatic erythrocyte: A newly formed erythrocyte (reticulocyte) containing RNA.

Prophage lambda ( $\lambda$ ): A virus in Escherichia coli (E. coli) bacteria that has integrated itself into the host $E$. coli DNA.

Proto-oncogene: A gene involved in normal cell growth. Mutations (changes) in a protooncogene may cause it to become an oncogene, which can cause the growth of cancer cells.

ptrend: Level of statistical significance of a change over time in a group selected to represent a larger population.

Pyknotic shrinkage: A thickening, especially the degeneration of a cell in which the nucleus shrinks in size and the chromatin condenses to a solid, structureless mass or masses.

Pyrolysis: The chemical and physical decomposition of organic material that occurs at high temperatures in the absence of oxygen.

QUOSA: A collection of scientific literature management software and services for researchers and information professionals in the life sciences and related scientific and medical areas designed to retrieve, organize, and analyze full-text articles and documents.

Selection bias: An error in choosing the individuals or groups to take part in a study. Ideally, the subjects in a study should be very similar to one another and to the larger population from which 
they are drawn (for example, all individuals with the same disease or condition). If there are important differences, the results of the study may not be valid.

Sister-chromatid exchange: The exchange during mitosis of homologous genetic material between sister chromatids; increased as a result of inordinate chromosomal fragility due to genetic or environmental factors.

SKF-525A: An inhibitor of drug metabolism and cytochrome P-450 activity.

Soft tissue sarcoma: A cancer that begins in the muscle, fat, fibrous tissue, blood vessels, or other supporting tissue of the body.

Solubility: The ability of a substance to dissolve in another substance and form a solution. The Report on Carcinogens uses the following definitions (and concentration ranges) for degrees of solubility: (1) miscible (see definition), (2) freely soluble-capable of being dissolved in a specified solvent to a high degree $(>1,000 \mathrm{~g} / \mathrm{L})$, (3) soluble- capable of being dissolved in a specified solvent (10-1,000 g/L), (4) slightly soluble-capable of being dissolved in a specified solvent to a limited degree (1-10 g/L), and (5) practically insoluble- incapable of dissolving to any significant extent in a specified solvent $(<1 \mathrm{~g} / \mathrm{L})$.

Specific gravity: The ratio of the density of a material to the density of a standard material, such as water at a specific temperature; when two temperatures are specified, the first is the temperature of the material and the second is the temperature of water.

Spot test: Qualitative assay in which a small amount of test chemical is added directly to a selective agar medium plate seeded with the test organism, e.g., Salmonella. As the chemical diffuses into the agar, a concentration gradient is formed. A mutagenic chemical will give rise to a ring of revertant colonies surrounding the area where the chemical was applied; if the chemical is toxic, a zone of growth inhibition will also be observed.

Steric bulk: An indicator of the stability of the spatial arrangement of atoms in a molecule.

T-helper cell: A type of immune cell that stimulates killer T cells, macrophages, and B cells to make immune responses. A helper T cell is a type of white blood cell and a type of lymphocyte. Also called CD4-positive T lymphocyte.

Tg.AC: A transgenic mouse model with the ability to mount a tumorigenic response within 6 months in skin paint assays when dosed topically with nonmutagenic carcinogens.

Time-weighted average: The average exposure concentration of a chemical measured over a period of time (not an instantaneous concentration).

Toxicokinetics: The mathematical description (toxicokinetic models) of the time course of disposition of a chemical in the body.

Transitions: DNA nucleotide substitution mutation in which a purine base is substituted for another purine base (adenine $\rightarrow$ guanine or guanine $\rightarrow$ adenine) or a pyrimidine base for another pyrimidine base (cytosine $\rightarrow$ thymine or thymine $\rightarrow$ cytosine).

Transversions: DNA nucleotide substitution mutation in which a purine base (adenine or guanine) is substituted for a pyrimidine base (cytosine or thymine) or vice versa. 
Two-compartment pharmacokinetic model: A two-compartment pharmacokinetic model resolves the body into a central compartment and a peripheral compartment. The central compartment generally comprises tissues that are highly perfused, such as heart, lungs, kidneys, liver, and brain. The peripheral compartment comprises less well-perfused tissues, such as muscle, fat, and skin. A two-compartment model assumes that, following drug administration into the central compartment, the drug distributes between that compartment and the peripheral compartment. However, the drug does not achieve instantaneous distribution (i.e., equilibrium), between the two compartments. After a time interval ( $t$ ), distribution equilibrium is achieved between the central and peripheral compartments, and elimination of the drug is assumed to occur from the central compartment.

Type-I error: The error of rejecting a true null hypothesis, i.e., declaring that a difference exists when it does not.

Type-II error: The error of failing to reject a false null hypothesis, i.e., declaring that a difference does not exist when in fact it does.

Vapor degreasing: A type of cleaning procedure using a refrigerated cooling coil around the top of the interior of a vapor chamber to condense solvent vapor into liquid droplets on the surface of parts to remove surface impurities. Excess solvent drips back into the solvent sump and is recycled as the parts ascend from the vapor to condensing zones.

Vapor density, relative: A value that indicates how many times a gas (or vapor) is heavier than air at the same temperature. If the substance is a liquid or solid, the value applies only to the vapor formed from the boiling liquid.

Vapor pressure: The pressure of the vapor over a liquid (and some solids) at equilibrium, usually expressed as $\mathrm{mm} \mathrm{Hg}$ at a specific temperature $\left({ }^{\circ} \mathrm{C}\right)$. 


\section{Appendix A. Literature Search Strategy}

\section{Figures}

Figure A-1. Literature Search Strategy and Review

A-2 
The data sources, search terms, and search strategies that were used to identify literature for the draft monograph on trichloroethylene are described in the "Trichloroethylene Protocol" (http://ntp.niehs.nih.gov/ntp/roc/thirteenth/protocols/tce protocol12-31-13_508.pdf).

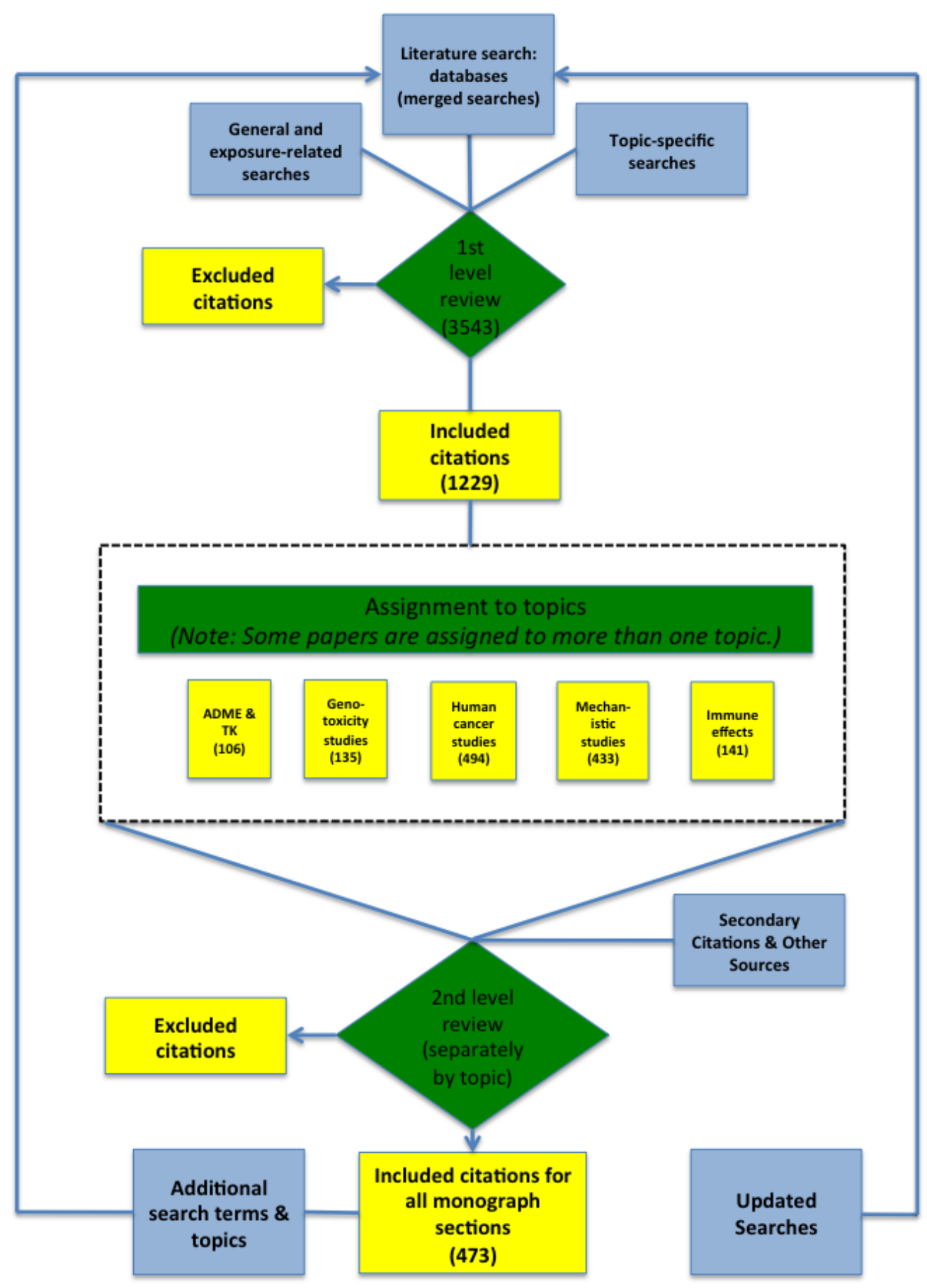

Figure A-1. Literature Search Strategy and Review 


\section{Appendix B. ADME Tables}

\section{Tables}

Table B-1. In Vitro Kinetics of Oxidative Metabolism of Trichloroethylene

Table B-2. In Vitro Kinetics of Chloral Hydrate and Dichloroacetic Acid

Biotransformation

Table B-3. Rates of DCVG Formation from Trichloroethylene Conjugation ${ }^{\mathrm{a}}$.

Table B-4. Kinetics of In Vitro Glutathione Conjugation of Trichloroethylene

Table B-5. $\beta$-Lyase Activity from Human, Rat, and Mouse Kidney Cytosol ....

Table B-6. Comparison of Hepatic In Vitro Oxidation and Glutathione Conjugation of

Trichloroethylene in Human Hepatocytes and Liver Subcellular Fractions 
RoC Monograph on Trichloroethylene

Table B-1. In Vitro Kinetics of Oxidative Metabolism of Trichloroethylene

\begin{tabular}{|c|c|c|c|c|c|}
\hline System & $\mathbf{N}$ & $\mathbf{K}_{\mathbf{m}}(\boldsymbol{\mu} \mathbf{M})$ & $\begin{array}{c}V_{\max }(n m o l \\
\text { TCE/min/mg Protein) }\end{array}$ & $1,000 \times V_{\max } / K_{m}$ & Reference \\
\hline \multicolumn{6}{|l|}{ Human } \\
\hline Hepatocytes & 6 & $210 \pm 159^{a}$ & $1.5 \pm 1.2^{b}$ & {$[13.7 \pm 12.8]$} & $\begin{array}{l}\text { Lipscomb et al. } \\
\text { (1998a) }\end{array}$ \\
\hline Liver microsomes & $\begin{array}{c}10 \\
9 \\
4 \\
23\end{array}$ & $\begin{array}{c}16.7 \pm 2.45^{\mathrm{c}} \\
30.9 \pm 3.3^{\mathrm{d}} \\
51.1 \pm 3.77^{\mathrm{e}} \\
28.3 \pm 12.9^{\mathrm{f}}\end{array}$ & $\begin{array}{l}1.25 \pm 0.81^{\mathrm{c}} \\
1.44 \pm 0.46^{\mathrm{d}} \\
2.77 \pm 0.58^{\mathrm{e}} \\
1.59 \pm 0.84^{\mathrm{f}}\end{array}$ & $\begin{array}{l}{[74.1 \pm 44.1]} \\
{[47.0 \pm 16.0]} \\
{[54.9 \pm 14.1]} \\
{[60.2 \pm 32.9]}\end{array}$ & Lipscomb et al. (1997) \\
\hline Liver microsomes & 7 & 24.6 & 1.44 & 58.5 & $\begin{array}{l}\text { Lipscomb et al. } \\
\text { (1998b) }\end{array}$ \\
\hline $\begin{array}{l}\text { Liver microsomes (high } \\
\text { affinity) }\end{array}$ & $\begin{array}{l}3 \\
3\end{array}$ & $\begin{array}{c}12 \pm 3 \\
26 \pm 17\end{array}$ & $\begin{array}{c}0.52 \pm 0.17 \text { (males) } \\
0.33 \pm 0.15 \text { (females) }\end{array}$ & $\begin{array}{l}48.0 \pm 23.1 \\
15.3 \pm 10.1\end{array}$ & Elfarra et al. (1998) \\
\hline $\begin{array}{l}\text { Liver microsomes (low } \\
\text { affinity) }\end{array}$ & $\begin{array}{l}3 \\
3\end{array}$ & $\begin{aligned} 93 & \pm 26 \\
160 & \pm 162\end{aligned}$ & $\begin{array}{c}0.93 \pm 0.17 \text { (males) } \\
0.72 \pm 0.60 \text { (females) }\end{array}$ & $\begin{array}{c}10.7 \pm 3.9 \\
6.8 \pm 5.6\end{array}$ & Elfarra et al. (1998) \\
\hline \multicolumn{6}{|l|}{ Rat } \\
\hline Liver microsomes & 5 & $55.5^{\mathrm{g}}$ & 4.83 & 87.0 & $\begin{array}{l}\text { Lipscomb et al. } \\
\text { (1998b) }\end{array}$ \\
\hline $\begin{array}{l}\text { Liver microsomes (high } \\
\text { affinity) }\end{array}$ & $\begin{array}{l}5 \\
3\end{array}$ & $\begin{array}{l}72 \pm 82 \\
42 \pm 21\end{array}$ & $\begin{array}{c}0.96 \pm 0.65 \text { (males) } \\
2.91 \pm 0.71 \text { (females) }\end{array}$ & $\begin{array}{l}23.8 \pm 20.6 \\
80.0 \pm 33.9\end{array}$ & Elfarra et al. (1998) \\
\hline $\begin{array}{l}\text { Liver microsomes (low } \\
\text { affinity) }\end{array}$ & $\begin{array}{l}5 \\
3\end{array}$ & $\begin{array}{c}482 \pm 104 \\
111 \pm 27\end{array}$ & $\begin{array}{c}2.48 \pm 0.97 \text { (males) } \\
4.31 \pm 0.31 \text { (females) }\end{array}$ & $\begin{array}{c}5.3 \pm 2.2 \\
40.1 \pm 7.1\end{array}$ & Elfarra et al. (1998) \\
\hline Kidney microsomes & 3 & $940^{\mathrm{h}}$ & 0.154 & {$[0.164]$} & $\begin{array}{l}\text { Cummings et al. } \\
(2001)\end{array}$ \\
\hline \multicolumn{6}{|l|}{ Mouse } \\
\hline Liver microsomes & 5 & $35.4^{\mathrm{g}}$ & 5.43 & 153.4 & $\begin{array}{l}\text { Lipscomb et al. } \\
\text { (1998b) }\end{array}$ \\
\hline Liver microsomes & $\begin{array}{l}5 \\
3\end{array}$ & $\begin{array}{c}378 \pm 414 \\
161 \pm 29\end{array}$ & $\begin{array}{c}8.6 \pm 4.5 \text { (males) } \\
26.1 \pm 7.29 \text { (females) }\end{array}$ & $\begin{array}{c}42.0 \pm 28.5 \\
162.8 \pm 36.7\end{array}$ & Elfarra et al. (1998) \\
\hline $\begin{array}{l}\text { Values in brackets were calc } \\
{ }^{a} \text { Converted from ppm trichlc } \\
{ }^{\mathrm{b}} \text { Converted from nmol/h/10 } \\
{ }^{\mathrm{c}} \text { Low } \mathrm{K}_{\mathrm{m}}(12-20) \text { group. } \\
{ }^{\mathrm{d}} \mathrm{Mid} \mathrm{K}_{\mathrm{m}}(26-37) \text { group. } \\
{ }^{\mathrm{e}} \mathrm{High} \mathrm{K}_{\mathrm{m}}(>46) \text { group. } \\
{ }^{\mathrm{f}} \text { Combined across all } \mathrm{K}_{\mathrm{m}} \text { gro } \\
{ }^{\mathrm{g}} \mathrm{K}_{\mathrm{m}} \text { value for } 0-5,000 \mu \mathrm{M}\end{array}$ & $\begin{array}{l}\text { lated } \\
\text { oethy }\end{array}$ & $\begin{array}{l}\text { y NTP. } \\
\text { ne in headspace. } \\
\text { ytes. } \\
\text { entration. }\end{array}$ & 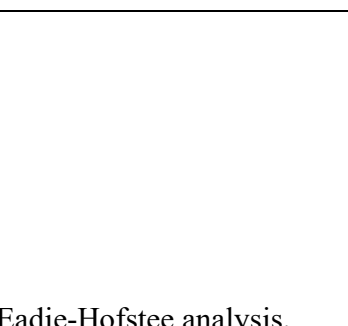 & & \\
\hline
\end{tabular}


Table B-2. In Vitro Kinetics of Chloral Hydrate and Dichloroacetic Acid Biotransformation

\begin{tabular}{|c|c|c|c|c|}
\hline Metabolic Step & System & $\mathbf{K}_{\mathrm{m}}(\boldsymbol{\mu M})$ & $\begin{array}{c}\mathrm{V}_{\max }(\mathrm{nmol} / \mathrm{min} / \mathrm{mg} \\
\text { Protein) }\end{array}$ & $1,000 \times V_{\max } / K_{m}$ \\
\hline \multirow[t]{5}{*}{$\mathrm{CH}$ to $\mathrm{TCOH}$} & Human & 1,340 & 34.7 & 25.9 \\
\hline & Rat & 520 & 24.3 & 46.7 \\
\hline & Mouse & 190 & 11.3 & 59.5 \\
\hline & high affinity & 120 & 6.3 & 52.5 \\
\hline & low affinity & 510 & 6.1 & 12.0 \\
\hline \multirow[t]{3}{*}{$\mathrm{CH}$ to TCA } & Human & 23,900 & 65.2 & 2.7 \\
\hline & Rat & 16,400 & 4.0 & 0.24 \\
\hline & Mouse & 3,500 & 10.6 & 3.0 \\
\hline \multirow[t]{3}{*}{ DCA to glyoxylate } & Human & 71 & 0.37 & 5.2 \\
\hline & Rat & 280 & 11.6 & 41.4 \\
\hline & Mouse & 350 & 13.1 & 37.4 \\
\hline
\end{tabular}

Sources: Adapted from EPA (2011a), Lash et al. (2000a).

$\mathrm{CH}=$ chloral hydrate; $\mathrm{DCA}=$ dichloroacetic acid; $\mathrm{TCA}=$ trichloroacetic acid; $\mathrm{TCOH}=$ trichloroethanol.

Table B-3. Rates of DCVG Formation from Trichloroethylene Conjugation ${ }^{\text {a }}$

\begin{tabular}{lccl}
\hline \multicolumn{1}{c}{ System } & Male & Female & Reference \\
\hline Human & & & \\
Hepatocytes (0.9 mM, pooled) & $11 \pm 3^{\mathrm{b}}$ & & Lash et al. (1999a) \\
Liver cytosol (1 mM, individual samples) & $156 \pm 16$ & $174 \pm 13$ & Lash et al. (1999a) \\
Liver cytosol (1 mM, pooled) & $420^{\mathrm{b}}$ & & Lash et al. (1999a) \\
Liver cytosol (2 mM, pooled) & $346^{\mathrm{b}}$ & & Lash et al. (1999a) \\
Liver cytosol (1.9 mM) & $0.011^{\mathrm{c}}$ & & Green et al. (1997) \\
Liver microsomes (1 mM, individual samples) & $108 \pm 24$ & $83 \pm 11$ & Lash et al. (1999a) \\
Liver microsomes (1 mM, pooled) & $146^{\mathrm{b}}$ & & Lash et al. (1999a) \\
Kidney cytosol (2 mM, pooled) & $42^{\mathrm{b}}$ & & Lash et al. (1999a) \\
Kidney microsomes (1 mM, pooled) & $320^{\mathrm{b}}$ & & Lash et al. (1999a) \\
Rat & & & \\
Hepatocytes (2 mM) & $9.7 \pm 0.29^{*}$ & $2.67 \pm 0.69$ & Lash et al. (1998) \\
Liver cytosol (2 mM) & $7.3 \pm 2.8$ & $4.86 \pm 0.14$ & Lash et al. (1998) \\
Liver cytosol (1.9 mM) & $0.097^{\mathrm{c}}$ & & Green (1997) \\
Liver cytosol (4 mM) & $\mathrm{nd}$ & & Dekant et al. (1990) \\
Liver microsomes (2 mM) & $10.3 \pm 2.8$ & $7.24 \pm 0.24$ & Lash et al. (1998) \\
Liver microsomes (4 mM) & 0.12 & & Dekant et al. (1990) \\
Kidney cortical cells (2 mM) & & & Lash et al. (1998) \\
Kidney cytosol (2 mM) & $0.48 \pm 0.02$ & $0.65 \pm 0.15$ & Lash et al. (1998) \\
Kidney microsomes (2 mM) & $0.45 \pm 0.22$ & $0.32 \pm 0.02$ & Lash et al. (1998) \\
\hline
\end{tabular}




\begin{tabular}{|c|c|c|c|c|}
\hline \multirow{2}{*}{$\begin{array}{ll} & \text { System } \\
\text { Mouse } & \end{array}$} & \multicolumn{2}{|c|}{ Male } & Female & Reference \\
\hline & & & & \\
\hline Liver cytosol (2 mM) & \multicolumn{2}{|c|}{$24.5 \pm 2.4$} & \multirow[t]{2}{*}{$21.7 \pm 0.9$} & Lash et al. (1998) \\
\hline Liver cytosol (1.9 mM) & \multicolumn{2}{|c|}{$0.15^{\mathrm{c}}$} & & Green (1997) \\
\hline Liver microsomes $(2 \mathrm{mM})$ & \multicolumn{2}{|c|}{$40.0 \pm 3.1^{*}$} & $25.6 \pm 0.8$ & Lash et al. (1998) \\
\hline Kidney cytosol (2 mM) & \multicolumn{2}{|c|}{$5.6 \pm 0.24 *$} & $3.7 \pm 0.48$ & Lash et al. (1998) \\
\hline Kidney microsomes (2 mM) & \multicolumn{2}{|c|}{$5.47 \pm 1.41^{*}$} & $16.7 \pm 4.7$ & Lash et al. (1998) \\
\hline \multicolumn{5}{|c|}{$\begin{array}{l}{ }^{*} \mathrm{p}<0.05 \text { (compared to corresponding tissue sample in females). } \\
\text { Source: Adapted from EPA (2011a). } \\
\text { nd = not detected. } \\
\text { aUnits are nmol/hr/mg protein or } 10^{6} \text { cells. } \\
\text { bPooled samples include preparations derived from both sexes. } \\
{ }^{\circ} \text { Converted from } \mathrm{pmol} / \mathrm{min} / \mathrm{mg} \text { protein. }\end{array}$} \\
\hline \multicolumn{5}{|c|}{ Table B-4. Kinetics of In Vitro Glutathione Conjugation of Trichloroethylene } \\
\hline System & $\begin{array}{c}\mathbf{K}_{\mathbf{m}} \\
(\mu \mathrm{M} \text { TCE })\end{array}$ & $\begin{array}{r}V_{\text {max }} \\
P\end{array}$ & $\begin{array}{l}\text { nol DCVG/min/mg } \\
\text { ein or } 10^{6} \mathrm{Cells} \text { ) }\end{array}$ & $1,000 \times V_{\max } / K_{m}$ \\
\hline \multicolumn{5}{|l|}{ Human } \\
\hline Hepatocytes & $37 \sim 106$ & & $0.16 \sim 0.26$ & $2.4 \sim 4.5$ \\
\hline Liver cytosol: high affinity & 22.7 & & 4.27 & 190 \\
\hline Liver cytosol: low affinity & 333 & & 8.77 & 26.3 \\
\hline Liver microsomes: high affinity & 29.4 & & 1.42 & 48.3 \\
\hline Liver microsomes: low affinity & 250 & & 3.1 & 12.4 \\
\hline Kidney proximal tubular cells: high affinity & 580 & & 0.11 & 0.19 \\
\hline Kidney proximal tubular cells: low affinity & 29,400 & & 1.35 & 0.046 \\
\hline Kidney cytosol & 26.3 & & 0.81 & 31 \\
\hline Kidney microsomes & 167 & & 6.29 & 38 \\
\hline \multicolumn{5}{|l|}{ Rat } \\
\hline Kidney proximal tubular cells: high affinity & 460 & & 0.47 & 1.0 \\
\hline Kidney proximal tubular cells: low affinity & 2,910 & & 0.65 & 0.22 \\
\hline
\end{tabular}

Sources: Cummings and Lash (2000), Cummings et al. (2000), Lash et al. (1999a), EPA (2011a).

Table B-5. $\beta$-Lyase Activity from Human, Rat, and Mouse Kidney Cytosol

\begin{tabular}{llccl}
\hline \multicolumn{1}{c}{ System } & Substrate & $\begin{array}{c}\text { Km } \\
(\mathbf{m M} \text { TCE) }\end{array}$ & $\begin{array}{c}\mathbf{V}_{\mathbf{m a x}}(\mathbf{n m o l} \\
\mathbf{T C E} / \mathbf{m i n} / \mathbf{m g} \\
\text { Protein) }\end{array}$ & Reference \\
\hline Human & & & & \\
Male & TCVC & $2.53 \pm 0.09$ & $0.49 \pm 0.07$ & Green et al. (1990) \\
Female & TCVC & $2.67 \pm 2.11$ & $0.64 \pm 0.54$ & Green et al. (1990) \\
F344 Rat & & & & \\
Male & BTC & $1.66 \pm 0.19$ & $74.8 \pm 6.5$ & Lash et al. (1986) \\
\hline
\end{tabular}




\begin{tabular}{llccl}
\hline \multicolumn{1}{c}{ System } & \multicolumn{1}{c}{ Substrate } & $\begin{array}{c}\mathbf{K}_{\mathbf{m}} \\
(\mathbf{m M} \text { TCE) }\end{array}$ & $\begin{array}{c}\mathbf{V}_{\mathbf{m a x}}(\mathbf{n m o l} \\
\mathbf{T C E} / \mathbf{m i n} / \mathbf{m g} \\
\text { Protein) }\end{array}$ & Reference \\
\hline Male & CTFC & $1.78 \pm 0.17$ & $11.6 \pm 1.6$ & Lash et al. (1986) \\
Male & DCVC & $1.36 \pm 0.05$ & $38.3 \pm 1.4$ & Lash et al. (1986) \\
Male & DCVC & 0.26 & 2.2 & Stevens et al. (1989) \\
Male & TCVC & $0.68 \pm 0.06$ & $4.00 \pm 0.11$ & Green et al. (1990) \\
Female & TCVC & $1.26 \pm 0.21$ & $3.64 \pm 0.41$ & Green et al. (1990) \\
B6C3F 1 Mouse & & & & \\
Male & TCVC & $5.69 \pm 2.22$ & $1.15 \pm 0.31$ & Green et al. (1990) \\
Female & TCVC & $4.43 \pm 1.42$ & $1.66 \pm 0.27$ & Green et al. (1990) \\
\hline
\end{tabular}

Sources: Adapted from Lash et al. (2000a).

$\mathrm{BTC}=S$-(2-benzothiazolyl)-L-cysteine; CTFC $=S$-(2-chloro-1,1,2-trifluoroethyl)-L-cysteine; DCVC $=S$-dichlorovinyl-Lcysteine; TCVC $=S$-(1,2,2-trichlorovinyl)-L-cysteine.

Table B-6. Comparison of Hepatic In Vitro Oxidation and Glutathione Conjugation of Trichloroethylene in Human Hepatocytes and Liver Subcellular Fractions ${ }^{\mathrm{a}}$

\begin{tabular}{|c|c|c|c|c|}
\hline System & Pathway & $\begin{array}{c}\mathbf{K}_{\mathrm{m}} \\
(\mu \mathrm{M} \text { in Blood })\end{array}$ & $\begin{array}{c}\mathrm{V}_{\max }(\mathrm{nmol} \mathrm{TCE} / \mathrm{min} / \mathrm{g} \\
\text { Tissue }\end{array}$ & $\begin{array}{c}\mathrm{V}_{\max } / \mathbf{K}_{\mathrm{m}}(\mathrm{mL} / \mathrm{min} / \mathrm{g} \\
\text { Tissue })\end{array}$ \\
\hline Hepatocytes & $\begin{array}{l}\text { Oxidation } \\
\text { Conjugation }\end{array}$ & $\begin{array}{c}22.1-198 \\
16-47\end{array}$ & $\begin{array}{c}10-68.4 \\
16-25\end{array}$ & $\begin{array}{c}0.087-1.12 \\
0.55-1.0\end{array}$ \\
\hline $\begin{array}{l}\text { Microsomes } \\
\text { (option } 1)^{\mathrm{b}}\end{array}$ & $\begin{array}{l}\text { Oxidation } \\
\text { Conjugation }\end{array}$ & $\begin{array}{c}2.66-11.1 \\
5.9\end{array}$ & $\begin{array}{c}6.1-111 \\
45\end{array}$ & $\begin{array}{l}1.71-28.2 \\
7.6\end{array}$ \\
\hline $\begin{array}{l}\text { Microsomes } \\
\text { (option 2) }\end{array}$ & $\begin{array}{l}\text { Oxidation } \\
\text { Conjugation }\end{array}$ & $\begin{array}{c}71-297 \\
157\end{array}$ & $\begin{array}{c}6.1-111 \\
45\end{array}$ & $\begin{array}{l}0.064-1.06 \\
0.29\end{array}$ \\
\hline 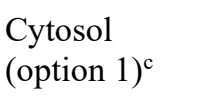 & $\begin{array}{l}\text { Oxidation } \\
\text { Conjugation }\end{array}$ & $\begin{array}{l}\mathrm{na} \\
4.5\end{array}$ & $\begin{array}{c}\mathrm{na} \\
380\end{array}$ & $\begin{array}{l}\text { na } \\
84\end{array}$ \\
\hline $\begin{array}{l}\text { Cytosol } \\
\text { (option 2) }^{\mathrm{c}}\end{array}$ & $\begin{array}{l}\text { Oxidation } \\
\text { Conjugation }\end{array}$ & $\begin{array}{c}\text { na } \\
22.7\end{array}$ & $\begin{array}{c}\text { na } \\
380\end{array}$ & $\begin{array}{c}\text { na } \\
16.7\end{array}$ \\
\hline
\end{tabular}

Sources: Adapted from EPA (2011a).

na $=$ not applicable.

${ }^{a}$ When biphasic metabolism was reported, only the high affinity pathway is shown.

${ }^{b} K_{m}$ values for microsomal protein calculated using different conversion assumptions: option 1 assumes $\mathrm{K}_{\mathrm{m}}$ in medium is equal to $\mathrm{K}_{\mathrm{m}}$ in tissue and converts to $\mathrm{K}_{\mathrm{m}}$ in blood by using a liver:blood partition coefficient of 5; option 2 converts $\mathrm{K}_{\mathrm{m}}$ in medium to $\mathrm{K}_{\mathrm{m}}$ in air using the measured microsomal protein:air partition coefficient of 1.78 , then converts to $\mathrm{K}_{\mathrm{m}}$ in blood using blood:air partition coefficient of 9.5 .

${ }^{c} K_{m}$ values for cytosolic protein calculated using different conversion assumptions: option 1 assumes $\mathrm{K}_{\mathrm{m}}$ in medium is equal to $\mathrm{K}_{\mathrm{m}}$ in tissue and converts $\mathrm{K}_{\mathrm{m}}$ in blood by using a liver:blood partition coefficient of 5; option 2 assumes $\mathrm{K}_{\mathrm{m}}$ in medium is equal to $\mathrm{K}_{\mathrm{m}}$ in blood, thus no conversion was necessary. 


\section{Appendix C. Genetic Toxicology}

\section{Tables}

Table C-1. In Vitro Mutagenicity Studies of Trichloroethylene in Bacteria C-3

Table C-2. In Vitro Genotoxicity Studies of Trichloroethylene in Non-mammalian

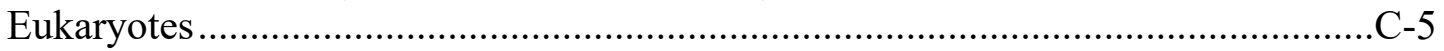

Table C-3. In Vitro Studies of Genotoxicity and Related Effects of Trichloroethylene in Mammalian (Including Human) Cells C-6

Table C-4. Studies of DNA and Protein Binding of Trichloroethylene in Mammalian Cells or Rodents

Table C-5. In Vivo Studies of Genotoxicity of Trichloroethylene in Rodents C-11

Table C-6. Cytogenetic Studies in Peripheral Blood Lymphocytes from Trichloroethylene-exposed Workers .C-15 
The tables on the following pages contain data discussed in the section "Genotoxicity and Related Effects" for trichloroethylene (Section 2).

Data are reported for in vitro studies of trichloroethylene, including mutagenicity in bacteria (Table C-1) and genotoxicity studies in non-mammalian eukaryotes (Table C-2) and mammalian cells (Table C-3). Studies on DNA and protein binding related to trichloroethylene exposure are included in Table C-4. In vivo studies of cytogenetic effects after trichloroethylene exposure in rodents are presented in Table $\mathrm{C}-5$; studies of cytogenetic effects in peripheral blood lymphocytes from trichloroethylene-exposed workers are provided in Table C-6. The chemical purity of the test samples of trichloroethylene used in the studies is included here if available, or it is noted if the sample is of unknown purity/contained stabilizers or pure/did not contain stabilizers. 
Table C-1. In Vitro Mutagenicity Studies of Trichloroethylene in Bacteria

\begin{tabular}{|c|c|c|c|c|c|}
\hline Test System/Endpoint & $\mathrm{LEC} / \mathrm{HIC} \mathbf{a}^{\mathrm{a}}$ & Without Activation & With Activation & Purity, Methods and Comments ${ }^{b}$ & References \\
\hline S. typhimurium TA100 & 14,650 & - & - & $\begin{array}{l}\text { Stabilizers (not epoxides), no DMSO } \\
\text { Plate incorporation assay }\end{array}$ & $\begin{array}{l}\text { Henschler et al. } \\
\text { (1977) }\end{array}$ \\
\hline $\begin{array}{l}\text { S. typhimurium TA100, } \\
\text { TA1535 }\end{array}$ & $\begin{array}{c}160 \text { (vapor) } \\
2,800 \text { (vapor) }\end{array}$ & - & $\begin{array}{l}(+) \\
-\end{array}$ & $\begin{array}{l}\text { No stabilizers, no DMSO } \\
\text { Desiccator assay; rat and mouse S9 } \\
\text { For TA100, increased revertants but not } \\
\text { doubled; effect greater with mouse S9 }\end{array}$ & $\begin{array}{l}\text { Simmon et al. } \\
(1977)\end{array}$ \\
\hline $\begin{array}{l}\text { S. typhimurium TA98, } \\
\text { TA100 }\end{array}$ & 525 (vapor) & - all strains & - all strains & $\begin{array}{l}\text { Stabilizers, no DMSO } \\
\text { Study conducted in sealed desiccator vials }\end{array}$ & Waskell (1978) \\
\hline $\begin{array}{l}\text { S. typhimurium TA100, } \\
\text { TA1535 }\end{array}$ & $\begin{array}{l}160 \text { (vapor) } \\
526 \text { (vapor) }\end{array}$ & $\begin{array}{l}\text { - both strains; } \\
\text { both methods }\end{array}$ & $\begin{array}{l}(+) \text { TA100 } \\
- \text { TA1535 }\end{array}$ & $\begin{array}{l}\text { No stabilizers, } \geq 99.5 \% \text { purity, no DMSO } \\
\text { Two methods: plate incorporation in } \\
\text { desiccator and preincubation; rat S9 } \\
\text { Increased revertants but not doubled; effect } \\
\text { only for plate incorporation in desiccator }\end{array}$ & Baden et al. (1979) \\
\hline S. typhimurium TA100 & $\begin{array}{c}420 \text { (8\% vapor) } \\
16 \mathrm{hr}\end{array}$ & - plate incorporation & $\begin{array}{l}\text { - plate } \\
\text { incorporation } \\
+ \text { preincubation }\end{array}$ & $\begin{array}{l}\text { No stabilizers; purity } 99.5 \% \text {, DMSO used as } \\
\text { solvent } \\
\text { Two methods: plate incorporation in } \\
\text { desiccator and preincubation; mouse S9 } \\
\text { Revertants doubled for preincubation assay }\end{array}$ & $\begin{array}{l}\text { Bartsch et al. } \\
(1979)\end{array}$ \\
\hline S. typhimurium TA 1535 & & $(+)$ & & $\begin{array}{l}\text { No stabilizers; purity } 99.5 \% \text {, no DMSO } \\
\text { Plate incorporation }\end{array}$ & $\begin{array}{l}\text { Kringstad et al. } \\
(1981)\end{array}$ \\
\hline S. typhimurium TA100 & 18 (vapor) & - & + & No stabilizers (epoxide-free), no DMSO & $\begin{array}{l}\text { Crebelli et al. } \\
(1982)\end{array}$ \\
\hline $\begin{array}{l}\text { S. typhimurium TA1535, } \\
\text { TA100 }\end{array}$ & 50 (vapor) & $\begin{array}{l}\mathrm{H}:- \text { both strains } \\
\mathrm{L}:(+) \text { both strains }\end{array}$ & $\begin{array}{l}\mathrm{H}:- \text { both strains } \\
\mathrm{L}:(+) \text { both strains }\end{array}$ & $\begin{array}{l}\text { No stabilizers, purity } 99.98 \%(\mathrm{~L}) \text { and } 99.5 \% \\
(\mathrm{H}) \text {; tested high }(\mathrm{H} \text {, Trichlor } 136) \text { and low }(\mathrm{L} \text {, } \\
\text { Trichlor } 119) \text { stabilized samples, no DMSO } \\
\text { TA100 } \pm \text { S9 positive only at top dose and } 3 \% \\
\text { survival }\end{array}$ & $\begin{array}{l}\text { Shimada et al. } \\
(1985)\end{array}$ \\
\hline $\begin{array}{l}\text { S. typhimurium TA98, } \\
\text { TA100, TA1535, } \\
\text { TA1537, TA97 }\end{array}$ & 167 & - all strains & - all strains & $\begin{array}{l}\text { No stabilizers; purity }>99 \% \text {, DMSO used as } \\
\text { solvent } \\
\text { Preincubation assay }\end{array}$ & $\begin{array}{l}\text { Mortelmans et al. } \\
(1986)\end{array}$ \\
\hline $\begin{array}{l}\text { S. typhimurium TA } 1535 \text {, } \\
\text { TA } 100, \text { TA } 1537, \text { TA } 98\end{array}$ & Not reported & $\begin{array}{l}\text { + TA1535, TA100 } \\
\text { - TA1537, TA98 }\end{array}$ & $\begin{array}{l}\text { + TA1535, TA100 } \\
\text { - TA } 1537, \text { TA98 }\end{array}$ & $\begin{array}{l}\text { Purity not specified (97\%-99\%), no DMSO } \\
\text { Desiccator (vapor) assay }\end{array}$ & $\begin{array}{l}\text { Milman et al. } \\
\text { (1988) }\end{array}$ \\
\hline
\end{tabular}


RoC Monograph on Trichloroethylene

\begin{tabular}{|c|c|c|c|c|c|}
\hline Test System/Endpoint & LEC/HIC a & Without Activation & With Activation & Purity, Methods and Comments ${ }^{b}$ & References \\
\hline $\begin{array}{l}\text { S. typhimurium TA98, } \\
\text { TA100 }\end{array}$ & 1,050 (vapor) & & - & $\begin{array}{l}\text { No stabilizers, purity }>99.9 \% \text {, DMSO used as } \\
\text { solvent } \\
\text { Desiccator (vapor) assay; S9 from rat and } \\
\text { hamster }\end{array}$ & $\begin{array}{l}\text { McGregor et al. } \\
(1989)\end{array}$ \\
\hline $\begin{array}{l}\text { S. typhimurium TA98, } \\
\text { TA100 }\end{array}$ & 1,050 (vapor) & - & - & $\begin{array}{l}\text { No epoxybutane and epichlorohydrin, oxirane } \\
\text { stabilized, purity }>99.9 \% \text {, DMSO used as } \\
\text { solvent } \\
\text { Preincubation assay }\end{array}$ & $\begin{array}{l}\text { McGregor et al. } \\
(1989)\end{array}$ \\
\hline $\begin{array}{l}\text { S. typhimurium TA1535, } \\
\text { TA100, TA98 }\end{array}$ & $\begin{array}{l}33 \text { (vapor) } \\
130 \text { (vapor) } \\
65 \text { (vapor) }\end{array}$ & $\begin{array}{l}+ \text { TA1535 } \\
+ \text { TA100 } \\
\text { - TA98 }\end{array}$ & $\begin{array}{l}+ \text { TA1535 } \\
+ \text { TA100 } \\
\text { - TA98 }\end{array}$ & $\begin{array}{l}\text { Stabilizers (epoxybutane and epichlorohydrin } \\
\text { and oxirane), DMSO used as solvent } \\
\text { Desiccator (vapor) assay }\end{array}$ & $\begin{array}{l}\text { McGregor et al. } \\
(1989)\end{array}$ \\
\hline S. typhimurium BAL13 & 190 (vapor) & - & - & $\begin{array}{l}\text { No stabilizers, purity } 99 \% \text {, DMSO used as } \\
\text { solvent } \\
\text { Forward mutation assay (ara test) }\end{array}$ & $\begin{array}{l}\text { Roldan-Arjona et } \\
\text { al. (1991) }\end{array}$ \\
\hline S. typhimurium YG7108 & $3,000 \mu \mathrm{g} / \mathrm{plate}$ & + & & $\begin{array}{l}\text { Purity } \geq 99.5 \% \text {, DMSO used as solvent } \\
\text { CYP E1 metabolically competent strain } \\
\text { microcolony assay/revertants }\end{array}$ & $\begin{array}{l}\text { Emmert et al. } \\
(2006)\end{array}$ \\
\hline $\begin{array}{l}\text { Escherichia coli } \mathrm{K} 12 \text {, } \\
\text { reverse mutation } \arg ^{+}\end{array}$ & 434 & - & + & $\begin{array}{l}\text { Purity unknown; analytical grade, no DMSO } \\
\text { Reverse mutation }\left(\arg ^{+}\right)\end{array}$ & Greim et al. (1975) \\
\hline Escherichia coli $\mathrm{PQ} 37$ & 13,140 & - & - & $\begin{array}{l}\text { Purity unknown, use of DMSO unknown } \\
\text { SOS chromotest }\end{array}$ & $\begin{array}{l}\text { von der Hude et al. } \\
\text { (1988) }\end{array}$ \\
\hline Escherichia coli $\mathrm{PQ} 37$ & 7,325 & - & - & $\begin{array}{l}\text { No stabilizers, purity unknown, use of DMSO } \\
\text { unknown } \\
\text { SOS chromotest }\end{array}$ & $\begin{array}{l}\text { Mersch- } \\
\text { Sundermann et al. } \\
\text { (1989) }\end{array}$ \\
\hline
\end{tabular}

Sources: EPA (2011a), IARC (2014); primary papers were reviewed for additional details on purity of test substance and solvent used.

$+=$ positive $(+)=$ weak positive; $-=$ negative.

aTreatment concentrations are $\mu \mathrm{g} / \mathrm{mL}$ unless noted otherwise.

bresence of DMSO in test sample is indicated when noted by authors. 
Table C-2. In Vitro Genotoxicity Studies of Trichloroethylene in Non-mammalian Eukaryotes

\begin{tabular}{|c|c|c|c|c|c|}
\hline $\begin{array}{l}\text { Test System/ } \\
\text { Endpoint }\end{array}$ & LEC/HIC & $\begin{array}{l}\text { Without } \\
\text { Activation }\end{array}$ & $\begin{array}{c}\text { With } \\
\text { Activation }\end{array}$ & Purity, Methods, and Comments ${ }^{\mathrm{a}}$ & References \\
\hline \multicolumn{6}{|l|}{ Gene mutation } \\
\hline S. cerevisiae D7 & 1,300 & - & + & $\begin{array}{l}\text { No stabilizers, solvent corn oil, no DMSO } \\
\text { Reverse mutation }\end{array}$ & $\begin{array}{l}\text { Bronzetti et al. } \\
\text { (1978) }\end{array}$ \\
\hline S. cerevisiae D7 & 1,970 & & + & $\begin{array}{l}\text { Purity unknown, contained } 0.01 \% \text { thymol as } \\
\text { stabilizer, no DMSO } \\
\text { Reverse mutation, log phase }\end{array}$ & Callen et al. (1980) \\
\hline S. cerevisiae D7 & 725 & - & $(+)$ & $\begin{array}{l}\text { Purity unknown, analytical grade, no DMSO } \\
\text { Reverse mutation, log phase and stationary }\end{array}$ & Koch et al. (1988) \\
\hline $\begin{array}{l}\text { A. nidulans haploid } \\
\text { strain } 35\end{array}$ & $\begin{array}{l}100 \text { ppm (quiescent) } \\
13 \text { ppm (growing) }\end{array}$ & $\begin{array}{l}- \\
+\end{array}$ & & $\begin{array}{l}\text { No stabilizers, purity unknown, no DMSO } \\
\text { Forward mutation, desiccator (vapor) }\end{array}$ & Crebelli et al. (1985) \\
\hline $\begin{array}{l}\text { Schizosaccharomyces } \\
\text { pombe } \mathrm{P} 1\end{array}$ & $\begin{array}{l}3,280 \text { (quiescent) } \\
13,140 \text { (growing) }\end{array}$ & - & - & $\begin{array}{l}\text { Measured forward mutation } \\
\text { Tested both technical grade and pure (without } \\
\text { stabilizers), DMSO ( } \leq 2 \%) \text { used as solvent } \\
\text { Negative for both sample purities and growth } \\
\text { conditions }\end{array}$ & Rossi et al. (1983) \\
\hline \multicolumn{6}{|l|}{ Gene conversion } \\
\hline $\begin{array}{l}\text { Saccharomyces } \\
\text { cerevisiae }\end{array}$ & $\begin{array}{l}\text { Strain D7: } 1,970 \\
\text { Strain D4: } 2,900\end{array}$ & & $\begin{array}{l}+ \\
-\end{array}$ & $\begin{array}{l}\text { Purity unknown, contained } 0.01 \% \text { thymol as } \\
\text { stabilizer, no DMSO } \\
\text { Log-phase cultures } \\
\text { CYP content } 5 \text {-fold greater in D7 than D } 4 \\
\text { Strain D7 had high cytotoxicity at } 2,900 \mu \mathrm{g} / \mathrm{mL}\end{array}$ & Callen et al. (1980) \\
\hline S. cerevisiae D7 & 2,900 & - & - & $\begin{array}{l}\text { Purity unknown, analytical grade, no DMSO } \\
\text { Stationary and log phase cultures } \\
\text { Production of phototropic colonies }\end{array}$ & Koch et al. (1988) \\
\hline S. cerevisiae D7 & 2,600 & - & + & No stabilizers, solvent corn oil, no DMSO & $\begin{array}{l}\text { Bronzetti et al. } \\
(1978)\end{array}$ \\
\hline \multicolumn{6}{|c|}{ Recombination and mitotic crossover } \\
\hline S. cerevisiae D7 & 1,970 & & + & $\begin{array}{l}\text { Purity unknown, contained } 0.01 \% \text { thymol as } \\
\text { stabilizer, no DMSO }\end{array}$ & Callen et al. (1980) \\
\hline
\end{tabular}




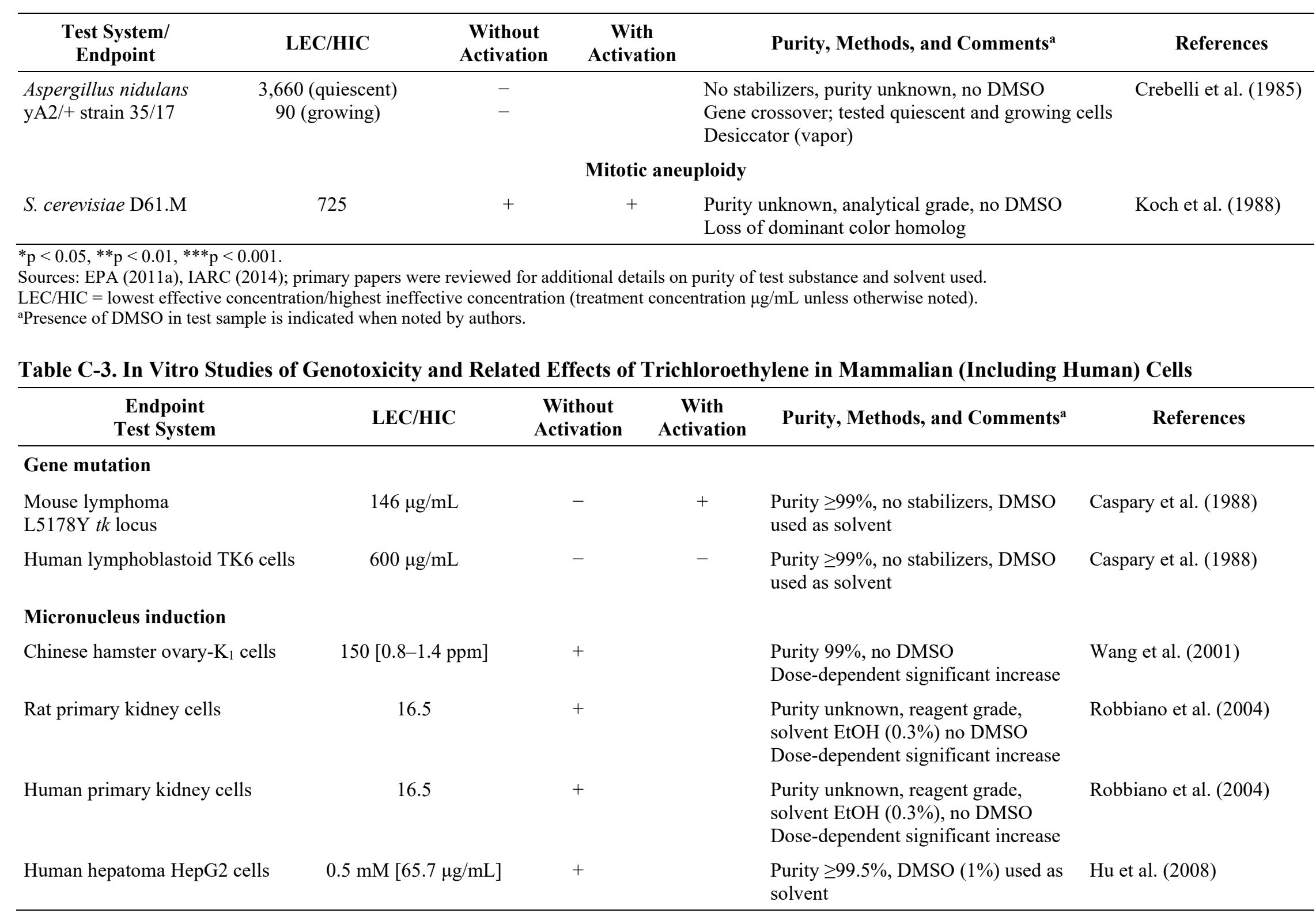




\begin{tabular}{|c|c|c|c|c|c|}
\hline $\begin{array}{l}\text { Endpoint } \\
\text { Test System }\end{array}$ & LEC/HIC & $\begin{array}{c}\text { Without } \\
\text { Activation }\end{array}$ & $\begin{array}{c}\text { With } \\
\text { Activation }\end{array}$ & Purity, Methods, and Comments ${ }^{\mathrm{a}}$ & References \\
\hline Human lymphocytes & $6 \mathrm{mM}$ & - & & $\begin{array}{l}\text { Purity unknown, DMSO }(0.3 \%) \text { used } \\
\text { as solvent } \\
\text { Cytokinesis-block assay }\end{array}$ & Kumar et al. (2009) \\
\hline \multicolumn{6}{|l|}{ Chromosomal aberrations } \\
\hline Chinese hamster lung cells & $1,000 \mu \mathrm{g} / \mathrm{mL}$ & - & - & $\begin{array}{l}\text { Purity unknown, use of DMSO not } \\
\text { known }\end{array}$ & Sofuni et al. (1985) \\
\hline Chinese hamster ovary cells & $14,900 \mu \mathrm{g} / \mathrm{mL}$ & - & - & $\begin{array}{l}\text { No stabilizers, use of DMSO not } \\
\text { known but probable }\end{array}$ & Galloway et al. (1987) \\
\hline Human lymphocytes & $6 \mathrm{mM}$ & - & & $\begin{array}{l}\text { Purity unknown, DMSO }(0.3 \%) \text { used } \\
\text { as solvent }\end{array}$ & Kumar et al. (2009) \\
\hline \multicolumn{6}{|l|}{ Sister chromatid exchange } \\
\hline Chinese hamster ovary cells & 9 & & - & $\begin{array}{l}\text { Purity unknown, no DMSO } \\
1 \text { hr (vapor) } \\
\text { Limitations: short exposure time, few } \\
\text { doses, no positive control }\end{array}$ & White et al. (1979) \\
\hline Chinese hamster ovary cells & $\begin{array}{l}\text { +S9: } 401 \mu \mathrm{g} / \mathrm{mL} \\
\text {-S9: } 700 \mu \mathrm{g} / \mathrm{mL}\end{array}$ & $(+)$ & + & $\begin{array}{l}\text { Purity } \geq 99 \% \text {, use of DMSO not } \\
\text { known but probable }\end{array}$ & Galloway et al. (1987) \\
\hline Human lymphocytes & $178 \mu \mathrm{g} / \mathrm{mL}$ & + & & $\begin{array}{l}\text { No stabilizers, use of DMSO } \\
\text { unknown }\end{array}$ & Gu et al. (1981) \\
\hline \multicolumn{6}{|l|}{ DNA strand breaks } \\
\hline Human hepatoma HepG2 cells & $0.5 \mathrm{mM}[65.7 \mathrm{~g} / \mathrm{mL}]$ & + & & $\begin{array}{l}\text { Purity } \geq 99.5 \% \text {, DMSO (1\%) used as } \\
\text { solvent } \\
\text { Comet assay }\end{array}$ & Hu et al. (2008) \\
\hline Rat primary kidney cells & 16.5 & + & & $\begin{array}{l}\text { Purity unknown (reagent grade), } \\
\text { Solvent EtOH }(0.3 \%) \text {, no DMSO } \\
\text { Comet assay } \\
\text { Dose-dependent significant increase }\end{array}$ & Robbiano et al. (2004) \\
\hline Human primary kidney cells & 16.5 & + & & $\begin{array}{l}\text { Purity unknown (reagent grade), } \\
\text { Solvent EtOH }(0.3 \%) \text {, no DMSO } \\
\text { Comet assay } \\
\text { Dose-dependent significant increase }\end{array}$ & Robbiano et al. (2004) \\
\hline
\end{tabular}


RoC Monograph on Trichloroethylene

\begin{tabular}{|c|c|c|c|c|c|}
\hline $\begin{array}{l}\text { Endpoint } \\
\text { Test System }\end{array}$ & LEC/HIC & $\begin{array}{l}\text { Without } \\
\text { Activation }\end{array}$ & $\begin{array}{c}\text { With } \\
\text { Activation }\end{array}$ & Purity, Methods, and Comments ${ }^{\mathrm{a}}$ & References \\
\hline \multicolumn{6}{|l|}{ UDS (DNA repair) } \\
\hline $\begin{array}{l}\text { Rat hepatocytes, } \\
\text { phenobarbital-induced }\end{array}$ & 368 & + & & Purity unknown, no DMSO & Costa and Ivanetich (1984) \\
\hline Rat primary hepatocytes & 130 (vapor) & $\begin{array}{l}\text { - without } \\
\text { stabilizers } \\
\text { - with } \\
\text { stabilizers }\end{array}$ & & $\begin{array}{l}\text { Tested samples with and without } \\
\text { stabilizers, no DMSO } \\
\text { Cytotoxic }\end{array}$ & Shimada et al. (1985) \\
\hline Rat primary hepatocytes & $\begin{array}{c}56.77 \text { (without } \\
\text { stabilizer) } \\
1,445 \text { (with or without } \\
\text { stabilizer) }\end{array}$ & $\begin{array}{l}\text { + without } \\
\text { stabilizers } \\
\text { - vapor phase } \\
\text { testing }\end{array}$ & & $\begin{array}{l}\text { No stabilizers, purity unknown, no } \\
\text { DMSO, standard test } \\
\text { Tested vapor phase for samples both } \\
\text { with and without stabilizers }\end{array}$ & Williams et al. (1989) \\
\hline $\begin{array}{l}\mathrm{B} 6 \mathrm{C}_{3} \mathrm{~F}_{1} \text { mouse primary } \\
\text { hepatocytes }\end{array}$ & NR & + & & $\begin{array}{l}\text { Stabilizers; purity unknown, no } \\
\text { DMSO }\end{array}$ & Milman et al. (1988) \\
\hline Rat primary hepatocytes & NR & - & & $\begin{array}{l}\text { Stabilizers; purity unknown, no } \\
\text { DMSO }\end{array}$ & Milman et al. (1988) \\
\hline Human lymphocytes & $2.5 \mu \mathrm{l} / \mathrm{mL}$ & $(+)$ & & $\begin{array}{l}\text { No stabilizers; purity } 97 \%-99 \% \text {, } \\
\text { DMSO (1\%) used as solvent }\end{array}$ & Perocco and Prodi (1981) \\
\hline \multicolumn{6}{|l|}{ Cell transformation } \\
\hline $\begin{array}{l}\text { RLV/Fischer rat F1706 embryo } \\
\text { cells }\end{array}$ & 144 & + & & Purity $99.9 \%$, no DMSO & Price et al. (1978) \\
\hline Syrian hamster embryo cells & 25 & $(+)$ & & $\begin{array}{l}\text { Purity unknown, DMSO used as } \\
\text { solvent }\end{array}$ & $\begin{array}{l}\text { Amacher and Zelljadt } \\
\text { (1983) }\end{array}$ \\
\hline BALB/C-3T3 mouse cells & 250 & $(+)$ & & $\begin{array}{l}\text { Purity not specified }(97 \%-99 \%) \text {, no } \\
\text { DMSO }\end{array}$ & Tu et al. (1985) \\
\hline
\end{tabular}


Table C-4. Studies of DNA and Protein Binding of Trichloroethylene in Mammalian Cells or Rodents

\begin{tabular}{|c|c|c|c|c|c|}
\hline $\begin{array}{l}\text { Endpoint/ } \\
\text { Test System }\end{array}$ & LED/HID & $\begin{array}{l}\text { Without } \\
\text { Activation }\end{array}$ & $\begin{array}{c}\text { With } \\
\text { Activation }\end{array}$ & Purity, Methods, and Comments ${ }^{a}$ & References \\
\hline \multicolumn{6}{|l|}{ In vitro } \\
\hline $\begin{array}{l}\text { Covalent binding } \\
\text { Calf thymus DNA }\end{array}$ & 131 & & + & No stabilizers, purity unknown, no DMSO & DiRenzo et al. (1982) \\
\hline $\begin{array}{l}\text { Covalent binding } \\
\text { Calf thymus DNA }\end{array}$ & 340 & - & + & No stabilizers, purity $>99 \%$, no DMSO & Bergman (1983) \\
\hline $\begin{array}{l}\text { Covalent binding } \\
\text { Calf thymus DNA }\end{array}$ & 13 & & + & No stabilizers, purity $>99 \%$, no DMSO & $\begin{array}{l}\text { Miller and Guengerich } \\
\text { (1983) }\end{array}$ \\
\hline $\begin{array}{l}\text { Covalent binding } \\
\text { Rat hepatocyte DNA }\end{array}$ & 13 & + & & No stabilizers, purity $>99 \%$, no DMSO & $\begin{array}{l}\text { Miller and Guengerich } \\
\text { (1983) }\end{array}$ \\
\hline $\begin{array}{l}\text { Covalent binding } \\
\text { Mouse hepatocyte DNA }\end{array}$ & 13 & + & & No stabilizers, purity $>99 \%$, no DMSO & $\begin{array}{l}\text { Miller and Guengerich } \\
\text { (1983) }\end{array}$ \\
\hline $\begin{array}{l}\text { Covalent binding } \\
\text { Calf thymus DNA }\end{array}$ & 3.2 & & + & $\begin{array}{l}\text { Purity } 98.9 \% \text {, no DMSO } \\
\text { Mediated by phenobarbitone-induced } \\
\text { microsomal and/or cytosolic fractions from rat } \\
\text { and mouse organs (mainly liver; also kidney, } \\
\text { lung, stomach) }\end{array}$ & Mazzullo et al. (1992) \\
\hline $\begin{array}{l}\text { Covalent binding DNA } \\
\text { Salmon sperm DNA }\end{array}$ & 270 & - & + & No stabilizers, purity $>99 \%$, no DMSO & $\begin{array}{l}\text { Banerjee and Van Duuren } \\
\text { (1978) }\end{array}$ \\
\hline $\begin{array}{l}\text { Protein binding } \\
\text { Liver, lung, stomach, kidney } \\
\text { microsomes } \\
\text { Sprague-Dawley, Osborne- } \\
\text { Mendel, and Fischer } 344 \text { rats (M } \\
\text { \& F) }\end{array}$ & & + & & $\begin{array}{l}\text { No stabilizers, purity }>99 \% \text {, no DMSO } \\
\text { Sprague-Dawley-rats had higher binding levels } \\
\text { than Osborne-Mendel and Fischer } 344 \text { rats } \\
\text { Binding was greater for males than females in } \\
\text { Osborne-Mendel rats but not other strains }\end{array}$ & $\begin{array}{l}\text { Banerjee and Van Duuren } \\
\text { (1978) }\end{array}$ \\
\hline $\begin{array}{l}\text { Protein binding } \\
\text { Liver, lung, stomach, kidney } \\
\text { microsomes } \\
\text { B6C } 3 F_{1} \text { mouse }\end{array}$ & & + & & $\begin{array}{l}\text { No stabilizers, purity }>99 \% \text {, no DMSO } \\
\text { Liver, lung stomach, kidney } \\
\text { Binding was greater in mouse than rat (all } \\
\text { strains) in same study } \\
\text { Binding was greater in male than female mice }\end{array}$ & $\begin{array}{l}\text { Banerjee and Van Duuren } \\
\text { (1978) }\end{array}$ \\
\hline
\end{tabular}


RoC Monograph on Trichloroethylene

\begin{tabular}{|c|c|c|c|c|c|}
\hline $\begin{array}{l}\text { Endpoint/ } \\
\text { Test System }\end{array}$ & LED/HID & $\begin{array}{l}\text { Without } \\
\text { Activation }\end{array}$ & $\begin{array}{c}\text { With } \\
\text { Activation }\end{array}$ & Purity, Methods, and Comments ${ }^{\mathrm{a}}$ & References \\
\hline $\begin{array}{l}\text { Protein binding } \\
\text { Liver and lung microsomes } \\
\text { Osborne-Mendel rat }\end{array}$ & & + & & $\begin{array}{l}\text { No stabilizers, purity }>99 \% \text {, no DMSO } \\
\text { Binding to TCE oxide }\end{array}$ & $\begin{array}{l}\text { Miller and Guengerich } \\
\text { (1983) }\end{array}$ \\
\hline 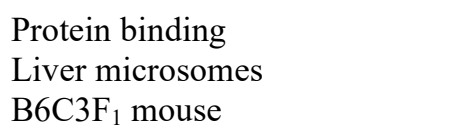 & & + & & $\begin{array}{l}\text { No stabilizers, purity }>99 \% \text {, no DMSO } \\
\text { Binding to TCE oxide }\end{array}$ & $\begin{array}{l}\text { Miller and Guengerich } \\
\text { (1983) }\end{array}$ \\
\hline $\begin{array}{l}\text { Protein binding } \\
\text { Insulin and adrenocorticotropic } \\
\text { hormone } \\
\text { Rabbit }\end{array}$ & & + & & $\begin{array}{l}\text { No stabilizers, purity unknown, no DMSO } \\
\text { Binding to TCE oxide }\end{array}$ & Cai and Guengerich (2001) \\
\hline $\begin{array}{l}\text { Protein binding } \\
\text { Liver microsomes } \\
\text { Human }\end{array}$ & & + & & $\begin{array}{l}\text { No stabilizers, purity }>99 \% \text {, no DMSO } \\
\text { Binding to TCE oxide }\end{array}$ & $\begin{array}{l}\text { Miller and Guengerich } \\
\text { (1983) }\end{array}$ \\
\hline \multicolumn{6}{|l|}{ In vivo } \\
\hline $\begin{array}{l}\text { Protein binding } \\
\text { Liver } \\
\text { Kidney } \\
\text { B6C3F }_{1} \text { mouse (male) }\end{array}$ & $\begin{array}{l}10 \mathrm{ppm} \\
600 \mathrm{ppm}\end{array}$ & $\begin{array}{l}+ \\
+\end{array}$ & & $\begin{array}{l}\text { Purity }>99.9 \% \text {, no DMSO } \\
\text { Amine stabilized } \\
\text { inh. } 6 \mathrm{hr}(10 \text { or } 600 \mathrm{ppm}) \\
\text { Measured reactive metabolite } \\
\text { Mouse greater binding than rat in same study, } \\
\text { for both doses and tissues }\end{array}$ & Stott et al. (1982) \\
\hline $\begin{array}{l}\text { Protein binding } \\
\text { Liver, kidney } \\
\text { Osborne-Mendel rat (male) }\end{array}$ & $600 \mathrm{ppm}$ & - & & $\begin{array}{l}\text { Purity }>99.9 \% \text {, no DMSO } \\
\text { Amine stabilized } \\
\text { inh. } 6 \mathrm{hr}(10 \text { or } 600 \mathrm{ppm}) \\
\text { Measured reactive metabolite }\end{array}$ & Stott et al. (1982) \\
\hline $\begin{array}{l}\text { Covalent binding } \\
\text { Liver DNA } \\
\text { B6C3 } 3 \mathrm{~F}_{1} \text { mouse (male) }\end{array}$ & 1,200 & $?$ & & $\begin{array}{l}\text { Purity }>99.9 \% \text {, no DMSO } \\
\text { Amine stabilized } \\
\text { p.o. } \times 1\end{array}$ & Stott et al. (1982) \\
\hline $\begin{array}{l}\text { Covalent binding } \\
\text { Liver, kidney, lung, stomach } \\
\text { DNA } \\
\text { BALB/c mouse (male) }\end{array}$ & 0.76 & $(+)$ & & $\begin{array}{l}\text { Purity } 98.9 \% \text {, no DMSO } \\
\text { i.p. } \times 1\end{array}$ & Mazzullo et al. (1992) \\
\hline
\end{tabular}




\begin{tabular}{|c|c|c|c|c|c|}
\hline $\begin{array}{l}\text { Endpoint/ } \\
\text { Test System }\end{array}$ & LED/HID & $\begin{array}{l}\text { Without } \\
\text { Activation }\end{array}$ & $\begin{array}{c}\text { With } \\
\text { Activation }\end{array}$ & Purity, Methods, and Comments ${ }^{\mathrm{a}}$ & References \\
\hline $\begin{array}{l}\text { Covalent binding } \\
\text { Liver, kidney, lung, stomach } \\
\text { DNA } \\
\text { Wistar rat (male) }\end{array}$ & 0.76 & $(+)$ & & $\begin{array}{l}\text { Purity } 98.9 \% \text {, no DMSO } \\
\text { i.p. } \times 1\end{array}$ & Mazzullo et al. (1992) \\
\hline $\begin{array}{l}\text { Covalent binding } \\
\text { Spleen, lung, kidney, pancreas, } \\
\text { testis, brain DNA } \\
\text { NMRI mouse }\end{array}$ & 67 & - & & $\begin{array}{l}\text { No stabilizers, purity }>99 \% \text {, solvent peanut oil, } \\
\text { no DMSO } \\
\text { i.p. } \times 5 \\
\text { Metabolic incorporation of }{ }^{14} \mathrm{C} \text { into nucleotides } \\
\text { was observed; findings for liver inconclusive }\end{array}$ & Bergman (1983) \\
\hline $\begin{array}{l}\text { Covalent binding } \\
\text { Spleen, lung, liver, kidney, } \\
\text { pancreas, testis, brain RNA } \\
\text { NMRI mouse }\end{array}$ & 67 & - & & $\begin{array}{l}\text { No stabilizers, purity }>99 \% \text {, solvent peanut oil, } \\
\text { no DMSO } \\
\text { i.p. } \times 5 \\
\text { Metabolic incorporation of }{ }^{14} \mathrm{C} \text { into nucleotides } \\
\text { observed }\end{array}$ & Bergman (1983) \\
\hline
\end{tabular}

Sources: EPA (2011a), IARC (2014); primary papers were reviewed for additional details on purity of test substance and solvent used; studies considered to be inconclusive are not included here.

Exposure in vitro, $\mu \mathrm{g} / \mathrm{mL}$, unless otherwise indicated; in vivo, doses in ppm; p.o. = orally; i.p. $=$ intraperitoneal injection in $\mathrm{mg} / \mathrm{kg}$ body $\mathrm{weight}$; inh. $=$ inhalation; $+=$ positive,

$(+)=$ weak positive; $-=$ negative; $?=$ inconclusive.

apresence of DMSO in test sample is indicated when noted by authors.

\section{Table C-5. In Vivo Studies of Genotoxicity of Trichloroethylene in Rodents}

\begin{tabular}{|c|c|c|c|c|}
\hline $\begin{array}{l}\text { Test System/ } \\
\text { Endpoint }\end{array}$ & LED/HID & Results & Purity, Methods, and Comments & Reference \\
\hline \multicolumn{5}{|l|}{ Gene mutation } \\
\hline $\begin{array}{l}\text { NMRI-Hans/BGA mouse (male) } \\
\text { Dominant lethal }\end{array}$ & 3,400 & - & $\begin{array}{l}\text { No stabilizers, purity } 99.5 \% \text {, no DMSO } \\
\text { inh. } 24 \mathrm{hr} \times 1\end{array}$ & Slacik-Erben et al. (1980) \\
\hline $\begin{array}{l}\text { Lac } Z \text { transgenic mouse (male } \\
\text { and female) } \\
\text { Lung, liver, spleen, kidney, } \\
\text { testicular germ cells }\end{array}$ & 3,144 & - & $\begin{array}{l}\text { No stabilizers, purity }>99 \% \text {, no DMSO } \\
\text { inh. } 6 \mathrm{hr} / \mathrm{d} \times 6 \mathrm{~d} \\
\text { No base changes or small deletions }\end{array}$ & Douglas et al. (1999) \\
\hline \multicolumn{5}{|l|}{ Micronucleus induction } \\
\hline $\begin{array}{l}\text { Mouse } \\
\text { Bone-marrow erythrocytes }\end{array}$ & 750 & + & $\begin{array}{l}\text { No stabilizers, use of DMSO unknown } \\
\text { p.o. in gum arabic } \times 2\end{array}$ & Duprat and Gradiski (1980) \\
\hline
\end{tabular}


RoC Monograph on Trichloroethylene

\begin{tabular}{|c|c|c|c|c|}
\hline $\begin{array}{l}\text { Test System/ } \\
\text { Endpoint }\end{array}$ & LED/HID & Results & Purity, Methods, and Comments & Reference \\
\hline $\begin{array}{l}\mathrm{B} 6 \mathrm{C}_{3} \mathrm{~F}_{1} \text { mouse (male) } \\
\text { Bone-marrow erythrocytes }\end{array}$ & 2,500 & - & $\begin{array}{l}\text { No stabilizers, purity not reported, no DMSO } \\
\text { i.p. in corn oil } \times 3\end{array}$ & Shelby et al. (1993) \\
\hline $\begin{array}{l}\text { C57BL/6J mouse (male) } \\
\text { Bone marrow erythrocytes }\end{array}$ & 9,800 & - & $\begin{array}{l}\text { No stabilizers, purity }>99 \% \text {, no DMSO } \\
\text { inh. } 6 \mathrm{hr} \times 1\end{array}$ & Kligerman et al. (1994) \\
\hline $\begin{array}{l}\text { CD-1 mouse (male) } \\
\text { Bone-marrow erythrocytes }\end{array}$ & 460 & + & $\begin{array}{l}\text { Purity not reported, no DMSO } \\
\text { i.p. in corn oil } \times 1 \\
\text { Correlated with urinary TCOH }\end{array}$ & Hrelia et al. (1994) \\
\hline $\begin{array}{l}\text { C57B1/6J mouse (male) } \\
\text { Spermatocytes }\end{array}$ & 565 & - & $\begin{array}{l}\text { No stabilizers, purity } \geq 99 \% \text {, no DMSO } \\
\text { inh. } 6 \mathrm{hr} / \mathrm{d} \times 5 \mathrm{~d} \\
\text { Spermatids examined }\end{array}$ & Allen et al. (1994) \\
\hline $\begin{array}{l}\text { C57BL/6J mouse (male) } \\
\text { Splenocytes }\end{array}$ & 9,800 & - & $\begin{array}{l}\text { No stabilizers, purity }>99 \% \text {, no DMSO } \\
\text { inh. } 6 \mathrm{hr} \times 1\end{array}$ & Kligerman et al. (1994) \\
\hline $\begin{array}{l}\text { Sprague-Dawley CD rat (male) } \\
\text { Bone-marrow erythrocytes }\end{array}$ & 5 & + & $\begin{array}{l}\text { No stabilizers, purity }>99 \% \text {, no DMSO } \\
\text { inh. } 6 \mathrm{hr} \times 1 \\
\text { Dose-related increases from } 5 \text { to } 5,000 \mathrm{ppm} \text {; findings } \\
\text { confirmed in repeated study of high dose }\end{array}$ & Kligerman et al. (1994) \\
\hline $\begin{array}{l}\text { Sprague-Dawley CD rat (male) } \\
\text { Bone-marrow erythrocytes }\end{array}$ & 960 & - & $\begin{array}{l}\text { No stabilizers, purity }>99 \% \text {, no DMSO } \\
\text { inh. } 6 \mathrm{hr} \times 4 \\
\text { Authors note concurrent controls were unusually high }\end{array}$ & Kligerman et al. (1994) \\
\hline $\begin{array}{l}\text { Sprague-Dawley CD rat (male) } \\
\text { Bone marrow erythrocytes }\end{array}$ & 5,000 & - & $\begin{array}{l}\text { Purity } 99.97 \% \text {, no DMSO } \\
\text { inh. } 6 \mathrm{hr} \times 1\end{array}$ & Wilmer et al. (2014) \\
\hline $\begin{array}{l}\text { Sprague-Dawley CD rat (male) } \\
\text { Peripheral blood lymphocytes }\end{array}$ & 8,800 & - & $\begin{array}{l}\text { No stabilizers, purity }>99 \% \text {, no DMSO } \\
\text { inh. } 6 \mathrm{hr} \times 1\end{array}$ & Kligerman et al. (1994) \\
\hline $\begin{array}{l}\text { Sprague-Dawley CD rat (male) } \\
\text { Peripheral blood lymphocytes }\end{array}$ & 960 & - & $\begin{array}{l}\text { No stabilizers, purity }>99 \% \text {, no DMSO } \\
\text { inh. } 6 \mathrm{hr} \times 4\end{array}$ & Kligerman et al. (1994) \\
\hline $\begin{array}{l}\text { Sprague-Dawley CD rat (male) } \\
\text { Kidney cells }\end{array}$ & 3,591 & + & $\begin{array}{l}\text { Purity unknown, reagent grade, no DMSO } \\
\text { p.o. in EtOH } \times 1\end{array}$ & Robbiano et al. (2004) \\
\hline
\end{tabular}


RoC Monograph on Trichloroethylene

\begin{tabular}{|c|c|c|c|c|}
\hline $\begin{array}{l}\text { Test System/ } \\
\text { Endpoint }\end{array}$ & LED/HID & Results & Purity, Methods, and Comments & Reference \\
\hline \multicolumn{5}{|l|}{ Chromosomal aberrations } \\
\hline $\begin{array}{l}\text { C57BL/6J mouse (male) } \\
\text { Splenocytes }\end{array}$ & 9,800 & - & $\begin{array}{l}\text { No stabilizers, purity }>99 \% \text {, no DMSO } \\
\text { inh. } 6 \mathrm{hr} \times 1\end{array}$ & Kligerman et al. (1994) \\
\hline $\begin{array}{l}\text { Sprague-Dawley CD rat (male) } \\
\text { Peripheral blood lymphocytes }\end{array}$ & 8,800 & - & $\begin{array}{l}\text { No stabilizers, purity }>99 \% \text {, no DMSO } \\
\text { inh. } 6 \mathrm{hr} \times 1\end{array}$ & Kligerman et al. (1994) \\
\hline $\begin{array}{l}\text { Sprague-Dawley CD rat (male) } \\
\text { Peripheral blood lymphocytes }\end{array}$ & 960 & - & $\begin{array}{l}\text { No stabilizers, purity }>99 \% \text {, no DMSO } \\
\text { inh. } 6 \mathrm{hr} \times 4\end{array}$ & Kligerman et al. (1994) \\
\hline $\begin{array}{l}\text { CD-1 mouse } \\
\text { Bone-marrow cells }\end{array}$ & 1,000 & - & $\begin{array}{l}\text { Purity unknown, use of DMSO unknown } \\
\text { p.o. } \times 1\end{array}$ & $\begin{array}{l}\text { Loprieno and Abbondandolo } \\
\text { (1980) }\end{array}$ \\
\hline \multicolumn{5}{|l|}{ Sister chromatid exchange } \\
\hline $\begin{array}{l}\text { C57BL/6J mouse (male) } \\
\text { Splenocytes }\end{array}$ & 9,800 & - & $\begin{array}{l}\text { No stabilizers, purity }>99 \% \text {, no DMSO } \\
\text { inh. } 6 \mathrm{hr} \times 1\end{array}$ & Kligerman et al. (1994) \\
\hline $\begin{array}{l}\text { Sprague-Dawley CD rat (male) } \\
\text { Peripheral blood lymphocytes }\end{array}$ & 8,800 & - & $\begin{array}{l}\text { No stabilizers, purity }>99 \% \text {, no DMSO } \\
\text { inh. } 6 \mathrm{hr} \times 1\end{array}$ & Kligerman et al. (1994) \\
\hline $\begin{array}{l}\text { Sprague-Dawley CD rat (male) } \\
\text { Peripheral blood lymphocytes }\end{array}$ & 960 & - & $\begin{array}{l}\text { No stabilizers, purity }>99 \% \text {, no DMSO } \\
\text { inh. } 6 \mathrm{hr} \times 4\end{array}$ & Kligerman et al. (1994) \\
\hline \multicolumn{5}{|l|}{ DNA single-strand breaks } \\
\hline $\begin{array}{l}\mathrm{B} 6 \mathrm{C} 3 \mathrm{~F}_{1} \text { mouse (male) } \\
\text { Liver }\end{array}$ & 2,000 & - & $\begin{array}{l}\text { No stabilizers, purity unknown, use of DMSO unknown } \\
\text { i.p. } \times 1\end{array}$ & Parchman and Magee (1982) \\
\hline $\begin{array}{l}\text { NMRI mouse (male) } \\
\text { Kidney, liver, lung }\end{array}$ & $\begin{array}{c}790 \\
1,300\end{array}$ & $\begin{array}{l}+ \text { (kidney, liver) } \\
\quad-\text { (lung) }\end{array}$ & $\begin{array}{l}\text { No stabilizers, purity } 99.5 \% \text {, no DMSO } \\
\text { Alkaline unwinding } \\
\text { i.p. in Tween- } 80 \times 1\end{array}$ & Walles (1986) \\
\hline $\begin{array}{l}\mathrm{B} 6 \mathrm{C} 3 \mathrm{~F}_{1} \text { mouse (male) } \\
\text { Liver }\end{array}$ & 1,500 & + & $\begin{array}{l}\text { Purity }>99 \% \text {, no DMSO } \\
\text { DNA single strand breaks } \\
\text { Alkaline unwinding } \\
\text { p.o. in Tween- } 80(1 \%) \times 1\end{array}$ & Nelson and Bull (1988) \\
\hline $\begin{array}{l}\text { Mouse spot test in (DNA } \\
\text { alternations) embryos from } \\
\text { treated dams }\end{array}$ & 350 & - & $\begin{array}{l}\text { No stabilizers, purity } 99.5 \% \text {, no DMSO } \\
\text { i.p. } \times 1\end{array}$ & Fahrig (1977) \\
\hline
\end{tabular}


RoC Monograph on Trichloroethylene

\begin{tabular}{|c|c|c|c|c|}
\hline $\begin{array}{l}\text { Test System/ } \\
\text { Endpoint }\end{array}$ & LED/HID & Results & Purity, Methods, and Comments & Reference \\
\hline $\begin{array}{l}\text { Sprague-Dawley rat (male) } \\
\text { Liver }\end{array}$ & 3,000 & + & $\begin{array}{l}\text { Purity }>99 \% \text {, no DMSO } \\
\text { Alkaline unwinding } \\
\text { p.o. in Tween- } 80(1 \%) \times 1\end{array}$ & Nelson and Bull (1988) \\
\hline $\begin{array}{l}\text { Sprague-Dawley CD rat (male) } \\
\text { Kidney }\end{array}$ & 3,591 & + & $\begin{array}{l}\text { Purity reagent grade, no DMSO } \\
\text { Comet assay } \\
\text { p.o. in EtOH }\end{array}$ & Robbiano et al. (2004) \\
\hline $\begin{array}{l}\text { Sprague-Dawley CD rat (male) } \\
\text { Kidney }\end{array}$ & $\begin{array}{c}2,000 \mathrm{ppm} \\
{\left[\begin{array}{c}\sim 0,800 \mathrm{mg} / \\
\left.\mathrm{kg} / \text { day }^{\mathrm{a}}\right]\end{array}\right.}\end{array}$ & - & $\begin{array}{l}\text { Purity } 99.5 \% \text {, no DMSO } \\
\text { Comet assay } \\
\text { inh. } 6 \mathrm{hr} \times 5\end{array}$ & Clay (2008) \\
\hline \multicolumn{5}{|l|}{ UDS (DNA repair) } \\
\hline $\begin{array}{l}\text { Fischer } 344 \text { rat (male) } \\
\text { Primary hepatocytes }\end{array}$ & 1,000 & - & $\begin{array}{l}\text { Purity unknown, no DMSO } \\
\text { p.o. corn oil or water } \times 1\end{array}$ & Mirsalis et al. (1989) \\
\hline $\begin{array}{l}\mathrm{B} 6 \mathrm{C} 3 \mathrm{~F}_{1} \text { mouse (male and female) } \\
\text { Primary hepatocytes }\end{array}$ & 1,000 & - & $\begin{array}{l}\text { Purity unknown, no DMSO } \\
\text { p.o. corn oil or water } \times 1\end{array}$ & Mirsalis et al. (1989) \\
\hline $\begin{array}{l}\text { CD-1 mouse (male) } \\
\text { Primary hepatocytes }\end{array}$ & 1,000 & - & $\begin{array}{l}\text { No stabilizers, no DMSO } \\
\text { p.o. in corn oil } \times 1\end{array}$ & Doolittle et al. (1987) \\
\hline
\end{tabular}


RoC Monograph on Trichloroethylene

Table C-6. Cytogenetic Studies in Peripheral Blood Lymphocytes from Trichloroethylene-exposed Workers

\begin{tabular}{|c|c|c|c|c|}
\hline Reference & Population & Exposure Group & Findings & Comments \\
\hline \multirow{3}{*}{$\begin{array}{l}\text { Rasmussen et al. } \\
\text { (1988) } \\
\text { Denmark }\end{array}$} & \multirow{3}{*}{$\begin{array}{l}15 \text { metal degreaser workers exposed } \\
\text { to TCE }>20 \mathrm{hr} / \mathrm{wk} \\
669 \text { controls from population-based } \\
\text { study and survey of parents with } \\
\text { offspring with stable chromosomal } \\
\text { abnormalities in same geographical } \\
\text { location }\end{array}$} & \multirow{2}{*}{$\begin{array}{l}\text { Exposed } \\
\text { Controls }\end{array}$} & $\begin{array}{l}\text { CA: breaks } \\
1.90(1.22-2.84) \\
0.85(0.66-1.08)^{* * *}\end{array}$ & $\begin{array}{l}15 \text { workers identified from a total of } 116 \\
\text { workers }\end{array}$ \\
\hline & & & $\begin{array}{l}\text { CA: other } \\
1.35(0.79-2.16)^{* * *} \\
0.15(0.08-0.27)\end{array}$ & $\begin{array}{l}\text { Control population may not be comparable to } \\
\text { workers although the large size may reduce } \\
\text { any potential selection bias } \\
\text { Other chromosomal aberrations include } \\
\text { translocations, deletions, and inversions }\end{array}$ \\
\hline & & $\begin{array}{l}\text { Exposed } \\
\text { Controls }\end{array}$ & $\begin{array}{l}\text { CA: hyperdiploid } \\
0.79(0.38-1.46)^{*} \\
0.24(0.15-0.38)\end{array}$ & \\
\hline \multirow{3}{*}{$\begin{array}{l}\text { Seiji et al. (1990) } \\
\text { Japan }\end{array}$} & \multirow{3}{*}{$\begin{array}{l}38 \text { TCE-exposed }(22 \mathrm{M}, 16 \mathrm{~W}) \\
7 \mathrm{ppm} \\
51 \text { controls }(26 \mathrm{M}, 25 \mathrm{~W}) \text { matched } \\
\text { on age, sex, smoking habits, and } \\
\text { geographical location }\end{array}$} & Men & \multirow[b]{2}{*}{$\begin{array}{l}S C E \\
7.06 \pm 1.38^{* *} \\
5.10 \pm 1.16 \\
6.46 \pm 1.25 \\
5.78 \pm 1.64\end{array}$} & Workers TCE synthesis and degreasers \\
\hline & & $\begin{array}{l}\text { Exposed smoker (8) } \\
\text { Control smokers }(7) \\
\text { Exposed non-smokers (14) } \\
\text { Control non-smokers (19) }\end{array}$ & & $\begin{array}{l}\text { TCE exposure levels }(\mathrm{ppm}) \text { were higher in } \\
\text { women }(3-32 \mathrm{ppm}) \text { than men }(2-10 \mathrm{ppm}) \text {; } \\
\text { duration was shorter in women }(\sim 70 \text { months }) \\
\text { than men ( } 120 \text { months) }\end{array}$ \\
\hline & & $\begin{array}{l}\text { Women } \\
\text { Exposed non-smokers (16) } \\
\text { Control non-smokers (25) }\end{array}$ & $\begin{array}{l}6.15 \pm 1.34 \\
6.25 \pm 1.42\end{array}$ & No independent effect of smoking \\
\hline \multirow{3}{*}{$\begin{array}{l}\text { Nagaya et al. } \\
(1989) \\
\text { Japan }\end{array}$} & \multicolumn{2}{|c|}{22 TCE-exposed workers ( $\sim 30 \mathrm{ppm})$} & \multirow{3}{*}{$\begin{array}{l}S C E \\
7.7 \pm 1.3 \\
8.0 \pm 1.4\end{array}$} & \multirow{2}{*}{$\begin{array}{l}\text { Employment duration } 0.7-34 \text { years, mean } \\
9.7 \text { years }\end{array}$} \\
\hline & \multirow{2}{*}{$\begin{array}{l}22 \text { workers without exposure to } \\
\text { solvents and matched on age, and } \\
\text { sex, and smoking habits }\end{array}$} & \multirow{2}{*}{$\begin{array}{l}\text { Exposed } \\
\text { Controls }\end{array}$} & & \\
\hline & & & & $\begin{array}{l}\text { Estimated exposure } 30 \mathrm{ppm} \text {, based on urinary } \\
\text { total trichloro compounds, but large range in } \\
\text { exposure levels }\end{array}$ \\
\hline \multirow{2}{*}{$\begin{array}{l}\text { Gu et al. (1981) } \\
\text { (Cited from IARC } \\
\text { 2014) }\end{array}$} & \multirow{2}{*}{$\begin{array}{l}6 \text { TCE-exposed workers } \\
9 \text { controls }\end{array}$} & & $S C E$ & \multirow{2}{*}{$\begin{array}{l}\text { Exposure assessed by measurement of TCE } \\
\text { and metabolite (U-TCA) in blood }\end{array}$} \\
\hline & & $\begin{array}{l}\text { Exposed } \\
\text { Controls }\end{array}$ & $\begin{array}{l}9.045 \pm 4.898 \\
7.910 \pm 2890\end{array}$ & \\
\hline
\end{tabular}




\section{Appendix D. Human Cancer Study Tables}

\section{Table of Contents}

D.1. Methodologies and Study Characteristics of the Selected Epidemiologic Studies and Identification of Cancer Endpoints..................................................................... D-2

D.2. Assessment of Potential Biases and Other Characteristics ......................................... D-31

\section{Tables}

Table D-1. Study Descriptions and Methodologies: Cohort Studies of Trichloroethylene

Exposure

Table D-2. Study Descriptions and Methodologies: Case-control Studies of

Trichloroethylene Exposure and Kidney Cancer

Table D-3. Study Descriptions and Methodologies: Case-control Studies of

Trichloroethylene Exposure and NHL and Related Subtypes

Table D-4. Cohort and Nested Case-control Studies of Trichloroethylene Exposure:

Summary of Study Quality....

Table D-5. Cohort Studies: Study Sensitivity and Exposure-response Analyses

Table D-6. Case-control Studies of Trichloroethylene and Kidney or Liver Cancer: Study Quality....

Table D-7. Kidney Case-control Studies: Study Sensitivity and Exposure Response Analyses

Table D-8. Case-control Studies of Trichloroethylene and NHL and Related Subtypes:

Summary of Study Quality.

Table D-9. NHL Case-control Studies: Study Sensitivity and Exposure Response Analysis

Table D-10. Studies Included in Three Meta-analyses by Cancer Site 
This appendix contains background information related to the cancer assessment on trichloroethylene in humans including detailed (1) data information on study design, methods, and findings for human cancer studies (Table D-1, Table D-2, and Table D-3) and (2) detailed information on the quality assessment of the individual studies (Table D-4, Table D-5, Table D-6, Table D-7, Table D-8, and Table D-9) and (3) studies included in several metaanalyses (Table D-10).

\section{D.1. Methodologies and Study Characteristics of the Selected Epidemiologic Studies and Identification of Cancer Endpoints}

The data from the 16 cohort studies, which include two nested case-control studies (Table D-1), 7 kidney case-control studies, including 1 that reported on liver cancer (Table D-2), and 11 NHL or related subtypes case-control studies (Table D-3), which includes 1 pooled study and the 3 constituent studies, were systematically extracted from relevant publications and are summarized in the tables below. The cohort studies are organized according to several broad occupational groups related to the exposure scenarios or occupations similar to Table 3-1, and the case-control studies are organized similar to Table 3-2 and Table 3-3.

Table D-1. Study Descriptions and Methodologies: Cohort Studies of Trichloroethylene Exposure

\begin{tabular}{|c|c|}
\hline & Study Description and Methodology \\
\hline \multicolumn{2}{|l|}{ Vlaanderen et al. (2013) } \\
\hline Related References & Kauppinen et al. (2009); Pukkala et al. (2009) \\
\hline Geographic Location & Denmark, Finland, Iceland, Norway, Sweden \\
\hline \multicolumn{2}{|c|}{ Population Characteristics } \\
\hline \multirow[t]{4}{*}{$\begin{array}{l}\text { Cases: selection and } \\
\text { ascertainment }\end{array}$} & $\begin{array}{l}\text { Cases: Kidney cancer: 44,708 M, 31,422 F; Liver cancer: 14,702 M, 9,194 F; NHL: } \\
\text { 36,487 M, 32,767 F; MM 187,777 M, 16,757 F }\end{array}$ \\
\hline & $\begin{array}{l}\text { Eligibility criteria (cohort): All men and women aged } 30-64 \text { years old participating in } \\
\text { the } 1960,1970,1980-1981 \text { and/or } 1990 \text { censuses in participating countries and alive on } \\
\text { Jan } 1 \text { of year following the census }\end{array}$ \\
\hline & $\begin{array}{l}\text { Cohort: NOCCA Cohort : } 45 \text { years of cancer incidence and follow-up for } 15 \text { million } \\
\text { people }\end{array}$ \\
\hline & $\begin{array}{l}\text { Case identification and ascertainment: Linkage to cancer registries (incident cases) and } \\
\text { followed by linkage to population registries }\end{array}$ \\
\hline \multirow[t]{3}{*}{$\begin{array}{l}\text { Controls: selection and } \\
\text { ascertainment }\end{array}$} & $\begin{array}{l}\text { Referents: Kidney cancer: 223,540 M, 157,110 F; Liver cancer: 73,510 M, 45,970 F; } \\
\text { NHL: } 182,435 \text { M, 163,835 F; MM: 93,885 M, 83,785 F }\end{array}$ \\
\hline & $\begin{array}{l}\text { Referent eligibility criteria: } \\
\text { cancer }\end{array}$ \\
\hline & $\begin{array}{l}\text { Matching criteria: Age }( \pm 1 \mathrm{yr}) \text {, sex, country; } 5 \text { controls per case and without cancer at } \\
\text { time of case diagnosis }\end{array}$ \\
\hline Follow-up & $\begin{array}{l}\text { Date of } 1 \text { st entry into census to emigration, death, or end of } 2003 \text { (Norway), } 2004 \\
\text { (Iceland), } 2005 \text { (Sweden, Finland) }\end{array}$ \\
\hline $\begin{array}{l}\text { Study Design and } \\
\text { Analytical Methods }\end{array}$ & $\begin{array}{l}\text { Cancer registry and census record linkage study (Pukkala et al. 2009); Nested case- } \\
\text { control design; Analysis of hazard ratio by exposure to TCE using conditional logistic } \\
\text { regression for tertiles of cumulative exposure and continuous cumulative exposure } \\
\text { (spline or linear); Non-exposed participants as internal controls; } 1-, 5-, 10 \text { - and } 20 \text {-year } \\
\text { lagging explored but had minimal effect and thus unlagged models used }\end{array}$ \\
\hline
\end{tabular}




\section{Study Description and Methodology}

Exposure Data and Information Assessment

Exposure: levels and co- Levels: NR. Overall prevalence of exposure to TCE low (5\%) exposures

High estimated levels of exposure to TCE were found in shoe and leather industry workers, mechanics, laundry and smelting workers

Moderate correlation between TCE and tetrachloroethylene $(\mathrm{r}=0.58-0.63)$, chlorinated hydrocarbons $(\mathrm{r}=0.56-0.61)$ and $1,1,1$-trichloroethane $(\mathrm{r}=0.37-0.43)$; No association between TCE and benzene and ionizing radiation

Exposure assessment Census questionnaire data was used to construct country-specific and calendar timespecific quantitative JEM for 29 agents for NOCCA

Person-yr of exposure started at age 20 or age at first job until death, emigration, cancer diagnosis or age 65. Assume same exposure between census reports. Exposures before first census report assumed same since age of first entry into cohort

Cumulative exposure $=$ prevalence $\times$ level of exposure by calendar year $\times$ lifetime employment duration. High exposure $=$ exposure to levels $>90$ th percentile of cumulative exposure or average intensity $\times$ prevalence

Assessment of potential NR confounders

Disease assessment

ICD-7; NHL 200+202, MM 203

Hansen et al. (2013)

Related References

Anttila et al. (1995); Axelson et al. (1978); Axelson et al. (1994); Hansen et al. (2001); Tola et al. (1980)

Geographic Location Sweden, Finland, Denmark

Population Characteristics

Exposed cohort and

Eligibility criteria: All workers provided with urine TCA monitoring in Sweden (1955ascertainment

1975), Finland (1965-1982), and Denmark (1947-1989) $\geq 1$ U-TCA measurement

Exposed cohort: 5,553 workers (3,776 men; 1,777 women); total 154,778 person-yr of observation

Follow-up: Sweden, 1958-2003; Finland, 1967-2004; Denmark, 1968-2008

Loss to follow-up: $0.1 \%$

Reference population National rates (Sweden, Finland, Denmark)

All-cause and all-cancer All-cause incidence (SIR): NR

mortality/incidence

All-cancer incidence $($ SIR $)=1.06(0.99-1.13) ; 997$

Study Design and Analytical Pooled and extended analysis of three historical cohort cancer incidence (registry) studies

Methods/Control for Confounding

External analysis (by sex, age, and calendar period) with 10- and 20-year exposure lagging; Internal analysis by U-TCA category (average level) using Cox regression to estimate hazard rate ratios adjusted for age, sex, calendar period, country; Indirectly evaluated potential confounding by smoking and alcohol consumption by calculating combined SIR of smoking- or alcohol-related cancers)

Additional analyses in earlier updates for the 3 cohorts: Swedish study evaluated risk (SIR, SMR) of U-TCA stratified by exposure time ( $<$ and $>2$ years). Danish study also evaluated cancer risk by period of first employment, duration of employment, mean and cumulative exposure (air calculated from U-TCA) with 10- and 20-year lagging and stratified by sex 


\section{Study Description and Methodology}

Exposure Data and Information Assessment

Exposure: levels and co- Mean/median urine TCA levels $(\mathrm{mg} / \mathrm{L})^{\mathrm{a}}$ exposures

Mean $\pm \mathrm{SD}$ Median \% samples $>50 \mathrm{mg} / \mathrm{L}$

Sweden: $\quad 28.2 \pm 40 \quad 13.0 \quad 17.7$

Finland: $\quad 30.3 \pm 82 \quad 9.2 \quad 13.3$

Denmark: $39.2 \pm 78 \quad 15.0 \quad 20.7$

No data on cumulative exposure or exposure duration; Mean duration of employment: $5.5 \mathrm{yr}$ (Sweden) and 6.3 (Denmark), NR for Finland, 81\% of Swedish cohort with $<20$ ppm ambient TCE

Finland: Estimated TCE exposures were $\sim 4 \mathrm{ppm}$ (median) and $6 \mathrm{ppm}$ (mean). Denmark: Overall calculated air concentrations (urinary TCA to air) $=1-2 \mathrm{ppm}\left(65 \mathrm{mg} / \mathrm{m}^{3}\right)$ mean, $3.53 \mathrm{ppm}\left(19 \mathrm{mg} / \mathrm{m}^{3}\right)$ median

Exposure assessment Individual Urine TCA measurements (national surveillance program); Most (65\%-66\%) of the first U-TCA samples were taken after 1970. Few measurements (usually 2 or 3 ) were available for each individual. Employment history was available in the Denmark cohort

Co-exposures (Finland)

$\begin{array}{llll} & \underline{\text { TCA }} & \underline{\text { Perc }} & \underline{\text { TCE }} \\ \text { Urine }(\mu \mathrm{mol} / \mathrm{L}) & 48-53 & \text { NR } & \text { NR } \\ \text { Air }(\mathrm{ppm}) & 6 \text { avg } & <50 & 79 \text { avg } \\ \text { Blood }(\mu \mathrm{mol} / \mathrm{L}) & \mathrm{NR} & 0.4-0.7 & 20-25\end{array}$

Assessment of potential NR confounders

Disease assessment Personal identification number linked to Central Person Registers to ascertain vital status; linkage to national cancer registries. ICD-7 (modified)

\section{Raaschou-Nielsen et al. (2003)}

Related References Raaschou-Nielsen et al. (2001); Raaschou-Nielsen et al. (2002); Note: cohort partly overlaps that of Hansen et al. (2001)

Geographic Location Denmark

Population Characteristics

Exposed cohort and ascertainment
Eligibility criteria: All male and female blue-collar workers employed $\geq 3$ months in 347 companies using TCE with $<200$ employees; Potentially higher exposed workers had $>1$ year duration of employment and were first employed before 1980

Potentially exposed cohort: 40,049 blue-collar workers in 347 TCE-using companies; 588,047 person-yr of exposure (men), 118,270 person-yr (women); 14,360 with potential higher exposure to TCE; TCE-using companies identified via Danish Institute for Occupational Health, Danish Product Registry, dry cleaning survey and files of main TCE producer

Follow-up: 1968-1997

Loss to follow-up: NR, "Virtually complete"

Reference population
Danish population 


\section{Study Description and Methodology}

All-cause and all-cancer All cause incidence (SIR): NR

mortality/incidence

All-cancer incidence:

SIR: 1.08 (1.04-1.12); 2,620 (men)

SIR: 1.23 (1.14-1.33); 624 (women)

Study Design and Analytical

Historical cohort incidence (registry) study; External SIR analysis, adjusted for sex, age, Methods/Control for Confounding and calendar year, by lag time, calendar period, duration of employment, size of company; Separate analysis on potential high exposure by the same variables; No analysis for potential confounding from co-exposure or lifestyle factors; Sensitivity analysis on excluded workers ( $<3$ months employment)

Exposure Data and Information Assessment

Exposure: levels and co- All workers in Danish TCE measurement registry: (Raaschou-Nielsen et al. 2001; exposures

Raaschou-Nielsen et al. 2002)

1960-1964: mean U-TCA $=58 \mathrm{mg} / \mathrm{L}(21 \mathrm{ppm} \text { TCE air })^{\mathrm{a}}$

1960s: mean air TCE $=318 \mathrm{mg} / \mathrm{m}^{3}$ (59 ppm TCE air)

1980-1985: mean U-TCA $=14 \mathrm{mg} / \mathrm{L}(5 \mathrm{ppm} \text { TCE air })^{\mathrm{a}}$

1980s: mean air TCE $=75 \mathrm{mg} / \mathrm{m}^{3}(14 \mathrm{ppm})$

Co-exposures NR, Industries include iron and metal (>50\%), electronics, painting, printing, chemicals, dry cleaning

Exposure assessment Potentially exposed workers identified from Central Population Registry (1968 on) and Danish Pension Fund (compulsory membership since 1964)

Job title and individual employment history (duration and year of first employment) obtained from Danish Pension Fund. Size of company also used as a surrogate for prevalence of TCE. $81 \%, 51 \%$, and $19 \%$ of the blue-collar workers in small $(<50$ employees) medium (50-100) and large (>100) companies, respectively, estimated to be exposed to TCE

No exposure data on individual workers

Assessment: other

NR

exposures

Disease assessment

Danish Cancer Registry

Modified ICD-7

Lipworth et al. (2011)

Related References

Boice et al. (1999) (errata published in Boice and McLaughlin (2001)); Marano et al. (2000)

Geographic Location Burbank, CA (USA)

Population Characteristics

Exposed cohort and

Eligibility criteria: All aircraft manufacturing workers employed $\geq 1$ year from 1960 ascertainment

Exposed cohort: 5,443 M+F (180,704 person-yr)

Total cohort: 77,943 Aircraft mfg. workers at Lockheed Martin (Burbank)

Follow-up: 1960-2008 or age 95 (avg 32 yr)

Loss to follow-up: $1.7 \%$ total cohort

Reference population California (white workers) and USA (non-white workers) 


\section{Study Description and Methodology}

All-cause and all-cancer All-cause mortality: SMR: 0.91 (0.88-0.93); 4,070

mortality/incidence $\quad$ All-cancer mortality: SMR: 0.92 (0.86-0.97); 986

Study Design and Historical cohort mortality study; External analysis: adjusted for age, sex, and calendar Analytical Methods/Control for Confounding period; Internal analyses: Cox proportional hazard models for specific cancer by duration of exposure and exposure pattern adjusting for age, date of birth, date of hire, termination date, sex, and race; No control for potential confounding from co-exposures and lifestyle factors

Exposure Data and Information Assessment

Exposure: levels and co- No quantitative exposure assessment exposures

TCE used for vapor degreasing up to 1966 , replaced by tetrachloroethylene

Approx. $12 \%$ workers with routine TCE exposure, $30 \%$ routine or intermittent TCE exposure

Co-exposures: PCE, chromate, mixed solvents (including methyl ethyl ketone, alcohols, petroleum distillates, 1,1,1-trichloroethane, methylene chloride, methyl isobutyl ketone, acetone, toluene, xylene, freons), cutting fluids, lubricants

Exposure assessment Qualitative JEM; Occupational job groups developed by industrial hygienists based on walk-through survey, veteran employee interviews and historical industrial hygiene surveys and reports

Individuals classified as ever/never, routine, or intermittent exposure to TCE and coexposures (PCE, and mixed solvents) (Boice et al. 1999) and by duration of potential exposure to each substance (Lipworth et al. 2011)

Assessment: other NR

exposures

Disease assessment

California death files, National Death Index, state vital statistics records, vital records search company; Pension Benefit Information Files, Social Security Death Index, Health Care Financing Administration beneficiary files, California DMV, work and pension records

Nosologist coded cause of death from death certificates using ICD in use at time of death, underlying cause of death

Radican et al. (2008)

Related References

Spirtas et al. (1991), Stewart et al. (1991), Blair et al. (1998) (mortality and incidence)

Geographic Location

Utah (USA)

Population Characteristics

Exposed cohort and ascertainment

Reference population
Eligibility criteria: employed $\geq 1$ year $1952-1956$

Exposed cohort: 7,204 (6,153 men, 1,051 women) TCE-exposed workers

Total cohort: 10,730 male and 3,725 female civilian aircraft maintenance workers (at Hill Force military base)

Follow-up: mortality1991-2000; incidence 1973-1990

Loss to follow-up: NR

USA (mortality; Radican et al. (2008)) and Utah (cancer registry; Blair et al. (1998))

Non-chemical-exposed workers (internal analysis) 


\section{Study Description and Methodology}

All-cause and all-cancer Radican et al. (2008) (internal analysis)

mortality/incidence

Study Design and Analytical Methods/Control for Confounding
All-cause mortality HR = $1.04(0.98-1.11) ; 3,628$

All-cancer mortality HR = $1.12(0.96-1.30) ; 729$

Historical cohort mortality/incidence study; Internal analyses (External analysis reported for 1990 follow-up for mortality only)

2000 follow-up (mortality): Cox proportional hazards model, using age as variable and adjusting for race, stratified by sex and considering calendar year; 1990 follow-up (mortality and incidence): Poisson multivariate regression analysis adjusted for age, calendar year, and sex. Both models were used to evaluate TCE exposure-response by cumulative exposure and exposure patterns (mortality only). Separate analysis for other chemical exposures, no multivariate analysis controlling for potential confounding from exposure to other substances or lifestyle factors

Exposure Data and Information Assessment

Exposure: level and co- No quantitative exposure (air) assessment specific for TCE, but air measurements exposures

available on vapor degreasing and other solvents. Estimated TCE exposures (ppm) were:

$$
\text { Peak Low level }
$$

1939-54 $600 \quad 10$

$1955-67 \quad 400 \quad 10$

$1968-78 \quad 200 \quad 0$

1979-83 $0 \quad 0$

Cherrie et al. (2001) estimated long-term exposure as $50 \mathrm{ppm}$ and short term up to $600 \mathrm{ppm}$. The NAS concluded the cohort had a modest number of highly exposed ( $\sim 100 \mathrm{ppm})$, but most were exposed to low TCE concentrations ( $10 \mathrm{ppm})$

Co-exposures: Ever/never exposure for 1,1,1-trichloroethane, methylene chloride, carbon tetrachloride, freon, isopropyl alcohol, acetone, ketone, toluene, methyl ethyl ketone, $o$-dichlorobenzene, perchloroethylene, chloroform, Stoddard solvent, xylene, styrene, JP4 gasoline, metal fumes/dust, silica, zinc chromate, nitroglycerine, solder flux

Exposure assessment All exposures: Walk-through surveys; veteran employee assessment; individual work and job histories from personnel records; Process descriptions were used to develop ever vs. never exposure to 21 solvents and chemicals for each job-department combination

TCE: Semi-quantitative individual exposure assessment, calendar-year specific; Detailed job task descriptions used to develop exposure score for each job based on relative exposure intensity for each calendar period, frequency of use and duration of use. Cumulative exposure (unit-years) was the sum of exposure scores $\times$ job duration across jobs (Stewart et al. 1991). Workers also assigned to patterns or types of exposure (e.g., low level, peak, continuous, or intermittent)

\section{Boice et al. (2006)}

Related References

Overlaps cohort of Ritz et al. (1999) and Zhao et al. (2005) (see above)

Geographic Location
Los Angeles (USA) 


\section{Study Description and Methodology}

Population Characteristics

Exposed cohort and ascertainment
Eligibility criteria: male Rocketdyne rocket engine testing workers employed $\geq 6$ months from Jan 1, 1948 on and with adequate work histories and identifying data

Exposed cohort: 1,111 test stand mechanics with any estimated exposure to TCE or hydrazine

Total cohort: 8,372 Rocketdyne Aerospace workers (7,083 M, 1,289 F) at the SSFL facility; 1,651 were test-stand mechanics

Follow-up: $1948-1999 ; \sim 88 \%$ of test stand mechanics were followed for over 20 years.

Loss to follow-up: $0.4 \%$ test stand mechanics

Reference population External: US population

Internal: Hourly non-administrative Rocketdyne workers at SSFL and adjacent facilities

All-cause and all-cancer All-cause mortality: SMR $=0.87(0.78-0.96) ; 391$

mortality/incidence

All-cancer mortality: SMR $=1.00(0.83-1.19) ; 121$

Study Design and

Analytical

Methods/Control for

Confounding

Historical cohort mortality study; External (all cancers) adjusted for age, race, calendar year. Internal analysis (selected cancers including kidney but not NHL or liver cancer) using Cox proportional hazard models, adjusting for date of birth, year of hire, pay type (surrogate for SES) and exposure to hydrazine (for TCE analyses and TCE for hydrazine analyses) for any exposure, duration of employment with potential exposure to TCE or hydrazine, and number of engine test flushes using TCE

Exposure Data and Information Assessment

Exposure: levels and co- No quantitative exposure assessment exposures

TCE used for engine flush to mid-1960s, used as utility solvent to 1974. Approx. 58\% exposed to TCE during engine flushing/cleaning (high exposure); Approx. $42 \%$ exposed to TCE during utility cleaning (lower exposure). PPE only used in later years

Co-exposures: hydrazine, mixed solvents, rocket fuels, oxidizers, exhaust gases, other chemicals, radiation

$8.4 \%(\mathrm{~N}=121)$ exposed to both hydrazine and TCE

Exposure assessment Qualitative exposure assessment to TCE; Walk-through surveys and veteran employees' assessments used to determine dates that TCE was used at test strands as a utility solvent or to flush engines. Did not consider PPE. Comprehensive job history based on dates and job titles used to assign workers to specific test stands

Workers assigned to (1) duration of employment for potential exposure to TCE and (2) duration (test years) of exposure to TCE from engine flush, which was weighted by number of engine tests per year accounting for the number of workers that year

Assessment: other exposures

Disease assessment
Smoking history (pack-yr) among subsample of 600 workers

SSA, California death index, NDI, state vital records, Pension Benefit Information Files, Medicare and Medicaid Services data, company personnel, pension and retirement records

ICD in use at time of death

\section{Zhao et al. (2005)}

Related References

Geographic Location
Morgenstern et al. (1997); Ritz et al. (1999). Members of cohort also part of separate larger mortality cohort study of Boice et al. (2006)

Los Angeles, CA (USA) 


\section{Study Description and Methodology}

Population Characteristics

Exposed cohort and Eligibility criteria: Male workers at Rocketdyne aerospace facility 1950-1980 with ascertainment $\geq 2$ years' employment and no radiation exposure

Exposed cohort: 6,107 male aerospace workers at SSFL; mortality: 6,044; incidence: 5,049 workers; TCE-exposed workers (greater than intensity score 3): mortality 2,648; incidence 2,236

Total cohort: 55,000 Rockwell/Rocketdyne aerospace workers

Follow-up:

1950-2001 (mortality)

1988-2000 (incidence)

Average follow-up $29 \mathrm{yr}$

Loss to follow-up: $<1 \%$ for mortality

Reference population Mortality: US population

Incidence: California and 8 other state incidence rates

Internal analysis: Low TCE exposure category

All-cause and all-cancer All-cause and all cancer mortality (SMR): NR mortality/incidence

All-cause and all cancer incidence (SIR): NR

Study Design and Analytical Methods/Control for Confounding

Historical cohort mortality/incidence study; internal analyses; proportional hazards modeling with fixed and time-dependent variables; multivariate models for cumulative exposure (low-, medium-, and high-exposure intensity) in lagged and unlagged analyses included pay type (surrogate for SES), time since first employment (surrogate for survival), age, and co-exposures to other chemicals

Exposure Data and Information Assessment

Exposure: levels and co- Limited quantitative exposure assessment exposures Co-exposures: hydrazine, PAH, benzene (early years), mineral oil, gasoline, fuel oils

Exposure assessment Semi-quantitative JEM developed by industrial hygienists based on walk-through surveys; employees' assessments, job task manuals, review of company records for TCE, hydrazine, PAH, mineral oil; Work histories for each individual linked to JEM to generate calendar time-dependent intensity scores for each chemical exposure for each worker. Individual cumulative intensity scores (low/medium/high) based on estimated intensity of exposure in job/task $\times$ time in job

$1 \%$ workers missing job description; $3 \%$ workers with insufficient job descriptionexposure imputed from job title

Assessment: other Smoking data for subsample of 200 workers with medical questionnaire data exposures

Disease assessment Mortality: ICD-9 and 10; underlying and contributing causes of death Incidence: California Cancer Registry and 8 other state cancer registries. ICD-O (incidence). Reports all lymphohematopoietic cancers (excluding CLL) only, not NHL

Morgan et al. (1998)

Related References

Wong and Morgan (1990)

Geographic Location Arizona (USA) 


\section{Study Description and Methodology}

Population Characteristics

Exposed cohort and Eligibility criteria: All male and female aircraft manufacturing workers employed ascertainment $\geq 6$ months $1950-1985$

Exposed cohort: 4,733 (2,555 men; 2,178 women)

Total cohort: 20,508 aircraft manufacturing workers at the Hughes Aircraft

Manufacturing Site

Follow-up: 1950(?)-1993 (approx. 66\% followed for $>20 \mathrm{yr}$ )

Loss to follow-up: $0.1 \%$ excluded due to missing data (not clear if vital status or other data)

Reference population External analysis: NR (assume U.S. population)

Internal analysis: 11,187 male and 4,588 female unexposed workers; peak exposureused unexposed and low exposed workers as the reference group

All-cause and all-cancer All-cause mortality: SMR $=0.84$ (0.79-0.90); 917

mortality/incidence

All-cancer mortality/incidence:

SMR: 0.92 (0.81-1.03); 270

Study Design and

Analytical

Methods/Control for

Confounding
Historical cohort mortality study; External (SMR) analysis for TCE-exposed cohort, low and high exposure for multiple cancer sites; Internal analyses using Cox proportional hazards adjusting for age at hire, and sex used to evaluate cumulative (low and high) and peak exposure and selected cancer sites. No control for potential confounding from coexposures and lifestyle habits

Exposure Data and Information Assessment

Exposure: levels and co- Limited quantitative exposure levels available, especially before 1975. Before 1981, exposures plant had contaminated well water estimated between 730 and 2,200 ppb TCE for showers and drinking

High exposure $=$ work on degreaser machines using TCE (estimated to be $50 \mathrm{ppm}$ ); medium exposure $=$ jobs near degreasing area (occasional contact); low exposure $=$ jobs away from degreaser work

TCE used for vapor degreasing 1952-1977

Co-exposures: NR

Exposure assessment Semi-quantitative individual JEM based on veteran employees' plus company industrial hygienists' exposure rankings. Jobs classified into no, low, medium, high exposure scores

Cumulative exposure score (low, high) $=$ exposure category $\times$ duration of exposure.

Peak exposure $=$ jobs with medium and high exposure

Medium/low exposures may be misclassified

Assessment: other $\quad$ NR

exposures

Disease assessment

SSA; NDI; State death certificates; ICD-7, 8 or 9 in use at time of death

Silver et al. (2014)

Related References Clapp and Hoffman (2008); Fleming et al. (2014)

Geographic Location New York State, USA

Population Characteristics 


\section{Study Description and Methodology}

Exposed cohort and ascertainment
Eligibility criteria: Workers with 91 or more days of employment 1969-2001; contract and foreign national workers (or without SSN) excluded

Exposed cohort: 3,113 ever exposed to TCE

Total cohort: 34,494 (24,037 men, 10,457 women) employed a microelectronics business facility; hourly workers $=15,447 \mathrm{M}$ and 8,934 W

Follow-up: 1969-2009; average 25.7 years (total cohort)

Loss to follow-up: NR

Reference population US mortality rates, NY State mortality rates (excluding New York City)

All-cause and all-cancer All-cause mortality: SMR (all hourly workers)

mortality/incidence

M: 0.76 (0.73-0.78) 3571; F: $0.73(0.68-0.79) 823$

All-cancer mortality: SMR (all hourly workers)

M: 0.83 (0.78-0.88) 1005; F: $0.86(0.76-0.96) 291$

Study Design and Analytical Methods/Control for Confounding
Historical cohort mortality study. External analyses: SMR for all workers only calculated using NIOSH life table analysis system (race, sex, and calendar year) and Poisson distribution

Internal (conditional forward Cox regression) analyses for workers exposed to TCE, tetrachloroethylene, methylene chloride, lead, or classes of agents, by cumulative probability of exposure or duration of exposure; analyses controlled for age in risk set selection. Univariate models included sex, paycode, and chemical exposure.

Multivariate models included those variables with significant findings in univariate models and birth cohort, time since last exposure (healthy worker survivor), employment duration prior to 1966, and hire era. No control for other potential confounders

Exposure Data and Information Assessment

Exposure: levels and co- Trichloroethylene was used from 1969 to 1987. Some sampling of levels available from exposures 1980 to 1984 , which was not thought to be representative of earlier time periods (levels NR)

Lead, tetrachloroethylene, methylene chloride, methyl chloroform, classes of chlorinated and other hydrocarbons, acids, bases used in plant. Information on co-exposures not reported

Exposure assessment Company industrial hygiene monitoring and related records, veteran employees' information and expert assessment used to identify dept. in which chemical agents were used over time

Individual work history linked to dept.-year exposure matrix. Cumulative exposure score for each worker assigned by (i) extent of chemical use based on dept. (none, intermittent, routine), (ii) employment duration in dept. (iii) potential of exposure based on broad job category within department (processing vs. clerical or administrative), and (iv) chemical usage in dept.-during time period

Assessment: other NR

exposures

Disease assessment State vital records or NDI; ICD code in use at time of death

Yiin et al. (2009)

Related References None

Geographic Location Tennessee (USA) 


\section{Study Description and Methodology}

\section{Population Characteristics}

Exposed cohort and ascertainment
Eligibility criteria: hired before 1985, employed 30 days or longer

Total cohort: 47,941 Uranium enrichment (gaseous diffusion) plant workers; TCEexposed NR

Cases: 98 multiple myeloma deaths

Follow-up: 1985-1998

\section{Loss to follow-up: NR}

Reference population 419 controls (219 deaths) 5:1 controls to cases, matched on age, sex, race

Selected by incidence density sampling from risk set of all workers at risk of mortality from multiple myeloma

All-cause and all-cancer Not applicable mortality/incidence

Study Design and Analytical Methods/Control for Confounding

Nested case-control mortality study

Conditional logistic regression (univariate and multivariate analyses focusing on ionizing radiation dose adjusted for external radiation, X-rays, and TCE, mercury, and nickel as potential confounders; 15 year lagging. Also conducted separate analyses for TCE and other chemicals

Exposure Data and Information Assessment

Exposure: levels and co- Historical area air monitoring data available but inadequate information (e.g., building exposures work location) to link to employees

Estimated cumulative exposure levels to TCE (mean); 183.8 cases, and 113.4 controls (units not reported)

Internal and external radiation dose estimated: average cumulative exposure $=0.026 \mathrm{mGy}$ cases, $0.012 \mathrm{mGy}$ controls

Other exposures: Mercury and nickel

Exposure assessment Exposure to TCE, mercury, nickel: modified job-exposure matrix using site records to identify exposure activities for TCE and other chemicals. Mean air levels estimated for each activity by decade. Activities associated with dept. based on workforce information

Cumulative exposure scores (ranks) based on estimated exposure level for activity, employment duration (days) in dept. associated with exposure activity, and fraction of the day in exposure activity work area based on expert assessment (industrial hygienists)

Assessment: other NR

exposures

Disease assessment Source of mortality data NR

ICD-8 203; underlying and contributory cause of death

\section{Ritz (1999)}

Related References None

Geographic Location Ohio (USA)

Population Characteristics 


\section{Study Description and Methodology}

Exposed cohort and Eligibility criteria: All white males employed from 1951 to 1972 for $\geq 3$ months with ascertainment chemical exposure data and monitoring data for radiation $(\mathrm{N}=3,814)$

Exposed cohort: 2,971 (of 3,814 eligible) white male uranium-processing workers Follow-up: 1951-1989; mean length: 31.5 years

Loss to follow-up: NR

Reference population U.S. population; NIOSH-CORPS reference data (Zahm 1992)

All-cause and all-cancer Total cohort only: mortality (SMR)

mortality/incidence

All-cause mortality: 0.84 (0.79-0.90); 1,045 deaths

All-cancer mortality: $1.10(0.99-1.23) 328$ deaths

Study Design and Analytical

Historical cohort mortality study; external (SMR) adjusted for age and calendar year. Internal (risk-set) analyses by level (category) and duration of exposure in 15 lagged and unlagged analyses using conditional Cox proportional hazards modeling matching by age to index case age, and adjusting for time since first hired, pay status (surrogate for SES), using time since first hired (surrogate for healthy worker survival effects, radiation dose and exposure to other chemicals)

Exposure Data and Information Assessment

Exposure: levels and co- No quantitative exposure assessment exposures

Only $6 \%$ of cohort had moderate exposure and no workers had heavy exposure Co-exposures: cutting fluids, kerosene, carbon (approx. 50\% TCE-exposed workers exposed to cutting fluids, some to kerosene or carbon), and external and internal radiation (badge dosimetry, urine, area monitoring) (mainly uranium and thorium isotopes)

287 workers excluded because of missing radiation exposure data

Exposure assessment JEM based on in-plant industrial hygiene assessment by hygienists, veteran workers, engineers in 1970s and 1980s to estimate probability of chemical exposures by job title and department

Workers classified by estimated exposure level categories (light, moderate, heavy) and exposure duration

Assessment: other exposures

Disease assessment

Related References None

Geographic Location Germany
Henschler et al. (1995)
Smoking history available for approx. 20\% subsample of workers from 1968; used to indirectly estimate smoking prevalence by exposure status among workforce

Social Security Administration (prior to 1979)

National Death Index

Internal analysis: ICD-9 codes 


\section{Study Description and Methodology}

\section{Population Characteristics}

Exposed cohort and ascertainment
Eligibility criteria: All workers exposed to TCE $\geq 1$ year 1956-1975

Exposed cohort: 169 (of eligible 183) male cardboard manufacturing workers exposed to TCE $(5,188$ person-yr)

Total cohort: 169 TCE exposed and 190 unexposed workers (6,100 person-yr); Median age 59 years for exposed and 62 for unexposed

Follow-up: 1956-1992; Average follow-up >30 years for both exposed and unexposed; (Note: 2 exposed cases identified outside follow-up period, included in additional analysis)

Loss to follow-up: $7.7 \%$ (169 of 183 analyzed) includes refusals, unable to participate, loss to follow-up) for exposed cohort; NR for unexposed workers

Reference population External analysis: local population (mortality) Danish and German Democratic Republic cancer rates (renal-cell cancer incidence)

Internal analysis: 190 workers in factory not exposed to TCE matched as group for age and physical activity; excluding office workers

All-cause and all-cancer All-cause mortality mortality/incidence

Exposed: SMR $=0.68(0.48-0.93) ; 50$ deaths

Unexposed: SMR $=1.03$ (0.77-1.35); 52 deaths

All-cancer mortality:

Exposed: SMR $=0.96$ (0.51-1.67); 15 deaths

Unexposed: SMR $=1.16(0.65-1.91): 15$ deaths

All cause and all cancer incidence not reported

Study Design and

Analytical

Methods/Control for

Confounding

Exposure Data and Information Assessment

Exposure: levels and co- No quantitative air levels or urine measurements of TCE. Subanaesthetic symptoms exposures
Historical cohort mortality and incidence study (renal cancer); external and internal analysis (Mantel-Haenszel test statistics ignoring age stratification) usually associated with TCE concentrations above $37 \mathrm{ppm}$; Cherrie et al. (2001) estimate peak exposures were $>2,000 \mathrm{ppm}$ with average long-term exposure 10 to $225 \mathrm{ppm}$. In cold degreasing process, estimated average chronic exposure was $\sim 100 \mathrm{ppm}$

Long exposure periods (17.8 months)

TCE used from 1956-1975

Cardboard machine area cleaned with TCE every 2 weeks for 4-5 hours, open system, and poor ventilation, no protective gloves and presumed high exposure, odor recorded and sweet taste in mouth and adverse effects (headache, dizziness, vertigo)

TCE used in locksmith's and electrical workshop for degreasing metal parts and involved "continuous exposure" at lower levels than in cardboard machine area without personal protective equipment

Other solvents, including halogenated and non-halogenated hydrocarbons, pentachlorophenol, 1,1,1-trichloroethane, tetrachloroethane used in "negligible" amounts compared with TCE from 1967 


\begin{tabular}{ll}
\hline Exposure assessment & \multicolumn{1}{c}{ Study Description and Methodology } \\
& $\begin{array}{l}\text { Walk-through survey and employee interview used to identify three locations of } \\
\text { exposure: cardboard machine area, locksmith's area, and electrical workshop areas. TCE } \\
\text { also used for general cleaning purpose to clean floors, clothes, and hands } \\
\text { Individual employee questionnaire on job history, tasks, materials used }\end{array}$ \\
$\begin{array}{l}\text { Assessment: other } \\
\text { exposures }\end{array}$ & $\begin{array}{l}\text { Smoking, alcohol intake, diuretics, body weight, height, blood pressure (individual } \\
\text { employee questionnaire) }\end{array}$ \\
Disease assessment & $\begin{array}{l}\text { Mortality: vital status from medical, personnel, and pension depts, relatives' interview; } \\
\text { cause of death from hospital or physician records, not based on death certificates (ICD- } \\
\text { 9) } \\
\text { Incidence: hospital and physician records; Physical examination by abdominal } \\
\text { sonography; Renal tumors histologically confirmed }\end{array}$
\end{tabular}

\section{Greenland et al. (1994)}

$\begin{array}{ll}\text { Related References } & \text { None } \\ \text { Geographic Location } & \text { Massachusetts (USA) } \\ \text { Population Characteristics } & \end{array}$

Exposed cohort and Eligibility criteria ("cohort"): 1,821 white males at electrical manufacturing plant ascertainment (transformers, plastics, ordnance systems) employed before 1984, terminated working after 1946, 21-90 years old, date of death benefit claims between 1969-1984 with insurance death records and exposure information; (total \# NR)

Cases: 512 cancer deaths, 15 NHL and Hodgkin lymphoma combined, 12 kidney, 9 liver cancers

Follow-up: Workers who died between 1969-1984

Loss to follow-up: NR

Reference population Controls: 1,202 non-cancer deaths "unrelated to exposures under study" (primarily circulatory [78\%], respiratory [10\%], injury [6\%], and other causes [6\%])

All-cause and all-cancer All-cause mortality: SMR NR mortality/incidence

Study Design and Analytical Methods/Control for Confounding

Nested case-control analysis among workers at a plant with death benefit claims Separate analyses by specific exposure for cancers with more than 8 cases that adjusted for age and date of death, and covariates (related to employment that changed the risk estimate by $>20 \%$ ); No multivariate control for potential confounding from coexposures or lifestyle habits

Exposure Data and Information Assessment

Exposure: levels and co- No industrial hygiene data exposures

TCE used 1930-1977

NAS (2006) noted low likelihood of TCE potential exposure among subjects 30 chemicals with carcinogenic potential identified; 6 selected with large volume or number of jobs in addition to TCE: Pyranol (PCBs and trichlorobenzene), benzene, other solvents, machining fluids, asbestos, resins (mostly phenol formaldehyde, polyvinyl resin)

Exposure assessment Interviews with employees and combination of job titles and department and building used by industrial hygienist to construct qualitative JEM for seven exposures. JEM combined with work history to assign exposure to TCE to each individual (ever/never exposure) 


\section{Study Description and Methodology}

Assessment: other NR

exposures

Disease assessment Death records in company pensions system; subset of death certificate diagnoses for cancers with $<90 \%$ confirmation rate verified using hospital records

ICDA-8 (combined NHL and Hodgkin lymphomas only)

\section{Wilcosky et al. (1984)}

Related References

Geographic Location

Population Characteristics

Exposed cohort and ascertainment
Arp et al. (1983); McMichael et al. (1974); McMichael et al. (1976)

Ohio (USA)

Eligibility criteria (exposed cohort): 6,678 current and retired rubber manufacturing workers 40-84 years old in 1964 exposed to selected solvents $>1$ year

Cases: Deaths for cancers in excess in cohort study (McMichael et al. 1976); NHL (ICD 200): stomach (30), prostate (333), lymphosarcoma and reticulum cell sarcoma (9) and lymphatic leukemia (10)

Follow-up: 1964-1974

Loss to follow-up: NR

Reference population $\quad$ Controls: $20 \%$ age-stratified sample of cohort

All-cause and all-cancer N/A

mortality/incidence

All-cancer mortality/incidence: N/A

SMR: N/A

SIR: N/A

Study Design and Analytical Methods/Control for Confounding

Nested case-control study; separate age-adjusted analyses stratified by race for any vs. no exposure to each of $20+$ solvents; No adjustment for potential confounding from coexposure or lifestyle factors

Exposure Data and Information Assessment

Exposure: levels and co- No quantitative exposure assessment or industrial hygiene measurements available exposures

Co-exposures: 25 solvents identified in different processes

Exposure assessment Review of product specifications for solvents authorized for use in specified processes and operations by calendar year used to develop JEM. It is not known whether the solvents were actually used. Work histories constructed from job title/dept. (company records) and linked to JEM

Exposure defined as ever/never work in a process area where one or more of 25 solvents (including TCE) authorized for use

Assessment: other NR

exposures

Disease assessment Death certificates; ICD-8 (coded by nosologist)

Bove et al. (2014)

Related References None

Geographic Location Camp Lejeune, NC, Camp Pendleton, CA (USA)

Population Characteristics 


\section{Study Description and Methodology}

Exposed cohort and $\quad$ Eligibility criteria: All Marine and Navy personnel on active duty and stationed at Camp ascertainment Lejeune between April 1975-December 1985

Exposed cohort: 154,932 M+F stationed at Camp Lejeune; 97\% under the age of 55 and $<6 \%$ had died by the end of the study

Follow-up: 1979-2008

Loss to follow-up: 1.3\% Camp Lejeune, 1.5\% Camp Pendleton

Reference population Eligibility criteria: All Marine and Navy personnel on active duty April 1975-December 1985 and stationed at Camp Pendleton, CA any time during this period

"Unexposed cohort": Camp Pendleton

All-cause and all-cancer All-cause mortality: SMR $=0.83(0.81-0.84) ; 8,964$

mortality/incidence

All-cancer mortality: SMR $=0.85(0.80-0.90) ; 1,078$

Study Design and

Analytical

Retrospective cohort study using ecological exposure assessment; Two types of

Methods/Control for

Confounding analyses:

Evaluation of contaminated water comparing the exposed (Camp Lejeune) and nonexposed population (Camp Pendleton): Hazard Ratio using Cox extended regression models with age and time as a variable that compared mortality rates (SMR) between the 2 cohorts. SMR were calculated using Life Table Analysis System that adjusted for age, sex, and calendar period and accounted for latency

Evaluation of individual water contaminants within the Camp Lejeune cohort: Evaluation of exposure response relationships of cumulative exposure (untransformed, $\log 10$ transformed and continuous) for each contaminant using Cox extended regression adjusting for age, and accounting for latency. Other analyses included duration of exposure and restricted cubic spline

Models were adjusted for sex, race, and education. Other variables considered in the model (did not change risk estimates by $10 \%$ ) include marital status, birth cohort, date of death, duty occupation. Smoking was considered by subtracting the log HR among smoking-related diseases from the log HR of disease of interest

Exposure Data and Information Assessment

Exposure: levels and co- Two of the eight drinking water systems at Camp Lejeune were contaminated with exposures solvents based on sampling data from 1980 to 1984

Tarawa Terrace: Contaminated by off-base dry-cleaning business: Primary contaminant PCE

Estimated mean level $(\mu \mathrm{g} / \mathrm{L})$ : TCE: 3.1, PCE: 75.7

Hadnot Point: Contaminated by on-base sources (underground storage tank, industrial spills): Primary contaminant TCE (up to $1,400 \mu \mathrm{g} / \mathrm{L}, \sim 0.04 \mathrm{ppm}$ air equivalent ${ }^{\mathrm{b}}$ )

Estimated mean levels ( $\mu \mathrm{g} / \mathrm{L}$ ): TCE: 358.7, PCE: 14.7, Vinyl Chloride: 24, Benzene: 5.4

TCE and PCE highly correlated with each other

Overall cumulative exposure, $\mu \mathrm{g} / \mathrm{L}$-months (ppm-months ${ }^{\mathrm{b}}$ ), for TCE, mean $=6,369$

$(0.17)$; median $=5,289(0.14) ; 20 \%$ were exposed to levels between 7,700 and 39,745

(0.21-1.06)

Potential daily exposure from Hadnot Point could be as high as $3.6 \mathrm{mg} / \mathrm{day}\left(0.07 \mathrm{ppm}^{\mathrm{b}}\right)$ (showering and drinking water) 


\section{Study Description and Methodology}

Exposure assessment TCE and other contaminant levels: Historical reconstruction using historical samples, and modeling based on water fate and distribution modeling

Tarawa Terrace water system served on-base houses and Hadnot Point mainly served bachelor quarters

Each individual at Camp Lejeune was assigned an estimated average contaminant concentration in the drinking water system serving their residence for the period of their residence. Several sources were used to determine the individual residence. Probability and matching were used to link married cohort members to base housing

Cumulative exposure ( $\mu \mathrm{g} / \mathrm{L}$-months) was calculated using the estimated monthly average contaminants, in the water serving the individual residence and occupancy dates. No information on water consumption

Assessment: other exposures

Disease assessment
Tetrachloroethylene

1,2-Dichloroethylene

Vinyl chloride

\section{used to determine vital status}

$\mathrm{NHL}=$ non-Hodgkin lymphoma; $\mathrm{M}=$ males/men; $\mathrm{F}=$ females; $\mathrm{W}=$ women; $\mathrm{MM}=$ multiple myeloma; $\mathrm{NOCCA}=\mathrm{Nordic}$ Occupational Cancer; TCE = trichloroethylene; $\mathrm{NR}=$ not reported; JEM = job exposure matrix; ICD = International Classification of Diseases; TCA = trichloroacetic acid; SIR = standardized incidence ratio; U-TCA = urine trichloroacetic acid; $\mathrm{SMR}=$ standardized mortality ratio; $\mathrm{SD}=$ standard deviation; $\mathrm{ppm}=$ parts per million; Perc = perchloroethylene

(tetrachloroethylene); PCE = perchloroethylene (tetrachloroethylene); DMV = Department of Motor Vehicles; NAS = National Academy of Science; JP4 = jet propellant-4; SSFL = Santa Susanna Field Laboratory; SES = socioeconomic status; $\mathrm{PPE}=$ personal protective equipment; SSA = Social Security Administration; NDI = National Death Index; PAH = polycyclic aromatic hydrocarbon; $\mathrm{CLL}=$ chronic lymphocytic lymphoma; $\mathrm{ppb}=$ parts per billion; $\mathrm{SSN}=$ Social Security Number; $\mathrm{mGy}=$ milligray; NIOSH $=$ National Institute for Occupational Safety and Health; CORPS = Computerized Occupational Referent Population System; N/A = not applicable; HR = hazard ratio; TWA = time-weighted average; PCBs = polychlorinated biphenyls; $\mathrm{EAC}=$ equivalent airborne concentrations $\left(\mathrm{mg} / \mathrm{m}^{3}\right) ; \mathrm{OD}=$ oral dose $(\mathrm{mg} / \mathrm{kg}) ; \mathrm{BW}=$ body weight; $\mathrm{MV}=\mathrm{minute}$ volume $(\mathrm{mL} / \mathrm{min}) ; \mathrm{AF}=$ fraction of inhaled substance absorbed (default $=1) ; \mathrm{EL}=$ exposure length $(\mathrm{min})$.

${ }^{a} \mathrm{U}-\mathrm{TCA}$ (half-life $100 \mathrm{hrs}$ ) is approx. linear with air TCE $<375 \mathrm{mg} / \mathrm{m}^{3}(70 \mathrm{ppm})$, according to formula TCE $\mathrm{mg} / \mathrm{m}^{3}=[1.96 \times \mathrm{U}-$ TCA (mg/L) - 0.7] (Hansen et al. 2001).

${ }^{\mathrm{b}}$ Oral dose of TCE in drinking water converted to equivalent of airborne occupational exposure (ppm, 8-hr TWA) assuming average $1.5 \mathrm{~L}$ day intake, $70 \mathrm{~kg}$ body weight, minute volume for typical $8-\mathrm{hr}$ shift $=10 \mathrm{~m}^{3}$, according to formula:

$\mathrm{EAC}=(\mathrm{OD} \times \mathrm{BW}) /(\mathrm{MV} \times \mathrm{AF} \times \mathrm{EL} \times 10 \mathrm{E}-06) ; 10 \mathrm{E}-06=$ conversion factor $\left(\mathrm{mL}\right.$ to $\left.\mathrm{m}^{3}\right) ; 1 \mathrm{mg} / \mathrm{m}^{3} \mathrm{TCE}=0.186 \mathrm{ppm}$.

Table D-2. Study Descriptions and Methodologies: Case-control Studies of Trichloroethylene Exposure and Kidney Cancer

\section{Study Description and Methodology}

\section{Moore et al. (2010)}

Related References Brennan et al. (2008); Hung et al. (2007)

Geographic Location Central and Eastern Europe (7 centers, 4 countries)

Population Characteristics

Cases: selection and ascertainment

Cases: 1,097 RCC

Case eligibility criteria: Cases at participating hospitals 1999-2003; living in area for at least $1 \mathrm{yr}$

Participation rate: NR 


\section{Study Description and Methodology}

Controls: selection and Referents: 1,476

ascertainment

Study Design and Analytical Methods

Referent eligibility criteria: Inpatients or outpatients with non-tobacco-related conditions at same hospitals without cancer or genitourinary disorders (except benign prostate hyperplasia)

Matching criteria: age, sex, study center

Hospital-based: Unconditional logistic regression evaluating ever and categories of different exposure metrics: duration (hr, yr), average intensity and cumulative for all subjects and for subjects with high confidence exposure assessment; Lagged analysis: Analyses were lagged and controlled for sex, age, study center; residence, smoking, BMI, and history of hypertension considered but did not affect risk estimate. Analysis by GST genotypes

Exposure Data and Information Assessment

Exposure: levels and co-exposures

Exposure assessment

Chlorinated and other solvents

Intensity and prevalence of occupational exposures have been higher in central and eastern Europe than other industrial areas

Estimated median exposure and IQR

Cumulative exposure (ppm-yr): IQR $=0.77-2.87$ for controls; median $=1.95$, $\mathrm{IQR}=0.83-7.25$ for cases

Average intensity (ppm): IQR $=0.08-0.16$ for controls; median $=0.08, \mathrm{IQR}=0.08-0.44$ for cases

In-person interviews using structured and occupational specific and detailed questionnaires (e.g., tasks, working environment, time on each task) for lifetime jobs held at least 1 year. Expert assessment (blinded) by exposure assessment teams (with knowledge of plants in their study area) coded jobs for exposure to specific agent and assessed the frequency, confidence, intensity of exposure. Cumulative exposure defined as product of intensity, duration, and frequency of exposure. Confidence $=$ expected percent of workers exposed in a given job (possible, probable, definite)

Assessment of organic exposures were reevaluated at a later date blinded to the previous assessment and disease status. For TCE, the agreement was $83 \%$ in 1 country and $100 \%$ in 2 countries (not done in the 4th country because of unlikely exposure to TCE)

Assessment of potential Interviews: Lifestyle factors, especially tobacco consumption, anthropometric measures confounders 1 year before diagnosis, and personal and familial medical history

Disease assessment Histologically confirmed by study experts using standard confirmation and disease classification. ICD-0-2, Code C.64

Charbotel et al. (2006); Charbotel et al. (2009)

Related References

Geographic Location

Population Characteristics

Cases: selection and ascertainment
Fevotte et al. (2006)

Arve Valley, France

Cases: 86 cases RCC (19 deceased), 67\% men

Case eligibility criteria: Deceased or living identified retrospectively from medical (urology and oncology) practitioners 1993-2003

Participation rate: Cases 74\%; controls 78\%; follow-up questionnaire sent to nonparticipants 


\section{Study Description and Methodology}

Controls: selection and $\underline{\text { Referents: }} 326$ (7 deceased); 70\% men

ascertainment

Study Design and Analytical Methods
Referent eligibility criteria: Patients (without kidney cancer or disease or urinary tract cancer) randomly selected from the same practitioners as cases

Matching criteria: Age, gender (matched 4:1)

Hospital-based: Multivariate analysis using variables with 10\% differences (4 classes of smoking and 3 classes of BMI) between cases and controls; Analysis performed for ever exposure, cumulative exposure, and combined cumulative \& peak exposure. Additional analyses for higher TWA exposure thresholds $(35,50$, and $75 \mathrm{ppm})$ and for co-exposure to cutting, petroleum and/or other mineral oils (Charbotel et al. 2009); Sensitivity analysis to assess sources of misclassification (proxy, older patients, jobs with high confidence)

Exposure Data and Information Assessment

Exposure: levels and co-exposures

Region has high prevalence and high degree of exposure to TCE because of its use as a degreaser in the widespread screw-cutting industry

Estimated TCE concentrations associated with quantitative ranking: low $=5-150 \mathrm{ppm}$ yr; medium $=155-335$ ppm-yr; high $\geq 335$ ppm-yr. Among controls the median exposure for low, medium, and high categories $=60,252$, and $630 \mathrm{ppm}$, respectively. Among cases median exposure $=30,300$, and 885 ppm, respectively

Co-exposure to cutting oils, petroleum oils, and other mineral oils

Exposure assessment Telephone interviews using medical and occupational questionnaires, with subject or next of kin, focusing on screw-cutting industry (TCE used); Exposure to TCE and other substances assigned using expert and task exposure matrix (JTEM) for screw-cutting industry. Exposure to TCE was semi-quantitative; ranked categories

Assessment of potential Questionnaire: familial kidney disease and medical history, such as kidney stones, confounders infection, chronic dialysis, hypertension and use of drugs (anti-hypertensive drugs, diuretics, and analgesics); BMI, lifestyle considered smoking habits (pack-years) and coffee consumption. No significant differences in most of these characteristics were found in univariate analysis except for BMI and smoking

Disease assessment

Mainz classification; histologically confirmed

Brüning et al. (2003)

Related References

Geographic Location

Vamvakas et al. (1998) (same area but no overlap)

Arnsberg and $30 \mathrm{~km}$ surrounding area, Germany

Population Characteristics

Cases: selection and ascertainment

Cases: 134 RCC (113 incident, 21 deceased)

Case eligibility criteria: People with nephrectomy 1992-2000 from urology department serving the area (1 hospital)

Participation rate: $83 \%$ cases, controls NR

Controls: selection and Referents: 401

ascertainment

Referent eligibility criteria: People in hospital for surgery or geriatrics without dementia or diagnosis of cancer

Matching criteria: sex, age (5 yr) 3:1 ratio (frequency-matched)

Study Design and Analytical Methods
Hospital-based: Conditional logistic regression for three types of exposure assessments, and for duration and time since first and last exposure for self-assessed exposure

Adjusted for sex, age (from matching), and smoking 


\section{Study Description and Methodology}

Exposure Data and Information Assessment

Exposure: levels and Levels NR but estimated to be 400 to 600 ppm during hot dipping and $>100$ ppm overall co-exposures

(Cherrie et al. 2001)

Arnsberg area is a small geographical area with large number of companies in the metal process industries. TCE use was widespread and only two solvents were used in the industry. Exposure prevalence among controls varied by exposure assessment: Very high (for jobs associated with TCE exposure) using CAREX (80\%) to 10\% using selfreported exposure

Regulatory measures were enforced starting in the 1980s

Exposure assessment Interview questionnaire (as used by Vamvakas et al. (1998)) (approx. 16\% proxies for deceased cases used, no proxies for controls); No information on whether interviewers blinded to case status

Exposure assessed via three methods: (1) job/industry (ever and longest held) associated with exposure using CAREX database (which is based on expert assessment) and applying JEM, (2) agent-specific (not specific for TCE) using British JEM (duration, probability, and intensity) for jobs held for $>1$ year, (3) self-assessed frequency and duration of exposure to TCE and narcotic symptoms (comparable to Vamvakas et al. (1998) exposure assessment)

Assessment of potential Questionnaire/interview: Smoking, BMI, analgesics use. Cases and controls similar with confounders respect to obesity (BMI $>30)$, analgesics use, sex, and age

Disease assessment

Histologically confirmed

Vamvakas et al. (1998)

Related References None (same area but no overlap with Brüning et al. (2003))

Geographic Location Arnsberg (city), Germany

Population Characteristics

Cases: selection and ascertainment

\section{Cases: $58 \mathrm{RCC}$}

Case eligibility criteria: People with nephrectomy between 1987 and 1992 at a county hospital

Participation rate: $87 \%$ cases, $75 \%$ controls

Controls: selection and ascertainment

Study Design and Analytical Methods

\section{Referents: 84}

Referent eligibility criteria: Accident patients at 3 nearby hospitals (not the same hospital as cases) in 1993 without kidney cancer (sonograph)

Matching criteria: None

Hospital-based: Multivariate logistic regression by exposure category (no, low, medium, high). Stratified by age analysis (Mantel-Haenszel). Adjusted for age, gender, smoking, blood pressure, and diuretic intake

Exposure Data and Information Assessment 


\begin{tabular}{|c|c|}
\hline & Study Description and Methodology \\
\hline \multirow[t]{3}{*}{$\begin{array}{l}\text { Exposure: levels and } \\
\text { co-exposures }\end{array}$} & $\begin{array}{l}\text { Levels NR but estimated to be } 400 \text { to } 600 \text { ppm during hot dipping and }>100 \text { ppm over } \\
\text { (Cherrie et al. 2001) }\end{array}$ \\
\hline & $\begin{array}{l}\text { Arnsberg area is a small geographical area with a large number of companies doing } \\
\text { metal and electronics work. TCE use was widespread and one of only two solvents } \\
\text { (other was carbon tetrachloride) used in the industry }\end{array}$ \\
\hline & $\begin{array}{l}\text { Most subjects were involved in metal degreasing, without personal protective } \\
\text { equipment. According to walkthrough surveys and interviews with employees and hea } \\
\text { professionals, degreasing procedures were done in open conditions above } 60^{\circ} \mathrm{C} \text {, and } \\
\text { TCE was used to clean arms and hands, cloths, floors, etc. }\end{array}$ \\
\hline \multirow[t]{2}{*}{ Exposure assessment } & $\begin{array}{l}\text { Interview using structured questionnaire (not blinded) by physician on occupational } \\
\text { history and exposure to multiple substances with subject or proxy. Follow-up info on } \\
\text { exposure to TCE and tetrachloroethylene; Detailed info obtained from employer liabil } \\
\text { insurance }\end{array}$ \\
\hline & $\begin{array}{l}\text { Exposure level based on combination of exposure duration and frequency and severity } \\
\text { of acute pre-narcotic symptoms }\end{array}$ \\
\hline $\begin{array}{l}\text { Assessment of potential } \\
\text { confounders }\end{array}$ & $\begin{array}{l}\text { Interview: smoking, alcohol consumption, BMI, blood pressure, diuretics intake, and } \\
\text { exposure to other known carcinogens-asbestos, cadmium, gasoline and/or other } \\
\text { petroleum products. Cases and controls similar with respect to alcohol consumption, } \\
\text { BMI, percentage of males }\end{array}$ \\
\hline Disease assessment & Histologically re-confirmed (double blind). All cases arose from tubule epithelium \\
\hline
\end{tabular}

Christensen et al. (2013)

Related References $\quad$ Siemiatycki (1991)
Geographic Location
Population Characteristics (Canada)

Cases: selection and ascertainment

Cases: 177 RCC; 48 liver cancer

Case eligibility criteria: Male Canadian citizens, ages 35-70, incident cases 1979-1985 identified from 18 major hospitals

Participation rate: $82 \%$ cases, $72 \%$ controls (total study population)

Controls: selection and Referents: 533 population controls; 1,999 cancer controls for kidney cancer and 1,834 ascertainment for liver cancer

Referent eligibility criteria: Population controls randomly selected from electoral records. Cancer controls - no more than $20 \%$ of one cancer, excluded lung and contiguous sites for the index cancer; Specific cancers not reported

\section{Matching criteria: Age, sex}

Study Design and Analytical Methods

Hospital and population-based: Unconditional logistic regression using each type of control and pooled (weighted) controls and controlling for SES, ethnicity, interview type (self or proxy), smoking, coffee, alcohol; Risk calculated for any and substantial exposure. Exposures occurring 5 years before diagnosis were excluded

Exposure Data and Information Assessment

Exposure: levels and NR co-exposures

Exposure prevalence to TCE: $\leq 2 \%$ of cancer controls or population controls had substantial exposure and 3\% had any exposure

Occupations considered to have the highest exposure were mechanics and repairmen, metal machining occupations, electrical and electronics and metal shaping and formulation 


\section{Study Description and Methodology}

Exposure assessment In person interviews (with subject or proxy) obtaining detailed information on lifetime occupational history and duration in 13 specific occupations/industries and seven jobs with specific exposures, and PPE. Proxy interviews were done for $12 \%$ of population controls and $14 \%$ of kidney cancer cases

Expert (team) assigned exposures based on reported job histories for close to 300 substances and rated the confidence, frequency, and intensity of each exposure. Assessor blinded to case-control status. Substantial exposure based on duration, frequency, confidence, and concentration

Assessment of potential Questionnaire/interviews: age, SES, ethnicity, interview type (self or proxy), and confounders lifestyle factors (such as smoking, alcohol consumption)

Disease assessment Histologically confirmed

Pesch et al. (2000b)

Related References Pesch et al. (2000a)

Geographic Location Germany

Population Characteristics

Cases: selection and ascertainment

Controls: selection and Referents: 4,298 ascertainment

Study Design and Analytical Methods registers
Cases: 935 RCC (570 males and 365 females)

Case eligibility criteria: German nationals (no age limit) from large hospitals 1990-1995 Participation rate: $88 \%$ cases, $71 \%$ controls

Referent eligibility criteria: German nationals randomly selected from local residency

Matching criteria: Region, sex, age (5 yr) (1:4)

Population-based: Conditional logistic regression adjusting for smoking (pack-years) and matching variables (region, sex, age) for exposure index (medium, high, substantial) using the low-exposure group as the reference

Exposure Data and Information Assessment

Exposure: levels and co-exposures

Exposure assessment

NR; no information on the types of job exposed to TCE

Prevalence of substantial TCE exposure was low among cases (4\% to $10 \%)$ and varied by type of JEM

Includes the Arnsberg and other regions; NAS (2006) estimated that most subjects had minimal contact with TCE, averaging concentrations of $10 \mathrm{ppm}$ or less

Interview using structured questionnaires; Exposure assessed using two JEM (British, German) and a job task-exposure matrix (JTEM), which provided an expert assessment of probability of exposure and intensity to a given agent

Life-time exposure (exposure index) was the product of probability, duration, and intensity of exposure summarized across jobs for both JEM and JTEM

Assessment of potential Questionnaire/interview: various measures of smoking, socioeconomic status, analgesics confounders use, and BMI. SES was an independent risk factor for kidney cancer among women. Cases and controls did not differ in BMI, education, age, region, and smoking status, and analgesics use

Disease assessment Histologically (95\%) or sonographically (5\%) confirmed

Dosemeci et al. (1999)

Related References

Chow et al. (1994) 


\begin{tabular}{|c|c|}
\hline & Study Description and Methodology \\
\hline Geographic Location & Minnesota (USA) \\
\hline \multicolumn{2}{|l|}{ Population Characteristics } \\
\hline \multirow{3}{*}{$\begin{array}{l}\text { Cases: selection and } \\
\text { ascertainment }\end{array}$} & Cases: 438 (273 men, 165 women) \\
\hline & $\begin{array}{l}\text { Case eligibility criteria: Newly diagnosed white cases RCC } 1988 \text { to } 1990 \text { from state } \\
\text { cancer registry; deceased cases excluded }\end{array}$ \\
\hline & $\begin{array}{l}\text { Participation rate: } 87 \% \text { cases, } 86 \% \text { controls; Occupational analysis with complete } \\
\text { personal interviews: } 64 \% \text { cases; } 97 \% \text { controls }\end{array}$ \\
\hline \multirow{3}{*}{$\begin{array}{l}\text { Controls: selection and } \\
\text { ascertainment }\end{array}$} & Referents: 687 (462 men, 225 women) \\
\hline & $\begin{array}{l}\text { Referent eligibility criteria: Randomly selected (random digit dialing) (ages } 20 \text { to } 64 \text { ) or } \\
\text { systematic selection from health care financing agency (ages } 65 \text { to } 85 \text { yr old) white } \\
\text { controls }\end{array}$ \\
\hline & Matching criteria: Age and sex stratified \\
\hline $\begin{array}{l}\text { Study Design and } \\
\text { Analytical Methods }\end{array}$ & $\begin{array}{l}\text { Population-based: Logistic regression controlling for age, smoking, hypertension status, } \\
\text { use of diuretic or anti-hypertension drugs, BMI; Risk for ever-exposed reported } \\
\text { separately for men and women }\end{array}$ \\
\hline
\end{tabular}

Exposure Data and Information Assessment

Exposure: levels and NR

co-exposures

Exposure assessment In-person interview with questionnaire for usual and most recent occupation, employment duration and duration for industries with specific exposure; Exposure assigned using JEM which linked occupation/industry code to exposure to chemicals (TCE and other chlorinated hydrocarbons). Interviewer blinded to case/control status and proxy interviews excluded from analysis

Assessment of potential Questionnaire/interview: demographic and ethnic variables, residential history, smoking confounders habits, medical history, and drug use

Disease assessment Histologically confirmed

$\mathrm{RCC}$ = renal-cell cancer; NR = not reported; BMI = body mass index; GST = glutathione-S-transferase; IQR = interquartile range; $\mathrm{ppm}=$ parts per million; TCE = trichloroethylene; $\mathrm{ICD}=$ International Classification of Diseases; TWA = time-weighted average; JTEM = job-task exposure matrix; CAREX = CARcinogen EXposure (Canada); JEM = job exposure matrix; $\mathrm{SES}=$ socioeconomic status; $\mathrm{PPE}=$ personal protective equipment; NAS = National Academy of Science.

Table D-3. Study Descriptions and Methodologies: Case-control Studies of Trichloroethylene Exposure and NHL and Related Subtypes

\begin{tabular}{ll}
\hline \multicolumn{1}{c}{ Christensen et al. (2013) } & \multicolumn{1}{c}{ Study Description and Methodology } \\
$\begin{array}{l}\text { Related References } \\
\text { Geographic Location }\end{array}$ & Siemiatycki (1991) \\
$\begin{array}{l}\text { Population Characteristics } \\
\text { Cases: selection and } \\
\text { ascertainment }\end{array}$ & Cases: 215 NHL \\
& Case eligibility criteria: Male Canadian citizens, ages 35-70, incident cases 1979-1985 \\
& $\begin{array}{l}\text { identified from 18 major hospitals } \\
\text { Participation rate: 82\% total cancer cases (also used as cancer controls), 72\% total }\end{array}$ \\
& population controls (used for analysis of 11 cancer sites) \\
\hline
\end{tabular}




\section{Study Description and Methodology}

Controls: selection and $\underline{\text { Referents: }}$ 2,341 cancer controls, 533 population controls ascertainment

Referent eligibility criteria: Population controls randomly selected from electoral records; cancer controls - no more than $20 \%$ of one cancer, excluded lung and contiguous sites for the index cancer; Specific cancers not reported

Matching criteria: Age, sex

Study Design and Analytical Methods
Hospital and population-based: Unconditional logistic regression using each type of control and pooled (weighted) controls controlling for age, ethnicity, SES, interview type (self or proxy); Risk calculated for any and substantial exposure. Exposures occurring 5 years before diagnosis were excluded

Exposure Data and Information Assessment

Exposure: levels and Levels NR co-exposures

Exposure assessment
Exposure prevalence to TCE very rare; $\leq 2 \%$ of cancer controls or population controls had substantial exposure and $3 \%$ had any exposure

Occupations considered to have the highest exposure were mechanics and repairmen, metal machining occupations, electrical and electronics and metal shaping and formulation

In-person interviews (with subject or proxy) obtaining detailed information on lifetime occupational history, PPE, and duration in 13 specific occupations/industries and seven jobs with specific exposures; Proxy interviews were done for $12 \%$ of population controls and $21.9 \%$ of cases

Expert assessment (team) translated jobs into potential exposure for close to 300 substances and rated the confidence, frequency, and intensity for each exposure. Assessor blinded to case-control status. Substantial exposure based on duration, frequency, confidence, and concentration

Assessment of potential Questionnaire/interviews: age, SES, ethnicity, interview type (self or proxy), and confounders lifestyle factors (such as smoking, alcohol consumption)

Disease assessment Histologically confirmed

Cocco et al. (2013)

Related References

Geographic Location

Includes populations reported by Cocco et al. (2010); Miligi et al. (2006); Orsi et al. (2010); Purdue et al. (2011a)

Population

Characteristics

Cases: selection and ascertainment

\section{Cases: 3,788}

Study eligibility criteria:

Study selected had (1) complete occupational history for all study participants, (2) expert exposure assessment to TCE, (3) incident NHL cases, and (4) histological information available for each case

Controls: selection and Referents: 4,279 ascertainment
Matching criteria: Age and sex (frequency or individually) except MIS, selected to represent age and sex distribution in general population

Participation rate: see individual studies 


\begin{tabular}{ll}
\hline & \multicolumn{1}{c}{ Study Description and Methodology } \\
\hline Study Design and & Pooled analysis of four case-control studies (EPILYMPH, NCI-SEER, ENGELA, MIS): \\
Analytical Methods & Risks calculated for ever, probability, intensity, frequency, and duration of exposure, \\
& and intensity, duration, and frequency among high probability subjects and all subjects \\
& using unexposed as reference group and calculating linear trend test. Unconditional \\
& logistic regression was used for NHL and NHL subtypes. Polytomous regression \\
& analysis was used for NHL adjusting for age, gender, and study. Fisher statistics using \\
& Bonferroni correction were conducted to test chance probability of trends for 4 exposure \\
& metrics. Sensitivity analysis also conducted; excluded subjects exposed to benzene.
\end{tabular}

Exposure Data and Information Assessment

Exposure: levels and $\quad 9 \%$ of subjects ever exposed to TCE and 1\% had high probability of exposure co-exposures

No assessment of exposure to other solvents but benzene not shown to be a confounder

Exposure assessment Expert assessment of questionnaire data, workplace inspection, industrial hygiene report and experience used to assign scores of intensity (4-point scale related to OSHA PEL), frequency (4-point scale on work time in contact with agent), duration, and probability of exposure (harmonized using a priori JEM). The objective was to harmonize the exposure assessment from the four studies

Assessment of potential NR in pooled analysis confounders

Disease assessment NHL incidence: InterLymph Consortium classification

Cocco et al. (2010)

Related References

Besson et al. (2006) (Same EPILYMPH study population-association of alcohol and smoking on NHL risk)

Included in pooled InterLymph analysis: Cocco et al. (2013)

Geographic Location Multiple centers in Europe (Czech Republic, France, German, Ireland, Spain)

Population Characteristics

Cases: selection and ascertainment

Study Design and Analytical Methods ascertainment
Cases: 2,348 lymphoma (analysis for histologic subtypes of B-NHL including multiple myeloma)

Case eligibility criteria: Consecutive adult lymphoma at participating centers 1998-2004

Participation rate: Cases $88 \%$; population controls 52\%; hospital controls $81 \%$

Controls: selection and Referents: 2,462

Referent eligibility criteria: Germany \& Italy: Randomly selected from population; Others: Hospital controls (diagnoses other than cancer, infectious and immunodeficiency diseases)

Matching criteria: Age (5 yr), sex, residence

Multi-center population and hospital-based (EPILYMPH Study): Unconditional logistic regression adjusting for age, education, and center using unexposed to any solvent as the reference group and calculated for ever exposed, combined confidence, intensity and frequency, and cumulative exposure (among subject with exposure assessed as having high degree of confidence) for histologic subtypes of NHL; Bonferroni correction for multiple comparisons

Exposure Data and Information Assessment 


\begin{tabular}{|c|c|}
\hline & Study Description and Methodology \\
\hline \multirow{3}{*}{$\begin{array}{l}\text { Exposure: levels and } \\
\text { co-exposures }\end{array}$} & NR \\
\hline & $\begin{array}{l}\text { TCE exposure prevalence is low: For B-cell lymphoma, } 5 \% \text { among controls with high } \\
\text { confidence of exposure, } 2 \% \text { had high cumulative exposure }\end{array}$ \\
\hline & $\begin{array}{l}\text { Approximately one third of chlorinated aliphatic solvent-exposed workers had } \\
\text { concurrent exposure to benzene, toluene, or xylene }\end{array}$ \\
\hline \multirow[t]{2}{*}{ Exposure assessment } & $\begin{array}{l}\text { In-person interviews with structured questionnaires: Detailed lifetime occupational } \\
\text { history for jobs held more than } 1 \text { year; Detailed questionnaire on tasks, processes, and } \\
\text { PPE for exposures of a priori concern }\end{array}$ \\
\hline & $\begin{array}{l}\text { Expert review of questionnaire and assessment of } 43 \text { agents according to confidence, } \\
\text { intensity, and frequency; Cumulative exposure scores were calculated based on } \\
\text { intensity, duration, and frequency }\end{array}$ \\
\hline
\end{tabular}

Assessment of potential Questionnaire/interview on social and demographic variables, lifestyle, health history confounders

Disease assessment 2001 WHO Classification, 20\% centrally reviewed by pathologist, B-NHL and major subtypes and Hodgkin lymphoma including multiple myeloma

Deng et al. (2013)/Wang et al. (2009b)

$\begin{array}{ll}\text { Related References } & \text { Morton et al. (2003); Zhang et al. (2004) } \\ \text { Geographic Location } & \text { Connecticut (USA) }\end{array}$

Population Characteristics

Cases: selection and ascertainment

Cases: 601 NHL (inc. DLBCL, FL, CLL/small lymphocytic-lymphoma); 518 for genotype analysis

Case eligibility criteria: Women 21-24 years old without history of other cancer (except non-melanoma skin cancer) and residents of Connecticut

Participation rate: Cases 72\%; Controls - RDD 69\%, Health care 47\%

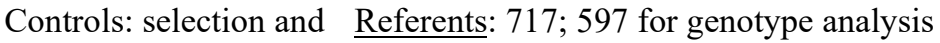

ascertainment

Referent eligibility criteria: Selected via RDD $(<65)$ or Medicare/Medicaid service files $(\geq 65)$ in Connecticut

Matching criteria: Age (5 yr frequency)

Study Design and

Population-cancer registry-based: Unconditional logistic regression adjusting for age,

Analytical Methods

family history of LHC (Wang only), alcohol consumption, race was used to calculate risks by ever, average (intensity and/or probability). Smoking, medical history, income, education levels and LHC history (Deng only) were not included in final models because they did not change the risk estimates. Polytomous logistic regression was used to evaluate using tertiles of cumulative exposure and histological subtype of NHL. Trends using continuous exposures; Deng reported risk estimates for ever vs. never stratified by immune gene polymorphisms

Exposure Data and Information Assessment

Exposure: levels and co-exposures
NR

TCE exposure prevalence among controls was $11 \%$ for ever-exposed and $1.8 \%$ for medium/high exposure 


\section{Study Description and Methodology}

Exposure assessment Interviews using structured questionnaire on detailed lifetime occupational history on job titles, companies, and activities (jobs 1 yr or longer); Jobs were linked to a JEM, which assigned probability and intensity index of exposure to solvents for each occupation/industry. Individual assigned to exposure categories that combined duration with probability and intensity to estimate ever exposure, cumulative intensity, cumulative probability for each job, and the average intensity, average probability exposure across jobs. Exposure assessment was blinded to case/control status

Assessment of potential Age, family history of LHC, alcohol consumption, race (considered smoking, education, confounders income, family history of immune disease)

Disease assessment Histologically confirmed by study pathologists using 2001 WHO (REAL) classification ICD-O-2, M-9590-9642, 9690-9701, 9740-9750

Gold et al. (2011)

Related References

Chatterjee et al. (2004)

Geographic Location

Seattle, WA and Detroit, MI SEER sites

Population Characteristics

Cases: selection and ascertainment

\section{Cases: $181 \mathrm{MM}$}

Case eligibility criteria: $\mathrm{M}+\mathrm{F}$ alive at time of study, 35-74 years old, resident in SEER area and diagnosed between 2000 and 2002

Participation rate: $60 \%$ eligible cases alive at study date; $71 \%$ of contacted cases, $52 \%$ eligible (living) controls

Controls: selection and Referents: 481 (used for study of NHL; see Chatterjee et al. (2004)) ascertainment

Referent eligibility criteria: Randomly selected via random digit dialing ( $<65 \mathrm{yr})$ or Medicare files ( $>65 \mathrm{yr}$ ) from two SEER regions, 35-74 yr old with no previous HIV infection, multiple myeloma, or plasmacytoma

Matching criteria: Age, sex, residence

Study Design and Analytical Methods

Population-based: Unconditional logistic regression, adjusted for age, sex, race, education, residence (SEER site) used to calculate risks for exposure categories-ever, exposure duration and cumulative exposure (unlagged and 10-yr lagged) for TCE and other chlorinated solvents. Sensitivity analysis considering low-exposed jobs as unexposed

Exposure Data and Information Assessment

Exposure: levels and co-exposures

Exposure assessment
Exposure prevalence of TCE among controls was $29 \%$ for ever-exposed and $14 \%$ in highest cumulative exposure category

Highest cumulative exposure category $>7,794 \mathrm{ppm}$

Separate analyses for methylene chloride, tetrachloroethylene, 1,1,1-trichloroethane, chloroform and carbon tetrachloride (not clear if co-exposures)

In-person interview with subjects using questionnaires on work history ( $>1 \mathrm{yr}$ from 1,941 cases, 1,946 controls); Job-specific questionnaires (tasks and work environment) for 20 solvent-related occupations for jobs held for at least 2 years

Exposure metrics (probability, frequency, and intensity) were assigned by experts using questionnaire data and calendar-specific JEM for industries related to solvent exposure based on extensive literature review. Cumulative exposure was calculated as sum of the intensity, frequency, and duration of all exposed jobs with a probability of exposure $>2$ for each solvent

Reviewer blind to case-control status 


\section{Study Description and Methodology}

Assessment of potential NR

confounders

Disease assessment

SEER cancer registry (data from hospitals, physicians, laboratories, death certificates); ICD-O 2/3

Costantini et al. (2008)

Related References Costantini et al. (2001); same population base as Miligi et al. (2006)

Geographic Location 11 centers, Italy

Population Characteristics

Cases: selection and $\quad$ Cases: MM ( 6 centers): 263 cases; CLL: NR ( 7 centers), 2,737 total

ascertainment $\quad$ lymphohematopoietic (11 centers)

Case eligibility criteria: All LH cancers (M+F) in 11 centers, 20-74 years of age 19911993

Participation rate: $83 \% \mathrm{MM}$ cases, $76 \%$ controls; CLL NR

Controls: selection and Referents: MM: 1,100 (6 centers); CLL: NR (7 centers); total: 1,799 (11 centers) ascertainment

Referent eligibility criteria: Random sample of population registers

Matching criteria: Age (5 yr), sex, region

Study Design and

Analytical Methods

Population-based: Multiple logistic regression models controlling for age, sex, education, region; Analyses for exposure intensity (very low/low and medium/high) and duration ( $<$ and $>15$ years) using individuals without exposure to any of the listed chemicals as the referent group

Exposure Data and Information Assessment

Exposure: levels and Study regions chosen because of large presence of manufacturing industries using co-exposures

solvents or they were agricultural areas

TCE prevalence among controls was $2.5 \%$ for medium/high and $3.5 \%$ for low/very low exposure

Exposure assessment In-person interviews (subject or proxy) using job/industry specific questionnaires; Expert assessment (ranked) by regional industrial hygienists of job information on the probability (3 levels) and intensity (4-point scale) of exposure to solvents. Experts blinded to case-control status

Assessment of potential Interviews: Education, relevant lifestyle factors (such as smoking), residential history, confounders extra occupational history, medical history (including X-rays, medications, diseases, and reproductive history)

Characteristics (demographics and ever smoking) were similar among cases and controls

Disease assessment Hospitals

ICD-9 MM 203, CLL 204.1

\section{Persson and Fredrikson}

(1999)

Related References Pooled analysis of two studies Persson et al. (1989); Persson et al. (1993)

Geographic Location Regional, Sweden

Population Characteristics

Cases: selection and ascertainment
Cases: 199 NHL, 106 (1989) + 93 (1993)

Case eligibility criteria: NHL in 2 hospital registries 1989 study: 1964-1986; 1993 study: 1975-1984. 20-80 years old, resident in hospital catchment area, Swedish-born

Participation rate: 1989: cases 96\%; 1993: cases 90\%, controls NR 


\section{Study Description and Methodology}

Controls: selection and $\underline{\text { Referents: }} 479$ population controls ascertainment

Referent eligibility criteria: population registry, 20-80 years old, resident in same catchment area as cases, Swedish-born; Unclear which years cases were recruited

Matching criteria: No matching specified; eligibility criteria required same age range, similar residence, and citizenship

Study Design and Analytical Methods

Population-based: Mantel-Haenszel OR stratified by age and sex with 5-yr lag. Logistic regression if $\mathrm{OR}>1.5$ on separate analyses by exposures and occupations with at least 10 cases

Exposure Data and Information Assessment

Exposure: levels and co-exposures

Exposure assessment TCE exposure prevalence among referents $\sim 7 \%$

Mailed questionnaire on occupational and leisure exposures, medical data. Self-reported exposure by rank category; Minimum 1 yr of exposure and exposure window of 5 to $45 \mathrm{yr}$ before disease diagnosis; Not clear if interviewers were blinded to case-control status

Assessment of potential Smoking, medication, X-rays, pets assessed by questionnaire and evaluated in separate confounders analyses. Unclear whether case and controls varied on demographic variables

Disease assessment Cancer registry; 1989 study: not histologically confirmed; 1993 study: 2 histologically confirmed with $4 \%$ misclassification rate cf. clinical diagnosis ICD code NR

Nordström et al. (1998)

$\begin{array}{ll}\text { Related References } & \text { None } \\ \text { Geographic Location } & \text { Sweden } \\ \text { Population Characteristics } & \end{array}$

Cases: selection and ascertainment

Cases: $111 \mathrm{HCL}$

Case eligibility criteria: Men identified from Swedish Cancer Registry 1987-1992

Participation rate: cases $91 \%$; controls $83 \%$

Controls: selection and Referents: 400 ascertainment

Referent eligibility criteria: National Population Registry Matching criteria: Age, sex, county

Study Design and Analytical Methods

Population-based: TCE: Logistic regression controlling for age; matching dissolved in analysis. Total solvents: multivariate analysis, controlling for exposure to herbicides, fungicides, impregnating agents, all exhausts for ever-exposure and univariate exposure, controlling for age and duration of exposure

Exposure Data and Information Assessment

Exposure: levels and Level: NR co-exposures

TCE exposure prevalence among controls 7\%

Exposure assessment Mailed questionnaire on complete working history, information on leisure activity and protective equipment. Ever exposed - at least 1 working day and induction of at least 1 yr.; Reviewer of questionnaire data blinded to case-control status. Proxy answers for 3 cases and 5 controls

Assessment of potential Smoking not a risk factor for HCL. No information on other lifestyle habits confounders 


\begin{tabular}{|c|c|}
\hline & Study Description and Methodology \\
\hline Disease assessment & $\begin{array}{l}\text { NCI classification, } 20 \% \text { and doubtful diagnosis reviewed by } 3 \text { pathologists; histological } \\
\text { subtypes }\end{array}$ \\
\hline \multicolumn{2}{|l|}{ Hardell et al. (1994) } \\
\hline Related References & Hardell et al. (1981) \\
\hline Geographic Location & Umeå region, Sweden \\
\hline \multicolumn{2}{|l|}{ Population Characteristics } \\
\hline \multirow{3}{*}{$\begin{array}{l}\text { Cases: selection and } \\
\text { ascertainment }\end{array}$} & Cases: $105 \mathrm{NHL}$ \\
\hline & Case eligibility criteria: Men 25-85 yr old, diagnosed at hospital pathology dept. 1974- \\
\hline & $\underline{\text { Participation rate: }}$ NR \\
\hline \multirow{3}{*}{$\begin{array}{l}\text { Controls: selection and } \\
\text { ascertainment }\end{array}$} & Referents: 355 NHL \\
\hline & $\begin{array}{l}\text { Referent eligibility criteria: National Population Registry, National Registry for Causes } \\
\text { of Death }\end{array}$ \\
\hline & $\begin{array}{l}\text { Matching criteria: Age, sex, place of residence, vital status; deceased subjects also } \\
\text { matched by year of death }\end{array}$ \\
\hline \multirow[t]{2}{*}{$\begin{array}{l}\text { Study Design and } \\
\text { Analytical Methods }\end{array}$} & $\begin{array}{l}\text { Population-based: TCE-specific analysis: Mantel-Haenszel stratified analysis by age and } \\
\text { vital status. }\end{array}$ \\
\hline & $\begin{array}{l}\text { Organic solvents class analysis: Multivariate logistic regression controlling for } \\
\text { phenoxyacetic acids, chlorophenols, DDT, asbestos, for ever-exposed, and univariate } \\
\text { analysis for subtype and stage of NHL }\end{array}$ \\
\hline \multicolumn{2}{|c|}{ Exposure Data and Information Assessment } \\
\hline \multirow{2}{*}{$\begin{array}{l}\text { Exposure: levels and } \\
\text { co-exposures }\end{array}$} & NR \\
\hline & Prevalence of TCE exposure among controls was $1 \%$ \\
\hline Exposure assessment & $\begin{array}{l}\text { Mailed questionnaire to subjects and next of kin: self-reported complete working } \\
\text { history, information on leisure activity and protective equipment; Low grade exposure- } \\
<1 \text { wk continuous or } 1 \mathrm{mo} \text {; high-grade greater than that; Reviewer of questionnaire data } \\
\text { (not clear that reviewer was an expert in exposure assessment) blinded to case-control } \\
\text { status }\end{array}$ \\
\hline
\end{tabular}

Assessment of potential NR confounders

Disease assessment Histologically confirmed; reexamined Rappaport classification

$\mathrm{NHL}=$ non-Hodgkin lymphoma; SES = socioeconomic status; NR = not reported; TCE = trichloroethylene; PPE = personal protective equipment; SEER = Surveillance, Epidemiology and End Results Program; MIS = Multicentre Italian Study; $\mathrm{NCI}=$ National Cancer Institute; OSHA = Occupational Safety and Health Administration; PEL = permissible exposure limit; $\mathrm{WHO}=$ World Health Organization; DLBCL = diffuse large B-cell lymphoma; FL = follicular lymphoma; CLL = chronic lymphocytic lymphoma; RDD = random digit dialing; LHC = lymphohematopoietic cancers; REAL = Revised EuropeanAmerican Lymphoma classification; $\mathrm{MM}=$ multiple myeloma; $\mathrm{M}=$ males; $\mathrm{F}$ = females; $\mathrm{HIV}=$ human immunodeficiency virus; $\mathrm{ppm}=$ parts per million; $\mathrm{OR}=$ odds ratio; $\mathrm{HCL}=$ hairy-cell leukemia.

\section{D.2. Assessment of Potential Biases and Other Characteristics}

Each primary study was systematically evaluated for its ability to inform the cancer hazard identification using similar questions and guidelines outlined in the protocol (see http:/ntp.niehs.nih.gov/NTP/roc/thirteenth/Protocols/TCE_Protocol12-31-13_508.pdf). Studies were evaluated for elements of study quality (potential for biases), study sensitivity, the ability to 
evaluate exposure-response relationships, and the potential for confounding (see Section 3.3.2). The guidelines describe the ideal methods and design for each study element. Two reviewers evaluated study quality in concert with input from technical advisors and from a public webinar(https://ntp.niehs.nih.gov/go/711176).

The study quality elements for each individual study that contribute to its ability to inform the cancer hazard evaluation are evaluated and summarized in Table D-4 (cohort and nested casecontrol studies), Table D-6 (kidney and liver cancer case-control studies) and Table D-8 (NHL case-control studies). These elements include the following: (1) the potential for selection and attrition bias (unlikely, possible, or probable), (2) the quality of the exposure and disease assessment (good, adequate, limited to adequate, and limited) and the (3) likelihood of and concern for exposure or disease misclassification, and whether such misclassification is considered differential or nondifferential. The general terms used for defining the potential for selection or information bias (exposure and disease misclassification) are as follows:

- Unlikely/minimal: Information from study designs and methodologies indicate that they are close to the ideal study characteristics and the potential for bias is unlikely or minimal. (See below for a description of the ideal characteristics for each specific study element.)

- Possible: Study designs or methodologies are close to but less than ideal, recognizing that in observational studies, there is almost always some methodological or informational limitation and thus some potential for certain types of bias.

- Probable: Study designs or methodologies suggest that the potential for a specific type of bias is likely.

In some cases, there is insufficient information to evaluate the level of concern. If adequate information is available, each type of bias is also characterized as to whether it is differential or non-differential. Differential (systematic) biases in the selection of study participants or information assessment are related to both exposure and disease status and have the potential to bias findings in one direction or another, whereas non-differential (random) biases, which are not related to both exposure and disease, tend to reduce the precision of the risk estimates and often bias the findings toward the null. For example, occupational cohort studies may have limited exposure data across exposure groups, increasing the potential for non-differential exposure misclassification, and may also have the potential for a healthy worker (hire or survival) effect, a type of selection bias that tends to bias findings away from finding an effect (if present) in studies where the comparison group comes from the general population.

The presence of a potential bias in a study does not necessarily mean that the findings of the study should be disregarded. For example, the effect of confounding may only account for a small percentage of the magnitude of the risk estimate. Therefore, an important step in the process of evaluating biases is to determine the probable impact of the described biases on study results - that is, the magnitude of distortion and the direction in which each bias is likely to affect the outcome of interest (if known). The impact of the potential bias or confounding on the study findings is discussed in the cancer hazard assessment (see Sections 4.1, 5.1, and 6.1). 


\section{D.2.1. Selection Bias}

Selection or attrition bias was considered unlikely if cohorts or cases and controls represented the same underlying population, there was little or no evidence of a healthy worker hire or survival effect, cases and controls were selected by similar criteria that were not related to trichloroethylene exposure, participation was high and not related to exposure or disease status, and loss to follow-up was low (preferably $<5 \%$ ) and similar in both groups.

\section{D.2.2. Quality of Exposure Assessment and Misclassification of Exposure}

Exposure assessment: A ranking of good was given to studies having many of the following elements: industrial hygiene or biomonitoring data, individual detailed job-task exposure matrices, job or task descriptions, knowledge of the exposure setting, consideration of frequency, confidence and intensity, expert assessment, and/or calendar period-specific exposure data. It should be noted, for example, that not all job-exposure or job-task exposure matrices are of equal quality; some are based only on generic occupational or industrial categories or codes, rather than information specific for the plant or industry under investigation, and this may result in substantial misclassification of exposure.

The assessment of exposure misclassification is complex and involves multiple factors such as the likelihood that subjects were ever exposed and misclassification of exposure level, and thus labels such as unlikely, possible, or probable, do not adequately capture the complexity of exposure misclassification; thus, his study element is evaluated qualitatively rather than by category.

\section{D.2.3. Quality of Case Ascertainment and Disease Misclassification}

Case ascertainment: A ranking of good was given to studies where multiple or verified sources were used to identify vital status and/or cases/deaths and ascertainment of cases/deaths was complete or close to complete.

Disease misclassification, for each endpoint of concern, is ranked as unlikely, possible, or probable, based on the sensitivity and specificity of the disease diagnosis, i.e., whether cases were histologically confirmed and whether the system of disease classification was based on newer ICD classifications. The potential for bias in case or death misclassification is typically nondifferential, but can also be differential, i.e., differ by exposure status.

\section{D.2.4. Study Sensitivity and Exposure-response Relationships}

The study sensitivity and exposure-response elements evaluated and summarized in Table D-5 (cohort and nested case-control studies), Table D-7 (kidney and liver cancer case-control studies) and Table D-9 (NHL case-control studies). A study's sensitivity is defined as the ability to detect an effect of exposure, which is principally a function of study size (specifically, the numbers of trichloroethylene-exposed subjects in cohort studies or the numbers of trichloroethylene-exposed controls in case-control studies), the length of follow-up and levels of exposure to trichloroethylene. A ranking of good was given to studies having many of the following elements: larger numbers of exposed subjects or cases, adequate length of follow-up, high levels of exposure, long exposure duration, large groups or subgroups with a range of exposures from low/medium to high to permit the evaluation of exposure-response relationships and little concern about exposure misclassification. Factual information on these elements is also 
presented in these tables. Studies less than ideal were assigned rankings of adequate or limited. The adequacy of data (range of exposure) and methods used to evaluate exposure-response relationships were also evaluated.

\section{D.2.5. Overall Ranking of Studies}

In general, studies given the most weight in the cancer hazard evaluation had the following characteristics:

- little evidence of the potential for selection bias

- adequate to good exposure assessment with little evidence for exposure misclassification

- incidence studies, histologically confirmed case or use of more recent classification codes

- adequate sensitivity (e.g., sufficient power, length of follow-up and adequate levels of exposure) to detect an effect of exposure

- potential confounding is considered minimal

- appropriate methods for evaluating exposure-response relationships

The ranking of study sensitivity considered multiple factors. For example, very low (or uncertain) exposure levels or duration and/or a high probability of exposure misclassification may result in the study being inadequate to evaluate cancer risk despite adequate study size, or a lack of other biases or evidence of potential confounding. Conversely, high exposure levels may partly compensate for smaller study sizes in some studies.

Based on the overall evaluation, studies were broadly grouped according to their ability to inform the cancer hazard evaluation based on the above characteristics, as follows:

- high utility: most elements were ranked as having little concern for biases or misclassification or the quality of the element was ranked as good to adequate

- moderate utility: most elements were ranked as having some concern bias or information misclassification, or the quality of the element was ranked as limited

- low to moderate utility: similar to moderate but lower study sensitivity and somewhat greater concerns for exposure or disease misclassification

- low utility: considerable concerns about exposure misclassification or systematic biases, and low study sensitivity

Not all elements may equally affect the overall ability of a given study to inform the evaluation. The quality of the exposure assessment and potential for exposure misclassification was given considerable weight in ranking the studies. In addition, studies with high probability of systematic biases were rated low. The impact of identified biases, in terms of both direction and magnitude, and potential for confounding, is evaluated in the cancer hazard assessment in the light of the study findings (Sections 4, 5, and 6). For example, the potential for selection or participation bias, or confounding does not always negate a positive association, if the observed risk estimate is high. 
Table D-4. Cohort and Nested Case-control Studies of Trichloroethylene Exposure: Summary of Study Quality

\begin{tabular}{|c|c|c|c|}
\hline $\begin{array}{c}\text { Study and Number of } \\
\text { TCE-exposed } \\
\text { Subjects }\end{array}$ & $\begin{array}{c}\text { Selection Bias and Completeness of } \\
\text { Follow-up }\end{array}$ & $\begin{array}{l}\text { Quality of Exposure Assessment and } \\
\text { Misclassification of Exposure }\end{array}$ & $\begin{array}{l}\text { Quality of Case Ascertainment and } \\
\text { Misclassification of Diagnosis }\end{array}$ \\
\hline Nordic Studies & & & \\
\hline $\begin{array}{l}\text { Vlaanderen et al. } \\
\text { (2013) } \\
\text { Cancer registry-based } \\
\text { (Nordic Occupational } \\
\text { Cohort); Nested case- } \\
\text { control analysis } \\
76,130 \text { kidney cancer } \\
\text { cases ( } 41 \% \mathrm{~F}) ; 380,650 \\
\text { controls (41\% F); } \\
23,896 \text { liver cancer } \\
\text { cases ( } 38 \% \mathrm{~F}), 119,480 \\
\text { controls (38\% F) } \\
\text { 1960-90 to 2003-05 } \\
\text { Mortality }\end{array}$ & $\begin{array}{l}\text { Unlikely } \\
\text { Adequate methods (census, cancer registry, } \\
\text { population registries) for identifying } \\
\text { cohort; Controls matched to cases by age, } \\
\text { sex, country } \\
\text { Loss to follow-up: Not reported; assume } \\
\text { complete because of linkage with registry } \\
\text { data }\end{array}$ & $\begin{array}{l}\text { Limited: Quantitative, calendar-year specific, } \\
\text { country specific, generic JEM; Exposure was } \\
\text { assigned based on limited occupation } \\
\text { information on specific jobs from census data } \\
\text { and assumed no changes in jobs between } \\
\text { censuses. The JEM had poor sensitivity and did } \\
\text { not account for job tasks, heterogeneity within } \\
\text { jobs and changes over time } \\
\text { Use of population-wide occupational exposure } \\
\text { database may lack precision for individual } \\
\text { participants }\end{array}$ & $\begin{array}{l}\text { Case ascertainment: Adequate } \\
\text { Linkage via cancer registry } \\
\text { Misclassification of diagnosis: } \\
\text { Possible for NHL, unlikely for kidney } \\
\text { and liver } \\
\text { RCC (histologically confirmed), liver } \\
\text { and MM; Diagnosis of NHL based on } \\
\text { broad ICD-7 classification that } \\
\text { includes several diseases and does not } \\
\text { differentiate subtypes }\end{array}$ \\
\hline
\end{tabular}




\begin{tabular}{|c|c|c|c|}
\hline $\begin{array}{l}\text { Study and Number of } \\
\text { TCE-exposed } \\
\text { Subjects }\end{array}$ & $\begin{array}{l}\text { Selection Bias and Completeness of } \\
\text { Follow-up }\end{array}$ & $\begin{array}{l}\text { Quality of Exposure Assessment and } \\
\text { Misclassification of Exposure }\end{array}$ & $\begin{array}{l}\text { Quality of Case Ascertainment and } \\
\text { Misclassification of Diagnosis }\end{array}$ \\
\hline Hansen et al. (2013) & Unlikely & \multirow{2}{*}{$\begin{array}{l}\text { Adequate to good: Biomonitoring at the } \\
\text { individual level (urine-TCA); Few data on } \\
\text { individual industries or jobs of workers, } \\
\text { cumulative exposure, and exposure duration; } \\
\text { Diverse TCE-using industries included }\end{array}$} & Case ascertainment: Adequate \\
\hline $\begin{array}{l}\text { Pooled Nordic cohort } \\
\text { incidence analysis; } \\
5,553 \text { workers }(3,776\end{array}$ & $\begin{array}{l}\text { Adequate methods to select cohort } \\
\text { members. All workers with } \geq 1 \text { urine TCA } \\
\text { or air TCE measurement included in }\end{array}$ & & $\begin{array}{l}\text { Cases identified in cancer registries via } \\
\text { ID linkage; only } 0.1 \% \text { of the cohort } \\
\text { was lost to follow-up }\end{array}$ \\
\hline \multirow[t]{2}{*}{$\begin{array}{l}\text { Axelson et al. (1994), } \\
\text { Anttila et al. (1995), } \\
\text { Hansen et al. (2001) }\end{array}$} & \multirow[t]{2}{*}{ Loss to follow-up: Minimal; $(<1 \%)$. } & \multirow{2}{*}{$\begin{array}{l}\text { The U-TCA exposure assessment is expected to } \\
\text { have high sensitivity, but specificity may be a } \\
\text { concern if workers were exposed to other } \\
\text { chlorinated solvents that are metabolized to } \\
\text { TCA. In addition, because few measurements ( } 2 \\
\text { to 3) were available for most subjects and many } \\
\text { subjects (55\% of Swedish study) had only } 1 \\
\text { measurement, individuals classified as } \\
\text { unexposed could have been exposed to TCE and } \\
\text { U-TCA and U-TCA exposure misclassification } \\
\text { related to intensity level may occur }\end{array}$} & $\begin{array}{l}\text { Misclassification of diagnosis: } \\
\text { Possible for NHL, unlikely for kidney } \\
\text { and liver }\end{array}$ \\
\hline & & & $\begin{array}{l}\text { Histologically confirmed in Swedish } \\
\text { study; Diagnosis of NHL based on } \\
\text { broad ICD-7 classification that } \\
\text { includes several diseases and does not } \\
\text { differentiate subtypes }\end{array}$ \\
\hline \multirow{2}{*}{$\begin{array}{l}\text { Raaschou-Nielsen et al. } \\
(2003)\end{array}$} & Possible & \multirow{4}{*}{$\begin{array}{l}\text { Limited: Employment as a blue-collar worker in } \\
\text { a TCE-using company used as a surrogate for } \\
\text { potential TCE exposure and size of company } \\
\text { used as surrogate for estimated percentage of } \\
\text { workers exposed to TCE. Limited } \\
\text { characterization of exposure: Urine TCA and air } \\
\text { TCE data for some workers but not used in } \\
\text { exposure assessment }\end{array}$} & Case ascertainment: Adequate \\
\hline & \multirow{4}{*}{$\begin{array}{l}\text { Cohort and comparison group differ with } \\
\text { respect to socioeconomic status. Cohort } \\
\text { included all "blue-collar" workers whereas } \\
\text { reference population (Danish population) } \\
\text { included both blue- and white-collar } \\
\text { workers, which could lead to an under- or } \\
\text { overestimate of expected cases for cancer } \\
\text { sites that are associated with SES. } \\
\text { Differences in SES may explain significant } \\
\text { increase in all-cancer incidence (M and F) } \\
\text { and of smoking-related cancers. }\end{array}$} & & $\begin{array}{l}\text { Cases identified via ID linkage with } \\
\text { cancer registry }\end{array}$ \\
\hline $\begin{array}{l}\text { collar worker cohort; } \\
40,049 \text { workers } \\
\text { approx. } 70 \% \text { men) }\end{array}$ & & & $\begin{array}{l}\text { Misclassification of diagnosis: } \\
\text { Possible for NHL, unlikely for kidney } \\
\text { and liver }\end{array}$ \\
\hline & & & \\
\hline & & \multirow{2}{*}{$\begin{array}{l}\text { Exposure misclassification (non-differential): is } \\
\text { a concern. Only } 19 \%-81 \%(41 \% \text { overall) with } \\
\text { estimated exposure to TCE (working in the same } \\
\text { room that TCE was used); Employment duration } \\
\text { before } 1964 \text { was not considered, which could } \\
\text { attenuate exposure-duration relationships }\end{array}$} & $\begin{array}{l}\text { ICD-7 classification includes several } \\
\text { diseases and does not differentiate } \\
\text { subtypes }\end{array}$ \\
\hline & $\begin{array}{l}\text { Loss to follow-up: Minimal; authors report } \\
\text { follow-up as being virtually complete }\end{array}$ & & \\
\hline
\end{tabular}




\begin{tabular}{cccc}
\hline $\begin{array}{c}\text { Study and Number of } \\
\text { TCE-exposed } \\
\text { Subjects }\end{array}$ & $\begin{array}{c}\text { Selection Bias and Completeness of } \\
\text { Follow-up }\end{array}$ & $\begin{array}{c}\text { Quality of Exposure Assessment and } \\
\text { Misclassification of Exposure }\end{array}$ & $\begin{array}{c}\text { Quality of Case Ascertainment and } \\
\text { Misclassification of Diagnosis }\end{array}$ \\
\hline
\end{tabular}

\section{Rocket engine testing or aircraft manufacturing workers}

Lipworth et al. (2011) Possible

Burbank, CA (USA) Adequate methods to select cohort: All aircraft manufacturing workers with minimum of 1 year workers cohort; 5,443 employment. Some evidence for HWE (approx. 80\% male)

Mortality Study based on $9 \%$ decrease in all-cause and allcancer mortality than CA and U.S. population

Loss to follow-up: Minimal; $1.7 \%$ total cohort
Limited to adequate: Qualitative JEM for occupational job groups based on plant data; Workers classified by ever exposure, type of exposure (routine or intermittent) and duration of potential exposure. No quantitative exposure assessment or ranking of relative intensity of exposure

Exposure misclassification is a concern (nondifferential) for all analyses

Adequate to good: Semi-quantitative calendar year specific JEM constructed from detailed occupational information used to develop exposure scores for each job based on intensity, frequency, and duration of exposure. Each worker classified by cumulative exposure score and pattern of exposure; however, exposure records that specific subjects were missing, and information was based on position descriptions. Some limited air monitoring by job/task but not used in exposure assessment

Exposure misclassification (with respect to whether workers were ever exposed) is a concern (non-differential). Misclassification regarding intensity level would most likely attenuate any exposure-response relationships
Case ascertainment: Adequate

Multiple sources used to determine vital status

Misclassification of diagnosis: Possible (non-differential) for some tumor sites

NDI using ICD at the time of diagnosis; possible concern for diagnosis of NHL

Potential for missing cases that do not result in death for cancers with long survival (kidney and NHL), which would decrease precision

Case ascertainment: Adequate Use of state vital records and NDI for vital status (missing data NR)

Misclassification of diagnosis: Possible (non-differential) for some tumor sites in mortality study

NDI using ICDA-8 or 9, ICD-10. Underlying and contributing causes of death; possible concern for diagnosis of NHL; SEER (Utah) registry used for incident cases (1973-1999) so possible concern about earlier ICD classifications of NHL

Potential for missing cases that do not result in death for cancers with long survival (kidney and NHL), which would decrease precision 


\begin{tabular}{|c|c|c|c|}
\hline $\begin{array}{c}\text { Study and Number of } \\
\text { TCE-exposed } \\
\text { Subjects }\end{array}$ & $\begin{array}{l}\text { Selection Bias and Completeness of } \\
\text { Follow-up }\end{array}$ & $\begin{array}{l}\text { Quality of Exposure Assessment and } \\
\text { Misclassification of Exposure }\end{array}$ & $\begin{array}{l}\text { Quality of Case Ascertainment and } \\
\text { Misclassification of Diagnosis }\end{array}$ \\
\hline $\begin{array}{l}\text { Boice et al. (2006) } \\
\text { Los Angeles (USA) } \\
\text { rocket engine testing } \\
\text { workers cohort; } 1,111 \\
\text { men } \\
\text { Mortality study } \\
\text { Overlap with Zhao et } \\
\text { al. }(2005) \text { cohort }\end{array}$ & $\begin{array}{l}\text { Possible for external analyses } \\
\text { Adequate methods to select cohort; all } \\
\text { workers with adequate employment data } \\
\text { included in cohort. Evidence of HWE } \\
\text { based on } 13 \% \text { (significant decrease in all- } \\
\text { cause mortality among test stand mechanics } \\
\text { with any exposure to TCE) } \\
\text { Loss to follow-up: Minimal; } 3.1 \% \text { missing } \\
\text { vital status }\end{array}$ & $\begin{array}{l}\text { Limited to adequate: Qualitative assessment of } \\
\text { TCE exposure using test stand mechanics as a } \\
\text { surrogate of exposure, exposure-based duration } \\
\text { of employment using TCE, walk-through } \\
\text { surveys and dates that TCE was used and } \\
\text { duration of exposure from engine flush. No } \\
\text { assessment of exposure intensity } \\
\text { The probability of being exposed to TCE is } \\
\text { greatest in analyses by test engine flush; } \\
\text { however, exposure misclassification is still } \\
\text { possible }\end{array}$ & $\begin{array}{l}\text { Case ascertainment: Adequate } \\
\text { Use of state vital records and NDI for } \\
\text { vital status } \\
\text { Misclassification of diagnosis: } \\
\text { Possible (non-differential) for some } \\
\text { tumor sites } \\
\text { Death certificate using ICD at the time } \\
\text { of diagnosis; possible concern for } \\
\text { diagnosis of NHL } \\
\text { Potential for missing cases that do not } \\
\text { result in death for cancers with long } \\
\text { survival (kidney and NHL), which } \\
\text { would decrease precision. }\end{array}$ \\
\hline $\begin{array}{l}\text { Zhao et al. (2005) } \\
\text { Los Angeles (USA) } \\
\text { aerospace workers } \\
\text { cohort } \\
\text { Mortality; 6,044 men } \\
\text { Incidence; 5,049 men }\end{array}$ & $\begin{array}{l}\text { Unlikely } \\
\text { Adequate methods to select cohort; all } \\
\text { workers with potential exposure to TCE } \\
\text { included in cohort } \\
\text { Loss to follow-up: Minimal; }(<0.1 \%)\end{array}$ & $\begin{array}{l}\text { Adequate to good: Semi-quantitative JEM } \\
\text { (relative intensity), which was calendar-year } \\
\text { specific, constructed using job titles and detailed } \\
\text { description of job tasks. Each worker's exposure } \\
\text { classified by cumulative relative intensity scores } \\
\text { to TCE and co-exposures, by calendar period. } \\
\text { No quantitative exposure measurements } \\
\text { Exposure misclassification is not a concern, } \\
\text { especially among individuals with the highest } \\
\text { cumulative exposure. Exposure misclassification } \\
\text { between levels of cumulative exposure would } \\
\text { most likely attenuate any exposure }\end{array}$ & $\begin{array}{l}\text { Case ascertainment: Adequate } \\
\text { NDI for cause of death (missing data } \\
\text { NR) and multiple cancer registries } \\
\text { used for diagnosis (missing data NR) } \\
\text { Misclassification of diagnosis: } \\
\text { Unlikely for incidence } \\
\text { Incidence: ICD-O (extension of ICD- } \\
\text { 10). Deaths: ICD-9 and 10; } \\
\text { Underlying and contributing causes of } \\
\text { death }\end{array}$ \\
\hline
\end{tabular}




\begin{tabular}{|c|c|c|c|}
\hline $\begin{array}{l}\text { Study and Number of } \\
\text { TCE-exposed } \\
\text { Subjects }\end{array}$ & $\begin{array}{l}\text { Selection Bias and Completeness of } \\
\text { Follow-up }\end{array}$ & $\begin{array}{l}\text { Quality of Exposure Assessment and } \\
\text { Misclassification of Exposure }\end{array}$ & $\begin{array}{l}\text { Quality of Case Ascertainment and } \\
\text { Misclassification of Diagnosis }\end{array}$ \\
\hline $\begin{array}{l}\text { Morgan et al. (1998) } \\
\text { Arizona (USA) aircraft } \\
\text { manufacturing workers } \\
\text { cohort; } 4,733 \text { (2,555 } \\
\text { men, 2,178 women) } \\
\text { Mortality study }\end{array}$ & $\begin{array}{l}\text { Possible for external analysis } \\
\text { Adequate methods to select cohort (all } \\
\text { workers employed for specific dates) but } \\
\text { evidence of HWE based on } 15 \% \text { significant } \\
\text { decrease in all-cause mortality for TCE- } \\
\text { exposed subcohort } \\
\text { Loss to follow up: Minimal; appears to be } \\
0.1 \% \text { (excluded due to "missing } \\
\text { information" but not clear if applies to vital } \\
\text { status or other data) }\end{array}$ & $\begin{array}{l}\text { Adequate: Semi-quantitative expert assessment, } \\
\text { using JEM by job title and based on location of } \\
\text { jobs in proximity to degreaser area, used to } \\
\text { estimate exposure category scores. Exposure } \\
\text { assessment is limited with respect to calendar } \\
\text { year, confidence, frequency, or probability of } \\
\text { exposure and information on tasks. Limited } \\
\text { quantitative exposure measurements available } \\
\text { during most of period TCE used (not reported or } \\
\text { used in exposure assessment) } \\
\text { The probability of being exposed to TCE is } \\
\text { greatest among workers in the "high" and } \\
\text { "peak" exposure categories. Exposure } \\
\text { misclassification (with respect to whether } \\
\text { workers were ever exposed) is more of a concern } \\
\text { (non-differential) in the med/low exposure } \\
\text { categories }\end{array}$ & $\begin{array}{l}\text { Case ascertainment: Adequate } \\
\text { Use of SSA, NDI, or state vital records } \\
\text { Potential for missing cases that do not } \\
\text { result in death for cancers with long } \\
\text { survival (kidney and NHL), which } \\
\text { would decrease precision } \\
\text { Misclassification of diagnosis: } \\
\text { Possible (non-differential) for some } \\
\text { tumor sites } \\
\text { Death certificate using ICD at the time } \\
\text { of diagnosis (7 to 9); possible concern } \\
\text { for diagnosis of NHL }\end{array}$ \\
\hline
\end{tabular}




\begin{tabular}{cccc}
\hline $\begin{array}{c}\text { Study and Number of } \\
\text { TCE-exposed } \\
\text { Subjects }\end{array}$ & $\begin{array}{c}\text { Selection Bias and Completeness of } \\
\text { Follow-up }\end{array}$ & $\begin{array}{c}\text { Quality of Exposure Assessment and } \\
\text { Misclassification of Exposure }\end{array}$ & $\begin{array}{c}\text { Quality of Case Ascertainment and } \\
\text { Misclassification of Diagnosis }\end{array}$ \\
\hline
\end{tabular}

\section{Other industries: Cohort and nested case-control studies}

Silver et al. (2014) Unknown for internal analysis used for

New York State (USA) TCE-exposed subcohort

electronics Strong evidence for healthy worker effect

manufacturing workers in total cohort; not clear if there is a healthy cohort; 24,037 men, worker survival effect

10,457 women (total cohort)

Mortality study
Bahr et al. (2011)

Kentucky (USA)

uranium enrichment

workers cohort; 5,535 men

Mortality study

\section{Probable}

Difficult to evaluate because of limited information. Selection of workers based on complete work histories; however, information about other eligibility criteria (e.g., age of workers, enrollment, number of subjects excluded due to missing data) were not provided. Evidence of HWE, based on $20 \%$ to $30 \%$ decrease in allcause mortality in TCE-exposed groups. Evidence of healthy worker survival effect Loss to follow-up: Not reported
Limited: Exposure assessment based on work history and dept.-year JEM, cumulative exposure assigned based on potential exposure to TCE (based on dept. use and board categories of position) and duration of TCE. No information on job tasks or exposure condition or levels of use. Exposure intensity could vary within a dept. and over time. Position title could not be used to compare exposure across dept. (except for classifying admin. staff as unexposed). Company records and work history incomplete, especially for time periods before 1974. Incomplete and contradictory work history records

Exposure misclassification (with respect to whether workers were ever exposed) is a concern (non-differential)

Limited: Generic qualitative JEM based on work Case ascertainment: Unknown history but little data provided on ranking of probability of TCE exposure. No quantitative exposure measurements

Exposure misclassification (non-differential) is a concern and likely to be substantial
Case ascertainment: Adequate

Use of appropriate methods (State vital records or NDI) to ascertain vital status

Young cohort (17\% deaths) and potential for missing cases of cancers with long survival (especially kidney and NHL), which would decrease precision

Misclassification of diagnosis: Possible (non-differential) for some tumor sites

Death certificate using ICD code at time of death used; possible concern for diagnosis of NHL

Source and completeness of vital status and cause of death data NR Misclassification of diagnosis:

Possible (non-differential) for some tumor sites

Death certificate using ICD code at time of death used; possible concern for diagnosis of NHL

Potential for missing cases of cancers with long survival (kidney and NHL), which would decrease precision 


\begin{tabular}{|c|c|c|c|}
\hline $\begin{array}{l}\text { Study and Number of } \\
\text { TCE-exposed } \\
\text { Subjects }\end{array}$ & $\begin{array}{c}\text { Selection Bias and Completeness of } \\
\text { Follow-up }\end{array}$ & $\begin{array}{l}\text { Quality of Exposure Assessment and } \\
\text { Misclassification of Exposure }\end{array}$ & $\begin{array}{l}\text { Quality of Case Ascertainment and } \\
\text { Misclassification of Diagnosis }\end{array}$ \\
\hline $\begin{array}{l}\text { Yiin et al. (2009) } \\
\text { Tennessee (USA) } \\
\text { Uranium enrichment } \\
\text { workers } \\
\text { Nested case-control } \\
\text { mortality study; 47,941 } \\
\text { men and women } \\
114 \text { cases of multiple } \\
\text { myeloma (ICD 203) }\end{array}$ & $\begin{array}{l}\text { Possible } \\
\text { Cohort selection based on employee roster } \\
\text { for all workers employed in gaseous } \\
\text { diffusion plant prior to } 1985 \text { (plant closing } \\
\text { date) and employed }>30 \text { days; cases and } \\
\text { controls selected based on availability of } \\
\text { uranium dose data (appears complete) } \\
\text { Loss to follow-up: NR }\end{array}$ & $\begin{array}{l}\text { Limited to adequate: Individual cumulative } \\
\text { exposure score for TCE assigned based on } \\
\text { modified JEM that estimated levels for exposure } \\
\text { activities by decade. Inadequate information to } \\
\text { link monitoring data to workers and work } \\
\text { history data missing information on } \\
\text { building/work location. Limited information } \\
\text { available on assessment } \\
\text { Exposure misclassification (non-differential) is a } \\
\text { concern }\end{array}$ & $\begin{array}{l}\text { Misclassification of diagnosis of } \\
\text { cases: Possible (non-differential) } \\
\text { Cases of multiple myeloma } \\
\text { (underlying and contributory causes of } \\
\text { death, ICD 203) identified from death } \\
\text { certificates (no other details reported) }\end{array}$ \\
\hline $\begin{array}{l}\text { Ritz (1999) } \\
\text { Ohio (USA) uranium } \\
\text { processing workers } \\
\text { cohort; } 2,972 \text { men } \\
\text { Mortality study }\end{array}$ & $\begin{array}{l}\text { Possible } \\
\text { Selection of workers based on all workers } \\
\text { with data on chemical exposure and } \\
\text { monitored for radiation exposure included } \\
\text { in cohort, but } 35 \% \text { total cohort excluded } \\
\text { due to absence of radiation records. Some } \\
\text { evidence of HWE based on } \sim 15 \% \\
\text { statistically significant decrease in all- } \\
\text { cause mortality. Also, a possible bias if } \\
\text { radiation exposure associated with TCE } \\
\text { exposure } \\
\text { Loss to follow up: Not reported }\end{array}$ & $\begin{array}{l}\text { Limited to adequate: Semi-quantitative JEM for } \\
\text { individual workers based on verified job title } \\
\text { and department using company industrial } \\
\text { hygienists and workers but does not appear to be } \\
\text { calendar-period specific. Exposure categorized } \\
\text { by } 2 \text { levels (light and moderate) and } 2 \text { categories } \\
\text { of duration. No quantitative exposure } \\
\text { measurements } \\
\text { Exposure misclassification (with respect to } \\
\text { whether workers were ever exposed) is a } \\
\text { concern (non-differential). Most of the workers } \\
\text { in this study had low levels of exposure }\end{array}$ & $\begin{array}{l}\text { Case ascertainment: Adequate } \\
\text { Use of appropriate methods (SSA or } \\
\text { NDI Records) to ascertain vital status } \\
\text { Misclassification of diagnosis: } \\
\text { Possible (non-differential) for some } \\
\text { tumor sites } \\
\text { Death certificate (NDI) using ICDA-8 } \\
\text { (external analysis) and ICD-9 (interna } \\
\text { analysis); possible concern for } \\
\text { diagnosis of NHL } \\
\text { Potential for missing cases that do not } \\
\text { result in death for cancers with long } \\
\text { survival (kidney and NHL) which } \\
\text { would decrease precision }\end{array}$ \\
\hline
\end{tabular}




\begin{tabular}{|c|c|c|c|}
\hline $\begin{array}{c}\text { Study and Number of } \\
\text { TCE-exposed } \\
\text { Subjects }\end{array}$ & $\begin{array}{l}\text { Selection Bias and Completeness of } \\
\text { Follow-up }\end{array}$ & $\begin{array}{l}\text { Quality of Exposure Assessment and } \\
\text { Misclassification of Exposure }\end{array}$ & $\begin{array}{l}\text { Quality of Case Ascertainment and } \\
\text { Misclassification of Diagnosis }\end{array}$ \\
\hline $\begin{array}{l}\text { Henschler et al. (1995) } \\
\text { German cardboard } \\
\text { manufacturing cohort; } \\
169 \text { men } \\
\text { Incidence \& mortality } \\
\text { study of kidney cancer }\end{array}$ & $\begin{array}{l}\text { Probable } \\
\text { Selection of cohort may be based on cluster } \\
\text { of renal cancers, which would bias towards } \\
\text { an overestimate of the risk estimate. } \\
\text { Comparison group from different countries } \\
\text { from exposed cohort (if a bias, the direction } \\
\text { would most likely be towards } \\
\text { underestimating the risk estimate from } \\
\text { using possibly inflated expected rates); } \\
\text { Evidence for a HWE based on statistically } \\
\text { significant } 30 \% \text { decrease in all cancer } \\
\text { mortality } \\
\text { Loss to follow-up: Minimal; } 8 \% \text { (refusal, } \\
\text { ill-health, or untraced) suggesting most } \\
\text { cases and deaths were identified }\end{array}$ & $\begin{array}{l}\text { Limited: Exposure assigned based on job } \\
\text { location in the plant and descriptions of plant } \\
\text { conditions (walk-through and interview) for ever } \\
\text { exposure only. Level and duration of exposure } \\
\text { not characterized } \\
\text { Although the exposure assessment was of } \\
\text { limited quality (based on workspace), exposure } \\
\text { to TCE occurred in an open system. Thus, } \\
\text { exposure misclassification is not a concern for } \\
\text { most workers, although exposure duration and } \\
\text { intensity are likely to vary among workers. It is } \\
\text { not clear if the unexposed workers included in } \\
\text { the internal analysis could have had some } \\
\text { exposure to TCE (no details are provided) }\end{array}$ & $\begin{array}{l}\text { Case ascertainment: Limited } \\
\text { Multiple methods used to identify } \\
\text { deaths and cases such as } \\
\text { hospital/medical records, rather than } \\
\text { central death records or cancer } \\
\text { registry. Different methods may have } \\
\text { been used to assign cause of death or } \\
\text { cases for exposed cohort (physicians } \\
\text { and records and abdominal sonogram) } \\
\text { than the general population in external } \\
\text { analysis, which could potentially bias } \\
\text { external (but not internal) analyses } \\
\text { towards an overestimate of the risk } \\
\text { estimate } \\
\text { Misclassification of diagnosis: } \\
\text { Unlikely for incidence, possible for } \\
\text { deaths (but only } 2 \text { deaths reported) } \\
\text { Deaths classified from medical records } \\
\text { or physicians using ICD-9; different } \\
\text { sources may vary in reliability. } \\
\text { Histological confirmation of renal-cell } \\
\text { cancer from incident cases (the only } \\
\text { tumors that were reported) }\end{array}$ \\
\hline
\end{tabular}




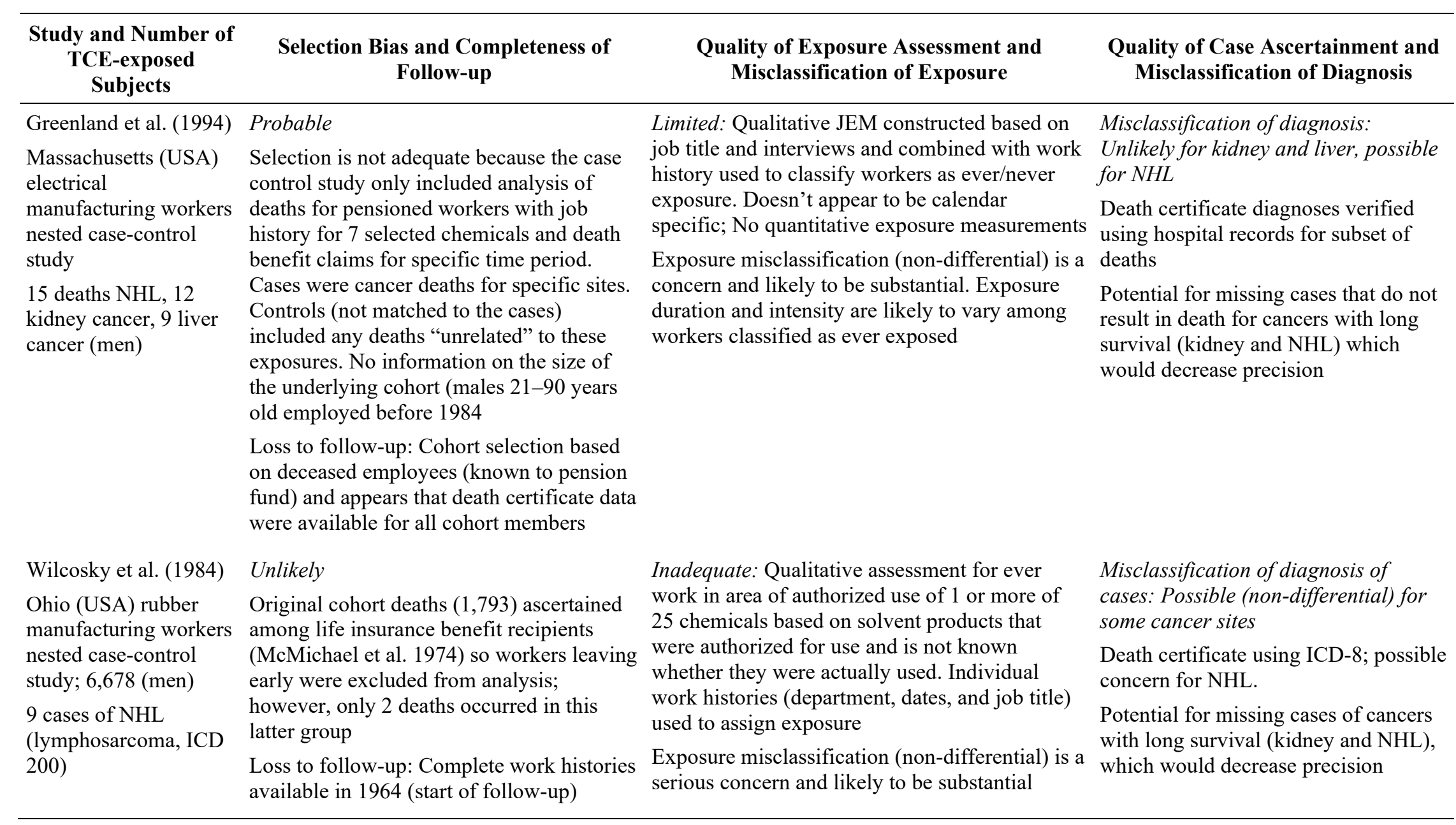




\begin{tabular}{|c|c|c|c|}
\hline $\begin{array}{c}\text { Study and Number of } \\
\text { TCE-exposed } \\
\text { Subjects }\end{array}$ & $\begin{array}{c}\text { Selection Bias and Completeness of } \\
\text { Follow-up }\end{array}$ & $\begin{array}{l}\text { Quality of Exposure Assessment and } \\
\text { Misclassification of Exposure }\end{array}$ & $\begin{array}{l}\text { Quality of Case Ascertainment and } \\
\text { Misclassification of Diagnosis }\end{array}$ \\
\hline \multicolumn{4}{|l|}{ Drinking Water Study } \\
\hline Bove et al. (2014) & Unlikely & \multirow{6}{*}{$\begin{array}{l}\text { Limited: Reconstruction of exposure is based on } \\
\text { historical sampling of two water supply systems } \\
\text { in defined regions. Estimate of cumulative } \\
\text { exposure based on duration at residence and } \\
\text { modeled TCE concentration levels from the } \\
\text { water supply system associated with the } \\
\text { residence. No data on individual consumption; } \\
\text { May have had errors in assignment of residential } \\
\text { location }\end{array}$} & Case-ascertainment: Adequate \\
\hline $\begin{array}{l}\text { Cohort study (drinking } \\
\text { water contamination) }\end{array}$ & \multirow{5}{*}{$\begin{array}{l}\text { Cohort: All active service personnel } \\
\text { eligible } \\
\text { Loss to follow-up: Minimal; }<2 \%\end{array}$} & & $\begin{array}{l}\text { Multiple sources used to determine } \\
\text { vital status including the NDI }\end{array}$ \\
\hline Camp Lejeune, $\mathrm{NC}$ and & & & Potential for missing cases of cancers \\
\hline $\begin{array}{l}\text { Camp Pendleton, CA } \\
\text { (USA) }\end{array}$ & & & $\begin{array}{l}\text { with long survival (kidney and NHL), } \\
\text { which would decrease precision }\end{array}$ \\
\hline $\begin{array}{l}154,932 \text { (Lejeune) } \\
154969 \text { (Pendleton) }\end{array}$ & & & $\begin{array}{l}\text { Misclassification of diagnosis: } \\
\text { possible for some tumor sites. }\end{array}$ \\
\hline 154,969 (Pendleton) & & & \\
\hline Mortality Study & & $\begin{array}{l}\text { wheosure misclassification (with respect to } \\
\text { concern) although to a lesser degree among } \\
\text { individuals with higher estimated cumulative } \\
\text { exposure. Exposure misclassification regarding } \\
\text { cumulative exposure would most likely attenuate } \\
\text { any exposure-response relationship }\end{array}$ & $\begin{array}{l}\text { Death certiticate; underlying and } \\
\text { contributing causes; ICD NR; possible } \\
\text { concern for NHL }\end{array}$ \\
\hline
\end{tabular}

TCE = trichloroethylene; F = females; JEM = job exposure matrix; NHL = non-Hodgkin lymphoma; $\mathrm{RCC}=$ renal-cell cancer; $\mathrm{MM}=$ multiple myeloma; ICD = International Classification of Diseases; TCA = trichloroacetic acid; HWE = healthy worker effect; U-TCA = urine trichloroacetic acid; SES = socioeconomic status; $\mathrm{M}=$ male; $\mathrm{NDI}=\mathrm{National}$ Death Index; NR = not reported; ICDA = International Classification of Diseases-Adjusted; SEER = Surveillance, Epidemiology and End Results Program (National Cancer Institute); SSA = Social Security Administration. 
Table D-5. Cohort Studies: Study Sensitivity and Exposure-response Analyses

\begin{tabular}{|c|c|c|c|}
\hline $\begin{array}{l}\text { Study Summary (Study } \\
\text { Sensitivity) }\end{array}$ & Study Size/Length of Follow-up & $\begin{array}{c}\text { Reported or Estimated Exposure Levels } \\
\text { or Duration }\end{array}$ & $\begin{array}{l}\text { Exposure-response Analyses: Dose } \\
\text { Metrics/Range Lagging Analyses }\end{array}$ \\
\hline Nordic studies & & & \\
\hline $\begin{array}{l}\text { Vlaanderen et al. (2013) } \\
\text { Cancer registry-based, Nordic } \\
\text { countries, nested case-control } \\
\text { study } \\
\text { Limited } \\
\text { Large number of exposed cases and } \\
\text { controls; however, exposure levels } \\
\text { were very low }\end{array}$ & $\begin{array}{l}\text { Large cohort: number of exposed } \\
\text { cases: } 4,145 \text { kidney, 1,610 liver, } \\
3,607 \text { NHL, 1,583 multiple myeloma } \\
\text { Follow-up: up to } 45 \text { years }\end{array}$ & $\begin{array}{l}\text { Levels not reported. Estimated median } \\
\text { exposure (unit-yr) }{ }^{\mathrm{b}} \text { for the cumulative } \\
\text { exposure categories: } \\
\text { 1st tertile: } 0.04 \text { (for liver, kidney, NHL, } \\
\text { MM) } \\
\text { 2nd tertile: } 0.25 \text { (liver), } 0.13 \text { (kidney), } 0.12 \\
\text { (NHL), } 0.13 \text { (MM) } \\
\text { 3rd tertile: } 0.77 \text { (liver), } 0.72 \text { (kidney), } 0.72 \\
\text { (NHL), } 0.74 \text { (MM) } \\
\text { Estimated cumulative levels of exposure } \\
\text { based on occupational group (not individual } \\
\text { job data) } \\
\text { High exposure was assigned to shoe and } \\
\text { leather industry workers, mechanics, } \\
\text { laundry workers. Laundry workers may not } \\
\text { be a good population to evaluate exposure } \\
\text { to TCE } \\
\text { Estimated exposure group for highest } \\
\text { cumulative exposure: assumed low } \\
\text { (uncertain because calculation includes } \\
\text { prevalence) }\end{array}$ & $\begin{array}{l}\text { Cumulative exposure (categorical } \\
\text { and continuous models) (units-yr }{ }^{\text {b }} \text { ) } \\
\text { Range not reported; tertiles of } \\
\text { estimated cumulative exposure only } \\
\text { used to evaluate for exposure- } \\
\text { response relationship } \\
\text { Lagging, } 0,1,5,10 \mathrm{yr}\end{array}$ \\
\hline
\end{tabular}




\begin{tabular}{|c|c|c|c|}
\hline $\begin{array}{l}\text { Study Summary (Study } \\
\text { Sensitivity) }\end{array}$ & Study Size/Length of Follow-up & $\begin{array}{c}\text { Reported or Estimated Exposure Levels } \\
\text { or Duration }\end{array}$ & $\begin{array}{l}\text { Exposure-response Analyses: Dose } \\
\text { Metrics/Range Lagging Analyses }\end{array}$ \\
\hline $\begin{array}{l}\text { Hansen et al. (2013) } \\
\text { Pooled Nordic cohort incidence } \\
\text { analysis } \\
\text { Limited for high exposure effects } \\
\text { Large numbers of exposed cases } \\
\text { that were ever exposed to TCE but } \\
\text { few cases with high exposure } \\
\text { (especially liver or NHL); Most of } \\
\text { the cohort was exposed to low } \\
\text { levels for short time periods. }\end{array}$ & $\begin{array}{l}\text { Medium size cohort: } 5,553 \text { workers; } \\
\sim 1,000 \text { cancer cases } \\
\text { Number of exposed cases: } 32 \text { kidney } \\
36 \text { liver, } 38 \text { NHL ever exposed; } 9 \\
\text { kidney, } 3 \text { liver, } 4 \text { NHL in highest } \\
\text { exposure group } \\
49 \% \text { total workers with }>30 \text { years of } \\
\text { follow-up }\end{array}$ & $\begin{array}{l}\text { Low exposure levels and short exposure } \\
\text { duration } \\
\text { Estimated TCE ambient levels: } 4 \mathrm{ppm} \\
\text { (median, Finland), } 12 \mathrm{ppm} \text { (median, } \\
\text { Denmark); }>80 \% \text { of Swedish study with } \\
<20 \mathrm{ppm} \\
\text { Only } 20 \% \text { of subjects had U-TCA levels } \\
>50 \mathrm{mg} / \mathrm{L} \text { (equivalent to } 20 \mathrm{ppm} \text { ) } \\
\text { Median duration of employment (yr): } 5.5 \\
\text { (Sweden) and } 6.3 \text { (Denmark), NR for } \\
\text { Finland } \\
\text { Estimated exposure group }{ }^{\mathrm{a}} \text { for highest U- } \\
\text { TCA exposure group ( } 20 \text { ppm): moderate }\end{array}$ & $\begin{array}{l}\text { Average U-TCA (mg/L, } 4 \text { levels) } \\
\text { Range: Appears adequate based on } \\
\text { U-TCA in exposure groups } \\
\text { Lagging: } 0,10,20 \mathrm{yr}\end{array}$ \\
\hline $\begin{array}{l}\text { Raaschou-Nielsen et al. (2003) } \\
\text { Danish TCE blue-collar worker } \\
\text { cohort } \\
\text { Record linkage incidence study } \\
\text { Adequate in subcohort of higher } \\
\text { exposed subjects } \\
\text { Large number of exposed cases for } \\
\text { NHL and kidney cancer in both } \\
\text { cohort and subcohort analysis; } \\
\text { fewer deaths from liver cancer }\end{array}$ & $\begin{array}{l}\text { Large cohort: }>40,000 \text { workers, } \\
\sim 14,000 \text { subcohort considered to } \\
\text { have higher exposure; }>3,000 \text { cancer } \\
\text { cases; } 76 \text { RCC, } 25 \text { liver, } 96 \mathrm{NHL} \\
\text { Follow-up to approx. } 30 \text { years but } \\
\text { cohort is relatively young; } 56 \% \text { were } \\
38 \text { to } 57 \text { years old at end of follow- } \\
\text { up; } 29 \% \text { of subjects were older than } \\
57 \text { years of age }\end{array}$ & $\begin{array}{l}\text { Low exposure levels after } 1980 \\
\text { Median exposures to TCE (ppm) [NAS } \\
\text { (2006)] } \\
\text { 1960-1969: } 49 \\
\text { 1970-1979: } 20 \\
\text { 1980-1989: 4 } \\
\text { Only 21\% of workers began employment } \\
\text { before 1970 (highest levels). Only } 42 \% \text { of } \\
\text { the cohort was considered to be exposed to } \\
\text { TCE } \\
\text { Estimated exposure group for high } \\
\text { exposure group (since 1970): moderate }\end{array}$ & $\begin{array}{l}\text { Exposure duration (yr), year of first } \\
\text { employment (crude surrogate for } \\
\text { level), company size (surrogate for } \\
\text { probability of exposure), lag time. } \\
\text { Analysis on presumed higher } \\
\text { exposed workers } \\
\text { Range: Appears to be wide based on } \\
\text { exposure changes over time. } \\
\text { Lagging } 0-9,10-20,>20 \mathrm{yr}\end{array}$ \\
\hline
\end{tabular}




\begin{tabular}{|c|c|c|c|}
\hline $\begin{array}{l}\text { Study Summary (Study } \\
\text { Sensitivity) }\end{array}$ & Study Size/Length of Follow-up & $\begin{array}{c}\text { Reported or Estimated Exposure Levels } \\
\text { or Duration }\end{array}$ & $\begin{array}{l}\text { Exposure-response Analyses: Dose } \\
\text { Metrics/Range Lagging Analyses }\end{array}$ \\
\hline \multicolumn{4}{|c|}{ Rocket engine or aircraft manufacturing workers } \\
\hline $\begin{array}{l}\text { Lipworth et al. (2011) } \\
\text { Burbank, CA (USA) aircraft } \\
\text { manufacturing workers cohort } \\
\text { Mortality study } \\
\text { Limited } \\
\text { Adequate numbers of exposed cases } \\
\text { but exposure duration may be } \\
\text { relatively short; few exposed cases } \\
\text { worked longer than } 5 \text { years. No } \\
\text { information or analysis by exposure } \\
\text { intensity }\end{array}$ & $\begin{array}{l}\text { Medium size cohort: } 5,443 ; \sim 1,000 \\
\text { cancer deaths; Exposed deaths: } 16 \\
\text { kidney, } 24 \text { liver, over } 50 \mathrm{NHL} \\
\text { Follow-up: Average } 32 \text { years }\end{array}$ & $\begin{array}{l}\text { No information on reported levels } \\
\text { Exposure duration most likely short for } \\
\text { unknown portion of the workers; Cohort } \\
\text { includes workers employed since } 1960 \text {, but } \\
\text { TCE exposure ceased in } 1966 \text {. Enrollment } \\
\text { of cohort started in } 1960 \text {, so maximum } \\
\text { possible exposure duration was } 6 \text { years. } \\
12 \% \text { of the cohort with potential exposure to } \\
\text { TCE } \\
\text { Estimated exposure group }{ }^{\text {a for longest }} \\
\text { duration: low (includes workers with high } \\
\text { and low exposure) }\end{array}$ & $\begin{array}{l}\text { Duration of exposure (yr) } \\
\text { Range: limited for duration, highest } \\
\text { category } 5 \text { years } \\
\text { Lagging: no analysis }\end{array}$ \\
\hline $\begin{array}{l}\text { Radican et al. (2008) (mortality } \\
\text { update); Blair et al. (1998) } \\
\text { (incidence) } \\
\text { Utah (USA) aircraft maintenance } \\
\text { workers cohort } \\
\text { Mortality and incidence study } \\
\text { Limited for subgroup analysis } \\
\text { Adequate number of exposed deaths } \\
\text { but few deaths or cases among } \\
\text { highest exposed group (especially } \\
\text { for kidney and liver cancer) }\end{array}$ & $\begin{array}{l}\text { Medium size cohort: } 7,204 ; 729 \\
\text { cancer deaths; } 528 \text { cancer cases. } \\
\text { Exposed deaths/cases (men): kidney: } \\
16 \text { deaths/13 cases; NHL: } 37 \\
\text { deaths/21 cases; liver: } 28 \text { deaths/12 } \\
\text { cases). Few cases or deaths }(\geq 5) \text { for } \\
\text { kidney \& liver in highest exposure } \\
\text { category } \\
\text { Follow-up: Average length of follow- } \\
\text { up not reported, but extended follow- } \\
\text { up approx. } 44 \text { years after latest date } \\
\text { of first employment (1956-2000) }\end{array}$ & $\begin{array}{l}\text { Cherrie et al. (2001) estimated long-term } \\
\text { exposure as } 50 \text { ppm and short term up to } \\
600 \text { ppm TCE. The NAS ( } 2006) \text { concluded } \\
\text { the cohort had a modest number of highly } \\
\text { exposed ( } 100 \text { ppm) but most were exposed } \\
\text { to low TCE concentrations ( } \sim 10 \mathrm{ppm}) \text {. } \\
\text { Other estimates for cumulative exposure are } \\
\text { up to } 38 \mathrm{ppm} \text {-yr from degreasing and up to } \\
15 \mathrm{ppm} \text {-yr from benchwork. Intensity would } \\
\text { be high } \\
\text { Estimated exposure group } \\
\text { cumulative exposure: moderate }\end{array}$ & $\begin{array}{l}\text { Cumulative exposure (unit-yr) and } \\
\text { exposure pattern (peak and } \\
\text { intermittent exposure) } \\
\text { Range. Appears adequate (categories } \\
\text { of exposure ranged up to } 25 \text { units- } \\
\text { year) }\end{array}$ \\
\hline
\end{tabular}




\begin{tabular}{|c|c|c|c|}
\hline $\begin{array}{l}\text { Study Summary (Study } \\
\text { Sensitivity) }\end{array}$ & Study Size/Length of Follow-up & $\begin{array}{c}\text { Reported or Estimated Exposure Levels } \\
\text { or Duration }\end{array}$ & $\begin{array}{l}\text { Exposure-response Analyses: Dose } \\
\text { Metrics/Range Lagging Analyses }\end{array}$ \\
\hline $\begin{array}{l}\text { Boice et al. (2006) } \\
\text { Los Angeles (USA) rocket engine } \\
\text { testing workers cohort } \\
\text { Mortality study } \\
\text { Limited } \\
\text { Few exposed deaths but } \\
\text { presumably high exposure. }\end{array}$ & $\begin{array}{l}\text { Small cohort: } 1,111 \text { workers; } 121 \\
\text { cancer deaths; Exposed deaths: } \\
\text { kidney: } 7 \text { deaths } \\
\text { Follow-up: } 88 \% \text { of test mechanics } \\
\text { followed for over } 20 \text { years }\end{array}$ & $\begin{array}{l}\text { Approx. } 58 \% \text { exposed to TCE during engine } \\
\text { flushing/cleaning (high exposure); approx. } \\
42 \% \text { exposed to TCE during utility cleaning } \\
\text { (lower exposure) }\end{array}$ & $\begin{array}{l}\text { Exposure duration (yr) } \\
\text { Range: Unknown, only two exposure } \\
\text { duration categories } \\
\text { Lagging: no analysis }\end{array}$ \\
\hline $\begin{array}{l}\text { Zhao et al. (2005) } \\
\text { Los Angeles (USA) aerospace } \\
\text { workers cohort } \\
\text { Mortality and incidence study } \\
\text { Limited } \\
\text { Small numbers of cases for } \\
\text { subgroup analysis for kidney; } \\
\text { however, strengths are analysis of } \\
\text { risks for high exposed workers and } \\
\text { exposure-response relationships }\end{array}$ & $\begin{array}{l}\text { Medium size cohort: } 6,107 \text {; Exposed } \\
\text { deaths/cases: Kidney: } 17 \text { deaths/ } 16 \\
\text { cases; NHL: } 60 \text { deaths/45 cases } \\
\text { Follow-up: Average } 29 \mathrm{yr}\end{array}$ & $\begin{array}{l}\text { Workers with job titles indicating technical } \\
\text { or mechanical work on rocket engines were } \\
\text { presumed to have high hydrazine rocket fuel } \\
\text { exposure and high TCE exposure, which } \\
\text { was used in cleaning rocket engines and } \\
\text { parts. } \\
80 \% \text { of workers employed before } 1970 \\
\text { when exposure levels were high. Intensity } \\
\text { estimated to be }>200 \text { ppm for } 1970 \text { and } 400 \\
\text { to } 600 \text { ppm for intensity. Cumulative } \\
\text { exposure estimated to range up to } 38 \text { ppm- } \\
\text { yrc. } \\
\text { Estimated exposure group }{ }^{\text {a }} \text { for cumulative } \\
\text { exposure: moderate }\end{array}$ & $\begin{array}{l}\text { Cumulative exposure score (ranked } \\
\text { categories) lagged and unlagged. } \\
\text { Range: Adequate } \\
\text { Lagging: } 0,20 \mathrm{yr}\end{array}$ \\
\hline $\begin{array}{l}\text { Morgan et al. (1998) } \\
\text { Arizona (USA) aircraft } \\
\text { manufacturing workers cohort } \\
\text { Mortality study } \\
\text { Limited statistical power in overall } \\
\text { and subgroup analysis; Some } \\
\text { workers with potential for exposure } \\
\text { to high levels but number not } \\
\text { known }\end{array}$ & $\begin{array}{l}\text { Median size cohort: } 4,733 ; 270 \\
\text { cancer deaths. Exposed deaths: } \\
\text { kidney: } 8 \text {, liver: } 6 \text {, NHL: } 3 \\
\text { Follow-up: not reported }\end{array}$ & $\begin{array}{l}\text { High exposure jobs were considered to be } \\
>50 \text { ppm TCE. Unclear on the number of } \\
\text { workers in high exposed jobs } \\
\text { Estimated exposure group }{ }^{\text {a for highest }} \\
\text { exposure group (peak/cumulative): } \\
\text { moderate }\end{array}$ & $\begin{array}{l}\text { Cumulative exposure score ranked } \\
\text { (two levels), peak exposure } \\
\text { Range: Not known, but only } \\
\text { analyzed low vs. high } \\
\text { Lagging: no analysis }\end{array}$ \\
\hline
\end{tabular}




\begin{tabular}{|c|c|c|c|}
\hline $\begin{array}{c}\text { Study Summary (Study } \\
\text { Sensitivity) }\end{array}$ & Study Size/Length of Follow-up & $\begin{array}{c}\text { Reported or Estimated Exposure Levels } \\
\text { or Duration }\end{array}$ & $\begin{array}{l}\text { Exposure-response Analyses: Dose } \\
\text { Metrics/Range Lagging Analyses }\end{array}$ \\
\hline Other cohorts & & & \\
\hline $\begin{array}{l}\text { Silver et al. (2014) } \\
\text { New York State (USA) electronics } \\
\text { manufacturing workers cohort } \\
\text { Mortality study } \\
\text { Limited } \\
\text { Exposure prevalence in total cohort } \\
\text { low, \# exposed deaths and exposure } \\
\text { levels NR. Analysis by } 1 \text { cumulative } \\
\text { exposure score }\end{array}$ & $\begin{array}{l}\text { Medium size exposed cohort: } 3,113 \\
\text { ever exposed to TCE } \\
\text { Follow-up: Average } 26 \text { years, but } \\
\text { young cohort with only } 17 \% \text { deaths } \\
\text { in total cohort at end of follow-up }\end{array}$ & $\begin{array}{l}\text { Level of exposure NR. Only } 13.9 \% \text { of male } \\
\text { hourly workers exposed to TCE }\end{array}$ & $\begin{array}{l}\text { Cumulative exposure score (1 } \\
\text { category) } \\
\text { Range: Not known } \\
\text { Lagging: } 10 \mathrm{yr}\end{array}$ \\
\hline $\begin{array}{l}\text { Bahr et al. (2011) } \\
\text { Kentucky (USA) uranium } \\
\text { enrichment workers cohort } \\
\text { Mortality study } \\
\text { Unclear } \\
\text { Inadequate information to evaluate }\end{array}$ & $\begin{array}{l}\text { Medium size cohort: } 5,335 \text { men; } 32 \\
\text { NHL deaths } \\
\text { Follow-up: Information not reported; } \\
\text { up to } 50 \text { years for some workers, but } \\
\text { may be more limited for others }\end{array}$ & $\begin{array}{l}\text { No information on exposure levels or nature } \\
\text { of work. Exposure scores and categories not } \\
\text { clear }\end{array}$ & $\begin{array}{l}\text { Exposure score and category } \\
\text { (ranked) } \\
\text { Range: not known } \\
\text { Lagging: no analysis }\end{array}$ \\
\hline $\begin{array}{l}\text { Yiin et al. (2009) } \\
\text { Tennessee (USA) uranium } \\
\text { enrichment workers nested case- } \\
\text { control study } \\
\text { Limited } \\
\text { Number of exposed deaths and } \\
\text { exposure levels unknown }\end{array}$ & $\begin{array}{l}\text { Number exposed to TCE unknown } \\
\text { Follow-up: NR, minimum of } \\
13 \text { years, analysis lagged } 15 \text { years }\end{array}$ & $\begin{array}{l}\text { Exposure levels or duration not reported. } \\
\text { Mean cumulative exposure in cases } \\
183.8 \pm 668.2 \text { for cases and } 113.4 \pm 558.3 \\
\text { for controls. Units not reported }\end{array}$ & $\begin{array}{l}\text { Average cumulative exposure score } \\
\text { ( } 1 \text { category) } \\
\text { Range: Wide range of estimated } \\
\text { cumulative exposure, No analyses by } \\
\text { exposure category. } \\
\text { Lagging: } 0,5,50,20 \mathrm{yr}\end{array}$ \\
\hline
\end{tabular}




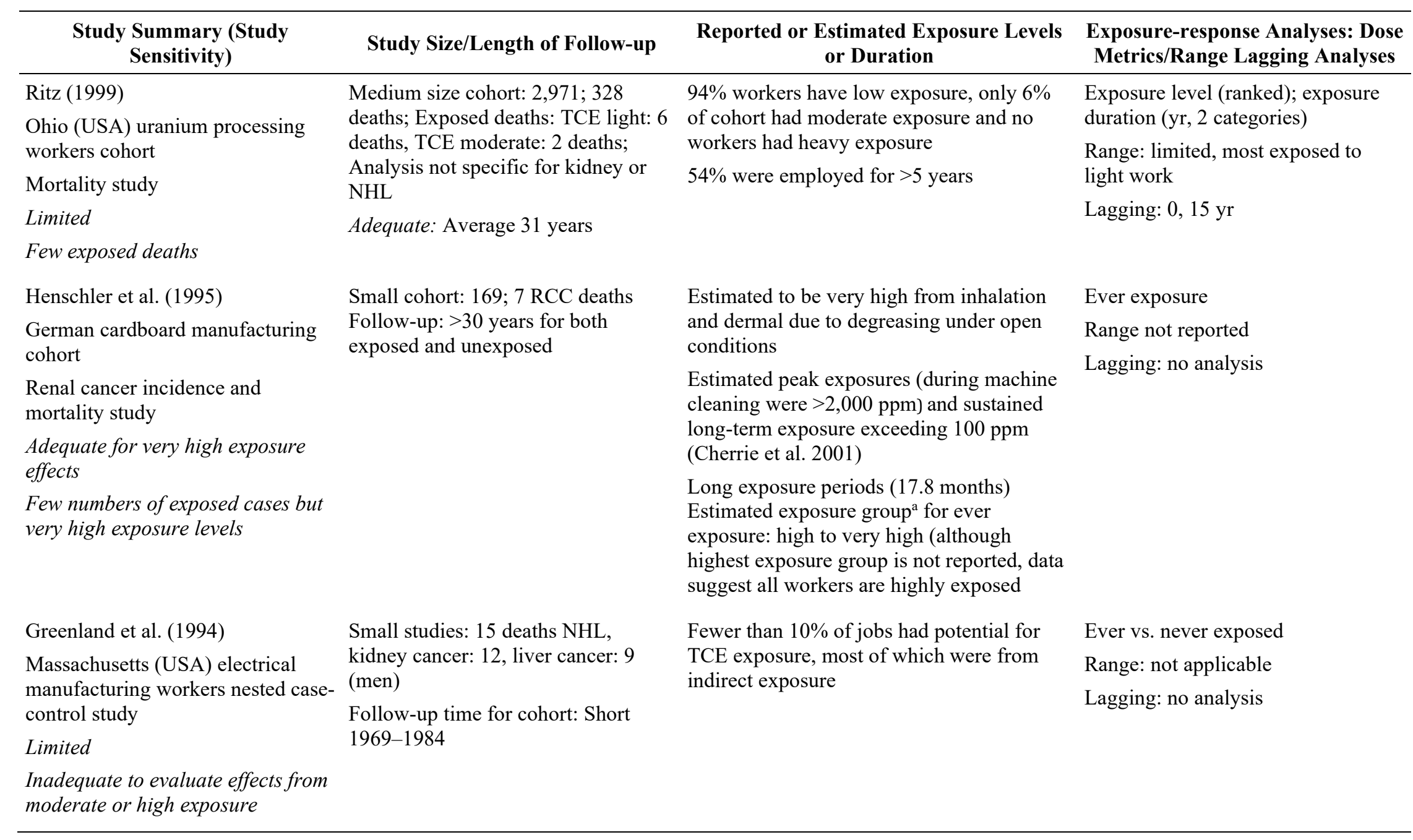




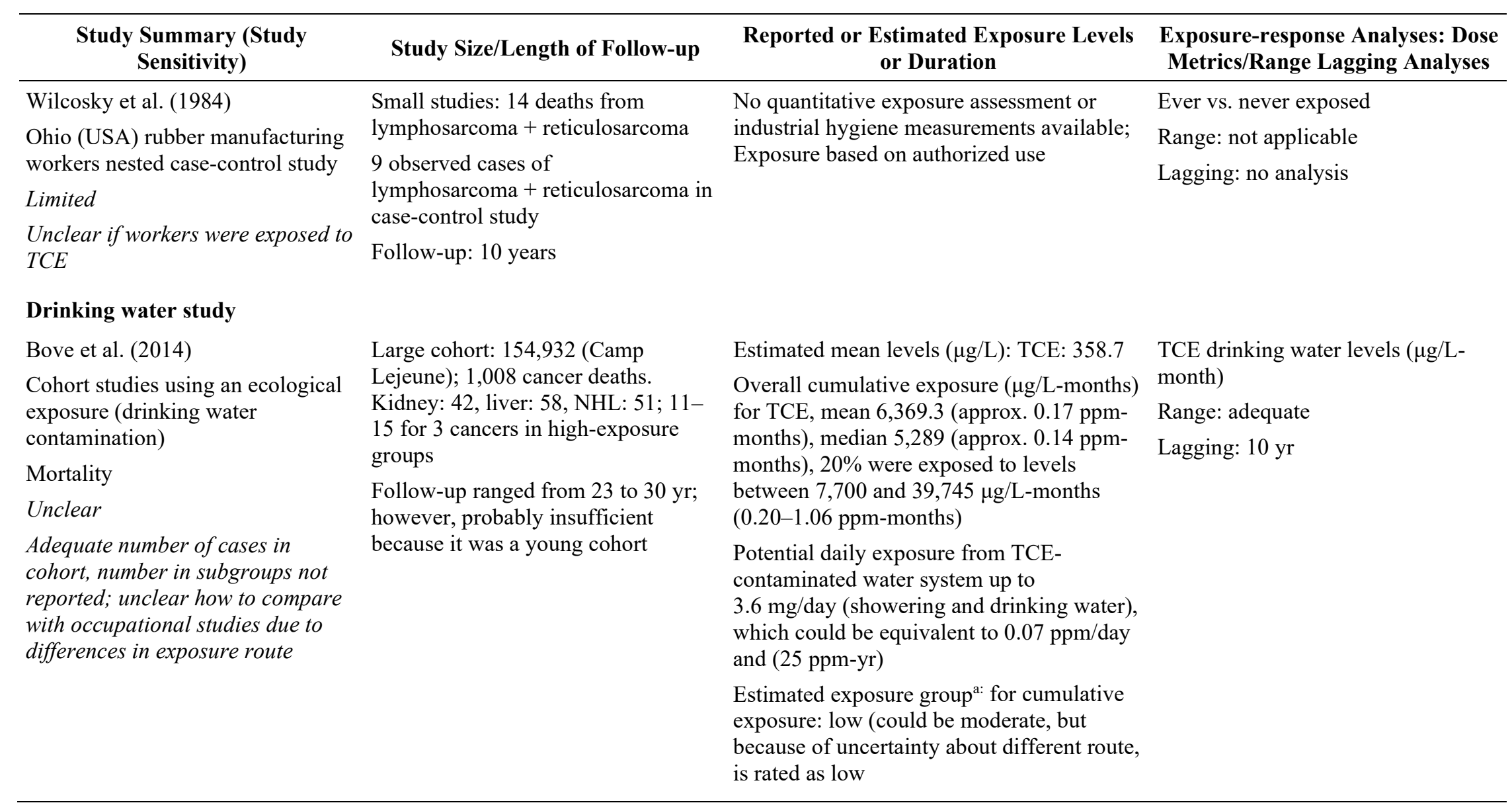

$\mathrm{NHL}=$ non-Hodgkin lymphoma; $\mathrm{MM}=$ multiple myeloma; $\mathrm{TCE}=$ trichloroethylene; $\mathrm{ppm}=$ parts per million; $\mathrm{U}-\mathrm{TCA}=$ urine trichloroacetic acid; $\mathrm{RCC}=$ renal-cell cancer; $\mathrm{NR}=$ not reported; NOCCA $=$ Nordic Occupational Cancer; JEM $=$ job exposure matrix.

${ }^{a}$ Estimated exposure groups across studies for forest plot of kidney cancer and highest exposure group reported in the study (Figure 4-2). This information is only provided for studies reporting a risk estimate for highest exposure and kidney cancer.

${ }^{b}$ NOCCA-JEM estimates exposure as ppm-yr but author reported as units per year because of uncertainty in the estimates (personal communication with authors).

'Personal communication from technical advisor. 
Table D-6. Case-control Studies of Trichloroethylene and Kidney or Liver Cancer: Study Quality

Study

Selection Bias

Participation Rates
TCE Exposure Assessment: Quality and Misclassification

Misclassification of Disease

\section{Studies in specific areas with knowledge of local industries.}

Moore et al. (2010) Possible (direction unclear)

Hospital-based, Hospital controls excluded smoking-

Central and Eastern related diseases

Europe

1,097 cases RCC,

1,476 controls

1999-2003

\section{Charbotel et al. \\ (2006); Charbotel \\ et al. (2009)}

Population-based,

France

86 cases RCC, 326

controls

1993-2003
Participation bias: Unknown: NR

\section{Unlikely}

Cases and controls (matched on area of residence, sex, and age) were randomly selected from same practitioners (excluding patients with kidney or bladder cancer, or chronic kidney disease)

Participation rate similar among cases and controls
Good: Structured, special job-specific questionnaire (job titles, tasks, working conditions) and expert assessment (with knowledge of plants in area) of intensity, frequency, and confidence; Assessment re-evaluated at a later time period with $83 \%$ agreement for TCE in 1 country and $100 \%$ in 2 countries

Exposure misclassification with respect to whether workers were ever exposed to TCE is not a concern among workers $(\sim 50 \%)$ with high confidence assessment (especially among workers with higher or longer exposure) but is more a concern for analysis of all workers

Good: Semi-quantitative estimates of TCE exposure based on detailed questionnaire, JTEM, and exposure monitoring data (air and urine) of industries in the area; Temporal trends were considered

Exposure misclassification (with respect to whether workers were ever exposed) is not a concern especially among individuals in the highest exposure categories (e.g., cumulative, cumulative + peaks). Study was conducted in a localized area with screw-cutting industry. Exposure prevalence and intensity was high, which increases the probability of exposure among the exposed group

\section{Unlikely}

RCC cases histologically confirmed 


\begin{tabular}{|c|c|c|c|}
\hline Study & $\begin{array}{c}\text { Selection Bias } \\
\text { Participation Rates }\end{array}$ & TCE Exposure Assessment: Quality and Misclassification & Misclassification of Disease \\
\hline $\begin{array}{l}\text { Brüning et al. (2003) } \\
\text { Hospital-based, } \\
\text { Germany } \\
134 \text { cases RCC, } 401 \\
\text { controls } \\
1992-2000\end{array}$ & $\begin{array}{l}\text { Possible } \\
\text { Prevalent cases from different hospital } \\
\text { departments (presumably most from the } \\
\text { same hospital) than residual controls. } \\
\text { Cases and control matched by age and } \\
\text { gender } \\
\text { Participation rate high among cases but } \\
\text { not reported for controls }\end{array}$ & $\begin{array}{l}\text { Limited: Exposure assessed via } 3 \text { methods: Self-reported } \\
\text { exposure, including narcotic symptoms using subjects (cases } \\
\text { and controls) and/or proxies (cases only); CAREX database } \\
\text { (expert assessment of occupation groups using TCE) and agent } \\
\text { specific (solvents as a group); British JEM; British JEM and } \\
\text { CAREX are broad and not country or calendar-year specific. } \\
\text { No information was provided on whether the interviewers were } \\
\text { blinded to disease status but may not have been blinded } \\
\text { The potential for recall bias (differential, over- or under- } \\
\text { estimate of the risk estimate) is usually a concern for self- } \\
\text { reported exposure. Self-reported exposure can also be } \\
\text { associated with non-differential misclassification; however, it } \\
\text { is less likely in this study because exposure to TCE was } \\
\text { probably high among at least some (symptomatic) workers, and } \\
\text { common knowledge. It seems reasonable that most of the } \\
\text { workers with self-reported exposure had high exposure. } \\
\text { Exposure misclassification (non-differential) is a concern for } \\
\text { subjects classified by the CAREX and JEM assessment. } \\
\text { Exposure prevalence varied greatly depending on the methods } \\
\text { ( } 80 \% \text { for CAREX versus } 18 \% \text { for self-reported) }\end{array}$ & $\begin{array}{l}\text { Unlikely } \\
\text { RCC cases histologically } \\
\text { confirmed }\end{array}$ \\
\hline
\end{tabular}




\begin{tabular}{|c|c|c|c|}
\hline Study & $\begin{array}{c}\text { Selection Bias } \\
\text { Participation Rates }\end{array}$ & TCE Exposure Assessment: Quality and Misclassification & Misclassification of Disease \\
\hline $\begin{array}{l}\text { Vamvakas et al. } \\
(1998) \\
\text { Hospital-based, } \\
\text { Germany } \\
58 \text { cases RCC, } 84 \\
\text { controls } \\
1987-2002\end{array}$ & $\begin{array}{l}\text { Probable (differential) } \\
\text { Differences in case and control } \\
\text { selection. Cases were selected from a } \\
\text { hospital in a highly industrial area with } \\
\text { small industries from } 1987 \text { to } 1993 \text {. } \\
\text { Unmatched controls selected from } \\
\text { different hospitals in adjacent } \\
\text { geographical region and at a later time } \\
\text { period (time of case-interview) than } \\
\text { prevalent cases. If potential bias } \\
\text { (differential, overestimate of the risk } \\
\text { estimate) could occur if TCE exposure } \\
\text { prevalence was lower in these areas and } \\
\text { time periods. Cases were older than } \\
\text { controls. Study done during period in } \\
\text { which legal proceedings were in } \\
\text { progress }\end{array}$ & $\begin{array}{l}\text { Potential for recall and interviewer bias (differential, } \\
\text { overestimate of risk estimate), especially for reporting } \\
\text { symptoms due to a legal investigation; However, estimated } \\
\text { exposure levels were very high in this study, which may } \\
\text { mitigate this concern }\end{array}$ & $\begin{array}{l}\text { RCC cases histologically } \\
\text { confirmed }\end{array}$ \\
\hline \multicolumn{4}{|l|}{ Other studies } \\
\hline $\begin{array}{l}\text { Christensen et al. } \\
\text { (2013) } \\
\text { Hospital and } \\
\text { population-based, } \\
\text { Canada } \\
177 \text { cases } R C C, 48 \\
\text { liver cancer cases; } \\
533 \text { population } \\
\text { controls, } 2,299 \\
\text { cancer controls } \\
1975-1985\end{array}$ & $\begin{array}{l}\text { Unlikely for population controls } \\
\text { Cases and cancer controls selected from } \\
\text { same hospital and controls randomly } \\
\text { from same underlying population using } \\
\text { similar inclusion criteria } \\
\text { Insufficient data regarding the tissue } \\
\text { sites of cancer controls, but }<20 \% \text { of } \\
\text { any given cancer site used } \\
\text { Participation rates were } 82 \% \text { for cancer } \\
\text { cases (both cancer cases and controls) } \\
\text { and } 72 \% \text { for population controls }\end{array}$ & $\begin{array}{l}\text { The use of a population-wide occupational database may } \\
\text { decrease the probability of exposure and the precision of } \\
\text { exposure estimates for individuals. Although expert assessment } \\
\text { is detailed and systematic, exposure misclassification (non- } \\
\text { differential) is still possible }\end{array}$ & $\begin{array}{l}\text { Unlikely } \\
\text { RCC, liver cases } \\
\text { histologically confirmed }\end{array}$ \\
\hline
\end{tabular}




\begin{tabular}{|c|c|c|c|}
\hline Study & $\begin{array}{c}\text { Selection Bias } \\
\text { Participation Rates }\end{array}$ & TCE Exposure Assessment: Quality and Misclassification & Misclassification of Disease \\
\hline $\begin{array}{l}\text { Pesch et al. }(2000 \mathrm{~b}) \\
\text { Population-based, } \\
\text { Germany } \\
935 \text { cases } \mathrm{RCC}, \\
4,298 \text { controls } \\
1991-1995\end{array}$ & $\begin{array}{l}\text { Unlikely } \\
\text { Cases and controls selected from same } \\
\text { population using the same inclusion } \\
\text { criteria } \\
\text { Participation rates high for cases and } \\
\text { controls ( } 88 \% \text { cases, } 71 \% \text { controls) }\end{array}$ & $\begin{array}{l}\text { Adequate: Questionnaire and expert assessment using JEM and } \\
\text { JTEM which ranked probability and intensity to a given agent } \\
\text { but few details on job tasks; Self-reported exposure also used; } \\
\text { The JTEM is considered to be a better assessment than JEM. } \\
\text { The British JEM may not reflect differences in occupational } \\
\text { exposures across studies } \\
\text { Exposure misclassification (non-differential) is a concern } \\
\text { because of the lower probability of exposure and limited JEM. } \\
\text { The level of concern is greater for subjects classified by JEM } \\
\text { than individuals classified by JTEM. Exposure } \\
\text { misclassification is probably the highest among individual in } \\
\text { the lower exposure categories for both matrices. Exposure } \\
\text { misclassification regarding exposure group (e.g., low, medium, } \\
\text { high) would most likely attenuate any exposure-response } \\
\text { relationships }\end{array}$ & $\begin{array}{l}\text { Unlikely } \\
\text { Most RCC cases } \\
\text { histologically confirmed; } \\
\text { some sonographically } \\
\text { confirmed }\end{array}$ \\
\hline $\begin{array}{l}\text { Dosemeci et al. } \\
\text { (1999) } \\
\text { Population-based, } \\
\text { Minnesota (USA) } \\
438 \text { cases RCC, } 687 \\
\text { controls } \\
\text { 1988-1999 }\end{array}$ & $\begin{array}{l}\text { Unlikely } \\
\text { Cases identified via state cancer registry } \\
\text { and controls randomly selected from the } \\
\text { same underlying population using } \\
\text { similar inclusion criteria } \\
\text { Participation rate was lower among } \\
\text { cases }(64 \%) \text { than controls }(97 \%) \text { but no } \\
\text { information to suspect that participation } \\
\text { was related to exposure and thus the } \\
\text { lower participation rate would most } \\
\text { likely reduce precision }\end{array}$ & $\begin{array}{l}\text { Limited: JEM assigned by expert but based on broad } \\
\text { occupational and industry codes; Only considered current and } \\
\text { usual jobs and duration of employment only assessed; Duration } \\
\text { by calendar period not considered } \\
\text { Exposure misclassification (non-differential) for ever-exposure } \\
\text { to TCE is a concern because of the limited JEM and lower } \\
\text { probability of exposure }\end{array}$ & $\begin{array}{l}\text { Unlikely } \\
\text { RCC cases histologically } \\
\text { confirmed }\end{array}$ \\
\hline
\end{tabular}

$\overline{\mathrm{TCE}}=$ trichloroethylene; $\mathrm{RCC}$ = renal-cell cancer; NR = not reported; JTEM = job-task exposure matrix; CAREX = CARcinogen EXposure (Canada); JEM = job exposure matrix. 
Table D-7. Kidney Case-control Studies: Study Sensitivity and Exposure Response Analyses

\begin{tabular}{|c|c|c|c|}
\hline Study Summary & $\begin{array}{c}\text { Study Size/Exposure } \\
\text { Prevalence }\end{array}$ & $\begin{array}{c}\text { Reported or Estimated Exposure Levels } \\
\text { or Duration }\end{array}$ & $\begin{array}{c}\text { Exposure Response Analyses: Dose } \\
\text { Metrics/Range Lagging }\end{array}$ \\
\hline \multicolumn{4}{|c|}{ Studies in specific areas with knowledge of local industries } \\
\hline $\begin{array}{l}\text { Moore et al. (2010) } \\
\text { Hospital-based, Central and } \\
\text { Eastern Europe } \\
\text { 1999-2003 } \\
\text { Adequate } \\
\text { Adequate cases and controls } \\
\text { with high confidence of } \\
\text { exposure; Ability to evaluate } \\
\text { effects of high exposure is } \\
\text { increased by stratifying on } \\
\text { probability and exposure } \\
\text { intensity or duration }\end{array}$ & $\begin{array}{l}\text { Large study: } 1,097 \mathrm{RCC} \\
\text { cases } / 1,476 \text { controls } \\
\text { Exposure prevalence: } 5.8 \% \\
(\mathrm{~N}=48) \text { cases and } 3.4 \% \\
(\mathrm{~N}=40) \text { controls for any } \\
\text { exposure and } \sim 2 \%-4 \%(\mathrm{~N}=17- \\
31) \text { cases and } 1 \%-2 \% \text { controls } \\
(\mathrm{N}=10-21) \text { for high exposure } \\
\text { categories }\end{array}$ & $\begin{array}{l}\text { No information on actual exposure } \\
\text { Estimated TCE intensity in JEM were } \\
\text { coded into } 3 \text { categories: } 0 \text { to }<5 \text { ppm, } 5 \text { to } \\
50 \text { ppm, and }>50 \text { ppm }(2.5,25 \text {, and } 75 \mathrm{ppm} \\
\text { midpoints) } \\
\text { Duration (years): } 1.35 \text { ( } 6.3-26.3 \text { for } \\
\text { controls) } 19.5 \text { (5.8-31) for cases } \\
\text { Estimated exposure group for individuals } \\
\text { with highest average exposure: moderate to } \\
\text { high }\end{array}$ & $\begin{array}{l}\text { Ever, cumulative (ppm-yr), average-intensity } \\
\text { (ppm), duration (hours and years); Separate } \\
\text { analyses conducted for all and high confidence } \\
\text { exposure assessments ( }>40 \% \text { workers probably } \\
\text { or definitely exposed jobs) } \\
\text { Range: Appears to be adequate based on } \\
\text { estimated interquartile range and differences in } \\
\text { exposure intensity among jobs; however, only } \\
\text { two exposure groups for each metric } \\
\text { Lagging: } 0,20 \mathrm{yr}\end{array}$ \\
\hline
\end{tabular}




\begin{tabular}{|c|c|c|c|}
\hline Study Summary & $\begin{array}{l}\text { Study Size/Exposure } \\
\text { Prevalence }\end{array}$ & $\begin{array}{c}\text { Reported or Estimated Exposure Levels } \\
\text { or Duration }\end{array}$ & $\begin{array}{c}\text { Exposure Response Analyses: Dose } \\
\text { Metrics/Range Lagging }\end{array}$ \\
\hline $\begin{array}{l}\text { Charbotel et al. (2006); } \\
\text { Charbotel et al. (2009) } \\
\text { Population-based, France } \\
\text { 1993-2003 } \\
\text { Adequate to good } \\
\text { Adequate number of subjects } \\
\text { exposed to high levels of TCE. } \\
\text { May not have adequate } \\
\text { statistical power in subgroup } \\
\text { analysis but good range in } \\
\text { exposure intensity }\end{array}$ & $\begin{array}{l}\text { Small study: } 86 \text { RCC cases; } 326 \\
\text { referents } \\
\text { Exposure prevalence: } 43 \% \\
(\mathrm{~N}=37) \text { cases, } 35 \%(\mathrm{~N}=110) \\
\text { controls for ever exposed, and } \\
19 \%(\mathrm{~N}=16) \text { cases, and } 11.7 \% \\
(\mathrm{~N}=37) \text { among highest } \\
\text { exposure group }\end{array}$ & $\begin{array}{l}\text { High intensity of exposure (duration NR); } \\
\text { Among controls the median exposure for } \\
\text { low, medium, and high categories = 60,252 } \\
\text { and } 630 \text { ppm, respectively; Among cases, } \\
\text { median exposure = 30, 300, and } 885 \mathrm{ppm} \text {, } \\
\text { respectively } \\
\text { Estimated TCE intensities (ppm) for } \\
\text { specific jobs } \\
\text { • } \quad 15-18 \text { for open cold degreasing } \\
\text { • } \quad 120 \text { for jobs near open hot } \\
\quad \text { degreasing machines } \\
\text { - up to } 300 \text { for work directly above } \\
\quad \text { tank } \\
\quad 300-600 \text { for emptying, cleaning, } \\
\text { and refilling degreasers } \\
\text { Cumulative exposure categories: low } 1- \\
150 \text { ppm-yr, medium } 155-335 \text { ppm-yr and } \\
>335 \text { ppm-yr } \\
\text { Estimated exposure group for highest } \\
\text { cumulative exposure: very high }\end{array}$ & $\begin{array}{l}\text { Ever exposed, cumulative exposure (ranked), } \\
\text { and combined cumulative and peak exposure, } \\
\text { trend analysis } \\
\text { Range: good (see previous column) } \\
\text { Lagging: no analysis }\end{array}$ \\
\hline $\begin{array}{l}\text { Brüning et al. (2003) } \\
\text { Hospital-based, Germany } \\
\text { 1992-2000 } \\
\text { Adequate to good } \\
\text { Adequate number of subjects } \\
\text { exposed to high levels of TCE }\end{array}$ & $\begin{array}{l}\text { Small/medium study: } 134 \mathrm{RCC} \\
\text { cases/401 controls } \\
\text { Exposure prevalence: } 18.7 \% \\
(\mathrm{~N}=25) \text { cases, } 9.5 \%(\mathrm{~N}=38) \\
\text { using self-assessment } \\
87 \% \text { cases, } 79 \% \text { controls using } \\
\text { CAREX (less confidence) }\end{array}$ & $\begin{array}{l}\text { Very high exposure and long exposures } \\
\text { Estimated to be } 400-600 \text { ppm during peak } \\
\text { (hot dipping) and }>100 \text { ppm overall } \\
\text { (Cherrie et al. } 2001 \text { ) } \\
\text { Approx. } 50 \% \text { cases }>10 \text { years' exposure } \\
\text { Estimated exposure group }{ }^{\text {a for workers }} \\
\text { with daily narcotic symptoms: very high }\end{array}$ & $\begin{array}{l}\text { Jobs using TCE (CAREX), exposure to solvent } \\
\text { (JEM) } \\
\text { Self-assessed: exposure + narcotic symptoms, } \\
\text { duration (yr) and time since first and last } \\
\text { exposure } \\
\text { Range: not known, but may be shallow due to } \\
\text { exposure from open conditions } \\
\text { Lagging 5-9, 10-19, } 20 \mathrm{yr}\end{array}$ \\
\hline
\end{tabular}




\begin{tabular}{|c|c|c|c|}
\hline Study Summary & $\begin{array}{l}\text { Study Size/Exposure } \\
\text { Prevalence }\end{array}$ & $\begin{array}{c}\text { Reported or Estimated Exposure Levels } \\
\text { or Duration }\end{array}$ & $\begin{array}{c}\text { Exposure Response Analyses: Dose } \\
\text { Metrics/Range Lagging }\end{array}$ \\
\hline $\begin{array}{l}\text { Vamvakas et al. (1998) } \\
\text { Hospital-based, Germany } \\
\text { 1987-2002 } \\
\text { Adequate } \\
\text { Limited number of subjects but } \\
\text { exposed to high levels of TCE }\end{array}$ & $\begin{array}{l}\text { Small study: } 58 \mathrm{RCC} \text { cases/84 } \\
\text { controls } \\
\text { Exposure prevalence: } 33 \% \\
(\mathrm{~N}=19) \text { cases; } 6 \%(\mathrm{~N}=5) \\
\text { controls }\end{array}$ & $\begin{array}{l}\text { Very high exposure and long exposures } \\
\text { Estimated to be } 400 \text { to } 600 \mathrm{ppm} \text { during } \\
\text { peak (hot dipping) and }>100 \mathrm{ppm} \text { overall } \\
\text { (Cherrie et al. 2001) } \\
\text { Mean duration of exposure among cases } \\
\text { was } 16 \text { years and } 7 \text { years among controls } \\
\text { Estimated exposure group }{ }^{\text {a for highest rank }} \\
\text { exposure category: very high }\end{array}$ & $\begin{array}{l}\text { Ever/never and exposure category (ranked, } \\
\text { integration of exposure time and symptoms) } \\
\text { Range: not known, but may be shallow due to } \\
\text { exposure from open conditions } \\
\text { Lagging: no analysis }\end{array}$ \\
\hline Other studies & & & \\
\hline $\begin{array}{l}\text { Christensen et al. (2013) } \\
\text { Hospital and population-based, } \\
\text { Canada } \\
1975-1985 \\
\text { Limited } \\
\text { Few exposed cases and } \\
\text { controls with substantial } \\
\text { exposure }\end{array}$ & $\begin{array}{l}\text { Moderate size: } 177 \mathrm{RCC} \text { cases/ } \\
1,999 \text { cancer controls, } 533 \\
\text { population controls } \\
\text { Small size: } 48 \text { liver cases, } 1,834 \\
\text { liver cancer controls and } 533 \\
\text { population controls } \\
\text { Exposure prevalence: }<3 \% \\
(\mathrm{~N}=15 \text { population controls; } 63 \\
\text { cancer cases, and } 5 \mathrm{RCC} \text { cases, } \\
1 \text { liver cancer) for any exposure } \\
\text { and controls and }<2(\mathrm{~N}=9 \\
\text { population controls, } \mathrm{N}=2 \mathrm{RCC} \text {, } \\
1 \text { liver cancer) for substantial } \\
\text { exposure }\end{array}$ & $\begin{array}{l}\text { Levels and duration not reported } \\
\text { Occupations considered to have the highest } \\
\text { exposure were mechanics and repairmen, } \\
\text { metal machining occupations, electrical } \\
\text { and electronics and metal shaping and } \\
\text { formulation } \\
\text { Estimated exposure group for individuals } \\
\text { with substantial exposure: assumed low } \\
\text { (unclear because category includes } \\
\text { confidence of exposure) }\end{array}$ & $\begin{array}{l}\text { Any and substantial (integration of probability, } \\
\text { frequency, concentration, and duration) } \\
\text { Range: not applicable } \\
\text { Lagging: no analysis }\end{array}$ \\
\hline
\end{tabular}




\begin{tabular}{|c|c|c|c|}
\hline Study Summary & $\begin{array}{l}\text { Study Size/Exposure } \\
\text { Prevalence }\end{array}$ & $\begin{array}{c}\text { Reported or Estimated Exposure Levels } \\
\text { or Duration }\end{array}$ & $\begin{array}{c}\text { Exposure Response Analyses: Dose } \\
\text { Metrics/Range Lagging }\end{array}$ \\
\hline $\begin{array}{l}\text { Pesch et al. (2000b) } \\
\text { Population-based, } 5 \text { German } \\
\text { regions } \\
\text { 1991-1995 } \\
\text { Limited } \\
\text { Few exposed cases and } \\
\text { controls, most of which were } \\
\text { likely exposed to low levels of } \\
\text { TCE }\end{array}$ & $\begin{array}{l}\text { Large study size: } 935(570 \text { men } \\
\& 375 \text { women }) \text { cases } / 4,298 \\
\text { controls } \\
\text { Prevalence of substantial TCE } \\
\text { exposure was low among male } \\
\text { cases and varied by type of } \\
\text { JEM: } 10 \%(\mathrm{~N}=55) \text { males } \\
\text { German JEM } 3.9 \%(\mathrm{~N}=15) \\
(\mathrm{JTEM}) . \text { Prevalence was }<2 \% \\
(\mathrm{~N}<5) \text { in females. Exposed } \\
\text { controls NR }\end{array}$ & $\begin{array}{l}\text { No information on the types of job that } \\
\text { were considered to be exposed to TCE or } \\
\text { on estimated exposure levels } \\
\text { Includes the Arnsberg and other regions; } \\
\text { NAS (2006) estimated that most subjects } \\
\text { had minimal contact with TCE averaging } \\
\text { concentration of } 10 \text { ppm or less } \\
\text { Estimated exposure group for individuals } \\
\text { with substantial exposure: assumed low } \\
\text { (unclear because category includes } \\
\text { probability of exposure) }\end{array}$ & $\begin{array}{l}\text { Exposure index (ranked; integration of } \\
\text { probability, duration, and intensity) for two } \\
\text { JEM and JTEM; Reported separately for men } \\
\text { and women } \\
\text { Range: Not applicable } \\
\text { Lagging: no analysis }\end{array}$ \\
\hline $\begin{array}{l}\text { Dosemeci et al. (1999) } \\
\text { Population-based, Minnesota, } \\
\text { (USA) } \\
\text { 1988-1999 } \\
\text { Limited to adequate } \\
\text { Adequate numbers of exposed } \\
\text { cases and controls to evaluate } \\
\text { ever versus never exposure; No } \\
\text { evaluation of exposure level }\end{array}$ & $\begin{array}{l}\text { Moderate size: } 438(273 \text { men } \\
165 \text { women }) \text { cases; } 687(462 \\
\text { men, } 225 \text { women }) \text { controls } \\
\text { Exposure prevalence: } 13 \% \text { cases } \\
(\mathrm{N}=55) ; 10 \% \text { controls } \\
(\mathrm{N}=\sim 69)\end{array}$ & $\begin{array}{l}\text { No information on level duration or jobs } \\
\text { considered to have TCE exposure }\end{array}$ & $\begin{array}{l}\text { Ever-exposed reported separately for men and } \\
\text { women } \\
\text { Range: not applicable } \\
\text { Lagging: no analysis }\end{array}$ \\
\hline
\end{tabular}

$\mathrm{RCC}=$ renal-cell cancer; $\mathrm{N}=$ number; TCE =trichloroethylene; JEM = job exposure matrix; ppm = parts per million; $\mathrm{NR}=$ not reported; CAREX = CARcinogen EXposure (Canada); NAS = National Academy of Science; JTEM = job-task exposure matrix

aEstimated exposure groups across studies for forest plot of kidney cancer and highest exposure group reported in the study (Figure 4-2). This information is only provided for studies reporting a risk estimate for highest exposure and kidney cancer. 
Table D-8. Case-control Studies of Trichloroethylene and NHL and Related Subtypes: Summary of Study Quality

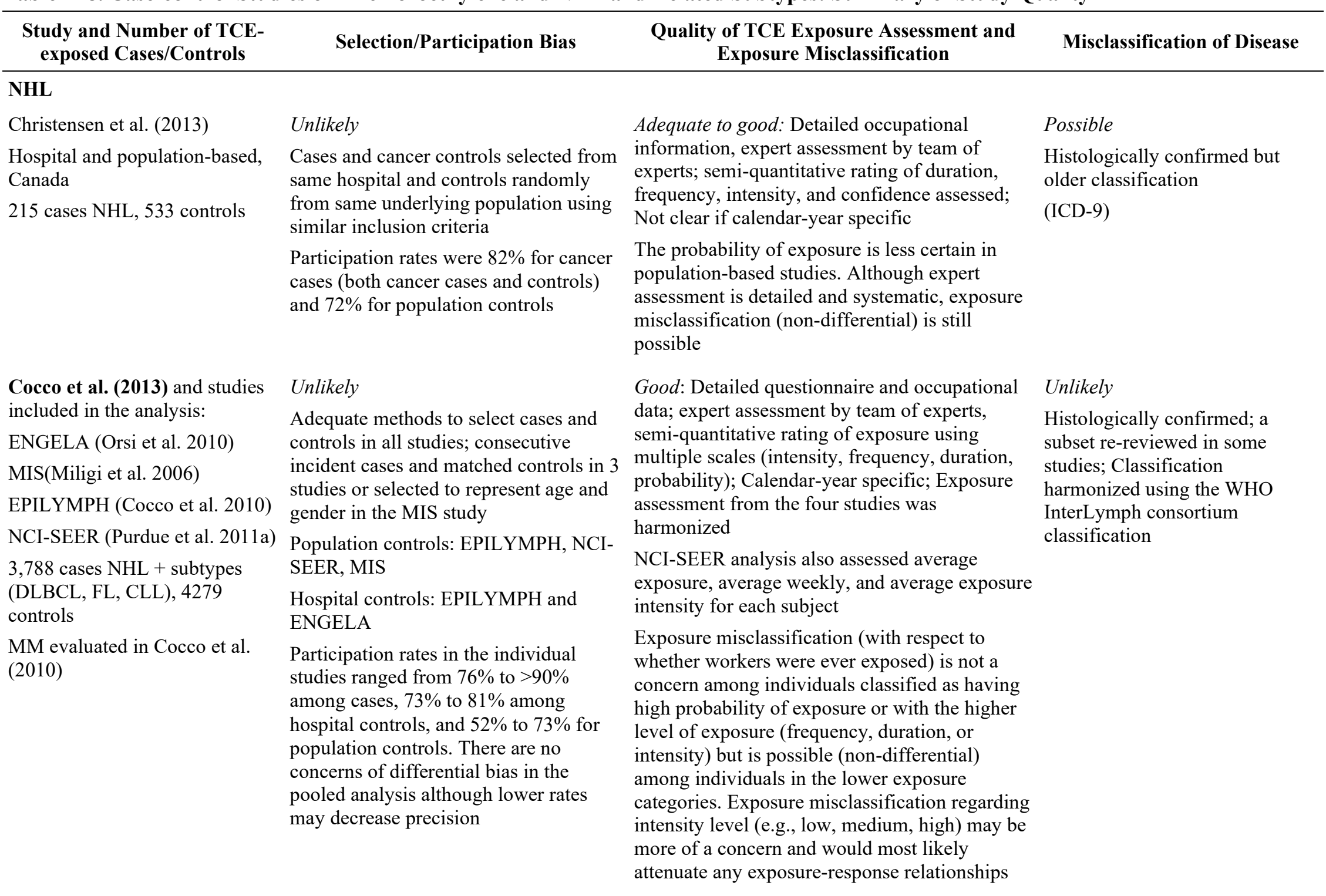




\begin{tabular}{|c|c|c|c|}
\hline $\begin{array}{l}\text { Study and Number of TCE- } \\
\text { exposed Cases/Controls }\end{array}$ & Selection/Participation Bias & $\begin{array}{c}\text { Quality of TCE Exposure Assessment and } \\
\text { Exposure Misclassification }\end{array}$ & Misclassification of Disease \\
\hline $\begin{array}{l}\text { Deng et al. (2013), Wang et al. } \\
\text { (2009b) } \\
\text { Population-based Connecticut } \\
\text { (USA) } \\
601 \mathrm{NHL}+\text { subtypes cases, } 7,171 \\
\text { controls }\end{array}$ & $\begin{array}{l}\text { Unlikely } \\
\text { Cases and matched controls selected } \\
\text { from the same underlying population } \\
\text { using similar inclusion criteria; Cases } \\
\text { selected from cancer registry } \\
\text { Participation rates: Cases } 72 \% \text {; } \\
\text { Controls: RDD } 69 \% \text {, health care } 47 \% \text {. } \\
\text { Low rates may decrease precision }\end{array}$ & $\begin{array}{l}\text { Limited to adequate: Occupational data on job } \\
\text { titles and companies, generic JEM based on } \\
\text { semi-quantitative rating of occupations (rather } \\
\text { than tasks); Not calendar-year specific } \\
\text { Overall, exposure misclassification (non- } \\
\text { differential) is a concern among individuals } \\
\text { classified as ever exposed. The likelihood of } \\
\text { exposure is increased among workers in the } \\
\text { higher probability or higher intensity categories }\end{array}$ & $\begin{array}{l}\text { Unlikely } \\
\text { Cases reviewed by pathologists; } \\
2001 \text { WHO (REAL) } \\
\text { classification }\end{array}$ \\
\hline $\begin{array}{l}\text { Persson and Fredrikson (1999) } \\
\text { Population-based (pooled study) } \\
\text { Sweden } \\
199 \text { cases NHL, } 479 \text { controls }\end{array}$ & $\begin{array}{l}\text { Unlikely } \\
\text { Cases and matched controls selected } \\
\text { from the same underlying population } \\
\text { using similar inclusion criteria } \\
\text { Controls drawn for other studies and } \\
\text { unclear which years controls were } \\
\text { recruited } \\
\text { Participation rate: } 90 \% \text { among cases but } \\
\text { NR for controls }\end{array}$ & $\begin{array}{l}\text { Limited: Self-reported ranked exposures ( } \sim 19 \\
\text { occupational exposures); Not clear if } \\
\text { interviewers were blinded to case-control status } \\
\text { Exposure misclassification is a concern and } \\
\text { likely to be substantial. Direction of potential of } \\
\text { bias is unknown since self-reported exposures } \\
\text { can vary between cases and control; however, } \\
\text { considerable non-differential misclassification } \\
\text { for cases and controls is also likely }\end{array}$ & $\begin{array}{l}\text { Possible } \\
\text { 2nd study histologically } \\
\text { confirmed but not } 1 \text { st study (not } \\
\text { histologically confirmed); ICD } \\
\text { coding NR }\end{array}$ \\
\hline $\begin{array}{l}\text { Nordström et al. (1998) } \\
\text { Population-based } \\
\text { Sweden } \\
121 \text { cases HCL, } 484 \text { controls }\end{array}$ & $\begin{array}{l}\text { Unlikely } \\
\text { Cases and matched controls selected } \\
\text { from the same underlying population } \\
\text { using similar inclusion criteria; Cancer } \\
\text { selected from cancer registry } \\
\text { Participation rates: cases } 91 \% \text {; controls } \\
83 \%\end{array}$ & $\begin{array}{l}\text { Limited: Complete occupational history and } \\
\text { self-reported exposure (primarily job titles, not } \\
\text { tasks or working conditions); Exposure assigned } \\
\text { based on self-report/occupation, qualitative; } \\
\text { Minimal requirements for ever exposure based } \\
\text { on very low exposure } \\
\text { Exposure misclassification is a concern and } \\
\text { likely to be substantial }\end{array}$ & $\begin{array}{l}\text { Possible } \\
\text { Subset of cases re-reviewed: NCI } \\
\text { classification }\end{array}$ \\
\hline
\end{tabular}




\begin{tabular}{|c|c|c|c|}
\hline $\begin{array}{l}\text { Study and Number of TCE- } \\
\text { exposed Cases/Controls }\end{array}$ & Selection/Participation Bias & $\begin{array}{l}\text { Quality of TCE Exposure Assessment and } \\
\text { Exposure Misclassification }\end{array}$ & Misclassification of Disease \\
\hline $\begin{array}{l}\text { Hardell et al. (1994) } \\
\text { Population-based } \\
\text { Sweden } \\
105 \text { cases NHL, } 355 \text { controls }\end{array}$ & $\begin{array}{l}\text { Unlikely } \\
\text { Cases and matched controls selected } \\
\text { from the same underlying population } \\
\text { using similar inclusion criteria; Cases } \\
\text { selected from hospital dept } \\
\text { Participation rates: unknown }\end{array}$ & $\begin{array}{l}\text { Limited: Complete occupational history } \\
\text { (primarily job titles, not tasks or working } \\
\text { conditions) and self-reported exposure; } \\
\text { Exposure assigned based on self- } \\
\text { report/occupation, qualitative; Minimal } \\
\text { requirements for ever exposure based on very } \\
\text { low exposure }\end{array}$ & $\begin{array}{l}\text { Cases histologically confirmed } \\
\text { by subtype, stage, and site but } \\
\text { older Rappaport classification }\end{array}$ \\
\hline \multicolumn{4}{|l|}{ Multiple Myeloma } \\
\hline $\begin{array}{l}\text { Gold et al. (2011) } \\
\text { Seattle, WA and Detroit, MI } \\
\text { (USA) SEER registry } \\
181 \text { cases MM, } 481 \text { controls }\end{array}$ & $\begin{array}{l}\text { Unlikely } \\
\text { Cases and matched controls selected } \\
\text { from the same underlying population } \\
\text { using similar inclusion criteria; Cases } \\
\text { selected from cancer registry } \\
\text { Participation rates: cases } 71 \% \text { and } \\
\text { controls } 52 \%\end{array}$ & $\begin{array}{l}\text { Good: Detailed occupational information, JTEM } \\
\text { specific for } 6 \text { solvents assigned by experts; } \\
\text { quantitative rating of exposure intensity and } \\
\text { assignment of cumulative exposure (based on } \\
\text { exposure measurement reported in the } \\
\text { literature); (Same exposure assessment as } \\
\text { Purdue et al. (2011a) for NHL) } \\
\text { Exposure misclassification is not a concern, } \\
\text { especially among individuals with the highest } \\
\text { cumulative exposure. Exposure } \\
\text { misclassification between levels of cumulative } \\
\text { exposure would most likely attenuate any } \\
\text { exposure-response }\end{array}$ & $\begin{array}{l}\text { Unlikely } \\
\text { Most SEER registry cases } \\
\text { histologically confirmed; ICD - } \\
\text { O-2 or } 3 \text { ) }\end{array}$ \\
\hline
\end{tabular}




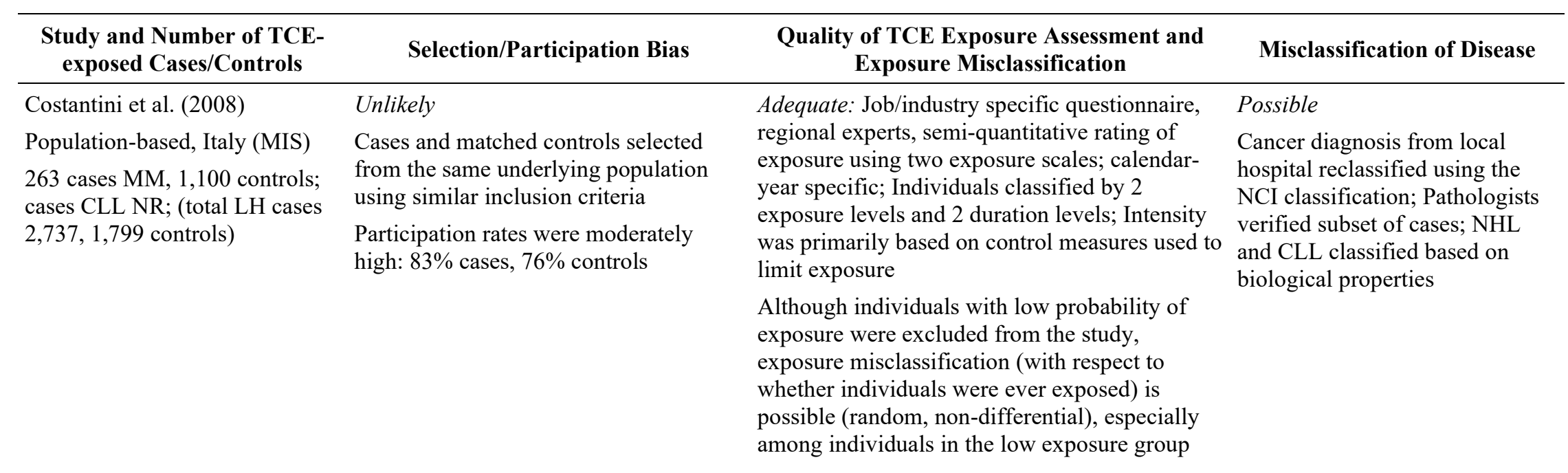

$\mathrm{NHL}=$ non-Hodgkin lymphoma; TCE = trichloroethylene; ICD = International Classification of Diseases; MIS = Multicentre Italian Study; NCI = National Cancer Institute; SEER = Surveillance, Epidemiology and End Results Program; DLBCL = diffuse large B-cell lymphoma; FL = follicular lymphoma; CLL = chronic lymphocytic lymphoma; $\mathrm{MM}=$ multiple myeloma; WHO = World Health Organization; RDD = random-digit dialing; JEM = job exposure matrix; REAL = Revised European-American Lymphoma classification; $\mathrm{NR}=$ not reported; $\mathrm{HCL}=$ hairy-cell leukemia; JTEM = job-task exposure matrix; $\mathrm{LH}=$ lymphohematopoietic.

\section{Table D-9. NHL Case-control Studies: Study Sensitivity and Exposure Response Analysis}

\begin{tabular}{|c|c|c|c|}
\hline $\begin{array}{l}\text { Study Summary (Study } \\
\text { Sensitivity) }\end{array}$ & Study Size/Exposure Prevalence & $\begin{array}{l}\text { Reported or Estimated Exposure } \\
\text { Levels or Duration }\end{array}$ & $\begin{array}{c}\text { Exposure Response Analyses: Dose } \\
\text { Metrics/Range Lagging }\end{array}$ \\
\hline \multicolumn{4}{|l|}{ NHL } \\
\hline $\begin{array}{l}\text { Christensen et al. (2013) } \\
\text { Hospital and population-based, } \\
\text { Canada } \\
\text { Limited } \\
\text { Small numbers of exposed cases } \\
\text { and controls }\end{array}$ & $\begin{array}{l}\text { Moderate size: } 215 \mathrm{NHL} \\
\text { cases/23,141 cancer controls, } 533 \\
\text { population controls } \\
\text { Exposure prevalence: }<3 \%(\mathrm{~N}=15 \\
\text { population controls; } 65 \text { cancer } \\
\text { cases, and } 7 \mathrm{NHL}) \text { for any exposure } \\
\text { and controls and }<2 \%(\mathrm{~N}=9 \\
\text { population controls, } \mathrm{N}=30 \text { cancer } \\
\text { controls, } \mathrm{N}=2 \mathrm{NHL}) \text { for } \\
\text { substantial exposure }\end{array}$ & $\begin{array}{l}\text { Levels and duration not reported } \\
\text { Occupations considered to have the } \\
\text { highest exposure were mechanics and } \\
\text { repairmen, metal machining occupations, } \\
\text { electrical and electronics and metal } \\
\text { shaping and formulation }\end{array}$ & $\begin{array}{l}\text { Any and substantial } \\
\text { Range: NA } \\
\text { Lagging: no analysis }\end{array}$ \\
\hline
\end{tabular}




\begin{tabular}{|c|c|c|c|}
\hline $\begin{array}{l}\text { Study Summary (Study } \\
\text { Sensitivity) }\end{array}$ & Study Size/Exposure Prevalence & $\begin{array}{c}\text { Reported or Estimated Exposure } \\
\text { Levels or Duration }\end{array}$ & $\begin{array}{c}\text { Exposure Response Analyses: Dose } \\
\text { Metrics/Range Lagging }\end{array}$ \\
\hline $\begin{array}{l}\text { Cocco et al. (2013) and studies } \\
\text { included in the pooled analysis: } \\
\text { ENGELA (Orsi et al. 2010) } \\
\text { MIS (Miligi et al. 2006) } \\
\text { EPILYMPH (Cocco et al. 2010) } \\
\text { NCI-SEER (Purdue et al. 2011a) } \\
\text { Adequate } \\
\text { Adequate number of cases and } \\
\text { controls all NHL but not all NHL } \\
\text { subtypes; Estimated levels } \\
\text { suggest levels relatively high for } \\
\text { the highest exposed workers }\end{array}$ & $\begin{array}{l}\text { Very large study: } 3,788 \text { cases } / 4,279 \\
\text { controls } \\
\text { Exposure prevalence in total } \\
\text { population: } 9 \%(\mathrm{~N}=711) \text { ever } \\
\text { exposed, } 1 \%(\mathrm{~N}=88) \text { definite } \\
\text { exposed } \\
\text { Exposure prevalence among highest } \\
\text { exposure intensity category: }<1.5 \% \\
(\mathrm{~N}=57 \text { controls, } 48 \text { cases }) \text { for total } \\
\text { population; }<10 \text { cases or controls } \\
\text { among those with high probability } \\
\text { of exposure }\end{array}$ & $\begin{array}{l}\text { Levels not reported: levels estimated for } \\
\text { analysis: Highest exposure intensity } \\
\text { category }>75 \mathrm{ppm} \\
\text { Estimated levels } \\
\text { NCI-SEER: levels not reported; levels } \\
\text { estimated for analysis } \\
\text { Highest cumulative exposure category: } \\
>234,000 \text { ppm-hr (prevalence: } 0.7 \% \\
\text { controls, } 2.5 \% \text { cases) } \\
\text { Highest average intensity exposure } \\
\text { category: }>99 \text { ppm (prevalence: } 2.3 \% \\
\text { controls, } 3.4 \% \text { cases) } \\
\text { MIS } \\
\text { Study regions chosen because of large } \\
\text { presence of manufacturing industries } \\
\text { using solvents or they were agricultural } \\
\text { areas }\end{array}$ & $\begin{array}{l}\text { Probability, intensity (ppm), frequency ( } \% \\
\text { work time), duration (yr) among all } \\
\text { subjects and high probability subjects } \\
\text { Additional metrics in individual studies } \\
\text { Cumulative exposure (ranked): } \\
\text { EPILYMPH, NCI-SEER (ppm-hr) } \\
\text { Average weekly ppm-hr/week): NCI-SEER } \\
\text { Sensitivity by latency, interviewing } \\
\text { variable, \& unemployment - NCI-SEER } \\
\text { Range: Adequate range based on estimates } \\
\text { of intensity, duration, and frequency of } \\
\text { exposure } \\
\text { Lagging: no analysis, although NCI-SEER } \\
\text { conducted 5- and 15-yr lagged analysis }\end{array}$ \\
\hline $\begin{array}{l}\text { Deng et al. (2013), Wang et al. } \\
\text { (2009b) } \\
\text { Population-based Connecticut, } \\
\text { (USA) (Women) } \\
\text { Limited } \\
\text { Few numbers of exposed cases } \\
\text { and controls; Limited ability to } \\
\text { detect an effect because there are } \\
\text { no workers with high exposure } \\
\text { and high probability of exposure }\end{array}$ & $\begin{array}{l}\text { Large study: } 601 \mathrm{NHL} / 717 \text { controls } \\
\text { Exposure prevalence: } 11 \% \text { controls } \\
(\mathrm{N}=79) \text { and } 13 \%(\mathrm{~N}=77) \text { for ever } \\
\text { exposed; }<2 \%(\mathrm{~N}=8 \text { controls and } \\
13 \text { cases }) \text { for medium high } \\
\text { exposure; and }(\mathrm{N}=31) \text { controls and } \\
5.7 \%(\mathrm{~N}=34) \text { cases for medium } \\
\text { high probability; } 0 \text { cases and } \\
\text { controls with high probability and } \\
\text { medium and high intensity }\end{array}$ & $\begin{array}{l}\text { No information on reported or estimated } \\
\text { level. }\end{array}$ & $\begin{array}{l}\text { Exposure intensity (ranked), exposure } \\
\text { probability } \\
\text { Range: No information } \\
\text { Lagging: no analysis }\end{array}$ \\
\hline
\end{tabular}




\begin{tabular}{|c|c|c|c|}
\hline $\begin{array}{c}\text { Study Summary (Study } \\
\text { Sensitivity) }\end{array}$ & Study Size/Exposure Prevalence & $\begin{array}{l}\text { Reported or Estimated Exposure } \\
\text { Levels or Duration }\end{array}$ & $\begin{array}{c}\text { Exposure Response Analyses: Dose } \\
\text { Metrics/Range Lagging }\end{array}$ \\
\hline $\begin{array}{l}\text { Gold et al. (2011) } \\
\text { Seattle, WA and Detroit, MI } \\
\text { (USA) SEER registry } \\
181 \text { cases MM, } 481 \text { controls } \\
\text { Adequate } \\
\text { Adequate number of cases in } \\
\text { control in subgroup analysis, } \\
\text { including the highest exposure } \\
\text { group }\end{array}$ & $\begin{array}{l}\text { Medium size study: } 181 \mathrm{MM} \text { cases, } \\
481 \text { controls } \\
\text { Exposure prevalence: } 29 \% \\
(\mathrm{~N}=138) \text { controls and } 37 \% \\
(\mathrm{~N}=66) \text { cases for ever-exposed and } \\
7.1(\mathrm{~N}=34) \text { controls and } 13 \% \\
(\mathrm{~N}=24) \text { in highest cumulative } \\
\text { exposure category }\end{array}$ & $\begin{array}{l}\text { Exposure levels not reported. Levels } \\
\text { estimated for analysis: Highest } \\
\text { cumulative exposure category }>7,794 \\
57,000 \text { ppm }\end{array}$ & $\begin{array}{l}\text { Exposure duration (yr) and cumulative } \\
\text { exposure (ppm-hr) } \\
\text { Range: adequate (estimated) range of } \\
\text { exposures } \\
\text { Lagging: } 0,10 \mathrm{yr}\end{array}$ \\
\hline $\begin{array}{l}\text { Costantini et al. (2008) } \\
\text { Population-based, Italy } \\
\text { Limited statistical power } \\
\text { Few exposed cases and controls }\end{array}$ & $\begin{array}{l}\text { Medium size study: } 263 \text { cases MM, } \\
1,100 \text { controls; cases CLL NR } \\
\text { TCE prevalence among controls } \\
\text { was } \sim 2.5 \% \text { ( } N=5 \text { cases and } 27 \\
\text { controls for medium/high and } 3.5 \% \\
(\mathrm{~N}=9 \text { cases and } 28 \text { controls) for } \\
\text { low/very low exposure }\end{array}$ & $\begin{array}{l}\text { Study regions chosen because of large } \\
\text { presence of manufacturing industries } \\
\text { using solvents or they were agricultural } \\
\text { areas }\end{array}$ & $\begin{array}{l}\text { Intensity and duration of exposure. } \\
\text { Range: No information } \\
\text { Lagging: no analysis }\end{array}$ \\
\hline $\begin{array}{l}\text { Persson and Fredrikson (1999) } \\
\text { Population-based (pooled study) } \\
\text { Sweden } \\
\text { Limited } \\
\text { Relatively small number of } \\
\text { exposed cases with possibly low } \\
\text { levels of exposure }\end{array}$ & $\begin{array}{l}\text { Medium size study: } 199 \text { cases } \\
\text { NHL, } 479 \text { controls } \\
\text { TCE exposure prevalence among } \\
\text { referents } \sim 7 \% \text { ( } 16 \text { cases } / 32 \\
\text { controls) }\end{array}$ & $\begin{array}{l}\text { No information on reported or estimated } \\
\text { levels or duration of exposure reported; } \\
\text { Authors state quantitative information } \\
\text { available but merged intensity categories } \\
\text { Minimum of 1-year exposure duration }\end{array}$ & $\begin{array}{l}\text { Ever/never exposure only. } \\
\text { Range: Not applicable }\end{array}$ \\
\hline
\end{tabular}




\begin{tabular}{|c|c|c|c|}
\hline $\begin{array}{l}\text { Study Summary (Study } \\
\text { Sensitivity) }\end{array}$ & Study Size/Exposure Prevalence & $\begin{array}{l}\text { Reported or Estimated Exposure } \\
\text { Levels or Duration }\end{array}$ & $\begin{array}{c}\text { Exposure Response Analyses: Dose } \\
\text { Metrics/Range Lagging }\end{array}$ \\
\hline $\begin{array}{l}\text { Nordström et al. (1998) } \\
\text { Population-based } \\
\text { Sweden } \\
\text { Limited } \\
\text { Relatively few exposed cases and } \\
\text { controls with possibly low levels } \\
\text { of exposure }\end{array}$ & $\begin{array}{l}\text { Small study: } 121 \text { cases NHL, } 484 \\
\text { controls } \\
\text { TCE exposure prevalence among } \\
\text { referents } \sim 7 \% \text { ( } 9 \text { cases and } 26 \\
\text { controls) }\end{array}$ & $\begin{array}{l}\text { No information on reported or estimated } \\
\text { levels or duration of exposure; Minimum } \\
\text { requirement for being classified as } \\
\text { exposed was } 1 \text { day }\end{array}$ & $\begin{array}{l}\text { Ever/never only } \\
\text { Range: Not applicable } \\
\text { Lagging: No analysis }\end{array}$ \\
\hline $\begin{array}{l}\text { Hardell et al. (1994) } \\
\text { Population-based } \\
\text { Sweden } \\
\text { Limited } \\
\text { Few cases and controls with } \\
\text { possibly low levels of exposure }\end{array}$ & $\begin{array}{l}\text { Small study: } 105 \text { cases and } 355 \\
\text { controls } \\
\text { Prevalence of TCE exposure among } \\
\text { controls was } 1 \% \text { ( } 4 \text { cases } / 4 \\
\text { controls) }\end{array}$ & $\begin{array}{l}\text { No information on exposure levels or } \\
\text { duration; Minimal criteria for being } \\
\text { considered exposed is low: }<1 \text { week } \\
\text { continuous exposure or }<1 \text { month total } \\
\text { exposure was considered low grade, and } \\
\text { more than that was considered high grade }\end{array}$ & $\begin{array}{l}\text { Ever/never only } \\
\text { Range: Not applicable } \\
\text { Lagging: No analysis }\end{array}$ \\
\hline
\end{tabular}

$\mathrm{NHL}=$ non-Hodgkin lymphoma; N = number; NA = not applicable; MIS = Multicentre Italian Study; NCI = National Cancer Institute; SEER = Surveillance, Epidemiology and End Results Program; ppm = parts per million; $\mathrm{MM}=$ multiple myeloma; $\mathrm{CLL}=$ chronic lymphocytic lymphoma; $\mathrm{NR}=$ not reported; TCE = trichloroethylene. 
Table D-10. Studies Included in Three Meta-analyses by Cancer Site

\begin{tabular}{|c|c|c|c|c|c|c|c|}
\hline \multirow[b]{2}{*}{ Studies Included } & \multicolumn{3}{|c|}{ Kidney } & \multicolumn{2}{|c|}{ Liver } & \multicolumn{2}{|c|}{ NHL } \\
\hline & $\begin{array}{c}\text { Scott and } \\
\text { Jinot } \\
(2011)^{\mathrm{a}}\end{array}$ & $\begin{array}{c}\text { Karami } \\
\text { et al. } \\
(2012)^{b}\end{array}$ & $\begin{array}{l}\text { Kelsh et } \\
\text { al. } \\
(2010)^{\mathrm{c}}\end{array}$ & $\begin{array}{c}\text { Scott and } \\
\text { Jinot } \\
(2011)^{\mathrm{a}}\end{array}$ & $\begin{array}{c}\text { Alexander } \\
\text { et al. } \\
(\mathbf{2 0 0 7})^{\mathrm{d}}\end{array}$ & $\begin{array}{c}\text { Scott and } \\
\text { Jinot } \\
(2011)^{\mathrm{a}}\end{array}$ & $\begin{array}{c}\text { Karami et al. } \\
(\mathbf{2 0 1 3})^{\mathbf{b}}\end{array}$ \\
\hline \multicolumn{8}{|c|}{ Cohort and nested case-control studies } \\
\hline Anttila et al. (1995) & $\mathrm{X}$ & $\mathrm{X}$ & $\mathrm{X}$ & $\mathrm{X}$ & $\mathrm{X}$ & $\mathrm{X}$ & $X$ \\
\hline Axelson et al. (1994) & $\mathrm{X}$ & $\mathrm{X}$ & $\mathrm{X}$ & $\mathrm{X}$ & $\mathrm{X}$ & $\mathrm{X}$ & $\mathrm{X}$ \\
\hline Bahr et al. (2011) & & & & & & & $\mathrm{X}$ \\
\hline Blair et al. (1998) & & & $\mathrm{X}$ & & $\mathrm{X}$ & & \\
\hline Boice et al. (1999) & $\mathrm{X}$ & & $\mathrm{X}$ & $\mathrm{X}$ & $\mathrm{X}$ & $\mathrm{X}$ & \\
\hline Boice et al. (2006) & & $\mathrm{X}$ & $\mathrm{X}$ & $\mathrm{X}$ & $\mathrm{X}$ & $\mathrm{X}$ & $\mathrm{X}$ \\
\hline Greenland et al. (1994) & $\mathrm{X}$ & $\mathrm{X}$ & & $\mathrm{X}$ & $\mathrm{X}$ & $\mathrm{X}$ & \\
\hline Hansen et al. (2001) & $\mathrm{X}$ & $\mathrm{X}$ & $\mathrm{X}$ & $\mathrm{X}$ & $\mathrm{X}$ & $\mathrm{X}$ & $\mathrm{X}$ \\
\hline Lipworth et al. (2011) & & $\mathrm{X}$ & & & & & $\mathrm{X}$ \\
\hline Morgan et al. (1998) & $\mathrm{X}$ & $\mathrm{X}$ & $\mathrm{X}$ & $\mathrm{X}$ & $X$ & $X$ & $X$ \\
\hline $\begin{array}{l}\text { Raaschou-Nielsen et al. } \\
(2003)\end{array}$ & $\mathrm{X}$ & $X$ & $\mathrm{X}$ & $\mathrm{X}$ & $X$ & $\mathrm{X}$ & $X$ \\
\hline Radican et al. (2008) & $\mathrm{X}$ & $\mathrm{X}$ & $\mathrm{X}$ & $\mathrm{X}$ & & $X$ & $X$ \\
\hline Ritz (1999) & & $\mathrm{X}$ & & & $\mathrm{X}$ & & $X$ \\
\hline Zhao et al. (2005) & $\mathrm{X}$ & & & $\mathrm{X}$ & & $\mathrm{X}$ & \\
\hline \multicolumn{8}{|l|}{ Case-control studies } \\
\hline Asal et al. (1988) & & $X$ & & & & & \\
\hline Brüning et al. (2003) & $\mathrm{X}$ & $\mathrm{X}$ & $\mathrm{X}$ & & & & \\
\hline Charbotel et al. (2006) & $\mathrm{X}$ & $\mathrm{X}$ & $\mathrm{X}$ & & & & \\
\hline Cocco et al. (2010) & & & & & & $X$ & $X$ \\
\hline Dosemeci et al. (1999) & $\mathrm{X}$ & $\mathrm{X}$ & $\mathrm{X}$ & & & & \\
\hline Hardell et al. (1994) & & & & & & $\mathrm{X}$ & $\mathrm{X}$ \\
\hline Harrington et al. (1989) & & $\mathrm{X}$ & & & & & \\
\hline Henschler et al. (1995) & & $\mathrm{X}$ & $\mathrm{X}$ & & & & \\
\hline Kato et al. (2005) & & & & & & & $X$ \\
\hline Moore et al. (2010) & $\mathrm{X}$ & $\mathrm{X}$ & & & & & \\
\hline
\end{tabular}


RoC Monograph on Trichloroethylene

\begin{tabular}{|c|c|c|c|c|c|c|c|}
\hline \multirow[b]{2}{*}{ Studies Included } & \multicolumn{3}{|c|}{ Kidney } & \multicolumn{2}{|c|}{ Liver } & \multicolumn{2}{|c|}{ NHL } \\
\hline & $\begin{array}{c}\text { Scott and } \\
\text { Jinot } \\
(2011)^{\mathrm{a}}\end{array}$ & $\begin{array}{c}\text { Karami } \\
\text { et al. } \\
(2012)^{b}\end{array}$ & $\begin{array}{l}\text { Kelsh et } \\
\text { al. } \\
(2010)^{\mathrm{c}}\end{array}$ & $\begin{array}{c}\text { Scott and } \\
\text { Jinot } \\
(2011)^{\mathrm{a}}\end{array}$ & $\begin{array}{l}\text { Alexander } \\
\text { et al. } \\
(\mathbf{2 0 0 7})^{\mathrm{d}}\end{array}$ & $\begin{array}{c}\text { Scott and } \\
\text { Jinot } \\
(2011)^{\mathrm{a}}\end{array}$ & $\begin{array}{c}\text { Karami et al. } \\
(\mathbf{2 0 1 3})^{\mathbf{b}}\end{array}$ \\
\hline Miligi et al. (2006) & & & & & & $\mathrm{X}$ & $\mathrm{X}$ \\
\hline Nordström et al. (1998) & & & & & & $X$ & $\mathrm{X}$ \\
\hline $\begin{array}{l}\text { Persson and Fredrikson } \\
\text { (1999) }\end{array}$ & & & & & & $\mathrm{X}$ & $X$ \\
\hline Pesch et al. (2000b) & $X$ & $X$ & $X$ & & & & \\
\hline Purdue et al. (2011a) & & & & & & $\mathrm{X}$ & $\mathrm{X}$ \\
\hline Siemiatycki (1991) & $\mathrm{X}$ & $X$ & $\mathrm{X}$ & & & $X$ & $X$ \\
\hline Vamvakas et al. (1998) & & $X$ & $X$ & & & & \\
\hline Wang et al. (2009b) & & & & & & $\mathrm{X}$ & $\mathrm{X}$ \\
\hline
\end{tabular}

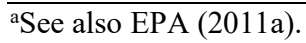

${ }^{b}$ Karami et al. (2012); Karami et al. (2013): Studies classified as TCE-exposed only; chlorinated solvent studies not included. 'Kelsh et al. (2010): Group I studies (classified as having adequate exposure data to identify workers with TCE exposure) only; Group II studies (limited exposure data) excluded.

${ }^{\mathrm{d} A l e x a n d e r}$ et al. (2007): Group 1 studies, TCE-exposed subgroup (classified as having adequate exposure data to identify subgroup of workers with TCE exposure) only; Group II studies (limited exposure data) excluded. 


\section{Appendix E. Immune Effects (Animals)}

\section{Tables}

Table E-1. Designs of Studies Evaluated for Trichloroethylene or Metabolite Induced Immunomodulation Relevant to Lymphoma and Liver Cancer ..............................

Table E-2. Immunomodulatory Endpoints Relevant to Cancer............................................... E-3

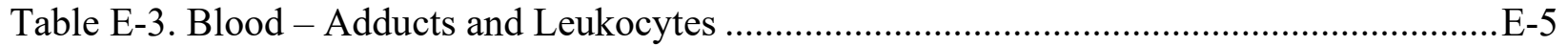

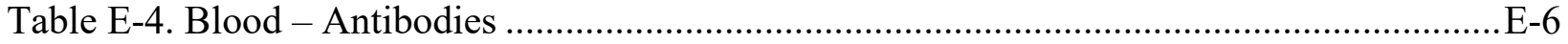

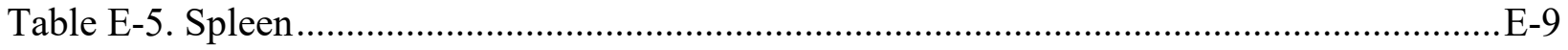

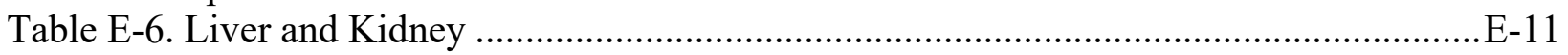

Table E-7. Splenic Ex Vivo Cytokines, Lymph Node, and Anti-bacterial Response ...............E-13 
This section has the tables summarizing the findings of immune effects in experimental animals. It also has tables related to methods, including study design and endpoints measured.

Table E-1. Designs of Studies Evaluated for Trichloroethylene or Metabolite Induced Immunomodulation Relevant to Lymphoma and Liver Cancer

\begin{tabular}{|c|c|c|c|c|}
\hline Species & Strain & Route & Chemical & $\begin{array}{l}\text { Number of } \\
\text { Studies }\end{array}$ \\
\hline \multirow[t]{20}{*}{ Mouse } & MRL+/+ & Drinking Water & TCE & $12^{\mathrm{a}}$ \\
\hline & & & TCA & 1 \\
\hline & & & TCAH & 2 \\
\hline & & IP & TCE & $5^{\mathrm{b}}$ \\
\hline & & & DCAA & 1 \\
\hline & & & DCAC & 3 \\
\hline & & $\mathrm{SC}$ & Formyl-albumin adduct & 1 \\
\hline & & & Dichloroacetyl-albumin adduct & 1 \\
\hline & & & $\begin{array}{l}\text { Trichloroethene oxide-albumin } \\
\text { adduct }\end{array}$ & 1 \\
\hline & & Inhalation & TCE & 1 \\
\hline & NOD/Born & Drinking water & TCE & 1 \\
\hline & NZBWF1 & Drinking water & TCE & 1 \\
\hline & $\begin{array}{l}\text { SV/129 (PPAR- } \\
\text { null) }\end{array}$ & Inhalation & TCE & 1 \\
\hline & $\mathrm{C} 3 \mathrm{H} / \mathrm{HeJ}$ & Drinking water & TCAH & 1 \\
\hline & $\mathrm{B} 6 \mathrm{C} 3 \mathrm{~F}_{1}$ & Drinking water & TCE & 2 \\
\hline & & IP & TCE & 1 \\
\hline & $\mathrm{CD}-1$ & Drinking water & TCE & 1 \\
\hline & & & $\mathrm{CH}$ & 1 \\
\hline & & Gavage & $\mathrm{CH}$ & 1 \\
\hline & & Inhalation & $\mathrm{TCE}+$ bacteria & 4 \\
\hline \multirow[t]{2}{*}{ Rat } & Sprague-Dawley & IP & TCE & 2 \\
\hline & & Intradermal & TCE & 1 \\
\hline \multirow[t]{2}{*}{ Guinea pig } & FMMU & Dermal & TCE & 1 \\
\hline & & Intradermal & TCE & 2 \\
\hline \multirow[t]{2}{*}{ Dog } & Cross-bred & Intratracheal intubation & TCE & 2 \\
\hline & & IV & TCE & 1 \\
\hline
\end{tabular}

TCE = trichloroethylene; TCA = trichloroacetic acid; TCAH = trichloroacetyl hydrate; DCAA = dichloroacetyl chloride; $\mathrm{DCAC}=$ dichloroacetyl anhydride; $\mathrm{CH}=$ chloral hydrate; $\mathrm{SC}=$ subcutaneous injection; $\mathrm{IP}=$ intraperitoneal injection; IV $=$ intravenous injection.

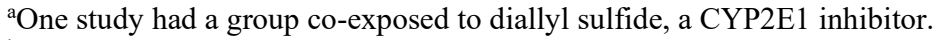

${ }^{b}$ One study had a group co-exposed to $N$-acetylcysteine, an enhancer of the antioxidant activity of glutathione. 
Table E-2. Immunomodulatory Endpoints Relevant to Cancer

\begin{tabular}{|c|c|c|c|c|}
\hline Endpoint & + & - & $=$ & $+1-$ \\
\hline \multicolumn{5}{|l|}{ Serum } \\
\hline dichloroacetyl-protein adducts & 1 & & & \\
\hline dichloroacetyl-albumin adducts & 1 & & & \\
\hline dichloroacetyl-CYP2E1 adducts & 1 & & & \\
\hline hydroxynonenal-protein adducts & 2 & & & \\
\hline malondialdehyde-protein adducts & 2 & & & \\
\hline $\operatorname{IgG}$ & 6 & 1 & 2 & \\
\hline anti-dichloroacetyl-protein adduct antibody & 2 & & 1 & \\
\hline anti-dichloroacetyl-albumin adduct antibody & $4^{\mathrm{e}}$ & & 1 & \\
\hline anti-formyl-albumin adduct antibody & $3^{\mathrm{e}}$ & & & \\
\hline anti-trichloroethene oxide-albumin adduct antibody & $3^{\mathrm{e}}$ & & & \\
\hline anti-albumin antibody & $3^{\mathrm{e}}$ & & 2 & \\
\hline anti-hydroxynonenal-protein adduct antibody & $4^{\mathrm{a}}$ & & & \\
\hline anti-malondialdehyde-protein adduct antibody & $5^{\mathrm{a}}$ & & 1 & \\
\hline anti-liver antibody & 1 & & 1 & \\
\hline anti-nuclear antibody & 9 & & 3 & \\
\hline anti-DNA antibody & $1^{\mathrm{a}}$ & & 2 & \\
\hline anti-ssDNA antibody & $6^{\mathrm{a}}$ & & $7^{\mathrm{d}}$ & \\
\hline anti-dsDNA antibody & $3^{\mathrm{a}}$ & & $4^{\mathrm{d}}$ & \\
\hline anti-sheep RBC IgM response & & & 1 & 1 \\
\hline \multicolumn{5}{|l|}{ Peripheral blood } \\
\hline leukocyte number & & 4 & 1 & \\
\hline neutrophil number & & 3 & & \\
\hline lymphocyte number & & 1 & 3 & \\
\hline CD4 T-cell number & & 2 & & \\
\hline CD8 T-cell number & & 1 & 1 & \\
\hline B-cell number & & 1 & & \\
\hline \multicolumn{5}{|l|}{ Spleen } \\
\hline lymphocyte number & 2 & & & \\
\hline lymphocyte proliferation & 1 & & & \\
\hline T-cell proliferation & & & $4^{\mathrm{d}}$ & \\
\hline CD4 T-cell number & & $2^{\mathrm{d}}$ & 1 & $1^{\mathrm{d}}$ \\
\hline CD4 T-cell proliferation & $2^{\mathrm{b}}$ & & $1^{\mathrm{d}}$ & \\
\hline CD8 T-cell number & & $1^{\mathrm{d}}$ & $6^{\mathrm{d}}$ & \\
\hline CD8 T-cell proliferation & & & $2^{\mathrm{a}, \mathrm{d}}$ & \\
\hline B-cell number & & $2^{\mathrm{d}}$ & 5 & \\
\hline
\end{tabular}




\begin{tabular}{|c|c|c|c|c|}
\hline Endpoint & + & - & $=$ & $+/-$ \\
\hline B-cell proliferation & & & $6^{\mathrm{d}}$ & \\
\hline B-cell activation & & & $4^{\mathrm{d}}$ & \\
\hline anti-sheep RBC IgM response & 1 & $2^{\mathrm{d}}$ & & \\
\hline NK cell proliferation & & & $2^{\mathrm{d}}$ & \\
\hline NK cell cytolytic activity & & & 3 & \\
\hline Splenocytes stimulated with hydroxynonenal-albumin adduct - IFN-gamma & 2 & & & \\
\hline Splenocytes stimulated with malondialdehyde-albumin adduct - IFN-gamma & 2 & & & \\
\hline Splenocytes stimulated with hydroxynonenal-albumin adduct - IL-2 & 2 & & 1 & \\
\hline Splenocytes stimulated with malondialdehyde-albumin adduct - IL-2 & 2 & & 1 & \\
\hline \multicolumn{5}{|l|}{ Lymph node } \\
\hline CD4 T-cell number & & & 5 & \\
\hline CD8 T-cell number & & & 4 & \\
\hline B-cell number & & & 2 & \\
\hline B-cell activation NOS & & & 2 & \\
\hline \multicolumn{5}{|l|}{ Liver } \\
\hline TCE-protein adduct & 1 & & & \\
\hline dichloroacetyl-protein adducts & $2^{\mathrm{b}}$ & & & \\
\hline dichloroacetyl-CYP2E1 adducts & 1 & & & \\
\hline hydroxynonenal-protein adducts & $2^{\mathrm{a}}$ & & & \\
\hline malondialdehyde-protein adducts & $2^{\mathrm{a}}$ & & & \\
\hline Inflammation & $7^{\mathrm{c}}$ & & 5 & \\
\hline T-cell infiltration & 2 & & & \\
\hline NK cell cytolytic activity & & 2 & & \\
\hline hepatocyte proliferation & $2^{\mathrm{c}}$ & & & \\
\hline \multicolumn{5}{|l|}{ Kidney } \\
\hline glomerular antibody deposits & 1 & & & \\
\hline hydroxynonenal-protein adducts & $1^{\mathrm{a}}$ & & & \\
\hline Malondialdehyde-protein adducts & $1^{\mathrm{a}}$ & & & \\
\hline Inflammation & 1 & & 1 & \\
\hline \multicolumn{5}{|l|}{ Bacterial infection } \\
\hline Death from bacterial infection & 2 & & & \\
\hline Lung - bacterial infection/bacteria clearance & 1 & & & 1 \\
\hline Lung - macrophage phagocytosis of bacteria & & 1 & & \\
\hline
\end{tabular}

$+=$ increased effect; $-=$ decreased effect; $==$ no change in effect; $+/-=$ both increases and decreases in effect were seen depending on dose or time point.

aPrevented by $N$-acetylcysteine.

bPrevented by diallyl sulfide.

cPPAR ${ }^{-/}$had no effect.

${ }^{\mathrm{d}}$ Exposure started before conception.

${ }^{\text {E}}$ Exposed to TCE albumin adducts (formyl-, trichlorethene oxide-, diacetyl-). 
The tables below provide study-by-study information on the immune effects of trichloroethylene in experimental animals (see Sections 5.2.2.2 and 6.2.1.5). The designs of 51 studies are reported along with the results of 62 endpoints. The five tables are divided by the endpoints studied (Table E-3. Blood - Adducts and Leukocytes; Table E-4. Blood - Antibodies; Table E-5. Spleen; Table E-6. Liver and Kidney; Table E-7. Splenic Ex Vivo Cytokines, Lymph Node, and Anti-bacterial Response).

Table E-3. Blood - Adducts and Leukocytes

\begin{tabular}{|c|c|c|c|c|c|c|c|c|c|c|c|c|}
\hline Reference & Design & 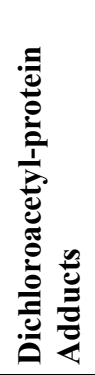 & 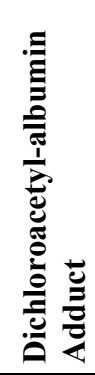 & 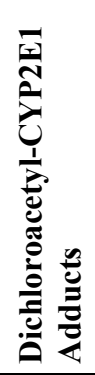 & 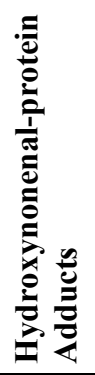 & 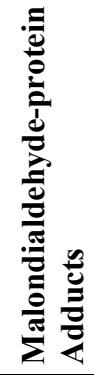 & 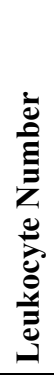 & 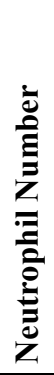 & 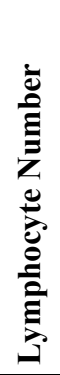 & 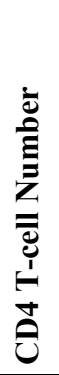 & 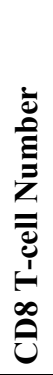 & 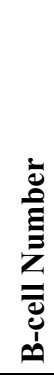 \\
\hline \multicolumn{13}{|c|}{ Trichloroethylene; Mouse (MRL+/+); Drinking water } \\
\hline Wang et al. (2007b) & $48 \mathrm{wk}$ & & & & + & + & & & & & & \\
\hline Wang et al. (2012a) & $12,24,36 \mathrm{wk}$ & & & & + & + & & & & & & \\
\hline \multicolumn{13}{|c|}{ Trichloroethylene; Mice (NOD/Born); Drinking water } \\
\hline Ravel et al. (2004) & $4,8,12 \mathrm{wk}$ & & & & & & - & & - & - & - & - \\
\hline \multicolumn{13}{|c|}{ Chloral hydrate; Mice (CD-1); Drinking water } \\
\hline Kauffmann et al. (1982) & $90 \mathrm{~d}$ & & & & & & $=$ & & & & & \\
\hline \multicolumn{13}{|c|}{ Trichloroethylene; Rat (Sprague-Dawley); IP } \\
\hline Halmes et al. (1997) & $4 \mathrm{hr}$ & + & + & + & & & & & & & & \\
\hline Chen et al. (2006) & $5,7 \mathrm{wk}$ & & & & & & & & & - & $=$ & \\
\hline \multicolumn{13}{|c|}{ Trichloroethylene; Dog (cross-bred); Intratracheal intubation } \\
\hline Hobara et al. (1984) & $1 \mathrm{hr}$ & & & & & & - & - & $=$ & & & \\
\hline Hobara et al. (1984) & $1,4 \mathrm{hr}$ & & & & & & - & - & $=$ & & & \\
\hline \multicolumn{13}{|c|}{ Trichloroethylene; Dog (cross-bred); IV } \\
\hline Hobara et al. (1984) & Single dose & & & & & & - & - & $=$ & & & \\
\hline
\end{tabular}




\begin{tabular}{|c|c|c|c|c|c|c|c|c|c|c|c|c|c|c|c|}
\hline Reference & Design & ל్ర & 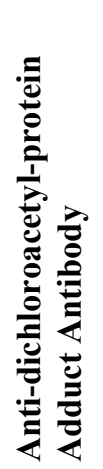 & 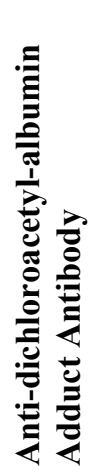 & 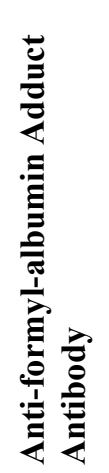 & 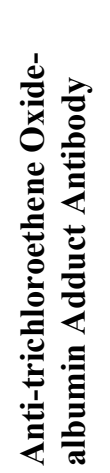 & 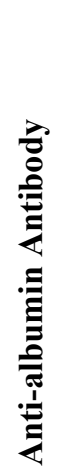 & 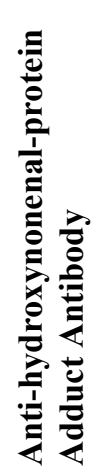 & 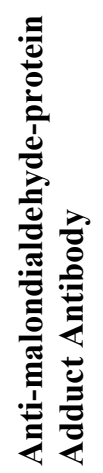 & 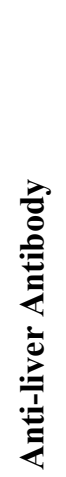 & 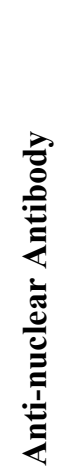 & 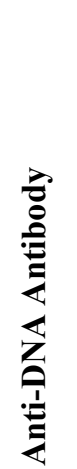 & 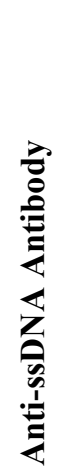 & 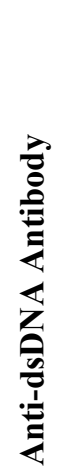 & 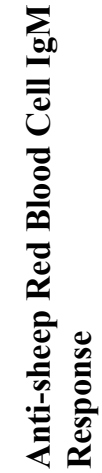 \\
\hline \multicolumn{16}{|c|}{ Trichloroethylene; Mice (MRL+/+); Drinking water } \\
\hline Blossom and Doss (2007) & $\begin{array}{l}\text { Preconception to } 4 \text {, } \\
6,8 \text { wk old }\end{array}$ & & & & & & & & & & & & $=$ & & \\
\hline Cai et al. (2008) & $36,48 \mathrm{wk}$ & & & & & & & & & & $=$ & & & & \\
\hline Gilbert et al. (2009) & $10,18,26 \mathrm{wk}$ & & & & & & & & & + & & & & & \\
\hline Gilbert et al. (2011) & $8 \mathrm{wk}$ & & & & & & & & & $=$ & $=$ & & & & \\
\hline Griffin et al. (2000a) & $4,6,8,22 \mathrm{wk}$ & + & + & & & & & & & & + & & & & \\
\hline Griffin et al. (2000c) & $4,32 \mathrm{wk}$ & & & & & & & & & & + & & & & \\
\hline Wang et al. (2007b) & 48 wk & & & & & & & & & & + & & $=$ & & \\
\hline Wang et al. (2012a) & $12,24,36 \mathrm{wk}$ & & & & & & & + & + & & + & & + & & \\
\hline \multicolumn{16}{|c|}{ Trichloroacetic acid; Mice (MRL $+/+)$; Drinking water } \\
\hline Blossom et al. (2004) & $4 \mathrm{wk}$ & & & & & & & & & & & & $=$ & & \\
\hline \multicolumn{16}{|c|}{ Trichloroacetaldehyde hydrate } \\
\hline Blossom et al. (2004) & $4 \mathrm{wk}$ & & & & & & & & & & & & $=$ & & \\
\hline Blossom et al. (2007) & $4,40 \mathrm{wk}$ & & & & & & & & & & & & $=$ & $=$ & \\
\hline \multicolumn{16}{|c|}{ Trichloroethylene; Mice (MRL+/+); IP } \\
\hline Khan et al. (1995) & $6 \mathrm{wk}$ & + & $=$ & & & & & & & & + & $=$ & + & & \\
\hline
\end{tabular}




\begin{tabular}{|c|c|c|c|c|c|c|c|c|c|c|c|c|c|c|c|}
\hline Reference & Design & ט్ర & 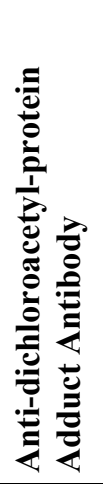 & 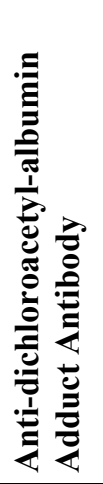 & 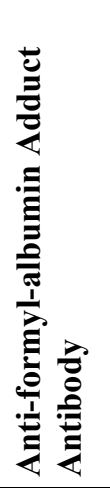 & 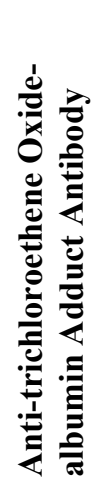 & 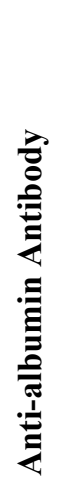 & 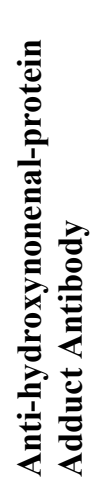 & 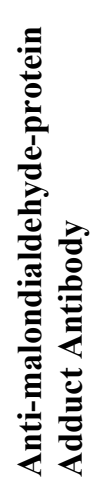 & 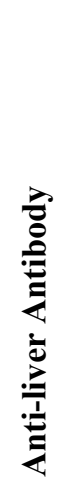 & 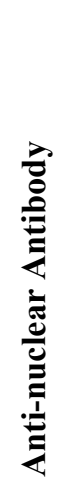 & 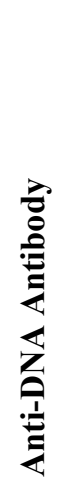 & 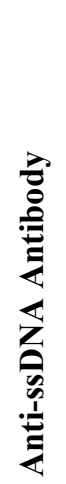 & 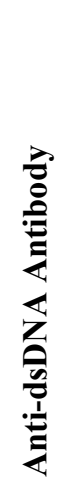 & 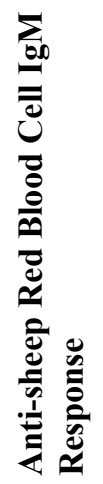 \\
\hline Khan et al. (2001) & $6 \mathrm{wk}$ & & & & & & & & $=$ & & & & & & \\
\hline Wang et al. (2007a) & $6,12 \mathrm{wk}$ & & & & & & & + & + & & + & & + & + & \\
\hline Wang et al. (2008) & $4 \mathrm{wk}$ & + & & & & & & + & + & & + & & & & \\
\hline Wang et al. $(2013)^{\mathrm{a}}$ & $6 \mathrm{wk}$ & & & & & & & + & + & & & + & + & + & \\
\hline \multicolumn{16}{|c|}{ Dichloroacetyl anhydride; Mice $(M R L+/+) ; I P$} \\
\hline Cai et al. (2006) & $6 \mathrm{wk}$ & $=$ & & $=$ & & & $=$ & & & & + & & & & \\
\hline \multicolumn{16}{|c|}{ Dichloroacetyl chloride; Mice (MRL+/+); IP } \\
\hline Cai et al. (2006) & $6 \mathrm{wk}$ & + & & + & & & $=$ & & & & + & & & & \\
\hline Khan et al. (1995) & $6 \mathrm{wk}$ & + & + & & & & & & & & $=$ & $=$ & + & & \\
\hline Khan et al. (2001) & $2,4,6,8 \mathrm{wk}$ & & & & & & & & + & & & & & & \\
\hline \multicolumn{16}{|c|}{ Trichloroethylene; Mice (MRL+/+); Inhalation } \\
\hline Kaneko et al. (2000) & $4,6,8 \mathrm{wk}$ & - & & & & & & & & & & & & & \\
\hline \multicolumn{16}{|c|}{ Formyl-albumin adduct; Mice $(M R L+/+) ; S C$} \\
\hline Cai et al. (2007b) & $4 \mathrm{wk}$ & & & + & + & + & + & & & & & & & & \\
\hline \multicolumn{16}{|c|}{ Dichloroacetyl-albumin adduct; Mice (MRL+/+); SC } \\
\hline Cai et al. (2007b) & $4 \mathrm{wk}$ & & & + & + & + & + & & & & & & & & \\
\hline \multicolumn{16}{|c|}{ Trichloroethene oxide-albumin adduct; Mice $(M R L+/+) ; S C$} \\
\hline Cai et al. (2007b) & $4 \mathrm{wk}$ & & & + & + & + & + & & & & & & & & \\
\hline
\end{tabular}




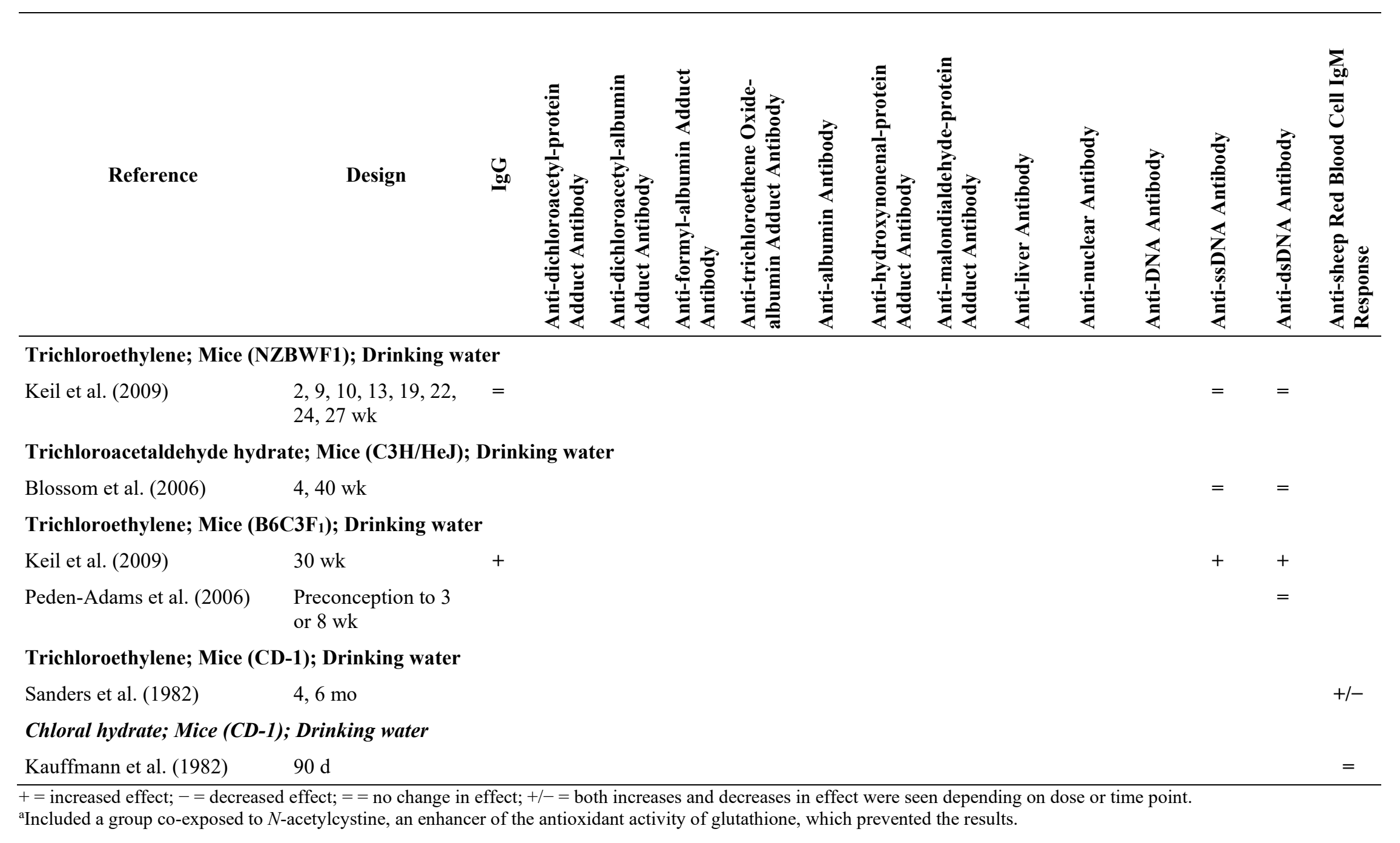




\begin{tabular}{|c|c|c|c|c|c|c|c|c|c|c|c|c|c|c|}
\hline Reference & Design & 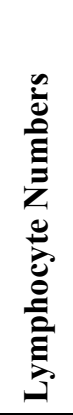 & 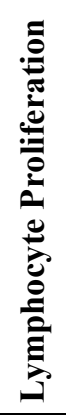 & 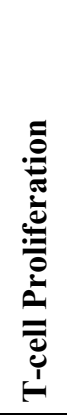 & 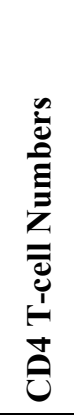 & 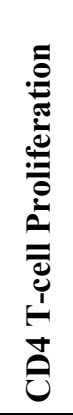 & 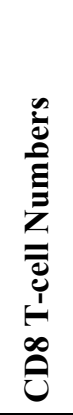 & 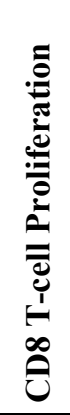 & 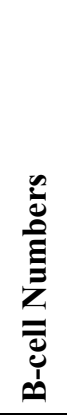 & 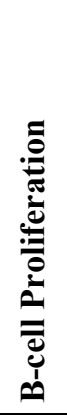 & 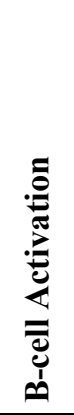 & 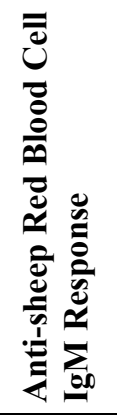 & 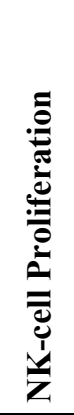 & 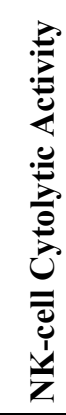 \\
\hline \multicolumn{15}{|c|}{ Trichloroethylene; Mice (MRL+/+); Drinking water } \\
\hline Blossom and Doss (2007) & $\begin{array}{l}\text { Preconception to } 4 \text {, } \\
6,8 \text { wk old }\end{array}$ & & & & - & & - & & - & & $=$ & & & \\
\hline Gilbert et al. (2011) & $8 \mathrm{wk}$ & & & & $=$ & & $=$ & & $=$ & & & & & \\
\hline Griffin et al. (2000a) & $4,6,8,22 \mathrm{wk}$ & & & & $=$ & & & & & & $=$ & & & \\
\hline Griffin et al. $(2000 \mathrm{~b})^{\mathrm{a}}$ & & & & & & + & & & & & & & & \\
\hline Peden-Adams et al. (2008) & $\begin{array}{l}\text { Preconception to } \\
12 \text { mo }\end{array}$ & & & & & $=$ & & $=$ & & $=$ & & & & \\
\hline \multicolumn{15}{|c|}{ Trichloroacetic acid; Mice $(M R L+/+)$; Drinking water } \\
\hline Blossom et al. (2004) & $4 \mathrm{wk}$ & & & & $=$ & & $=$ & & $=$ & & $=$ & & & \\
\hline \multicolumn{15}{|c|}{ Trichloroacetaldehyde hydrate; Mice $(M R L+/+)$; Drinking water } \\
\hline Blossom et al. (2004) & $4 \mathrm{wk}$ & & & & $=$ & & $=$ & & $=$ & & $=$ & & & \\
\hline Blossom et al. (2007) & $4,40 \mathrm{wk}$ & & & & - & & $=$ & & $=$ & & & & & \\
\hline \multicolumn{15}{|c|}{ Trichloroethylene; Mice (MRL+/+); IP } \\
\hline Wang et al. (2008) & $4 \mathrm{wk}$ & & & & & + & & $=$ & & $=$ & & & & \\
\hline \multicolumn{15}{|c|}{ Dichloroacetyl anhydride; Mice $(M R L+/+) ;$ IP } \\
\hline Cai et al. (2006) & $6 \mathrm{wk}$ & + & & & & & & & & & & & & \\
\hline \multicolumn{15}{|c|}{ Dichloroacetyl chloride; Mice $(M R L+/+) ; I P$} \\
\hline Cai et al. (2006) & $6 \mathrm{wk}$ & + & & & & & & & & & & & & \\
\hline
\end{tabular}




\begin{tabular}{|c|c|c|c|c|c|c|c|c|c|c|c|c|c|c|}
\hline Reference & Design & 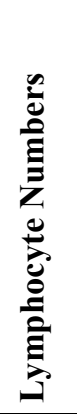 & 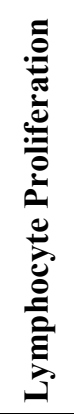 & 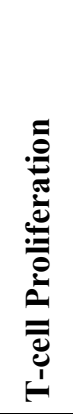 & 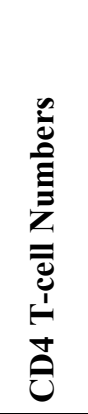 & 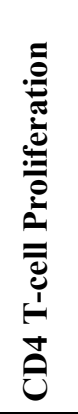 & 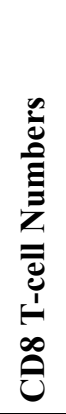 & 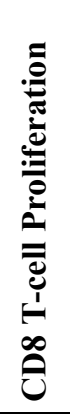 & 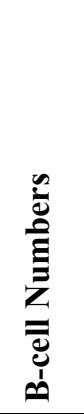 & 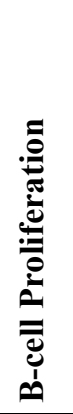 & 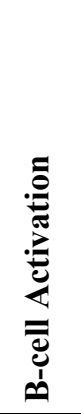 & 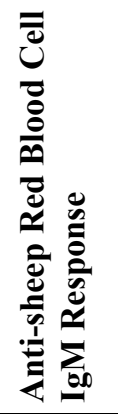 & 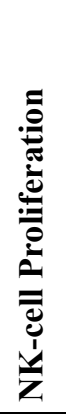 & 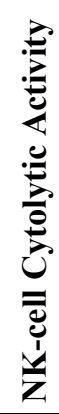 \\
\hline \multicolumn{15}{|c|}{ Trichloroethylene; Mice (NZBWF1); Drinking water } \\
\hline Keil et al. (2009) & $\begin{array}{l}2,9,10,13,19,22 \\
24,27 \mathrm{wk}\end{array}$ & & & $=$ & & & & & & $=$ & & & & $=$ \\
\hline \multicolumn{15}{|c|}{ Trichloroacetaldehyde hydrate; Mice (C3H/HeJ); Drinking water } \\
\hline Blossom et al. (2006) & $4,40 \mathrm{wk}$ & & & & $=$ & & $=$ & & & & & & & \\
\hline \multicolumn{15}{|c|}{ Trichloroethylene; Mice $\left(\mathrm{B} 6 \mathrm{C} 3 \mathrm{~F}_{1}\right)$; Drinking water } \\
\hline Peden-Adams et al. (2006) & $\begin{array}{l}\text { Preconception to } 3 \text {, } \\
8 \mathrm{wk}\end{array}$ & & & $=$ & $+/-$ & & $=$ & & - & $=$ & & - & $=$ & \\
\hline Keil et al. (2009) & $30 \mathrm{wk}$ & & & $=$ & & & & & & $=$ & & & $=$ & \\
\hline Wright et al. (1991) & $3 \mathrm{~d}$ & & & & & & & & & & & & & $=$ \\
\hline \multicolumn{15}{|c|}{ Trichloroethylene; Mice (CD-1); Drinking water } \\
\hline Sanders et al. (1982) & $4,6 \mathrm{mo}$ & & + & & & & & & & & & + & & \\
\hline \multicolumn{15}{|c|}{ Chloral hydrate; Mice (CD-1); Drinking water } \\
\hline Kauffmann et al. (1982) & $90 \mathrm{~d}$ & & & $=$ & & & & & & $=$ & & - & & \\
\hline \multicolumn{15}{|c|}{ Chloral hydrate; Mice (CD-1); Gavage } \\
\hline Kauffmann et al. (1982) & $15 \mathrm{~d}$ & & & & & & & & $=$ & & & & & \\
\hline \multicolumn{15}{|c|}{ Trichloroethylene; Rat (Sprague-Dawley); IP } \\
\hline Wright et al. (1991) & $3 \mathrm{~d}$ & & & & & & & & & & & & & $=$ \\
\hline
\end{tabular}


Table E-6. Liver and Kidney

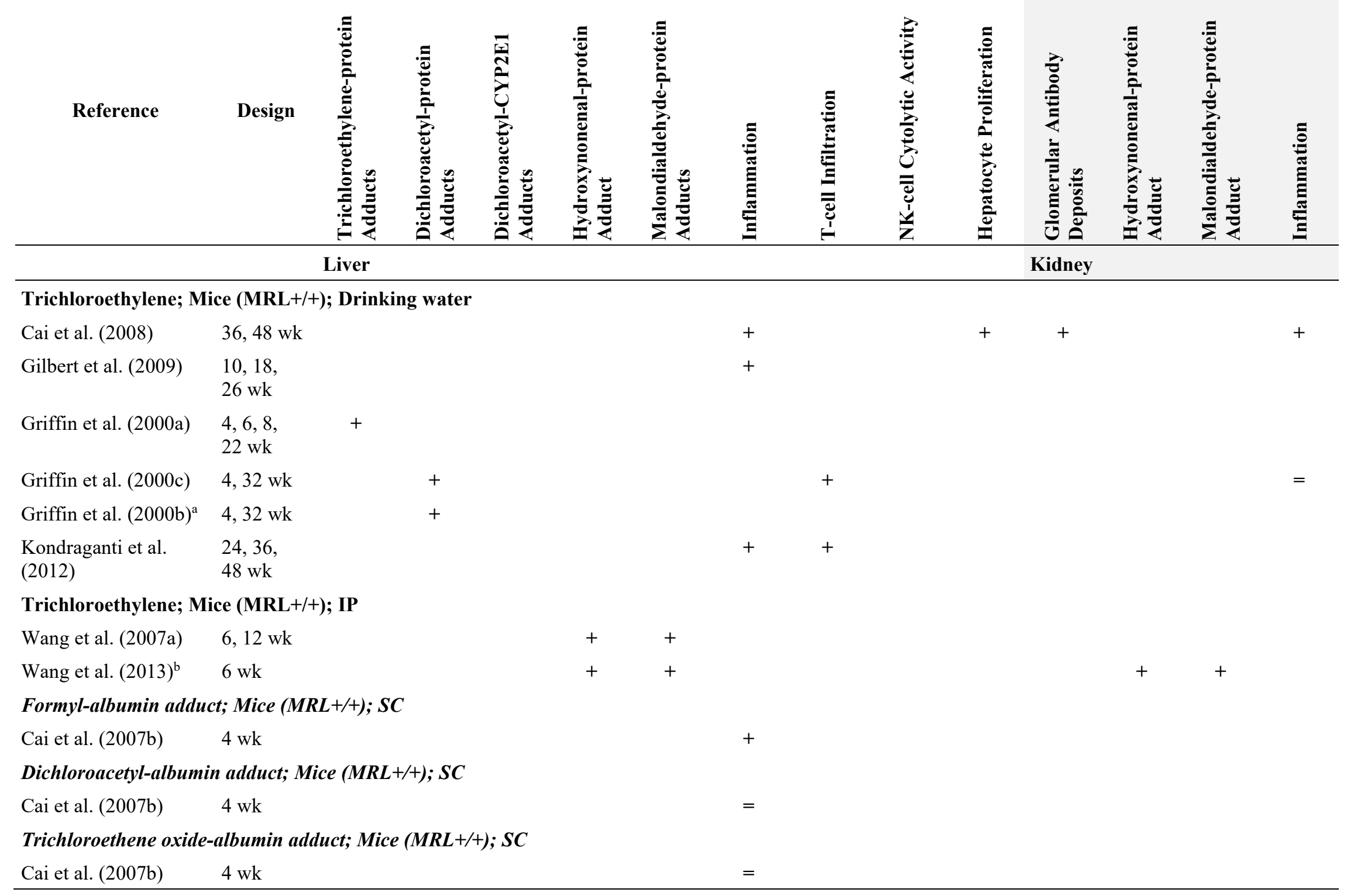




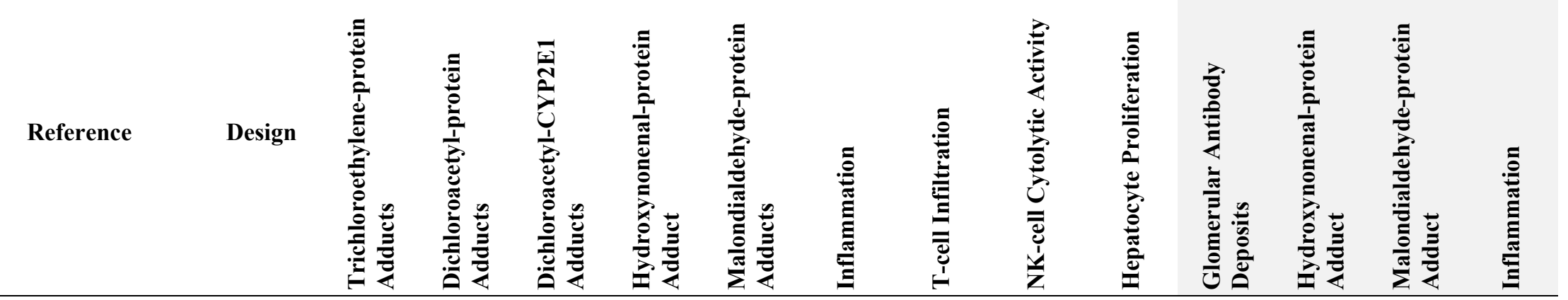

Trichloroethylene; Mice (MRL+/+); Inhalation

Kaneko et al. (2000) 4, 6, $8 \mathrm{wk}$

Trichloroethylene; Mice (NOD/Born); Drinking water

Ravel et al. (2004) 4,8, $12 \mathrm{wk}$

Trichloroethylene; Mice (SV/129) [wt/PPAR-null/PPAR-tet-off]; Inhalation

Ramdhan et al. (2010) $7 \mathrm{~d}$

Trichloroethylene; Mice (B6C3F 1$)$; IP

Wright et al. (1991) $3 \mathrm{~d}$

Trichloroethylene; Rat (Sprague-Dawley); IP

Halmes et al. (1997) $4 \mathrm{hr}$

Wright et al. (1991) $3 \mathrm{~d}$

Trichloroethylene; Guinea pig (FMMU); Dermal

Tang et al. (2008) $\quad 48 \mathrm{hr}$

Trichloroethylene; Guinea pig (FMMU); Intradermal/Dermal

Tang et al. (2008) $23 \mathrm{~d}$

Trichloroethylene; Guinea pig (FMMU); Intradermal

$\frac{\text { Tang et al. (2008) } \quad 48 \mathrm{hr}}{+=\text { increased effect; }-=\text { decreased effect; }==\text { no change in effect. }}$

ancluded a group co-exposed to diallyl sulfide, a CYP2E1 inhibitor, which prevented the results.

bIncluded a group co-exposed to $\mathrm{N}$-acetylcystine, an enhancer of the antioxidant activity of glutathione, which prevented the results. 


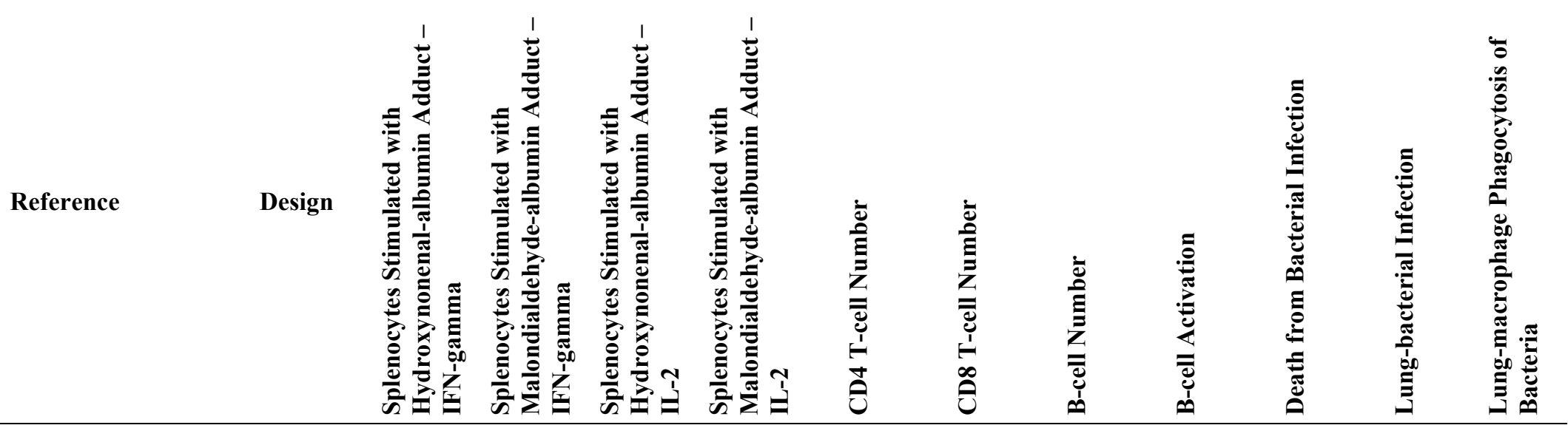

Trichloroethylene; Mice (MRL+/+); Drinking water

Gilbert et al. (2011) $\quad 8 \mathrm{wk}$

Gilbert et al. (2012)

$12,17 \mathrm{wk}$

Wang et al. (2012a)

$$
\begin{aligned}
& 12,24, \\
& 36 \mathrm{wk}
\end{aligned}
$$

Trichloroacetic acid; Mice (MRL+/+); Drinking water

\section{Blossom et al. (2004) $4 \mathrm{wk}$}

Trichloroacetaldehyde hydrate; Mice (MRL+/+); Drinking water

Blossom et al. (2004) $4 \mathrm{wk}$

Trichloroethylene; Mice (MRL+/+); IP

Wang et al. (2008) $4 \mathrm{wk}$

Dichloroacetyl anhydride; Mice $(M R L+/+)$; IP

Cai et al. (2006)

$6 \mathrm{wk}$

Dichloroacetyl chloride; Mice (MRL+/+); IP

Cai et al. (2006)

$6 \mathrm{wk}$

Trichloroethylene; Mice (C3H/HeJ); Drinking water 


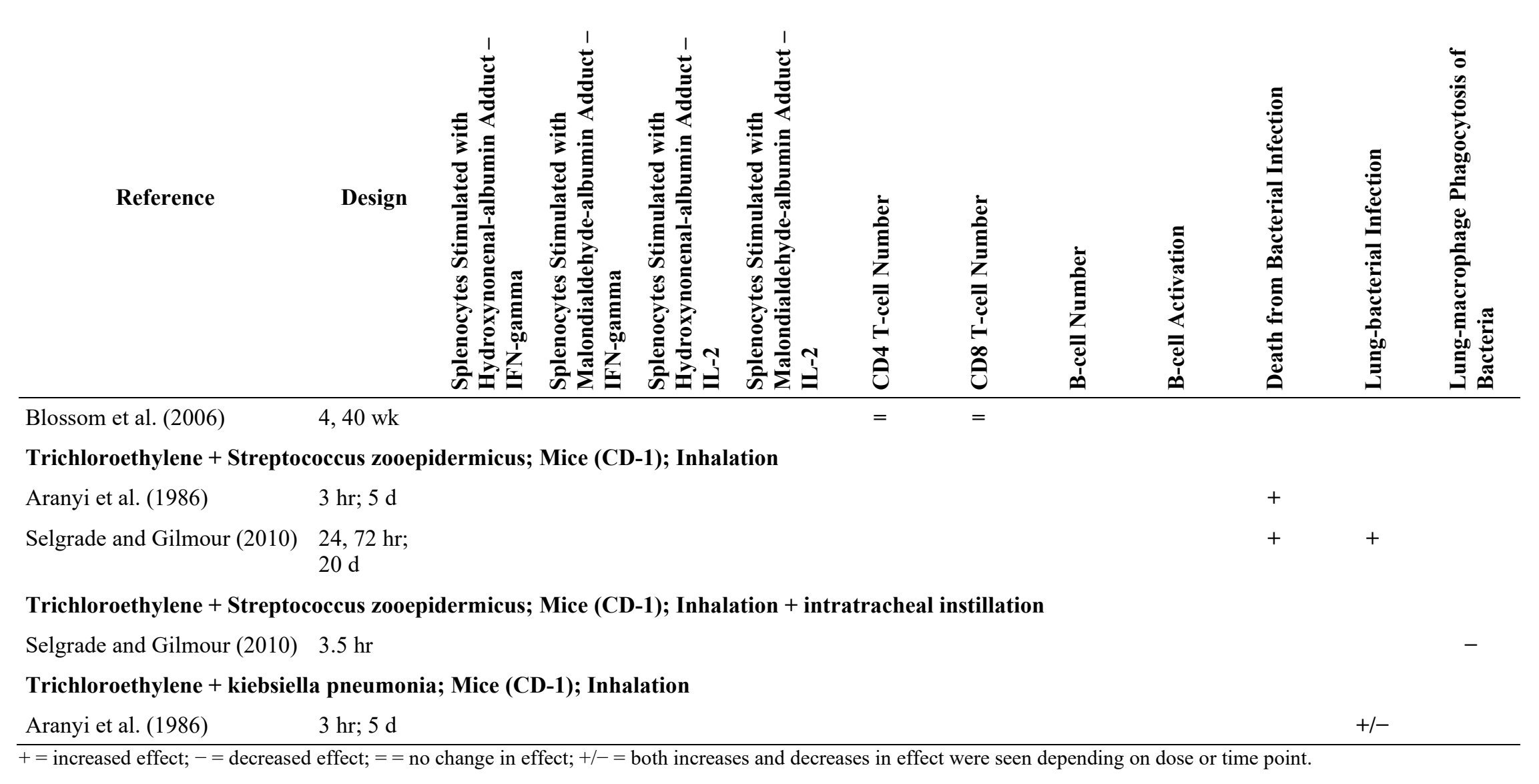




\section{Appendix F. Mechanisms of Action Tables}

\section{Tables}

Table F-1. Superoxide Anion Production in Male B6C3F 1 Mice Administered Acute, Subacute, and Subchronic Doses of Dichloroacetic Acid or Trichloroacetic Acid.

Table F-2. Lipid Peroxidation and DNA Single-strand Breaks in the Liver of Male

$\mathrm{B}_{6} \mathrm{C}_{3} \mathrm{~F}_{1}$ Mice Administered Dichloroacetic Acid or Trichloroacetic Acid Acutely, Subacutely, and Subchronically

Table F-3. Phagocytic Activation and Antioxidant Enzyme Activity in Peritoneal Lavage Cells from Male B6C3F 1 Mice Administered Subacute and Subchronic Doses of Dichloroacetic Acid or Trichloroacetic Acid.

Table F-4. Antioxidant Enzyme Activity in Liver from Male B6C3F 1 Mice Administered Dichloroacetic Acid or Trichloroacetic Acid Subacutely or Subchronically..... 
Table F-1. Superoxide Anion Production in Male B6C3F 1 Mice Administered Acute, Subacute, and Subchronic Doses of Dichloroacetic Acid or Trichloroacetic Acid

\begin{tabular}{|c|c|c|c|c|c|}
\hline Compound & $\begin{array}{c}\text { Dose } \\
(\mathrm{mg} / \mathrm{kg} / \mathrm{day})\end{array}$ & Time & $\mathbf{P L C s}^{\mathrm{a}}$ & Liver $^{\mathrm{a}}$ & Reference \\
\hline Dichloroacetic acid & 300 (single dose) & $\begin{array}{l}6 \mathrm{hr} \\
12 \mathrm{hr}\end{array}$ & $\begin{array}{l}1.5^{*} \\
1.4^{*}\end{array}$ & $\begin{array}{l}1.4^{*} \\
\text { INS }\end{array}$ & $\begin{array}{l}\text { Hassoun and Dey } \\
(2008)\end{array}$ \\
\hline Dichloroacetic acid & $\begin{array}{c}7.7 \\
77 \\
154 \\
410\end{array}$ & $4 \mathrm{wk}$ & $\begin{array}{l}\text { INS } \\
1.8^{*} \\
2.5^{*} \\
3.7^{*}\end{array}$ & $\begin{array}{l}1.2^{*} \\
2.5^{*} \\
4.0^{*} \\
4.3^{*}\end{array}$ & $\begin{array}{l}\text { Hassoun et al. (2010a); } \\
\text { Hassoun et al. } \\
\text { (2010b); Hassoun and } \\
\text { Cearfoss (2011) }\end{array}$ \\
\hline Dichloroacetic acid & $\begin{array}{c}7.7 \\
77 \\
154 \\
410\end{array}$ & $13 \mathrm{wk}$ & $\begin{array}{l}1.8^{*} \\
2.4^{*} \\
2.1^{*} \\
\text { INS }\end{array}$ & $\begin{array}{l}1.4^{*} \\
3.2^{*} \\
4.3^{*} \\
2.2^{*}\end{array}$ & $\begin{array}{l}\text { Hassoun et al. (2010a); } \\
\text { Hassoun et al. } \\
\text { (2010b); Hassoun and } \\
\text { Cearfoss (2011) }\end{array}$ \\
\hline Dichloroacetic acid & $\begin{array}{l}7.5 \\
15 \\
30\end{array}$ & 13 wk & $\begin{array}{l}1.8^{*} \\
2.0^{*} \\
2.2^{*}\end{array}$ & $\begin{array}{l}1.4^{*} \\
1.9^{*} \\
2.3^{*}\end{array}$ & $\begin{array}{l}\text { Hassoun et al. (2013) } \\
\text { Hassoun et al. (2014) }\end{array}$ \\
\hline Trichloroacetic acid & 300 (single dose) & $\begin{array}{l}6 \mathrm{hr} \\
12 \mathrm{hr}\end{array}$ & $\begin{array}{l}\text { INS } \\
1.5^{*}\end{array}$ & $\begin{array}{l}\text { INS } \\
1.2^{*}\end{array}$ & $\begin{array}{l}\text { Hassoun and Dey } \\
\text { (2008) }\end{array}$ \\
\hline Trichloroacetic acid & $\begin{array}{c}7.7 \\
77 \\
154 \\
410\end{array}$ & $4 \mathrm{wk}$ & $\begin{array}{l}\text { INS } \\
1.4^{*} \\
1.9^{*} \\
2.5^{*}\end{array}$ & $\begin{array}{l}\text { INS } \\
\text { INS } \\
1.3^{*} \\
2.8^{*}\end{array}$ & $\begin{array}{l}\text { Hassoun et al. (2010a); } \\
\text { Hassoun et al. } \\
\text { (2010b); Hassoun and } \\
\text { Cearfoss (2011) }\end{array}$ \\
\hline Trichloroacetic acid & $\begin{array}{c}7.7 \\
77 \\
154 \\
410\end{array}$ & 13 wk & $\begin{array}{l}\text { INS } \\
2.0^{*} \\
\text { INS } \\
\text { INS }\end{array}$ & $\begin{array}{l}1.2^{*} \\
1.8^{*} \\
2.5^{*} \\
2.8^{*}\end{array}$ & $\begin{array}{l}\text { Hassoun et al. (2010a); } \\
\text { Hassoun et al. } \\
\text { (2010b); Hassoun and } \\
\text { Cearfoss (2011) }\end{array}$ \\
\hline Trichloroacetic acid & $\begin{array}{c}12.5 \\
25 \\
50\end{array}$ & 13 wk & $\begin{array}{l}1.5^{*} \\
1.6^{*} \\
1.8^{*}\end{array}$ & $\begin{array}{l}1.3^{*} \\
1.5^{*} \\
1.7^{*}\end{array}$ & $\begin{array}{l}\text { Hassoun et al. (2013) } \\
\text { Hassoun et al. (2014) }\end{array}$ \\
\hline Mixtures & $\begin{array}{c}7.5 / 12.5^{b} \\
15 / 25 \\
30 / 50\end{array}$ & $13 \mathrm{wk}$ & $\begin{array}{l}2.1^{*} \\
2.7^{*} \\
2.6^{*}\end{array}$ & $\begin{array}{l}1.7^{*} \\
2.6^{*} \\
3.2^{*}\end{array}$ & $\begin{array}{l}\text { Hassoun et al. (2013) } \\
\text { Hassoun et al. (2014) }\end{array}$ \\
\hline
\end{tabular}

*p $<0.05$.

$-=$ Not measured; INS $=$ insignificant change compared to controls; PLCs = peritoneal lavage cells.

${ }^{\text {aS }}$ Superoxide anion production measured as cytochrome $\mathrm{c}$ reduced $/ \mathrm{min} / \mathrm{mg}$ protein and expressed as the approximate fold increase over control values (some values estimated from figures).

${ }^{\mathrm{b}}$ Concentration of dichloroacetic acid/trichloroacetic acid in the mixture.

Table F-2. Lipid Peroxidation and DNA Single-strand Breaks in the Liver of Male B6C3F 1 Mice Administered Dichloroacetic Acid or Trichloroacetic Acid Acutely, Subacutely, and Subchronically

\begin{tabular}{cccccc}
\hline Compound & $\begin{array}{c}\text { Dose } \\
(\mathbf{m g} / \mathbf{k g} / \mathbf{d a y})\end{array}$ & Time & $\mathbf{L P}^{\mathbf{a}}$ & $\mathbf{S S B s}^{\mathbf{a}}$ & Reference \\
\hline Dichloroacetic acid & 300 (single dose) & $6 \mathrm{hr}$ & $1.3^{*}$ & $2.6^{*}$ & Hassoun and Dey (2008) \\
& $12 \mathrm{hr}$ & $1.4^{*}$ & $3.9^{*}$ & \\
\hline
\end{tabular}




\begin{tabular}{|c|c|c|c|c|c|}
\hline Compound & $\begin{array}{c}\text { Dose } \\
\text { (mg/kg/day) }\end{array}$ & Time & $\mathbf{L P}^{\mathbf{a}}$ & $\mathbf{S S B s}^{\mathrm{a}}$ & Reference \\
\hline Dichloroacetic acid & $\begin{array}{c}7.7 \\
77 \\
154 \\
410\end{array}$ & $4 \mathrm{wk}$ & $\begin{array}{c}2.5^{*} \\
5.0^{*} \\
7.5^{*} \\
14.0^{*}\end{array}$ & $\begin{array}{l}\text { INS } \\
3.5^{*} \\
7.2^{*} \\
7.2^{*}\end{array}$ & $\begin{array}{l}\text { Hassoun et al. (2010a); } \\
\text { Hassoun and Cearfoss (2011) }\end{array}$ \\
\hline Dichloroacetic acid & $\begin{array}{c}7.7 \\
77 \\
154 \\
410\end{array}$ & $13 \mathrm{wk}$ & $\begin{array}{c}3.5^{*} \\
12.5^{*} \\
15.0^{*} \\
4.0^{*}\end{array}$ & $\begin{array}{l}1.6^{*} \\
5.6^{*} \\
5.6^{*} \\
4.0^{*}\end{array}$ & $\begin{array}{l}\text { Hassoun et al. (2010a); } \\
\text { Hassoun and Cearfoss (2011) }\end{array}$ \\
\hline Dichloroacetic acid & $\begin{array}{l}7.5 \\
15 \\
30\end{array}$ & $13 \mathrm{wk}$ & $\begin{array}{l}2.8^{*} \\
4.0^{*} \\
7.2^{*}\end{array}$ & $\begin{array}{l}1.6^{*} \\
2.8^{*} \\
4.0^{*}\end{array}$ & Hassoun et al. (2014) \\
\hline $\begin{array}{l}\text { Trichloroacetic } \\
\text { acid }\end{array}$ & 300 (single dose) & $\begin{array}{c}6 \mathrm{hr} \\
12 \mathrm{hr}\end{array}$ & $\begin{array}{l}\text { INS } \\
1.3^{*}\end{array}$ & $\begin{array}{l}\text { INS } \\
2.8^{*}\end{array}$ & Hassoun and Dey (2008) \\
\hline $\begin{array}{l}\text { Trichloroacetic } \\
\text { acid }\end{array}$ & $\begin{array}{c}7.7 \\
77 \\
154 \\
410\end{array}$ & $4 \mathrm{wk}$ & $\begin{array}{c}\text { INS } \\
2.0^{*} \\
2.5^{*} \\
11.0^{*}\end{array}$ & $\begin{array}{l}\text { INS } \\
1.8^{*} \\
2.3^{*} \\
4.3^{*}\end{array}$ & $\begin{array}{l}\text { Hassoun et al. (2010a); } \\
\text { Hassoun and Cearfoss (2011) }\end{array}$ \\
\hline $\begin{array}{l}\text { Trichloroacetic } \\
\text { acid }\end{array}$ & $\begin{array}{c}7.7 \\
77 \\
154 \\
410\end{array}$ & 13 wk & $\begin{array}{c}1.5^{*} \\
7.0^{*} \\
8.5^{*} \\
13.5^{*}\end{array}$ & $\begin{array}{l}\text { INS } \\
2.3^{*} \\
3.3^{*} \\
4.3^{*}\end{array}$ & $\begin{array}{l}\text { Hassoun et al. (2010a); } \\
\text { Hassoun and Cearfoss (2011) }\end{array}$ \\
\hline $\begin{array}{l}\text { Trichloroacetic } \\
\text { acid }\end{array}$ & $\begin{array}{l}12.5 \\
25 \\
50\end{array}$ & $13 \mathrm{wk}$ & $\begin{array}{l}1.6^{*} \\
2.6^{*} \\
4.0^{*}\end{array}$ & $\begin{array}{l}\text { INS } \\
1.6^{*} \\
2.0^{*}\end{array}$ & Hassoun et al. (2014) \\
\hline Mixture & $\begin{array}{l}7.5 / 12.5^{b} \\
15 / 25 \\
30 / 50\end{array}$ & 13 wk & $\begin{array}{l}3.2^{*} \\
6.2^{*} \\
13^{*}\end{array}$ & $\begin{array}{l}1.7^{*} \\
3.6^{*} \\
6.2^{*}\end{array}$ & Hassoun et al. (2014) \\
\hline
\end{tabular}

*p $<0.05$.

INS = insignificant change compared to controls; LP = lipid peroxidation (measured a nmole TBARS/mg protein and expressed as the approximate fold increase over control values); $\mathrm{SSBs}=$ single-strand breaks (alkaline elution technique, data reported as fold increase over control values).

${ }^{\text {a }}$ Data are the ratio of treated/controls (some values estimated from figures).

${ }^{b}$ Concentration of dichloroacetic acid/trichloroacetic acid in the mixture.

Table F-3. Phagocytic Activation and Antioxidant Enzyme Activity in Peritoneal Lavage Cells from Male B6C3F 1 Mice Administered Subacute and Subchronic Doses of Dichloroacetic Acid or Trichloroacetic Acid

\begin{tabular}{lcccccc}
\hline \multicolumn{1}{c}{ Compound } & $\begin{array}{c}\text { Dose } \\
\text { (mg/kg/day) }\end{array}$ & Time & SOD $^{\mathbf{a}}$ & MPO $^{\mathbf{a}}$ & TNF- $^{\mathbf{a}}$ & Reference \\
\hline Dichloroacetic & 7.7 & $4 \mathrm{wk}$ & INS & $5.0^{*}$ & INS & Hassoun et al. (2010b) \\
acid & 77 & & $2.5^{*}$ & $4.3^{*}$ & $2.0^{*}$ & \\
& 154 & & $4.1^{*}$ & $12.3^{*}$ & $3.0^{*}$ & \\
& 410 & & $6.5^{*}$ & $12.3^{*}$ & $6.8^{*}$ & \\
Dichloroacetic & 7.7 & \multirow{2}{*}{$\mathrm{wk}$} & $1.7^{*}$ & $6.3^{*}$ & $2.4^{*}$ & Hassoun et al. (2010b) \\
acid & 77 & & $3.7^{*}$ & $10.8^{*}$ & $6.2^{*}$ & \\
& 154 & & $4.5^{*}$ & $9.0^{*}$ & $5.2^{*}$ & \\
& 410 & & $5.2^{*}$ & INS & INS & \\
\hline
\end{tabular}


RoC Monograph on Trichloroethylene

\begin{tabular}{lcccccc}
\hline \multicolumn{1}{c}{ Compound } & $\begin{array}{c}\text { Dose } \\
\text { (mg/kg/day) }\end{array}$ & Time & SOD $^{\mathbf{a}}$ & MPO $^{\mathbf{a}}$ & TNF- $\mathbf{a}^{\mathbf{a}}$ & Reference \\
\hline Dichloroacetic & 7.5 & $13 \mathrm{wk}$ & - & $5.3^{*}$ & $2.1^{*}$ & Hassoun et al. (2013) \\
acid & 15 & & - & $7.8^{*}$ & $2.7^{*}$ & \\
& 30 & & - & $9.3^{*}$ & $3.3^{*}$ & \\
Trichloroacetic & 7.7 & $4 \mathrm{wk}$ & $\mathrm{INS}$ & $36^{* \mathrm{~b}}$ & $\mathrm{INS}$ & Hassoun et al. (2010b) \\
acid & 77 & & $2.1^{*}$ & $52^{* \mathrm{~b}}$ & $2.6^{*}$ & \\
& 154 & & $4.0^{*}$ & $66^{* \mathrm{~b}}$ & $4.3^{*}$ & \\
& 410 & & $5.0^{*}$ & $18^{* \mathrm{~b}}$ & $11.8^{*}$ & \\
Trichloroacetic & 7.7 & $13 \mathrm{wk}$ & $1.7^{*}$ & $6^{* \mathrm{~b}}$ & $\mathrm{INS}$ & Hassoun et al. (2010b) \\
acid & 77 & & $2.6^{*}$ & $16^{* \mathrm{~b}}$ & $3.0^{*}$ & \\
& 154 & & $4.2^{*}$ & $4^{* \mathrm{~b}}$ & $\mathrm{INS}$ & \\
Trichloroacetic & 410 & & $5.2^{*}$ & $\mathrm{INS}$ & $\mathrm{INS}$ & \\
acid & 12.5 & $13 \mathrm{wk}$ & - & $5.7^{*}$ & $1.9^{*}$ & Hassoun et al. (2013) \\
& 25 & & - & $7.0^{*}$ & $2.2^{*}$ & \\
Mixtures & 50 & & - & $9.5^{*}$ & $2.6^{*}$ & \\
& $7.5 / 12.5^{\mathrm{b}}$ & \multirow{2}{*}{$13 \mathrm{wk}$} & - & $9.5^{*}$ & $3.1^{*}$ & Hassoun et al. (2013) \\
& $15 / 25$ & & - & $13.2^{*}$ & $4.1^{*}$ & \\
\hline
\end{tabular}

*p $<0.05$.

$-=$ Not measured; INS = insignificant change compared to controls; $\mathrm{MPO}=$ myeloperoxidase (units/mg); $\mathrm{SOD}=$ superoxide dismutase (units/mg); TNF- $\alpha=$ tumor necrosis factor-alpha ( $\mathrm{pg} / \mathrm{mg}$ ).

${ }^{a}$ Data are the ratio of treated/controls (all values estimated from figures).

${ }^{b}$ Ratios are highly uncertain because the control levels were very small.

Table F-4. Antioxidant Enzyme Activity in Liver from Male B6C3F 1 Mice Administered Dichloroacetic Acid or Trichloroacetic Acid Subacutely or Subchronically

\begin{tabular}{ccccccc}
\hline Compound & $\begin{array}{c}\text { Dose } \\
(\mathbf{m g} / \mathbf{k g} / \mathbf{d a y})\end{array}$ & Time & SOD $^{\mathbf{a}}$ & CAT $^{\mathbf{a}}$ & GPO $^{\mathbf{a}}$ & GSH $^{\mathbf{a}}$ \\
\hline Dichloroacetic acid & 7.7 & $4 \mathrm{wk}$ & $0.05^{*}$ & INS & INS & INS \\
& 77 & & $0.05^{*}$ & INS & INS & INS \\
& 154 & & $0.1^{*}$ & INS & INS & INS \\
Dichloroacetic acid & 410 & & $0.5^{*}$ & INS & INS & INS \\
& 7.7 & \multirow{2}{*}{$13 \mathrm{wk}$} & $0.4^{*}$ & INS & $0.29^{*}$ & INS \\
& 77 & & $0.4^{*}$ & INS & $0.29^{*}$ & $0.73^{*}$ \\
Trichloroacetic acid & 154 & & $2.1^{*}$ & $1.9^{*}$ & $1.8^{*}$ & $0.66^{*}$ \\
& 410 & & $3.6^{*}$ & $2.2^{*}$ & $2.5^{*}$ & INS \\
& 7.7 & $4 \mathrm{wk}$ & $1.3^{*}$ & INS & $0.34^{*}$ & INS \\
Trichloroacetic acid & 77 & & $1.8^{*}$ & $1.5^{*}$ & $0.39^{*}$ & INS \\
& 154 & & $3.0^{*}$ & $1.7^{*}$ & $0.37^{*}$ & INS \\
& 410 & & $4.9^{*}$ & $1.9^{*}$ & $0.42^{*}$ & INS \\
& 7.7 & \multirow{2}{*}{$13 \mathrm{wk}$} & $2.4^{*}$ & $1.7^{*}$ & $0.62^{*}$ & INS \\
& 154 & & $3.6^{*}$ & $1.9^{*}$ & $0.30^{*}$ & INS \\
& 410 & & $6.4^{*}$ & $2.3^{*}$ & $0.20^{*}$ & INS \\
\hline
\end{tabular}

$*_{\mathrm{p}}<0.05$.

Source: Hassoun and Cearfoss (2011).

$\mathrm{CAT}=$ catalase (units $\times 10 / \mathrm{mg}$ protein); GPO $=$ glutathione peroxidase (nmoles NADPH oxidized $/ \mathrm{min} / \mathrm{mg}$ protein); GSH = total glutathione (nmoles/g tissue); INS = insignificant change compared to controls; SOD = superoxide dismutase (units $/ \mathrm{mg}$ ).

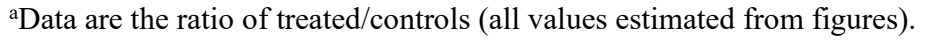




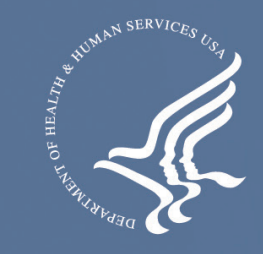

\section{National Toxicology Program}

National Institute of Environmental Health Sciences

National Institutes of Health

P.O. Box 12233, MD K2-05

Durham, NC 27709

Tel: 984-287-3211

ntpwebrequest@niehs.nih.gov

https://ntp.niehs.nih.gov 